\title{
Synthesis, Structure and Applications of Cationic Phosphonites
}

\author{
Dissertation \\ zur Erlangung des mathematisch-naturwissenschaftlichen Doktorgrades \\ "Doctor rerum naturalium" \\ der Georg-August-Universität Göttingen \\ im Promotionsprogramm: Chemie \\ der Georg-August-University School of Science (GAUSS)
}

vorgelegt von

Leo David Mwenya Nicholls

aus Leeds, vereinigtes Königreich

Göttingen, 2018 


\section{Betreuungsausschuss}

Prof. Dr. Manuel Alcarazo (Institut für Organische und Biomolekulare Chemie, Tammannstr. 2, 37077 Göttingen)

Prof. Dr. Lutz Ackermann (Institut für Organische und Biomolekulare Chemie, Tammannstr. 2, 37077 Göttingen)

\section{Mitglieder der Prüfungskommission}

Referent: Prof. Dr. Manuel Alcarazo (Institut für Organische und Biomolekulare Chemie, Tammannstr. 2, 37077 Göttingen)

Korreferent: Prof. Dr. Lutz Ackermann (Institut für Organische und Biomolekulare Chemie, Tammannstr. 2, 37077 Göttingen)

Weitere Mitglieder der Prüfungskommission:

Prof. Dr. Franc Meyer (Institut für Anorganische Chemie, Tammannstr. 4, 37077 Göttingen)

Prof. Dr. Dietmar Stalke (Institut für Anorganische Chemie, Tammannstr. 4, 37077 Göttingen)

Dr. Franziska Thomas (Institut für Organische und Biomolekulare Chemie, Tammannstr. 2, 37077 Göttingen)

Dr. Max. M. Hansmann (Institut für Organische und Biomolekulare Chemie, Tammannstr. 2, 37077 Göttingen)

Tag der mündlichen Prüfung: 22. Oktober 2018 
I hereby declare that this dissertation has been written independently and with no sources or aids other than those quoted. The parts performed by project collaborators have been clearly indicated.

Leo David Mwenya Nicholls 


\section{Abbreviations}

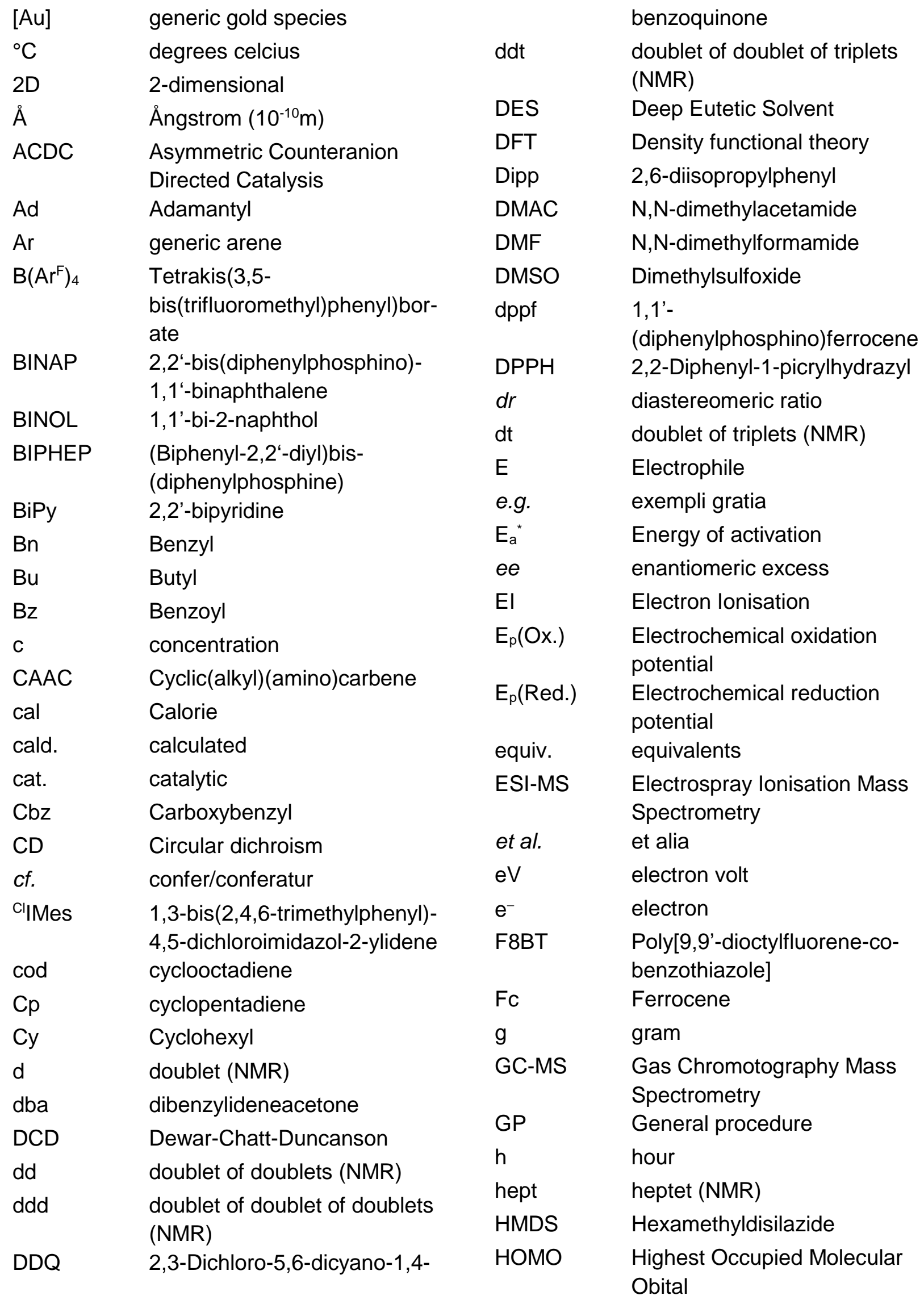




\begin{tabular}{|c|c|c|c|}
\hline & & MS & Molecular sieves \\
\hline HPLC & $\begin{array}{l}\text { High Performance Liquid } \\
\text { Chromotography }\end{array}$ & MTBE & Methyl-tert-butyl ether \\
\hline HRMS & High Resolution Mass & $n$ & generic number \\
\hline 年1 & Spectrometry & n.d. & not determined \\
\hline HSQC & Heteronuclear Single & NBS & N-bromosuccinamide \\
\hline & Quantum Coherence & $\mathrm{NHC}$ & N-heterocyclic carbene \\
\hline ho & Light irradiation & NMR & Nuclear Magnetic Resonance \\
\hline $\mathrm{Hz}$ & Hertz & $\mathrm{Nu}$ & Nucleophile \\
\hline i.e. & id est & 0 & ortho \\
\hline Ipc & Isopinocampheyl & oct & octet (NMR) \\
\hline IPr & $1,3-\operatorname{Bis}(2,6-$ & OLED & Organic Light Emitting Diode \\
\hline & diisopropylphenyl)imidazol-2- & $p$ & para \\
\hline & ylidene & $p$ & pentet (NMR) \\
\hline $\operatorname{Pr}$ & iso-propyl & $\mathrm{Pa}$ & Pascals \\
\hline $\mathrm{IR}$ & Infrared spectroscopy & PCC & Pyridinium chlorochromate \\
\hline Irel & Relative intensity & PDA & Photodiode Array \\
\hline IUPAC & $\begin{array}{l}\text { International Union of Pure } \\
\text { and Applied Chemistry }\end{array}$ & $\mathrm{Ph}$ & Phenyl \\
\hline J & Joule & Piv & pivaloyl \\
\hline$J$ & Coupling constant & ppm & parts per million \\
\hline K & Kelvin & $\operatorname{Pr}$ & Propyl \\
\hline $\mathrm{L}$ & Ligand & $p$-TSA & para-Toluenesulfonic acid \\
\hline LEP & Light Emitting Polymer & q & quartet (NMR) \\
\hline LUMO & $\begin{array}{l}\text { Lowest Unoccupied Molecular } \\
\text { Orbital }\end{array}$ & $\begin{array}{l}\text { quant. } \\
\mathrm{R}\end{array}$ & $\begin{array}{l}\text { quantitative } \\
\text { generic substituent }\end{array}$ \\
\hline$m$ & meta & rac & racemic \\
\hline M & Metal & $\mathrm{RCM}$ & Ring-Closing Alkene \\
\hline M & Molar $\left(\mathrm{mol} \mathrm{dm}^{-3}\right)$ & & Metathesis \\
\hline $\mathrm{m}$ & multiplet (NMR) & $\mathrm{rt}$ & room temperature \\
\hline $\mathrm{m}$ & meter & s & singlet (NMR) \\
\hline $\mathrm{m} / \mathrm{z}$ & mass to charge ratio & SEGPHOS & 5,5'-Bis(diphenylphosphino)- \\
\hline $\mathrm{Me}$ & Methyl & & $\begin{array}{l}\text { 4,4'-bi-1,3-benzodioxole } \\
\text { sextet (NMB) }\end{array}$ \\
\hline MeliPr & $\begin{array}{l}\text { 1,3-bis(isopropyl)-4,5- } \\
\text { dimethylimidazol-2-ylidene }\end{array}$ & $\begin{array}{l}\text { sext } \\
\text { Sphos }\end{array}$ & $\begin{array}{l}\text { sextet (IVIVIR) } \\
\text { 2-Dicyclohexylphosphino- }\end{array}$ \\
\hline MeIMes & $\begin{array}{l}\text { 1,3-bis(2,4,6-trimethylphenyl)- } \\
\text { 4,5-dimethylimidazol-2- } \\
\text { ylidene }\end{array}$ & STM & $\begin{array}{l}2^{\prime}, 6^{\prime} \text {-dimethoxybiphenyl } \\
\text { Scanning tunneling } \\
\text { microscope }\end{array}$ \\
\hline menth & menthyl & $\mathrm{T}$ & Temperature \\
\hline Mes & 2,4,6-Trimethylphenyl & $\mathrm{t}$ & time \\
\hline $\min$ & minutes & $t_{1 / 2}$ & half life \\
\hline $\mathrm{ml}$ & millitre & TADDOL & $\alpha, \alpha, \alpha^{\prime}, \alpha^{\prime}$-tetraaryl-2,2- \\
\hline MOM & Methoxymethyl & & 4,5-dimethanol \\
\hline
\end{tabular}




\begin{tabular}{|c|c|c|c|}
\hline TAPA & 2-(2,4,5,7-Tetranitro-9- & wt-\% & Weight percent \\
\hline & fluorenylideneaminooxy)propi- & X & Generic heteroatom \\
\hline & onic acid & $\mathrm{Y}$ & Generic substituent \\
\hline TBDMS & tert-butyldimethylsilyl & Z & atomic number \\
\hline tBu & tert-butyl & Z & Generic heteroatom \\
\hline TEP & Tolman Electronic Parameter & $\delta$ & Chemical shift \\
\hline $\begin{array}{l}\text { Tf } \\
\text { THF }\end{array}$ & $\begin{array}{l}\text { Trifluoromethanesulfonyl } \\
\text { Tetrahydrofuran }\end{array}$ & $\Delta \mathrm{G}^{\neq}$ & $\begin{array}{l}\text { Gibbs' free energy of } \\
\text { activation }\end{array}$ \\
\hline TIPS & Triisopropylsilyl & $\Delta \mathrm{H}^{\ddagger}$ & Enthalpy of activation \\
\hline TLC & Thin layer chromotography & $\Delta \mathrm{S}^{\ddagger}$ & Entropy of activation \\
\hline TMS & Trimethylsilyl & $\Delta \varepsilon$ & Molar circular dichroism \\
\hline$t_{\mathrm{R}}$ & retention time & $\varepsilon$ & extinction coefficient \\
\hline Tripp & 2,4,6-triisopropylphenyl & $\eta$ & Hapticity \\
\hline Ts & 4-Methylbenzenesulfonyl & $\lambda$ & wavelength \\
\hline $\begin{array}{l}\text { TS } \\
\text { tt }\end{array}$ & $\begin{array}{l}\text { Transition state } \\
\text { triplet of triplets (NMR) }\end{array}$ & $\lambda_{\operatorname{Max}}$ & $\begin{array}{l}\text { Wavelength at maxima } \\
\text { (UV/Vis) }\end{array}$ \\
\hline UV/Vis & Ultra violet/ visible & $\lambda_{\operatorname{Max}}$ & Excitation wavelength \\
\hline V & Volts & $\mu$-wave & Microwave \\
\hline$\tilde{v}$ & wavenumbers & $\Phi_{\mathrm{F}}$ & Fluorescence quantum yield \\
\hline $\mathrm{v} / \mathrm{v}$ & volume to volume & & \\
\hline vS. & versus & & \\
\hline
\end{tabular}




\section{Acknowledgments}

I would like to sincerely thank Prof. Dr. Manuel Alcarazo for the opportunity to conduct research in his group, the interesting research project he gave me and for his support and encouragement throughout my PhD. In addition, I would like to thank Prof. Dr. Alois Fürstner for allowing the utilisation of his group's resources in the initial stage of my PhD at the MaxPlanck-Institut für Kohlenforschung. I also thank Prof. Dr. Ackermann, Prof. Dr. Meyer, Prof. Dr. Stalke, Dr. Thomas and Dr. Hansmann for their participation in my thesis committee.

I would like to thank all technical staff from both the Alcarazo and Fürstner groups at The Max-Planck-Institut für Kohlenforschung, in particular Sigrid Lutz, Gerlinde Mehler and Christian Wille for their patient help and for teaching me new practical techniques in the laboratory. In addition, I would also like to thank Monica Lickfield for always quickly solving any administrative problems and for helping me on my arrival in Mülheim. I am grateful to all members of the Fürstner group, for the interesting discussions and motivating working atmosphere. I would also like to thank all personnel in the technical and service departments of The Max-Planck-Institut für Kohlenforschung for the measurement of samples and for assistance in solving various chemistry-related problems. In particular, Herr Dr. Richard Goddard, Herr Rust, Frau Schucht, Frau Dreher and Dr. Hendrik Tinnermann for solving XRay crystal structures, Frau Blumenthal, Herr Klein and Frau Margold for mass spectrometry measurements and Herr Farés, Herr Kochius, Frau Wirtz and Frau Philipps for the measurement and assignment of NMR samples. Furthermore, I would like to thank Alfred Deege, Heike Hinrichs, Sandra Kestermann, Marie Sophie Sterling and Sarah Henze for the measurement of HPLC samples, and particularly Alfred Deege and Heike Hinrichs for patiently helping me with HPLC and for continuing to accept samples for measurement even after the group had relocated to Göttingen. In addition, I would like to thank Dr. Elisa González Fernández for initially teaching me how to use HPLC.

I would like to thank all technical and administrative members of staff, as well as senior researchers of the Alcarazo group in Göttingen, including Martina Pretor, Martin Simon, Katja Grube, Sabine Schacht, Dr. Christopher Golz and Dr. Sergei I. Kozhushkov for their hard work in maintaining the every day running of the laboratory and the group. To the NMR and mass spectrometry departments in Göttingen, I am appreciative of the quick measurement of analytical samples. I would like to thank Dr. Christopher Golz for always being enthusiastic and optimistic in measuring and solving X-Ray crystallography samples. Prior to Christopher's arrival, I would like to thank Dr. Hendrik Tinnermann and Marvin Böhm for their work in measuring and solving X-Ray crystallography samples. For performing DFT calculations, I would alao like to thank Dr. Christopher Golz. 
I would like to sincerely thank all current and former members of the Alcarazo group for the excellent atmosphere, in and outside the lab, and for all the useful discussions and suggestions, which contributed to the working environment of the group and encouraged me to learn about new areas of chemistry. I am grateful to all those I shared a lab with during my PhD: Dr. Pawel Linowski, Dr. Garazi Talavera, Dr. Maria Del Rocio Lopez Rodriguez, Dr. Isaac Alonso, Dr. Jonathan Dube, Sigrid Lutz, Angus Rocha, Pascal Ortsack, Dr. Yin Zhang, Lukas Schaaf, Marvin Böhm, Tim Johannsen, Thierry Hartung, Maximillian Marx, Anja Brennecke, Lucas Paul and Steve Karreman. I would like to thank collaborators in the group for their work, especially Dr. Elisa González Fernández, who started the project on the enantioselective synthesis of [6]carbohelicenes, and also Alejandro García Barrado who worked on the synthesis of monocyanated helicenes. I am very thankful to the bachelor and master's students I worked with during my PhD for their hard work and enthusiasm: Lukas Schaaf, Maximillian Marx, Thierry Hartung, Tim Johannsen and Anja Brennecke. For the rapid correction of my $\mathrm{PhD}$ thesis and helpful suggestions I would like to thank Dr.Ágnes Kozma and Dr. Sergei I. Kozhushkov.

Finally, I would like to thank the friends I made in Mülheim and Göttingen for all the memories outside the laboratory and helping me to forget about chemistry once in a while through things like 'group trips', playing squash, going out for meals or drinks, bouldering and football. I am extremely grateful to my family, who from the beginning encouraged me to do something I enjoy. My biggest thanks goes to Ágnes, whose constant support throughout the last four years helped me to stay positive. 


\section{Table of Contents}

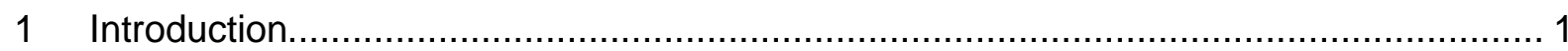

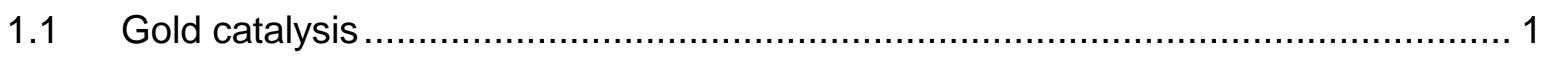

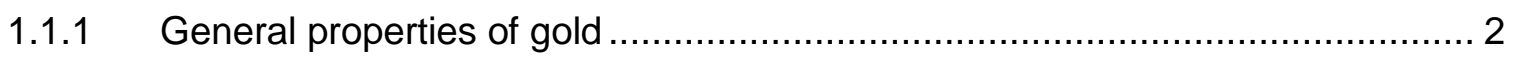

1.1.2 Gold as a carbophilic Lewis acid ........................................................... 4

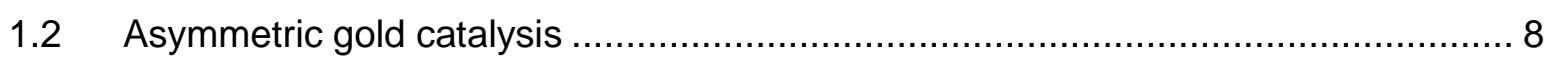

1.2.1 Chiral bis(phosphine) complexes ..................................................... 9

1.2.2 Asymmetric counterion-directed catalysis (ACDC) …............................... 11

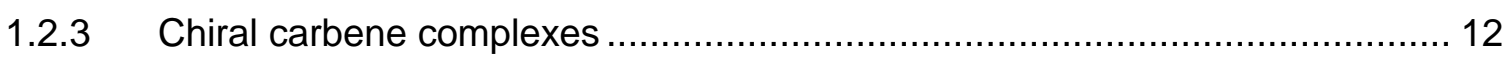

1.2.4 Chiral phosphoramidite complexes ..................................................... 14

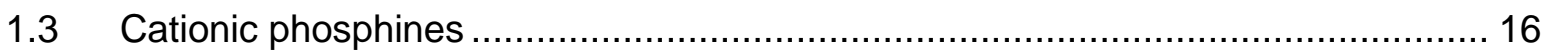

1.3.1 Synthesis of monocationic phosphines and phosphonites .......................... 17

1.3.2 Synthesis of polycationic phosphines and phosphonites ............................. 21

1.3.3 Structure and electronic properties of cationic phosphines and phosphonites 25

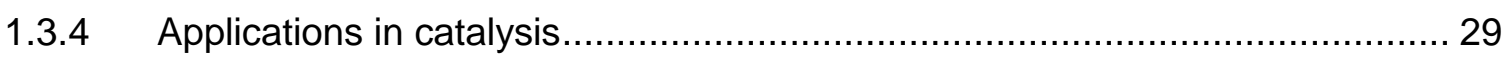

1.4 Synthesis, structure and applications of Helicenes ........................................... 35

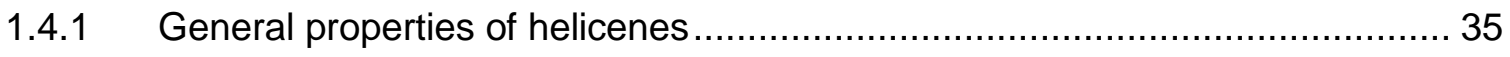

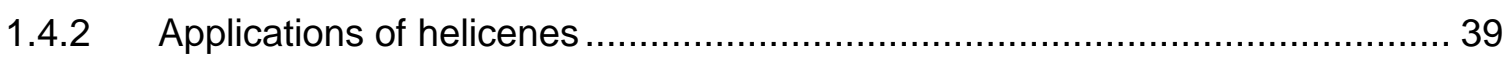

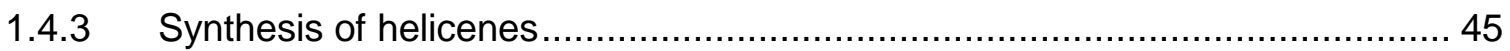

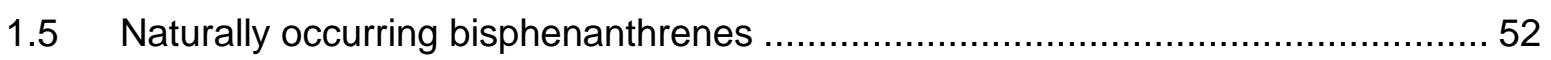

2 Previous research of our group in the synthesis of [6]helicenes............................... 55

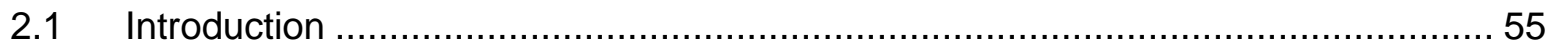

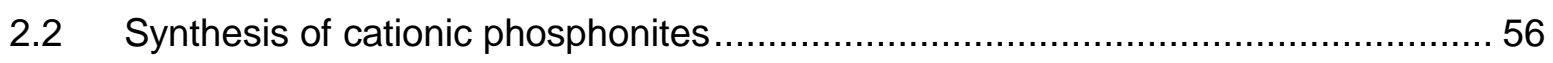

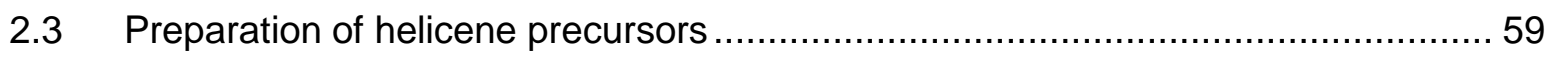

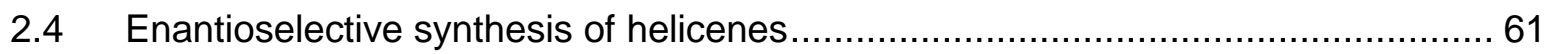

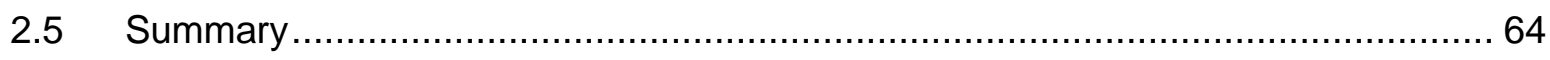

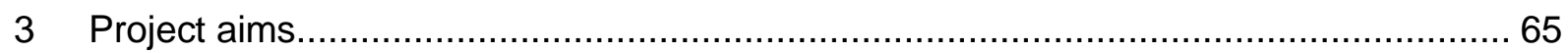

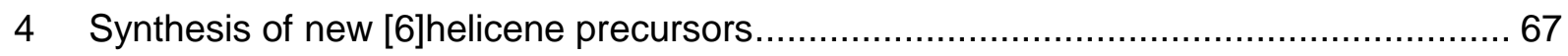


4.1 Synthesis of new [6]helicene precursors using existing methodology

4.2 Development of a new synthesis of [6]helicene precursors ...............................69

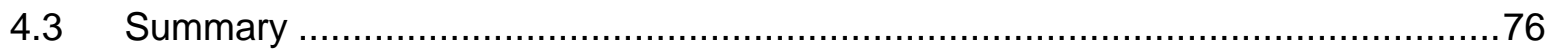

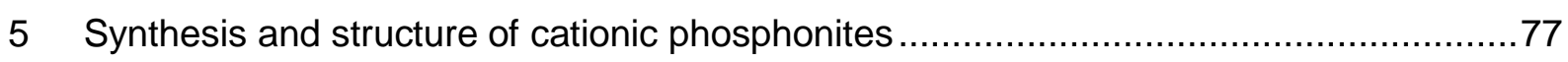

5.1 Synthesis of cationic phosphonites from TADDOL derivatives ............................77

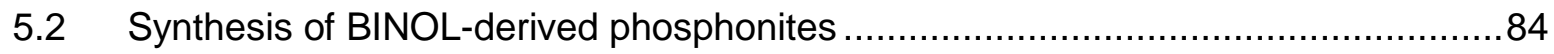

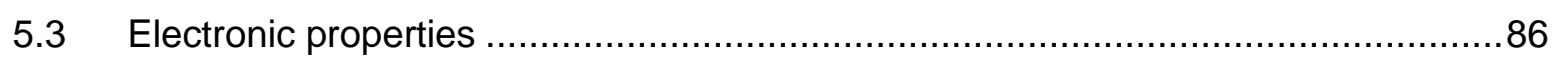

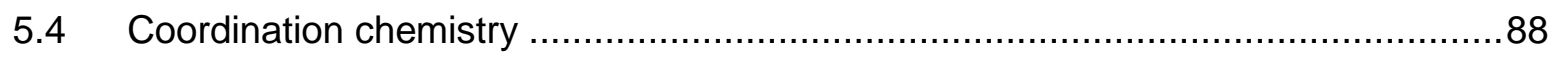

5.5 Synthesis of 1,2,4- and 1,2,3-triazolium-derived phosphonites and their

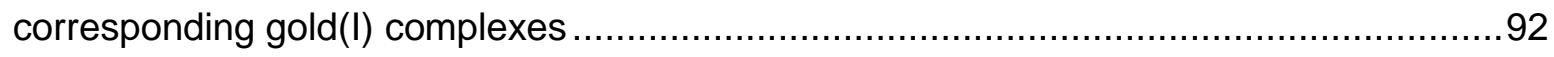

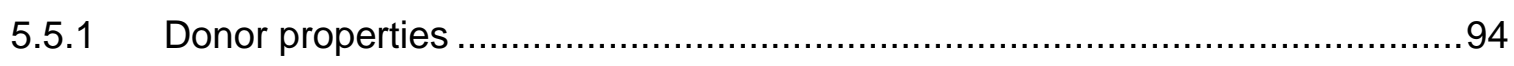

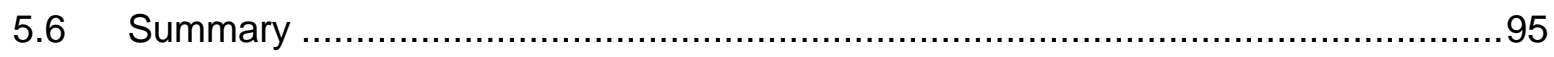

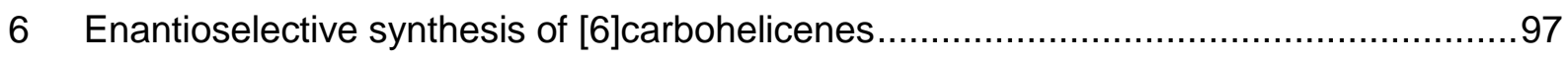

6.1 Enantioselective synthesis of [6]carbohelicenes using precatalyst $172 \mathrm{i} \ldots \ldots \ldots \ldots \ldots . . . . . . .97$

6.2 Model catalytic studies using new cationic phosphonite gold(I) complexes ..........101

6.3 Substrate scope of new precursors and comparison studies using cationic

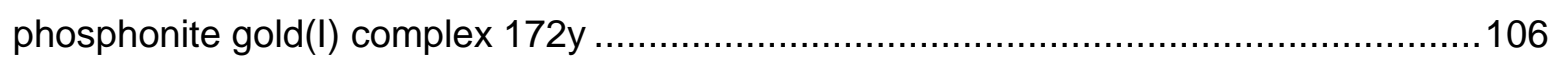

6.4 Enantioselective hydroarylation of cyanated tetrahelicenes..............................110

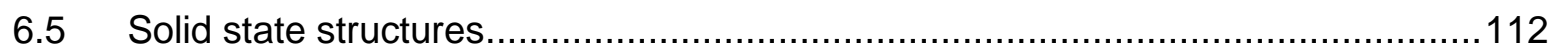

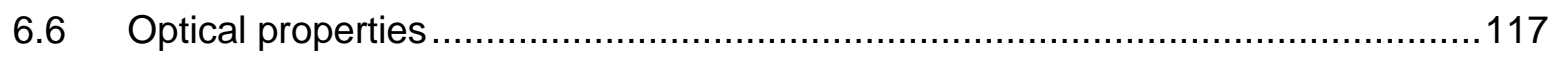

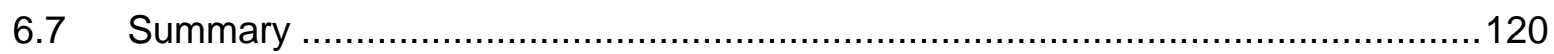

$7 \quad$ Towards the enantioselective total synthesis of Monbarbatain A ..............................121

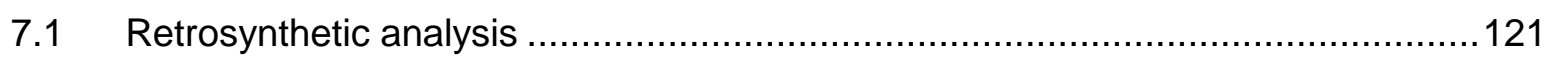

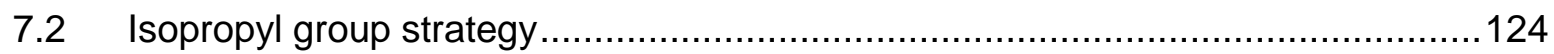

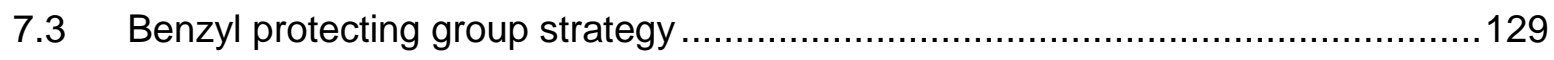

7.4 Synthesis of dimethyl Monbarbatain A ....................................................... 133

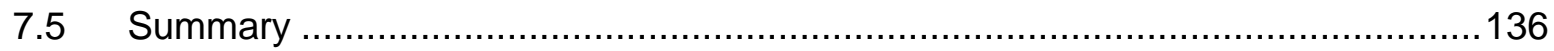

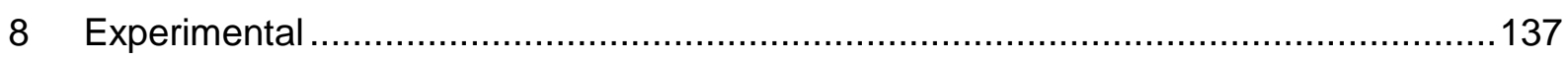

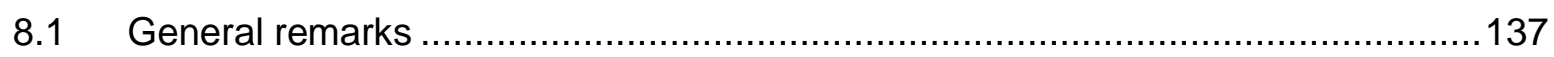

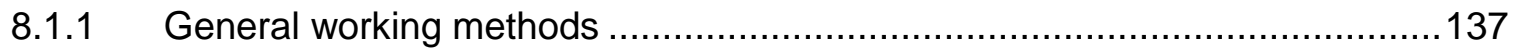




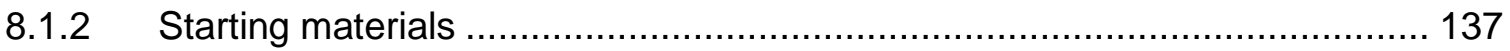

8.1.3 General analytical methods .......................................................... 138

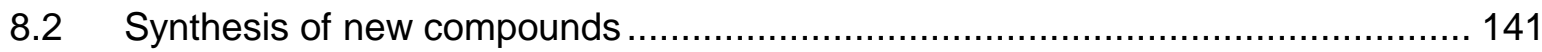

8.2.1 Synthesis of helicene precursors ...................................................... 141

8.2.2 Synthesis of chiral cationic phosphonites ............................................... 161

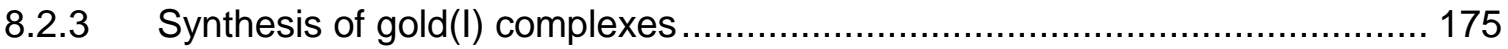

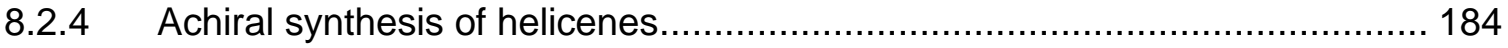

8.2.5 Enantioselective synthesis of helicenes................................................... 192

8.3 Towards the total synthesis of Monbarbatain A ............................................. 209

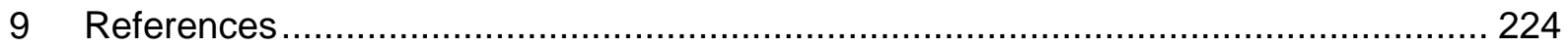




\section{Introduction}

\subsection{Gold catalysis}

For over a century, transition metals have proven to be integral to a variety of widely adopted catalytic reactions, featuring in important industrial processes such as the Fischer-Tropsch conversion of carbon monoxide and hydrogen into liquid hydrocarbons, the Haber-Bosch fixation of nitrogen and the Ziegler-Natta polymerization of alkenes into plastics. ${ }^{[1,2]}$ Towards the latter half of the $20^{\text {th }}$ century, homogenous transition metal-based catalysts ${ }^{[2]}$ were widely adopted in enantioselective catalysis, which based upon broadly applicable chiral scaffolds such as those developed by Noyori, ${ }^{[3]}$ Knowles $^{[4]}$ or Sharpless ${ }^{[5]}$ enabled a number of industrially scalable asymmetric processes with high efficiency, selectivity, atom economy and functional group tolerance.

Catalytic applications of gold, however, developed at a much slower place. Despite exhibiting a rich coordination chemistry, ${ }^{[6]}$ gold was deemed to be largely catalytically inactive by the chemical community. ${ }^{[7]}$ Gold has been, nevertheless, valued for millennia by mankind for its use in coins, jewellery and other items of value. Due to its high ductility and durability against degradation to chemicals or light, it is also a suitable material in areas such as medical implants and electronics. ${ }^{[8]}$ Much of this stems from the noble character of gold, with is defined as a reluctance to chemically bond with many elements, and a high oxidation potential. In fact, gold has the highest oxidation potential and electronegativity of any metal, ${ }^{[8]}$ and its reluctance to cycle between oxidation states and participate in the elementary steps of otherwise standard transition metal catalyzed reactions contributed to the notion of its catalytic inactivity.

This perspective however, gradually started to change, first of all with reports of heterogeneous gold catalysts in the hydrogenation of olefins in $1973^{[9]}$ and a decade later with the oxidation of $\mathrm{CO}^{[10]}$ and the hydrochlorination of alkynes, ${ }^{[11]}$ where gold outperformed other catalysts. Around the same time, the first report of homogenous gold catalysis was described by Ito et al, of the gold catalyzed aldol reaction between aldehydes and isocyanates to form oxazolines. Impressively, this was also the first report of asymmetric gold catalysis. ${ }^{[12]}$

However, the specialty of gold lies in its activity as a carbophilic, soft Lewis acid, and it has been widely applied within the arena of $\pi$-acid catalysis alongside metals such as platinum. ${ }^{[13,14]}$ Reports of gold in this regard began with the seminal work by Fukuda, Utimoto 
and Nozaki, ${ }^{[15]}$ where the $\mathrm{Au}(\mathrm{III})$ catalyzed hydroamination of alkynes was described (Scheme 1).

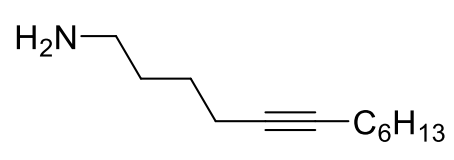

1

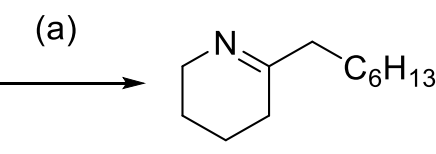

2: quant. yield

Scheme 1. $\mathrm{Au}$ (III)-catalyzed hydroamination of alkyne 1. Reagents and conditions: (a) $\mathrm{AuCl}_{4} \cdot 2 \mathrm{H}_{2} \mathrm{O}(5 \mathrm{~mol} \%)$, $\mathrm{MeCN}, \mathrm{rt}, 12 \mathrm{~h}$.

This was followed by other important studies, such as the gold catalyzed activation of alkynes to nucleophilic addition by water ${ }^{[16]}$ and alcohols, ${ }^{[16,17]}$ and later the activation of alkenes and alkynes ${ }^{[18]}$ and the synthesis of phenols through cycloisomerization reactions. ${ }^{[19]}$ Gold proved highly active and selective in these reactions, where its noble properties, namely a low oxo-philicitiy and redox activity, were highly advantageous. This sparked a huge interest in the field of gold catalyzed carbophilic activation, so-called the chemists "gold rush", ${ }^{[20]}$ which became one of the most intensively studied fields of chemistry of the $21^{\text {st }}$ century. ${ }^{[7,14,21]}$

\subsubsection{General properties of gold}

Gold is located in group 11 of the periodic table, in the 3rd row of the transition metals and exists most commonly in the oxidation states +1 and +3 . Gold(III) complexes tend to have a square planar geometry, while gold(I) complexes a predominantly linear geometry. In addition, gold can form strong aurophilic interactions, and a variety of gold clusters have been described. ${ }^{[22]}$

The qualities of gold as a noble metal can be attributed to relativistic effects. Because of the large gold nucleus, a large enough force is exerted on the orbiting electrons to bring their speed close to the speed of light. A relativistic consequence of this is that the mass of electrons approaching this speed increases. This can be described using the following equation:

$$
m=\frac{m_{0}}{\sqrt{\left[1-\left(\frac{v}{c}\right)^{2}\right]}}
$$

where $m$ is the corrected mass, $m_{0}$ is the non-relativistic mass, $v$ is the velocity and $c$ is the speed of light. The Bohr radius of an electron is inversely proportional to its mass, therefore if the mass increases, the orbital housing that electron will also contract. ${ }^{[23]}$ Gold is unique in having appreciably larger relativistic effects than its neighbors. This can be visualized by 
comparing the ratio between the calculated radius of the $6 \mathrm{~s}$ orbital, when relativistic effects are or are not taken into account (Figure 1). ${ }^{[24]}$

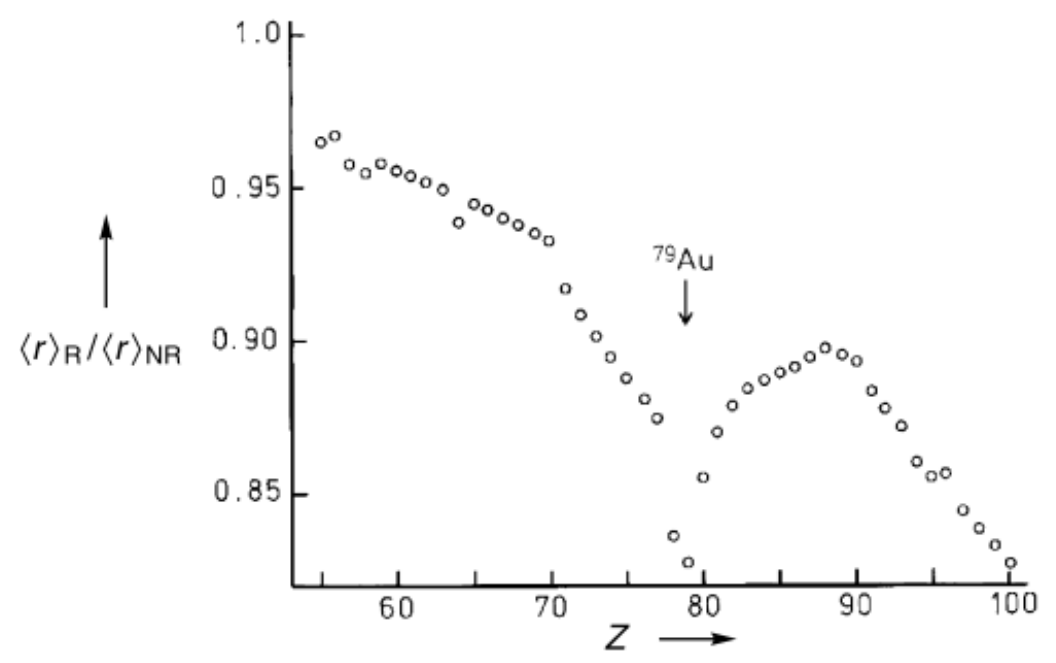

Figure 1. The ratio of non-relativistic and relativistic radii for the 6 s orbital for the elements of $Z=55-100$. Figure taken from P. Pyykkö, Angew. Chem. Int. Ed. 2004, 43, 4412.[24]

Relativistic effects account for some large differences between gold and silver, with which it shares group 11. For example, the first ionization energy of gold is much larger (9.22 eV vs $7.57 \mathrm{eV}$ ), as well as the electronegativity (2.4 vs 1.9). ${ }^{[23]}$ This also leads to generally stronger metal ligand bonds for gold complexes over silver complexes due a bond length contraction. ${ }^{[23]}$

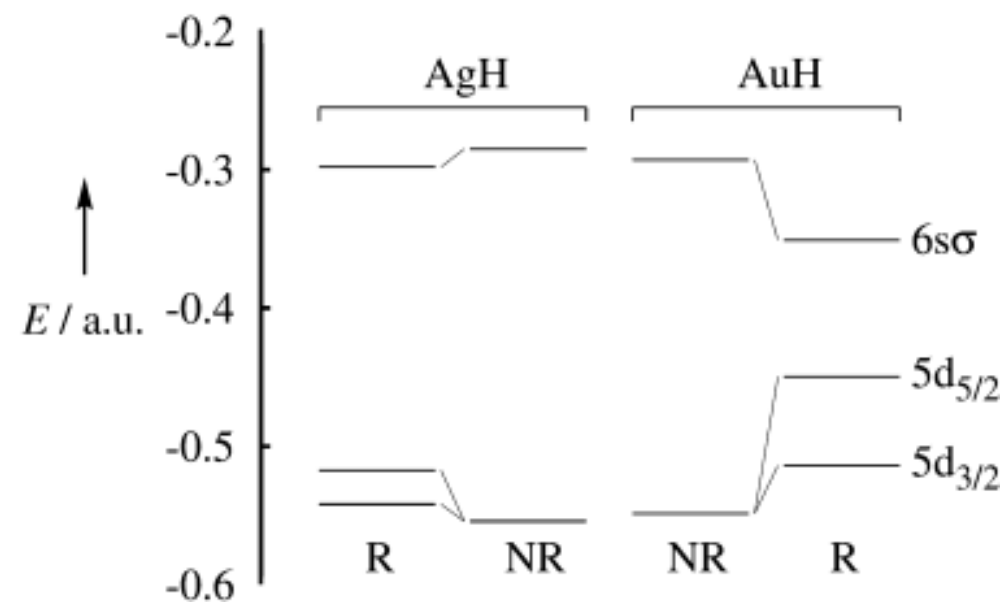

Figure 2. Calculated relativistic $(\mathrm{R})$ and non-relativistic $(\mathrm{NR})$ molecular orbital energies of $\mathrm{AuH}$ and $\mathrm{AgH}$. Figure taken from P. Pyykkö, Angew. Chem. Int. Ed. 2004, 43, 4412. ${ }^{[24]}$

While the $s$ and $p$ orbitals of gold experience a large relativistic contraction, the $d$ orbitals become destabilized and expand due to better shielding from the nuclear charge. This effect can be observed for the mononuclear complexes $\mathrm{AuH}$ and $\mathrm{AgH}$ (Figure 2). When the energies of the $6 \mathrm{~s} \sigma$ and $5 \mathrm{~d}$ orbitals for $\mathrm{AuH}$ and $\mathrm{AgH}$ are calculated without taking relativistic effects into consideration (NR in Figure 2), the orbital energies of the two compounds are 
comparable. However, when relativistic effects are considered ( $R$ in Figure 2$)$, the $6 s \sigma$ orbitals decrease in energy and the $5 d$ orbitals increase in energy. This effect is more pronounced for $\mathrm{AuH}$ over $\mathrm{AgH} .{ }^{[24]}$. A consequence of these effects is that gold is a chemically soft, carbophilic Lewis acid, due its low lying, large and polarizable empty orbitals. Another consequence is the stability of gold $(I)$ compounds towards redox processes, and although this closed off gold for many years from conventional transition metal catalysed reactions where the metal fluctuates between two oxidation states separated by two electrons, it opened new avenues in reactivity for $\pi$-acid catalysis, where gold demonstrates sometimes exquisite selectivity and orthogonality to other conventional redox reactions.

\subsubsection{Gold as a carbophilic Lewis acid}

The basic reaction mechanism of transformations catalyzed by a gold center is outlined in Figure $3{ }^{[25,26]}$ This will be discussed in detail over the course of this chapter with an aim to account for the exceptional properties of gold as a soft Lewis acid catalyst. In general, gold activates an incoming $\pi$-system towards nucleophilic attack, which results in the formation of the anti vinyl gold species D. Protodemetalation or other trapping reactions of the latter with an electrophile gives the product and regenerates the active gold species.

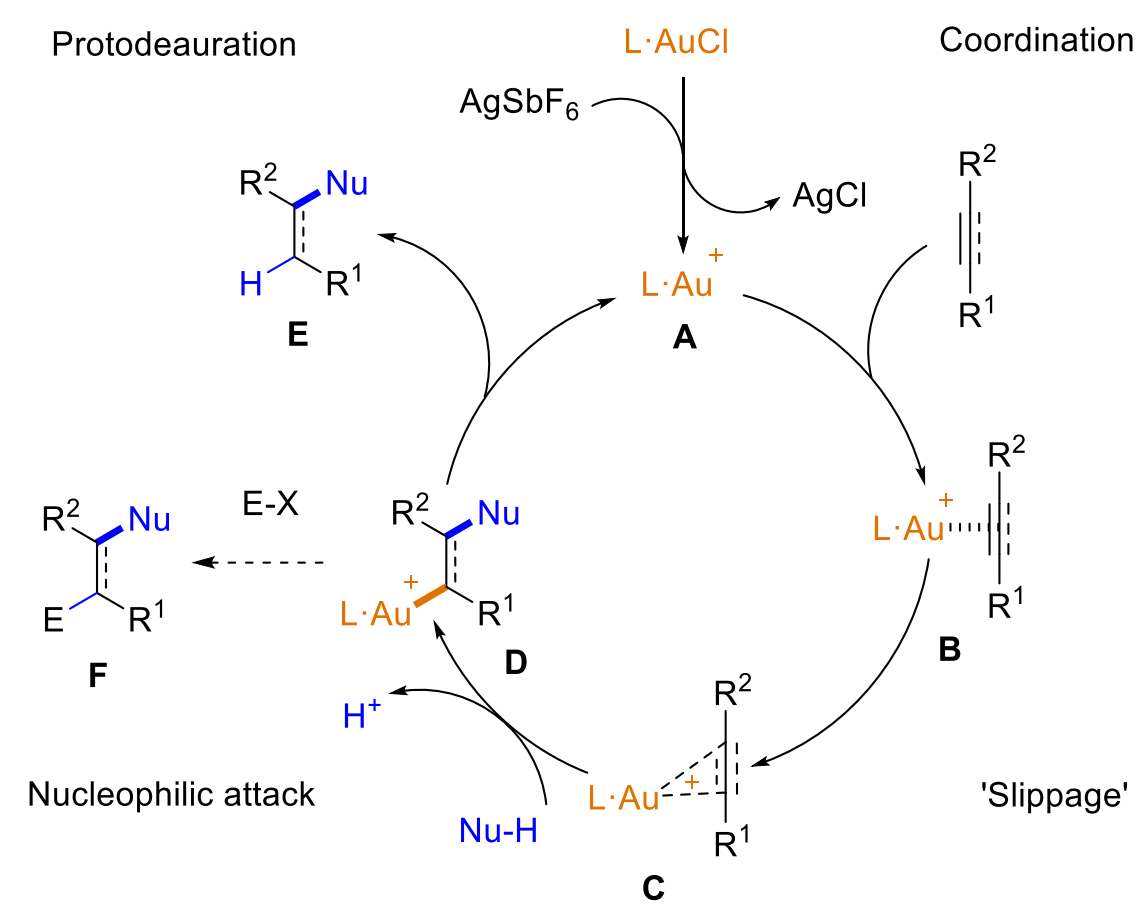

Figure 3. General gold catalysis mechanism of the activation of $\pi$-systems toward nucleophilic attack.

Many reactions in gold catalysis utilize gold(I) complexes of type (L·AuX), due to their stability and tuneability through the ancillary ligand $\mathrm{L}$, although $\mathrm{AuCl}_{3}$ is also a popular catalyst in cases where higher Lewis acidity may be required. When $\mathrm{X}$ is a non-coordinating counterion, for example triflamide, these complexes can be directly used in catalysis, as the 


\section{Introduction}

gold has a coordination vacancy. The corresponding cationic gold(I) complex can also be stabilized through neutral donors, such as acetonitrile or toluene, which are then easily displaced under catalytic conditions. Another approach is to remove the anion $\mathrm{X}$ in situ. This is commonly achieved, either by protonation with one equivalent of a Brønsted acid, for example when $\mathrm{X}=\mathrm{CH}_{3}$, or by silver-mediated abstraction, when $\mathrm{X}=\mathrm{Cl}$. In this case the gold $(I)$ precatalyst is commonly administered with a silver salt, which forms insoluble silver chloride and the active gold species. ${ }^{[25]}$ In many gold-catalyzed reactions, silver plays a noninnocent role and this has deserved comment in the chemical literature. ${ }^{[27]}$

The coordination vacancy of gold is then filled by formation of a $\pi$-complex with a suitable donor, such as an alkyne, allene or alkene. ${ }^{[24]}$ The bonding situation between transition metal complexes and carbon-carbon multiple bonds can be interpreted using the Dewar-ChattDuncanson model (DCD), ${ }^{[28]}$ which considers the bond as a donor acceptor interaction between two closed shell fragments. In general, a $\sigma$-bond is formed by donation of the $\pi$ system into empty, low lying orbitals on the metal of appropriate symmetry and m-backdonation occurs from filled metal orbitals into the $\pi^{*}$-orbital of the unsaturated ligand. In addition, a substantial electrostatic contribution has been calculated to exist between the two centers, with calculations for gold-acetylene and gold-ethylene predicting that this accounts for approximately half of the bond energy. ${ }^{[24]}$ As an example, the four predicted interactions between an alkyne and a metal are shown in Figure 4.

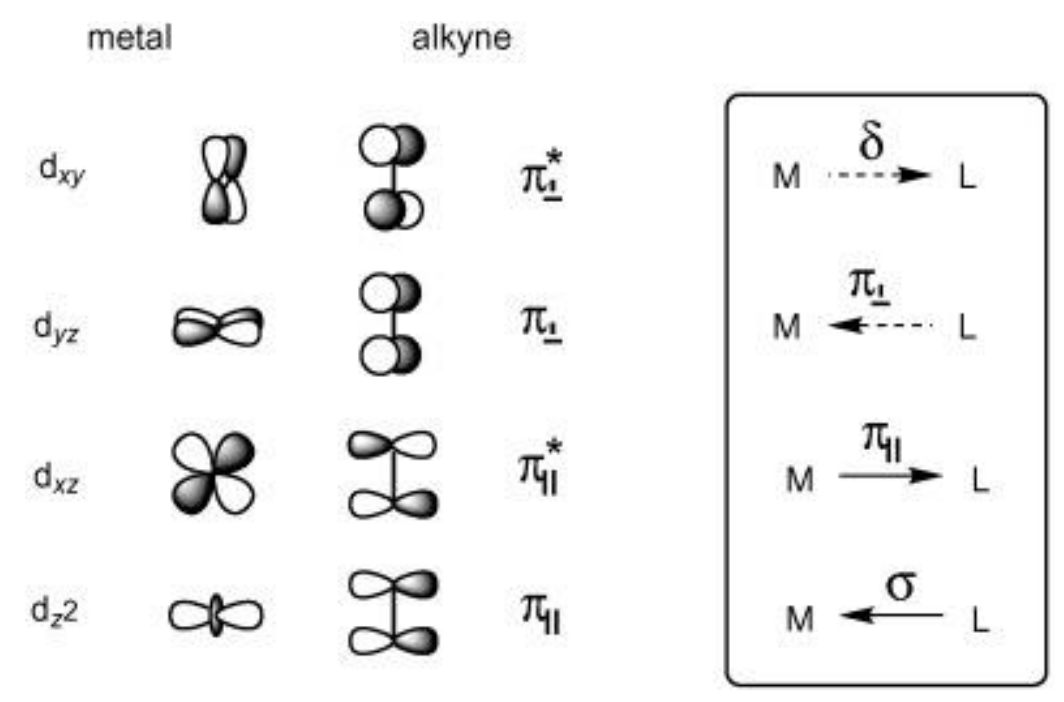

Figure 4. Schematic diagram showing bonding model between metal and an alkyne ligand. Figure taken from $A$. Fürstner, P. W. Davies, Angew. Chem. Int. Ed. 2007, 46, 3410.[14]

Firstly, the alkyne can form a $M \leftarrow L \sigma$-complex through donation of its filled in-plane $\pi$ orbital into the empty $d_{z} 2$ metal orbital; additionally $M \rightarrow L \pi^{*}$ back-donation can occur between the filled metal $d_{x z}$ orbital and the in-plane $\pi^{*}$ orbital of the alkyne. Other interactions that can also be considered are further $M \leftarrow L \pi$-donation from the orthogonal out-of-plane alkyne $\Pi$ - 
orbitals and the empty metal $d_{y z}$ orbital, as well as a possible $M \rightarrow L \delta$ interaction formed between the filled metal $d_{x y}$ orbital and the empty $\pi^{*}$ orbital of the alkyne. The calculated contributions from these four components for the $\left[\mathrm{Au}\left(\mathrm{C}_{2} \mathrm{H}_{2}\right)\right]^{+}$complex show that the $M \leftarrow L \sigma$ interaction accounts for the largest contribution to the orbital term (ca. 65\%). This is followed by the $M \rightarrow L \pi$-back donation (ca. 27\%), then the out of plane $M \leftarrow L \pi$ interaction (ca. $7 \%$ ) and the $\mathrm{M} \rightarrow \mathrm{L} \delta$ interaction (ca. 1\%). Additionally, an electrostatic contribution makes up approximately half of the total bond energy. ${ }^{[29]}$ What can be seen here is that apart from electrostatic interactions, the main contribution to the bond energy comes from donor interactions between the alkyne and the gold, although back donation from the gold still accounts for a non-negligible amount.

Experimentally, this can also be corroborated. The DCD model predicts that coordination of an alkyne to the gold center would increase the length of the carbon-carbon bond, due to net-donation from the alkyne to the metal and additional population of the antibonding $\pi^{*}$ orbital, as well as cause bending of the alkyne geometry from linearity. Fürstner and coworkers could synthesize and crystallize cyclododecyne gold chloride $\mathbf{4}$ and compared its structure to that of the free cyclododecyne (Figure 5). A clear elongation of the triple bond on coordination to gold could be seen between 1.196(4) $\AA$ in 3 to 1.224(5) $\AA$ in 4. Additionally, the $\mathrm{C} 3-\mathrm{C} 2-\mathrm{C} 1-\mathrm{C} 12$ angle was reduced from $175.9(9)^{\circ}$ in 3 to $165(1)^{\circ}$ in $4 .^{[30]}$

A)
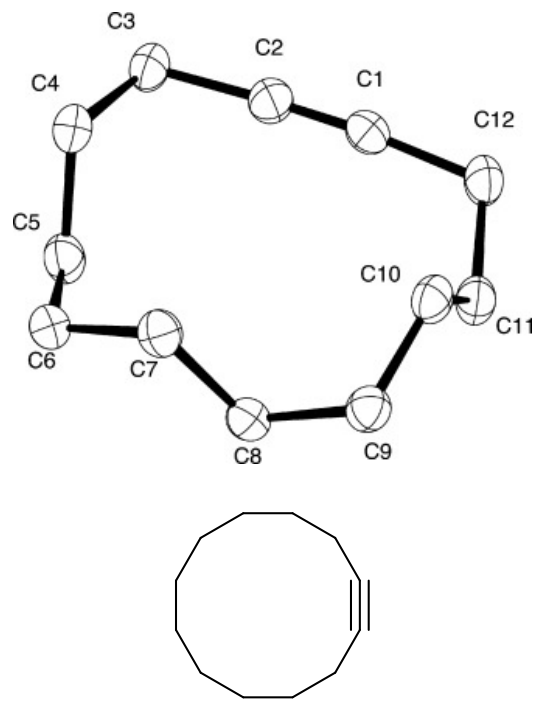

3
B)
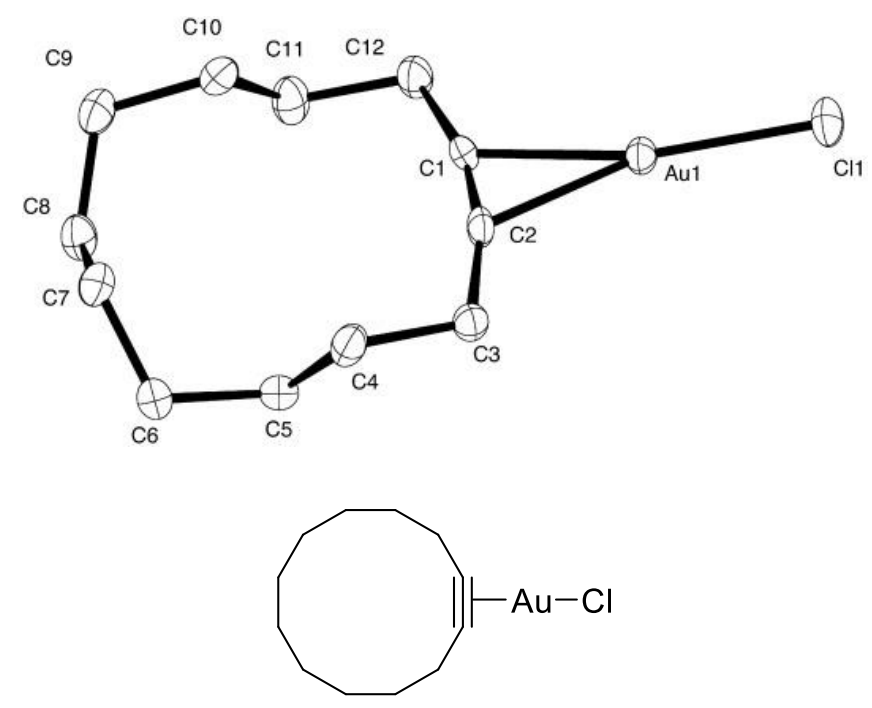

4

Figure 5. Solid state structures of A) cyclododecyne 3 and $B) \eta^{2}$-cyclododecyne-gold $(I)$ chloride 4 . Selected bond lengths and angles (a) C1-C2 = 1.196(4) $\AA$; $\Phi(C 3-C 2-C 1-C 12)=175.9^{\circ}$; (b) C1-C2 = 1.224(5) $\AA$; $\Phi$ (C3-C2$C 1-C 12)=165(1)^{\circ}$. Adapted from: S. Flügge, A. Anoop, R. Goddard, W. Thiel, A. Fürstner, Chem. Eur. J. 2009, $15,8558 .[30]$ 


\section{Introduction}

Overall, the effect of coordination of the $\pi$-system to the gold is to reduce its electron density and increase its electrophilicity towards nucleophilic attack. Interestingly, calculations have shown that between gold-acetylene and gold-ethylene, ethylene is the slightly better $\sigma$-donor. It is therefore unlikely that in polyunsaturated ene-yne substrates, the gold center would favor coordination of either one, and may even favor the carbon-carbon double bond. This contrasts with experimental evidence in ene-yne reactivity towards gold, where the olefin acts as a nucleophile towards the gold-coordinated alkyne. The apparent "alkynophilicity" of gold is therefore thought to be kinetic in origin, with a pronounced preference for nucleophilic attack at the coordinated triple bond. ${ }^{[14]}$

The process of nucleophilic attack towards a metal-coordinated $\pi$-system occurs via $\pi$ slippage, a process of electronic redistribution from a $\eta^{2}$ towards a $\eta^{1}$ structure. This facilitates charge transfer from the nucleophile to the ligand and consequently onto the metal center. ${ }^{[14]}$ If the alkyne has two different substituents, depending on their electronic or steric distribution or on the choice of ancillary gold ligand different regiomers can be formed. A variety of $\mathrm{O}, \mathrm{C}, \mathrm{N}$ or $\mathrm{S}$ centered nucleophiles can be successfully utilized together with alkynes, allenes or alkenes in this elementary step. ${ }^{\left[{ }^{31,32]}\right.}$ The nucleophile attacks the coordinated $\pi$-system in an outer sphere mechanism and gives an anti-oriented gold carbenoid intermediate. ${ }^{[33-35]}$ This can either simply undergo protodemetallation to give the desired product and regenerate the active gold catalyst, or through further inter- or intra molecular trapping reactions undergo a diverse number of cascades and rearrangements. ${ }^{[7,14,31,33-35]}$ In effect, this can quickly lead to a large increase in molecular complexity and is a defining advantage of gold catalysis. Moreover, in many cases the outcome of the reaction can be controlled through careful choice of ancillary ligand. ${ }^{[36]}$

One such example is the cycloisomerisation of allene-diene $\mathbf{5 a}$, which can selectively react to give the formal [4+3] or [4+2] cyclisation products $7 \mathbf{a}$ or $\mathbf{8 a}$, depending on the choice of ancillary ligand at the gold centre (Scheme 2). The Toste group demonstrated that when using the bulky biaryl phosphine 9 the product of the reaction was predominantly the [4+3] product $\mathbf{8 a}$, whereas when using the electron deficient phosphite 10 , the reaction favoured the formal [4+2] product 7a. ${ }^{[37]}$ The Mascareñas group described a similar effect for a related allene-diene substrate, comprising a sulfonamide tether. When using the ligand 10, the [4+2] product was predominantly formed, and a reversal of the selectivity was observed when using an $\mathrm{N}$-heterocyclic carbene $(\mathrm{NHC})$ as a ligand. ${ }^{[38]}$

DFT studies by both groups found that both products were formed via a concerted [4+3] cycloaddition to give the intermediate $6 \mathbf{a}$. Whereas electron withdrawing ligands such as phosphites favored a 1,2-carbon shift to give [4+2] product $\mathbf{8 a}$, sterically bulky ligands 
favored a 1,2-hydride migration to give [4+3] product 7a. The selectivity for the [4+2] product $8 \mathrm{a}$ when using phosphite ligands was found to be due to a reduction in the ability of the gold center to backbond towards the carbenoid carbon in $\mathbf{6 a}$ when ligated with an electron deficient ligand. This raised the energy of the 1,2-hydride shift, favoring the 1,2-carbon shift to take place instead. ${ }^{[37-39]}$ Additionally, the 1,2-carbon shift pathway was found to be strongly dependent on the nature of the substituents at the terminus of the allene, favoring a higher order of substitution. ${ }^{[38]}$

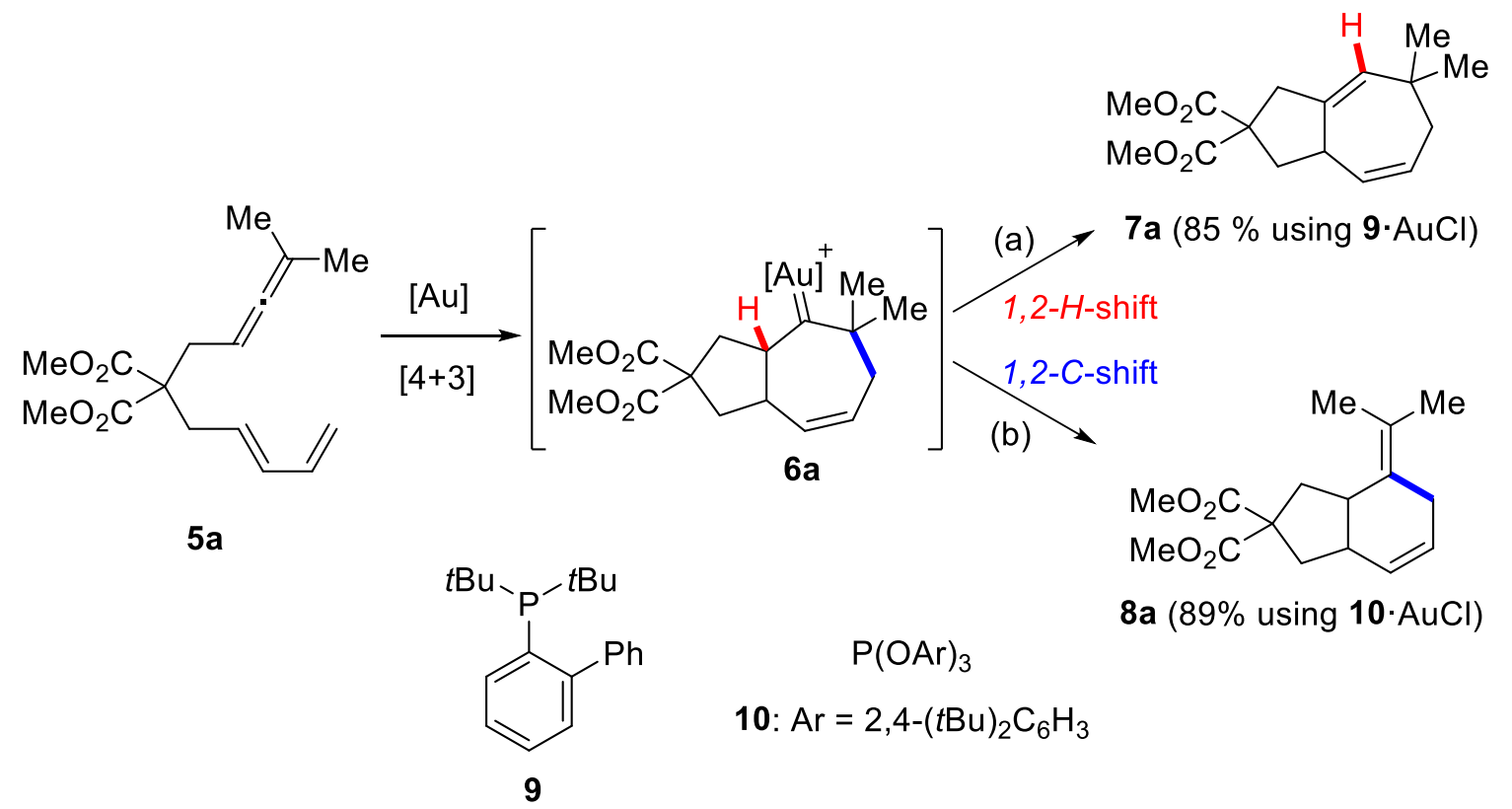

Scheme 2. Regiodivergent pathways in gold $(\mathrm{I})$-catalyzed cycloisomerization of allene-diene $\mathbf{5 a}$. Reagents and conditions (a) 9. AuCl (5 mol\%), $\mathrm{AgSbF}_{6}(5 \mathrm{~mol} \%), \mathrm{CH}_{2} \mathrm{Cl}_{2}, \mathrm{rt}, 24 \mathrm{~h}$; (b) 10. $\mathrm{AuCl}(5 \mathrm{~mol} \%), \mathrm{AgSbF}_{6}(5 \mathrm{~mol} \%$ ), $\mathrm{CH}_{2} \mathrm{Cl}_{2}, \mathrm{rt}, 30 \mathrm{~min}$.

Careful choice of ligand can also influence the diastereoselectivity and enantioselectivity of a given gold catalysed process. The following section will discuss the area of asymmetric gold catalysis in more detail.

\subsection{Asymmetric gold catalysis}

While a rapid acceleration of research activity occurred at the beginning of the $21^{\text {st }}$ century in gold catalysis, the field of enantioselective gold catalysis progressed at a slower pace. A much larger amount of asymmetric gold(I) cyclizations vs gold(III) or platinum(II) have been reported, ${ }^{[40-42]}$ however gold(I) complexes present a number of challenges. This is in part due to the linear geometry of gold(I) species, whereby any chiral information on the ligand is forced to lie at the opposite side of the gold to the substrate This is further exacerbated by the outer sphere anti mode of nucleophilic attack to the bound $\pi$-system. The mono- 


\section{Introduction}

coordinate geometry additionally permits a large degree of freedom in the ancillary ligand, with free rotation around the single bonds permittable(Figure 6). ${ }^{[40]}$

Although the first example of gold catalysis by Ito et al in 1986 also involved chiral ligands, ${ }^{[12]}$ the first communication on enantioselective gold catalysis through coordination of a $\pi$-system was reported much later by the group of Echavarren in 2005. ${ }^{[43]} \mathrm{A}$ number of approaches have led to significant advances in this field, achieving excellent levels of selectivity, and a selected number will be presented in this section. ${ }^{[40,42,44-46]}$

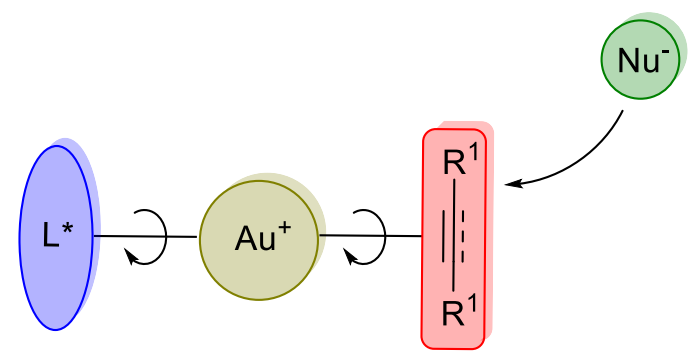

Figure 6. Linear coordination mode and outer sphere mode of nucleophilic attack in gold(I) catalysis.

\subsubsection{Chiral bis(phosphine) complexes}

One highly successful approach towards asymmetric gold catalysis is the use of chiral bis(phosphine) complexes, which have been applied in many areas of asymmetric catalysis as $\mathrm{C}_{2}$ symmetric, chelating ligands. ${ }^{[47]}$ In contrast, bis(phosphine) complexes form linear, binuclear species on coordination to gold. Generally speaking, the most widely used catalysts from this ligand class are shown in Figure 7, derived from the BINAP, MeO-BIPHEP and SEGPHOS scaffolds. ${ }^{[40,42,44-46]}$

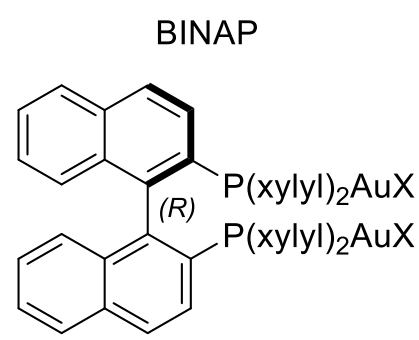

11

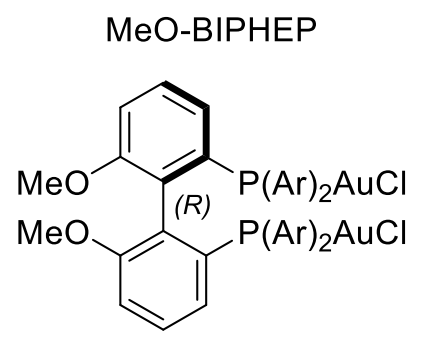

12
SEGPHOS

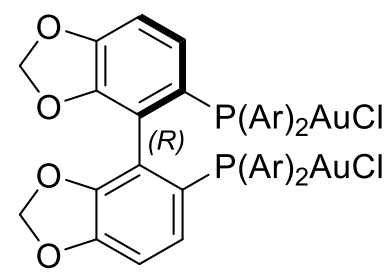

13

$$
\mathrm{Ar}=4-\mathrm{MeO}-3,5-(t \mathrm{Bu})_{2}\left(\mathrm{C}_{6} \mathrm{H}_{2}\right)
$$

Figure 7. Chiral bis(phosphines)-gold(I) complexes widely applicable in asymmetric catalysis.

The BINAP-derived precatalyst 11a was used by the Toste group in the highly enantioselective hydroamination reaction of allenes $14 a$ to give enantioenriched pyrrolidine derivatives such as $15 a$ (Scheme 3). ${ }^{[48]}$ A strong dependence on the counterion and equivalents of the added silver salt was observed, leading the authors to propose that the 
mono-cationic bis(gold) phosphine complex 11a could be responsible for the high enantioselectivity. Ultimately, a screening of counterions showed that the weakly coordinating para-nitrobenzoate gave the best results, with a sufficient amount of the catalytically active and highly enantioinducing monocationic species 11a existing in the reaction mixture to give high yields and ee's. It is likely that the additional gold center, though not catalytically active, contributes through sterics and potential aurophilic interactions to the chiral environment. A brominative variant of the same reaction was also later described, using the brominating agent 16 (Scheme 3). The authors commented that the lactam 16 achieved the perfect balance in being sufficiently reactive to give the halo-deauration product over proto-deauration, and not reactive enough to compete in a racemic background reaction. ${ }^{[49]}$
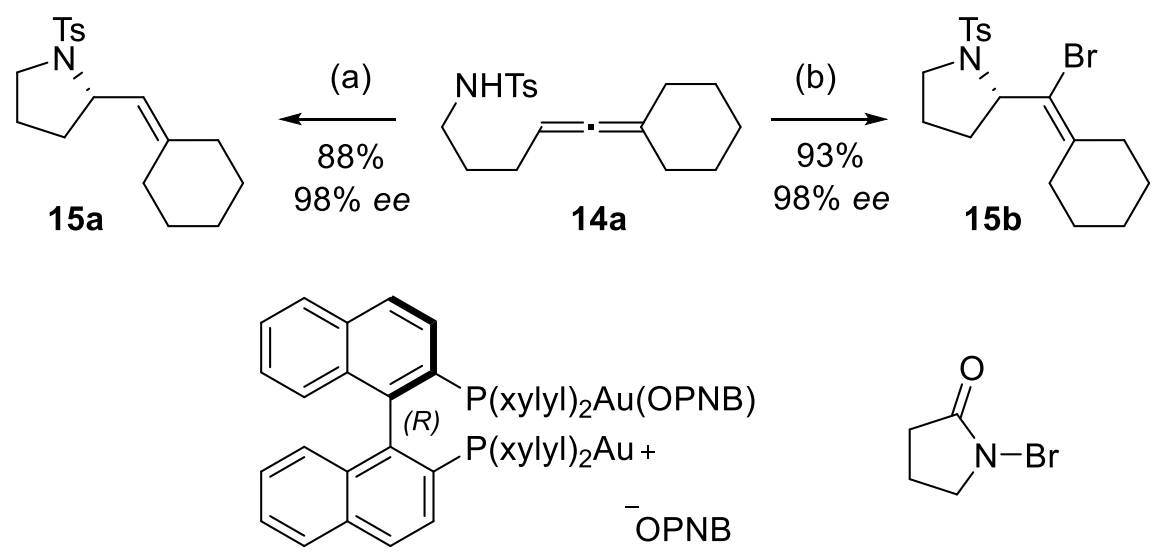

11a: proposed catalytically active species

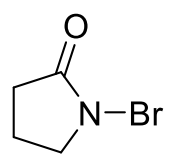

16

Scheme 3. Hydroamination of allene 14a catalyzed by 11a. Reagents and conditions: (a) 11a (3 mol\%), 1,2$\mathrm{Cl}_{2} \mathrm{C}_{2} \mathrm{H}_{4}$, rt; (b) 11a (5 mol\%), 16 (2.0 equiv.), $\mathrm{MeNO}_{2}$, rt, $12 \mathrm{~h}$.

Impressively, the Toste group applied the MeO-BIPHEP derived catalyst 9 towards the threefold cyclisation of polyene $\mathbf{1 7}$ to give tetracyclic product $\mathbf{1 8}$. The latter could be achieved in good yield, excellent enantioselectivity and perfect diastereoselectivity, in line with a proposed Stork-Eschemoser-type transition state (Scheme 4). ${ }^{[50]}$ Aside from arenes, amines, phenols and carboxylic acids were used with comparable levels of selectivity. 


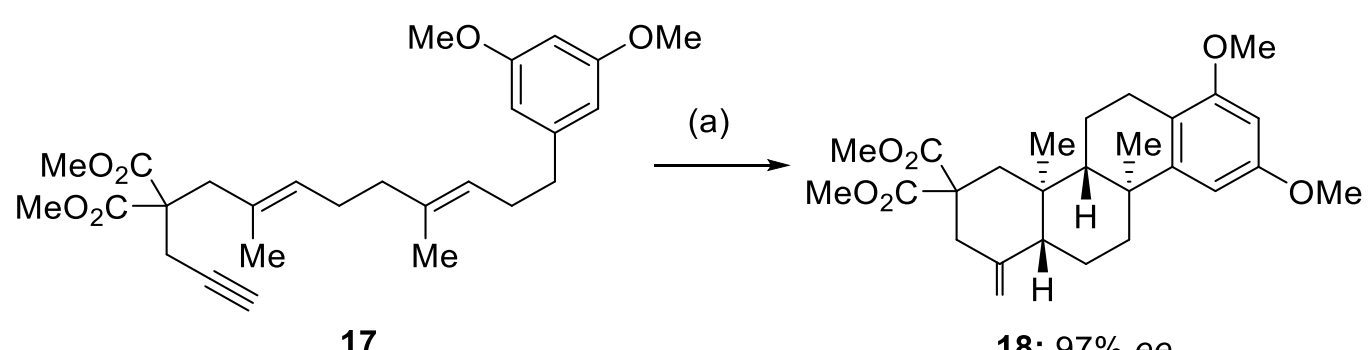

18: $97 \%$ ee

Scheme 4. Enantioselective gold(I)-catalyzed polycyclisation using the BIPHEP ligand scaffold. Reagents and conditions: (a) 12 (3 mol\%), AgSbF 6 (3 mol\%), m-xylene, rt.

The Toste group additionally described the highly selective intermolecular cyclopropanation reaction between styrenes and the propargylic pivolate 19, catalyzed by SEGPHOS derivative 13 (Scheme 5). ${ }^{[50-52]}$ The reaction proceeds first via the intramolecular rearrangement of $\mathbf{1 9}$ to give gold carbenoid species 22, which cyclopropanated styrenes $\mathbf{2 0}$ in excellent diastereoselectivity and enantioselectivity. This is still a rare example of a highly enantioselective intermolecular gold catalyzed reaction.

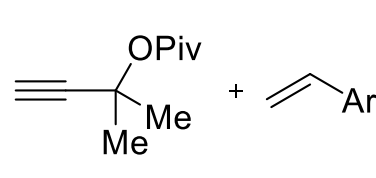

19

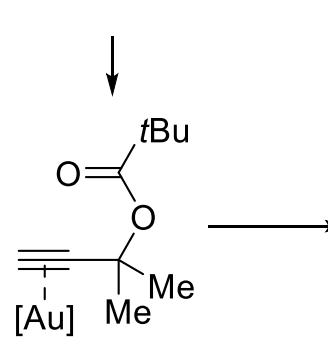

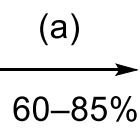

20<smiles>CC(C)=C(O[Na])C1CC1[Hg]</smiles>

21: $>20: 1 d r$ $76-94 \%$ ee

17<smiles>CC(C)=C(C=[Te][Te])OC(=O)C(C)(C)C</smiles>

Scheme 5. Gold(I)-catalyzed intermolecular cyclopropanation of styrenes 20. Reagents and conditions: (a) 13 (2.5 mol\%), $\mathrm{AgSbF}_{6}(5 \mathrm{~mol} \%), \mathrm{MeNO}_{2}, \mathrm{rt}, 20-40 \mathrm{~min}$.

\subsubsection{Asymmetric counterion-directed catalysis (ACDC)}

Having observed a strong counterion dependence in the gold $(I)$ catalyzed intramolecular hydroamination of allenes, ${ }^{[48,49]}$ the Toste group initiated efforts towards moving the source of chirality towards an outersphere counterion and studied if this could widen the scope of the transformation to other $\mathrm{N}$ - or O-centered nucleophiles. Utilizing the BINOL-derived phosphonate silver salt $\mathbf{2 3}$, the hydroalkoxylation of allene $\mathbf{2 4}$ bearing a hydroxyl functionality was investigated. ${ }^{[53]}$ In this case the bis(phosphine) gold complexes derived from SEGPHOS and BINAP had given almost racemic mixtures. Using the achiral bis(phosphine) 25 together 
with chiral silver salt $\mathbf{2 3}$ in an apolar solvent to maximize the ion pair led to the tetrahydrofuran product $\mathbf{2 6}$ in outstanding selectivity (Scheme 6A).

A)

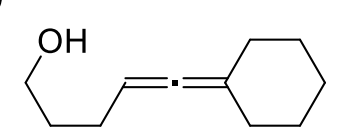

24

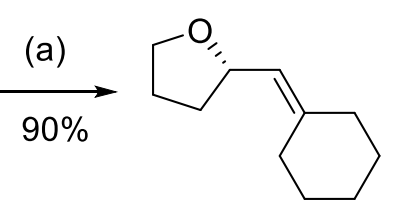

26: $97 \%$ ee

B)

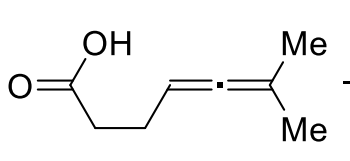

27

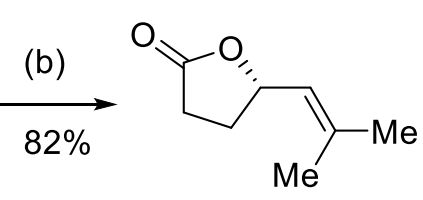

28: $82 \%$ ee

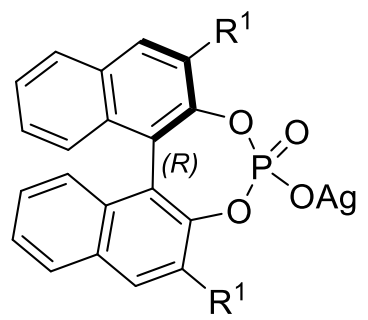

23: $\mathrm{R}^{1}=2,4,6-(\operatorname{Pr})_{3}\left(\mathrm{C}_{6} \mathrm{H}_{2}\right)$

$\mathrm{Ph}_{2} \mathrm{P} \smile \mathrm{PPh}_{2} 25$

Scheme 6. Asymmetric counteranion-directed catalysis in the cycloisomerization of allenes. Reagents and conditions: (a) 25. $(\mathrm{AuCl})_{2}(2.5 \mathrm{~mol} \%), 23$ (5 mol\%), $\mathrm{C}_{6} \mathrm{H}_{6}, \mathrm{rt}, 1 \mathrm{~h}$; (b) (S)-BINAP(AuCl) $2(2.5 \mathrm{~mol} \%), 23$ (5 mol\%), $\mathrm{C}_{6} \mathrm{H}_{6}, \mathrm{rt}, 24 \mathrm{~h}$.

This approach could also be extended to sulphonamides. For the use of carboxylates, a chiral phosphine/chiral silver salt pair was necessary to achieve high enantioselectivities (Scheme 6B). Here a strong matched/mismatched effect between the two was seen, with the combination of $(S)-\operatorname{BINAP}(\mathrm{AuCl})_{2}$ and $(R)-23$ giving the best results. The group also described a brominative variant of this reaction, accessing brominated tetrahydrofurans and lactones in high ee's, ${ }^{[49]}$ as well as an extension towards other nucleophiles, such as hydrazines and $\mathrm{O}$ - and $\mathrm{N}$-tethered hydroxylamines. ${ }^{[54]}$ In this way, a variety of synthetically useful heterocycles commonly found in biologically active compounds could be accessed using this strategy.

\subsubsection{Chiral carbene complexes}

Chiral carbenes are strong electron donor ligands and present an advantage when an electronic rich gold(I) is beneficial for the outcome of a given transformation. To this end, a variety of chiral carbene-gold complexes have been reported in highly asymmetric, regiodivergent transformations. ${ }^{[40-42,44,45]}$ One example is the rearrangement of propargylic alkynes 29, which when using carbene ligands selectively leads to the allene $\mathbf{3 0}$ over the gold-carbene intermediate 22. The Toste group exploited this reactivity in an intramolecular rearrangement/ hydroalkoxylation reaction using the BINOL derived bis(acylic diamino)carbene 31, which proceeded via a dynamic resolution of a chiral allene intermediate 30 . ${ }^{[55]}$ 


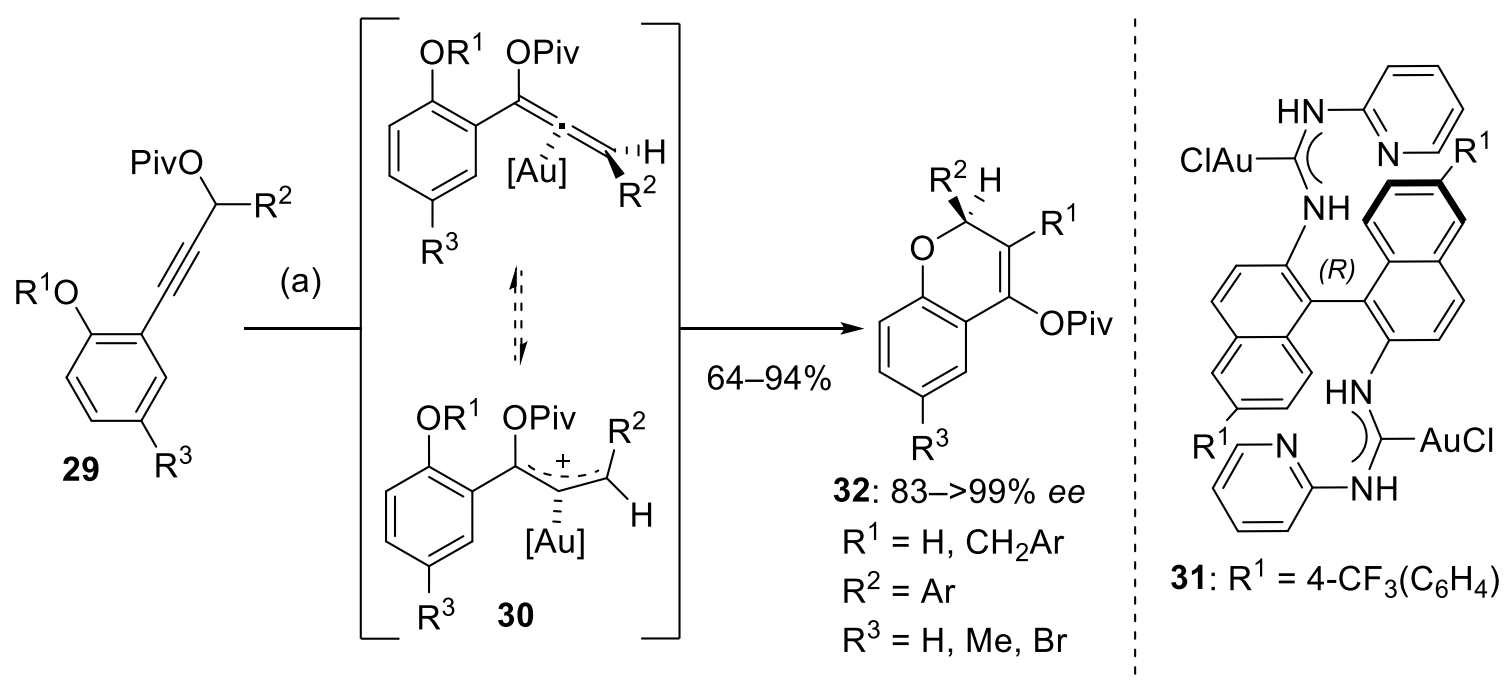

Scheme 7. Enantioselective kinetic dynamic resolution transformation of propargylic ethers 29 using the bis(carbene) 31. Reagents and conditions: (a) 31 (5 mol\%), AgOTf (10 mol\%), $\mathrm{CDCl}_{3}$, rt or $0{ }^{\circ} \mathrm{C}, 4 \mathrm{~h}$.

It was found that the electron rich carbene ligand on the gold accelerated a kinetic dynamic resolution of the intermediate chiral allene, which upon intramolecular hydroalkoxylation and protodeauration yielded the product $\mathbf{3 2}$ in excellent yield and enantioselectivity. Interestingly, when using benzylic ethers, the vinyl-gold species underwent an intramolecular benzylation, instead of protodeauration. In these cases, the enantioselectivity was even higher, ranging from 95 to $>99 \%$ ee.

In another example, the groups of Mascareñas, López, Fernández and Lassaletta reported a highly selective intermolecular [4+2] cyclisation of $\mathrm{N}$-alleneamides with dienes, leading to cyclohexenes such as 33. ${ }^{[56]}$ The reaction proceeded even at low temperatures and afforded the desired products in very good yields and excellent enantioselectivities. Furthermore, the [2+2] addition product could not be observed. ${ }^{[57]}$<smiles>C=C=CN1CCCC1=O</smiles>

34<smiles>C=C/C=C/c1ccccc1</smiles>

35 (a)<smiles>O=C1OCCN1C=C1CCC=C[C@H]1c1ccccc1</smiles>

33: $99 \%$ ee<smiles>O=C(c1cccc2ccccc12)C1N(Cl)N=C2C=c3ccccc3=C(Cl)N21</smiles>

36

Scheme 8. Gold catalyzed [4+2] addition of allenamides 34 and dienes $\mathbf{3 5}$ using chiral carbene gold complex $\mathbf{3 6}$. Reagents and conditions: (a) 36 (5 mol\%), $\mathrm{AgNTf}_{2}(5 \mathrm{~mol} \%), \mathrm{CH}_{2} \mathrm{Cl}_{2},-78{ }^{\circ} \mathrm{C}, 3 \mathrm{~h}$. 


\subsubsection{Chiral phosphoramidite complexes}

Another powerful tool in asymmetric gold catalysis, and indeed much farther afield ${ }^{[58]}$ is the emergence of monodentate phosphoramidites. In addition to their modular synthesis, these ligands are highly suitable for the restricted one-point binding of gold(I) complexes and, in contrast to bidentate phosphines, do not require the presence of two gold centers per catalyst. Additionally, their electron deficient nature makes them apt for transformations where an electron-poor gold(I) centre can influence the rate or selectivity of a given reaction.

The groups of Mascareñas, López and Ujaque exploited the ligand effect in the gold(I) catalysed cyclisation of sulfonamide tethered allene-diene $\mathbf{5}$, which selectively forms the formal [4+2] cycloaddition product 7 when using electron deficient ancillary ligands at the gold centre. ${ }^{[37,38]}$ In the presence of the bulky anthracene-substituted BINOL derived phosphoramidite ligand $\mathbf{4 1}$, the reaction of $\mathbf{5}$ to $\mathbf{7}$ proceeded with near perfect selectivity and excellent enantioselectivity (Scheme 9). ${ }^{[38,59]}$ The same group also found that allene-dienes which were only mono-substituted at the allene terminus were more prone to undergo a 1,2hydride shift and give the [4+3] product 8. Using the same catalyst, excellent levels of selectivity could be obtained (Scheme 8$).{ }^{[60]}$

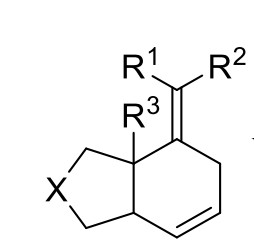

(a)

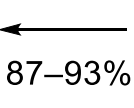

$$
\begin{gathered}
\text { 7: } \mathrm{X}=\mathrm{NTs} \\
\mathrm{R}^{1}=\mathrm{R}^{2}=\text { alkyl } \\
91-97 \% \text { ee }
\end{gathered}
$$

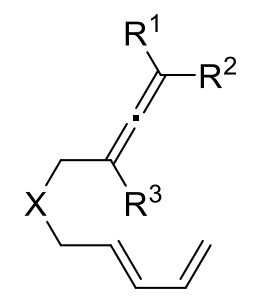

5

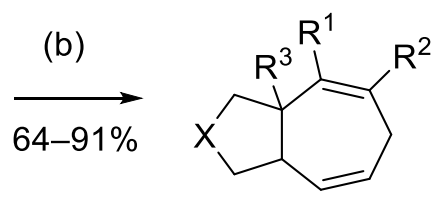

8: $\mathrm{X}=\mathrm{C}\left(\mathrm{CO}_{2} \mathrm{Me}\right), \mathrm{NTs}$

$\mathrm{R}^{1}=\mathrm{H}, \mathrm{R}^{2}=$ aryl

$\mathrm{R}^{3}=\mathrm{H}, \mathrm{Me}$

$87-98 \%$ ee

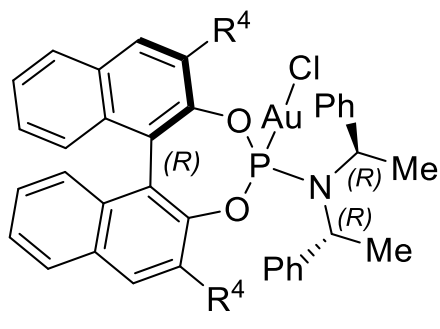

41: $\mathrm{R}^{4}=$ anthracenyl

Scheme 9. Regiodivergent substrate controlled pathways in allene-diene cyclizations. Reagents and conditions: (a) 41 (2-10 mol\%), AgSbF 6 (2-10 mol\%), $\mathrm{CH}_{2} \mathrm{Cl}_{2},-15{ }^{\circ} \mathrm{C}, 1-3 \mathrm{~h}$; (b) 41 (5 mol\%), $\mathrm{AgSbF}_{6}(5 \mathrm{~mol} \%), \mathrm{CH}_{2} \mathrm{Cl}_{2}$, $15^{\circ} \mathrm{C}$ to rt.

Six years ago, the group of Fürstner provided an outstanding mono-dentate catalytic system for a variety of gold(I)-catalyzed additions to allenes and alkynes, based on the TADDOL derived phosphoramidites 39 (Scheme 10). ${ }^{[52,61]}$ The group showed that modification of the backbone of the TADDOL towards the conformationally less rigid methoxy substituents gave improved enantioselectivities. The authors attributed this to the higher conformational freedom of the modified system, which enabled a tighter chiral pocket to form around the gold center, stabilized by secondary interactions. Impressively, catalysts of this type proved 


\section{Introduction}

not only to be highly selective in the gold catalyzed [4+2] cyclizations of allene-dienes $\mathbf{5 b}$ as well as the [2+2] cyclizations of allene-enes 42 , which rivalled those previously reported, but also the cyclopropanation reaction of ene-ynes $\mathbf{4 4}$ and $\mathbf{4 6}$ with $N$ and $\mathrm{O}$ tethers, respectively, as well as the hydroarylation, hydroamination and hydroalkoxylation of allenes 48 . ${ }^{[1]}$

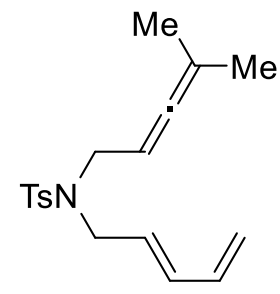

$5 \mathbf{b}$ (a)

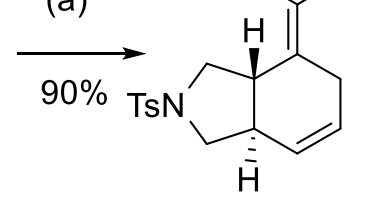

7b: $91 \%$ ee<smiles>CC(C)=C=CCC(C/C=C/Br)(C(=O)OBr)C(=O)OBr</smiles>

42<smiles>CC(C)=C1[C@H]2CC(C(=O)OBr)(C(=O)OCc3ccccc3)C[C@@H]2[C@H]1[Al]</smiles>

43: $\mathrm{Ar}=4-\mathrm{MeO}\left(\mathrm{C}_{6} \mathrm{H}_{4}\right)$ $99 \%$ ee<smiles>C=CCN(CC#CPc1ccccc1)C(=O)OC(=O)OCc1ccccc1</smiles>

44

45: $98 \%$ ee<smiles>CC(C)(OCC#CF)C1=CCCCO1</smiles>

46 (b)<smiles>COC=C[C@@]1(c2ccccc2)[C@@H]2CCCO[C@@]21OC</smiles>

47: $94 \%$ ee
49: $97 \%$ ee<smiles>COC(=O)N1C[C@H](C=C(C)C)c2c(OC)cc(OC)cc21</smiles>

50a: $\mathrm{R}^{1}=4-t \mathrm{Bu}\left(\mathrm{C}_{6} \mathrm{H}_{4}\right), \mathrm{R}^{2}=\mathrm{Me}$

50b: $\mathrm{R}^{1}=2$-naphthyl, $\mathrm{R}^{2}=\mathrm{Me}$

50c: $R^{1}=2$-naphthyl, $R^{2}=-\left(\mathrm{CH}_{2}\right)_{3}$ -

Scheme 10. Selected examples of gold(I)-catalyzed reactions using TADDOL-derived gold complexes $\mathbf{5 0}$. Reagents and conditions: (a) $(R, R, R, R) 50 \mathrm{a}(5.5 \mathrm{~mol} \%), \mathrm{AgBF}_{4}(5 \mathrm{~mol} \%), \mathrm{CH}_{2} \mathrm{Cl}_{2}, 0^{\circ} \mathrm{C}$; (b) $(S, S, S, S) 50 \mathrm{~b}(5.5$ mol\%), $\mathrm{AgBF}_{4}(5 \mathrm{~mol} \%)$, toluene, $0{ }^{\circ} \mathrm{C}$; (c) $(R, R, R, R) 50 \mathrm{c}(5.5 \mathrm{~mol} \%), \mathrm{AgBF}_{4}(5 \mathrm{~mol} \%), 1,2-\mathrm{C}_{2} \mathrm{H}_{4} \mathrm{Cl}_{2},-30{ }^{\circ} \mathrm{C}$.

In summary, a variety of different approaches can be employed for the development of new, highly enantioselective processes using gold $(I)$ catalysis, relying on an array of bis(phosphine), carbene or phosphoramidite gold complexes, depending on the nature of the reaction. The linear nature of gold(I) complexes presents a challenge, however, which necessitates a large distance between the substrate and ligand, meaning that usually in order to effectively transfer the chiral information, a steric "wall" must be designed to enclose the gold center. This can either be achieved using bulky substituents, such as in TADDOL and BINOL derived phosphoramidites, or in the use of a second gold center and/ or bulky chiral counterion. The electronic aspect of the ligand also allows control over the reaction outcome in some cases. Nevertheless, the development of new ligands can help to 
overcome as of yet unsolved challenges in this field and expand the possibilities presented by gold catalysis in the construction of complex molecular geometries through carbon-carbon bond formation. The next section will discuss the development of a new family of ligands bearing a cationic charge proximal to the phosphorus center, which present unique $\pi$ accepting properties, and their current applications in $\pi$-acid catalysis.

\subsection{Cationic phosphines}

The vast majority of ligands are either neutral or anionic, which naturally stems from a higher ability of these systems to coordinate an electropositive metal center and stabilize a metal complex. Cationically charged ligands however, have also been used in a variety of applications, including as ligands for the detection of reactive intermediates by ESI-MS, ${ }^{[62]}$ as easily recyclable water or ionic-liquid-soluble phase transfer catalysts, ${ }^{[63]}$ or as ion pairs in asymmetric catalysis (Figure 8) ${ }^{[64]}$ In all of these ligands, the charge is located far from the phosphorus center; and although the physicochemical properties are affected by the introduction of a cationic charge, the electronic properties do not significantly change. In cases when the cationic charge is installed adjacent to the phosphorus, the $\sigma$-donor ability of the phosphorus center decreases with a concurrent increase in its $\pi$-acceptor properties due to the strong electron-withdrawing effect of the positively charged substituent.

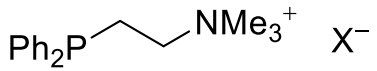

Phase-transfer catalysis

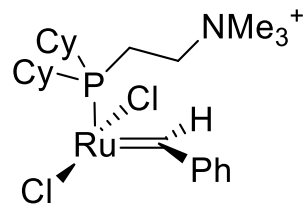

ESI-MS<smiles>[O-]c1c(-c2ccccc2)cc2ccccc2c1-c1c(O)c(-c2ccccc2)cc2ccccc12</smiles><smiles>CN(C)Cc1ccccc1P</smiles>

lon-paired asymmetric catalysis

Figure 8. Examples of cationic phosphines where the charge is located away from the phosphorus center.

Such phosphorus-based ligands display comparable donor abilities to strong-m-acceptor ligands such as polyhalogenated phosphines, trifluorophosphine or tris(trifluoromethyl)phosphine. However, in contrast to these last two, which are toxic and highly flammable gases, $\alpha$-cationic phosphines with a relatively inert phosphorus-carbon bond can be readily handled under ambient conditions. Strong $\pi$-acceptor ligands greatly deplete electron density from metals they coordinate. Therefore, if the rate-determining step of a catalytic cycle is influenced by a more electrophilic metal center, a dramatic enhancement of catalytic activity can be expected. The next section will discuss the synthesis of this class of ligands, the evaluation of their donor ability as well as their implications for catalysis. 


\section{Introduction}

\subsubsection{Synthesis of monocationic phosphines and phosphonites}

Although a-cationic phosphines had been mentioned in the literature in reports by Zoller in $1988^{[65]}$ and Komarov in $1995,{ }^{[66]}$ the first fully characterized a-cationic phosphine 51a, was described by Kuhn in 1999. ${ }^{[67]}$ Kuhn reported the direct condensation of imidazole-2-ylidenes with chlorophosphines, which had the advantage that both starting materials are readilyavailable. This method has since been applied using other imidazole-2-ylidenes, ${ }^{[68]}$ cyclic(alkyl)(amino)carbenes (CAAC's), ${ }^{[69,70]}$ dihydroimidazol-2-ylidenes ${ }^{[71]}$ and has also been extended to the synthesis of cationic phosphonites (Scheme 11). ${ }^{[72]}$ The carbene can even, in several cases, be generated in situ, via the base mediated deprotonation of suitable precursors. ${ }^{[70,73]}$<smiles></smiles>

51a: $\mathrm{R}^{2}=i \mathrm{Pr}, \mathrm{R}^{3}=\mathrm{Me}(87 \%)$ 51b: $R^{2}=\operatorname{Dipp}, R^{3}=\operatorname{Me}(77 \%)$ (a)<smiles>C1CC[Pb]C1</smiles>

(c)

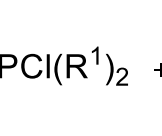<smiles>[X]C1([O])CCCCN1[18F]</smiles>

(b)<smiles>CCCCC(C)(C)C1CC(C)(C)N(c2ccccc2)C1P</smiles>

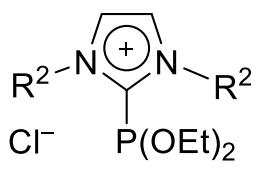

53a: $R^{2}=\operatorname{Mes}(70 \%)$

53b: $R^{2}=\operatorname{Dipp}(89 \%)$

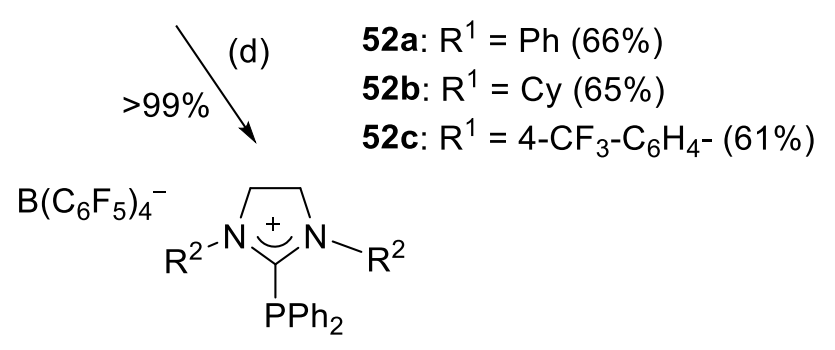

54

Scheme 11. Examples of cationic phosphines synthesis through condensation with free carbenes. Reagents and conditions: (a) NHC (1.0 equiv), Et2O, 30 min-1h; (b) CAAC (1.0 equiv.), THF, rt; (c) NHC (1.0 equiv.), Et2O, -78 ${ }^{\circ} \mathrm{C}, 15 \mathrm{~min}$; (d) NHC (1 equiv.), toluene, rt, $1 \mathrm{~h}$, then $\left[\mathrm{Et}_{3} \mathrm{Si}\right]\left[\mathrm{B}\left(\mathrm{C}_{6} \mathrm{~F}_{5}\right)_{4}\right]$ (1.0 equiv.), rt, $30 \mathrm{~min}$.

The outcome of this method, however strongly depends on the nature of the carbene used. In the formation of 2-imidazolium phosphine adducts such as $\mathbf{5 1 b}$, the addition of an additional equivalent of base facilitates a rearrangement, forming the 4-phosphino-imidazol2-ylidene 55 (Scheme 12). ${ }^{[68]}$ The Ruiz group has capitalized on this strategy to selectively synthesis $4^{[74]}$ and 4,5-[75] phosphino substituted imidazolium salts. In addition, the Weigand group has reported related rearrangements when using 4,5-dichloroimidazol-2-ylidenes, ${ }^{[76]}$ and postulated the same rearrangement in the presence of additional equivalents of imidazole-2-ylidenes. ${ }^{[77]}$ 


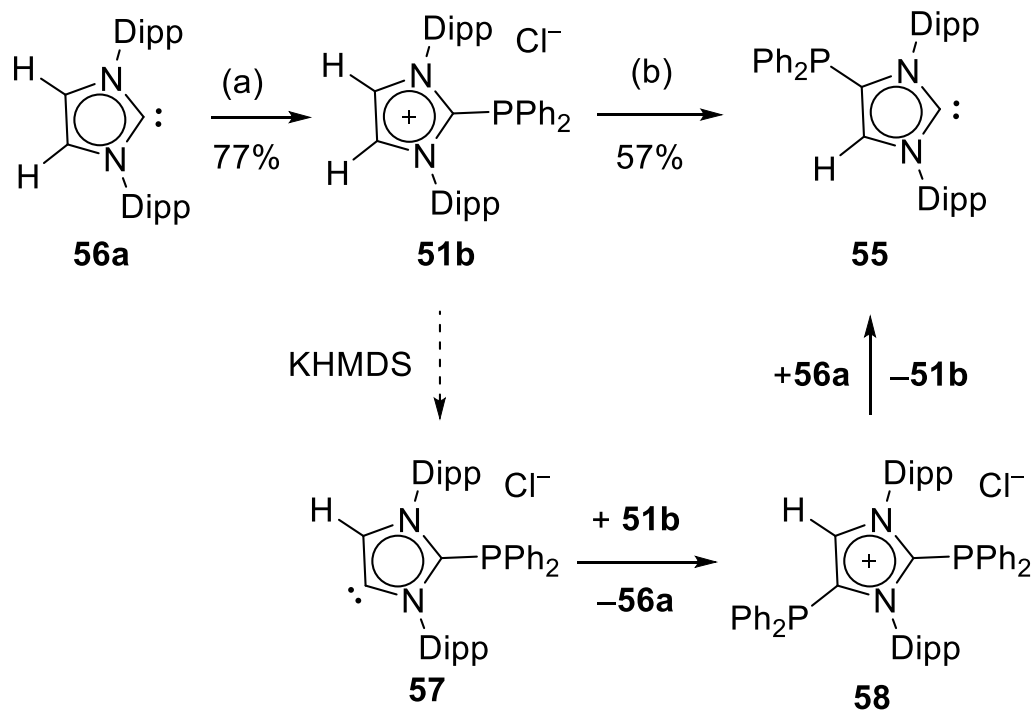

Scheme 12. Base mediated rearrangement of $\alpha$-cationic phosphine $\mathbf{5 1 b}$ to give the 4-phosphinoimidazol-2ylidene 55. Reagents and conditions (a) 56a (1.0 equiv.), Et $2 \mathrm{O}$, rt, $1 \mathrm{~h}$; KHMDS (1.0 equiv.), THF, $-78^{\circ} \mathrm{C}, 30 \mathrm{~min}$, then rt, 30 min.

The use of "masked" carbenes also selectively leads to a-cationic phosphines (Scheme 13). Thus, decarboxylation of $\mathbf{5 9}$ smoothly occurs at room temperature in the presence of chlorophosphines to give the imidazolium-derived cationic phosphines $51 \mathrm{c}-\mathbf{e}$, as reported by the group of Andrieu. ${ }^{[78]}$ Weigand and coworkers also developed a method using the carbene-trimethylsilyl triflate adduct $\mathbf{6 0}$. The product $\mathbf{6 1 a}$ could then be further functionalized with nitrile and azide substituents. ${ }^{[79]}$ Alternatively, reactive bis(carbene) silver complexes can be employed as carbene transfer reagents. This was demonstrated by Leitner and coworkers for the synthesis of a cationic phosphonite, ${ }^{[80]}$ and also by Stephan and coworkers via the direct transformation of the silver chloride complex $63 .{ }^{[70]}$ 
<smiles></smiles>

59

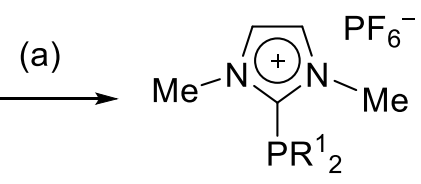

51c: $R^{1}=P h(91 \%)$

51d: $R^{1}=$ Cy $(58 \%)$

51e: $R^{1}=i \operatorname{Pr}(91 \%)$

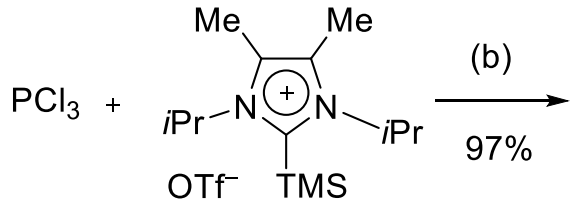

60<smiles></smiles>

$61 \mathrm{a}$<smiles></smiles>

51f: $R^{2}=C N(65 \%)$

51g: $R^{2}=N_{3}(43 \%)$<smiles></smiles>

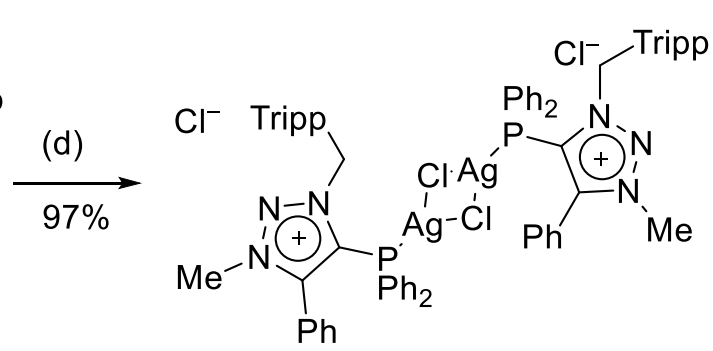

63

Scheme 13. Approaches towards monocationic phosphines or phosphonites using carbene carboxylates (59), silanes (60) or silver complexes (62a). Reagents and conditions: (a) 59 (1.0 equiv.), $\mathrm{CH}_{2} \mathrm{Cl}_{2}, \mathrm{rt}, 4 \mathrm{~h}$, then acetone, $\mathrm{KPF}_{6}$, rt, $48 \mathrm{~h}$; (b) $\mathrm{PCl}_{3}$ (excess), $\mathrm{C}_{6} \mathrm{H}_{5} \mathrm{~F}, 50{ }^{\circ} \mathrm{C}, 10 \mathrm{~h}$, ultrasonic bath; (c) TMSX (2.0 equiv), MeCN, $48 \mathrm{~h}$; (d) 62a (0.5 equiv.), $\mathrm{CH}_{2} \mathrm{Cl}_{2}$, rt, $2 \mathrm{~h}$.

Another approach stems from the selective $\mathrm{N}$-alkylation of the corresponding 2-(imidazoyl) phosphines using strong alkylating reagents. ${ }^{[6,81]}$ The group of Chauvin has used this method in the synthesis of phosphonites such as 50c and 50d (Scheme 14). ${ }^{[72]}$

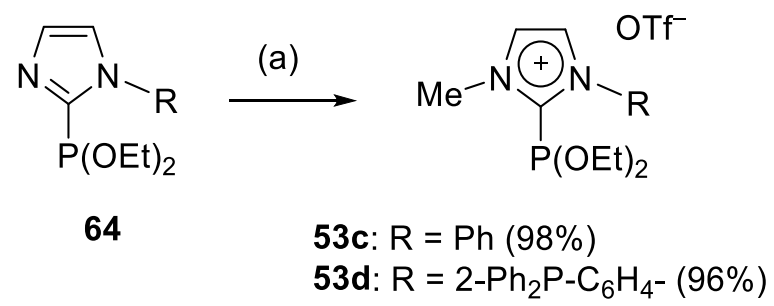

Scheme 14. N-Alkylation of 2-(imidazoyl)phosphines or -phosphites. Reagents and conditions: (a) MeOTf (1.0 equiv.), toluene, $\mathrm{rt}, 2 \mathrm{~h}$.

While these various methods allow the synthesis of cationic phosphines or phosphonites in good yields, an alternative approach, described by the Alcarazo group, greatly expanded upon the scope of different cationic phosphines that had not been previously reported. 
Starting from readily available secondary phosphines as well as Vilsmeier-type precursors and utilizing the so-called reverse "onio" strategy, the group first performed the synthesis of cyclopropenium-derived phosphines 61 by simply heating the two precursors together in THF. The procedure was also amenable to the use of more electron-withdrawing phosphines such as bis-(3,5-bis(trifluoromethyl)phenyl)phosphine by deprotonation of the phosphine with nbutyllithium followed by condensation. ${ }^{\left[{ }^{82]}\right.}$ The same strategy has also been extended towards dihydroimidazolium-[83] and formamidinium-derived ${ }^{[84]}$ salts (Scheme 15), in addition to cationic arsines. ${ }^{[85]}$

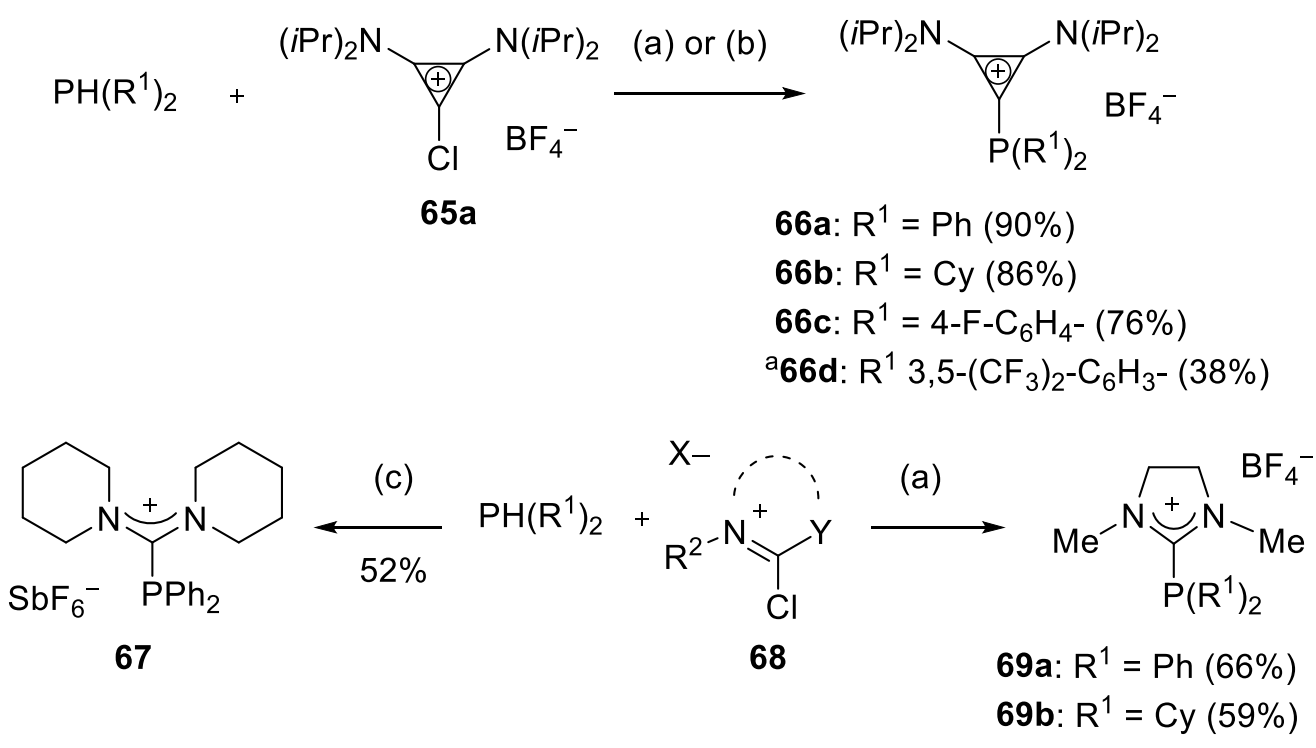

Scheme 15. Synthesis of cationic phosphines using reverse "onio" strategy. Reagents and conditions: (a) $\mathrm{PH}\left(\mathrm{R}^{1}\right)_{2}$ (3 equiv.), THF, $60{ }^{\circ} \mathrm{C}, 24 \mathrm{~h}$, then sat. $\mathrm{NaBF}_{4}$; (b) $\mathrm{PH}\left(\mathrm{R}^{1}\right)_{2}\left(1.0\right.$ equiv), nBuLi (1.0 equiv.), THF, $-78^{\circ} \mathrm{C}$, then $65 a$ (1.0 equiv.), $60{ }^{\circ} \mathrm{C}, 48 \mathrm{~h}$, then sat. $\mathrm{NaBF}_{4}$; (c) $\mathrm{PH}\left(\mathrm{R}^{1}\right)_{2}\left(3.0\right.$ equiv.), THF, $65{ }^{\circ} \mathrm{C}, 72 \mathrm{~h}$. ${ }^{\text {aAccording to }}$ method (b).

The group additionally described the incorporation of pyridinium substituents using the same strategy, either applying various $\mathrm{N}$-methylated or $\mathrm{N}$-arylated pyridinium chloride salts (Scheme 16). ${ }^{[86]}$ Bulky $\mathrm{N}$-aryl substituents, which were not amenable to simple direct $\mathrm{N}$ arylation protocols, could be synthesized using the Zincke reaction. Upon further elaboration, the pyridinium salts 65 were then condensed with various secondary phosphines at high temperatures in a microwave reactor. ${ }^{[87]}$ 


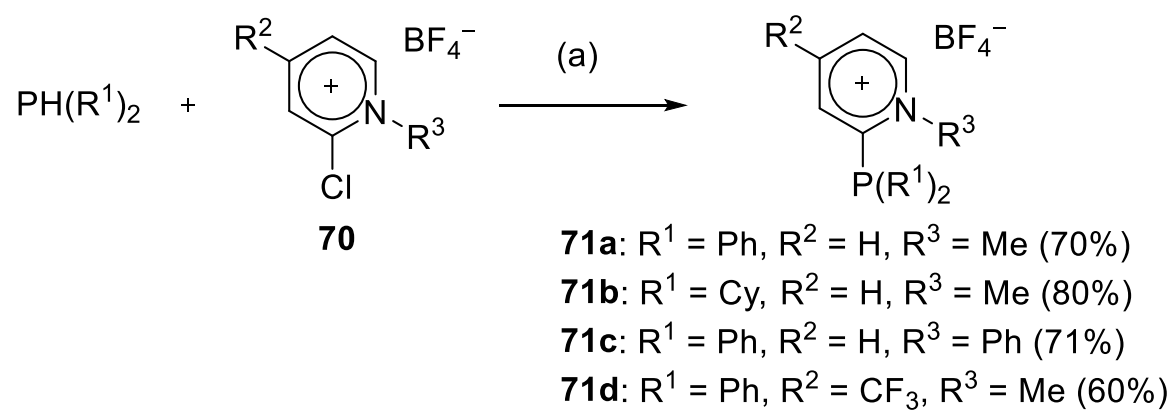

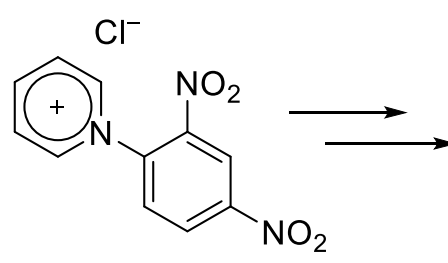

72

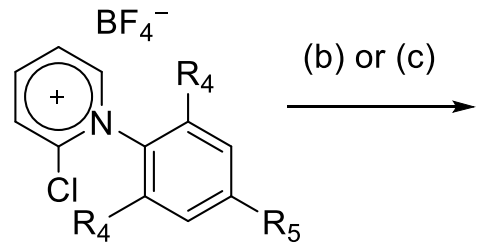

73

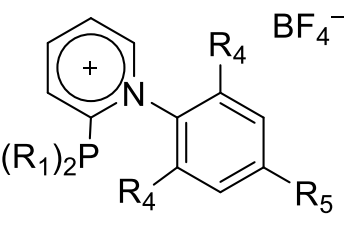

$$
\begin{aligned}
\text { 71e: } R_{1} & =P h, R_{4}=R_{5}=M e(50 \%) \\
\text { 71f: } R_{1} & =C y, R_{4}=R_{5}=M e(55 \%) \\
\text { 71g: } R_{1} & =C y, R_{4}=M e O, R_{5}=H(69 \%) \\
\text { a71h: } R_{1} & =3,5-\left(C_{3}\right)_{2}-C_{6} H_{3^{-}}, R_{4}=M e O \\
R_{5} & =H(45 \%)
\end{aligned}
$$

Scheme 16. Synthesis of pyridinium phosphines 71. Reagents and conditions: 70 (1.0 equiv.), $\mathrm{PH}\left(\mathrm{R}^{1}\right)_{2}(2.0$ equiv.), THF, $65^{\circ} \mathrm{C}, 1-3$ days; (b) 73 (1.0 equiv.), $\mathrm{PH}\left(\mathrm{R}^{1}\right)_{2}$ (3.0 equiv.), THF, $120-140{ }^{\circ} \mathrm{C}$, $\mu$-wave irradiation $12 \mathrm{~h}$, then $\mathrm{MeCN}, \mathrm{NaSb}_{6}$; (c) $\mathrm{PH}\left(\mathrm{R}^{1}\right)_{2}$ (1.0 equiv.), $\mathrm{KH}$ (8 equiv.), THF, $-78{ }^{\circ} \mathrm{C}, 1 \mathrm{~h}$, then 73 (1.0 equiv), $-78{ }^{\circ} \mathrm{C}$ to $\mathrm{rt}, 16$ $\mathrm{h}$, then MeCN, NaSbF 6 . ${ }^{a}$ According to method (c).

Despite possessing a cationic charge, monocationic phosphines display a rich coordination chemistry, with complexes to $\mathrm{Au}, \mathrm{Ag}, \mathrm{Cu}, \mathrm{Pt}, \mathrm{Ni}, \mathrm{Ir}, \mathrm{Pd}$ and $\mathrm{Rh}$ having been described to date. ${ }^{[88]}$ While the cationic charge significantly depletes electron density from the phosphorus center, the lone pair remains active, as evidenced by the pyramidal structure of these ligands in the solid state. However, unlike many other strong $\pi$-acceptor ligands, the presence of a relatively inert phosphorus-carbon bond allows many monocationic phosphines to be handled under air, or even be used in biphasic aqueous media.

\subsubsection{Synthesis of polycationic phosphines and phosphonites}

The synthesis of di and tricationic phosphines is amenable to many of the same methods outlined in the previous section. Dicationic imidazolium derived phosphines have been described by the groups of Andrieu ${ }^{[8]}$ and Weigand ${ }^{[00]}$ by reaction of two equivalents of either imidazolium carboxylate $\mathbf{5 9}$ or trimethylsilylimidazolium salt $\mathbf{6 0}$ with different dichlorophosphines. Additionally, double $\mathrm{N}$-alkyation of bis(2-imidazoyl)phosphines $\mathbf{7 4} 4^{[91]}$ can yield the corresponding dicationic salt $75 a$. 

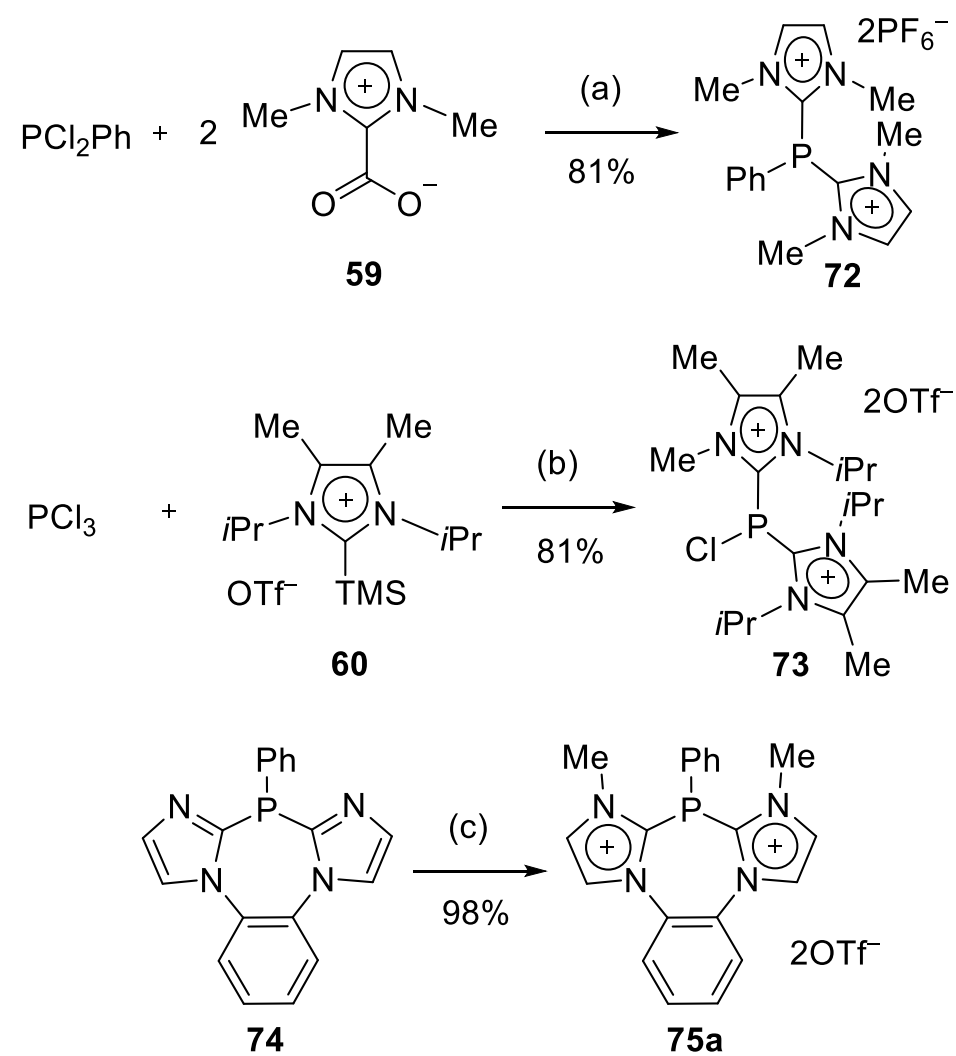

Scheme 17. Different approaches towards dicationic phosphines. Reagents and conditions: (a) 59 (2.0 equiv.), $\mathrm{CH}_{2} \mathrm{Cl}_{2}$, rt, 30 min, then $\mathrm{KPF}_{6}$, EtOH, rt, 24 h; (b) 60 (2.0 equiv.), PhF, $50{ }^{\circ} \mathrm{C}$, ultrasonic bath, 24 h; (c) MeOTf (2.0 equiv.), $\mathrm{CH}_{2} \mathrm{Cl}_{2},-78^{\circ} \mathrm{C}$ to rt, $2 \mathrm{~h}$.

Our group recently reported the direct reaction of bis(amino)cyclopropylidene 76, prepared by lithium-halogen exchange of $65 \mathrm{a}$, with dichlorophosphines to give the dicationic derivatives $\mathbf{7 7}$. Sterically bulky substituents such as adamantyl and mesityl were amenable to this protocol (Scheme 18). ${ }^{[92]}$ Recently the Chauvin group also described a similar method to synthesize dicationic phosphines. ${ }^{\left[{ }^{93]}\right.}$ Additionally, the in situ deprotonation of bis(imidazolium) salt 78 and condensation with dichloroethylphosphite to give dicationic $75 \mathrm{~b}$ has been described by the same group. ${ }^{[58]}$ The direct combination of dichlorophosphines with free carbenes, however depends strongly on the nature of the carbene used, as evidenced by competitive reductive processes when employing other imidazol-2-ylidenes. ${ }^{[94]}$ 


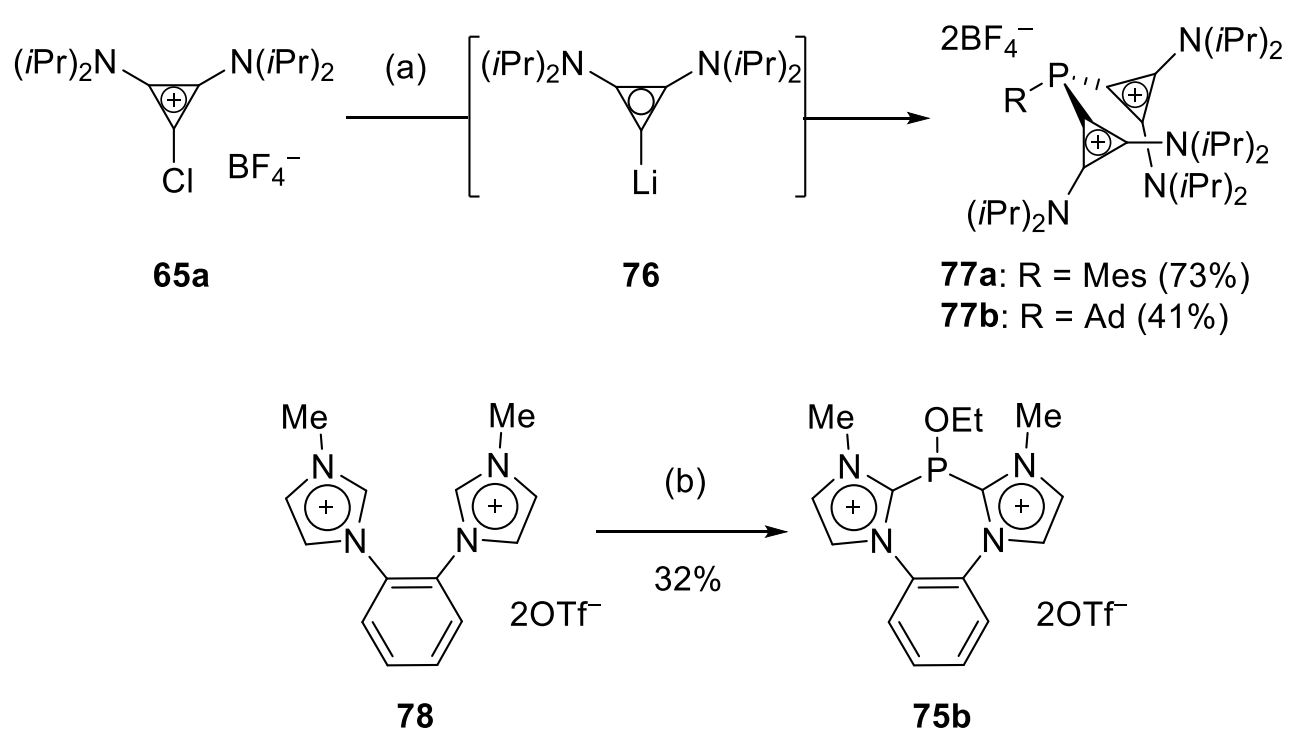

Scheme 18. Synthesis of dicationic phosphines. Reagents and conditions: (a) 65a (1.0 equiv.), $n \mathrm{BuLi}$ (1.0 equiv.), $\mathrm{THF},-78{ }^{\circ} \mathrm{C}, 15 \mathrm{~min}$, then $\mathrm{PCl}_{2} \mathrm{R}^{1}$ (0.5 equiv.), THF, $-78^{\circ} \mathrm{C}$ to rt, $16 \mathrm{~h}$; (b) EtOPCl 2 (1.0 equiv.), Et $3 \mathrm{~N}$ (2.0 equiv.), $\mathrm{CH}_{2} \mathrm{Cl}_{2},-78^{\circ} \mathrm{C}$ to $\mathrm{rt}, 2 \mathrm{~h}$.

The reverse "onio" strategy has also been utilized by the Alcarazo group towards the synthesis of dicationic cyclopropenium, ${ }^{[92,95]}$ formanidinium ${ }^{[84]}$ and dihydroimidazolium ${ }^{[84]}$ phosphines, again offering a complimentary scope of cationic substituents when compared with other methods (Scheme 19). Generally, formation of the first C-P bond proceeds more readily than the second one, where the corresponding monocationic phosphine 78 must be deprotonated with one equivalent of a base to improve conversion. This method therefore additionally allows the attachment of differently substituted cationic frameworks to the same phosphorus center, as evidenced in the synthesis of dicationic cyclopropenium salts 77 . The Alcarazo group recently described the dicationic bis(phosphines) $\mathbf{7 9 b}$ and (rac)-80, which were synthesized according to the same strategy, using triethylamine as a base. The presence of an axially chiral axis in $\mathbf{7 5}$ mimics the BIPHEP family of ligands. 


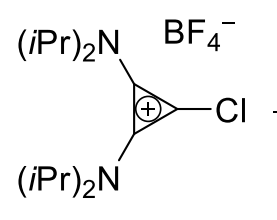

$65 a$

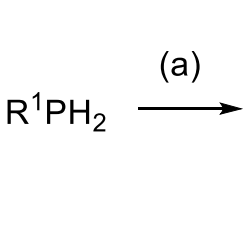

78a: $R^{1}=P h(76 \%)$

78b: $R^{1}=$ Cy $(65 \%)$

78c: $R^{1}=o-B i P h(61 \%)$ (b)

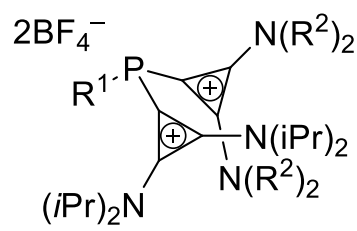

77c: $\mathrm{R}^{1}=\mathrm{Ph}, \mathrm{R}^{2}=i \operatorname{Pr}(69 \%)$

77d: $R^{1}=P h, R^{2}=\operatorname{Hex}(65 \%)$

77e: $\mathrm{R}^{1}=\mathrm{Ph}, \mathrm{R}^{2}=i \operatorname{Pr}(69 \%)$

77f: $R^{1}=o-B i P h, R^{2}=i \operatorname{Pr}(49 \%)$

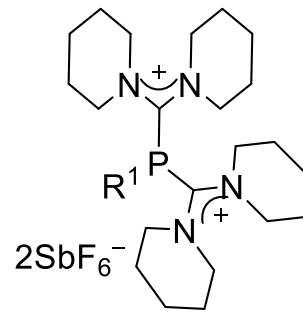

81a: $R^{1}=P h(41 \%)$

81b: $R^{1}=$ Cy $(73 \%)$ (c)

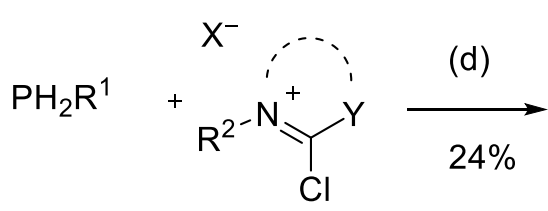

68

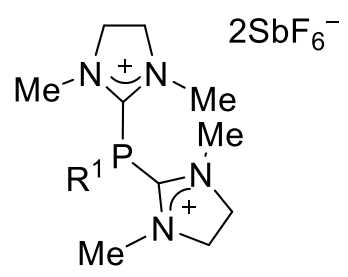

79a: $\mathrm{R}^{1}=0-\mathrm{BiPh}$

(e)

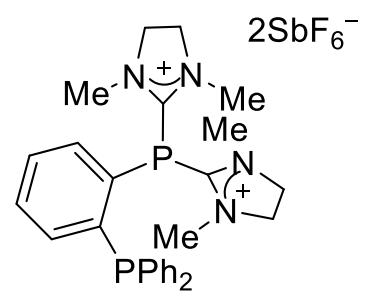

79b: $(72 \%)$

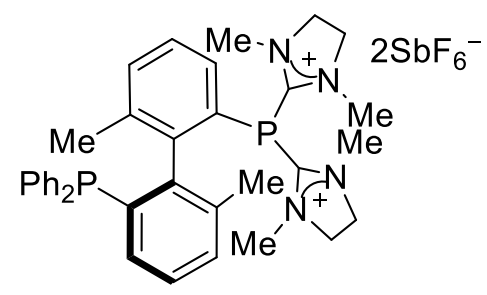

(rac)-80: (41\%)

Scheme 19. Reverse "onio" strategy towards dicationic phosphines. Reagents and conditions: (a) 65a (2.0-3.0 equiv.), THF or diglyme, $60-100^{\circ} \mathrm{C}, 16 \mathrm{~h}$; (b) KHMDS (1.0 equiv.), THF, $-40^{\circ} \mathrm{C}, 2 \mathrm{~h}$, then $65 \mathrm{a}$ ( 1.0 equiv.), rt, $16 \mathrm{~h}$; (c) 68 (2.0 equiv.), Et ${ }_{3} \mathrm{~N}$ (2.1 equiv), THF, $65^{\circ} \mathrm{C}, 16 \mathrm{~h}$, then aq. $\mathrm{NaBF}_{4}$; (d) 68 (2.0 equiv.), KHMDS, (2.0 equiv.), THF, $-78{ }^{\circ} \mathrm{C}$ to rt, $16 \mathrm{~h}$, then $\mathrm{MeCN}^{\mathrm{N}} \mathrm{NaSbF}_{6}, \mathrm{rt}, 16 \mathrm{~h}$; (e) 68 (2.0 equiv), Et $3 \mathrm{~N}\left(2.0\right.$ equiv.), THF, $60^{\circ} \mathrm{C}, 16 \mathrm{~h}$.

Dicationic phosphines and phosphonites display less rich coordination chemistry, owing to the diminished donor properties of the resulting compounds. Indeed, bis(imidazolium) derivatives such as $\mathbf{7 5 a}$ or $\mathbf{7 5 b}$ have not been found to coordinate any metal. Nevertheless, the complexes of $\mathrm{Au}(\mathrm{I}), \mathrm{Pd}(\mathrm{II})$ and $\mathrm{Pt}(\mathrm{II})$ with the cyclopropenium-derived dicationic phospines $\mathbf{7 7}$ have been described, most likely due to the comparatively less electronwithdrawing nature of thecyclopropenium substituent. ${ }^{[8]}$ One strategy to broaden the coordination chemistry of dicationic phosphines is to introduce a second chelating phosphorus centre, such as in the chelating dicationic phosphines 79b and (rac)-80 (cf. Scheme 19). Indeed, a wider variety of coordination complexes can be formed using these chelates, and includes $\mathrm{Mo}(0), \operatorname{Rh}(\mathrm{I}), \mathrm{Pd}(\mathrm{II})$ and $\mathrm{Pt}(\mathrm{II})$ centers. ${ }^{[83,96]}$

Only a limited number of tricationic phosphines have to date been described, by the groups of Alcarazo ${ }^{[97]}$ and Weigand. ${ }^{[90]}$ Treatment of tris(trimethylsilyl)phosphine with three 


\section{Introduction}

equivalents of chlorocyclopropenium salts $\mathbf{6 5}$ led to the smooth formation of trications $\mathbf{8 2}$. The steric bulk of the $\mathrm{N}$-alkyl substituents played a major role in this reaction, with higher yields being observed for the methyl derivative $\mathbf{8 2 b}$. Weigand and coworkers also described the trication $\mathbf{8 3}$, which was synthesized by refluxing the trimethylsilyl carbene adduct $\mathbf{6 0}$ with trichlorophosphine.

Unsurprisingly, no coordination chemistry for trication $\mathbf{8 3}$ has been described. Trication $\mathbf{8 2 b}$, however, was found to coordinate $\mathrm{Pt}(\mathrm{II})$ and even $\mathrm{Au}(\mathrm{I})$, although this complex was not stable at room temperature. ${ }^{[97]}$

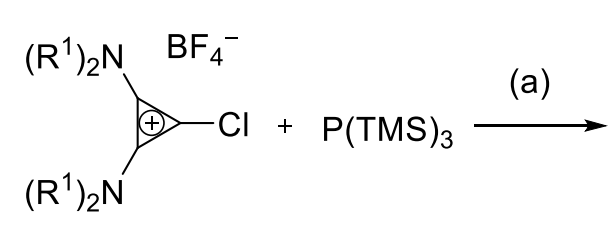

65

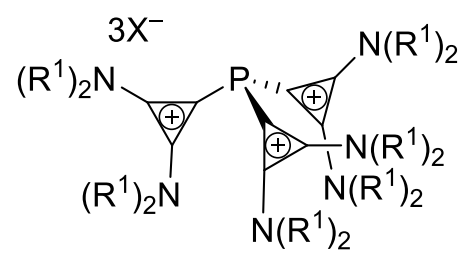

82a: $\mathrm{R}^{1}=i \mathrm{Pr}, \mathrm{X}=\mathrm{BF}_{4}(22 \%)$

82b: $R^{1}=M e, X=T f O(73 \%)$

(b)<smiles></smiles>

83

Scheme 20. Synthesis of tricationic phosphines 82 and 83. Reagents and conditions: (a) 60 (3.0 equiv.), PhF, 60 ${ }^{\circ} \mathrm{C}, 16-72 \mathrm{~h}$; (b) 60 (3.0 equiv.), $140{ }^{\circ} \mathrm{C}, 3 \mathrm{~h}$.

\subsubsection{Structure and electronic properties of cationic phosphines and phosphonites}

The strong $\pi$-accepting nature of cationic phosphines and phosphonites can be explained through an examination of their frontier orbitals. In phosphorus-based ligands, the HOMO is predominantly located at the phosphorus lone pair thus participating in their $\sigma$ donor ability, and the LUMO is comprised by the $\sigma^{*}$ orbitals relating of the three phosphorus substituents, which accounts for the $\pi$-accepting properties of the ligand. An a-cationic substituent would be expected to lower all the molecular orbitals of the corresponding phosphine, therefore reducing its overall net electron-donating ability. Secondary orbital interactions between the phosphorus lone pair and the low lying $\pi$-system of an aromatic cationic substituent should also be possible, contributing to a further reduction in the $\sigma$-donor ability (Figure 9a). Additionally, stabilization of the $\sigma^{*}$ orbitals of the other phosphorus substituents through 
constructive overlap with the $\pi$-system of the cationic substituent could also lead to a further increase in the $\pi$-accepting ability (Figure 9b).

a)

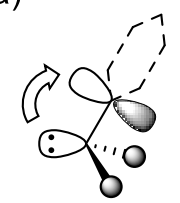

b)

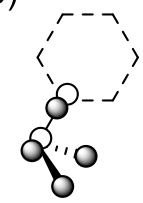

Figure 9. Secondary orbital interactions in cationic phosphorus based ligands.

An experimental quantification of the donor properties of phosphines can be found in the measurement of the carbonyl stretching frequencies of the corresponding $\mathrm{Ni}(\mathrm{L})(\mathrm{CO})_{3}$ complexes, where $L$ is the phosphine under study, in a technique known as the Tolman analysis. ${ }^{[98]}$ To avoid the handling of highly toxic nickel tetracarbonyl, calculation of the $\mathrm{NiL}(\mathrm{CO})_{3} \mathrm{CO}$ stretching frequency can be achieved, offering a viable alternative. ${ }^{[99]}$ In addition, synthesis of the corresponding $\mathrm{RhClCO}(\mathrm{L})_{2}$ has gained popularity. In these systems, the overall net donor character of the phosphine can be deduced, based on the shift in the stretching frequency of the metal bound carbonyl ligands. A stronger donor translates to increased $\pi$-backbonding to the carbonyl ligand from the metal into the $(C-O) \pi^{*}$ orbital. This decreases the bond strength and translates into a lowered $\mathrm{CO}$ stretching vibration. In many cases when the measured phosphine displays a large steric demand, formation of the trans-[RhClCO$\left.(\mathrm{L})_{2}\right]$ complex leads to a significant deviation of the ligands from an ideal square planar geometry. ${ }^{[86]}$ Because this has an additional impact on the orbital overlap in the complex, comparison of the resulting stretching frequencies is not always possible. ${ }^{[88]}$

A Tolman electronic map, comprising phosphines (orange), phosphites (purple) and cationic phosphines (green) or arsines (pink) is shown in Figure 10. Experimentally determined values are shown as red points, while calculated ones are shown as blue points. Generally, monocationic phosphines or arsines can be seen to occupy the region of the map between phosphites and polyhalogenated phosphines such as $\mathrm{PCl}_{3}$ or $\mathrm{PF}_{3}$. Introduction of one or more additional charge increases the stretching frequency still, placing polycationic phosphines 82b, 77c and 72 at the upper reaches of the map alongside $\mathrm{AsF}_{3}$ and $\mathrm{PF}_{3}$. Cationic ligands offer distinct advantages in comparison, however, with polyhalogenated phosphines or arsines being highly flammable and toxic gases or liquids. Cationic phosphines are in contrast solids that can be easily handled under ambient conditions. While dicationic 72 would be expected to possess the strongest m-accepting abilities, its lack of coordination ability limits its utility as a ligand. Only slightly less donating, $\mathbf{8 2 b}$ and $77 \mathrm{c}$ with 
cyclopropenium substituents still possess enough net donor ability to form a limited number of coordination complexes.

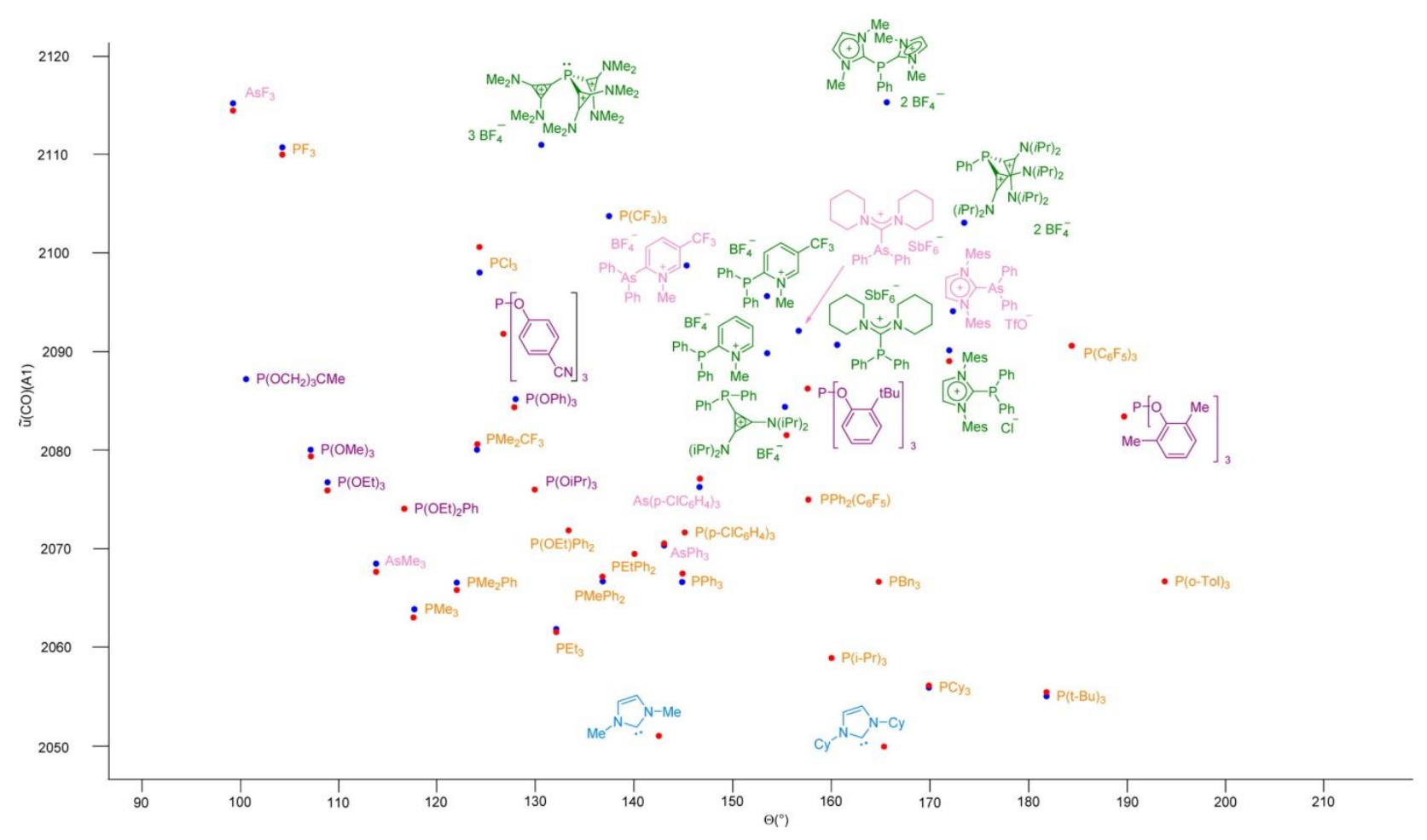

Figure 10 Tolman electronic map for a variety of different phosphorus based ligands. Experimentally determined values shown as points in red, calculated in blue. Adopted from: M. Alcarazo, Acc. Chem. Res. 2016, 49, 1797. ${ }^{[88]}$

An alternative method of measuring the donor ability of phosphines, which can avoid potential problems in the synthesis and measurement of metal carbonyl complexes, is the determination of oxidation $\left[E_{P}(0 x)\right]$ and reduction $\left[E_{P}(r e d)\right]$ potentials by cyclic voltammetry. $A$ variety of cationic ligands, as well as commercially available phosphines and phosphites are shown in Table 1. ${ }^{[88]}$ Although the oxidation potentials of the phosphines shown in Table 1 are irreversible, qualitatively they follow the same trends as shown in the Tolman electronic map (Figure 10). The monocationic phosphines 66a and 67 lie in the range of trimethyl phosphite, while others such as 71a and 69a exceed it. Similarly, the polycationic ligands display the largest oxidation potentials. Entries 10 and 12 show imidazoyl-/imidazoliumderived cationic phosphines $\mathbf{8 4 a}$ and $\mathbf{7 5 a}$, which were part of a wider study by the Chauvin group into the coordination limit of cationic ligands. ${ }^{[2]}$ It was found that, while the monocationic 84a would still coordinate some metal centers such as $[\mathrm{Rh}(\mathrm{cod}) \mathrm{Cl}]_{2}$, the dicationic 75a was completely inert towards coordination. They proposed that the coordination limit may therefore lie between these two ligands. 
Table 1. Oxidation potentials, calculated vs ferrocene/ferrocenium, $\mathrm{Bu}_{4} \mathrm{NPF}_{6}(0.1 \mathrm{M})$ in $\mathrm{CH}_{2} \mathrm{Cl}_{2}$.

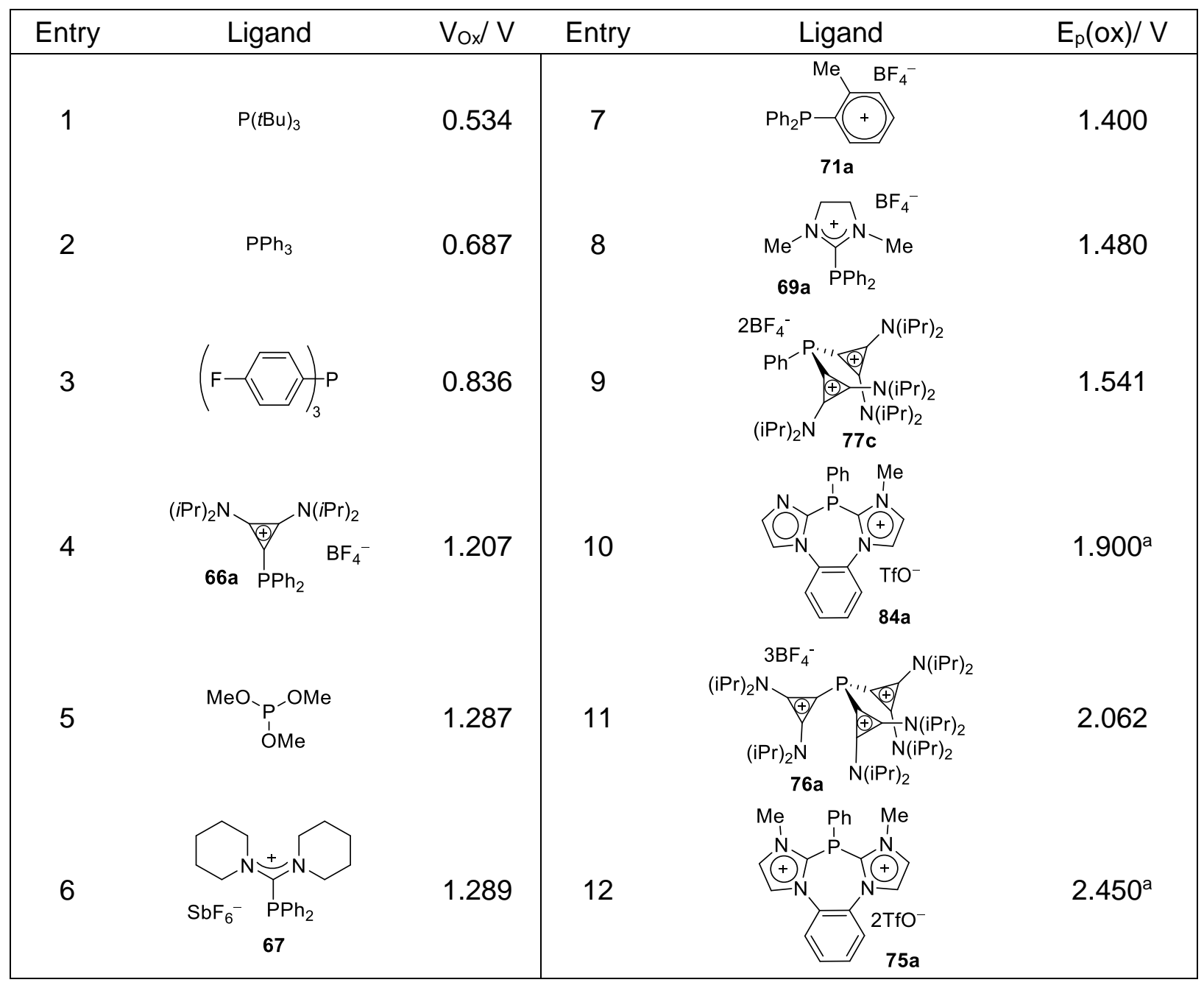

aMeasured in $\mathrm{CH}_{3} \mathrm{CN}$, adapted to same scale from ref. ${ }^{[72]}$ using conversion constant listed in ref. ${ }^{[100]}$. Unless otherwise stated, all oxidations were irreversible.

Although a qualitative picture of the net donor properties can be deduced from the aforementioned measurements, the relative contributions that different cationic substituents have with regards to the $\sigma$-donating and $\pi$-accepting properties of the resulting phosphine cannot be determined. The Alcarazo group recently calculated the HOMO and LUMO energies for a variety of cationic substituents, in an effort to shed light on these aspects, shown in Figure 11. ${ }^{[88]}$ As can be seen, introduction of any of the calculated cationic substituents significantly lowers both the HOMO and LUMO energy of the resulting phosphine, in comparison with triphenylphosphine. However, between the different cationic substituents, the position of the HOMO is not significantly affected, lying between -9.05 and $-9.85 \mathrm{eV}$ for the compounds in the series. It is the LUMO, which is strongly influenced by the nature of the cationic substituent, with the cyclopropenium $(-4.10 \mathrm{eV})$, imidazolium $(-4.37$ $\mathrm{eV})$, formamidinium $(5.00 \mathrm{eV})$ and pyridinium $(6.07 \mathrm{eV})$ substituents giving respectively decreasing LUMO energies, thus resulting in higher $\pi$-accepting properties for the 


\section{Introduction}

corresponding phosphines. In turn, it is therefore likely that the variation in the donor ability observed for the differently cationic phosphines may be primarily attributable to changes in their $\pi$-accepting character.

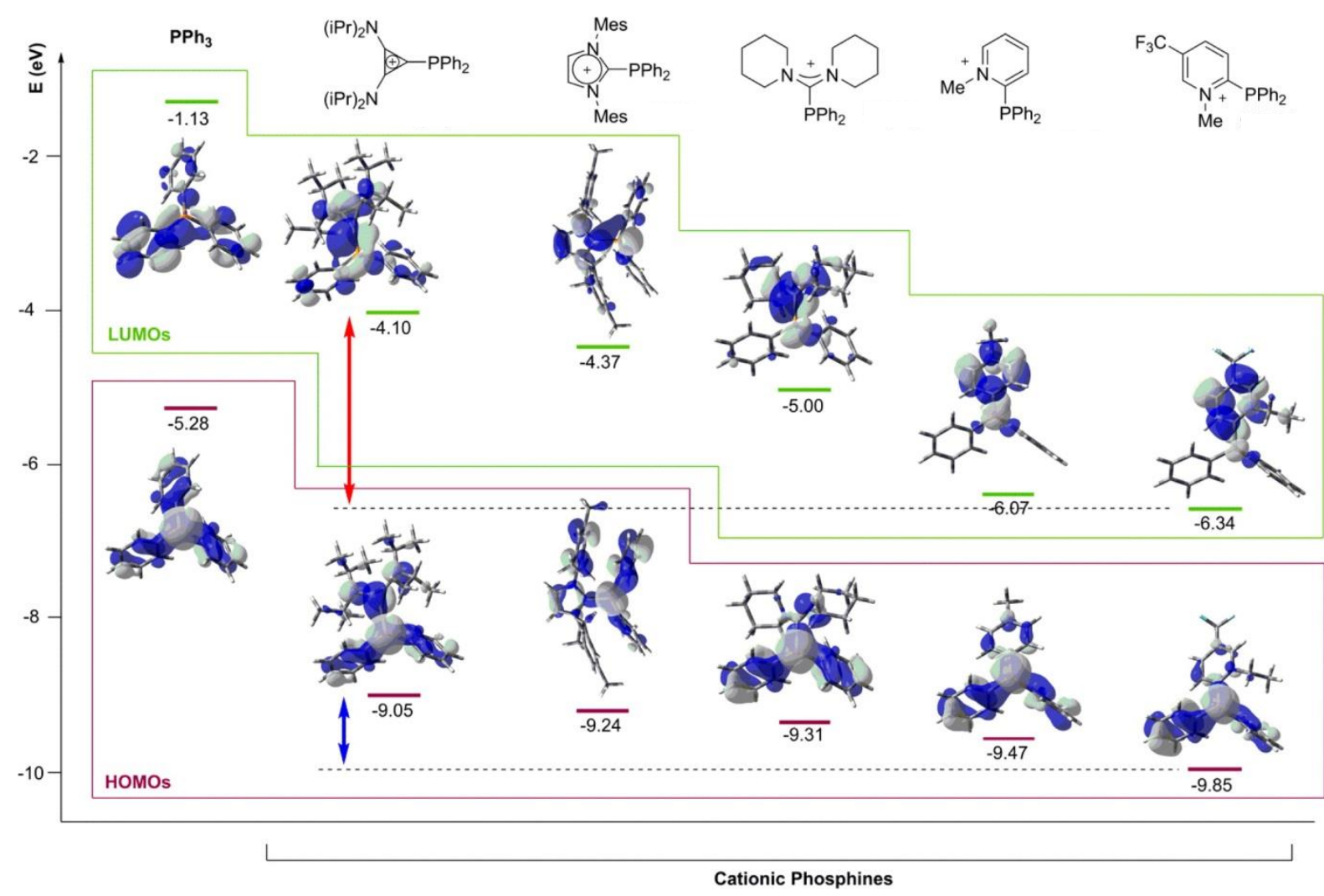

Figure 11. Calculated HOMO and LUMO energy levels for a variety of cationic phosphines. Figure taken from: M. Alcarazo, Acc. Chem. Res. 2016, 49, 1797.[69]

\subsubsection{Applications in catalysis}

Based on their increased polarity and high stability, initial applications of $\alpha$-cationic phosphine-containing complexes were primarily as recyclable phase-transfer catalysts in polar or biphasic media. This can be beneficial to save the use of volatile organic solvents and use easily reused and greener solvents such as ionic liquids. A cationic catalyst system should be highly soluble in such polar media, and ideally would enable easy separation of reaction products after simple phase separation or extraction. In this regard, a variety of reactions, including Negishi, ${ }^{[73]}$ Suzuki, Heck and Sonogashira cross couplings, ${ }^{[101]}$ alkynylation, ${ }^{[102]}$ hydroformylation, ${ }^{[103]}$ hydrogenation, ${ }^{102]}$ hydrosilylation ${ }^{[104]}$ and ene-yne cycloisomerisation $^{[105]}$ have been described and demonstrated the recyclability of cationic phosphines as catalysts in such a reaction medium. For example, a recent report by the group of Alonso and Ramón studied pyridinio phosphines as deep eutectic solvent (DES) compatible ligands for a variety of palladium catalysed cross couplings (Scheme 21). ${ }^{[101]} \mathrm{A}$ DES medium is formed on mixing different Lewis or Brønsted acids or bases and exhibit a 
lower melting or phase transition temperature than their individual components. The cationic pyridinio phosphines were superior to a variety of conventional phosphine ligands for an assortment of Suzuki couplings, such as in the formation of $\mathbf{8 7}$, when the reactions were conducted in a DES medium. Similarly, the same catalyst worked efficiently for Sonogashira and Heck couplings and the catalyst system could be recycled up to five times without loss of activity after simple extraction of the products with cyclopentylmethyl ether.<smiles>CC(=O)c1ccc(Br)cc1</smiles>

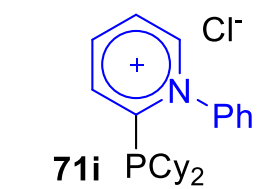<smiles>C#CP(C)c1ccccc1</smiles><smiles>C[N+](C)CCO</smiles>

94<smiles>OCC(O)CO</smiles><smiles>CCOC(=O)/C=C/c1ccc([N+](=O)[O-])cc1</smiles>

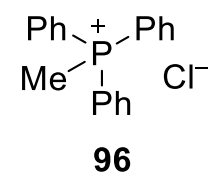

Scheme 21. Pyridinio-phosphines as deep eutectic solvent (DES) compatible ligands in palladium catalyzed Suzuki, Sonogashira and Heck couplings. Reagents and conditions (a) $\mathrm{PdCl}_{2}$ (0.1 mol\%), $71 \mathrm{i}(0.3 \mathrm{~mol} \%), \mathrm{K}_{2} \mathrm{CO}_{3}$ (3 equiv.), 94/ 95 1:2, $100{ }^{\circ} \mathrm{C}, 2 \mathrm{~h}$; (b) $\mathrm{PdCl}_{2}(0.1 \mathrm{~mol} \%), 71 \mathrm{i}(0.3 \mathrm{~mol} \%), \mathrm{Pr}_{2} \mathrm{NH}$ (2 equiv.), 96/ $951: 2,80{ }^{\circ} \mathrm{C}, 5 \mathrm{~h}$; (c) 91 (1.2 equiv.), $\mathrm{PdCl}_{2}\left(0.2 \mathrm{~mol}^{\circ}\right)$, $71 \mathrm{i}(1 \mathrm{~mol} \%), \mathrm{NaOAc}\left(1.5\right.$ equiv.) $94 / 951: 2,100^{\circ} \mathrm{C}, 3 \mathrm{~h}$.

As previously mentioned, the donor properties of $\alpha$-cationic phosphines also significantly alter the electronic properties metals they coordinate. Cationic ligands, as strongly $\pi-$ accepting moieties, would be expected to also be beneficial as ligands in catalysis when: either the outcome of the reaction can be influenced by the electronic nature of the catalyst; or when the rate determining step of a reaction can be influenced by increasing the electrophilicity of the catalyst. In $\pi$-acid catalysis, there exist a number of reactions which possess either of these qualities. The Alcarazo group has actively investigated the application of cationic ligands to a number of such reactions, with an interest in following up with applications towards total synthesis. ${ }^{[88,106]}$

The strongly $\pi$-acidic pyridinio-phosphines 71 have been utilized in a variety of mechanistically different gold- and platinum-catalyzed reactions. ${ }^{[86]}$ The formal $[2+2]$ cycloaddition in 1,8-ene-yne 97 nicely demonstrated the superior reactivity of the pyridiniophosphine gold complex $\mathbf{9 8 g}$, which afforded the bicyclic product $\mathbf{9 9}$ in only a few minutes. This was significantly faster than any previously reported system, for which a substantial 


\section{Introduction}

ligand screen had already been conducted. ${ }^{[107]}$ Additionally, due to stabilizing arene-gold interactions arising from the $N$-aryl substituent, the high catalyst stability enabled the reaction to be conducted with only $0.2 \mathrm{~mol} \%$ catalyst loading (Scheme 22). ${ }^{[87]}$<smiles>C#CCCCC(CC=C(C)C)(CC=C(C)C)C(=O)OCC</smiles>

97
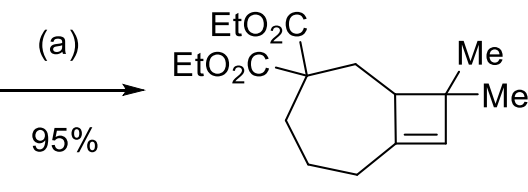

99

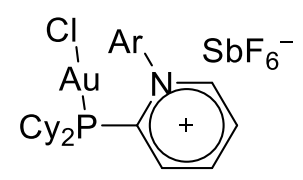

98g: $\mathrm{Ar}=2,6-(\mathrm{MeO})_{2}-\mathrm{C}_{6} \mathrm{H}_{3}-$

Scheme 22. Gold(I)-catalyzed reaction of ene-yne 97 . Reagents and conditions: (a) $\mathbf{9 8 g}\left(0.2 \mathrm{~mol}^{2}\right), \mathrm{AgSbF}_{6}(0.2$ $\mathrm{mol} \%), \mathrm{CH}_{2} \mathrm{Cl}_{2}$, rt, $16 \mathrm{~h}$.

Another interesting reaction was highlighted by the monocationic arsine-platinum complex $\mathbf{1 0 0}$, for the cyclopropanation reaction of 1,6-ene-yne 101 giving the highly strained tricyclic product 102 in $75 \%$ yield (Scheme 23). The evaluation of reactions of similar ene-ynes with terminal alkyne moieties had shown that even on heating with platinum the transformations proceeded slowly, ${ }^{[108]}$ and when using gold yields were low with the concomitant occurrence of polymerization products. For comparison, under the best conditions previously reported for the substrate 101 using gallium trichloride, compound 102 was obtained in $12 \%$ isolated yield. ${ }^{[109]}$ This result was greatly improved upon when using the cationic arsine 100, where the desired product could be obtained in $75 \%$ yield.<smiles>C#CCN([AsH3])C1C=CCCC1</smiles>

101 (a)

$75 \%$

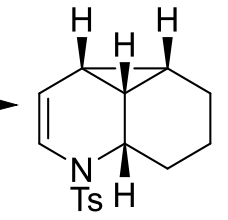

102

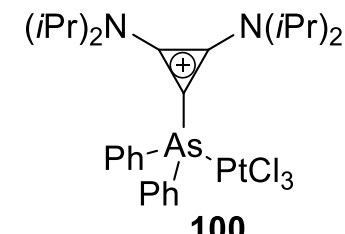

100

Scheme 23. Platinum-catalyzed cycloisomerization of ene-yne 101. Reagents and conditions: (a) 100 (2 mol\%), $\mathrm{AgSbF}_{6}(2 \mathrm{~mol} \%), 1,2-\mathrm{C}_{2} \mathrm{H}_{4} \mathrm{Cl}_{2}$, rt, $30 \mathrm{~min}$.

The dicationic bis(phosphine) $\mathbf{7 9 b}$ proved to be an excellent ligand in the $\mathrm{Rh}(\mathrm{I})$-catalyzed hydroarylation of aryl-substituted dienes. ${ }^{[83]}$ In this reaction both potential issues of reactivity as well as regioselectivity were overcome to give the products $\mathbf{1 0 3}$ in high yields and extend the scope of the reaction to the addition of azulenes, benzofurans as well as recorsinol derivatives. Importantly, the addition of $\mathrm{KB}\left(\mathrm{Ar}^{\mathrm{F}}\right)_{4}$ greatly improved conversion, due to the increased solubility of the resulting catalyst system (Scheme 24). 
(a)<smiles></smiles>

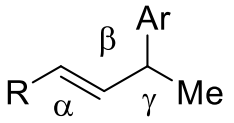

103

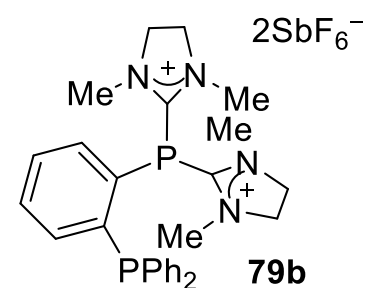

$\mathrm{Ph}$<smiles>COc1ccc(C(C)/C=C/c2ccccc2)c(OC)c1</smiles>

103a: (90\%), [98:2]<smiles>CC(/C=C/c1ccccc1)c1c[nH]c2cccc(F)c12</smiles>

103b: (65\%), [94:6]

103c: $(71 \%)$, [95:5]<smiles>CC(/C=C/c1c2ccccc2cc2ccccc12)c1ccc2cccccc1-2</smiles>

103: (68\%), [95:5]<smiles>CC(/C=C/c1ccccc1)c1coc2ccccc12</smiles>

103e: $(31 \%),[>99: 1]$

Scheme 24. Rhodium-catalyzed hydroarylation of dienes. Reagents and conditions: (a) $79 \mathrm{~b}$ (5 mol\%), $\left[\mathrm{RhCl}(\mathrm{CO})_{2}\right]_{2}(2.5 \mathrm{~mol} \%), \mathrm{KB}\left(\mathrm{Ar}^{\mathrm{F}}\right)_{4}(10 \mathrm{~mol} \%), 1,2-\mathrm{C}_{2} \mathrm{H}_{4} \mathrm{Cl}_{2}, 70{ }^{\circ} \mathrm{C}, 12 \mathrm{~h}$. Regiomers ratios are given in square parentheses.

An on-going topic of interest in the Alcarazo group, which has been studied in detail, is the $\pi-$ acid-catalyzed intramolecular hydroarylation reaction of ortho-alkynyl-substituted biphenyls such as 104 to give phenanthrenes $105 .{ }^{[110]}$ It was found that polycationic ligands were excellent catalysts in this transformation, using both platinum(II) ${ }^{[111]}$ and gold $(\mathrm{I})^{\left[{ }^{[5]}\right.}$ complexes. In the case of gold, the synthesis of highly strained phenanthrenes with two substituents in internal positions was possible, and proceeded with an exceptionally high rate when compared with other commercially available ligands (Figure 12).

The reaction mechanism, calculated on the DFT-D3 level of theory, indicated the nucleophilic attack of the arene towards the gold-complexed alkyne moiety as the rate-determining step of the reaction. The kinetic barrier to this step was greatly reduced when using dicationic $77 \mathrm{c}$ as a ligand (green pathway, Figure 13). 


\section{Introduction}<smiles>CC#Cc1cccc(C)c1-c1ccccc1C</smiles>

104a<smiles>Cc1cccc2ccc3cccc(C)c3c12</smiles>

$105 a$

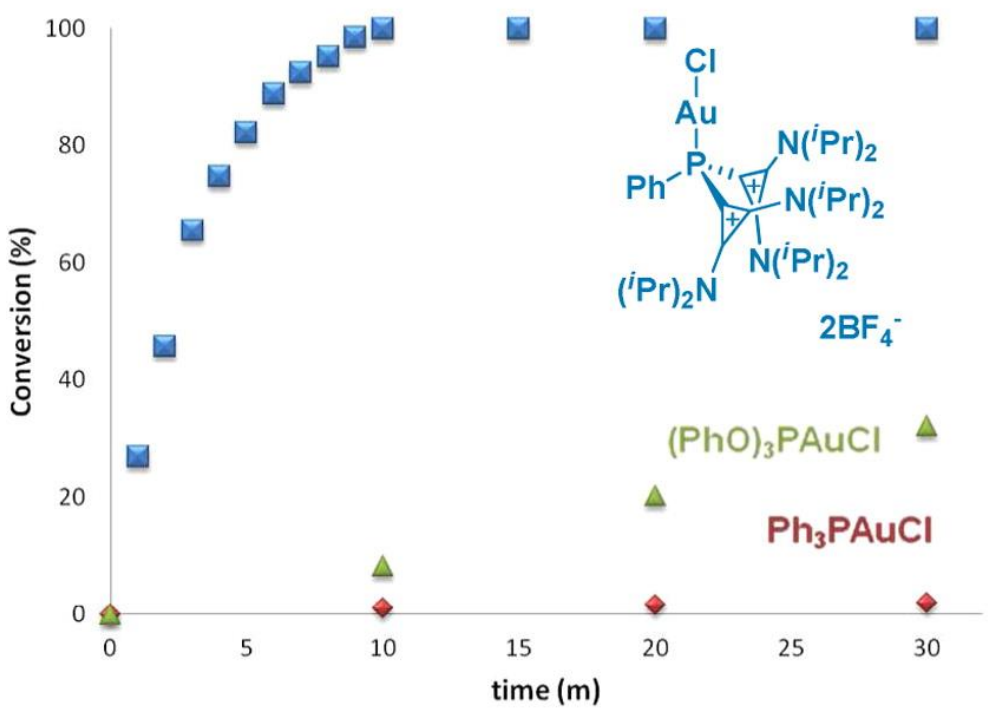

Figure 12. Gold-catalyzed intramolecular hydroarylation of o-alkynylbiphenyl 104a. Reagents and conditions: (a) gold precatalysts (2 mol\%), $\mathrm{AgSbF}_{6}(2 \mathrm{~mol} \%), \mathrm{CH}_{2} \mathrm{Cl}_{2}$, rt. Figure adapted from: J. Carreras, G. Gopakumar, L. Gu, L. Gu, A. Gimeno, P. Linowski, J. Petuškova, W. Thiel, M. Alcarazo, J. Am. Chem. Soc. 2013, 135, 18815. ${ }^{[76]}$

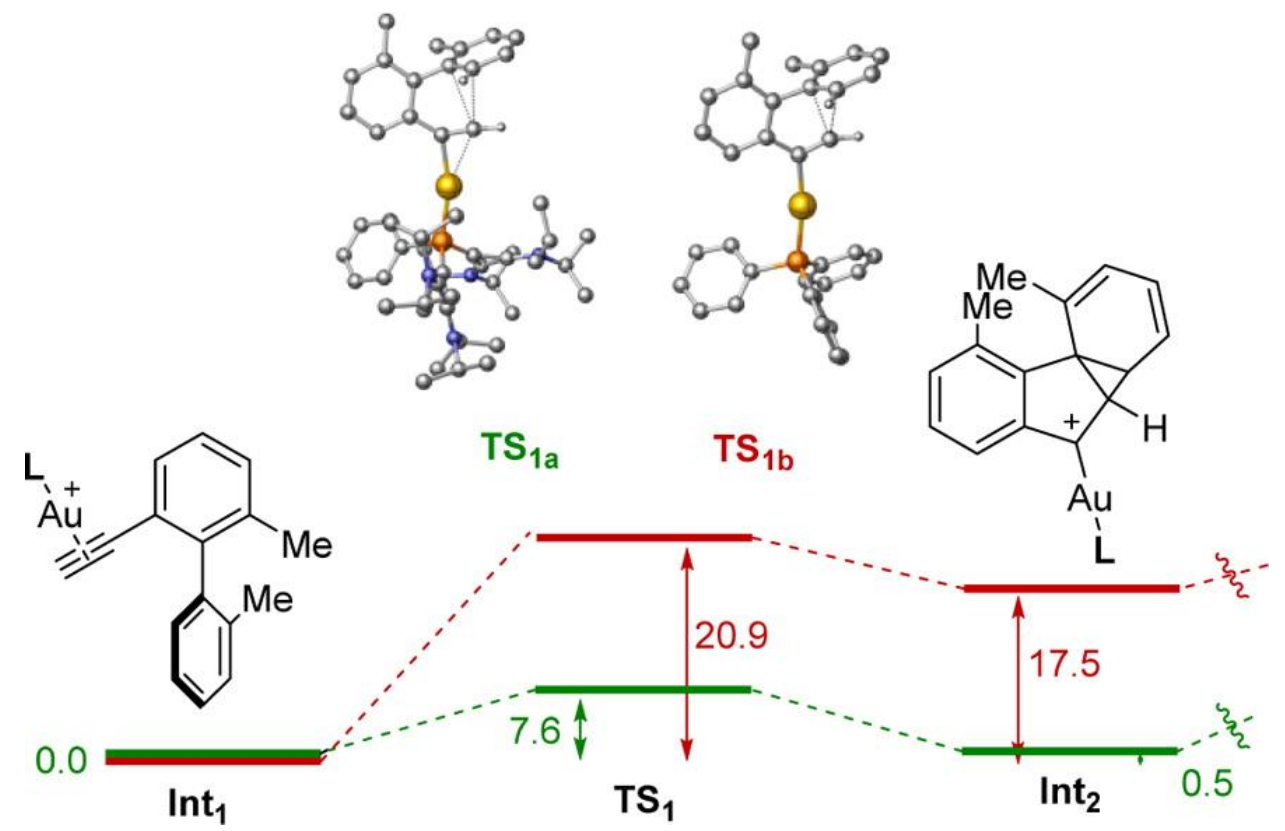

Figure 13. Calculated relative free energy profile of the rate-determining step of the gold(I)-catalyzed hydroarylation of $\mathbf{5}$. In red: using triphenylphosphine as a ligand; in green: using dicationic species $\mathbf{7 7 c}$ as a ligand. Figure taken from: J. Carreras, G. Gopakumar, L. Gu, L. Gu, A. Gimeno, P. Linowski, J. Petuškova, W. Thiel, M. Alcarazo, J. Am. Chem. Soc. 2013, 135, 18815. ${ }^{[76]}$ 
With this knowledge, intramolecular hydroarylations have been utilized to great effect by the group, exhibiting a broad scope for the synthesis of new condensed aromatics and heteroaromatics using arene-[95,111] furan-[92] thiophene-[84] and pyrrole-based ${ }^{[105]}$ nucleophiles. Since a number of naturally occurring phenanthrenes have been isolated from plants of the Orchidaceae family, the Alcarazo group has also attempted the synthesis of those compounds using this methodology. To this end, natural products such as Bulbophylantrin, ${ }^{[112]}$ Coeloginin, ${ }^{[13]}$ Epimedoicarisoside $A^{[114]}$ and Calanquinone $C^{[115]}$ have been synthesized to date (Figure 14).<smiles>COc1cc(CC(C)C)cc2cccc(O)c12</smiles>

Bulbophyllantrin

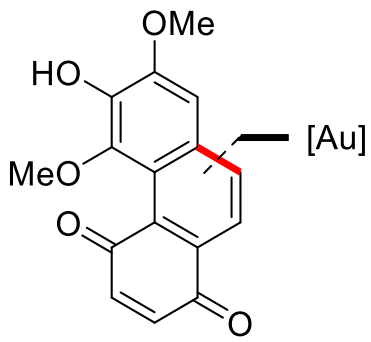

Calanquinone $\mathrm{C}$<smiles>COc1c(O)c(OC)c2c(=O)oc3cc(O)cc4c3c2c1CC4</smiles>

Coelognin<smiles></smiles>

Epimedoicarisoside A

Figure 14. Examples of naturally occurring phenanthrenes synthesized using cationic ligands.

In conclusion, $\alpha$-Cationic ligands have emerged as a rapidly growing class of exceptionally strong $\pi$-acceptor ligands, owing to their modular synthesis and high stability compared with other strongly electron withdrawing ligands. Despite their electron poor nature, many exhibit rich coordination chemistry. Numerous applications of these complexes have arisen in phase-transfer catalysis for their solubility in ionic liquids or aqueous media; however, until relatively recently, the applications of their electronic abilities in catalysis had not been explored. The intermolecular hydroarylation reaction of ortho-alkynyl biarenes is greatly influenced by this class of ligands and has been investigated, amongst other reactions, by the Alcarazo group. Using this methodology, a variety of new, highly twisted phenanthrenes have been prepared, as well as a variety of natural products. Key to these accomplishments is the lowering of the activation barrier for the nucleophilic attack of the arene to the goldactivated alkyne moiety, which is the rate-determining step when using strong $\pi$-acceptor ligands. Despite these advancements, the synthesis of chiral cationic ligands has yet to be 


\section{Introduction}

developed and investigated. Applications of such ligands in enantioselective hydroarylation reactions could have the potential to address challenges in the synthesis of highly strained polyaromatic systems through carbon-carbon bond formation.

\subsection{Synthesis, structure and applications of Helicenes}

One such class of polyaromatic compounds are helicenes; ${ }^{[116-124]}$ despite their numerous interesting properties and applications relating to their axis of chirality, the number of enantioselective methods applicable to their synthesis is limited. ${ }^{[117,120]}$ The following section will discuss their general properties, applications and selected methods towards their diastero- or enantioselective synthesis.

\subsubsection{General properties of helicenes}

Helicenes consist of a minimum of four ortho-fused benzene or other aromatic rings, which due to steric repulsions of the terminal rings form a helically chiral axis. The number of rings in a helicene is described by the number $n$ in brackets $[n]$, which indicates the number of ortho-fused aromatic rings. When a helicene is comprised solely of benzene rings in the backbone, it is denoted a carbohelicene; when the aromatic rings comprising its structure are thiophenes, pyrroles, pyridines or furans, the corresponding helicenes are denoted [n]thiahelicenes, [n]azahelicenes and [ $n$ ]oxahelicenes, respectively. The helical axis of a helicene can either wind clockwise or anti-clockwise. According to the Cahn-Ingold-Prelog rules, a left-handed winding of the helix (anti-clockwise) is denoted as the $M$ enantiomer, whereas a right-handed winding of the helix (clockwise) is denoted as the $P$ enantiomer. ${ }^{[125]}$ Similarly, the $M$ and $P$ enantiomers mostly give respective negative and positive optical rotation values.
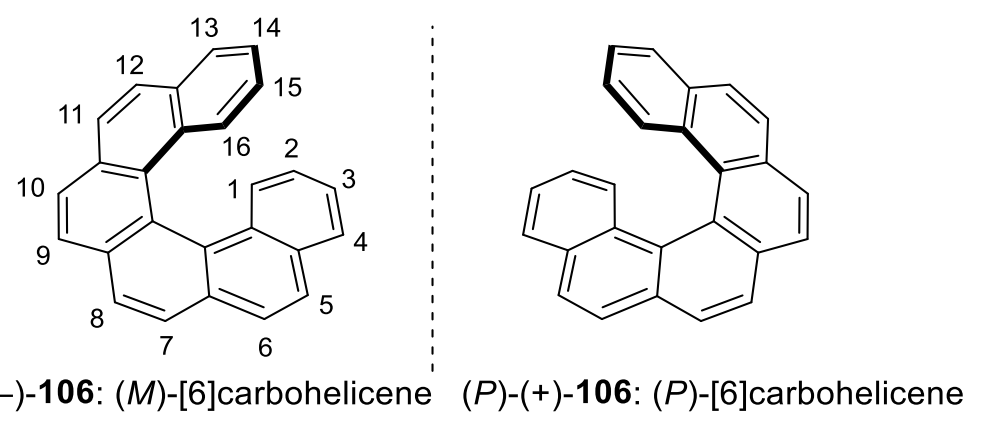

$(M)-(-)-106:(M)-[6]$ carbohelicene $(P)-(+)-106:(P)-[6]$ carbohelicene

Figure 15. Depiction of the two enantiomers of $\pi-[6]$ carbohelicene, including the standard numbering system for helicene carbons.

One of the striking properties of helicenes is their unusually high optical rotation values (Figure 16), which are likely due to the large spatial volume their chiral axis occupies. Combined with their extended, easily polarizable, aromatic systems, this has sparked interest 
in helicenes in a diverse array of fields, including asymmetric catalysis, supramolecular chemistry, macromolecular chemistry, nonlinear optics and optoelectronic materials, liquid crystals, nanoscience and biological chemistry. ${ }^{[116-124]}$

Generally, [6]carbohelicenes and higher [ $n$ ]carbohelicenes are considered to have a stable helical axis of chirality (Figure 16). Thus, while [5] helicene is known to racemize at $22{ }^{\circ} \mathrm{C}^{[126]}$ with a half-life of $1100 \mathrm{~min},{ }^{[127]}$ for [6]carbohelicene the reported $t_{1 / 2}($ rac.) is 187 min at 187.6 ${ }^{\circ} \mathrm{C} .{ }^{[128,129]}$ The racemization barrier dramatically increases however, on incorporating substituents into the internal "bay area" of the helix and 1-methyl-[5]helicene forms stable enantiomers. ${ }^{[130,131]}$

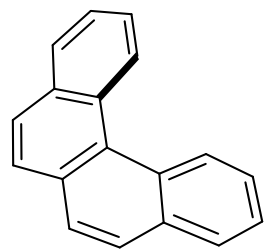<smiles>c1ccc2c(c1)ccc1ccc3ccc4ccccc4c3c12</smiles><smiles>c1ccc2c(c1)ccc1ccc3ccc4ccc5ccccc5c4c3c12</smiles>

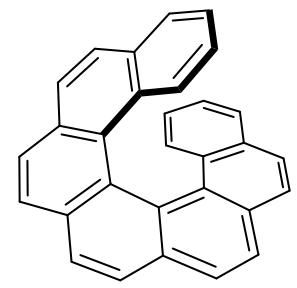

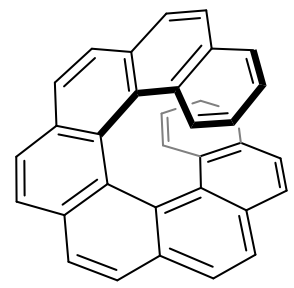

(M)-(-)-107:

(M)-(-)-108a:

$(M)-(-)-106:$

[5]helicene ${ }^{\mathrm{a}}$

[6]helicene ${ }^{b}$

$[\alpha]_{578}^{26}=-1670^{\circ}$
$($ iso-octane $)$

$[\alpha]_{589}^{22}=-3640^{\circ}$ ( $\mathrm{c}=0.098, \mathrm{CHCl}_{3}$ )

(M)-(-)-109:

[7] helicene ${ }^{\mathrm{c}}$ $[\alpha]_{579}^{25}=-5900^{\circ}$ $(\mathrm{c}=0.06$, $\left.\mathrm{CHCl}_{3}\right)$

$$
\begin{array}{rlcc}
E_{a}{ }^{*}=23.5 & E_{a}{ }^{*}=35.6 & E_{a}{ }^{*}=41.1 \quad E_{a}{ }^{*}= \\
\Delta\left(E_{a}^{*}\right)=12.1 \leftarrow \leftarrow \leftarrow\left(E_{a}^{*}\right)=5.5 \leftarrow \leftarrow \leftarrow \Delta\left(E_{a}^{*}\right)=0.5
\end{array}
$$$$
\text { (M)-(-)-110: }
$$$$
\text { [8]helicene }{ }^{\mathrm{d}}
$$

Figure 16. Optical rotations and racemization energies $\left(E_{a}^{*} / \mathrm{kcal}^{\mathrm{mol}}{ }^{-1}\right)^{[117]}$ of carbohelicenes. ${ }^{\mathrm{a} F r o m}$ ref. ${ }^{[126]}{ }^{\mathrm{b}}$ From ref. $^{[132]}$ cFrom ref. ${ }^{[129]}$ dFrom ref. ${ }^{[133]}$

When one or more five-membered rings make up the helicene, a higher number of rings is required to give a stable chiral axis. This is because of the smaller in-plane-turn of a five- $v s$. a six-membered ring. For example, the heterohelicenes 5,8-[6]dithiahelicene and 3,6,9[6]trithiahelicene have significantly lower barriers to racemization than [6]carbohelicene (Figure 17) and have been reported to not be configurationally stable at room temperature. ${ }^{[134]}$ [6]Carbohelicene racemizes via a symmetrical non-planar transition state, whereby the helix simultaneously widens to minimize the peripheral overlap (a process accommodated by all the aromatic rings of the molecule) and the last aromatic rings of the helix invert their respective positions. ${ }^{[135]}$ 
<smiles>c1ccc2c(c1)ccc1ccc3ccccc3c12</smiles>

108a: 24.6

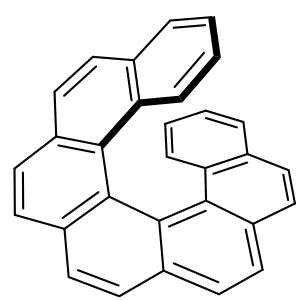

109: 41.7<smiles>Cc1cc(C)c2cc(C)c3ccc4ccc5ccccc5c4c3c2c1C</smiles>

108b: 38.7

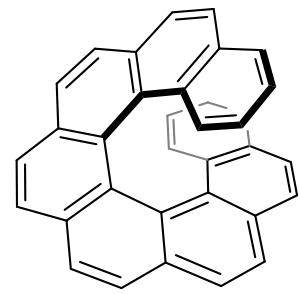

110: 42.4

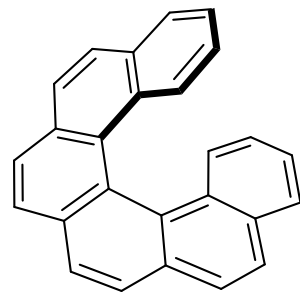

106: 36.9

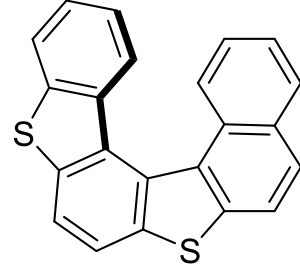

$111: 23.7$

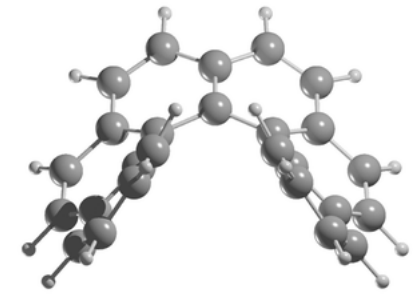

85: transition state

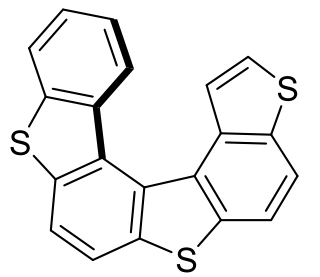

112: 22

Figure 17. Racemization barriers (in $\mathrm{kcal}^{\mathrm{mol}}{ }^{-1}$ ) for helicenes 108a, ${ }^{[126]}$ 108b, ${ }^{[130]} 106$ (including transition state), ${ }^{[135]} 109,110,{ }^{[128]} 111$ and $112 .{ }^{[134]}$

The helix of helicenes is, in addition, fairly flexible and can be extended and squashed with a relatively low energy barrier. A curious property of helicenes is that, unexpectantly, very few show a $\mathrm{C}_{2}$ symmetry in the solid state. Frequently, $\mathrm{X}$-ray crystallographic analysis shows that the different aromatic rings of the helix accommodate the torsional strain imposed by the helical geometry to a different extent, and that the structural features relating to the helical geometry can change depending on the crystallization conditions. ${ }^{[120]}$ The group of Sýkora recently investigated this phenomenon in greater detail, by studying hexahelicene and some of its derivatives in solution and in the solid state. ${ }^{[136]}$ These studies were corroborated by theoretical calculations and showed that the energy penalty to widen or narrow the pitch (defined as the height of one complete helical turn) of hexahelicene from the minimum energy value of $4.2 \AA$ was surprisingly low and to deviate by $0.4 \AA$ in either direction only required approximately $0.6 \mathrm{kcal} \cdot \mathrm{mol}^{-1}$ (Figure 18). The pitch of helicenes can therefore be expected to be reasonably fluctional in solution and to deviate in the solid state due to intermolecular packing forces. 


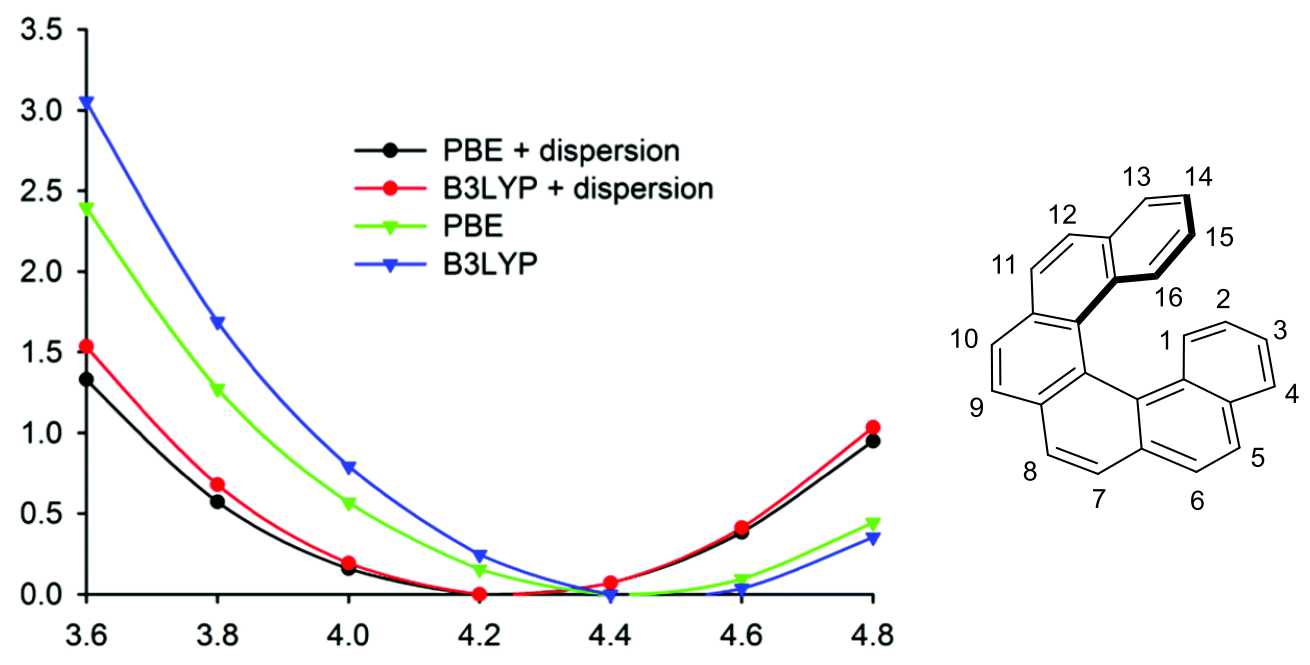

Figure 18. Calculated energy dependence $E_{\text {rel }} / \mathrm{kcal} \mathrm{mol}^{-1}$ vs. C2-C15 distance $(\AA)$ of hexahelicene. Figure taken from M. Dračinsky, J. Storch, V. Cirkva, I. Cisařova, J. Sýkora, Chem. Phys. Phys. Chem. 2017, 19, 2900.[105]

Another interesting feature of helicenes is the $\pi$-interactions of their polyaromatic system and the resulting absorbance and emission spectra. The helical twist still enables electronic delocalization throughout their structure, although the extent of $\pi$-conjugation is not as good as when compared to other planar polyaromatic systems. This is due to a slight loss of aromatic character because of the helical axis. ${ }^{[137]}$ Nevertheless the HOMO-LUMO gap of isomeric [ $n$ ] helicenes and phenacenes $(n \geq 6)$ has been calculated to be smaller for the helicenes, due to $\pi-\pi$ overlap across the helical pitches. This was calculated to steadily

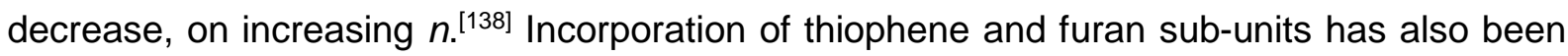
shown to decrease the HOMO-LUMO gap, so long as the thiophenes ${ }^{[139]}$ alternate with benzene rings to maintain effective $\pi$-conjugation. Other methods include annulating additional rings or helices onto the structure, therefore increasing the area of electron delocalization, ${ }^{[140,141]}$ or incorporating push-pull elements by introduction of electron donor/acceptor substituents. ${ }^{[142]}$ Finally, incorporation of an anti-aromatic subunit to the helicene also has a dramatic effect on the position of the frontier orbitals. ${ }^{[143]}$ Some selected helicenes and their HOMO-LUMO gaps are shown in Figure 19A.

Helicenes are also fluorescent compounds, although the quantum yield $\left(\Phi_{F}\right)$ is usually relatively low due to non-emissive quenching events decreasing the efficiency of fluorescence. For example, for [5]carbohelicene 87a $\Phi_{\mathrm{F}}=0.04 .{ }^{[144]}$. This can however be increased by incorporating long alkyl chains. Additionally, the wavelength of emission can be altered by modulation of the helicene substituents. ${ }^{[144,145]}$ Some examples of helicenes with high fluorescence quantum yields are shown in Figure 19B. 
A)<smiles>c1ccc2c(c1)ccc1ccc3ccc4ccccc4c3c12</smiles>

108a: $4.20 \mathrm{eV}$<smiles></smiles>

109: $3.87 \mathrm{eV}$<smiles>c1cc2c3c(ccc2s1)Sc1ccc2c-3c1-c1c(ccc3sccc13)S2</smiles>

113: $3.21 \mathrm{eV}$

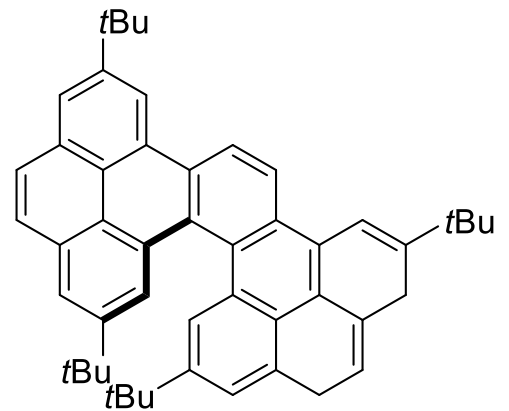

114: $3.61 \mathrm{eV}$

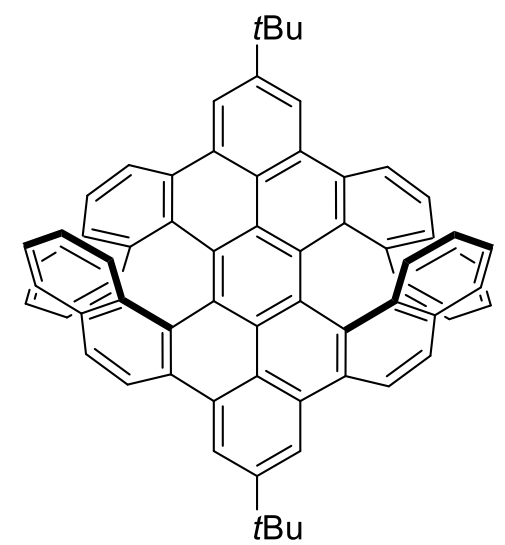

115: $2.65 \mathrm{eV}$<smiles>O=C1c2ccccc2-c2c1c1ccc3ccccc3c1c1c2ccc2ccccc21</smiles>

116: $2.43 \mathrm{eV}$

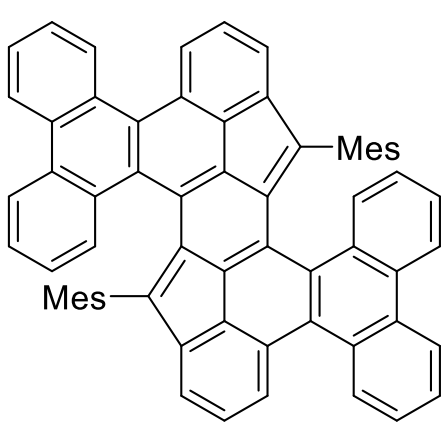

117: $1.88 \mathrm{eV}$

B)<smiles>CCCCc1ccc2nc(-c3ccc(C#N)cc3)oc3c4c(oc2c1)c4c1c3c2ccccc2n1CC(=O)O</smiles>

118: $\Phi_{F}=0.97$ (1,4-dioxane)

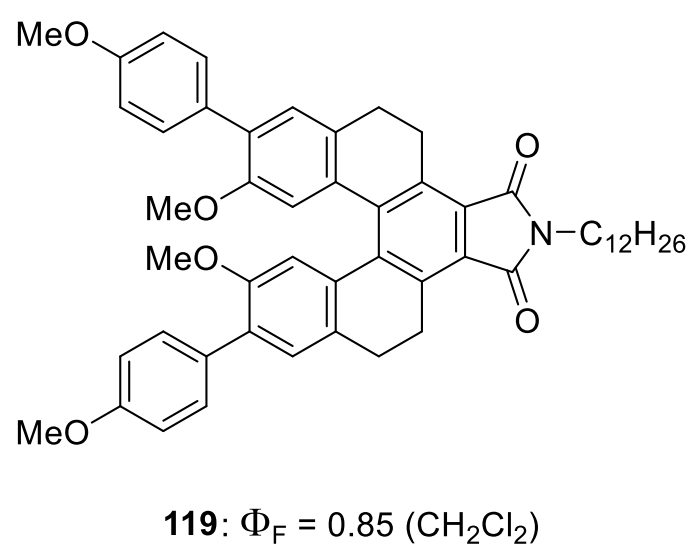

Figure 19. A) HOMO-LUMO gaps for the helicenes 108a, ${ }^{[142]} 109,{ }^{[146]} 113,{ }^{[139]} 114,,^{[141]} 115,{ }^{[140]} 116,,^{[142]} 117 .{ }^{[143]}$ B) examples of helicene derivatives with high fluorescence quantum yields $118^{[145]}$ and $119 .{ }^{[147]}$

\subsubsection{Applications of helicenes}

Helicenes exhibit a unique combination of exceptionally strong chiral transfer and a large aromatic $\pi$-system, which bestows them with interesting optical and electronic properties as well as the ability to readily form $\pi$-complexes and influence the formation of higher order structures. Some selected applications of helicenes will be described in this section to highlight the areas of research where they have attracted interest, maintaining an emphasis on recent examples as much as possible. 
One application of helicenes is in asymmetric catalysis. ${ }^{[116,119,148,149]}$ A recent series of examples of phosphahelicene-based catalysts was published by the group of Marinetti. These compounds were highly selective organocatalysts in the [3+2] cyclization of allenes and electron-poor olefins (Scheme 25A) ${ }^{[150]}$ and also as ligands in enantioselective gold(I) catalysis (Scheme 25B). ${ }^{[151,152]}$

A)

120<smiles>C1CCCCC1</smiles><smiles>[R][V]C1CCCCC1</smiles>

121<smiles>[R]C=C(C#N)C#N</smiles>

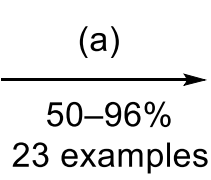

122: $R^{1}=A r ; R^{2}=\mathrm{CO}_{2} E t$, $\mathrm{CN} ; \mathrm{R}^{3}=\mathrm{Alk}, \mathrm{Ar}$ 82-97\% ee, $90-95 \% d r$

B)

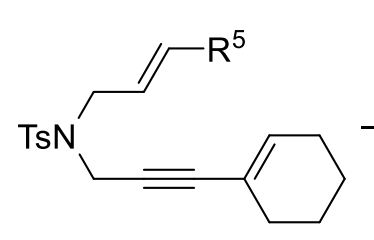

124 (b)

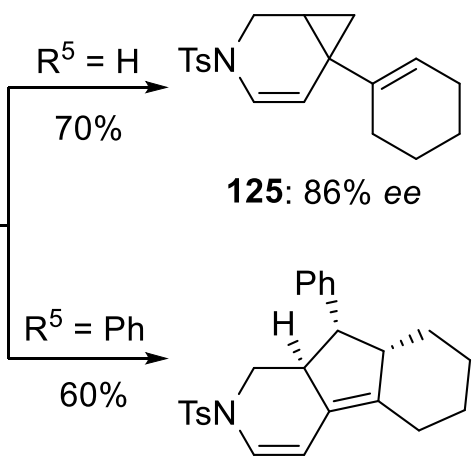

126: $84 \%$ ee<smiles></smiles>

$(P)-123 \mathrm{a}: \mathrm{R}^{4}=1 \mathrm{pc}$ *

$\left(S_{P}, P\right)-123 \mathbf{b}: \mathrm{R}^{4}=$ menth $^{*}$

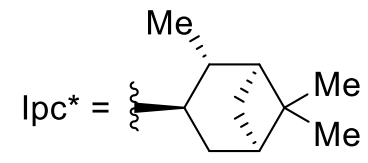

menth $=$

Scheme 25. Use of phosphahelicenes 123 as organocatalysts $(A)$ and in gold catalysis (B). Reagents and conditions: (a) (P)-123a (10 mol\%), toluene, $80{ }^{\circ} \mathrm{C}, 18-22 \mathrm{~h}$; (b) $\left(S_{P}, P\right)-123 b \cdot A u C l(4 \mathrm{~mol} \%), \mathrm{AgBF}_{4}(4 \mathrm{~mol} \%)$, toluene, rt, $16 \mathrm{~h}$.

For example, $\left(S_{P}, P\right)$-123a.AuCl efficiently catalyzed the ene-yne cyclisation of 124 into the products 125 and 126 with very good ee's. The reaction was found to be substrate dependent, and furnished the product 125 from terminal alkenes $\left(R^{5}=H\right)$ and 126 when $R^{5}=$ $\mathrm{Ph} .{ }^{[153]}$

The ability of helicenes to transfer chirality to other systems has additionally been explored in the field of chiral recognition, where helicenes have been utilized in the sensing of single chiral molecules. ${ }^{[116,119,148,149]}$ A tri-helicene organic cage was recently reported by Qu and co-workers (Figure 20). ${ }^{[154]}$ The cavity inside the cage could distinguish between enantiomers of racemic mixtures of chiral alcohols and amines, preferentially incorporating one enantiomer as a guest molecule that could then be later extracted. The enantiomeric excesses of the resulting extracts were impressive in comparison to the lower ee's obtained for other chiral cages. ${ }^{[155]}$ 

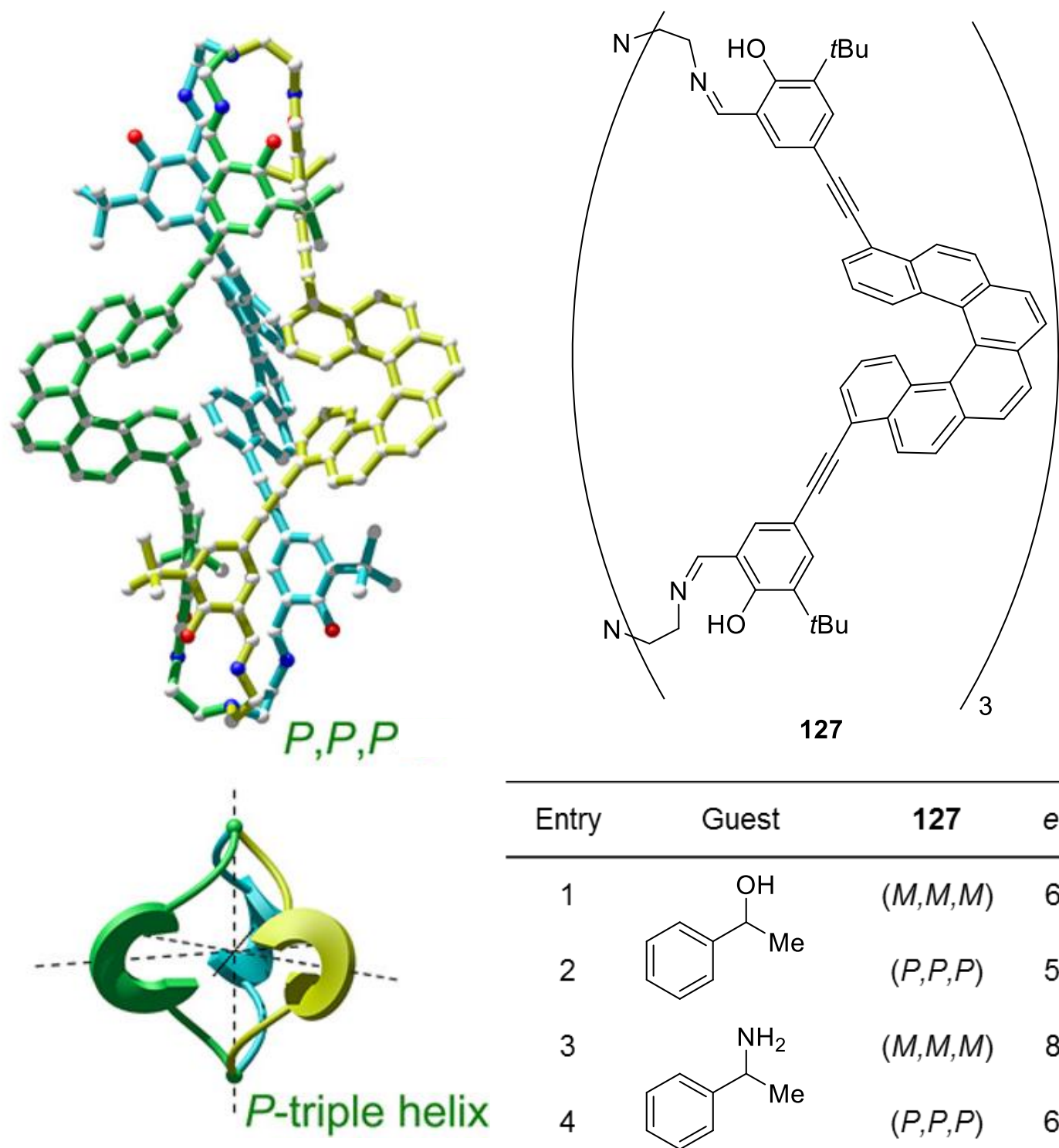

\begin{tabular}{cccc}
\hline Entry & Guest & 127 & ee (\%) \\
\hline & & \\
\hline
\end{tabular}

Figure 20. Host-guest complexes formed between 127 and racemic mixtures of chiral alcohols and amines. Crystal structure of $(P, P, P)-\mathbf{1 2 7}$ (top) and representation of triple helix geometry (bottom) are shown as well..154]

A variety of studies have also been conducted into the aggregative properties of helicenes, including as oligomers, covalently bonded materials and monomers that form higher structures. ${ }^{[116,119,121]}$ The group of Katz were important contributors to this field. In addition to other molecules, they synthesized the helicenebisquinone 128 $\mathbf{a}^{[156-158]}$ and found it to undergo self-assembly into large fibrous macrostructures (Figure 21). 
(A)

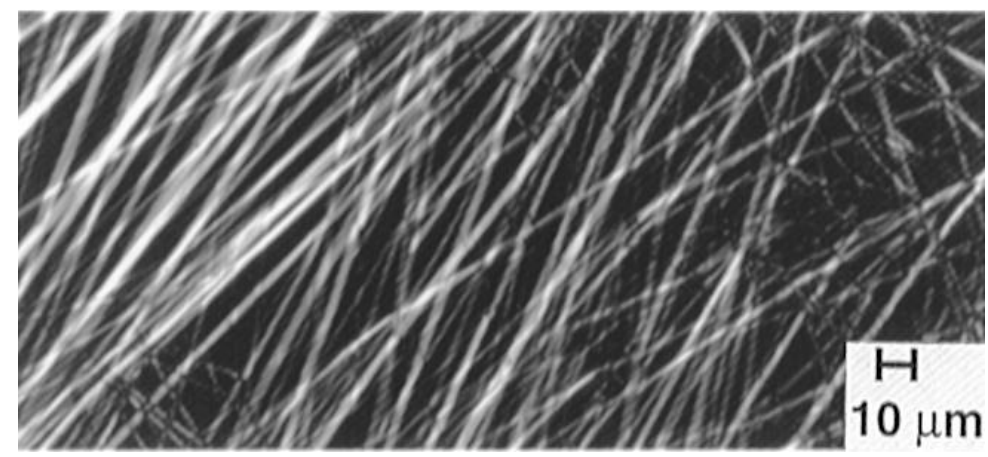

(C)

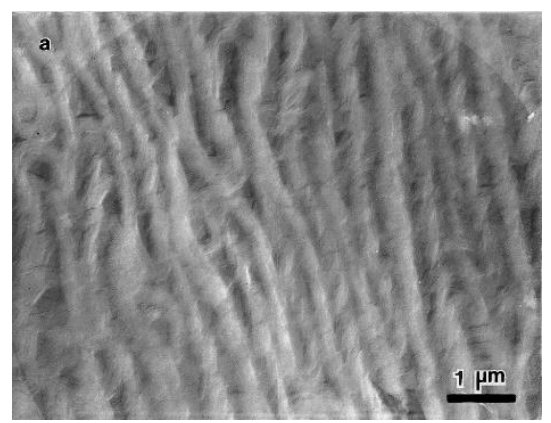

(D)
(B)
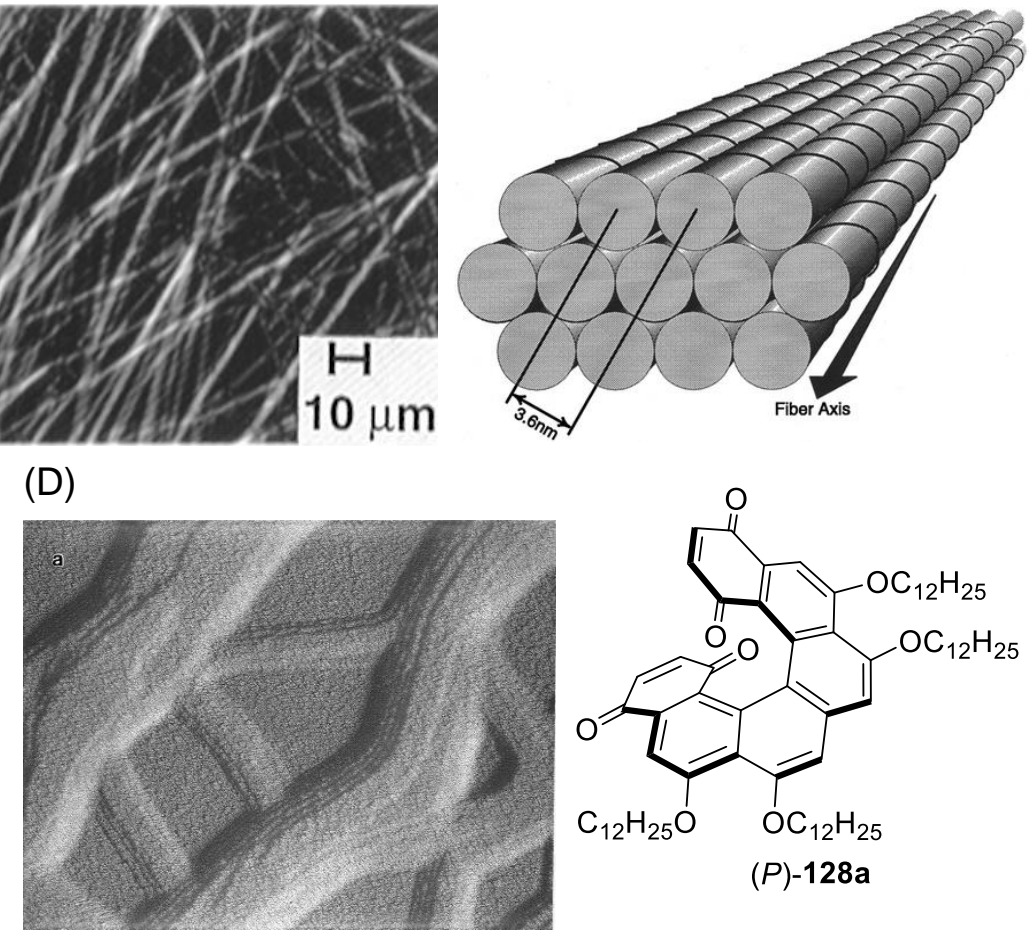

$(P)-128 \mathbf{a}$

Figure 21. Self-aggregation of 128a into fibres: (A) Optical micrograph; (B) representation of aggregation of fibres; (C) and (D) electron transmission microscopy of aggregates. Figure adapted from: C. Nuckolls, T. J. Katz, L. Castellanos, J. Am. Chem. Soc. 1996, 118, 3767-3768 ${ }^{[157]}$ and A. J. Lovinger, C. Nuckolls, T. J. Katz J. Am. Chem. Soc. 1998, 120, 264-268. ${ }^{[159]}$

This phenomenon, which could be detected using an optical microscope, was only observed by the authors for the enantiopure material (Figure 21A). ${ }^{[157]}$ Interestingly, the optical rotations and circular dichroisms were greatly enhanced for the aggregate material, compared with dilute solutions of 128a. Further analysis of a macroscopic liquid crystalline phase of $128 \mathrm{a}$ by X-ray diffraction, electron transmission microscopy and polarized light microscopy showed that the helicenes self-assembled end-to-end into helical fibres that aggregated into bundles (Figure 21B, 21C and 21D). ${ }^{[159]}$ Langmuir-Blodgett films of 128a also formed the same columnar aggregates, which again displayed significantly enhanced circular dichroism and optical rotations. The circular dichroism spectra could be measured for a single mono-layer of $\mathbf{1 2 8 a}$, which at that time was unprecedented. This is testament to the inherently large dichroisms of the helicene 128a and the following amplification of its chiroptical properties through aggregation. ${ }^{[160]}$ Furthermore, the aggregates of the LangmuirBlodgett film displayed a strong second order non-linear optical response, which was a direct consequence of the supramolecular chirality of the system. ${ }^{[161]}$

Another emerging field augmented by helicenes is so-called "chiranoscience", which studies chirality of systems on the nanoscale. ${ }^{[16]}$ An investigation of the absorption of 


\section{Introduction}

[7]carbohelicene onto a copper (110) surface by scanning tunneling microscopy (STM) showed that the racemic material self-organized into homochiral domains (denoted $\lambda$ and $\rho$ ), where even a small excess of one enantiomer led to a non-linear expression of chirality on the surface (Figure 22). ${ }^{[162]}$ The domains themselves were composed of both $(M)$ and $(P)$ enantiomers of the helicene alternating in a zig-zag geometry, but the lattice formed and the vectors of the respective domains were mirror images of each other, tilted by an angle of $\pm 10.9{ }^{\circ}$ with respect to the $\mathrm{Cu}(110)$ surface direction. This seminary work opened the possibility of building chiral surfaces that may lead to the development of future asymmetric heterogeneous processes.

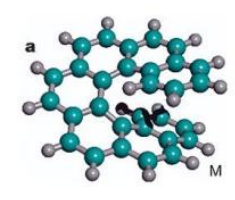

b
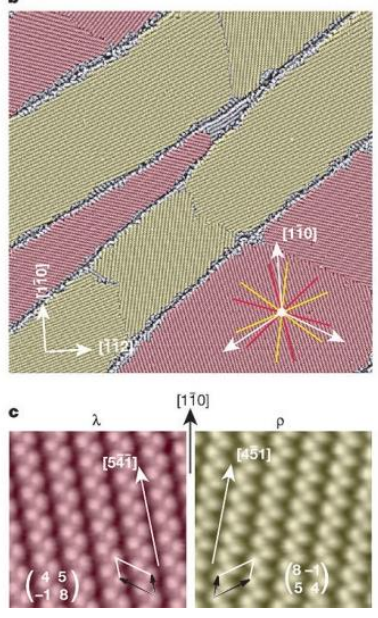
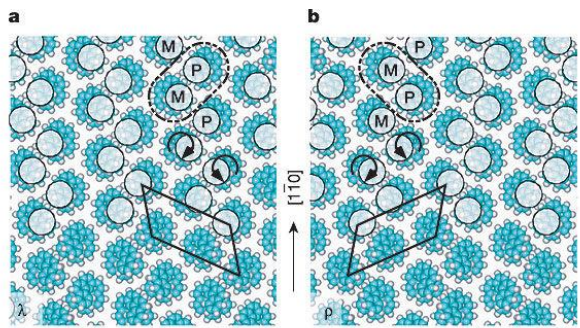

c
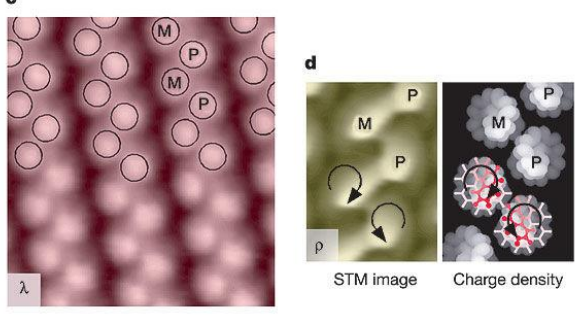

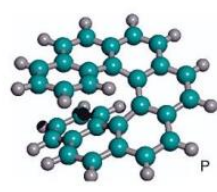

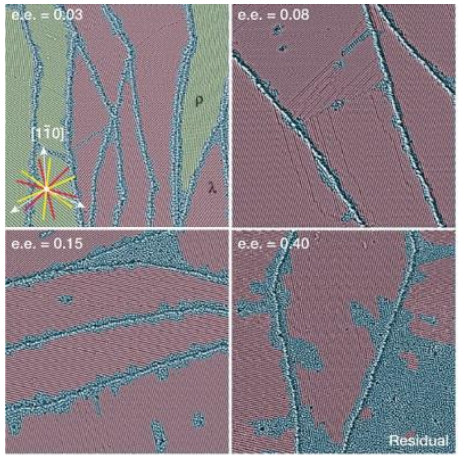

b

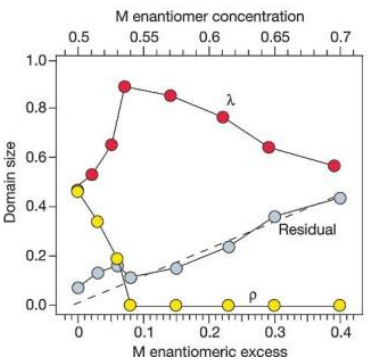

Figure 22. Absorption of [7]carbohelicene onto copper (110) surface and organization into homochiral domains: (left) STM image of $\lambda$ (red) and $\rho$ (yellow) domains; (middle) structure of domains, composed of zig-zag alternation of enantiomers and representation of vector of lattice; (right) composition of domains at carrying ee values. Figure taken from R. Fasel, M. Parschau, K. H. Ernst, Nature 2006, 439, 449. ${ }^{[162]}$

The tunable polyaromatic system of helicenes has attracted interest towards their possible applications in opto-electronic devices. Optical devices which directly emit circularly polarized light have been proposed to be more favorable in terms of simplicity, compactness, energy efficiency and cost. Fuchter and co-workers recently explored this concept, by doping a light emitting polymer (LEP) with either the enantiopure 1-aza[6]helicene or [7]carbohelicene and investigating the influence on the overall structure and the emissive properties (Figure 23). ${ }^{[163]}$ Prototypical devices doped with the enantiopure helicenes exhibited a strong brightness and emitted strongly circularly polarized light, indicating the helicene had a significant influence on the higher order structure of the material. The properties were comparable to other approaches using chiral light emitting polymers, ${ }^{[164]}$ however the 
advantages of this method are that the helicenes could be potentially doped into a variety of established PLED and OLED materials across the full visual spectrum and don't require bespoke chiral polymer synthesis. Other optical-electronic applications of helicenes include organic field-effect transistors, ${ }^{[165]}$ organic semi-conductors, ${ }^{[166]}$ dye sensitized solar cells, ${ }^{[145,167]}$ organic spin filters ${ }^{[168]}$ chiroptical switches, ${ }^{[169]}$ as well as biological imaging agents. ${ }^{[170]}$

A)

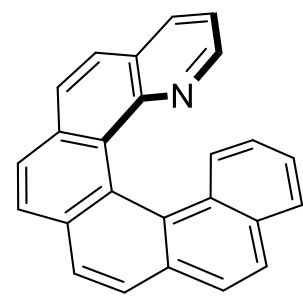

1-aza-[6]helicene

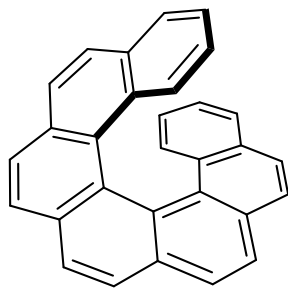

[7]carbohelicene

B)

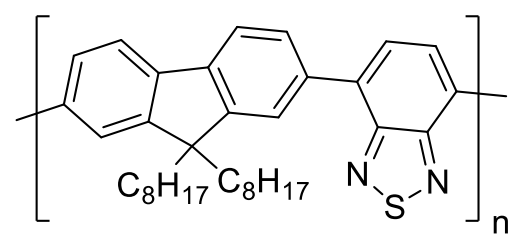

poly[9,9-dioctylfluorene-cobenzodithiazole] (F8BT)

C)

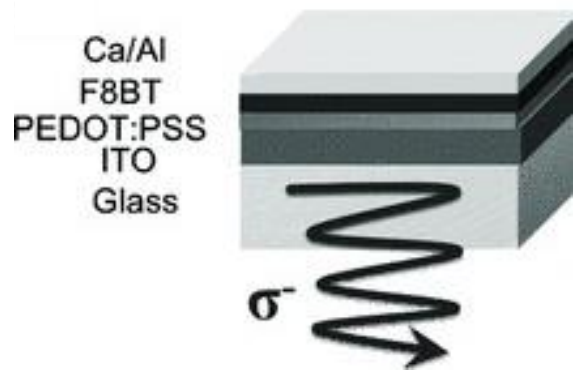

Figure 23. A) Helicenes doped into the circularly polarized light emitting device; B) light emitting polymer used in the study; C) schematic of circularly polarized light emitting device using $(M)$-helicenes. Figure taken from $\mathrm{Y}$. Yang, R. C. da Costa, D. M. Smilgies, A. J. Campbell, M. J. Fuchter, Adv. Mater. 2013, 25, 2624.[163]

All of the applications of helicenes mentioned in this section are a consequence of their unique chiroptical properties and the extent to which this has an influence on other systems be that in catalysis, in molecular recognition or chiral sensors. In many cases, helicenes display fascinating self-organization and amplification of their chiroptical properties on formation of higher order structures. Initiated by more fundamental studies, this is leading to an ever increasing number of applications, where helicenes could already be incorporated into a variety of efficient optical devices. Since their inherent chirality is at the forefront of their unique properties, it is interesting to note that all of the applications described in this section relied on the racemic synthesis of the helicene followed by its preparative chiral separation $^{[154,162,163]}$ or diastereoselective methods. ${ }^{[150,157]}$ In almost no cases was the helicene prepared enantioselectively. This highlights both the still limited number of ways in which helicenes can be accessed in their enantiopure forms as well as the need for ongoing 


\section{Introduction}

work on their enantioselective syntheses. The following sections will discuss the most prominent methods for the achiral, diastereoselective and finally enantioselective preparations of carbohelicenes up the writing of this thesis.

\subsubsection{Synthesis of helicenes}

\subsubsection{Transition metal free synthesis}

The synthesis of [6]carbohelicene was first described by Newman in 1955 ${ }^{[171]}$ and 1956, ${ }^{[132]}$ utilizing a 10-step Friedel-Crafts/dehydration sequence. Resolution could also be achieved by repeated crystallization with 2-(2,4,5,7-tetranitro-9-fluorenylideneaminooxy)propionic acid (TAPA). Although low yielding, this was highly innovative for its time and confirmed the helical chirality of [6]carbohelicene (Scheme 26).

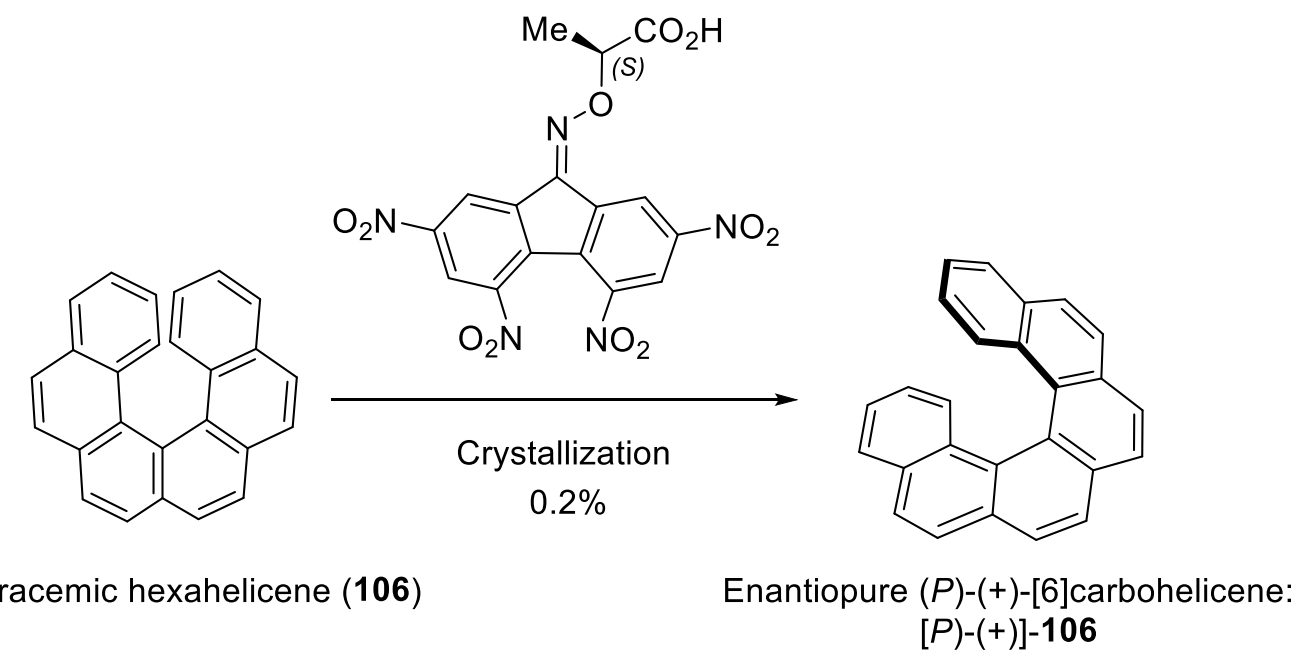

Scheme 26. First optical resolution of hexahelicene 106.

The first major breakthrough in helicene synthesis however, was the photochemical strategy reported by Schölz ${ }^{[172]}$ and Martin ${ }^{[173]}$ in 1967. The synthesis of [7]carbohelicene [(rac)-109] is shown in Scheme 27. ${ }^{[142]}$ Starting from 3-phenanthrylacetyl chloride (128) and phenanthrene (129), hydrocarbon (rac)-109 could be prepared in four steps with a 12.5\% yield for the photocyclization step. This enabled the preparation of functionalized [5]carbohelicenes, ${ }^{[174]}$ [6]-, [8]-, [9]-, ${ }^{[175]}[10]-$, [11]-, [12]-, [13]-[176] $[14]^{-[177]}$ and more recently [16]carbohelicenes, ${ }^{[178]}$ as well as other heterohelicenes. ${ }^{[120]}$ Later optimizations of Martin ${ }^{[176,179]}$ and Katz, ${ }^{[180,181,182]}$ led to improved yields and regioselectivities. ${ }^{[183]}$ Thus, [7]carbohelicene [(rac)-109] was prepared over four steps in $29 \%$ overall yield with a $75 \%$ yield for the photocyclization step. ${ }^{[181]}$

This method still stands out today as a way to quickly access small quantities of functionalized helicenes, ${ }^{[182]}$ since the synthetic precursors are easily prepared by Wittig olefination or Heck coupling and the reaction shows a good functional group tolerance. 
Drawbacks of helicene photochemical synthesis are that extremely high dilutions are necessary to avoid dimerization reactions, which limits the amount of helicene that can be obtained on a preparative scale. Additionally, highly pure solvents are required for the cyclization. Notably, no general enantioselective process for this type of reaction currently exists. $^{[17]}$

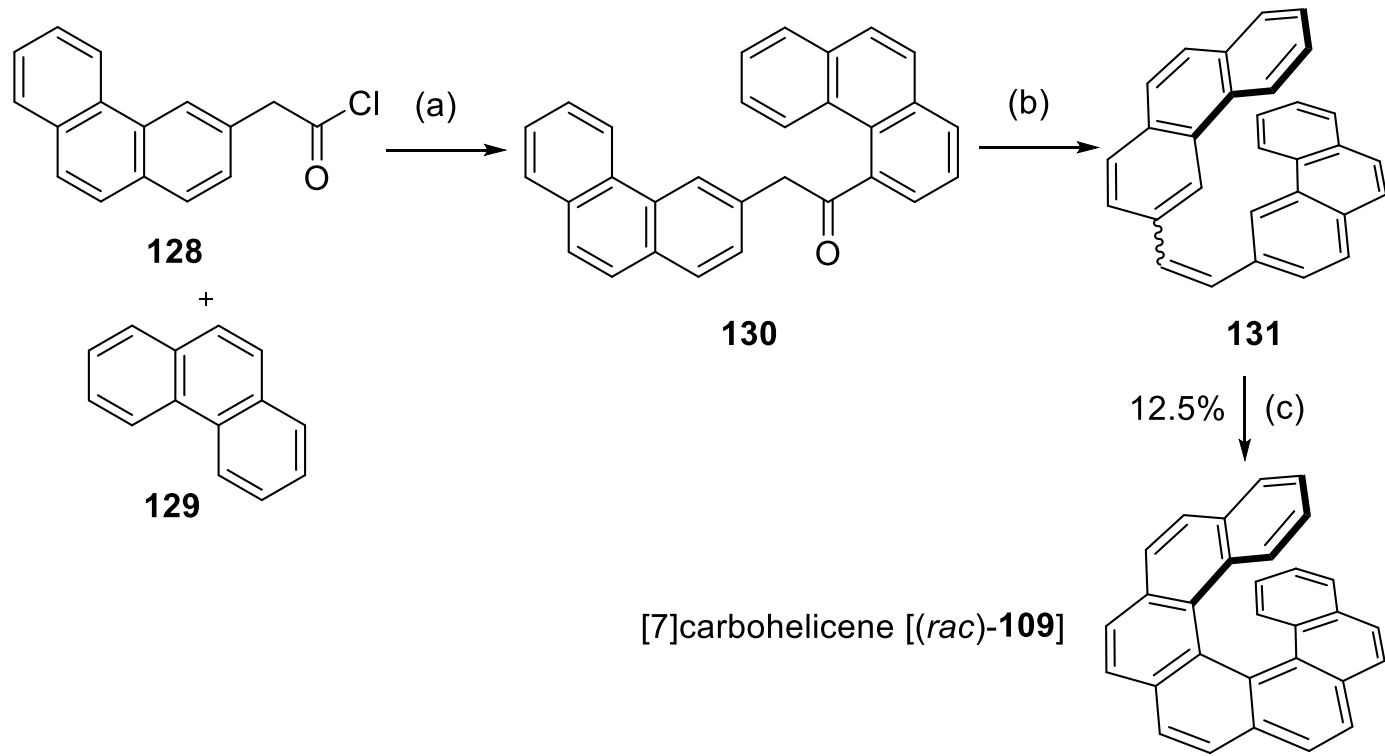

Scheme 27. Photochemical synthesis of [7]carbohelicene. Reagents and conditions: (a) 128, 129, $\mathrm{AlCl}_{3}$, $\mathrm{C}_{6} \mathrm{H}_{5} \mathrm{NO}_{2}, 20^{\circ} \mathrm{C}$; (b) $\mathrm{LiAlH}_{4}$, workup; then $\mathrm{HCO}_{2} \mathrm{H}$, heating (c) $h v\left(\mathrm{Hg}\right.$ middle pressure lamp), $\mathrm{l}_{2}, \mathrm{C}_{6} \mathrm{H}_{6}, 20^{\circ} \mathrm{C}, 8 \mathrm{~h}$, $12.5 \%$ yield.

Another breakthrough came in 1990 when Katz and coworkers described the synthesis of helicenes via a Diels-Alder reaction involving divinyl arenes 112 with an excess of $p$ benzoquinone (Scheme 28). ${ }^{[156,184]}$ The rates and yields of the reaction could be improved by adding electron-donating substituents to the diene molecule, especially at the vinyl moiety. Although the yields are in many cases diminished, this approach enabled rapid access to gram quantities of helicenes and accelerated further study of these compounds.

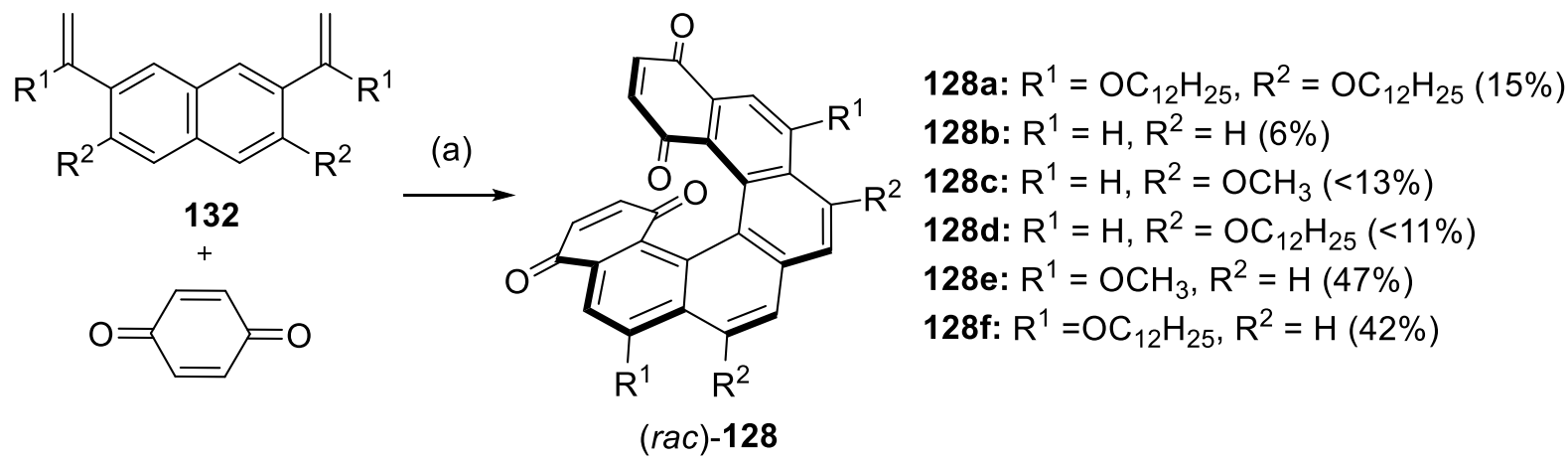

Scheme 28. Access to helicenebisquinones 128 through Diels-Alder strategy; selected examples. Reagents and conditions: (a)- $\mathrm{CCl}_{3} \mathrm{CO}_{2} \mathrm{H}$ or basic $\mathrm{Al}_{2} \mathrm{O}_{s}$ (cat.), toluene, reflux. 


\section{Introduction}

Importantly, the enantiomers of the racemic helicenes could be resolved by reduction to the bishydroquinone, ester formation with (-)-camphoryl chloride and diastereomeric resolution by crystallisation. The resolved enantiomers could then be reoxidised to the bisquinones without erosion of the enantiopurity. ${ }^{[157]}$ A diastereoselective variant of this Diels-Alder approach was reported by the group of Urbano and Carreño. The chiral information stems from a chiral sulfoxide auxiliary of quinone (S)-134 that was pyrolytically cleaved under the reaction conditions, giving helicenes such as $(M)-135$ in up to $88 \%$ ee (Scheme 29A). ${ }^{[185]}$ Considerably high pressures (4-12 kbar) were required for this reaction and compound $(M)$ 135a was furnished in low yields. The reactivity of the diene could be improved by using alicyclic variants such as 136, which furnished dihydrohelicenes 137 with good to excellent ee's (Scheme 29B). ${ }^{[186]}$ This approach was also extended towards partially saturated [4]-[187] and [7]carbohelicenes. ${ }^{[188]}$

A)<smiles>C=Cc1ccc(C=C)c2ccccc12</smiles>

133<smiles>C[Sb](=O)(O)C1=CC(=O)C=CC1=O</smiles>

$(S)-134$ (a)

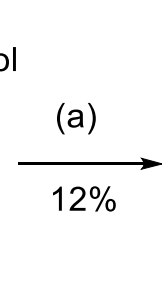

(1)<smiles>[R2]Oc1ccc(O[R])c2c3c(ccc12)CCC(C([R20])=C)=C3</smiles>

136<smiles>C[As](=O)(O)C1=CC(=O)C=CC1=O</smiles>

(b)

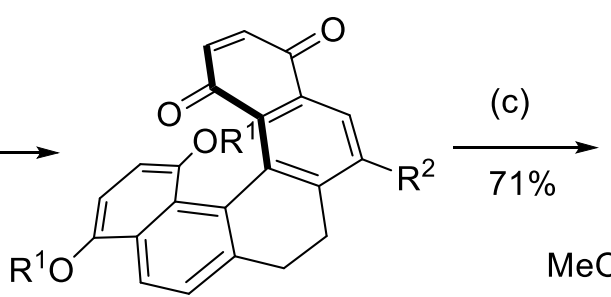

(S)-134

$(P)$-137a: $\mathrm{R}^{1}=\mathrm{Me}, \mathrm{R}^{2}=\mathrm{H}(72 \%): 84 \%$ ee

$(P)-137 b: R^{1}=M e, R^{2}=$ OEt $(62 \%): 92 \%$ ee

$(P)-137 \mathrm{c}: \mathrm{R}^{1}=$ OTBDMS, $\mathrm{R}^{2}=\mathrm{H}(75 \%):>98 \%$ ee

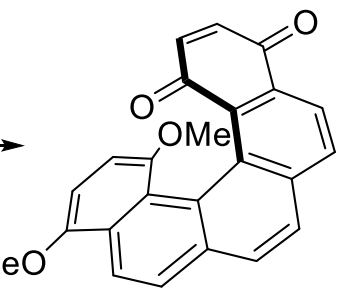

$(P)-138 a$

Scheme 29. Selected examples of diastereoselective Diels-Alder reactions to give carbohelicenes 135 and dihydrohelicenes 137. Reagents and conditions: (a) 134 (6.0 equiv.) $\mathrm{CH}_{2} \mathrm{Cl}_{2}, 4 \mathrm{kbar}, 4 \mathrm{~d}$; (b) 134 (2.0 equiv.) $\mathrm{CH}_{2} \mathrm{Cl}_{2},-40$ to $-60^{\circ} \mathrm{C}, 6-17 \mathrm{~d}$; (c) $D D Q, \mathrm{C}_{6} \mathrm{H}_{6}$, reflux, $4 \mathrm{~d}$.

\subsubsection{Transition metal-mediated synthesis}

A much larger number of transition metal-catalyzed synthetic transformations have been described for the synthesis of helicenes. Among them, Pd-catalyzed intramolecular $\mathrm{C}-\mathrm{H}$ bond arylations have been used, however, in racemic versions. ${ }^{[189]}$ Stará, Starý and coworkers pioneered a wide-ranging and largely successful approach, based on the $[2+2+2]$ cycloisomerization of triynes using $\mathrm{Co}(\mathrm{I})$ or $\mathrm{Ni}(0)$. Early reports, using $\mathrm{Co}(\mathrm{I})$ catalysis enabled the synthesis of a variety of partially saturated [5]-, [6]- and [7]- oxa-, aza- and 
carbohelicenes in mostly good yields. ${ }^{[190,191]}$ Later a variety of $O-, N-, S-$, and $P$ functionalized helicenes were described. Partially saturated helicenes such as $\mathbf{1 4 0}$ in Scheme 30 could be aromatized to the helicenes such as 141 using triphenylcarbenium tetrafluoroborate in excellent yields. ${ }^{[192]}$ This work was then extended to use $\mathrm{Ni}(0)$, which was generally more reactive, to the direct access of [6]- and [7]carbohelicenes from cis, cis-dienetriynes at room temperature. ${ }^{[193]}$ Generally, this method is widely applicable and amongst the types of helicenes accessible are [11]carbohelicene, ${ }^{[194]}$ a [19]oxahelicene, ${ }^{[195]}$ at this moment the largest known helicene that has been synthesized, as well as pyridio-[196] and pyridazino${ }^{[197]}[5]-$, [6]- and [7]helicenes from the corresponding cyanodiynes and ynedinitriles.

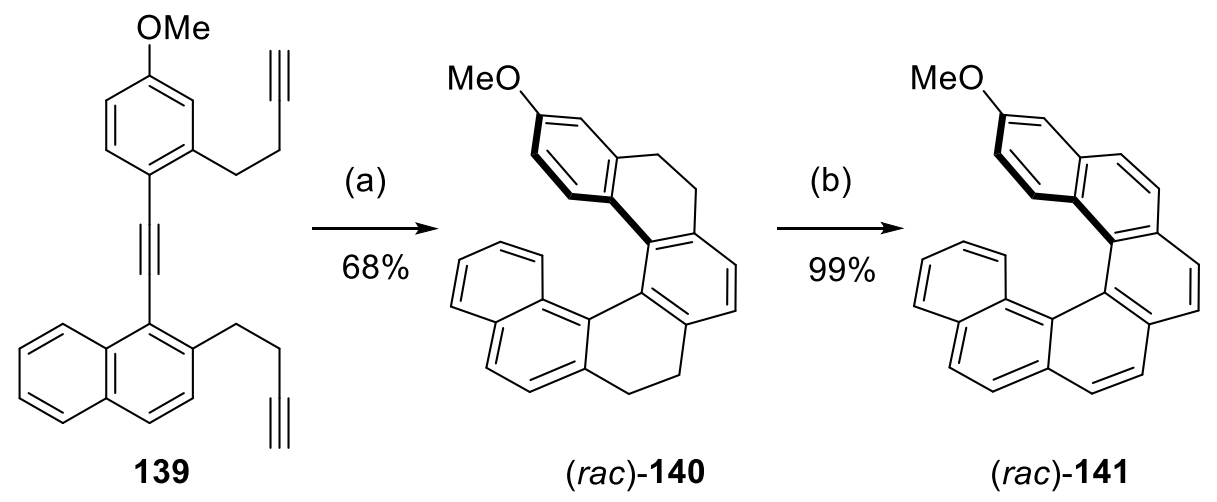

Scheme 30. Examples of carbohelicenes accessed through $2+2+2$ cyclotrimerization. (a) $\mathrm{CpCo}(\mathrm{CO})_{2}(20 \mathrm{~mol} \%)$, $\mathrm{PPh}_{3}$ (40 mol\%), decane, halogen lamp, $140{ }^{\circ} \mathrm{C}, 2.5 \mathrm{~h}$; (b) $\mathrm{Ph}_{3} \mathrm{BF}_{4}$ (3.4 equiv.), 1,2-DCE, $80{ }^{\circ} \mathrm{C}, 2 \mathrm{~h}$.

Recently published reports have described the highly diastereoselective synthesis of carbohelicenes 144. ${ }^{[198]}$ Thus, enzymatically resolved propargylic alcohols, when incorporated into triyne substrates 142 and subjected to the cobalt catalyzed $[2+2+2]$ cycloisomerization, enabled a highly efficient transfer of chirality to the helical backbone $\mathbf{1 4 3}$ (Scheme 31).<smiles>CO[C@H](C#C[Ge])Cc1ccc2ccccc2[X]1C#Cc1c([C@H](CC#C[Ge])OC)ccc2ccccc12</smiles>

142: $X=C, N$ Tol $=p$-tolyl (a)

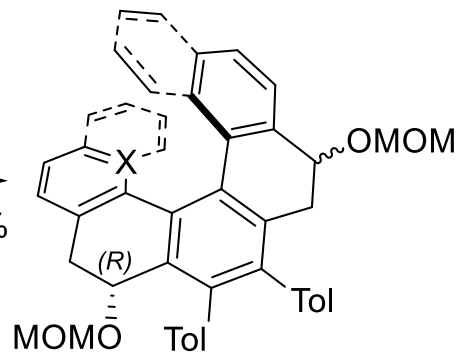

143: $>99 \% d r$ (b)

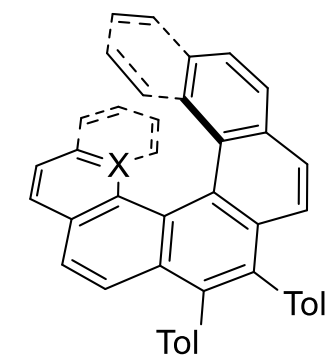

144: $>99 \%$ ee

Scheme 31. Diastereoselective $2+2+2$ cycloisomerization to access enantiopure [5]-, [6]- and [7]helicenes. ${ }^{[198]}$ Reagents and conditions: (a) $\mathrm{CpCo}(\mathrm{CO})$ (fum) (50 mol\%), 1-butyl-2,3-dimethylimidazolium tetrafluoroborate in THF, microwave irradiation, $150-180^{\circ} \mathrm{C}, 10-20 \mathrm{~min}$; (b) $p$-TSA or $\mathrm{HCl}, \mathrm{MeCN}$. 


\section{Introduction}

The authors showed that the high reaction temperatures enabled enantioinversion of the helical axis, which then arranged itself to give the most stable diastereomer in all cases, driven by the central chirality of the OMOM ether. An elimination procedure then yielded the helicenes 144, which included [6]- and [7]- aza- and carbohelicenes with a variety of substituents. Although highly diastereoselective, this procedure still has the drawback that a chiral center must be introduced and later removed to access the enantiopure helicenes. This is still an inherently wasteful method when compared to directly enantioselective reactions.

Numerous efforts have so far been described in the enantioselective [2+2+2] cycloisomerization of triynes such as $\mathbf{1 4 5}$ to give enantioenriched helicenes using nickel catalysts, as they are generally more reactive and do not require such high temperatures, ${ }^{[191,199,200]}$ although in these cases the number of substrates described has so far been limited.
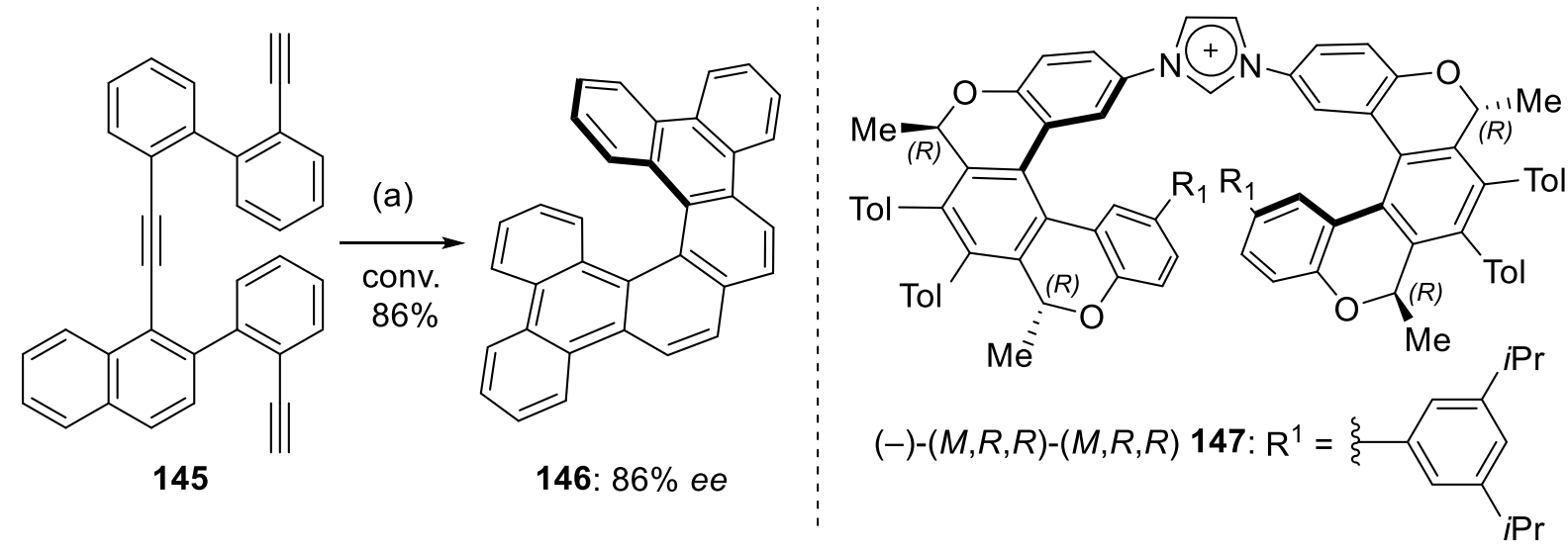

Scheme 32. Enantioselective [2+2+2] cycloisomerization to give carbohelicene 146 using oxahelicene-NHCbased ligand 147. Reagents and conditions: (a) Ni(acac) 2 (20 mol\%), 147 (44 mol\%), EtMgCl (0.9 equiv.), THF, rt, $2 \mathrm{~h}$.

One recent example details the synthesis and catalytic behavior of oxahelicenes-substituted imidazolium salts of the type 147 (Scheme 32). ${ }^{[201]}$ These helicene-based ligands were, in turn, themselves synthesized according to a diastereoselective [2+2+2] cyclisation. Although the application of $\mathbf{1 4 7}$ to the cyclisation of $\mathbf{1 4 5}$ reached a promising ee of $86 \%$, it is strange that the evaluation of no further substrates was described. Additionally, an analogous method using Rh(I)-BINAP complexes has been recently described by the group of Tanaka in the enantioselective synthesis of dibenzo[7]helicenes, although the selectivity of the transformation was lower and a limited scope was reported. ${ }^{[202]}$

Other transition metal-based strategies that have been applied to helicene synthesis include the $\operatorname{Pd}(0)$ catalyzed $[2+2+2]$ cycloisomerization of arynes and electron-deficient alkynes, of 
which an enantioselective variant has been reported. ${ }^{[203]} A$ variety of chiral ligands were screened; although a high ee for one substrate was described, very low conversion and poor selectivity towards the desired product remain significant challenges for this transformation. An approach using ring closing alkene metathesis (RCM) has also been investigated by the group of Collins, where the synthesis of a variety of functionalized racemic [5]- and [6]- and [7]carbohelicenes could be performed in good yields. ${ }^{[204]}$ An enantioselective variant using chiral carbene-derived catalysts aimed to furnish [7] helicene from the racemic precursor 148 via a kinetic resolution. ${ }^{[205]}$ After multiple rounds of optimization, the targeted [7]helicene [(M)109] could be accessed in an $80 \%$ ee and $38 \%$ conversion, with a significant solvent effect seen for hexafluorobenzene and the addition of vinylcyclohexane as an additive. Nevertheless, an inherent drawback of this reaction is that the starting material is already chiral and therefore the reaction proceeds via a kinetic resolution. Moreover, no comment in the paper is given on the ease in which starting material can be separated from the product. Some examples of [4]carbohelicenes have also recently been described using the emerging olefin-carbonyl metathesis reaction. ${ }^{[206]}$
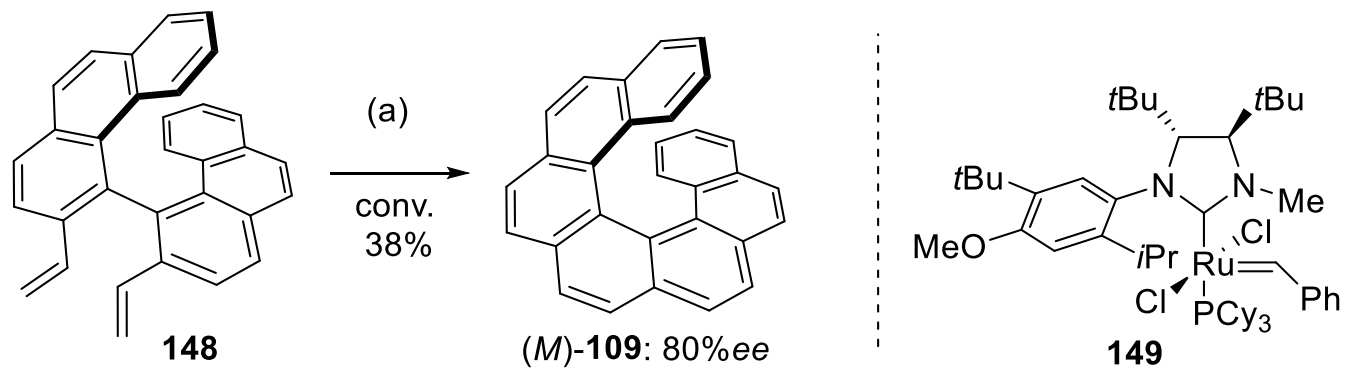

Scheme 33. Synthesis of [7]helicene using RCM. Reagents and conditions: (a) 149 (4 mol\%), vinylcyclohexane (10 equiv.), $\mathrm{C}_{6} \mathrm{~F}_{6}, \mathrm{rt}, 2 \mathrm{~h}$.

Another effective strategy derives from the $\pi$-acid-catalyzed cycloisomerization of aryl alkynes. ${ }^{[110,207]}$ Some asymmetric variants of this reaction have also been attempted by Tanaka and coworkers, in the synthesis of [6]- (151) and S-shaped double [6]carbohelicenes 154. ${ }^{[202]}$ This reaction proceeds through a tandem gold-catalyzed hydroarylation/carbocyclization of diyneones 150 (Scheme 34A) or tetraynediones 153 (Scheme 34B) and yields the corresponding [6]carbohelicenes 151 after methylation in moderate to good yields, with ee's between 37 and 74\%. An extension towards the synthesis of S-shaped double [6]carbohelicenes 154 via the twice-repeated tandem gold-catalyzed hydroarylation/carbocyclization of tetraynediones 153 was also described, although ee's did not increase and very high catalyst loadings of gold and silver $(60 \mathrm{~mol} \%$ and $90 \mathrm{~mol} \%$, respectively) were required, when compared with the isolated yield of 151 for $29-34 \%$ (Scheme 34B). 


\section{Introduction}

Additionally, the group has demonstrated the enantioselective synthesis of mono and double S-shaped aza[6]helicenes, ${ }^{[208]}$ as well as a [10]azahelicene ${ }^{[209]}$ from the corresponding substrates. In these cases, the ee's were very good for the double azahelicene and [10]helicene (up to $>99 \%$ ), although catalyst loadings were still high and yields remained low.

A)<smiles>[R]C#Cc1ccc2ccccc2c1C#CC(=O)Cc1ccc2ccccc2c1</smiles>

150 (a)<smiles>[R]c1cc2ccc3ccccc3c2c2c1c(OC)cc1ccc3ccccc3c12</smiles><smiles>FC(F)(F)OC1(F)Oc2ccc(-c3ccccc3)c(-c3c(-c4ccccc4)ccc4c3OC(F)(F)O4)c2O1</smiles>

B)

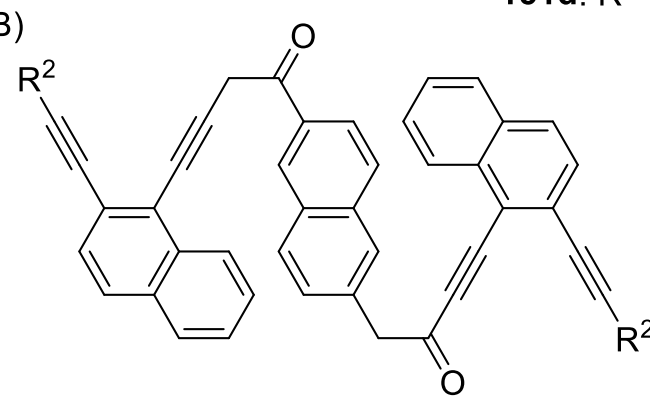

153 (b)

151a: $\mathrm{R}^{1}=2-\mathrm{MeO}-\mathrm{C}_{6} \mathrm{H}_{4^{-}}(69 \%): 64 \%$ ee

151b: $\mathrm{R}^{1}=4-\mathrm{MeO}-\mathrm{C}_{6} \mathrm{H}_{4}-(50 \%): 54 \%$ ee

151c: $\mathrm{R}^{1}=\mathrm{Ph}(21 \%): 37 \%$ ee

151d: $R^{1}=2$-thienyl (63\%): $74 \%$ ee

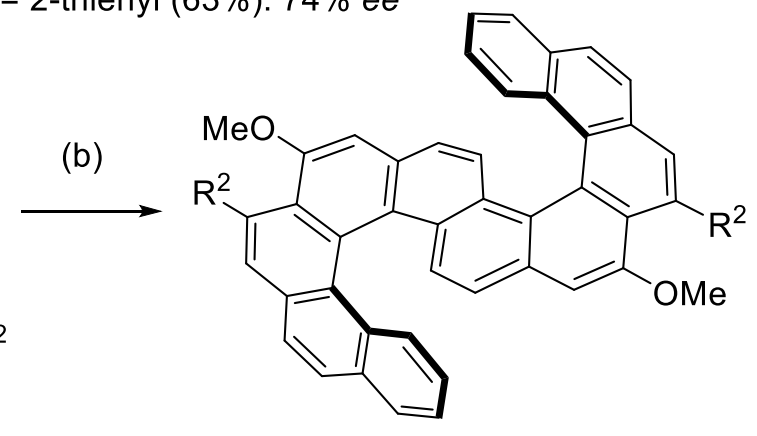

154a: $\mathrm{R}^{2}=4-\mathrm{MeO}-\mathrm{C}_{6} \mathrm{H}_{4^{-}}(34 \%): 66 \%$ ee 154b: $R^{2}=2$-thienyl (29\%): $64 \%$ ee

Scheme 34. Enantioselective gold(I)-catalyzed intramolecular hydroarylation of diynones $150(A)$ and ii). tetraynediones 153 (B). Reagents and conditions: (a) i). AuCl.SMe (20 mol\%), AgNTf 2 (30 mol\%), 152 (12 mol\%), $\mathrm{CH}_{2} \mathrm{Cl}_{2}$, rt, 16 h. ii). TMS-diazomethane, $\mathrm{MeOH} /$ toluene, $0{ }^{\circ} \mathrm{C}$ to rt, $16 \mathrm{~h}$; (b) i). AuCl.SMe $(60 \mathrm{~mol} \%), \mathrm{AgNTf}_{2}(90$ mol\%), (R)-BINAP (36 mol\%), $\mathrm{CH}_{2} \mathrm{Cl}_{2}$, rt, $16 \mathrm{~h}$. ii). TMS-diazomethane, $\mathrm{MeOH} /$ toluene, $0{ }^{\circ} \mathrm{C}$ to rt, $16 \mathrm{~h}(154 \mathrm{a})$ or Mel, $\mathrm{K}_{2} \mathrm{CO}_{3}$ (154b).

In summary, although the applications of helicenes are closely related to their intrinsic chirality, there is still a deficiency in effective asymmetric methods to access them. The reactions that have been reported, up to the date of writing this thesis, mostly do not display a broad scope, with variant ee's depending on substitution of the substrate or very few substrates reported. Additionally, in many cases high catalyst loadings are required, sometimes higher than the yield of the products, which are also in many cases also low. This is especially true for carbohelicenes, for which there is currently no general method that furnishes carbohelicenes with high yields and ee. This highlights the fundamental difficulty in transferring chirality efficiently to form a helically chiral axis. Considering the previous work of the Alcarazo group in the hydroarylation of alkynes catalyzed by strong $\pi$-accepting $\pi$-acid catalysts, ${ }^{[88]}$ it was envisaged that the synthesis of [6]carbohelicenes through an 
enantioselective gold(I)-catalyzed hydroarylation reaction could provide an exciting new entry to this field.

\subsection{Naturally occurring bisphenanthrenes}

The work conducted by the Alcarazo group into $\pi$-acid catalyzed hydroarylation reactions has resulted in the synthesis of a number of natural products comprising a phenanthrene core. ${ }^{[88,106]}$ In nature, a variety of phenanthrenes have been isolated from the Orchidaceae family. Many of these plants have been used in traditional folk medicine and phenanthrenes have therefore been studied for their antimicrobial, spasmolytic, anti-inflammatory, antiplatelet aggregative, anti-allergic and phytotoxic activity. ${ }^{[210]}$ Phenanthrenes are classified as secondary metabolites and are widely thought to be synthesized by plants through the oxidative cyclisation of stilbene precursors. Their structure is often highly oxygenated and a variety of dihydroderivatives also exist, which are thought to occur through bibenzyl precursors. ${ }^{[211]}$ As many of the monomers of dimeric phenanthrenes are also isolated from the same plant, it is probable that these occur through dimerization pathways.

Naturally occurring phenanthrenes can be subdivided into three groups: monophenanthrenes, diphenanthrenes and triphenanthrenes. By far, the most phenanthrenes occur in monomeric form; however, diphenathrenes still comprise approximately 40 different naturally occurring species. ${ }^{[210]} A$ series of bisphenanthrenes, the Blestriarenes family 155 (Figure 24), were isolated from Bletilla striata, the plant used in Chinese folk medicine to treat pulmonary disorders. They were found to exhibit antibacterial efficacy against a variety of microorganisms, with Blestriarene B (155b) showing the highest activity. ${ }^{[212]}$<smiles>COc1cc(O)c(-c2c(O)cc(OC)c3c2CCc2cc(O)ccc2-3)c2c1-c1ccc(O)cc1CC2</smiles>

155a: Blestriarene A<smiles>COc1cc(O)c(-c2c(O)cc(OC)c3c4c(ccc23)CCc2cc(O)ccc2-4)c2ccc(O)cc12</smiles>

155b: Blestriarene B<smiles></smiles>

155c: Blestriarene C

Figure 24. Structures of Blestriarenes A, B and C.

Blestriarene $C$ (155c) was recently the target of a diastereoselective total synthesis by the group of Miyano and was obtained via an oxidative coupling of the two phenanthrene subunits, followed by diastereomeric resolution. ${ }^{[213]}$ The authors reported that on isolation of 


\section{Introduction}

the enantiopure natural product, rapid racemization was observed, the rate of which was strongly dependant on whether or not the sample was exposed to light and oxygen. A follow up study concluded that the highly oxygenated phenanthrenes were susceptible to oxidation by atmospheric oxygen and could racemize through a cationic radical species (Scheme 35). ${ }^{[214]}$ The propensity of photoracemization depended strongly on the substituents, and the authors could correlate the oxidation potentials of a number of biphenanthrene derivatives with their rates of racemization.<smiles></smiles>

$(S)-155 c$<smiles>COc1cc(O)c(-c2c(O)cc(OC)c3c2ccc2cc(O)ccc23)c2ccc3cc(O)ccc3c12</smiles>

$(R)-155 c$<smiles></smiles><smiles>CCCO[C@H](O)C(=O)O</smiles>

$(R)-156 \mathrm{c}$

Scheme 35. Proposed racemization pathway of Blestriarene C (155c).

While many biphenanthrenes possess free hydroxyl groups in the 2,2' positions and therefore could also potentially participate in such photoracemizations with similar pathways; in a selected group of naturally occurring biphenanthrenes these 2,2' positions are protected with methyl ether groups (Figure 25).

Due to hindered rotation about the carbon-carbon between the two phenanthrene units, these natural products should be chiral. Indeed, the natural biphenanthrenes Monbarbatain A (156a) and Monbarbatain B (156b), isolated from Monomeria babarta Lindl of the Orchidaceae family, were both optically active and showed significant cytotoxic activity against liver carcinoma HepG-2 and promyelocytic leukaemia HL-60 cell lines. ${ }^{[15]}$ The bulb of Monomeria babarta Lindl is used in traditional Chinese folk medicine to treat cough, pulmonary tuberculosis and trauma. The tetrahydro biphenanthrene 157 was isolated from 
Deondrobium plicatile, another plant used in traditional folk medicine as a tonic and to treat fever; its structure was established as 2,2'-dimethoxy-9,9',10,10'-tetrahydro-[1,1'biphenanthrene]-4,4',7,7'-tetraol. ${ }^{[216]}$ Finally, the prenylated biphenanthrene 158 was isolated from the orchid Prosthechea michuacana, traditionally used plant in folk medicine in Mexico as a kidney anti-inflammatory, diuretic, in wound healing and in the treatment of diabetes. This compound exhibited anti-oxidant activity as a scavenger of the DPPH radical. ${ }^{[17]}$<smiles>COc1cc(O)c2c(ccc3cc(O)ccc32)c1-c1c(OC)cc(O)c2c1ccc1cc(O)ccc12</smiles>

Monbarbatain A<smiles>COc1cc(O)c2c(c1-c1c(OC)cc(O)c3c1-c1ccc(O)cc1CC3)CCc1cc(O)ccc1-2</smiles>

157<smiles>COc1cc(O)c2c(c1-c1c(OC)cc(O)c3c1ccc1cc(O)ccc13)CCc1cc(O)ccc1-2</smiles>

$156 b$

Monbarbatain B<smiles></smiles>

158

Figure 25. Naturally occurring biphenanthrenes Monbarbatain A (156a), Monbarbatain B (156b), 157 and 158.

To the best of our knowledge, no synthesis of this class of natural products has been previously attempted. The structure of Monbarbatain A, with an axis of chirality formed from the two fused phenanthrene moieties, could make an interesting target for total synthesis using a gold(I)-catalyzed hydroarylation as the key step. Moreover, an approach to access larger amounts of this compound could allow the possibility of further evaluating its biological activity. 


\section{Previous research of our group in the synthesis of [6]helicenes}

\subsection{Introduction}

The Alcarazo group had previously studied the $\mathrm{Au}(\mathrm{I})$ or $\mathrm{Pt}(\mathrm{II})$ catalysed intramolecular hydroarylation of alkynes in the synthesis of condensed polyaromatic compounds, ${ }^{[88,106]}$ demonstrating a significant enhancement of the reaction rate when a-cationic phosphines were used as ancillary ligands. This approach enabled the synthesis of a variety of highly twisted phenanthrenes. It was envisaged that the related prochiral diyne 159 could act as a suitable substrate for a $A u(I)$ catalysed, intramolecular double hydroarylation sequence, which would then afford [6]carbohelicenes 160 (Scheme 36). Because the precursor 159 is prochiral, an axis of helical chirality would be formed in this reaction.

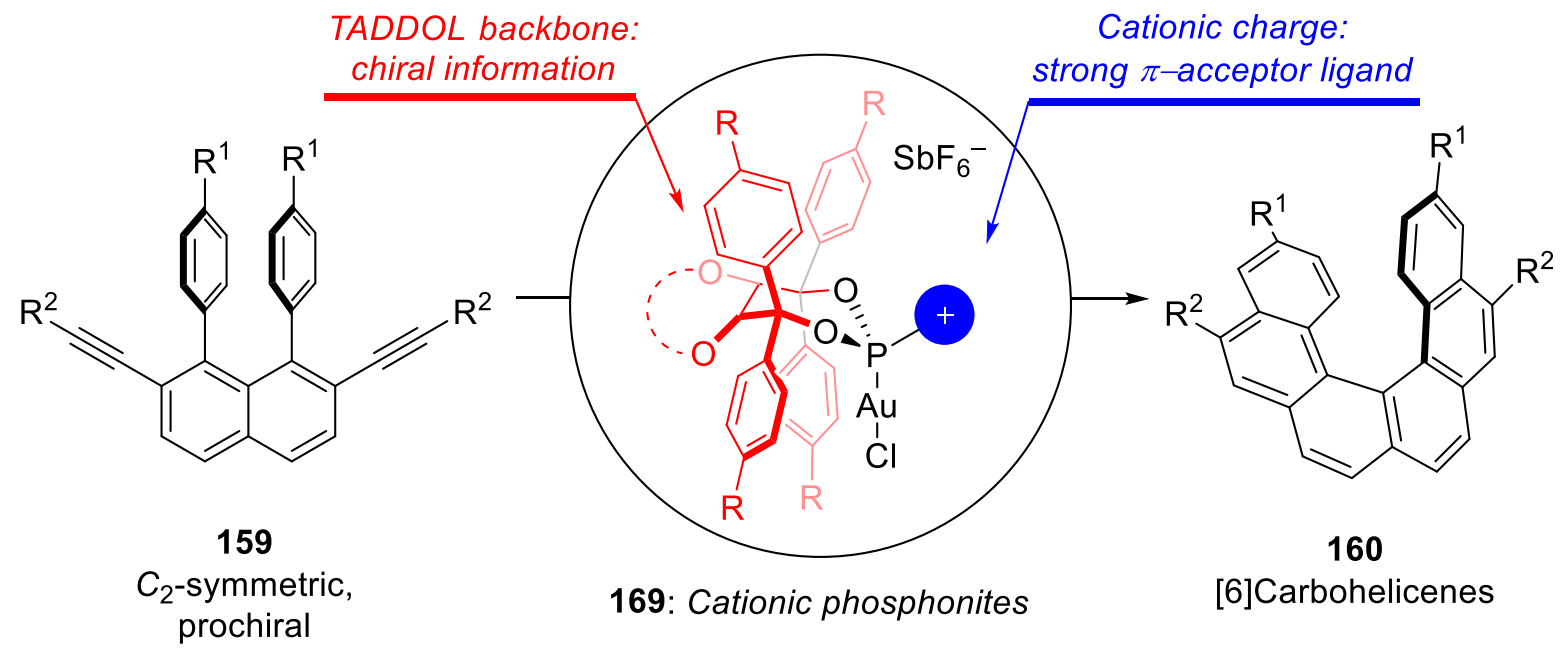

Scheme 36. Proposed approach to access [6]helicenes through asymmetric gold catalysis using cationic phosphonites.

Previous work in enantioselective gold catalysis had highlighted the utility of monodentate TADDOL derived phosphoramidites, which as ligands in gold catalysis had provided high enantioselectivities in a variety of mechanistically different processes. ${ }^{[58,61,218]} A$ novel family of chiral cationic phosphonites was therefore proposed (Scheme 36), combining a chiral TADDOL framework and a cationic substituent. Due to the electron-withdrawing nature of both the oxo- and cationic substituents, it was envisaged that this class of ligands would have strong $\pi$-acceptor properties and that the corresponding gold complexes would efficiently promote a gold $(\mathrm{I})$ catalysed hydroarylation sequence to afford [6]carbohelicenes. Moreover, the modular synthesis of TADDOL derivatives would allow the preparation of a variety of ligands to be screened in this reaction. The following section will discuss the 
2. Previous research of our group in the synthesis of [6]helicenes

Alcarazo group's previous work in the synthesis of cationic phosphonites and the applications of their gold(I) complexes in the enantioselective synthesis of [6]carbohelicenes. ${ }^{[219,220]}$

\subsection{Synthesis of cationic phosphonites}

The cationic phosphonite motif in the targeted ligand family had been previously described in two literature reports. The first, by the group of Chauvin, ${ }^{[72]}$ offered two different approaches: by condensation of $\mathrm{N}$-phenyl-1 $\mathrm{H}$-imidazole and chlorodiethylphosphite followed by selective $\mathrm{N}$-methylation (Scheme 37A), or through direct condensation of the $\mathrm{N}$-heterocyclic carbenes 56 and chlorodiethylphosphite at low temperatures (Scheme 37B). Another report by the group of Leitner described the metathesis of bis(carbene)silver complexes with the BINOL derived chlorophosphite 159a (Scheme 37C). ${ }^{[80]}$

A)

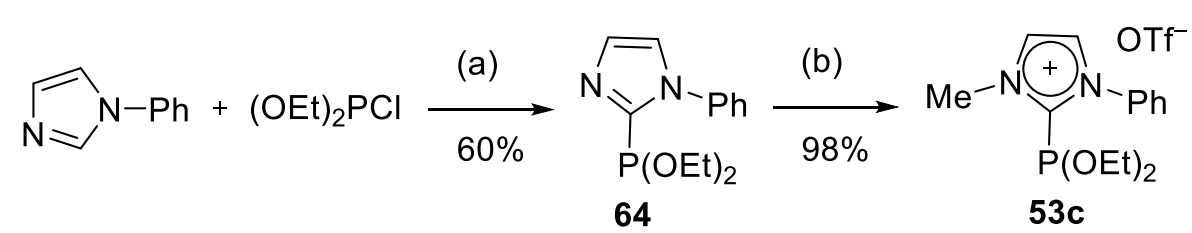

B)

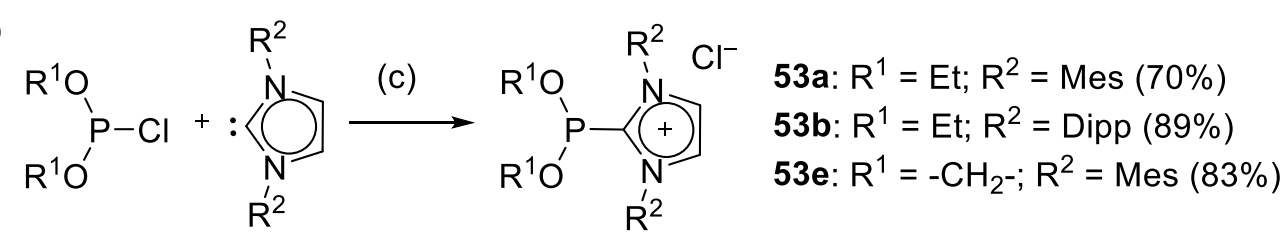

56

C)<smiles></smiles>

Scheme 37. Literature reported methods $(A)-(C)$ to synthesize cationic phosphonites 53 and 160a. Reagents and conditions: (a) 1-phenylimidazole (1.0 equiv.), $n \mathrm{BuLi}\left(1.0\right.$ equiv.), $\mathrm{Et}_{2} \mathrm{O},-78{ }^{\circ} \mathrm{C}$ to $\mathrm{rt}, 4 \mathrm{~h}$, then $(\mathrm{EtO}){ }_{2} \mathrm{PCl}(1.0$ equiv.), $-78^{\circ} \mathrm{C}$ to rt, $2 \mathrm{~h}$; (b) MeOTf, toluene, rt, $2 \mathrm{~h}$; (c) 56 (1.0 equiv.), Et $2 \mathrm{O},-78^{\circ} \mathrm{C}, 15 \mathrm{~min}$; (d) THF, rt, $2 \mathrm{~h}$; the yield is not reported.

From these options, the most preferable approach would be to allow free carbenes to directly react with chlorophosphites, (Scheme 35B) which would permit the incorporation of bulky and readily available $\mathrm{N}$-heterocyclic carbenes (NHCs). Following the aforementioned strategy, the cationic phosphonites $169 \mathbf{a}-\mathbf{k}$ were synthesized using TADDOL derivatives with different aryl substituents and acyclic or cyclic backbones, as well as the NHC 56b and the 
cyclic(alkyl)(amino)carbene (CAAC) 56c (Scheme 38). This was achieved by addition of the carbenes to the corresponding chlorophosphites at $-78{ }^{\circ} \mathrm{C}$ and allowing the reaction mixture to warm up to room temperature overnight, before exchanging the counter ion to hexafluoroantimonate and purification by column chromatography.<smiles>OC(Br)(Br)C1OCCCOC1C(Br)(Br)Br</smiles>

170 (a) or (b)<smiles>[Al]C1(Br)OP(Cl)OC(Br)(Br)C2OCCCOC21</smiles>

171

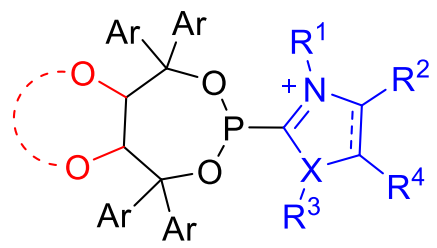

169

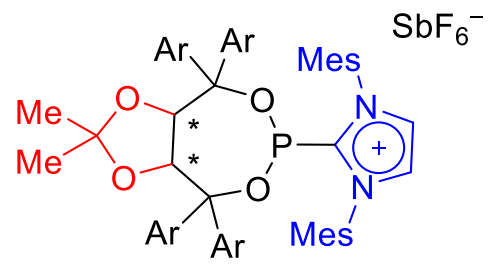

\begin{abstract}
169a $(S, S) ; \mathrm{Ar}=4-\mathrm{Bu}^{-\mathrm{C}_{6}} \mathrm{H}_{4}-$
169b $(S, S) ; \mathrm{Ar}=4-\mathrm{Ph}_{-} \mathrm{C}_{6} \mathrm{H}_{4}-$

169c $(S, S) ; \mathrm{Ar}=4-\mathrm{CF}_{3}-\mathrm{C}_{6} \mathrm{H}_{4^{-}}$

169d $(S, S) ; \mathrm{Ar}=2$-naphthyl

169e $(R, R) ; \mathrm{Ar}=3,5-\left(\mathrm{CF}_{3}\right)_{2}-\mathrm{C}_{6} \mathrm{H}_{3}-(55 \%)^{\mathrm{b}}$

$(29 \%)^{a}$

$(48 \%)^{b}$

$(72 \%)^{b}$

$(64 \%)^{b}$
\end{abstract}

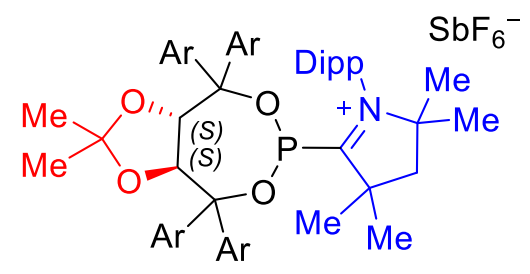

$169 f(S, S) ; A r=2-n a p h t h y l(40 \%)^{a}$

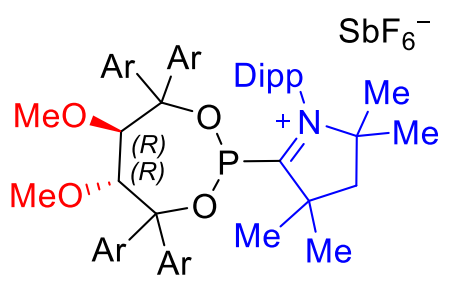

169k $(R, R) ; \mathrm{Ar}=4-t \mathrm{Bu}-\mathrm{C}_{6} \mathrm{H}_{4}-(34 \%)^{\mathrm{a}}$

Scheme 38. Synthesis of cationic phosphonites $169 \mathbf{a}-\mathbf{i}, \mathbf{k}$ from chiral TADDOL derivatives. Reagents and conditions: (a) 170 (1.0 equiv.), molecular sieves $4 \AA$, $\mathrm{Et}_{3} \mathrm{~N}$ (3 equiv.), $\mathrm{PCl}_{3}\left(1.05\right.$ equiv.), toluene, $60^{\circ} \mathrm{C}, 1 \mathrm{~h}$; (b) 170 (1.0 equiv.), molecular sieves $4 \AA$ A, pyridine (3 equiv.), $\mathrm{PCl}_{3}\left(1.1\right.$ equiv.), toluene, $60{ }^{\circ} \mathrm{C}, 1 \mathrm{~h}$; (c) (i) 56 (1.0 equiv.), $\mathrm{Et}_{2} \mathrm{O},-78^{\circ}$ to rt, $16 \mathrm{~h}$; (ii) $\mathrm{NaSbF}_{6}$ (3.0 equiv), MeCN, rt, 2 h; (d) 56 or (0.7 to 0.9 equiv.), $\mathrm{NaSbF}_{6}(3.0$ equiv.) $\mathrm{Et}_{2} \mathrm{O},-78{ }^{\circ} \mathrm{C}$ to rt, $16 \mathrm{~h}$. aPrepared using conditions (a), then (c). bPrepared using conditions (b), then (d).

Certain optimizations led to improved yields in this protocol. ${ }^{[219]}$ Firstly, it was found that triethylamine hydrochloride, formed upon preparation of the chlorophosphites, was difficult to remove and subsequently protonated the free carbenes 56 in the next step. This could be avoided by using pyridine instead, as pyridinium chloride could be easily separated. Secondly, it was found that the ion exchange to hexafluoroantimonate could be performed in situ, reducing the number of synthetic steps in the sequence. Finally, greater yields could be 
obtained by purifying the crude reaction mixture by column chromatography at reduced temperatures.

The ligands were then coordinated to gold using the gold precursor (dimethyl sulfide)gold(I) chloride, affording the gold complexes $172 a-e$ and $172 \mathbf{i}$ in good to excellent yields (Scheme 39).
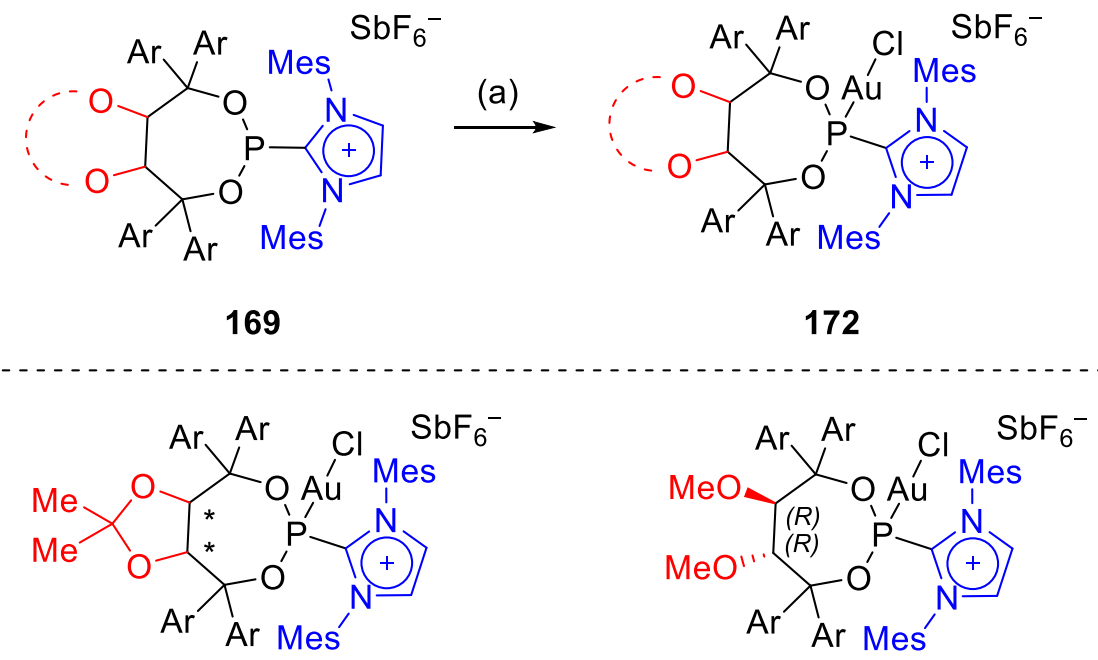

$$
\begin{aligned}
& (S, S)-172 a ; A r=4-t B u-\mathrm{C}_{6} \mathrm{H}_{4}- \\
& (S, S)-172 b ; \mathrm{Ar}=4-\mathrm{Ph}-\mathrm{C}_{6} \mathrm{H}_{4}- \\
& (S, S)-172 \mathrm{c} ; \mathrm{Ar}=4-\mathrm{CF}_{3}-\mathrm{C}_{6} \mathrm{H}_{4^{-}} \\
& (S, S) \text {-172d; } \mathrm{Ar}=\text { 2-naphthyl } \\
& (R, R)-172 \mathrm{e} ; \mathrm{Ar}=3,5-\left(\mathrm{CF}_{3}\right)_{2}-\mathrm{C}_{6} \mathrm{H}_{3}-(56 \%)^{a}
\end{aligned}
$$

Scheme 39. Synthesis of gold(I) complexes 146 from cationic phosphonites 145. Reagents and conditions: (a) 145 (1.0 equiv.), (Me2S)AuCl (1.0 equiv), $\mathrm{CH}_{2} \mathrm{Cl}_{2},-20^{\circ} \mathrm{C}$ to rt, $1 \mathrm{~h}$. ${ }^{a}(\mathrm{Me} 2 \mathrm{~S}) \mathrm{AuCl}$ (1.2 equiv.), $\mathrm{CH}_{2} \mathrm{Cl}_{2}$, rt, overnight.

Conversely, reactions with the ligands $169 \mathrm{f}-169 \mathrm{~h}$ and $169 \mathrm{k}$ led to complete decomposition. The well-defined species 173 and $174 a$, relating to the decomposition of $169 k$, as well as $175 \mathrm{a}$ and 174a, resulting from the decomposition of $169 \mathrm{~g}$ could be isolated and characterized (Scheme 40). The proposed mechanism followed previous work published by Seebach and coworkers on the decomposition of electron-rich TADDOLs in acidic media. ${ }^{[221]}$ In the case of $169 \mathrm{k}$ and $\mathbf{1 6 9} \mathrm{g}$, it was proposed that the gold(I) complex should indeed form, but would be unstable due to the possibility of forming transient diarylsubstituted carbocations, which would then decompose through a Friedel-Crafts-type alkylationelimination-1,2-aryl shift cascade leading to the compounds shown in Scheme 40. This process was proposed to be triggered by the highly electron deficient nature of the goldcoordinated phosphonite with a CAAC substituent, making this fragment an excellent leaving group. Additionally the higher conformational freedom of the TADDOL subunit with an acyclic backbone, would likely enable the gold complex to adopt conformations prone towards elimination processes more easily. ${ }^{[219]}$ 


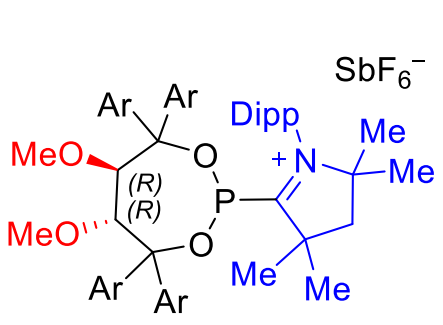

$169 k ; \mathrm{Ar}=4-\mathrm{tBu}^{-\mathrm{C}_{6} \mathrm{H}_{4}-}$ (a)

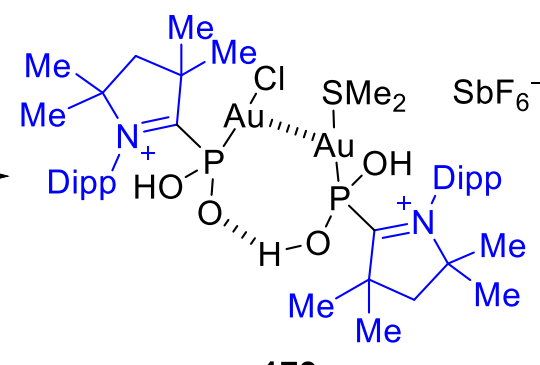

173<smiles>COc1c(-c2ccc(C(C)(C)C)cc2)c(-c2ccc(C(C)(C)C)cc2)c2ccc(C(C)(C)C)cc2c1-c1ccc(C(C)(C)C)cc1</smiles>

$\mathrm{SbF}_{6}^{-}$

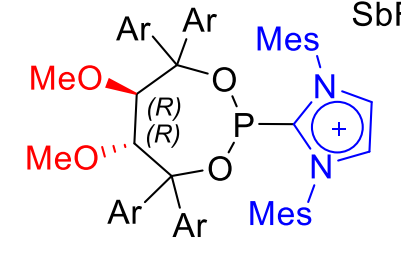

169g; $\mathrm{Ar}=4-\mathrm{tBu}-\mathrm{C}_{6} \mathrm{H}_{4}-$ (a)<smiles></smiles>

$174 a$

Scheme 40 . Decomposition of $169 \mathrm{k}$ and $169 \mathrm{~g}$ into phosphonous acids $173,175 \mathrm{a}$ and naphthalene $174 \mathrm{a}$ upon attempted coordination to gold(I). Reagents and conditions: (a) 169k,g (1.0 equiv.), ( $\mathrm{Me} 2 \mathrm{~S}$ )AuCl (1.0 equiv), $\mathrm{CH}_{2} \mathrm{Cl}_{2},-20^{\circ} \mathrm{C}$ to rt, $1 \mathrm{~h}$.

\subsection{Preparation of helicene precursors}

The substrate 159 with a 1,2,7,8-tetrasubstituted naphthalene core was synthesized through a six-step sequence from 2,7-dimethylnaphthalene (179), which in turn was prepared in two simple steps (Scheme 41). A regioselective bromination at the positions 1 and 8 allowed the subsequent introduction of aryl substituents through Suzuki-Miyaura coupling. Attachment of 2-naphthyl substituents led to syn- and anti-conformers due to hindered rotation of the two aryl substituents, which were visible in the ${ }^{1} \mathrm{H}$ NMR spectrum even upon heating to $50{ }^{\circ} \mathrm{C}$. Radical bromination, hydrolysis, oxidation and Seyferth-Gilbert homologation then gave access to the terminal diynes 186 , which could be further functionalized by alkylation or Sonogashira-Hagihara coupling. Although limited by the necessity of introducing aryl substituents close to the beginning of the synthetic sequence, which restricted installations of some functional groups in these positions, the robust chemistry involved, mostly high yields and the straightforward purification of many of the steps allowed the synthesis to be conducted on a multigram scale. A summary of the synthesized derivatives of $\mathbf{1 5 9}$ is shown inTable 2. ${ }^{[219]}$ 


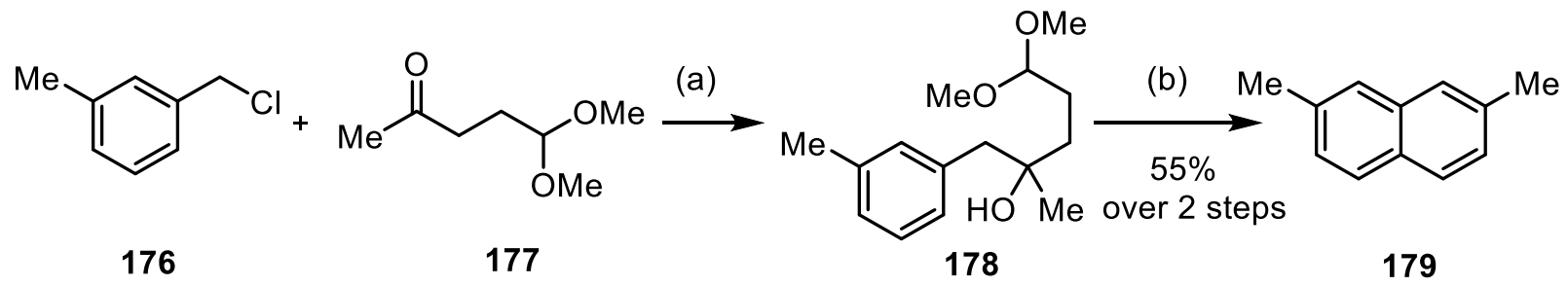

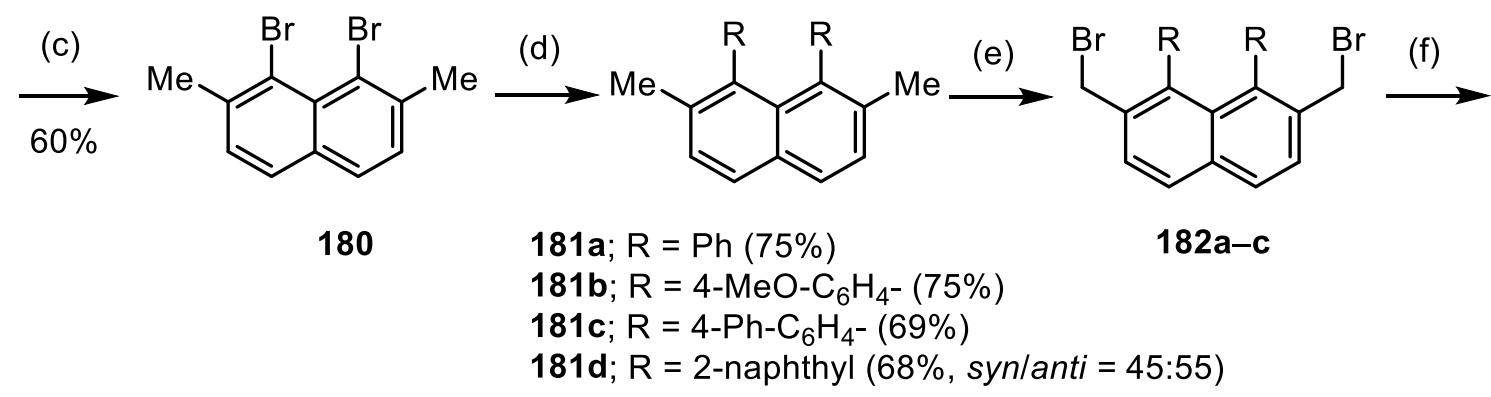<smiles>[R]c1c(C=O)ccc2ccc(CO)c([R])c12</smiles>

Scheme 41. Synthesis of terminal diynes 186a-d starting from compounds 176 and 177 . Reagents and conditions: (a) 176 ( 1.1 equiv.), $\mathrm{Mg}$ (1.5 equiv.), $\mathrm{Et}_{2} \mathrm{O}, 35^{\circ} \mathrm{C}, 3.5 \mathrm{~h}$, then 177 (1.0 equiv.), $15^{\circ} \mathrm{C}, 1 \mathrm{~h}$; (b) $\mathrm{AcOH}$ (glacial), $\mathrm{HBr}\left(40 \%\right.$ aq.), $100{ }^{\circ} \mathrm{C} 1 \mathrm{~h}$; (c) NBS (2.0 equiv.), DMF, rt, 12 days; (d) $\mathrm{RB}(\mathrm{OH})_{2}\left(4.0\right.$ equiv), $\mathrm{Cs}_{2} \mathrm{CO}_{3}(3.0$ equiv.), $\mathrm{Pd}_{2}$ (dba) 3 (4 mol\%), SPhos (8 mol\%), 1,4-dioxane/toluene, $100{ }^{\circ} \mathrm{C}, 2-16 \mathrm{~h}$;(e) $\mathrm{Bz}_{2} \mathrm{O}_{2}(5 \mathrm{~mol} \%), \mathrm{NBS}(2.0$ equiv.), benzene, reflux, $5 \mathrm{~h}$, (f) $\mathrm{CaCO}_{3}$ (5.5 equiv.), 1,4-dioxane/ $\mathrm{H}_{2} \mathrm{O}$, reflux, $48 \mathrm{~h}$; (g) $\mathrm{PCC}$ (3.0 equiv.) $\mathrm{CH}_{2} \mathrm{Cl}_{2}$, reflux, 1 h,; (h) 185 (3.0 equiv.), $\mathrm{K}_{2} \mathrm{CO}_{3}$ (5.0 equiv.), $\mathrm{MeOH}$, rt, $20 \mathrm{~h}$.

Table 2. Synthesis of substrates 159 according to Sonogashira-Hagihara coupling.<smiles>[Z]C#Cc1ccc2ccc(C#CC)c(-c3ccc([R])cc3)c2c1-c1ccc([R])cc1</smiles>

159aa; $R^{1}=H$

159ba; $\mathrm{R}^{1}=\mathrm{MeO}$ (a)<smiles>[R]c1ccc(-c2c(C#C)ccc3ccc(C#C)c(-c4ccc([R])cc4)c23)cc1</smiles>

186a-c (b)<smiles>[R]C#Cc1ccc2ccc(C#C[R])c(-c3ccc([R])cc3)c2c1-c1ccc([R])cc1</smiles> 
Table 3 (continued).

\begin{tabular}{|c|c|c|c|c|}
\hline Entry & $\mathrm{R}^{1}$ & $\mathrm{R}^{2}$ & 159 & Yield (\%) \\
\hline 1 & $\mathrm{H}$ & $\mathrm{Me}$ & 159aa & $80^{a}$ \\
\hline 2 & $\mathrm{H}$ & $p$-Tolyl & $159 a b$ & $80^{b}$ \\
\hline 3 & $\mathrm{H}$ & $\mathrm{Ph}$ & 159ac & $55^{b}$ \\
\hline 4 & $\mathrm{H}$ & $4-\mathrm{Cl}-\mathrm{C}_{6} \mathrm{H}_{4}{ }^{-}$ & 159ae & $65^{b}$ \\
\hline 5 & $\mathrm{H}$ & $3,5-\mathrm{Me}_{2}-\mathrm{C}_{6} \mathrm{H}_{3-}^{-}$ & 159ad & $86^{b}$ \\
\hline 6 & $\mathrm{MeO}$ & p-Tolyl & $159 \mathrm{bb}$ & $97^{b}$ \\
\hline 7 & $\mathrm{MeO}$ & (S)-(1-phenylethoxy)- $\mathrm{C}_{6} \mathrm{H}_{4-}$ & $159 b f$ & $75^{\mathrm{b}}$ \\
\hline 8 & $\mathrm{Ph}$ & $p$-Tolyl & $159 \mathrm{cb}$ & $73^{b}$ \\
\hline 9 & $\mathrm{Cl}$ & $p$-Tolyl & $159 o b$ & $66^{b}$ \\
\hline 10 & $\mathrm{MeO}$ & $\mathrm{Me}$ & $159 \mathrm{ba}$ & $62^{\mathrm{a}}$ \\
\hline 11 & $\mathrm{MeO}$ & $4-\mathrm{Cl}-\mathrm{C}_{6} \mathrm{H}_{4-}$ & 159be & $63^{b}$ \\
\hline 12 & $\mathrm{MeO}$ & 4-TMS- $\mathrm{C}_{6} \mathrm{H}_{4}-$ & $159 \mathrm{bg}$ & $61^{b}$ \\
\hline 13 & $\mathrm{MeO}$ & 4-TIPSOCH${ }_{2}-\mathrm{C}_{6} \mathrm{H}_{4}^{-}$ & $159 \mathrm{bh}$ & $92^{b}$ \\
\hline
\end{tabular}

Reagents and conditions: (a) LiHMDS (3.5 equiv.), Mel (5.0 equiv.), THF, $-20{ }^{\circ} \mathrm{C}$ to rt, $2.5-3 \mathrm{~h}$; (b) $\mathrm{R}^{2} \mathrm{l}$ (4.0 equiv.), $\mathrm{Pd}\left(\mathrm{PPh}_{3}\right)_{4}(5.5 \mathrm{~mol} \%)$, Cul (9 mol\%), $\mathrm{NH} / \mathrm{Pr}_{2} / \mathrm{THF}, \mathrm{rt}, 3 \mathrm{~h}$. aprepared according to conditions (a). bPrepared according to conditions (b).

\subsection{Enantioselective synthesis of helicenes}

With the helicene precursors 159 in hand, attention turned towards their transformation into [6]carbohelicenes 160. Following promising initial results of screening with achiral catalysts, ${ }^{[219]}$ the asymmetric gold(I)-catalyzed intramolecular hydroarylation was studied using the family of cationic phosphonite gold(I) complexes 172. In initial tests with the methylsubstituted diyne 159aa, consistently low ee's were obtained; however, switching to the arylsubstituted substrate 159ab dramatically improved the enantioselectivity. A rigorous screening of ligands, solvents and temperatures was conducted aiming to achieve: high conversion of both $159 \mathrm{ab}$ and the intermediate 187; high regioselectivity towards the desired helicene 160ab over the undesired 6-endo-dig/ 5-exo-dig product 188ab; and high enantiomeric excess of 188ab. ${ }^{[219]}$ Gratifyingly, the precatalyst $\mathbf{1 7 2 i}$, with an acyclic backbone and para-trifluoromethyl groups furnished the helicene 160ab with excellent yield, regio- and enantioselectivity. A comparative study using phosphoramidites with the same TADDOLsubstructure displayed poorer conversion and selectivities, demonstrating that the imidazolium moiety was not only key to the high activity of the catalysts, but also the regioand enantiocontrol. ${ }^{[219]}$ Under the optimized conditions, the scope of the reaction was evaluated for the substrates 159 (Table 3). An aromatic substituent proved to be important to maintain high ee's, with $63 \%$ and $30 \%$ ee being obtained for methylated diynes 159aa and 159ba, respectively (entries 1 and 10). The phenyl- and 3,5-(dimethyl)phenylsubstituted 
compounds 159ac and 159ad gave regio- and enantioselectivities similar to 159ab, although appreciable amounts of the intermediates $187 \mathrm{ac}$ and $187 \mathrm{ad}$ were detected due the poor solubility of both substrates in fluorobenzene (entries 3 and 4).

Table 3. Scope of the asymmetric gold-catalyzed synthesis of [6]carbohelicenes $\mathbf{1 6 0 .}$
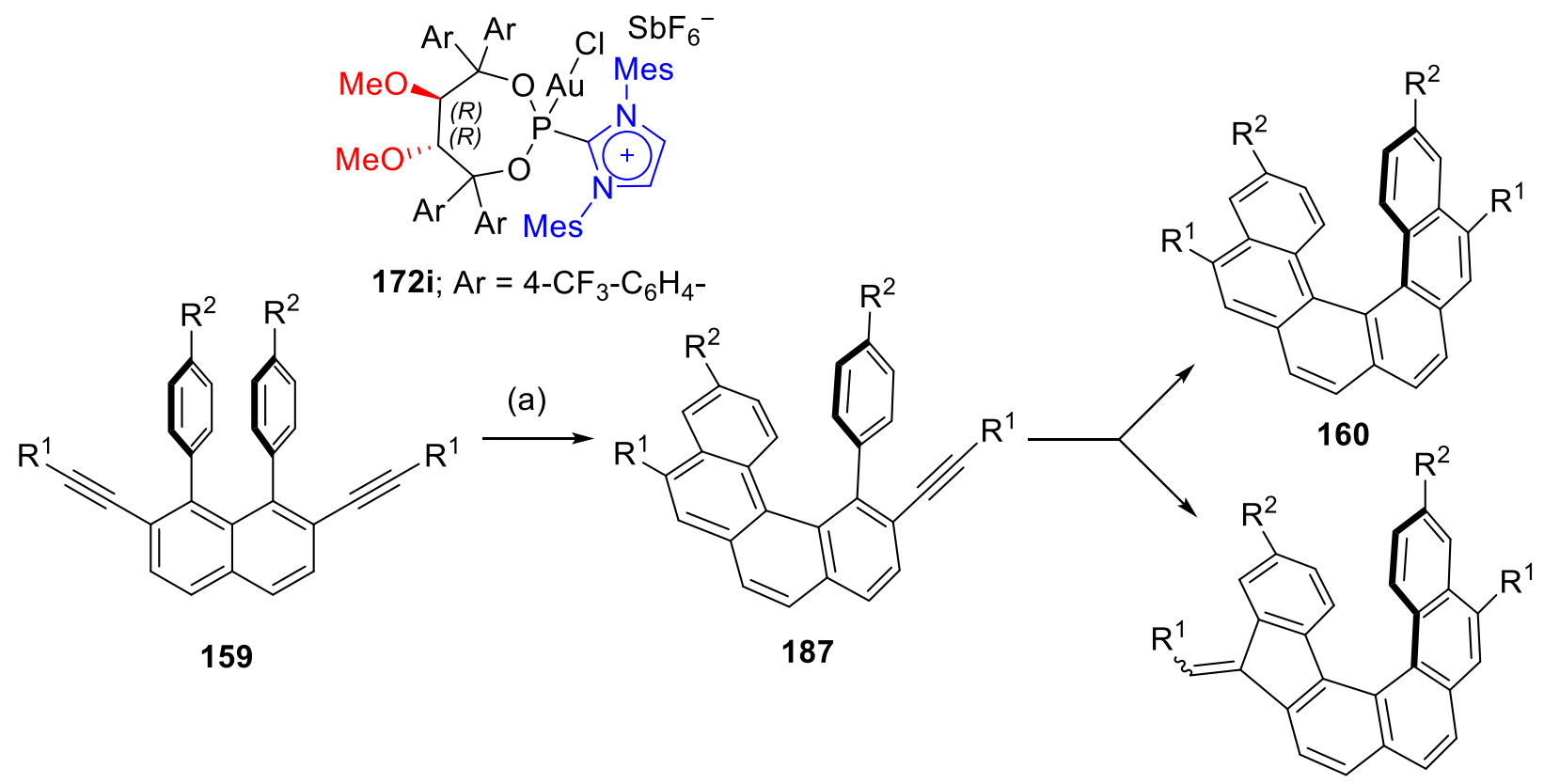

188

\begin{tabular}{|c|c|c|c|c|c|c|}
\hline Entry & 159 & $\mathrm{R}^{1}$ & $\mathrm{R}^{2}$ & Yield (\%) ${ }^{a}$ & $\begin{array}{c}\text { 160: } 188: \\
187\end{array}$ & $e e(\%)$ \\
\hline 1 & 159aa & $\mathrm{Me}$ & $\mathrm{H}$ & 82 & 99: 1:0 & +63 \\
\hline 2 & $159 a b$ & $p$-Tolyl & $\mathrm{H}$ & 88 & 97: 3: 0 & +91 \\
\hline 3 & 159ac & $\mathrm{Ph}$ & $\mathrm{H}$ & 77 & 82: 4: 14 & +92 \\
\hline 4 & 159ad & $3,5-\mathrm{Me}_{2}-\mathrm{C}_{6} \mathrm{H}_{3}-$ & $\mathrm{H}$ & 99 & $57: 4: 38^{b}$ & +89 \\
\hline 5 & $159 \mathrm{bb}$ & p-Tolyl & $\mathrm{MeO}$ & 99 & 88: 12: 0 & +78 \\
\hline 6 & $159 \mathrm{cb}$ & $p$-Tolyl & $\mathrm{Ph}$ & 84 & 97: $3: 0$ & +82 \\
\hline 7 & $159 \mathrm{ba}$ & $\mathrm{Me}$ & $\mathrm{MeO}$ & n. d. & 98: 2: 0 & +30 \\
\hline 8 & 159be & $4-\mathrm{Cl}-\mathrm{C}_{6} \mathrm{H}_{4-}$ & $\mathrm{MeO}$ & 92 & 87: 13: 0 & +95 \\
\hline 9 & $159 \mathrm{bg}$ & 4-TMS- $\mathrm{C}_{6} \mathrm{H}_{4-}$ & $\mathrm{MeO}$ & 64 & 73: $27: 0$ & +94 \\
\hline 10 & 159bh & 4-TIPSOCH${ }_{2}-\mathrm{C}_{6} \mathrm{H}_{4-}$ & $\mathrm{MeO}$ & 94 & 80: 20: 0 & +82 \\
\hline
\end{tabular}

Reagents and conditions: (a) 172i (10 mol\%), $\mathrm{AgSbF}_{6}(10 \mathrm{~mol} \%), \mathrm{C}_{6} \mathrm{H}_{5} \mathrm{~F},-20{ }^{\circ} \mathrm{C}, 96 \mathrm{~h}$. Unless otherwise stated, all reactions reached $>95 \%$ conversion of 159 . a Combined yields of inseparable isomers 160,188 and $187 .{ }^{b} 6 \%$ of $159 \mathrm{ad}$ remained in the reaction mixture.

Introducing a phenyl substituent to the helicene periphery in $159 \mathrm{cb}$ resulted in the same level of regioselectivity but lower enantioselectivity (entry 6), whereas methoxy groups in the same positions (entry 5) resulted in lower both regio- and enantioselectivity. Maintaining methoxy groups in these positions and varying the substituent on alkyne moieties (entries 8-10), 
resulted in consistently low regioselectivities, although excellent enantioselectivities were observed for the 4-chlorophenyl- and 4-(trimethylsilyl)phenylsubstituted derivatives 160be and $160 \mathrm{bg}$. ${ }^{[220]}$ In summary, of the substrates 159 tested in this screening, it could be seen that maintaining high conversion, selectivity and enantioselectivity remained challenging, as even small changes in the substrate structures could either affect solubility and reduce conversion, or have large impacts on the regio- or enantioselectivity of the reaction.

Finally, to investigate whether the first or second cyclisation of the process was enantiodetermining, the diastereomeric intermediate 187bf was prepared, bearing chiral (S)1-phenylethoxy substituents. On measuring variable temperature ${ }^{1} \mathrm{H}$ NMR, two coalescence phenomena could be seen (Figure 26).

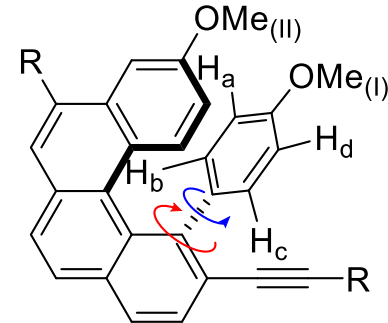

$187 \mathrm{bf}$
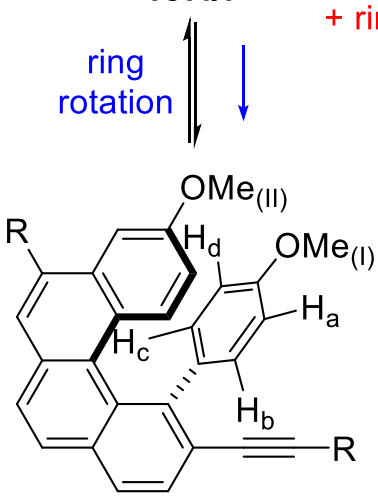

$187 \mathrm{bf}(\mathrm{IV})$

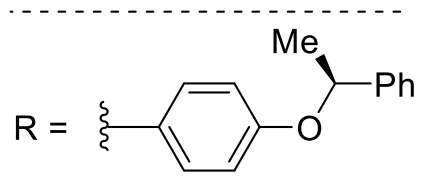

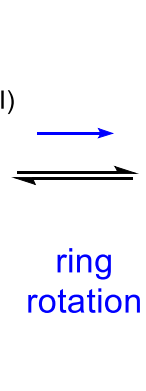

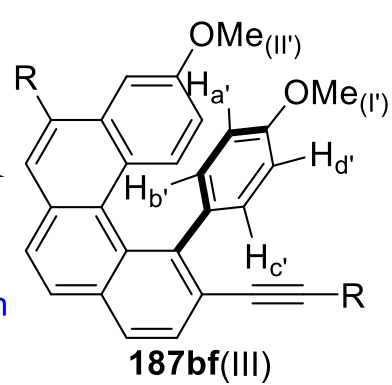

$$
+ \text { ring rotation }
$$
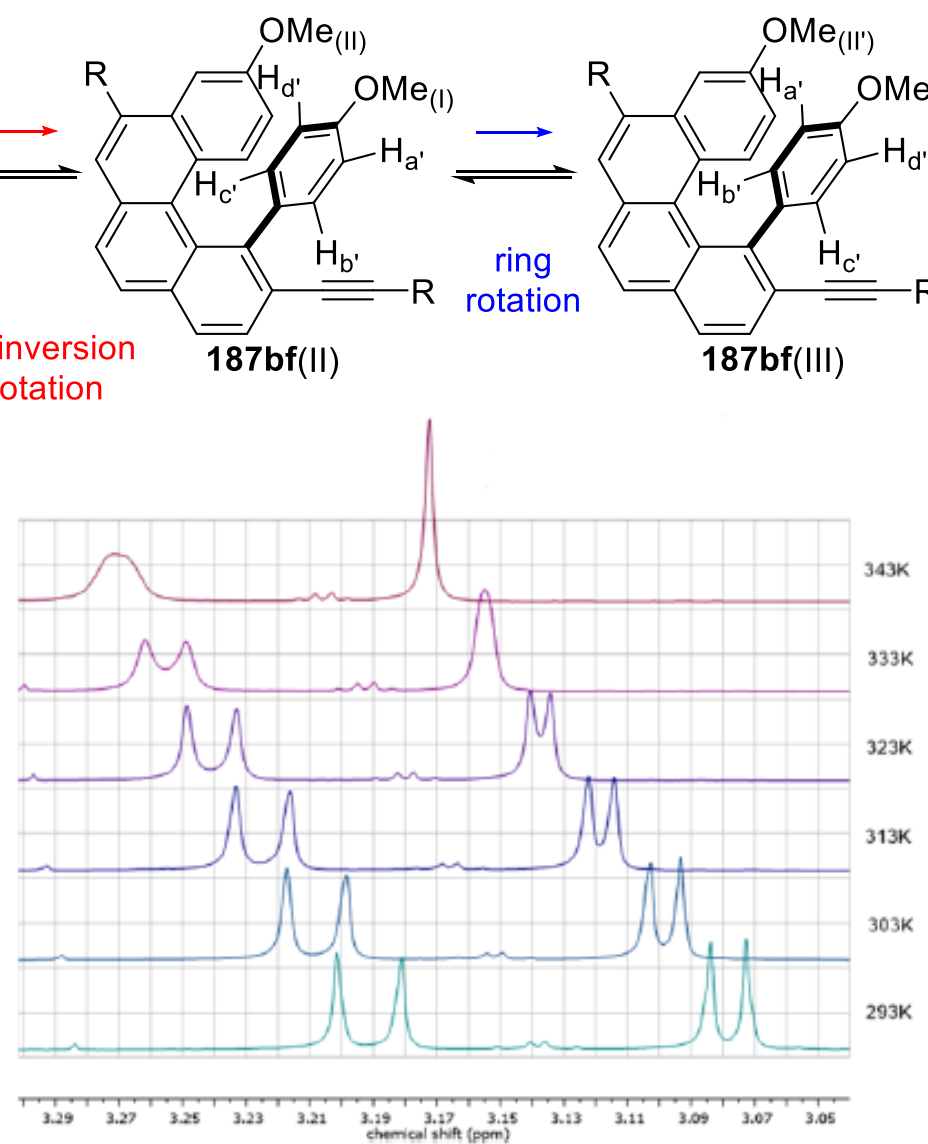

Figure 26. Coalescence phenomena of diastereomeric tetrahelicene intermediate 53bf.

The first $\left(313 \mathrm{~K}\right.$, blue arrows, interconversion of $\mathrm{H}_{a}$ with $\mathrm{H}_{d}$ and $\mathrm{H}_{\mathrm{b}}$ with $\left.\mathrm{H}_{c}\right)$, relating to rotation of the arene located in the bay of the tetrahelicene core does not lead to racemization; while the second ( $338 \mathrm{~K}$, red arrow) relating to the complete inversion of the tetrahelicene skeleton, leads to racemization. The ${ }^{1} \mathrm{H}$ NMR spectrum of the methoxy region in $187 \mathrm{bf}$ relating to $\mathrm{MeO}_{(I)}, \mathrm{MeO}_{(I I)}, \mathrm{MeO}_{\left(I^{\prime}\right)}$ and $\mathrm{MeO}_{\left(I^{\prime}\right)}$ is shown in Figure 26. At their respective coalescence temperatures, the activation barriers $\Delta \mathrm{G}^{\ddagger}$ were calculated to be 59.4 
and $74.5 \mathrm{~kJ} \mathrm{~mol}^{-1}$ for the ring rotation and skeleton inversion, respectively. These were in agreement for previously reported literature values for related tetrahelicenes. ${ }^{\text {[22] }}$ From the Eyring plot of the rates of exchange for skeletal inversion at varying temperatures, the thermodynamic parameters could be estimated. The enthalpy of activation was found to be relatively low $\left(\Delta \mathrm{H}^{\ddagger}=33.5 \mathrm{~kJ} \mathrm{~mol}^{-1}\right)$ and the entropic contribution was negative $\left(\Delta \mathrm{S}^{\ddagger}=-0.13\right.$ $\mathrm{kJ} \mathrm{mol}^{-1}$ ), indicating a highly ordered transition state. Because of this it was proposed that the racemization process of tetrahelicene $187 \mathrm{bf}$ occurs via a transition state where the tetrahelicene core and the phenyl substituent are perpendicular, i.e. where the skeletal inversion and the ring rotation occur simultaneously (shown as a red arrow in Figure 26). Finally, the half-life of diastereomeric interconversion was determined to be approximately 5 seconds at $-20{ }^{\circ} \mathrm{C}$, indicating that the intermediate of the reaction racemises much too quickly for the first cyclisation to be enantiodetermining. From these results it was concluded that the reaction proceeds via a dynamic kinetic resolution of the enantiomers of intermediates 187bf. ${ }^{[219]}$

\subsection{Summary}

In summary, an enantioselective sythesis of [6]carbohelicenes $\mathbf{1 6 0}$ from diyne precursors 159 was developed, utilising a novel family of TADDOL-derived phosphonite ligands with an $\alpha$-imidazolium substituent. These ligands benefit from a modular synthesis and can be accessed in high yields. The helicene precursors 159 were accessed in a 9-step procedure that is both robust and scalable, however suffers from the number of synthetic steps involved and a limited functional group tolerance and modularity in the arene substituents directly attached at the 1-and 8-positions of the naphthalene core. After a thorough screen of conditions and gold-complexes, the cationic phosphonite gold-complex 172i, with an acyclic backbone and para-trifluoromethyl groups at the TADDOL moiety was found to furnish the helicene 160ab from 159ab in excellent yield, regio- and enantioselectivity. Furthermore, control studies with structurally related phosphoramidite ligands found the cationic substituent to be crucial to these results. A preliminary screen of other helicene precursors however, showed that maintaining high conversion, regio- and enantioselectivity was a challenge in this transformation. Finally, an NMR study concluded that the reaction proceeds via a kinetic dynamic resolution of the tetrahelicene intermediates 187 , which under the reaction conditions rapidly racemise. 


\section{Project aims}

In light of the group's previous work in the synthesis of cationic phosphonite gold complexes and their applications towards enantioselective hydroarylation reactions, an expanded investigation will be conducted (Chart 1). The content of this thesis will target the following areas:

i. In order to evaluate the scope of the enantioselective transformation of 159 into $\mathbf{1 6 0}$, a wider variety of precursors 159 will be synthesised. Firstly, a greater variety of alkyne substituents will be incorporated using the established synthesis of 159 . Secondly, alternate synthetic routes towards 159 will be considered, aiming to reduce the number of synthetic steps and allow the easy introduction of different aromatic and heteroaromatic substituents at the 1- and 8-positions of the naphthalene core.

ii. To probe the wider influence of structural changes to the cationic phosphonite ligand family, in addition to further modulating the TADDOL back-bone structure, a number of additional cationic groups will be incorporated and studied. Furthermore, the synthesis of cationic phosphonites with a BINOL scaffold will be attempted.

iii. The gold complexes of the new cationic phosphonite ligands will be evaluated as precatalysts in the enantioselective synthesis of helicenes 160 , aiming to widen the scope of the transformation and afford consistently high conversion, regioselectivity and enantioselectivities. In addition, the absolute configuration of the helicenes formed using this methodology will be assigned.

iv. The new and existing cationic phosphonite gold(I) precatalysts will be evaluated in the enantioselective synthesis of the naturally occuring bisphenanthrene Monbarbatain A (156a). In order to achieve this, a retrosynthetic analysis will be conducted, focusing on a key asymmetric gold(I) catalysed intramolecular double hydroarylation reaction as the key step. 


\section{Project aims}

Proposed new chiral cationic phosphonites:

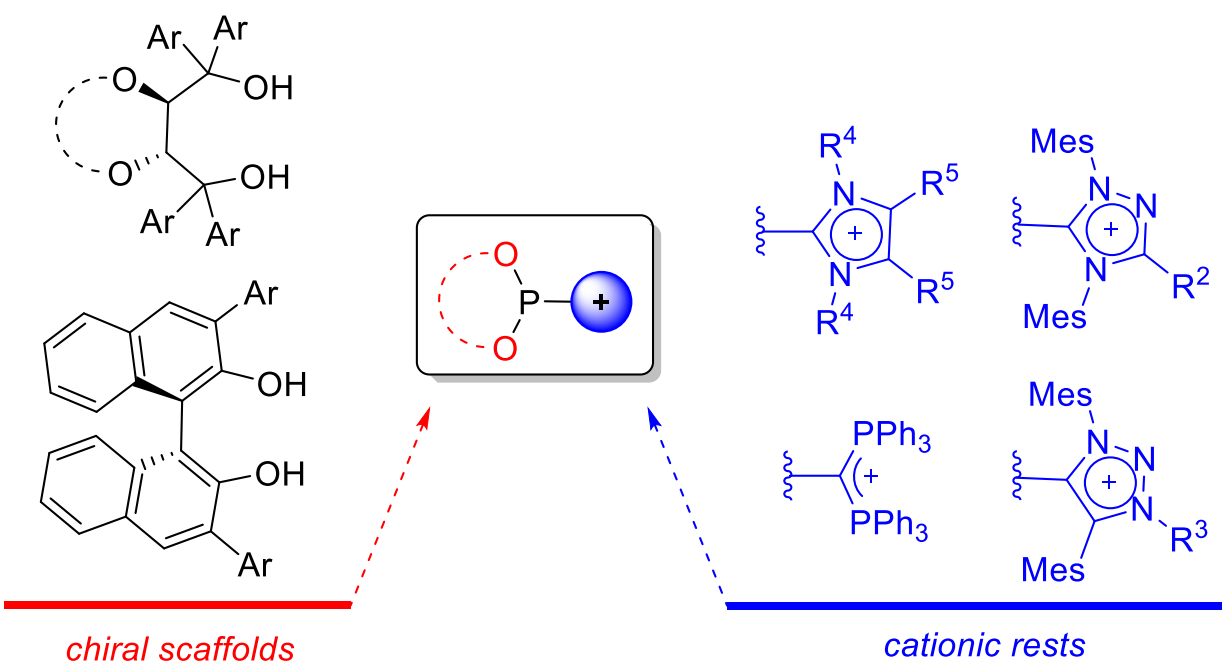

Application 1: enantioselective synthesis of [6]carbohelicenes

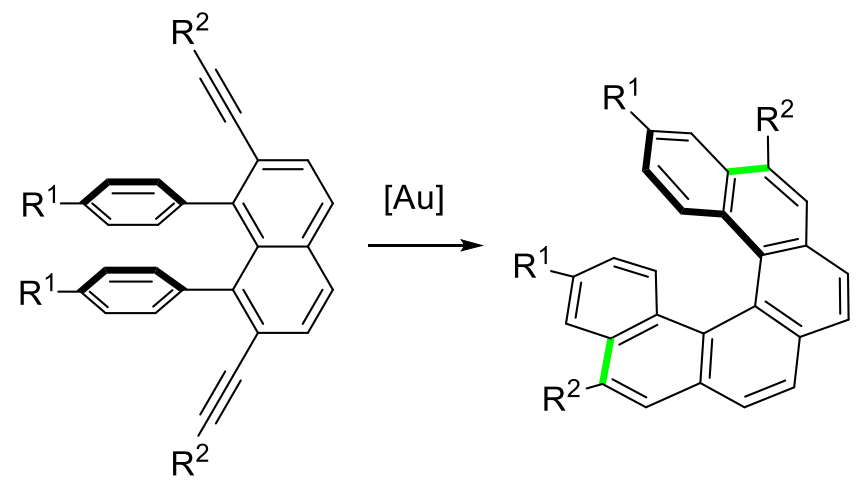

Application 2: enantioselective synthesis of Monbarbatain A

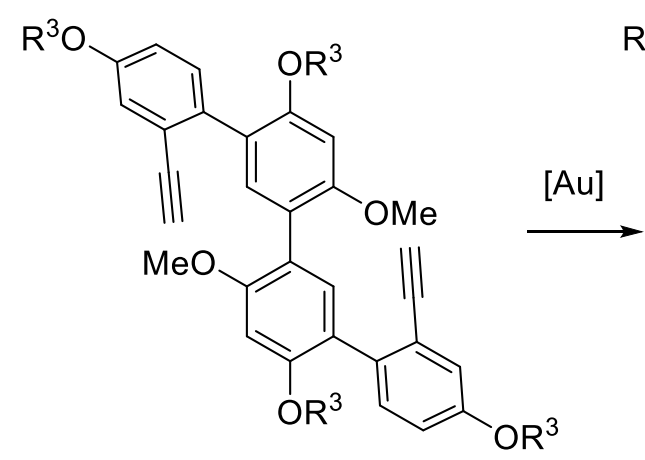

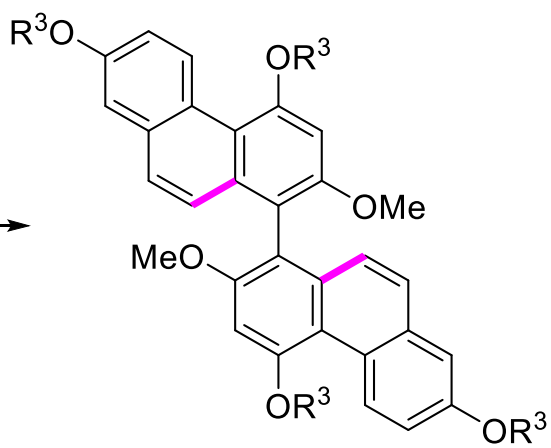

Chart 1. Proposed composition of chiral cationic phosphonites and the applications of their gold $(\mathrm{I})$ complexes towards enantioselective hydroarylation reactions. 


\section{Synthesis of new [6]helicene precursors}

\subsection{Synthesis of new [6]helicene precursors using existing methodology}

From a preliminary screening of different substrates 159 (see above), it was apparent that obtaining consistently high yields, regio- and enantioselectivities was a challenge in the synthesis of helicenes 160 . Considering the high potential of this system, the evaluation of a greater variety of substrates would be interesting to extend the scope of this transformation. Firstly, it was clear that aryl substituents at the alkyne were necessary for good ee's under the optimised conditions. Secondly, the regioselectivity was also not significantly affected on changing the substituents in this position. Fortunately, the existing substrate synthesis permitted diverse functionalization of the building block 186 with different arene substituents via Sonogashira-Hagihara coupling (Table 4).

Table 4. Synthesis of new substrates 159 via Sonigashira Hagihara coupling, bromination and silylation.<smiles>[R]C#Cc1ccc2ccc(C#C[R])c(-c3ccccc3)c2c1-c1ccccc1</smiles>

159 (a)
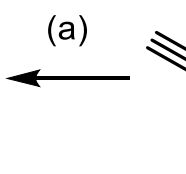<smiles>C#Cc1ccc2ccc(C#C)c(-c3ccccc3)c2c1-c1ccccc1</smiles>

$186 a$ (b) or (c)

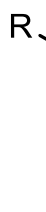<smiles>[R]C#Cc1ccc2ccc(C#C[R])c(-c3ccccc3)c2c1-c1ccccc1</smiles>

159an $\mathrm{R}=\mathrm{Br}$ 159ao $\mathrm{R}=\mathrm{TMS}$ 159ap $R=$ TIPS

\begin{tabular}{cccc}
\hline Entry & 159 & $\mathrm{R}$ & Yield (\%) \\
\hline 1 & 159 af & $4-\mathrm{F}-\mathrm{C}_{6} \mathrm{H}_{4^{-}}$ & $76^{\mathrm{a}}$ \\
2 & $159 \mathrm{ag}$ & $4-\mathrm{MeO}-\mathrm{C}_{6} \mathrm{H}_{4^{-}}$ & $85^{\mathrm{a}}$ \\
3 & $159 \mathrm{ah}$ & $4-\mathrm{BnO}^{\mathrm{C}} \mathrm{C}_{6} \mathrm{H}_{4^{-}}$ & $73^{\mathrm{a}}$ \\
4 & $159 \mathrm{ai}$ & $4-\mathrm{TMS}-\mathrm{C}_{6} \mathrm{H}_{4^{-}}$ & $53^{\mathrm{a}}$ \\
5 & $159 \mathrm{aj}$ & $4-\mathrm{Pr}-\mathrm{C}_{6} \mathrm{H}_{4^{-}}$ & $37^{\mathrm{a}}$ \\
6 & $159 \mathrm{ak}$ & $3,4-(\mathrm{Me})_{2}-\mathrm{C}_{6} \mathrm{H}_{3^{-}}$ & $64^{\mathrm{a}}$ \\
7 & $159 \mathrm{al}$ & $4-\mathrm{TIPSOCH}{ }_{2}-\mathrm{C}_{6} \mathrm{H}_{4^{-}}$ & $45^{\mathrm{a}}$ \\
8 & $159 \mathrm{am}$ & $4-\mathrm{MeOCH} \mathrm{C}_{2}-\mathrm{C}_{6} \mathrm{H}_{4^{-}}$ & $75^{\mathrm{a}}$ \\
9 & $159 \mathrm{an}$ & $\mathrm{Br}$ & $88^{\mathrm{b}}$ \\
10 & $159 \mathrm{ao}$ & $\mathrm{TMS}$ & $54^{\mathrm{c}}$ \\
11 & $159 \mathrm{ap}$ & $\mathrm{TIPS}$ & $39^{\mathrm{c}}$ \\
\hline
\end{tabular}

Reagents and conditions: (a) 186a (1.0 equiv.), Ar-I (4 equiv.), $\mathrm{Pd}\left(\mathrm{PPh}_{3}\right)_{4}(5.5 \mathrm{~mol} \%), \mathrm{Cul}(9 \mathrm{~mol} \%), \mathrm{Pr}_{2} \mathrm{NH} / \mathrm{THF}$, rt, 3-5 h; (b) 186a (1 equiv.), $\mathrm{AgNO}_{3}$ (13 mol\%, NBS (2.4 equiv.), acetone, rt, $30 \mathrm{~min}$; (c) 186a (1.0 equiv.), nBuLi (2.2 equiv.), $-78^{\circ} \mathrm{C}, 1 \mathrm{~h}$, then TMSCI or TIPSCl (2.2 equiv.), $-78^{\circ} \mathrm{C}, 30 \mathrm{~min}$, then rt, $3 \mathrm{~h}$. ${ }^{\text {a }}$ According to conditions (a). ${ }^{b}$ According to conditions (b). ${ }^{\mathrm{c}}$ According to conditions (c). 
A variety of electron-donating and electron-neutral substituents, such as protected phenols (entries 2 and 3), silanes (entry 4), alkanes (entries 5 and 6) and protected benzylic alcohols (entries 7 and 8), were installed (Table 6). For comparison, substrate 159af with a parafluorophenyl substituent was also synthesized (entry 1). The terminal alkyne 186a was additionally functionalized as the dibromide 159an as well as the trimethylsilyl and triisopropylsilyl derivatives 159ao and 159ap (entries 9-11).

Furthermore, motivated by the prospect of synthesizing dibenzohelicenes, the substrate $159 \mathrm{db}$, bearing two 2-naphthyl substituents, was prepared from the precursor $181 \mathrm{~d}$ following the sequence outlined in Scheme 42. Because of the instability of dialdehyde 184d toward purification by column chromatography, the terminal diyne $186 \mathrm{~d}$ was prepared according to a four-step procedure. As discussed above, compound 181d had demonstrated syn-anti conformerism in the ${ }^{1} \mathrm{H}$ NMR spectra. These conformers did not coalesce on heating up to $60{ }^{\circ} \mathrm{C}$, and their ratio remained the same until the final step of the sequence, with the final $p$ tolyl-substituted diyne $159 \mathrm{db}$ having a syn/anti ratio of $45: 55$.

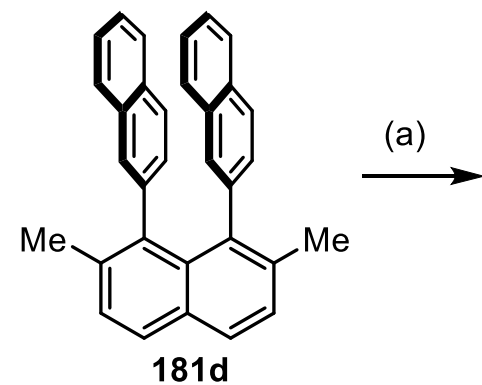

181d<smiles>BrCc1ccc2ccc(CBr)c(-c3cccc4ccccc34)c2c1-c1ccc2ccccc2c1</smiles>

182d (b)

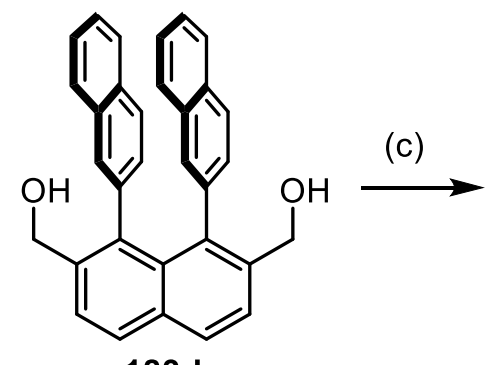<smiles>O=Cc1ccc2ccc(C=O)c(-c3cccc4ccccc34)c2c1-c1ccc2ccccc2c1</smiles>

184d

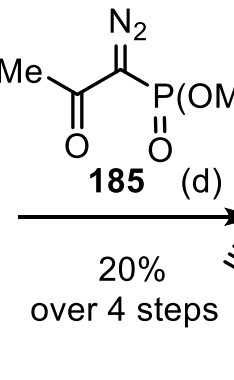

(d)<smiles>C#CCCC</smiles><smiles>C#Cc1ccc2ccc(I)c(-c3ccc4ccccc4c3)c2c1-c1ccc2ccccc2c1</smiles>

$186 \mathrm{~d}$

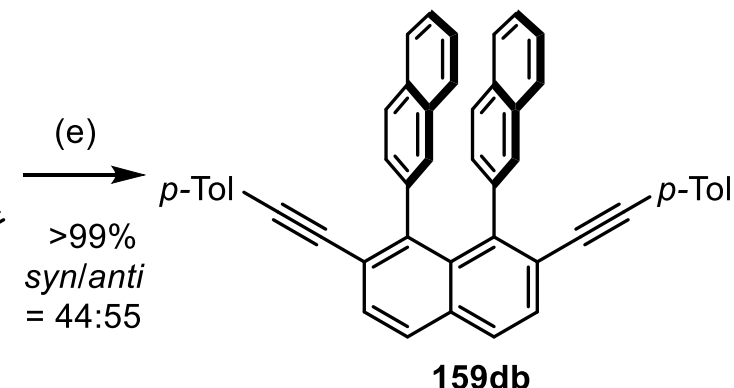

Scheme 42. Synthetic sequence to access alkyne 159db. Reagent and conditions: (a) $\mathrm{Bz}_{2} \mathrm{O}_{2}(6.7$ mol\%), NBS (2.2 equiv.), benzene, reflux, $7 \mathrm{~h}$, (b) $\mathrm{CaCO}_{3}$ (5.5 equiv.), 1,4-dioxane $/ \mathrm{H}_{2} \mathrm{O}$, reflux, $48 \mathrm{~h}$; (c) $\mathrm{PCC}$ (3.0 equiv.)

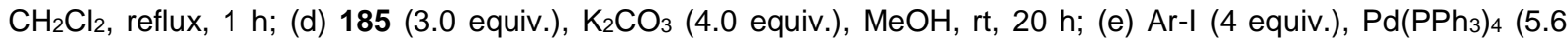
mol\%), Cul (9.0 mol\%), $\mathrm{Pr}_{2} \mathrm{NH} / \mathrm{THF}, \mathrm{rt}, 4 \mathrm{~h}$.

Although the naphthalene substituent in $159 \mathrm{db}$ has two reactive centers for possible nucleophilic attack, the position 1 is generally accepted to be the most nucleophilic (Figure 27). An initial 6-endo-dig cyclization from this position would result in tetrahelicene intermediate $180 \mathrm{db}$. This would likely have greater conformational freedom than the 
substrate $167 \mathrm{db}$ and be able to racemize according to skeletal inversion/rotation of the second 2-naphthyl substituent. If the kinetic dynamic resolution would still take place, then the product of the reaction would be enantioenriched (Figure 27). However, this would strongly depend on the regioselectivity of the reaction.

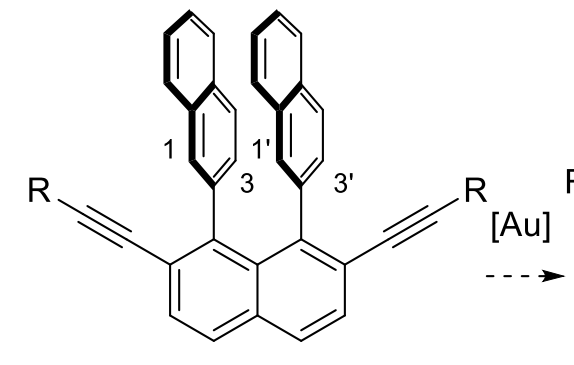

159db: $\mathrm{R}=p$-Tolyl syn/anti $=45: 55$

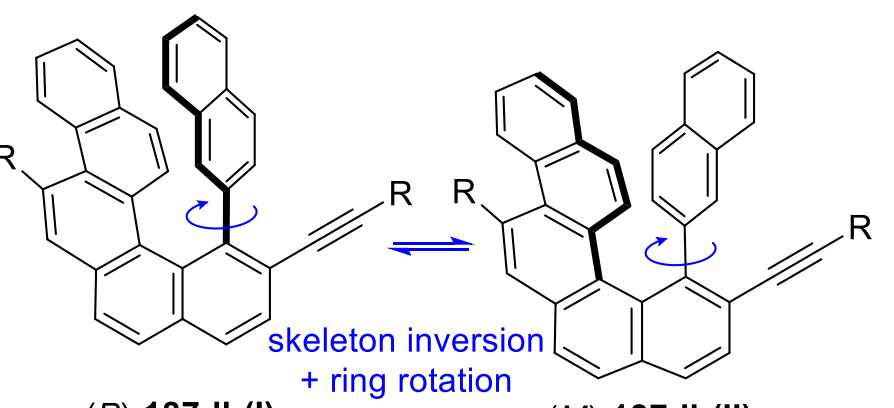

$(P)-187 \mathrm{db}(I)$

$(M)-187 \mathrm{db}(I I)$

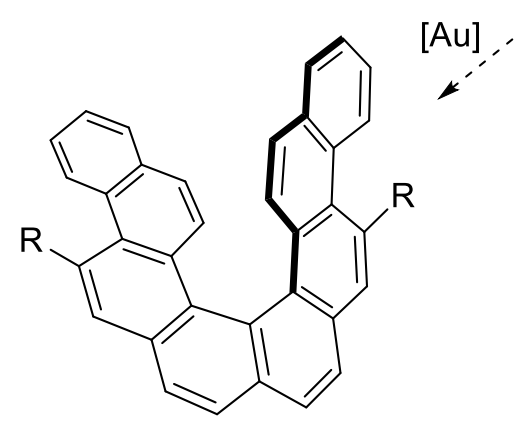

$160 \mathrm{db}$

Figure 27. Postulated racemization process of intermediate $180 \mathrm{db}$, formed after the first gold(I)-catalyzed intramolecular hydroarylation in substrate $167 \mathrm{db}$.

\subsection{Development of a new synthesis of [6]helicene precursors}

The existing synthesis of the helicenes substrates 159 involved nine synthetic steps, functional group interconversions and had a relatively limited scope. Moreover, the variation of the aryl substituents at the 1- and 8-positions of the naphthalene core was difficult, namely due to the necessary installation of these groups early on in the sequence. Despite of this, modulation of the substituents at the periphery of the helicene most probably has a larger impact on their electronic structure and thus on the possible applications of this interesting class of compounds. Therefore, a modified procedure was envisaged according to the retrosynthetic analysis shown in Scheme 43. This is based on previously reported strategies for the synthesis of ortho alkynyl biphenyls reported by Gevorgyan ${ }^{[223]}$ and Lautens. ${ }^{[224]}$ Starting from the commercially available 2,7-dihydroxynaphthalene and following a literature reported regioselective bromination at the 1 and 8 positions of the naphthalene with subsequent triflation of the free hydroxyl groups, a precursor 189 amenable to a chemoselective Sonogashira-Hagihara ethynylation followed by Suzuki-Miyaura coupling 
would be obtained. This approach would be ideally placed to easily modulate the aromatic groups at the 1 and 8 positions of the naphthalene core, enabling the access to a larger scope of diyne substrates. Efforts would be made to incorporate heteroaromatics using this methodology and add heterohelicenes to the list of substrates amenable to this enantioselective hydroarylation.
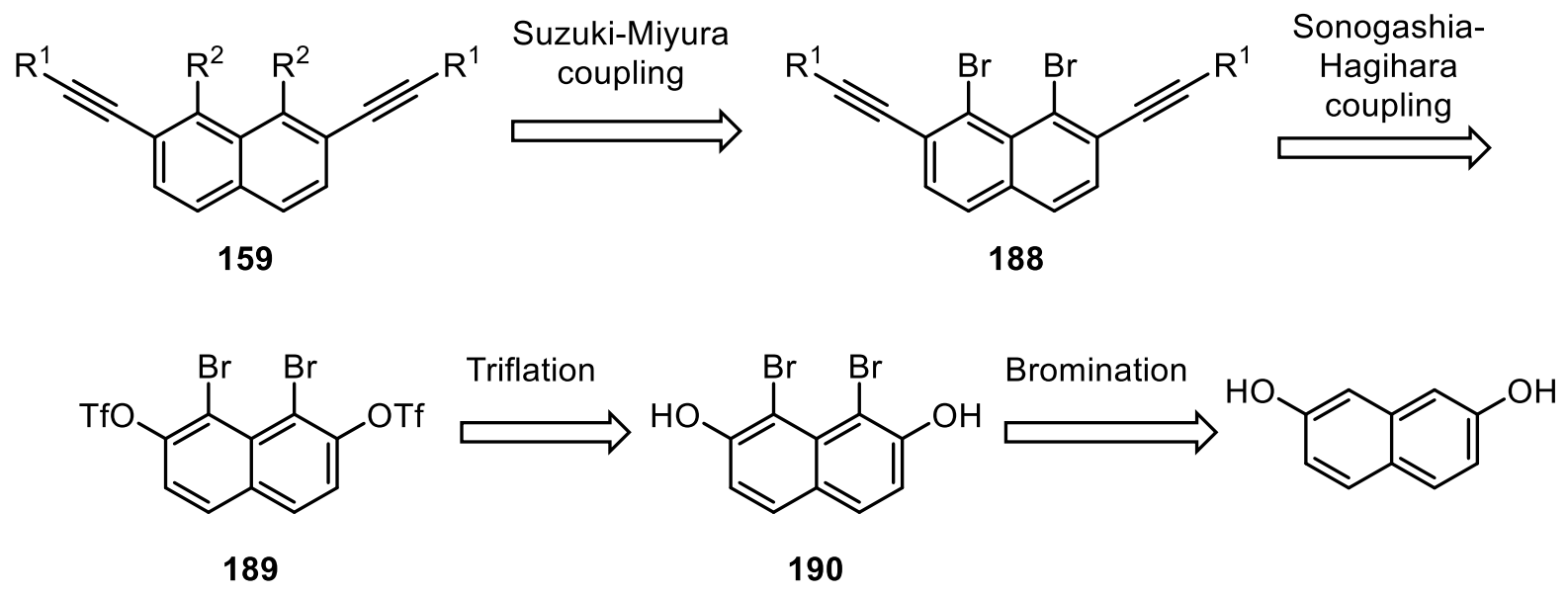

Scheme 43. Disconnection approach to access substrates 159 from 2,7-dihydroxynaphthalene.

The proposed synthesis was started by the selective bromination at the 1- and 8- positions of commercially available 2,7-dihydroxynaphthalene with N-bromosuccinamide (NBS). This had been described in the literature using two sets of conditions, firstly by Whiting, using substoichiometric pyridine in chloroform ${ }^{[225]}$ and later by Pérez, carrying out the reaction in acetonitrile at $10{ }^{\circ} \mathrm{C} .{ }^{[226]}$ Several variations of the conditions described were tried, including changing the order of reagents, method of addition and working with or without the exclusion of air and moisture. The best conditions in this study were found to be by adding NBS as a solid to a solution of 2,7-dihydroxynaphthalene at $10^{\circ} \mathrm{C}$ under argon. This method afforded an approximately $70 \%$ pure product 190 as a mixture with other isomers. Dibromide 190 was reported to be unstable for purification by column chromatography but could be conveniently converted into stable bis-triflate 9 . The latter was obtained in a yield of $26 \%$ over two steps. In spite of a poor yield, low starting material costs and short preparation times mean that larger amounts of this intermediate could be accessed relatively quickly and used in the following steps. 


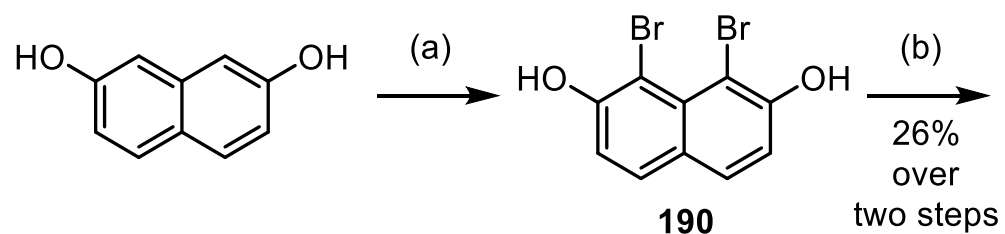<smiles>CCOc1ccc2ccc(Br)c(Br)c2c1Br</smiles>

189

Scheme 44. Two-step sequence to prepare bis-triflate 189. Reagents and conditions: (a) 2,7dihydroxynaphthalene (1.0 equiv.), NBS (2.0 equiv.), $\mathrm{MeCN}, 10^{\circ} \mathrm{C}, 2 \mathrm{~h}$; (b) crude 190 (1.0 equiv.), Tf $\mathrm{T}_{2} \mathrm{O}$ (2.3 equiv.), pyridine (3.0 equiv.), $\mathrm{CH}_{2} \mathrm{Cl}_{2}, 0^{\circ} \mathrm{C}, 30 \mathrm{~min}$.

The Sonogashira-Hagihara coupling proceeded chemoselectively to give the bis-alkynes 188a-c in moderate to good yields, with conservation of the two bromine substituents (Scheme 45). However, upon preparation of TMS-substituted diyne 188a, stirring of reaction mixture for longer than $1 \mathrm{~h}$ periods lead to the formation of additional side products, observable by GC-MS. Curiously, the selective mono-Sonogashira coupling of 189 to give TMS-substituted alkynylnaphthalene 191 could be carried out when using the less active palladium species $\mathrm{PdCl}_{2}\left(\mathrm{PPh}_{3}\right)_{2}$.<smiles>[R]C#Cc1ccc2ccc(C#C[R])c(Br)c2c1Br</smiles>

188a: $\mathrm{R}=\mathrm{TMS}(47 \%)$

188b: $\mathrm{R}=p$-Tolyl $(82 \%)$

188c: $\mathrm{R}=4-\mathrm{MeO}-\mathrm{C}_{6} \mathrm{H}_{4^{-}}(86 \%)$ (a)<smiles>COc1ccc2ccc(Br)c(Br)c2c1Br</smiles>

189 (b)<smiles>Cc1ccc2ccc(O)c(Br)c2c1Br</smiles>

191

Scheme 45. Sonogashira-Hagihara coupling to give alkynes 188a-c and 191. Reagents and conditions: (a) 189 (1.0 equiv.), arylacetylene (8-10 equiv.), $\mathrm{Pd}_{2} \mathrm{Cl}_{2}$ (dppf) (5 mol\%), Cul (10 mol\%), Et ${ }_{3} \mathrm{~N} / \mathrm{DMF}, \mathrm{rt}, 1-4 \mathrm{~h} ;$ (b) 189 (1.0 equiv.), TMS-acetylene (2.0 equiv.), $\mathrm{PdCl}_{2}\left(\mathrm{PPh}_{3}\right)_{2}$ (5 mol\%), Cul (10 mol\%), Et3 $\mathrm{N} / \mathrm{DMF}, \mathrm{rt}, 16 \mathrm{~h}$.

Single crystals of 188b were grown by slowly cooling a hot ethyl acetate solution. The solid state structure is shown in Figure 28. The two bromine atoms, forced into close proximity due to their position on the naphthalene ring, display a large steric repulsion, bending the whole naphthalene core out of the plane and resulting in a torsional angle of $30.0^{\circ}$ between the two bromine atoms. 

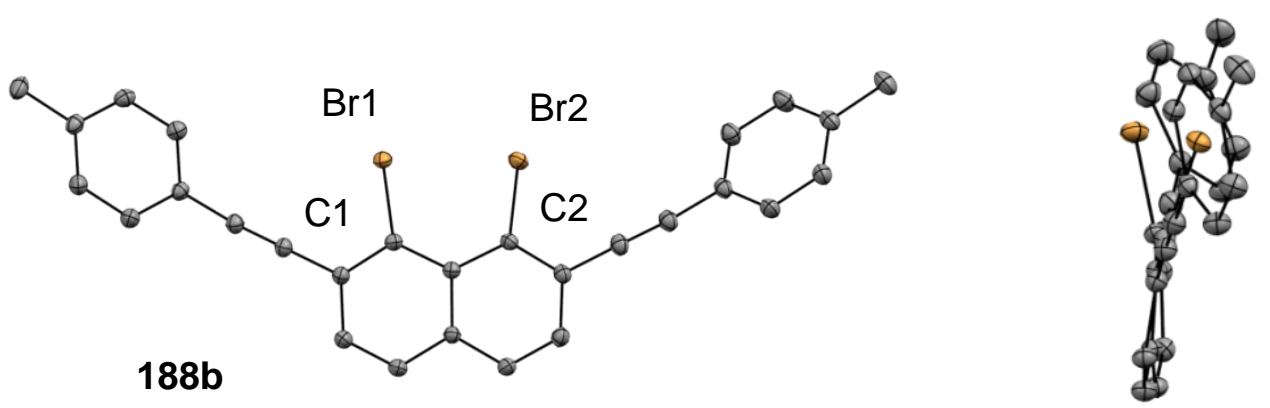

Figure 28. Structure of diyne 188b in the solid state. Solvent molecules and counterions are omitted for clarity. Thermal ellipsoids set at $50 \%$ probability, the numbering does not correspond to the IUPAC rules. Selected bond lengths $(\AA)$ and angles $\left({ }^{\circ}\right)$ : C1-Br1 = 1.897(1), C2-Br2 = 1.889(1), Br1-C1-C2-Br2 = -29.96(7).

Finally, the Suzuki-Miyaura coupling of the precursors 188 was evaluated for a variety of aromatics and heteroaromatics (Table 5).

Table 5. Suzuki-Miyaura coupling with compounds $188 \mathrm{~b}$ and $188 \mathrm{c}$.

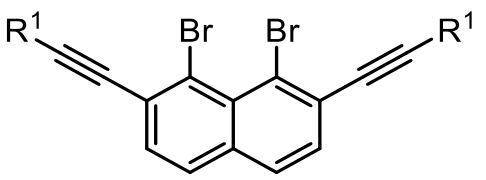

188 (a)<smiles>[R]C#Cc1ccc2ccc(C#C[R])c([R])c2c1[R]</smiles>

159

\begin{tabular}{|c|c|c|c|c|}
\hline Entry & 159 & $\mathrm{R}^{1}$ & $\mathrm{R}^{2}$ & Yield (\%) \\
\hline 1 & $159 \mathrm{eb}$ & p-Tolyl & 2-thienyl & 51 \\
\hline 2 & $159 \mathrm{fb}$ & p-Tolyl & 2-furanyl & 88 \\
\hline 3 & $159 \mathrm{gb}$ & p-Tolyl & 3-thienyl & 57 \\
\hline 4 & $159 \mathrm{hb}$ & p-Tolyl & 3-furanyl & 45 \\
\hline 5 & 159ib & p-Tolyl & 4-TMS- $\mathrm{C}_{6} \mathrm{H}_{4}-$ & 37 \\
\hline 6 & $159 \mathrm{jb}$ & p-Tolyl & $4-\mathrm{BnO}-\mathrm{C}_{6} \mathrm{H}_{4}^{-}$ & 46 \\
\hline 7 & $159 \mathrm{~kb}$ & p-Tolyl & $4-\mathrm{MeOCH}_{2}-\mathrm{C}_{6} \mathrm{H}_{4}-$ & 54 \\
\hline 8 & 159lb & p-Tolyl & 4-Ph- $\mathrm{C}_{6} \mathrm{H}_{4-}$ & 24 \\
\hline 9 & $159 \mathrm{mb}$ & p-Tolyl & $3,5-(\mathrm{MeO})_{2}-\mathrm{C}_{6} \mathrm{H}_{3^{-}}$ & 63 \\
\hline 10 & $159 \mathrm{nb}$ & p-Tolyl & $3,5-\mathrm{Me}_{2}-\mathrm{C}_{6} \mathrm{H}_{3^{-}}$ & 34 \\
\hline 11 & 159ag & 4-MeO- $\mathrm{C}_{6} \mathrm{H}_{4-}$ & $\mathrm{C}_{6} \mathrm{H}_{5}$ & 53 \\
\hline 12 & $159 \mathrm{pg}$ & $4-\mathrm{MeO}-\mathrm{C}_{6} \mathrm{H}_{4-}$ & $p$-Tolyl & 50 \\
\hline 13 & 159ig & 4-MeO- $\mathrm{C}_{6} \mathrm{H}_{4}-$ & 4-TMS- ${ }_{6} \mathrm{H}_{4-}$ & 25 \\
\hline 14 & $159 q g$ & 4- $\mathrm{MeO}-\mathrm{C}_{6} \mathrm{H}_{4-}$ & $4-\mathrm{F}-\mathrm{C}_{6} \mathrm{H}_{4-}^{-}$ & 34 \\
\hline 15 & 159ng & 4-MeO- $\mathrm{C}_{6} \mathrm{H}_{4-}$ & $3,5-\mathrm{Me}_{2}-\mathrm{C}_{6} \mathrm{H}_{3^{-}}$ & 39 \\
\hline
\end{tabular}

Reagents and conditions: (a) 188 (1.0 equiv.), $\mathrm{ArB}(\mathrm{OH})_{2}$ (3.0-4.3 equiv.), $\mathrm{Cs}_{2} \mathrm{CO}_{3}$ (3.0 equiv.), $\mathrm{Pd}_{2}\left(\mathrm{dba}_{3}\right)_{3}(4$ mol\%), Sphos (8 mol\%), $\mathrm{THF} / \mathrm{H}_{2} \mathrm{O}, 60^{\circ} \mathrm{C}, 1-16 \mathrm{~h}$.

This was carried out according to a modified procedure reported by Buchwald for the coupling of bulky participants, ${ }^{[227]}$ although using a ten to one mixture of tetrahydrofuran and 
water as solvent was found to improve conversion. A variety of aromatic and heteroaromatic groups could be incorporated, including thiophenes and furans. For these both, the 2- and 3substituted thienyl- and furanylboronic acids were employed. In addition, para-functionalized aromatics, including 4-trimethylsilyl-, 4-benzyloxy-, 4-methoxymethyl-, 4-phenyl-, 4-methyland 4-fluorophenyl, as well as the meta-substituted 3,5-dimethoxy- and 3-5-dimethylphenyl, could also be installed. Single crystals suitable for X-ray diffraction, could be grown for the products 159eb, 159fb, 159gb and 159ib, confirming their structural identity (Figure 29).

(A)

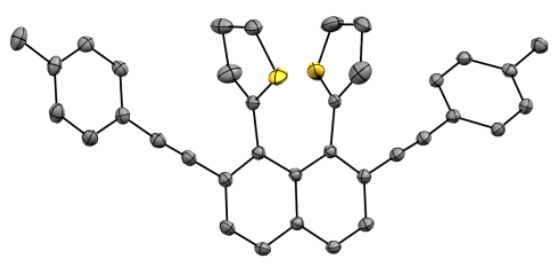

$159 \mathrm{eb}$

(C)

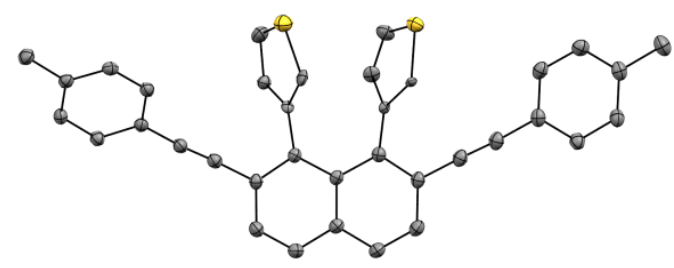

$159 \mathrm{gb}$
(B)

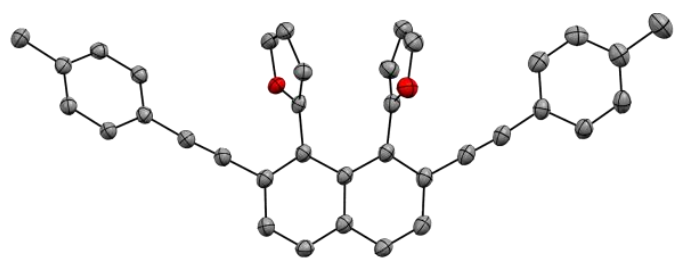

$159 \mathrm{fb}$

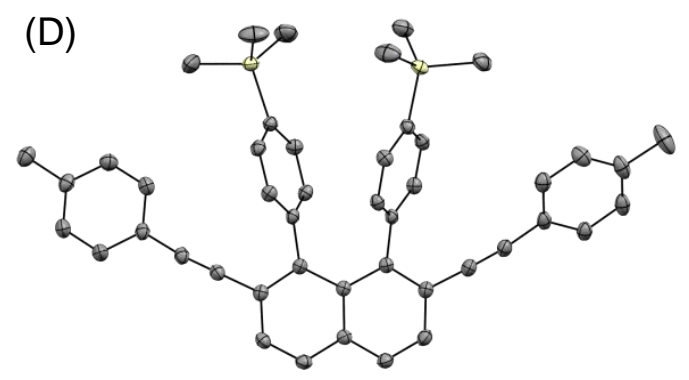

159ib

Figure 29. Solid state structures of compounds 159eb (A), 159fb (B), 159gb (C) and 159ib (D). Solvent molecules and counterions are omitted for clarity. Thermal ellipsoids set at $50 \%$ probability.

Unfortunately, the Suzuki-Miyaura couplings were not completely selective. Despite full conversion being reached in almost all cases, the yields of the transformations remained mostly poor or moderate. No single major side product could be identified in the crude reaction mixtures, although one clue as to the types of additional products formed could be elucidated from the structure of deep red dinaphthopentalene 192, which crystallized from the crude reaction mixture upon preparation of $159 \mathrm{~kb}$ (Figure 30). As they possess only 8 melectrons, pentalenes are formally anti-aromatic. Typically, pentalenes exhibit a relatively small HOMO-LUMO gap, due to the propensity to accept or donate electrons to relieve their anti-aromaticity. ${ }^{[138]}$ While helicenes containing a pentalene subunit, to the best of our knowledge, have not been reported, the incorporation of anti-aromatic subunits into polyaromatic structures such as helicenes has been previously shown to significantly alter their electronic properties. ${ }^{[143,228]}$ 

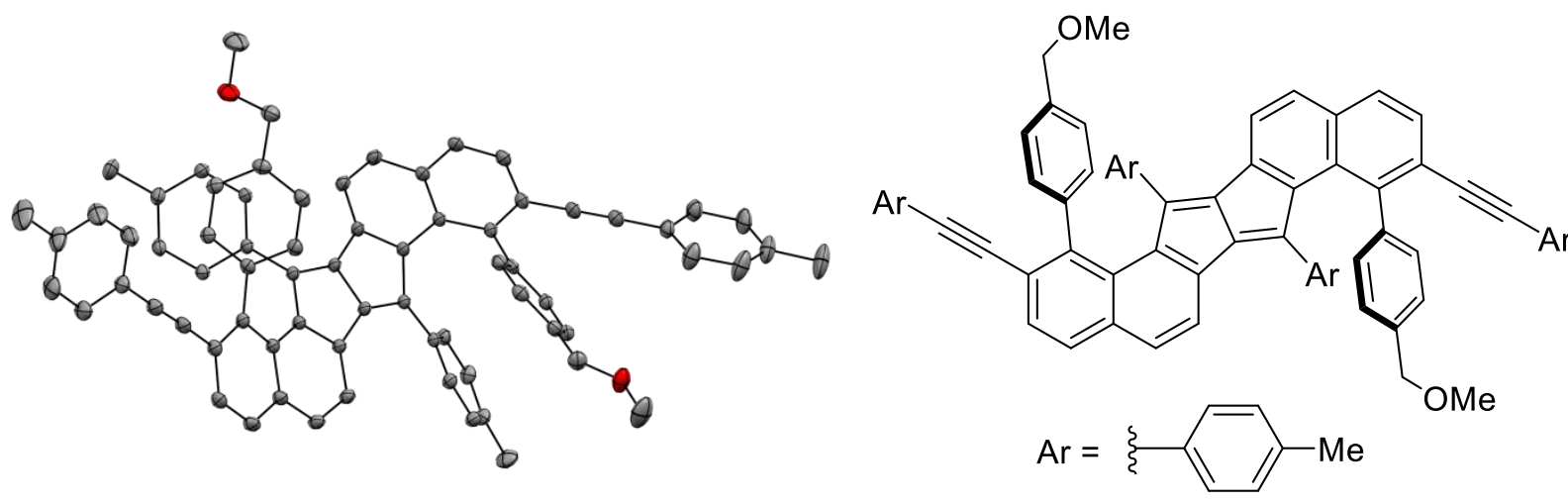

192

Figure 30. Solid state structure of 192. Solvent molecules and counter ions are omitted for clarity. Thermal ellipsoids set at $50 \%$ probability.

The group of Don Tilley has reported the synthesis of dibenzopentalenes via the palladiumcatalyzed homocoupling of ortho-alkynylaryl bromides under reductive conditions at high temperatures, with yields as high as $88 \% .^{[229]}$<smiles>[R]C#Cc1ccccc1Br</smiles>

193 (a)<smiles>[R]C1=C2C(=C([R])c3ccccc32)c2ccccc21</smiles>

194: $\mathrm{R}=\mathrm{Ph}, 4-\mathrm{BBu}-\mathrm{C}_{64^{-}}$, TMS, $p$-Tolyl

Scheme 46. Reductive homodimerization to give dibenzopentalenes. Reagents and conditions: (a) hydroquinone (2.0 equiv), $\mathrm{Cs}_{2} \mathrm{CO}_{3}$ (2.0 equiv), $\mathrm{CsF}$ (2.2 equiv.), $\mathrm{Pd}_{2}(\mathrm{dba})_{3}(1.5 \mathrm{~mol} \%), \mathrm{P}(t \mathrm{Bu})_{3}$ (6 mol\%), 1,4-dioxane, 135 ${ }^{\circ} \mathrm{C}$. 229$]$

Presumably, the product $\mathbf{1 9 2}$ is formed through the mechanism shown below, which is based on the findings of the group of Don Tilley (Scheme 47). Firstly, a Suzuki coupling would take place, giving monobrominated intermediate 195. After oxidative addition to the second carbon-bromine bond, instead of proceeding through a second transmetalation, the $\mathrm{Pd}(\mathrm{II})$ species 196 would undergo a migratory insertion with a second equivalent of the intermediate 195. The vinyl palladium(II) species 197 would then participate in a second migratory insertion, completing the first cyclopentadiene ring and giving intermediate 198. Finally, after oxidative addition to the closely proximal carbon-bromine bond in 198, reductive elimination from the resulting palladium (IV) species 199 would afford the dinaphthopentalene product 192and a palladium(II) species. The low yields of this product 
192 may result from the lack of reductant in the reaction mixture, as the palladium(II) should be reduced back to palladium( 0 ) to continue the catalytic cycle.

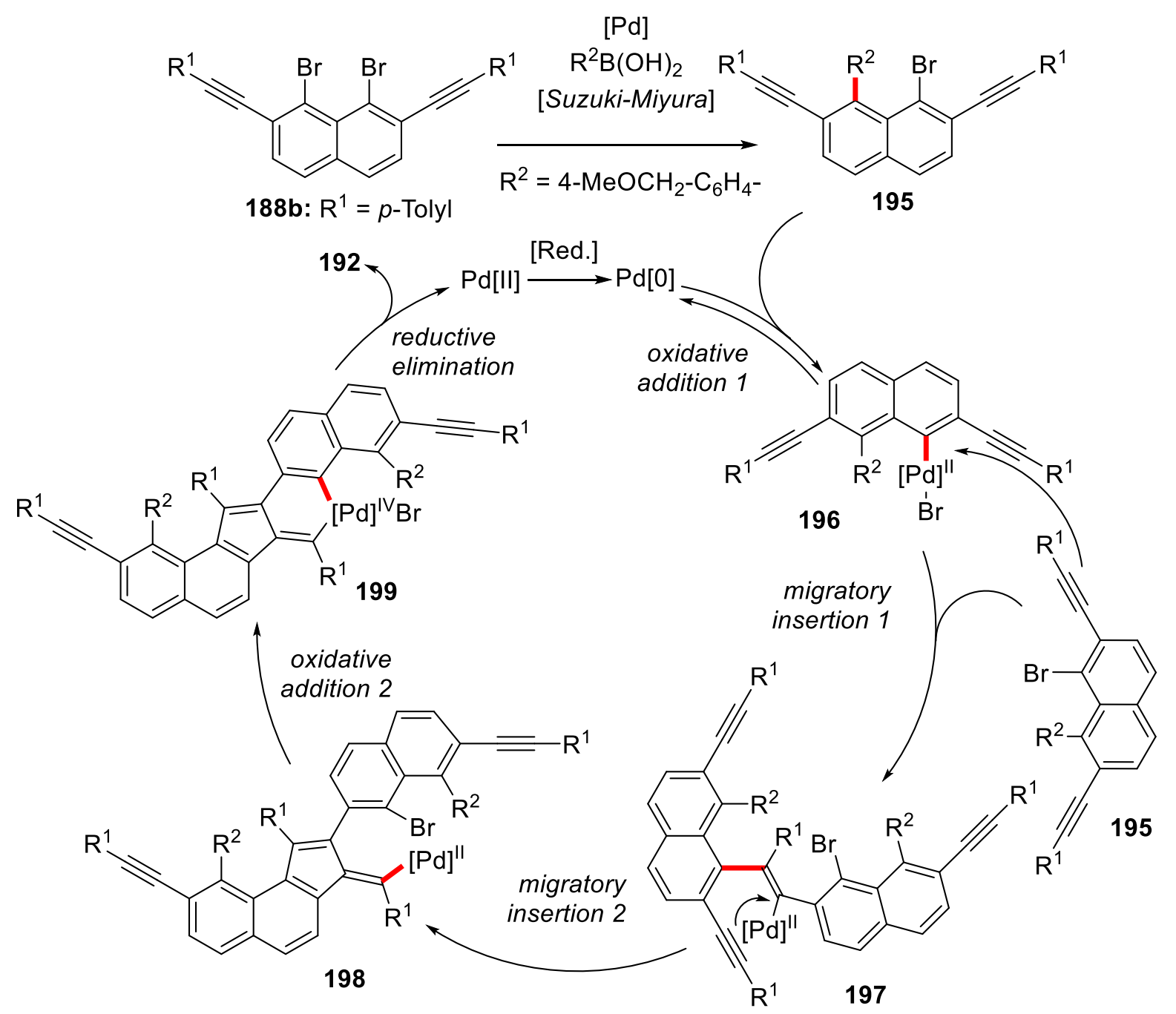

Scheme 47. Proposed mechanism for the formation of compound 192.

The solid-state structure of the product 192 suggests that the molecule may not yet be chiral, as the aromatic substituents in the two bay areas lie parallel to one another. Moreover, it can be considered as a potential substrate for a gold-catalyzed intramolecular hydroarylation, which would give the S-shaped fused double [5]helicene 200 (Scheme 48). The synthesis of fused helicenes and higher order polyaromatic structures has attracted a great deal of attention recently:[140,230,231] however to the best of our knowledge, only one example of an attempted enantioselective synthesis of a fused dihelicene ${ }^{[232]}$ as well as one example of a fused diheterohelicene ${ }^{[208]}$ have been reported up to now. If the compound 192 could be synthesized in better yield, it may offer an exciting entry to this emerging field of research. 
<smiles>[R]C#Cc1ccc2ccc3c(c2c1-c1ccc([R])cc1)-c1c([R])c-3c2ccc3ccc(C#C[R])c(-c4ccc([R])cc4)c3c2c1[R]</smiles>

Scheme 48. Possible transformation of 192 in gold(I)-catalyzed hydroarylation reactions.

\subsection{Summary}

In summary, a variety of new helicene precursors 159 were synthesised. Because the existing synthesis for helicene precursors 159 enabled modulation of the alkyne substituent; a broad range of aromatic substituents were installed using this methodology, incorporating functional groups amenable to further transformations such as ethers, protected phenols, silanes and halogens. The alkyne was additonally brominated and silylated in the interest of further study.

In addition, a new synthetic route to diynes 159 was developed, which significantly lowered the number of synthetic steps required and allowed the introduction of a diverse range of aromatic and heteroaromatic groups directly onto the naphthalene core, which was a challenge when using the existing precursor synthesis. The new synthesis of 159 proceeded through a regioselective bromination of 2,7-dihydroxynaphthalene, followed by triflation, chemoselective Sonogashira-Hagihara coupling and finally Suzuki-Miyaura coupling. The final Suzuki-Miyaura coupling however, gave trace amounts of side products, one of which could be identified as the dinaphtho pentalene 192. This is likely formed via a palladium catalysed, reductive dimerisation reaction, which if it could be optimsied further may provide an entry towards fused dihelicenes with an anti-aromatic core. 


\section{Synthesis and structure of cationic phosphonites}

\subsection{Synthesis of cationic phosphonites from TADDOL derivatives}

Following an interest in further diversifying additional aspects of the cationic phosphonite ligand structure, wider modulation of the cationic substituents of the aromatic groups of the TADDOL subunit, as well as the cyclic or acyclic nature of the TADDOL backbone was targeted. Accordingly, the phenyl-substituted TADDOL $(S, S)-1701,{ }^{[233]}$ its acyclic analogue $(R, R)-\mathbf{1 7 0} \mathbf{q}^{[52]}$ as well as the cyclohexyl-acetal derivative $(R, R)-\mathbf{1 7 0} \mathbf{t}^{[234]}$ were synthesised to systemically evaluate the effect of the backbone on the ligand structure (Scheme 49).

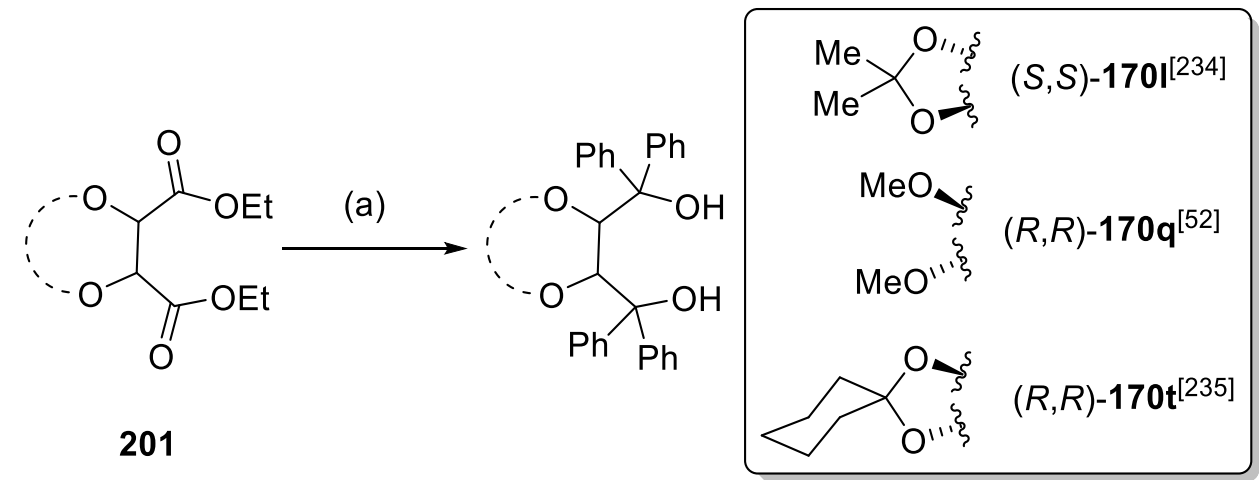

Scheme 49. Targeted TADDOL derivatives bearing different cyclic or acylic substituents at the backbone. Reagents and conditions: (a) $\mathrm{PhMgBr}$ (5 equiv.), THF, reflux, $2 \mathrm{~h}$.

Furthermore, the electron-deficient TADDOL derivative $(R, R)$-170s, decorated with 3,5bis(trifluoromethyl)phenyl substituents, was synthesized in $72 \%$ yield according to a modified literature procedure ${ }^{[186-188]}$ by adding a solution of freshly prepared 3,5-bis(trifluoromethyl)phenylmagnesiumbromide to a solution of $\mathbf{2 0 1 b}$ in tetrahydrofuran and refluxing for 2 hours (Scheme 50).<smiles>CCOC(=O)C(=O)OC</smiles>

201b<smiles>CO[C@@H]([C@@H](OC)C(O)(Br)Br)C(O)(Br)Br</smiles>

$170 \mathrm{~s}$

Scheme 50. Synthesis of TADDOL derivative 170s. Reagents and conditions: (a) 3,5-bis(trifluoromethyl)bromobenzene (5.0 equiv.), $\mathrm{Mg}$ (5.0 equiv.), I (cat.), THF, $70{ }^{\circ} \mathrm{C}, 2 \mathrm{~h}$, then $201 \mathrm{~b}$ (1.0 equiv.), $70{ }^{\circ} \mathrm{C}, 2 \mathrm{~h}$.

A variety of new cationic groups were proposed, comprising a variety of electronic and steric environments (Scheme 51). Accordingly, the carbodiphosphorane $(\mathbf{2 0 2}),{ }^{[235,236]}$ and the $\mathrm{N}$ - 
heterocyclic carbenes $\operatorname{IPr}(\mathbf{5 6 a})$, IMes (56b), ${ }^{[237]} \mathrm{Me} / \mathrm{IPr}(\mathbf{5 6 d}),{ }^{[238]}{ }^{\mathrm{C}} \mathrm{IMes}(\mathbf{5 6 e})^{[239]}$ and MeIMes $(\mathbf{5 6 f})^{[240]}$ were prepared in gram scale following established literature procedures.

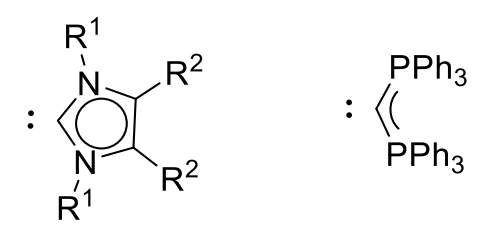

$$
\begin{aligned}
& \text { 56a: } R^{1}=\text { Mes, } R^{2}=H \\
& \text { 56b: } R^{1}=\operatorname{Dipp}, R^{2}=H \\
& \text { 56d: } R^{1}=i \operatorname{Pr}, R^{2}=M e \\
& \text { 56e: } R^{1}=\text { Mes, } R^{2}=C l \\
& \text { 56f: } R^{1}=\text { Mes, } R^{2}=M e
\end{aligned}
$$

Scheme 51. Carbenes 56 and carbodiphosphorane 202 targeted for the synthesis of cationic phosphonites.

Attention then turned towards the synthesis of the cationic phosphonite ligands themselves. The new ligands were all prepared by condensation of the chlorophosphites with the carbenes or carbodiphosphorane, followed by ion exchange to hexafluoroantimonate, in analogy to the synthesis of phosphonites $169 a-i, k$ described in the second chapter of this thesis. Chlorophosphites $171 \mathrm{l}$ and $\mathbf{1 7 1 m}$ were prepared according a procedure originally reported by Fürstner; ${ }^{[52]}$ by heating suspensions of the TADDOL derivative, trichlophosphine, triethylamine and molecular sieves $4 \AA$ in toluene, followed by filtration to remove the triethylammonium chloride by-product and concentration in vacuo. However, as discussed above, it was found that the carbenes 56 used in the condensation were susceptible to protonation by residual amounts of triethylammonium chloride, which is known to be partially soluble in many organic solvents and thus difficult to completely remove from the crude chlorophosphites 171. Nevertheless, the carbodiphosphorane-derived cationic phosphines $169 \mathrm{I}$ and $169 \mathrm{~m}$ could be prepared in this fashion (Scheme 52). The formation of the new species was clear in the ${ }^{31} \mathrm{P}$ NMR, showing a characteristic doublet at 20.4-20.6 ppm and a triplet at 175.1-179.5 ppm, with coupling constants of 91.8 (169l) and $100.9(\mathbf{1 6 9 m}) \mathrm{Hz}$. Phosphonite 169 l could be easily purified by simply recrystallizing the crude mixture from dichloromethane and pentane after ion exchange. In the case of $169 \mathrm{~m}$, formation of the ligand was slow, and full conversion of the chlorophosphite was only reached on addition of two equivalents of carbodiphosphorane 201 and heating to $60^{\circ} \mathrm{C}$. To our satisfaction, after ion exchange to the hexafluoroantimonate anion, $169 \mathrm{~m}$ could be purified by column chromatography and isolated in a $32 \%$ yield. 


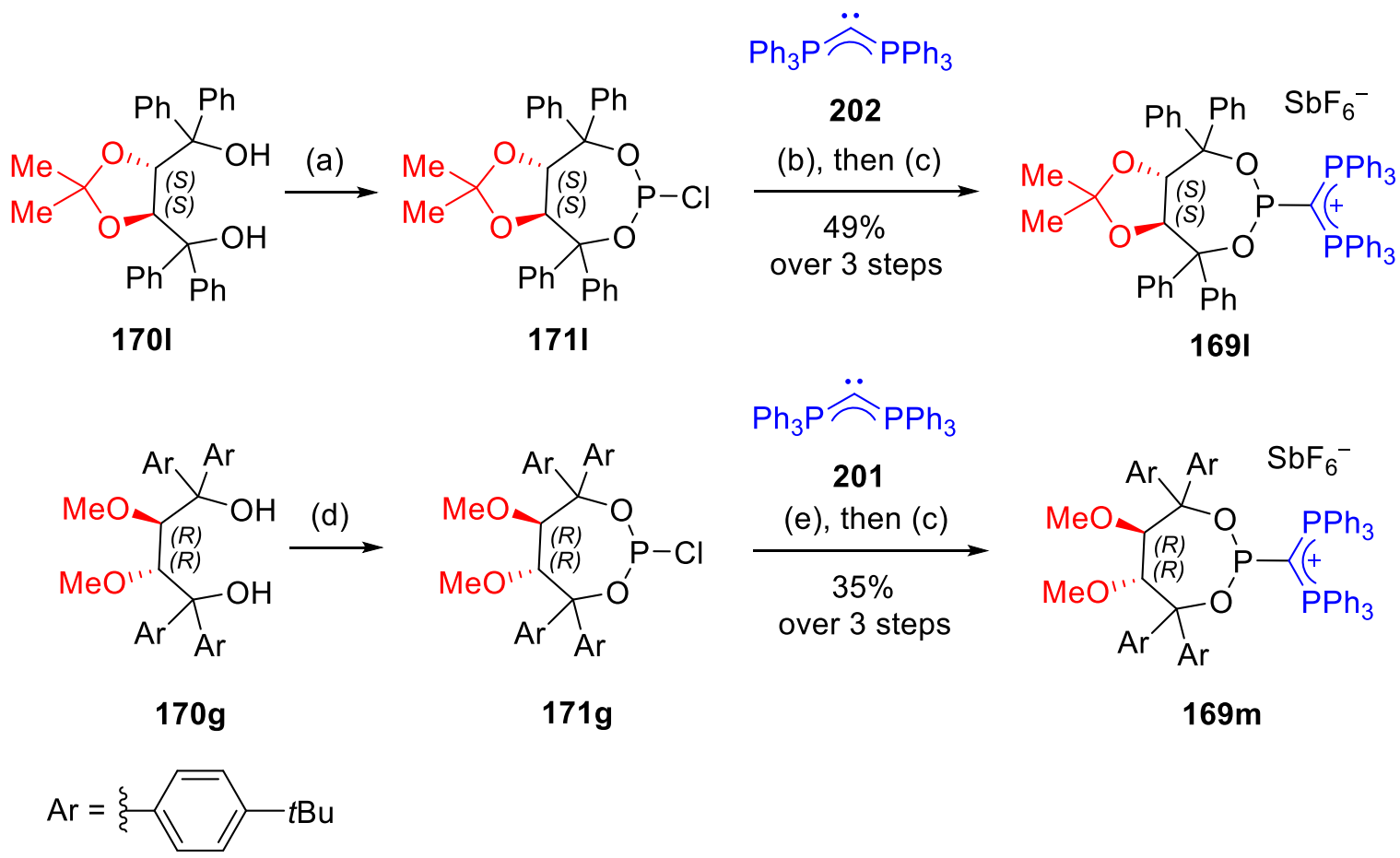

Scheme 52. Synthesis of cationic phosphonites $169 \mathrm{I}$ and $169 \mathrm{~m}$. Reagents and conditions: (a) $170 \mathrm{l}$ (1.0 equiv.), $\mathrm{PCl}_{3}$ (1.1 equiv.), NEt3 (2.2 equiv.), THF, $-78^{\circ} \mathrm{C}, 1 \mathrm{~h}$, then rt, 1h; (b) 202 (1.0 equiv.), THF, rt, $16 \mathrm{~h}$; (c) $\mathrm{NaSbF} 6$ (excess), MeCN, rt, $16 \mathrm{~h}$; (d) $\mathbf{1 7 0 g}$ (1.0 equiv.), $\mathrm{PCl}_{3}$ (1.2 equiv.), $\mathrm{NEt}_{3}$ (3.0 equiv.), toluene, $0^{\circ} \mathrm{C}$, then $60^{\circ} \mathrm{C}, 1 \mathrm{~h}$; 202 ( 0.9 equiv.), THF, rt, 24 h, 202 ( 0.9 equiv.), $60^{\circ} \mathrm{C}, 4 \mathrm{~h}$.

The ligands $169 n-t$ could be prepared in moderate to very good yields (Scheme 53), following the previously discussed optimisations outlined in the second chapter of this thesis. The formation of the ligand $169 p$ with larger IPr carbene moiety and bearing the bulky biphenyl substituents at the TADDOL backbone proved to proceed slower and gave poor conversion; however, adding the carbene to the chlorophosphite at $-40{ }^{\circ} \mathrm{C}$, followed by stirring the mixture for a further $24 \mathrm{~h}$ after allowing to warm up to room temperature enabled $169 p$ to be isolated in a yield of $40 \%$ after column chromatography. Similarly, due to the electron poor nature of the dichlorocarbene $56 \mathrm{e}$, longer reaction times were necessary to achieve full conversion of the chlorophosphite in the synthesis of the ligands $169 \mathrm{u}$ and $169 \mathrm{t}$. Interestingly, no conversion was detected when conducting this reaction in the absence of sodium hexafluoroantimonate. This might indicate pre-activation of the chlorophosphite by sodium cationic in the reaction mixture, facilitating nucelophilic attack by the carbene. In all cases a characteristic shift at between 138.1 and $153.0 \mathrm{ppm}$ in the ${ }^{31} \mathrm{P}$ NMR indicated formation of the ligand. 
5. Synthesis and structure of cationic phosphonites

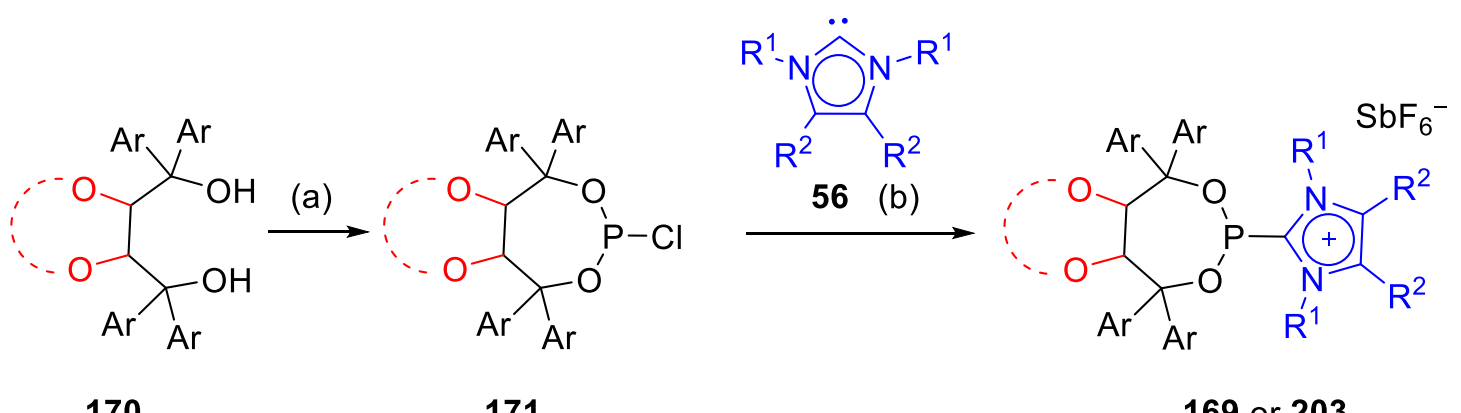

170

171

169 or 203

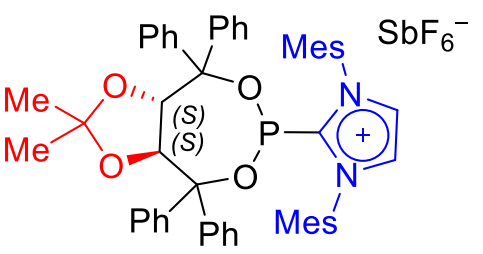

169n: $69 \%$

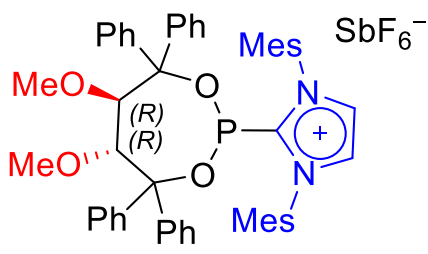

169q: $79 \%$

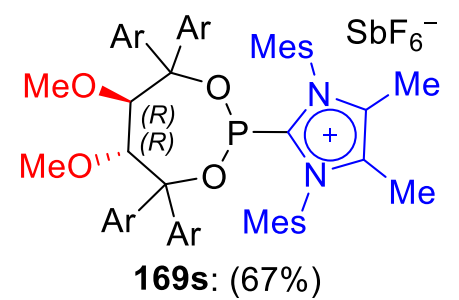<smiles>FC(F)(F)c1ccc(C=[Te])cc1</smiles><smiles>Cc1c(-c2ccccc2)c(P2OC(c3ccccc3)(c3ccccc3)C(C)(F)[C@H]3OC(C)(C)O[C@H]32)n(C(C)C)c1C</smiles>

169o: $51 \%$<smiles></smiles>

169r: $66 \%$

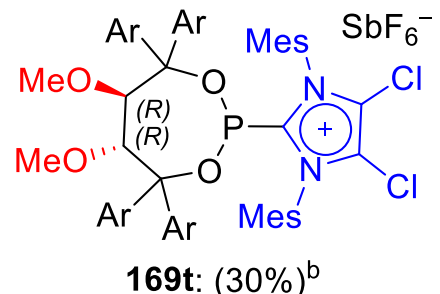<smiles>FC(F)(F)c1ccc(C=[Te])cc1</smiles>

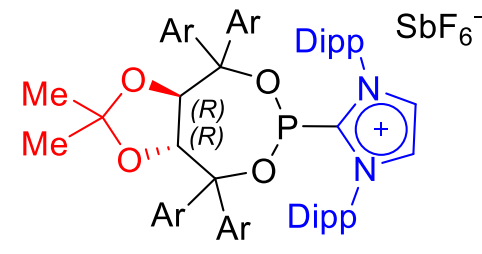

169p: $41 \%^{a}$<smiles>[Al]C(=[Al])c1ccc(-c2ccccc2)cc1</smiles>

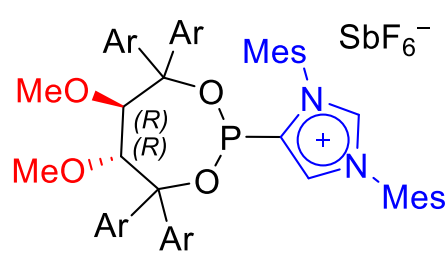

203a: $29 \%$<smiles>FC(F)(F)c1cc(C=[Zn])cc(C(F)(F)F)c1</smiles>

$\mathrm{CF}_{3}$

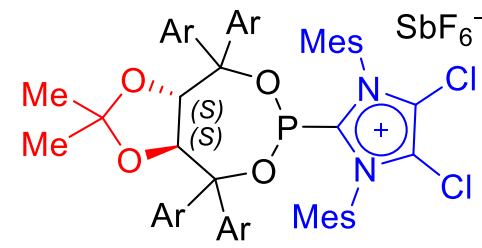

169u: $(31 \%)^{\mathrm{C}}$<smiles>CC(C)(C)c1ccc(C=[Te][Te])cc1</smiles>

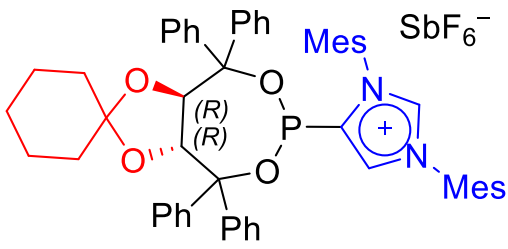

203b: $21 \%$

Scheme 53. Synthesis of cationic phosphonites $169 \mathrm{n}-\mathbf{u}, 202 \mathrm{a}$ and $202 \mathrm{~b}$ according to optimized protocol. Reagents and conditions: (a) diol 170 (1.0 equiv.), $\mathrm{PCl}_{3}$ (1.0-2.0 equiv.), pyridine (3.0-3.2 equiv.), toluene, $60{ }^{\circ} \mathrm{C}$,

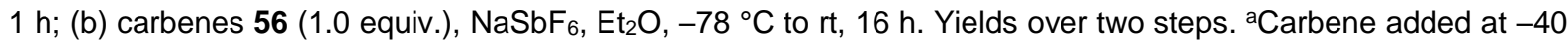
${ }^{\circ} \mathrm{C}$ and after allowing warming to rt stirred for another $24 \mathrm{~h}$. ${ }^{\mathrm{b}}$ After warming to room temperature, stirred for a further $48 \mathrm{~h}$ at ambient temperature. ${ }^{c}$ After warming to room temperature, stirred for a further $24 \mathrm{~h}$ at this temperature.

Single crystals suitable for X-ray structure determination of compounds $169 q$ and $169 r$ (Figure 31) were grown by slow diffusion of pentane into dichloromethane solutions. In 169q, disorder about the phosphorus center can be seen, indicating that two conformational 
isomers exist in the solid state. Because of the $\mathrm{C}_{2}$ symmetry of the structure however, the symmetry of the structure is independent with respect to this orientation. The P1a-C1 [1.864(4) $\AA$ ] and P1b-C1 [1.976(5) $\AA$ ] bond lengths are slightly longer than in previously published cationic phosphines with imidazolium substituents $\left(1.813-1.840 \AA\right.$ A.$^{[67,78,79]} \mathrm{A}$ slightly decreased $\mathrm{P} 1-\mathrm{C} 1$ bond length $[1.837(5) \AA]$ in $169 \mathrm{r}$ are in better agreement with the latter values. In both ligands, the phosphorus retains a pyramidal geometry (sum of angles for $169 q=298.1^{\circ}$; for $169 r=291.9^{\circ}$ ), although the steric shielding of the bis(mesityl)imidazolium substituent in $169 q$ is higher and the phosphorus atom in $169 \mathrm{r}$ is less shielded from the side of the cationic substituent.

(A)

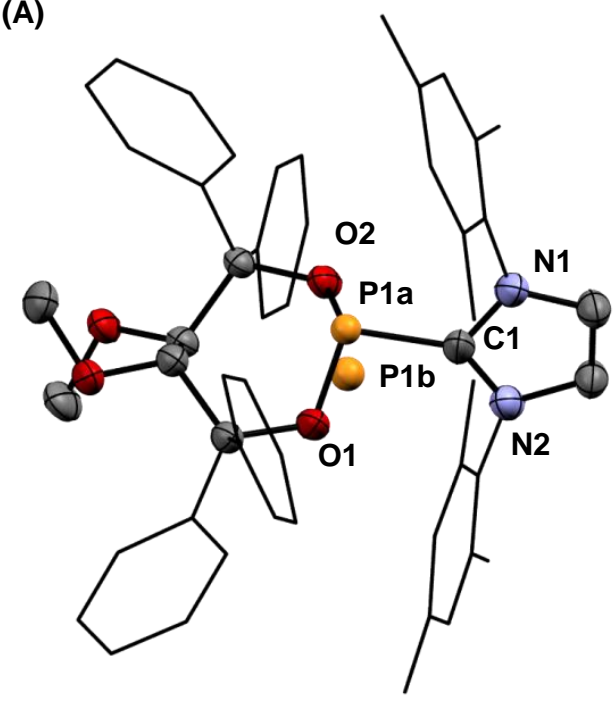

$169 q$
(B)

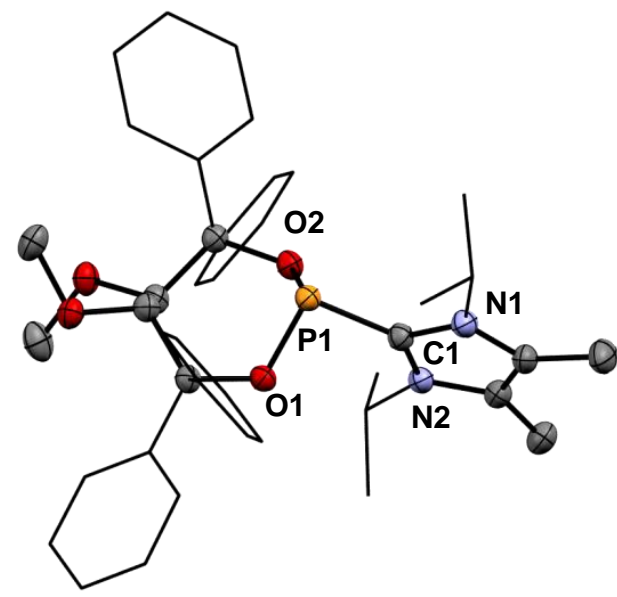

$169 \mathrm{r}$

Figure 31. Molecular structures of compounds 169q (A) and 169r (B). Counterions and solvent molecules are omitted for clarity. Thermal ellipsoids at $50 \%$ probability. Peripheral arene rings displayed as capped sticks for clarity. Selected bond distances $(\AA ̊):(A) \mathrm{P} 1 \mathrm{a}-\mathrm{C} 1=1.864(4), \mathrm{P} 1 \mathrm{~b}-\mathrm{C} 1=1.976(5), \mathrm{P} 1 \mathrm{a}-\mathrm{O} 1=1.595(3), \mathrm{P} 1 \mathrm{~b}-\mathrm{O} 1=$ $1.500(4), \mathrm{P} 1 \mathrm{a}-\mathrm{O} 2=1.582(3), \mathrm{P} 1 \mathrm{~b}-\mathrm{O} 2=1.447(5), \mathrm{N} 1-\mathrm{C} 1=1.332(5), \mathrm{N} 2-\mathrm{C} 1=1.340(5)$; (B) $\mathrm{P} 1-\mathrm{C} 1=1.837(5)$, $\mathrm{P} 1-\mathrm{O} 1=1.633(3), \mathrm{P} 1-\mathrm{O} 2=1.634(3), \mathrm{N} 1-\mathrm{C} 1=1.367(6), \mathrm{N} 2-\mathrm{C} 1=1.358(6)$; sum of angles around phosphorus $\left({ }^{\circ}\right):(\mathrm{A}) \mathrm{P} 1 \mathrm{a}=298.1, \mathrm{P} 1 \mathrm{~b}=309.6$; $(\mathrm{B}) \mathrm{P} 1=291.9$.

Interestingly the products of the reactions between the IMes 56b and chlorophosphites 171t, with a 1,1'-cyclohexyl linked backbone and 171s, with bulky 3,5-bis(trifluoromethyl)phenyl substituents, exhibited quite different ${ }^{1} \mathrm{H}$ NMR spectra when compared with the series of other synthesized ligands. In both cases, characteristic ${ }^{31} \mathrm{P}$ NMR shifts in the same range were seen $(\mathbf{2 0 3 a}=145.9 ; \mathbf{2 0 3} \mathbf{b}=143.1 \mathrm{ppm})$; however, whereas the two protons of the imidazolium backbone for the other IMes derivatives gave a single singlet at $7.78 \mathrm{ppm}(2 \mathrm{H})$ (169n) and 7.73 ppm (2H) (169q) in the ${ }^{1} \mathrm{H}$ NMR, in the ligand 203a two singlets at $8.85 \mathrm{ppm}$ and $8.83 \mathrm{ppm}$, each integrating to one proton, were observable. HSQC measurements could also correlate both of these signals with two signals in the ${ }^{13} \mathrm{C}$ NMR at quite different chemical shifts (141.7 ppm and 130.5 ppm). The former shift lays in the range normally 
associated with the carbon adjacent to phosphorus in the other IMes derivatives (169n: $\left.145.3 \mathrm{ppm}, \mathrm{d}, J_{C-P}=64.2 \mathrm{~Hz} ; 169 \mathrm{q}: 145.4 \mathrm{ppm}, J_{C-P}=66.4 \mathrm{~Hz}\right)$. Definitive proof of the structure of $203 \mathrm{a}$ could be confirmed by the analysis of single crystals by X-ray crystallography (Figure 32); the appropriate single crystals were grown by pentane diffusion into a dichloromethane solution.

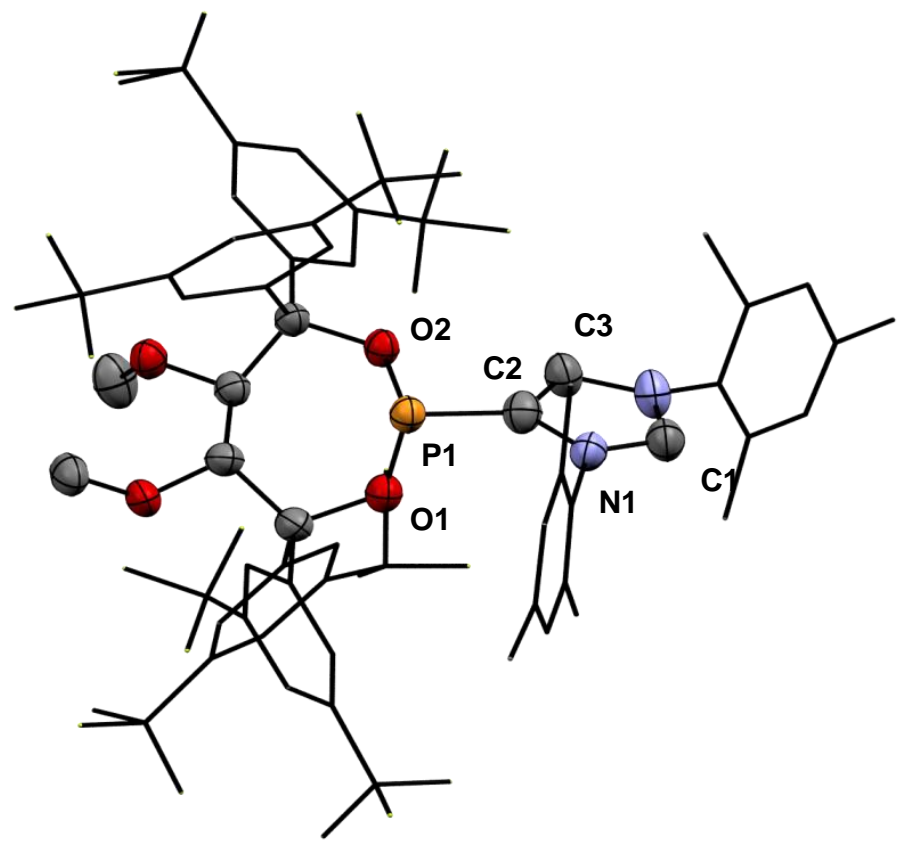

203a

Figure 32. Molecular structure of compound 152a. Counterions and solvent molecules omitted for clarity. Thermal ellipsoids at $50 \%$ probability. Peripheral arene rings displayed as capped sticks for clarity. Selected bond lengths $(\AA)$ : $\mathrm{P} 1-\mathrm{C} 2=1.807(8), \mathrm{P} 1-\mathrm{O} 1=1.641(5), \mathrm{P} 1-\mathrm{O} 2=1.631(5), \mathrm{C} 2-\mathrm{C} 3=1.35 \mathrm{X}(1), \mathrm{N} 1-\mathrm{C} 1=1.394(1)$; Sum of angles around phosphorus $=286.7^{\circ}$.

As can be seen, the connectivity of the imidazolium fragment to the phosphorus occurs not through the $\mathrm{C} 1$ carbon, but rather the $\mathrm{C} 2$ carbon (Figure 32). This would explain the observed discrepancies in the NMR spectra, as both methyne carbons of the imidazolium ring would be expected to lie in different environments and give different chemical shifts. When comparing the solid state structures of $169 q, 169 r$ and $203 a$, a shortening of the P1C2 bond length in 203a is discernible [1.807(8) $\AA$ ]; this may be because of the specific features of electronic density of the abnormally bound cationic substituent. The first abnormal carbene was synthesized by Bertrand. ${ }^{[241]}$ Calculations on its structure described this class of carbenes to be stronger $\sigma$-donors compared to their $\mathrm{NHC}$ analogues, with the free lone pair comprised of a higher s-character. This stronger $\sigma$-donor ability should increase the strength of the resulting carbon phosphorus bond and would result in a shortening of $\mathrm{P}-\mathrm{C}$ bond length in 203a. 
Considering previous reports by Bertrand ${ }^{[68]}$ and Weigand ${ }^{[76,77]}$ into the base mediated interconversions of NHC's, it is possible that the abnormal phosphonites 203a and 203b are formed via mechanisms related to those proposed by Bertrand and Weigand, with the IMes carbene 56b acting as external base (Scheme 54). Firstly the adduct 169 would form via the condensation between $\mathbf{5 6 b}$ and chlorophosphites $171 \mathrm{~s}$ or 171t. In the absence of any other external base other than an excess of carbene $\mathbf{5 6 b}$, the IMes would transiently deprotonate the backbone of the adduct $\mathbf{1 6 9}$ to give the mesoionic carbene 204 and the imidazolium salt 56b. $\mathrm{HX}\left(\mathrm{X}=\mathrm{Cl}^{-}\right.$or $\left.\mathrm{SbF}_{6}{ }^{-}\right)$. The mesoionic carbene 204 would then react with another equivalent of 169, giving IMes $56 \mathrm{~b}$ and the bis-adduct 205 . Finally, reaction with one more equivalent of the IMes $56 \mathrm{~b}$ or adduct 169 would regenerate the adduct 203 , as well as $56 \mathrm{~b}$ or 169 , continuing the cycle.

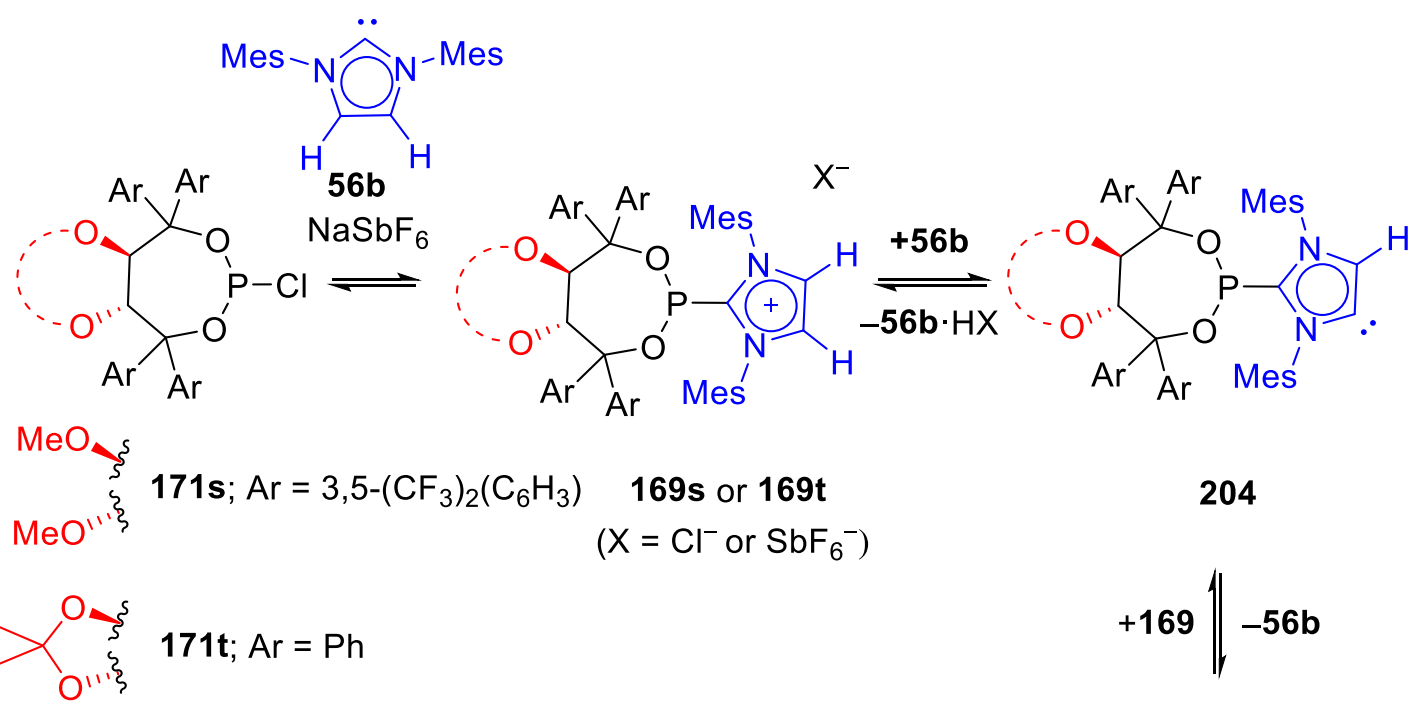

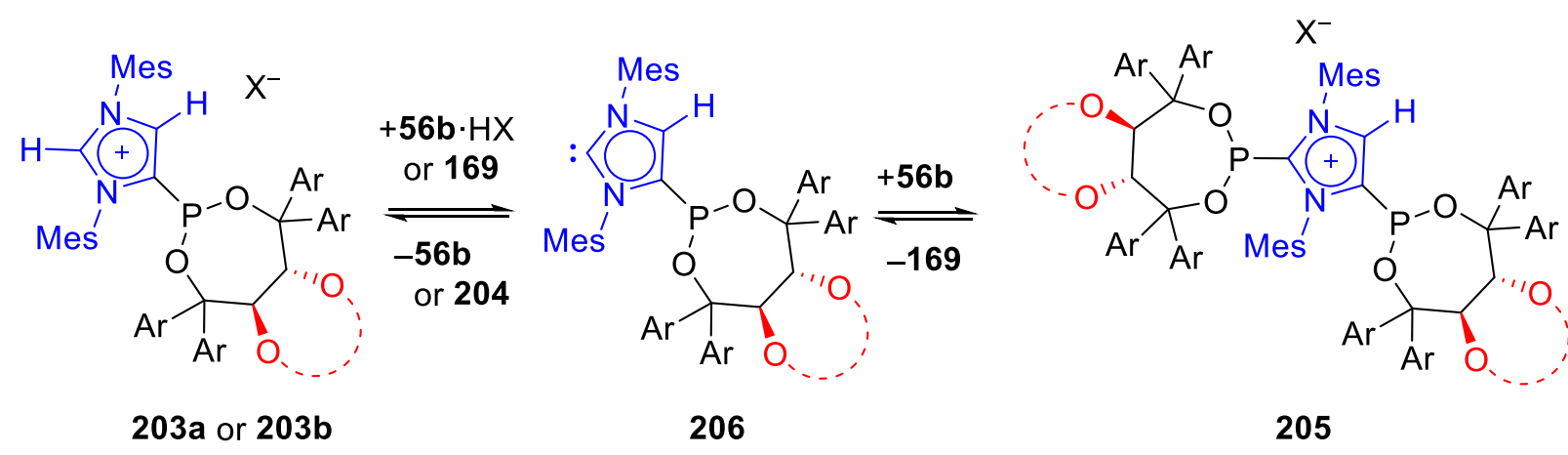

Scheme 54. Proposed mechanism for the formation of cationic phosphonites 203a and $\mathbf{2 0 3 b .}$

It is likely that these species exist in equilibrium with each other, as has also been proposed by Weigand. ${ }^{[76,77]}$ The higher $\sigma$-donor character of the mesoionic carbene substructure would confer a higher stability to the abnormal carbene adduct 204. Presumably, the backwards reaction, i.e. displacement of the abnormal carbene adduct 204 from the bis-adduct 205 by the carbene $\mathbf{5 6 b}$, would be less favorable, thus driving the reaction forward. 
The only difference between the TADDOL precursors $(S, S)-\mathbf{1 7 0 I},(R, R)-\mathbf{1 7 0} \mathbf{q}$ and $(R, R)-\mathbf{1 7 0 t}$ is the backbone linker. The cyclohexyl acetal linker in $(R, R)$-170t should confer a higher conformational rigidity to the corresponding chlorophosphite 171t, which would likely then result in a higher steric clash between the TADDOL and IMes subunits, potentially driving the formation of the 4-adduct instead. Similarly, the 3,5-bis(trifluoromethyl)phenyl substituents in the TADDOL $(R, R)-170$ s should also be quite sterically demanding. Furthermore, it is interesting that in both cases, the abnormal adducts were almost exclusively formed and could be readily purified. Nevertheless, it is still not clear why in other cases, when using sterically demanding systems such as those used in the synthesis of the ligands $169 p$ and $169 \mathrm{e}$, the normally expected C-2 adducts were exclusively formed instead.

\subsection{Synthesis of BINOL-derived phosphonites}

2,2'-Diphenylsubstituted diol 207, prepared according to a procedure by Jørgensen, ${ }^{[242]}$ was considered as a suitable precursor to evaluate the synthesis of BINOL-derived cationic phosphonites. When 207 was reacted with carbene 56b under the standard conditions, it was evident in the ${ }^{1} \mathrm{H}$ NMR data that two structurally related species, both containing imidazolium and BINOL fragments, were formed in almost equal amounts (Scheme 55). After careful purification and analysis, the structures of C-2 adduct 208a and C-4 adduct 208b could be elucidated. In analogy to the ligands 203a and 203b, this was achieved by assignment on the basis of $\{\mathrm{C}-\mathrm{H}\}$ interactions in the imidazolium ring through $2 \mathrm{D}$ NMR experiments and comparison of the chemical shifts.

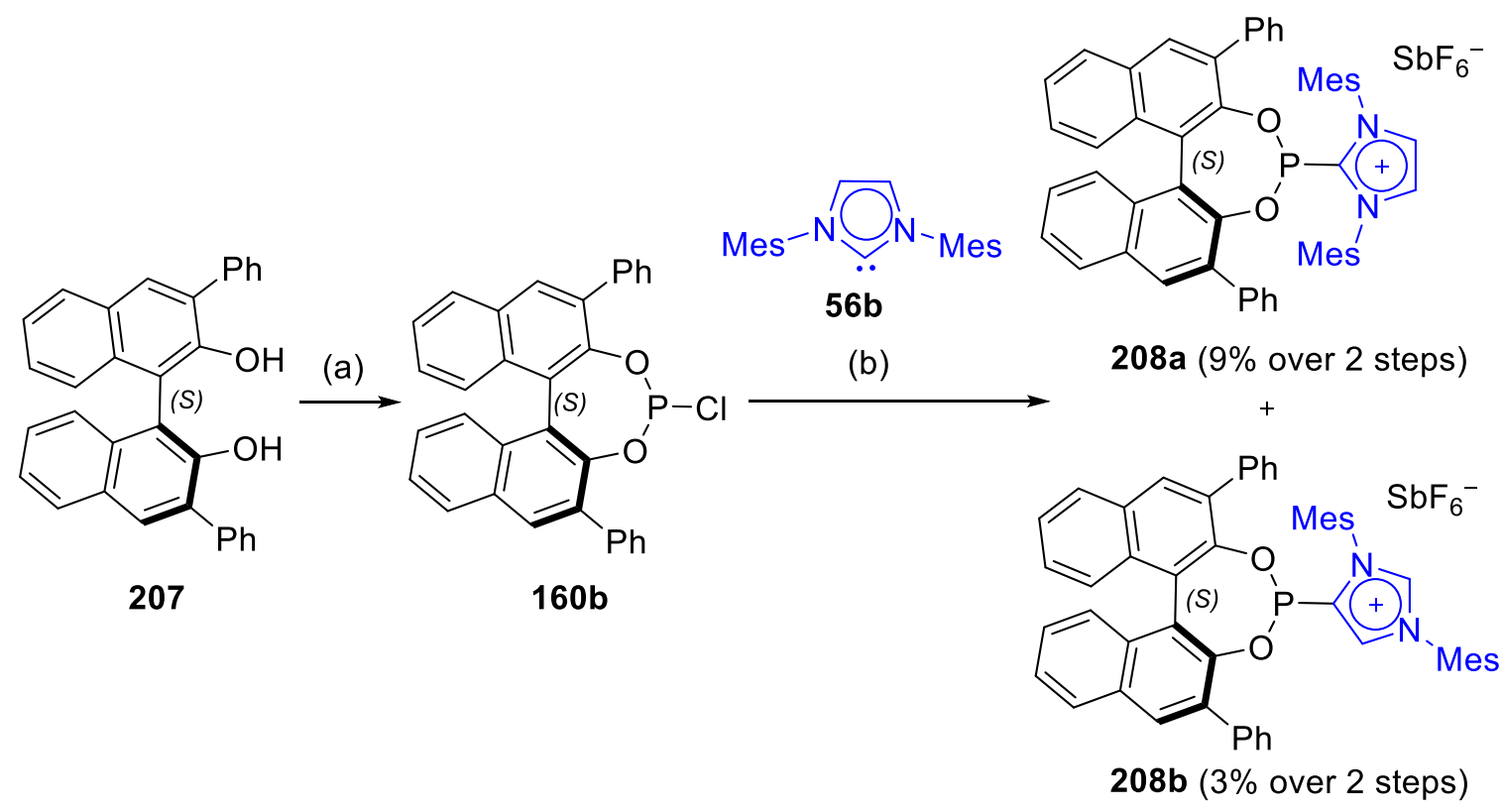

Scheme 55. Synthesis of compounds 208a and 208b. Reagents and conditions: (a) 207 (1.0 equiv.), $\mathrm{PCl}_{3}(1.5$ equiv.), pyridine (3.0 equiv.), toluene, $60^{\circ} \mathrm{C}, 1 \mathrm{~h}$; (b) $56 \mathrm{~b}$ (1.0 equiv.), $\mathrm{NaSbF}_{6}$ (3 equiv.), $\mathrm{Et}_{2} \mathrm{O},-78^{\circ} \mathrm{C}$ to rt, $16 \mathrm{~h}$. 
Additionally, single crystals of 208a suitable for X-ray diffraction could be grown by layering toluene over a solution in dichloromethane, unambiguously confirming its structure (Figure 33). Again, the C1-P1 bond length of $1.890(2) \AA$ is longer than would be normally expected for cationic phosphines with an imidazolium substituent. ${ }^{[67,78,79]}$ The phosphorus also retains a pyramidal geometry (sum of angles $=304.2^{\circ}$ ).

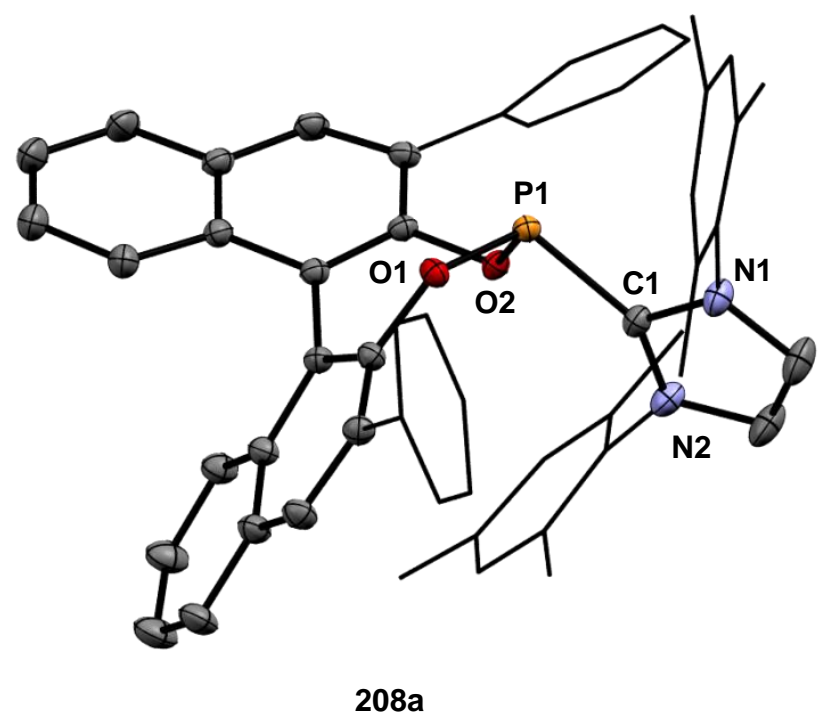

Figure 33. Molecular structure of compound 208a. Counterions and solvent molecules are omitted for clarity. Peripheral arene rings displayed as capped sticks for clarity. Thermal ellipsoids at $50 \%$ probability. Selected bond lengths $(\AA)$ : $\mathrm{P} 1-\mathrm{C} 1=1.890(2), \mathrm{P} 1-\mathrm{O} 1=1.621(1), \mathrm{P} 1-\mathrm{O} 2=1.631(1), \mathrm{N} 1-\mathrm{C} 1=1.358(2), \mathrm{N} 2-\mathrm{C} 1=1.354(2)$; degree of pyramidalization $\left(^{\circ}\right)=304.2$.

In analogy to ligands $203 a$ and $203 b$, these two products should be formed accordingly to the mechanism proposed in Scheme 54. However, in contrast to ligands 203a and 203b, where the C-4 adducts were almost exclusively formed and could be easily purified, a 1:1 mixture of both C-2 (208a) and C-4 (208b) products was obtained, which severely complicated their isolation. In order to avoid this problem, the reactivity of the carbene participant was blocked by using its 4,5-dimethyl derivative MeIMes (56f). ${ }^{[240]}$ Accordingly, this carbene was subjected to the optimised conditions with chlorophosphite 160b. In this case, no rearrangements were observed and the corresponding BINOL-derived cationic phosphonite 208c could be prepared in a dramatically improved yield of $73 \%$ (Scheme 56 ). 
<smiles>Oc1c(-c2ccccc2)cc2cc(-c3ccccc3)cc3ccccc3c1-2</smiles>

207 (a)<smiles>ClP(Cl)Oc1c(-c2ccccc2)cc2ccccc2c1-c1c(-c2ccccc2)cc2ccccc2c1-c1ccccc1</smiles><smiles></smiles>

$73 \%$ over 2 steps<smiles></smiles>

208c

Scheme 56. Synthesis of ligand 208c. Reagents and conditions: (a) diol 207 (1.0 equiv.), $\mathrm{PCl}_{3}$ (1.1 equiv.), pyridine ( 3.2 equiv.), toluene, $60^{\circ} \mathrm{C}, 1 \mathrm{~h}$; (b) $56 f$ ( 0.95 equiv.), $\mathrm{NaSbF}_{6}$ (3 equiv.), $\mathrm{Et}_{2} \mathrm{O},-78^{\circ} \mathrm{C}$ to rt, $16 \mathrm{~h}$.

Single crystals of 208c suitable for X-ray diffraction could be grown by layering a dichloromethane solution with toluene. A comparison of bond lengths shows a very strong similarity to 208a (Figure 30 and Figure 29), albeit with the exception of the P1-C1 bond length, which is notably shorter for the latter [1.890(2) $\AA$ in 208c vs. 1.849(6) $\AA$ in 208a].

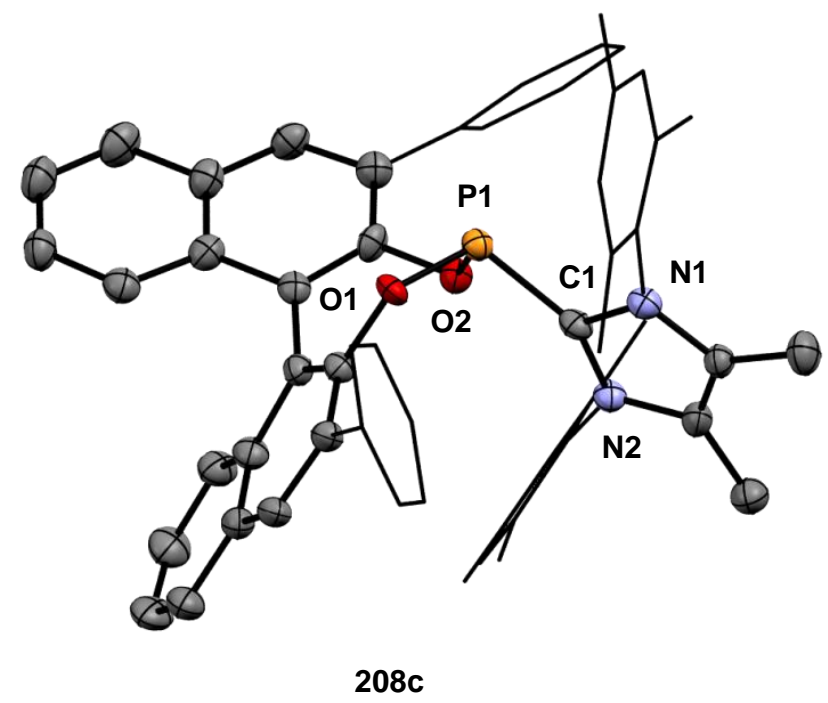

Figure 34. Molecular structure of 208c. Counterions and solvent molecules are omitted for clarity. Thermal ellipsoids at $50 \%$ probability. Peripheral arene rings displayed as capped sticks for clarity. Selected bond lengths $(\AA): \mathrm{P} 1-\mathrm{C} 1=1.849(6), \mathrm{P} 1-\mathrm{O} 1=1.620(4), \mathrm{P} 1-\mathrm{O} 2=1.638(4), \mathrm{N} 1-\mathrm{C} 1=1.365(7), \mathrm{N} 2-\mathrm{C} 1=1.364(7)$; degree of pyramidalization $\left(^{\circ}\right)=303.0$.

With a series of structurally varied cationic phosphonites in hand, the donor properties and coordination chemistry were investigated.

\subsection{Electronic properties}

The Chauvin group had previously described the calculated IR stretching frequencies for a series of cationic phosphines and phosphonites bearing imidazolium substituents. ${ }^{[72]}$ Based on these reports, cationic phosphonites with imidazolium substituents would be expected to be extremely good $\pi$-acceptors and weak $\sigma$-donors, lying approximately between dicationic 
77c and tricationic 82a, due to the cumulative electron withdrawing effect of the imidazolium and two neighboring oxygen atoms. Unfortunately, attempts to coordinate $169 \mathrm{n}$ to $[\mathrm{RhCl}(\mathrm{cod})]_{2}$ and $\left[\mathrm{IrCl}(\mathrm{cod})_{2}\right]_{2}$ were unsuccessful. The donor capacity of the chiral cationic phosphonites 169l, 169o-q and 169b was therefore probed by measuring their oxidation potentials using cyclic voltammetry. The results are summarized together with other illustrative examples in Table 6.

The imidazolium substituted 169o, 169p and 169q all undergo irreversible oxidations in the range of $1.76-1.88 \mathrm{~V}$, which is located close to the oxidation potential of the monocationic imidazoyl/imidazolium phosphine $\mathbf{8 4 a}$ and in between potentials of dicationic and tricationic phosphines 77c and 82a. The ligand 203a exhibits a slightly higher irreversible oxidation potential of $1.93 \mathrm{~V}$, which is likely a consequence of the electron-withdrawing 3,5bis(trifluoromethyl)phenyl substituents at the TADDOL backbone. This appears to off-set the expected higher $\sigma$-donor capacity of the abnormally bound imidazolium fragment. Conversely, the carbodiphosphorane-derived ligand 169l displays a significantly lower irreversible oxidation potential of $0.94 \mathrm{~V}$, which is in line with the ylidic nature of the carbon atom adjacent to the phosphorus.

Despite exhibiting high oxidation potentials, the solid state structures of the cationic phosphonites all showed a phosphorus center that was pyramidal, implying the existence of an active lone pair. Phosphorus-based ligands which exhibited similarly high oxidation potentials, such as tricationic phosphine $\mathbf{8 2 a}$, did not readily coordinate gold(I). ${ }^{[243]}$ It is possible that coulombic repulsion between the cationic ligands and the cationic gold atoms could have an additional influence. Being only monocationic, it was therefore more probable that this cationic phosphonite ligand family would readily coordinate metal centers. Additionally, previously observed arene-gold(I) interactions for TADDOL-based phoramidites would likely also favour coordination. ${ }^{[61]}$ 
5. Synthesis and structure of cationic phosphonites

Table 6. Oxidation and reduction potentials measured using cyclic voltammetry.

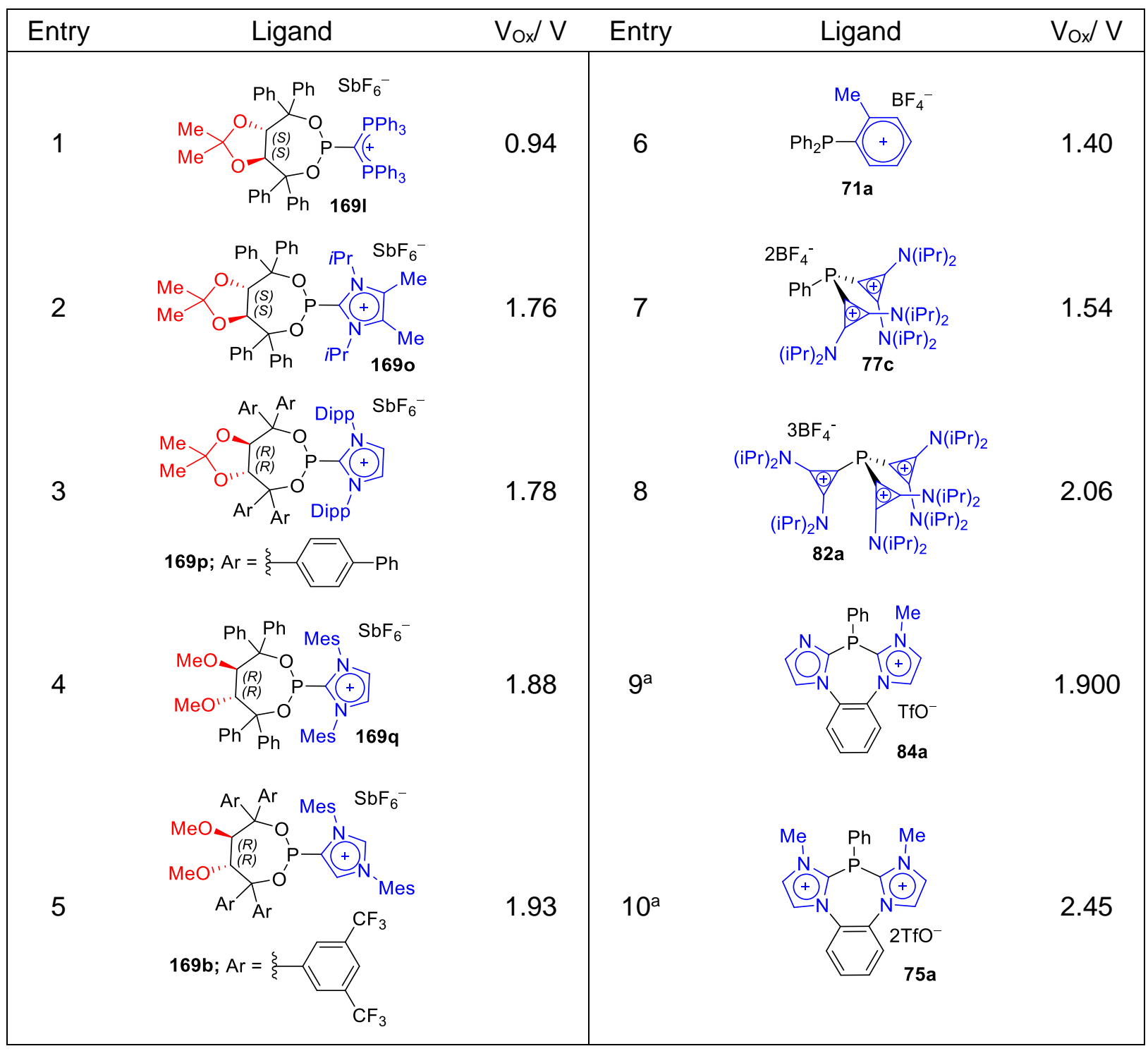

All voltammograms were measured using a $\mathrm{NBu}_{4} \mathrm{PF}_{6}$ electrolyte solution $\left(0.1 \mathrm{M}\right.$ in $\left.\mathrm{CH}_{2} \mathrm{Cl}_{2}\right)$ in the solvents indicated. Oxidation potentials were referenced to the ferrocene/ferrocenium oxidation potential. Unless otherwise

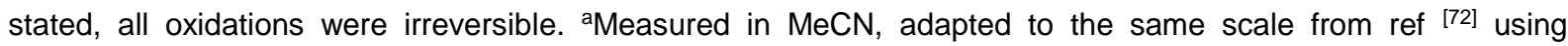
conversion tables listed in ref. ${ }^{[100]}$

\subsection{Coordination chemistry}

Fortunately, the gold $(\mathrm{I})$ complexes of both TADDOL derived ligands 169I-o, 169q, 169s-u, 203a,b and the BINOL-derived 164c could be prepared in good to excellent yields by adding (dimethyl sulfide)gold(I) chloride to dichloromethane solutions of the ligands at $-20{ }^{\circ} \mathrm{C}$ (Scheme 57). Formation of the desired gold(I) complexes was confirmed by ${ }^{31} \mathrm{P} N M R$, indicated by shifts to $132.5 \mathrm{ppm}$ (t, $J=38.2 \mathrm{~Hz}$ ) and $129.1 \mathrm{ppm}$ (t, $J=35.5 \mathrm{~Hz}$ ) for carbodiphosphorane derived complexes 172I,m and 106.8-112.8 ppm for imidazoliumderived complexes 172I-o,q,s-u, 209a,b, and 210a. 
5. Synthesis and structure of cationic phosphonites

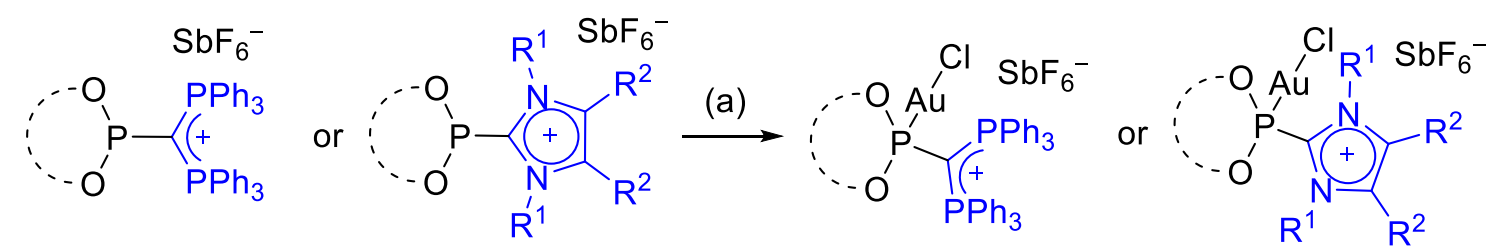

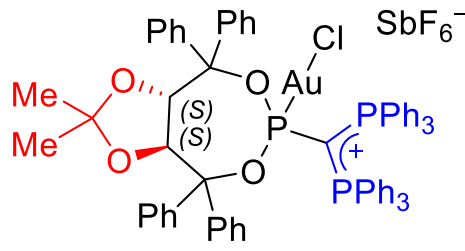

172I; (>99\%)

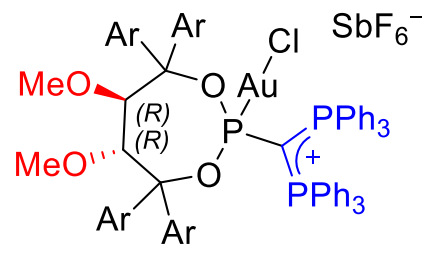

$172 \mathrm{~m} ;(99 \%)^{\mathrm{a}}$<smiles>CC(C)(C)c1ccc(C=[Te])cc1</smiles>

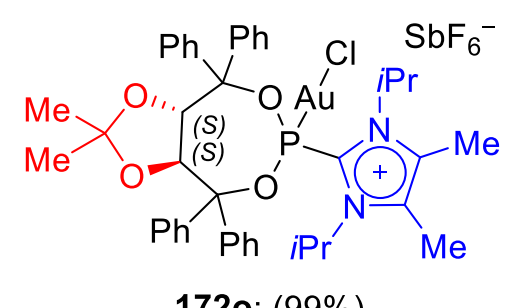

1720; (99\%)

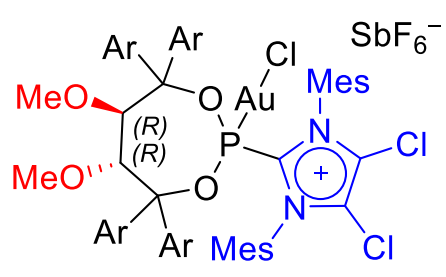

172t: $(92 \%)$<smiles>FC(F)(F)c1ccc(C=[Zn])cc1</smiles>

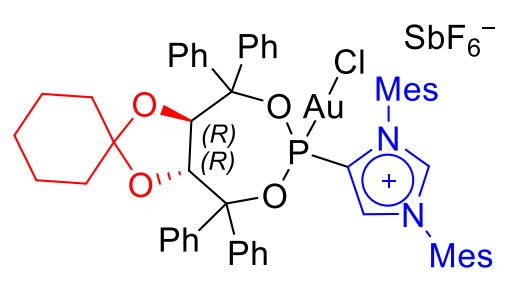

209b; (97\%)

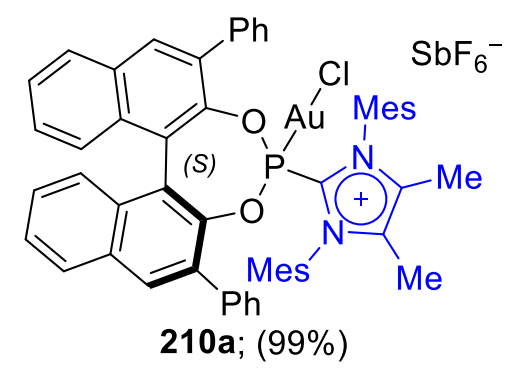

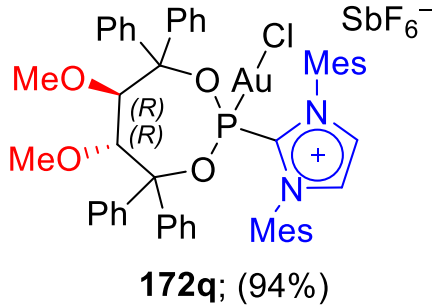
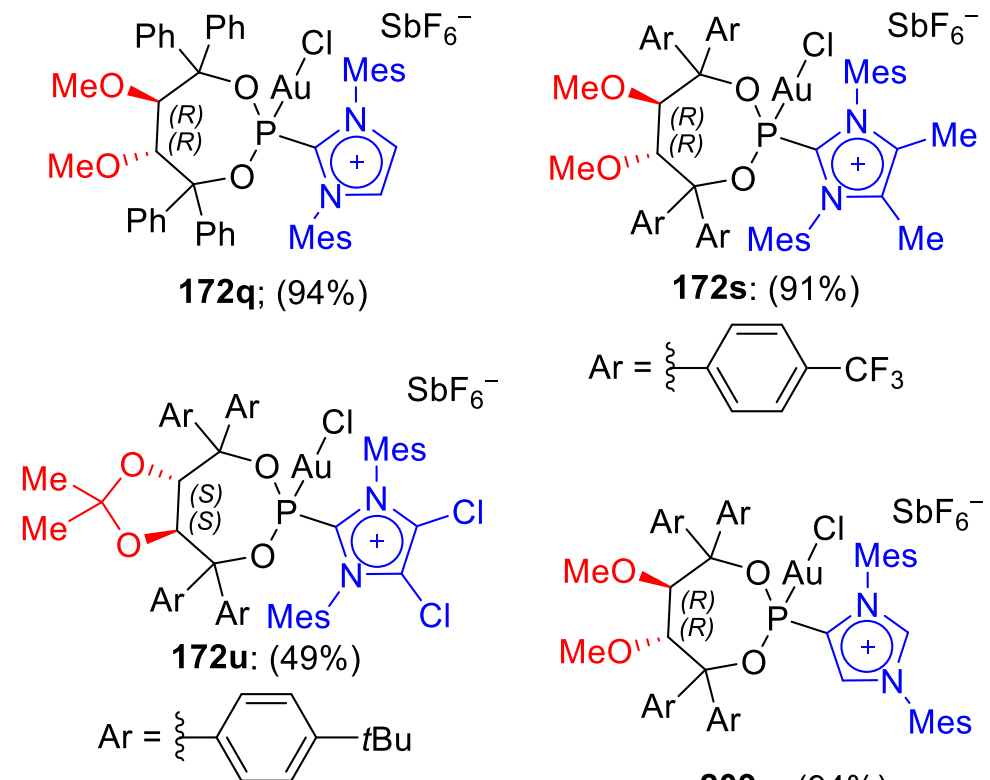

209a; (94\%)

Scheme 57. Synthesis of gold complexes 172I-0, 172q, 172s-u, 209a,b and 210a. Reagents and conditions: (a)

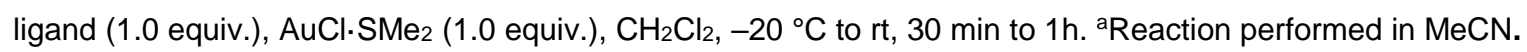

Single crystals of gold(I) complexes 172q, 209a, 172s and 172t for X-ray structure determinations (Figure 35) were grown by layering pentane over dichloromethane solutions. 
A comparison of selected bond lengths, including those in precatalyst $\mathbf{1 7 2} \mathbf{i},{ }^{[219]}$ is given in Table 7.

(A)

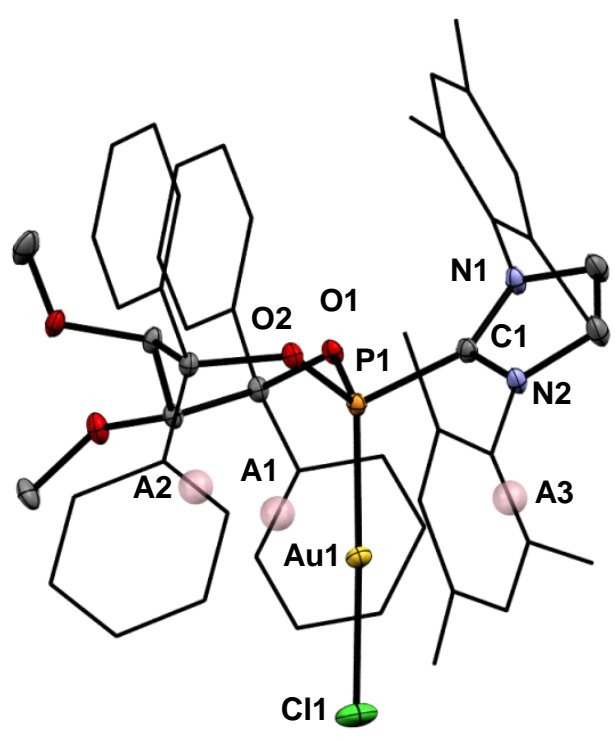

$172 q$

(C)

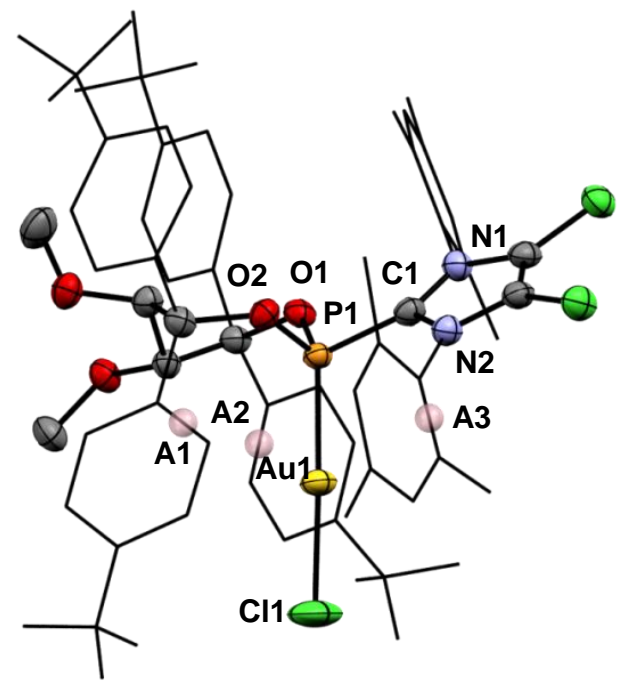

$172 t$
(B)

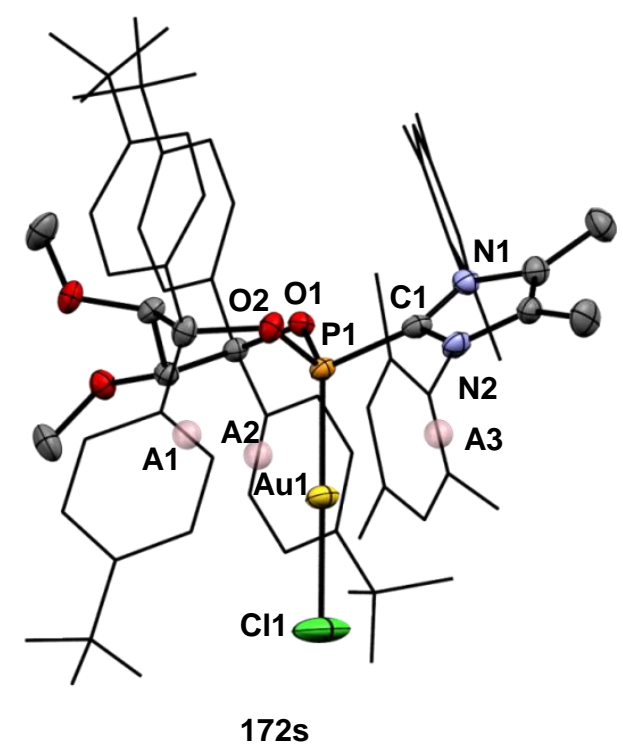

(D)

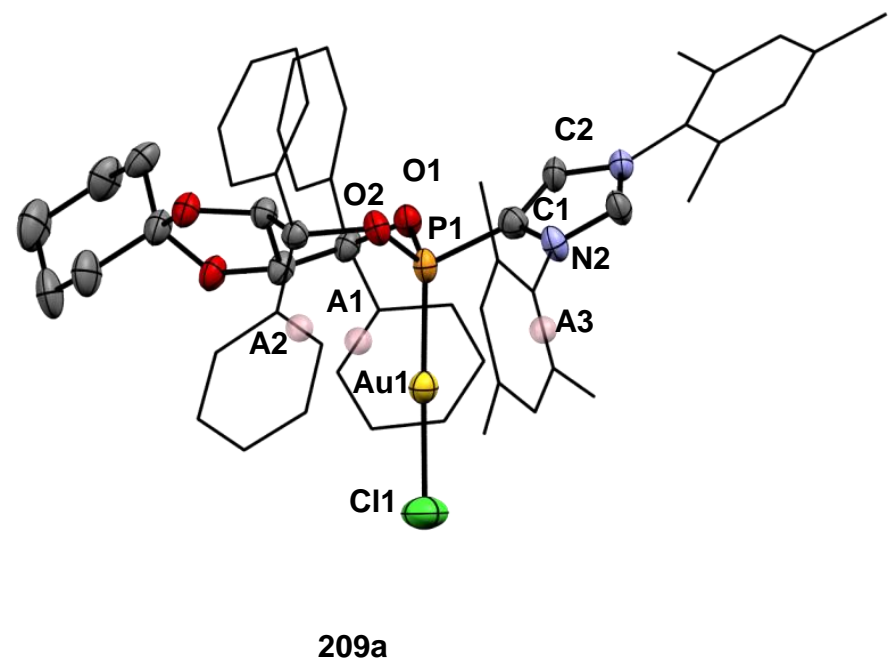

Figure 35. Molecular structures of gold $(\mathrm{I})$ complexes 172q, 209a, 172s and 172t. Solvent molecules and counter ions are omitted for clarity. Thermal ellipsoids set at 50\% probability. Points A1-A3 depict closest arene gold contacts. Peripheral aromatic rings depicted as wire frames for clarity. The numbering does not correspond to the IUPAC rules.

The solid state structure of all the gold(I) complexes shows the gold atom embedded in a deep chiral pocket, enclosed by two arene rings from the TADDOL backbone and one from the mesityl fragment of the imidazolium with a pseudo $C_{3}$ symmetry. This arrangement bears a structural similarity to the TADDOL-derived phosphoramidite gold complexes developed by the group of Fürstner, which performed exceptionally well in enantioselective gold(I) catalyzed transformations. ${ }^{[52]}$ It can also be seen that for the gold complexes $172 \mathrm{~s}$ and 172t, 
the para-trifluoromethyl groups at the arenes enclose the gold centre further. $\eta^{2}$-Arene-gold interactions can also be observed between the two rings on the TADDOL backbone and the gold center for the ligands $\mathbf{1 7 2 q}, \mathbf{1 7 2 s}$ and $\mathbf{1 7 2 t}$, as visualized by the points $A 1$ and $A 2$ in Figure 35 and Table 7, entries 8-10 (sum of van der Waals radii $=3.36 \AA$ ). Although the mesityl substituents clearly act to shield the gold centre, no short contacts with gold(I) centre could be observed in any cases. In addition, comparing all new gold(I) complexes with 172i, only a few changes in the structural properties are immediately apparent. The $\mathrm{P} 1-\mathrm{C} 1$ bond is slightly shortened in $\mathbf{1 7 2}$ s and 209a, compared with the other gold(I) complexes (entry 3 ). This may reflect the specific electronic strucutre of these cationic substituents, which are likely less electron withdrawing.

Table 7. Solid state structures and selected bond lengths of gold complexes 172q, 209a, 172s and $172 t$.

\begin{tabular}{ccccccc}
\hline Entry & Selected parameters & $\mathbf{1 7 2 q}$ & $\mathbf{2 0 9 a}$ & $\mathbf{1 7 2 s}$ & $\mathbf{1 7 2 t}$ & $\mathbf{1 7 2} \mathbf{i}^{[219]}$ \\
\hline 1 & Au1-Cl1 $(\AA)$ & $2.278(1)$ & $2.273(2)$ & $2.272(2)$ & $2.268(2)$ & $2.255(3)$ \\
2 & Au1-P1 $(\AA)$ & $2.200(1)$ & $2.201(4)$ & $2.193(1)$ & $2.191(2)$ & $2.185(2)$ \\
3 & $\mathrm{P} 1-\mathrm{C} 1(\AA)$ & $1.829(4)$ & $1.805(1)$ & $1.815(6)$ & $1.827(7)$ & $1.829(6)$ \\
4 & $\mathrm{P} 1-\mathrm{O} 1(\AA)$ & $1.588(3)$ & $1.578(9)$ & $1.585(4)$ & $1.588(5)$ & $1.581(4)$ \\
5 & $\mathrm{P} 1-\mathrm{O} 2(\AA)$ & $1.5890(3)$ & $1.584(9)$ & $1.595(4)$ & $1.593(5)$ & $1.584(6)$ \\
6 & $\mathrm{~N} 1-\mathrm{C} 1(\AA)$ & $1.343(6)$ & $1.335(2)$ & $1.349(7)$ & $1.344(1)$ & $1.351(7)$ \\
7 & $\mathrm{~N} 2-\mathrm{C} 1(\AA)$ & $1.350(6)$ & $1.404(2)$ & $1.368(7)$ & $1.358(9)$ & $1.351(7)$ \\
8 & $\mathrm{Au} 1-\mathrm{A} 1(\AA)$ & 3.259 & 3.431 & 3.282 & 3.282 & 3.368 \\
9 & $\mathrm{Au} 1-\mathrm{A} 2(\AA)$ & 3.325 & 3.403 & 3.326 & 3.289 & 3.438 \\
10 & $\mathrm{Au} 1-\mathrm{A} 3(\AA)$ & 3.394 & 3.579 & 3.460 & 3.464 & 3.351 \\
11 & $\mathrm{Au} 1-\mathrm{P} 1-\mathrm{C} 1-\mathrm{N} 2\left(^{\circ}\right)$ & 47.85 & 54.05 & $55.2(5)$ & $52.7(7)$ & $54.0(6)$ \\
\hline
\end{tabular}

Under otherwise identical conditions, ligands $169 \mathrm{p}$ and $169 \mathrm{r}$ did not afford the gold(I) complexes smoothly. While the ligands themselves were quite stable, complete decomposition on addition to the gold(I) precursor could be seen. In ${ }^{31} \mathrm{P}$ NMR spectrum of a mixture of products formed from the ligand 169r, a broad signal could be seen at 89.3 ppm, essentially, in the same range as for the previously reported phosphonous acid gold(I) chlorides 173 and 175a (cf. Scheme 40). ${ }^{[219]}$ In the case of 169p, the two well defined species, namely phosphonous acid $\mathbf{1 7 5 b}$ and polysubstituted naphthalene $\mathbf{1 7 4 b}$, could be isolated and characterized. By simply washing the crude reaction mixture with acetonitrile the naphthalene fragment $\mathbf{1 7 4 b}$ could be cleanly isolated. The phosphonous acid fragment $\mathbf{1 7 5 b}$ was then purified by crystallization from dichloromethane/ toluene and displays a characteristic ${ }^{31} \mathrm{P}$ NMR shift of $83.3 \mathrm{ppm}$. It is likely that the steric environment of the intermittently formed gold complex $169 p$ is too demanding to form a stable structure. 


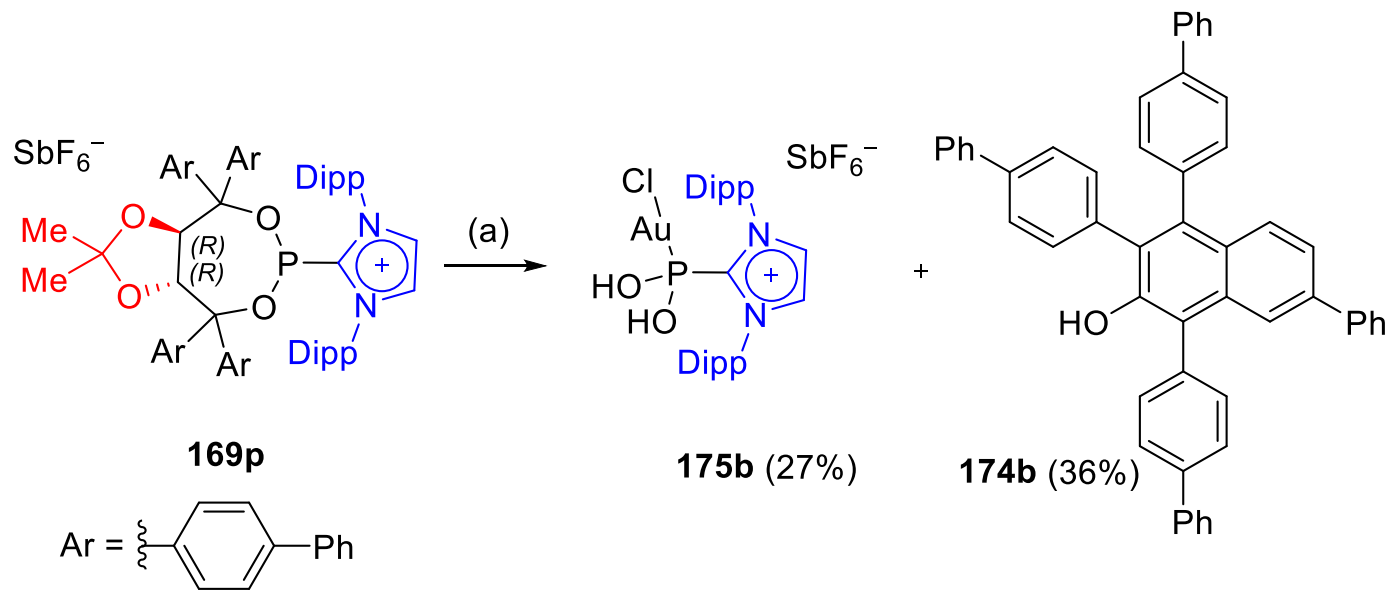

Scheme 58. Decomposition of ligand 169p on treatment with (dimethyl sulfide)gold(I) chloride, giving 175b and 174b. Reagent and conditions: (a) $169 \mathrm{p}$ (1.0 equiv.), $\mathrm{AuCl} \cdot \mathrm{SMe}_{2}\left(1.0\right.$ equiv.), $\mathrm{CH}_{2} \mathrm{Cl}_{2},-20^{\circ} \mathrm{C}$ to rt, $30 \mathrm{~min}$.

\subsection{Synthesis of 1,2,4- and 1,2,3-triazolium-derived phosphonites and their corresponding gold(I) complexes}

The work outlined in this section was carried out in collaboration with two Masters students: Maximillian Marx MSc. ${ }^{[24]}$ and Thierry Hartung MSc. ${ }^{[25]}$

In addition to the TADDOL-derived phosphonites previously described in this section, 1,2,4and 1,2,3- triazolium substituted phosphonites were targeted (Figure 36).

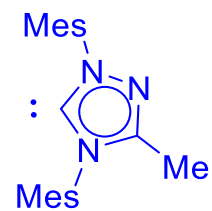

$56 \mathrm{~g}$

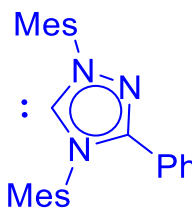

$56 h$<smiles></smiles>

$56 \mathbf{i}$<smiles></smiles>

56j

Figure 36. Targeted 1,24- and 1,2,3-triazol-5-ylidenes to be used in the synthesis of chiral cationic phosphonites. In the first instance, incorporation of additional nitrogen to the cationic substituent would most likely lower the energy of the $\pi$-system and make such substituents even more electron withdrawing than imidazolium substituents. Secondly, these carbenes were reported to be stable at room temperature and would therefore be compatible with the existing cationic phosphonite synthesis. ${ }^{[246,247]}$ With this in mind, the 1,2,4- and 1,2,3-triazol-5-ylidenes $56 \mathbf{g}$-j were synthesised. The 3-isopropyl and 3-mesityl 1,2,3-triazol-5-ylidenes were prepared according to modified literature procedures, ${ }^{[246]}$ while the 3-methyl- $(56 i)$ and 3-phenyl-1,2,4triazol-5-ylidene (56j) were prepared according to an optimized sequence proceeding via 1,3,4-oxadiazolium salts. ${ }^{[244,245]}$ 
These precursors were then condensed with chlorophosphite 171i under the standard conditions to give the ligands $169 x$-aa (Scheme 59 ).<smiles>[R]C([R])(OC)C(OC)C([R])(O)C([R])([R])O</smiles>

170i: $\mathrm{R}^{1}=4-\mathrm{CF}_{3}-\mathrm{C}_{6} \mathrm{H}_{4}-$
$171 \mathrm{i}$<smiles>[R][X]1(C)C=[Y](C)N(C)C1</smiles>

56g: $X=N, Y=C, R^{2}=M e$

56h: $X=N, Y=C, R^{2}=P h$

56i: $X=C, Y=N, R^{2}=i P r$

56j: $X=C, Y=N, R^{2}=$ Mes

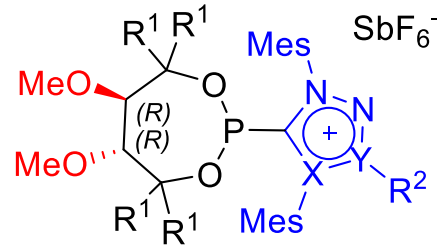

169

$$
\begin{array}{lll}
(\mathrm{C}) & \mathrm{MeO}
\end{array}
$$

Scheme 59. Synthesis of cationic phosphonite gold(I) chloride complexes 172v-y. Reagents and conditions: (a) diol (1.1 equiv.), $\mathrm{PCl}_{3}$ (1.2 equiv.), pyridine (3.2 equiv.), toluene, $60^{\circ} \mathrm{C}, 1 \mathrm{~h}$; (b) $171 \mathrm{i}$ (1.0 equiv.), $\mathrm{NaSbF}_{6}$ (2.1 equiv.), $\mathrm{Et}_{2} \mathrm{O},-78^{\circ} \mathrm{C}$ to rt, $22-24 \mathrm{~h}$; (c) ligand 169 (1.0 equiv.), $\mathrm{AuCl} \cdot \mathrm{SMe}_{2}\left(1.0\right.$ equiv.), $\mathrm{CH}_{2} \mathrm{Cl}_{2},-20^{\circ} \mathrm{C}$ to rt, $1 \mathrm{~h}$.

While the ligands $169 \mathrm{x}$ and $169 \mathrm{y}$ derived from 1,2,4-triazol-5-ylidenes $140 \mathrm{~g}$, h were quite unstable towards purification by column chromatography even at reduced temperatures, their gold $(\mathrm{I})$ complexes were more resistant and could be chromatographed at $-10^{\circ} \mathrm{C}$ giving pure 172x and 172y, both in $17 \%$ yields over three steps. The phosphonite gold(I) complexes $172 z$ and 172aa derived from 1,2,3-triazol-5-ylidenes $\mathbf{5 6 i , j}$ were similarly obtained in yields of 26 and $20 \%$, respectively, over two steps, as the crude ligands $169 z$ and 169 aa were still contaminated with inseparable impurities after attempted purification.

As the ligand 169a prepared from the para-tert-butylphenyl-substituted TADDOL 169a (Scheme 38) had shown promising enantioselectivities in the previous ligand screening for the gold(I)-catalyzed twofold hydroarylation reaction, ${ }^{[219]}$ this TADDOL framework might be another potentially promising ligand scaffold for the asymmetric helicene-forming synthesis. The 1,2,4-triazolium-based phosphonite $169 \mathrm{ab}$ proved to be more stable than its analogue $172 \mathrm{y}$, and was isolated in $44 \%$ yield before forming the gold $(\mathrm{I})$ complex $172 \mathrm{ab}$ in $85 \%$ yield (Scheme 60). However, when the same conditions were applied using the 4-methyl-1,2,4triazol-5-ylidene $(\mathbf{5 6 g})$, in addition to the desired ligand 169ac, which was detected by NMR but could not be isolated, the bisphosphonite $\mathbf{2 1 1}$ was formed. This presumably resulted from the in situ deprotonation of the methyl backbone in 169ac, followed by reaction with the second molecule of chlorophosphite 171a. Interestingly, this shows some resemblance to 
intermediate 205 proposed for the formation of 3-imidazolioadducts 203a and 203b (Scheme 54)<smiles>[R]C([R])(O)[C@@H]1OC(C)(C)O[C@@H]1C([R])(O)C([R])([R])O</smiles>
$170 \mathrm{a}: \mathrm{R}=4-\mathrm{tBu}-\mathrm{C}_{6} \mathrm{H}_{4^{-}}$

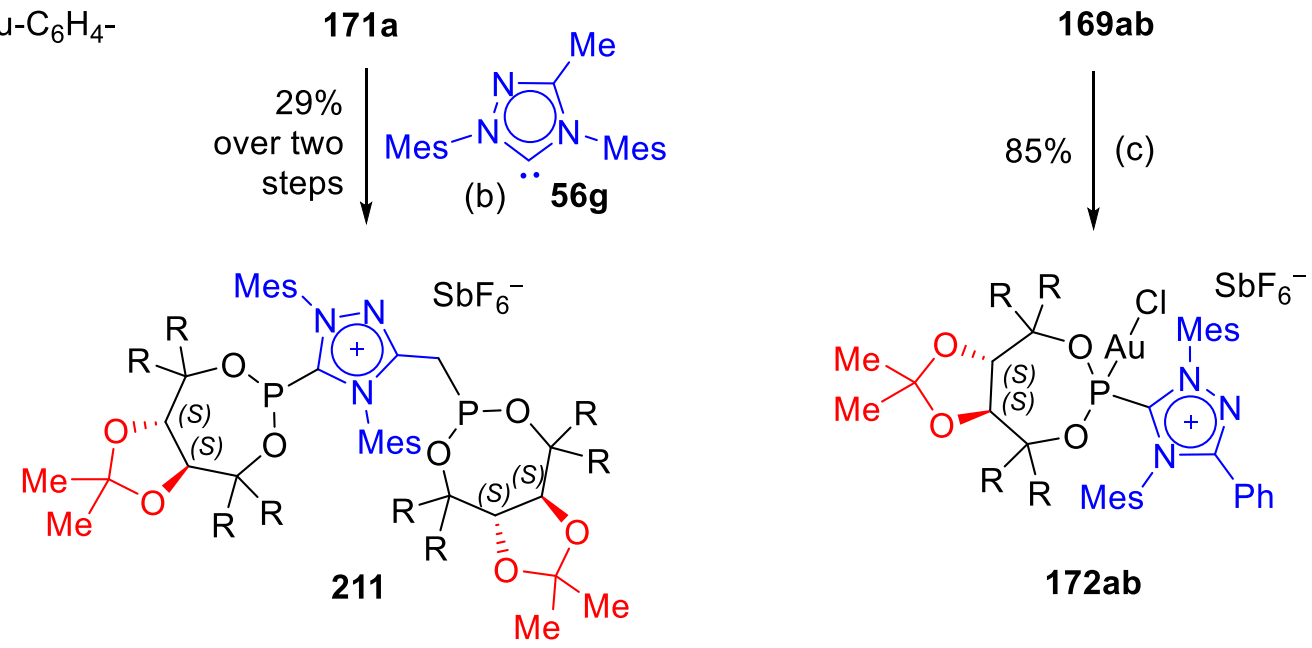

$169 \mathrm{ab}$

Scheme 60. Synthesis of gold(I) phosphonite complex 172ab and bis-phosphonite 211. Reagents and conditions: (a) 170a (1.1 equiv.), $\mathrm{PCl}_{3}\left(1.2\right.$ equiv.), pyridine (3.2 equiv.), toluene, $60^{\circ} \mathrm{C}, 1 \mathrm{~h}$; (b) carbene $56 \mathrm{~g}$ or 56h (1.0 equiv.), $\mathrm{NaSbF}_{6}$ (2.1 equiv.), $\mathrm{Et}_{2} \mathrm{O},-78{ }^{\circ} \mathrm{C}$ to rt, $22-24 \mathrm{~h}$; (c) ligand $169 \mathrm{ab}$ (1.0 equiv.), $\mathrm{AuCl}^{-\mathrm{SMe}_{2}}$ (1.0 equiv.), $\mathrm{CH}_{2} \mathrm{Cl}_{2},-20^{\circ} \mathrm{C}$ to rt, $1 \mathrm{~h}$.

\subsubsection{Donor properties}

The donor ability of the new ligand 169ab was also evaluated by cyclic voltammetry (Table $8)$.

Table 8. Cyclic voltammetry of triazolium-derived $169 \mathrm{ab}$ and $169 \mathrm{q}$.

\begin{tabular}{cccc}
\hline Entry & $\mathbf{L}$ & $\mathrm{E}_{\mathrm{Ox}} / \mathrm{V}$ & $\mathrm{E}_{\text {Red }} / \mathrm{V}$ \\
\hline 1 & $\mathbf{1 6 9 q}$ & 1.88 & -2.10 \\
2 & $\mathbf{1 6 9 a b}$ & - & $-1.71,-2.02$ (reversible) \\
\hline
\end{tabular}

Voltammograms conducted in $\mathrm{MeCN}$ with $\mathrm{Bu}_{4} \mathrm{PF}_{6}(0.2 \mathrm{M})$, referenced to ferrocene/ferrocinium.

Although no oxidation was seen for $169 \mathrm{ab}$, an irreversible reduction at -1.71 , followed by a reversible reduction at $2.02 \mathrm{~V}$ was observed. Comparison to the cationic phosphonite $\mathbf{1 6 9 q}$ showed that the first reduction potential was $0.39 \mathrm{~V}$ lower, indicating that the $\pi$-system in the triazolium moiety has a lower LUMO energy than the imidazolium structure and that the 
resulting cationic phosphonites may be even less electron rich. The second reversible reduction lies in the same range as a reversible reduction found for the Enders carbene, ${ }^{[248]}$ therefore it is likely that upon a one electron reduction of $169 \mathrm{ab}$, the corresponding radical species collapses to give the free triazol-5-ylidene carbene $56 \mathrm{~h}$, which can be further reversibly reduced.

\subsection{Summary}

A number of new chiral cationic phosphonites with different steric and electronic environments have been synthesized, including a variety of TADDOL derivatives and the first described BINOL derivative. In addition, the cationic substituent was modulated to include a diverse array of imidazolium, 1,2,4-triazolium, 1,2,3-triazolium and carbodiphosphonium substituents (Chart 2).
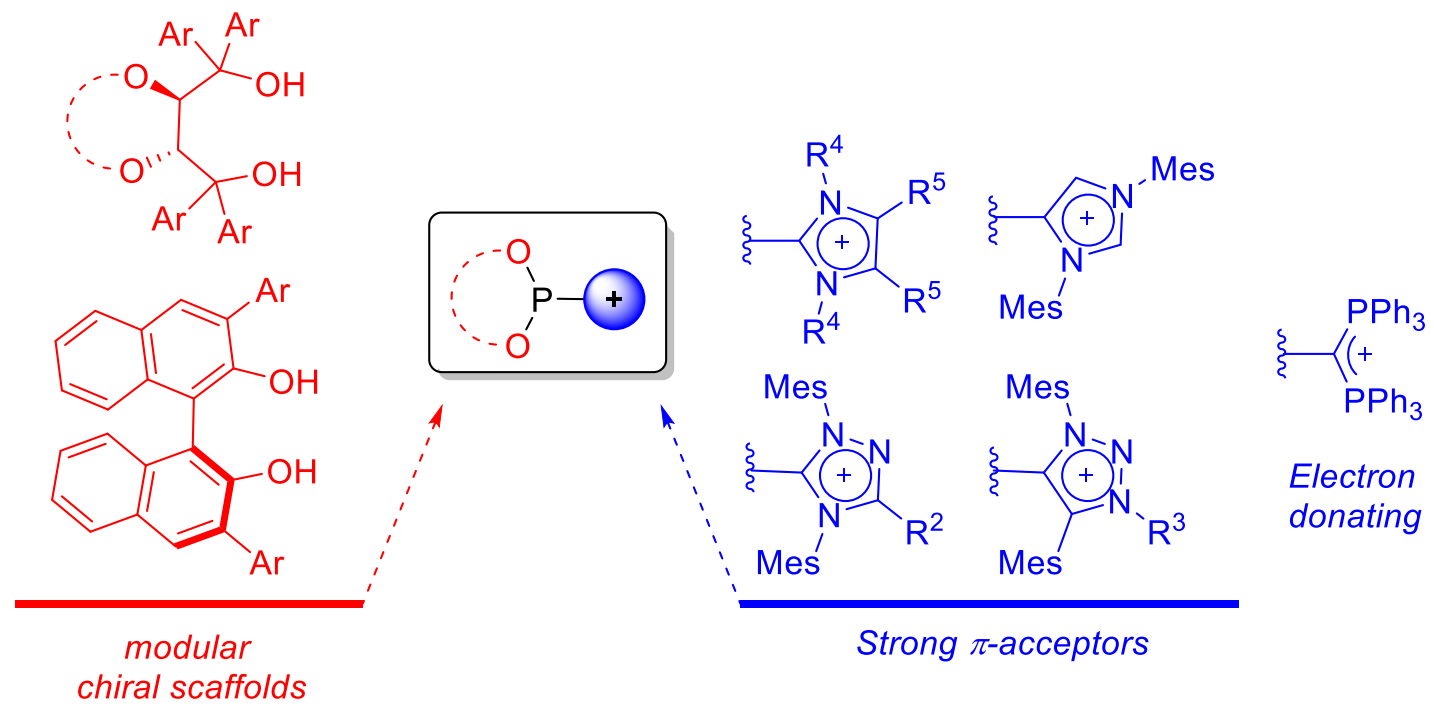

Chart 2. Chiral cationic phosphonites: Summary.

The reactions of the two TADDOL precursors $(R, R)-169 \mathrm{~s}$ and $(R, R)-\mathbf{1 6 9 t}$ led almost exclusively to the abnormally bound phosphonites $203 a$ and $203 b$, which likely form via a mechanism related to a base-mediated rearrangement reported by Bertrand and coworkers. ${ }^{[68]}$ Reactions between the BINOL precursor 207 and IMes 56b resulted in formation of a complex mixture consisting of both the normal and abnormal adducts 208a and $\mathbf{2 0 8 b}$, which could be circumvented by using the carbene $56 \mathrm{e}$ with blocked reactivity at the backbone, thus allowing the synthesis of BINOL-derived phosphonite $208 \mathrm{c}$ in very good yield.The donor properties were investigated using cyclic voltammetry and the imidazolium derived phosphonites displayed irreversible oxidation potentials in the range of $1.76-1.93 \mathrm{~V}$, indicating this class of ligands to be excellent $\pi$-acceptors and poor $\sigma$-donors. The carbodiphosphorane-derived $\mathbf{1 6 9}$ conversely conversely exhibited a decreased oxidation 
5. Synthesis and structure of cationic phosphonites

potential. Finally, the gold $(I)$ complexes $172 m-\mathbf{0}, \mathbf{q}, \mathbf{s}-\mathbf{u}, \mathbf{x}-\mathbf{a b}, 208 \mathbf{a}, \mathbf{b}$ and $209 \mathbf{a}$ could be synthesized, indicating hese compounds to be excellent ligands for gold. Interestingly, the gold(I) complexes of $169 p$ and $169 q$ could not be formed, with concommitant decomposition occuring instead. The isolated gold(I) phosphonous acid species $175 \mathrm{~b}$ and naphthalene 174a indicate that decomposition likely occurs via a previously described Friedel-Crafts-type alkylation-elimination-1,2-aryl shift cascade. 


\section{Enantioselective synthesis of [6]carbohelicenes}

\subsection{Enantioselective synthesis of [6]carbohelicenes using precatalyst $172 \mathrm{i}$}

Aiming to expand the scope of the gold $(I)$ catalysed enantioselective hydroarylation of precursors 159 into [6] helicenes 160, the substrates 159ae-am, with variable substituents at the alkyne, were evaluated using the chiral precatalyst 172i under the optimized reaction conditions. The conversion of the starting material (159) and the intermediate (187) after one 6 -endo-dig cyclisation were measured, in addition to the regioselectivity of the desired helicene (160) over the 6-endo-dig/ 5-exo-dig product (188) and the enantioselectivity of the desired helicene (160). A summary of the results is given in Table 9. For details of the racemic reactions, which were required to measure the enantiomeric excess, see the experimental section of this thesis.

Under the optimized conditions, almost all of the reactions reached full conversion. For reactions of the substrates 159af, 159ah and 159ae with 4-fluorophenyl, 4-benzyloxy and 4chlorophenyl substituents, respectively, the reaction mixture contained residual starting material (entries 1, 3 and 7). All of these substrates were generally less soluble, and this was most likely the determining factor in their lower conversion. Because conversion was so low for substrates 159ah and 159ae, the reactions were also conducted in dichloromethane, where full conversion for 159ah and an improved conversion for 159ae were obtained (entries 4 and 8).

Especially high regioselectivities over the undesired isomer 188 were obtained using substrates with substituents that favorably polarized the alkyne, such as 4-methoxyphenyl (entry 2), 4-benzyloxyphenyl (entries 3 and 4) and 3,4-dimethylphenyl (entry 9). Similarly, 4chlorophenyl-substituted substrate 159ae displayed a very good selectivity, although chlorine is generally considered to be a weakly electron-withdrawing group, taking into account its Hammet parameter. ${ }^{[249]}$ In the case of 159af, with 4-fluorophenyl substituents, the disclosed selectivity was slightly lower (entry 1). When steric bulk in the para position was increased, again generally lower selectivities were detected. This was seen for the substrates 159ai, 159al and 159aj containing 4-(trimethylsilyl)phenyl (entry 5), 4(triisopropylsilyloxymethyl)phenyl (entry 10) and 4-isopropylphenyl (entry 6) substituents. In these cases, separation of the desired helicene 160 could only be achieved by preparative HPLC separation. 
Fortunately, the enantioselectivities remained high across the tested series, for the most part lying in the range of $86-92 \%$ ee, and showing tolerance for the range of functional groups within the scope of this transformation. Exceptions to this were substrates 159ak and 159ag, bearing 3,4-dimethylphenyl and 4-methoxyphenyl substituents, which displayed ee's of 77 and $81 \%$, respectively. Conversely, substrate 159ah gave an astonishing $99 \%$ ee, even considering that the reaction was conducted in dichloromethane and not in fluorobenzene, which generally resulted in lower enantioselectivites for this reaction. Similarly, cyclization of 4-chlorophenyl-substituted diyne 159ae resulted in an excellent $92 \%$ ee.

Table 9. Enantioselective synthesis of helicenes.

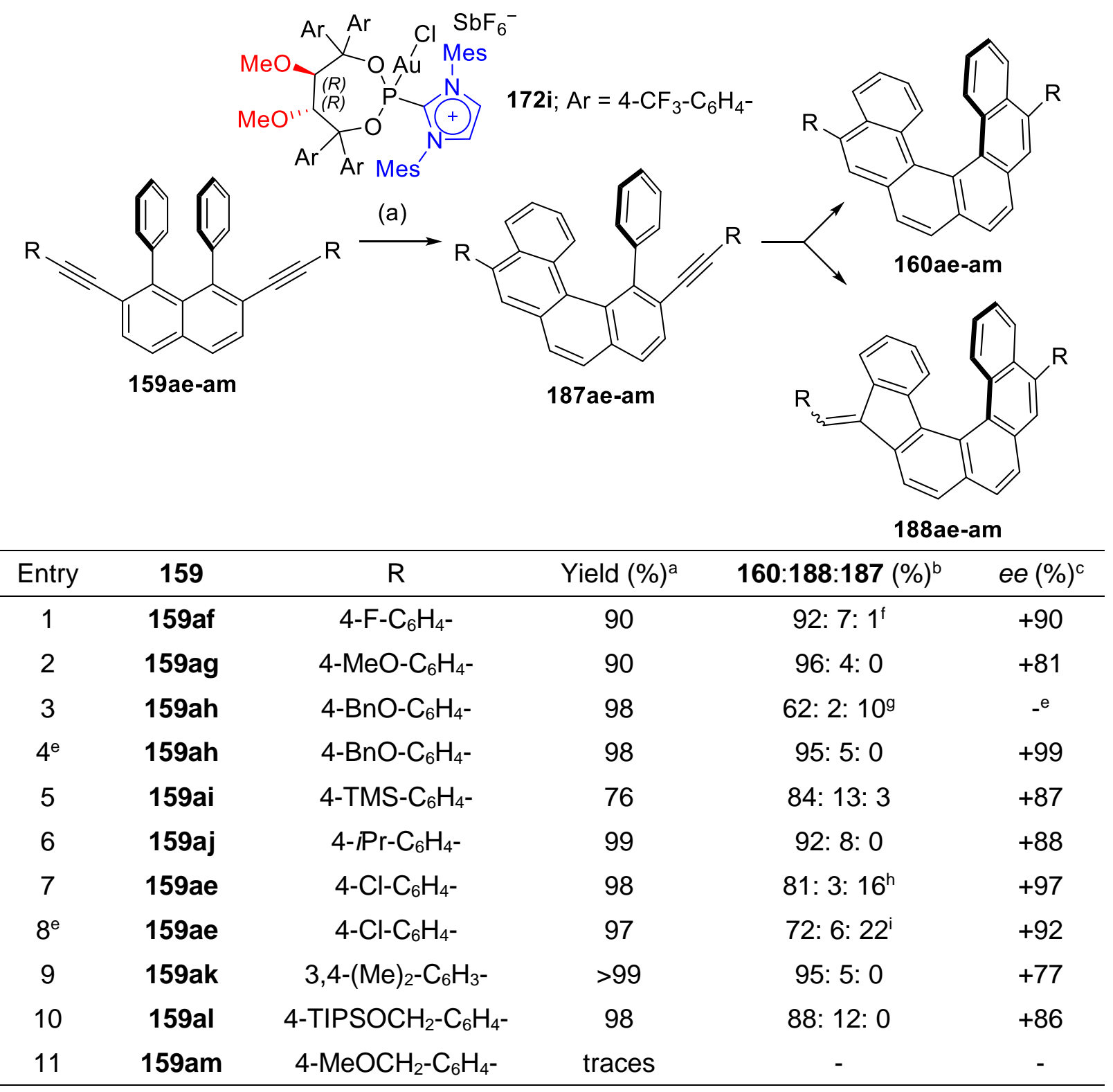

Reagents and conditions: (a) 159 (1 equiv.), 172i (10 mol\%), $\mathrm{AgSbF}_{6}(10 \mathrm{~mol} \%), \mathrm{C}_{6} \mathrm{H}_{5} \mathrm{~F},-20{ }^{\circ} \mathrm{C}, 72 \mathrm{~h} .{ }^{\text {aYields }}$ determined of mixtures of inseparable isomers. 'belectivity determined by NMR and/ or HPLC. "Enantiomeric excess determined by HPLC. ${ }^{\mathrm{d}}$ Reaction conducted in dichloromethane. eEnantiomeric excess could not be determined due to poor separation in HPLC. ${ }^{f} 8 \%$ residual 159 af. ${ }^{9} 36 \%$ residual 159 ah. ${ }^{h} 69 \%$ residual 159 ae. ${ }^{i} 15 \%$ residual 167ae. 
On submission of the 2-naphthyl-substituted compound $159 \mathrm{db}$ to the reaction conditions, one major product, approximately $68 \%$ of the crude reaction mixtrure, could be identified in the ${ }^{1} \mathrm{H}$ NMR. Upon preparative HPLC, the structure could be identified as the dibenzohelicene $160 \mathrm{db}$ (Scheme 61 ), with an enantiomeric excess of $46 \%$. Although this is rather low in comparison to the other helicenes, it is proof of dynamic interconversion of the enantiomers $(P)-\mathbf{1 8 7 d b}(I)$ and $(M)-187 d b(I I)$ of the tetrahelicene intermediate (cf. Figure 27).

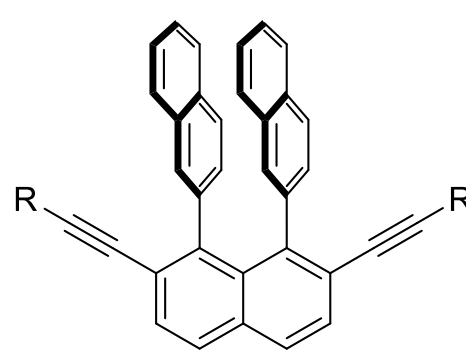

$159 \mathrm{db}: \mathrm{R}=p$-Tolyl syn/anti $=45: 55$ (a)<smiles></smiles>

$160 \mathrm{db}$

$35 \%$ (preparative separation) $+46 \%$ ee

Scheme 61. Enantioselective gold(I) catalysed hydroarylation on $159 \mathrm{db}$. Reagents and conditions (a) $159 \mathrm{db}(1$ equiv.), $172 \mathrm{i}(10 \mathrm{~mol} \%), \mathrm{AgSbF}_{6}(10 \mathrm{~mol} \%), \mathrm{C}_{6} \mathrm{H}_{5} \mathrm{~F},-20^{\circ} \mathrm{C}, 72 \mathrm{~h}$.

The enantioselective gold(I) catalysed hydroarylation reaction was next evaluated for bromosubstituted substrate 159an, under the optimised conditions using precatalyst 172i (Scheme 62). Full conversion of starting material was reached using the gold $(I)$ catalyst $\mathbf{1 7 2} \mathbf{i}$, also giving what appeared to be one major product in the crude NMR, with some additional small impurities. Although the product was $\mathrm{C}_{2}$ symmetric, its identity could not be confirmed soley using NMR techniques.

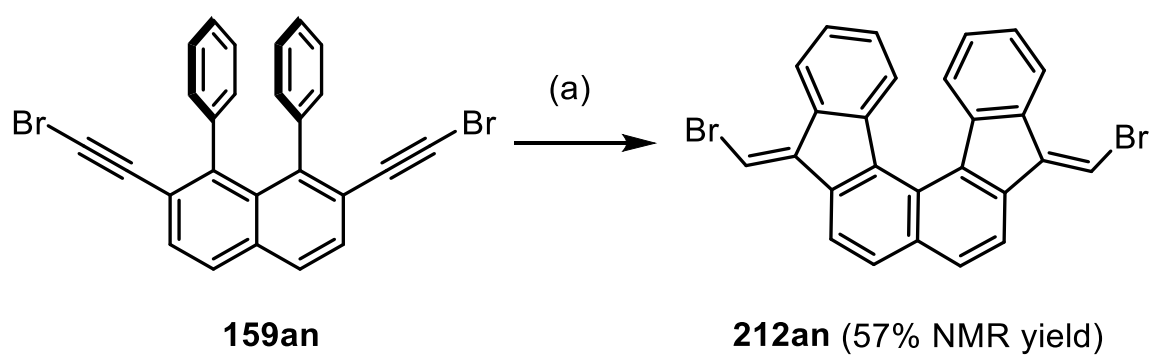

Scheme 62. Gold(I) catalysed intramolecular double hydroarylation of 159an.Reagents and conditions (a) 172i (10 mol\%), AgSb 6 (10 mol\%), $\mathrm{C}_{6} \mathrm{H}_{5} \mathrm{~F},-20^{\circ} \mathrm{C} .96 \mathrm{~h}$. aNMR yield determined using 1,4-dioxane as internal standard.

Attempts to separate the crude reaction mixture by preparative HPLC only decreased the purity and upon comparison with the original crude ${ }^{1} \mathrm{H}$ spectrum appeared to show formation of new signals, suggesting decomposition of the sample under the HPLC conditions. (For further details see experimental section) Because all the other samples had been stable to the HPLC conditions, it was likely that a different isomer formed under the reaction 
conditions. Gratifyingly, after crystallisation, the unambiguous identitiy of the product could be as the 5-exo-dig/ 5-exo-dig product 212an. Because dibromide 212an contains two fivemembered rings, the helix does not form a $360^{\circ}$ turn and the peripheral rings do not overlap. It is therefore likely that this product is not configurationally stable at room temperature. The Lewis acid catalysed cyclisation of haloalkynes has been previously studied by Fürstner and coworkers. ${ }^{[110]}$ It was found that for certain Lewis acids, such as $\mathrm{Au}(\mathrm{I}) \mathrm{Cl}$ a 1,2- migration of the halogen could occur. This was not observed for harder Lewis acids, such as $\ln (\mathrm{III}) \mathrm{Cl}_{3}{ }^{[250]}$ The authors proposed that back-bonding from a $\mathrm{Au}(\mathrm{I})$ centre could favour formation of a $\mathrm{Au}(\mathrm{I})$ vinylidene intermediate 213, in which the halogen has migrated to the other side of the alkyne, leading to the 9-bromo product 214 (Scheme 63). ${ }^{[251]}$ Presumably, a highly electronwithdrawing ligand at the gold center, such as $169 \mathbf{i}$, would diminish the gold's backbonding capability, disfavoring the formation of a gold(I) vinylidene species. Netherless, it is not immediately clear why a 5-exo-dig cyclisation would then be favoured when using precatalyst $\mathbf{1 7 2} \mathbf{i}$, as in previous studies a 6-endo-dig cyclisation has been favoured for bromoalkynes. ${ }^{[110,250]}$ Perhaps the large steric bulk of the bromo substituent and the ligated gold(I) catalyst cause a steric clash, which favours the isomer 212.

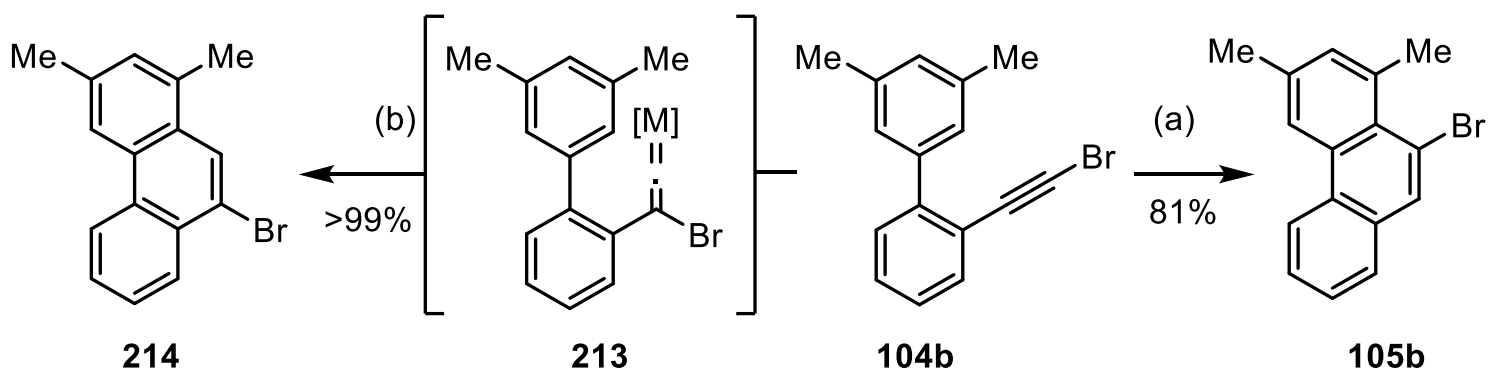

Scheme 63. Divergant, Lewis acid catalysed cycloisomerisations of haloalkynes using In(III) chloride (a) and $\mathrm{Au}(\mathrm{I}) \mathrm{Cl}$ (b), as reported by the Fürstner and corworkers. ${ }^{[110]}$ Reagents and conditions (a) In(III) $\mathrm{Cl}_{3}(1.0$ equiv.), toluene, $80^{\circ} \mathrm{C}, 16 \mathrm{~h}$; (b) $\mathrm{Au}(\mathrm{I}) \mathrm{Cl}(20 \mathrm{~mol} \%)$, toluene, $80^{\circ} \mathrm{C}, 20 \mathrm{~h}$.

In summary, the enantioselective gold(I)-catalyzed hydroarylation to give [6]carbohelicenes described herein demonstrates the highest enantioselectivities in the catalytic synthesis of [6]carbohelicenes obtained to date. The scope outlined in this section extends this transformation to include a variety of functional groups amenable to further conversions, including protected alcohols, silanes and halogens. Certain aspects of this transformation however, could still be optimized to further increase the applicability of this method. Firstly, although conversion of the starting material 159 and intermediate 187 formed in the reaction, as well as the ratio between the desired helicene product 160 and other isomers relating to the 6-endo-dig/5-exo-dig cyclization was mostly very good, in certain cases these undesired side-products could still be detected in the reaction mixture. In this instance, to isolate the helicene a semi-preparative HPLC separation was necessary, which afforded the pure 
helicenes in low yields. Moreover, several substrates still displayed poor conversions when using the catalyst system 172i. Secondly, the high enantioselectivities obtained were limited to aryl-substituted alkynes, with a significant drop seen for the alkyl-substituted substrates 159aa and 159ba (63 and 30\% ee, respectively, cf. Table 3). With this in mind, it was decided to try to further optimise this reaction, by evaluating the new chiral cationic phosphonites described in chapter 5 of this thesis in a series of model studies.

\subsection{Model catalytic studies using new cationic phosphonite gold(I) complexes}

The new series of phosphonites (Figure 37) was evaluated in a series of model reactions with the substrates 159ab, 159aa and 159ae.

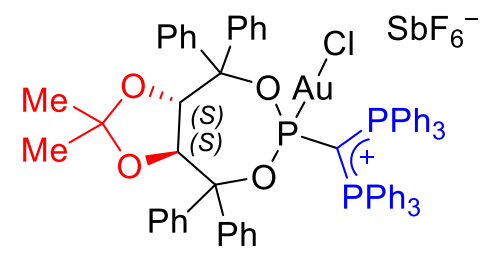

172I

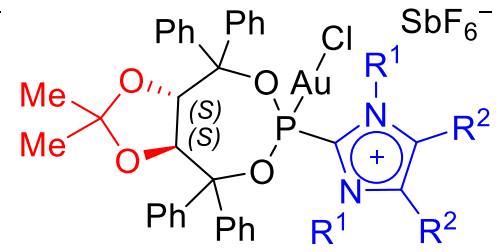

172m: $\mathrm{R}^{1}=$ Mes, $\mathrm{R}^{2}=\mathrm{H}$ 1720: $\mathrm{R}^{1}=i \mathrm{Pr}, \mathrm{R}^{2}=\mathrm{Me}$

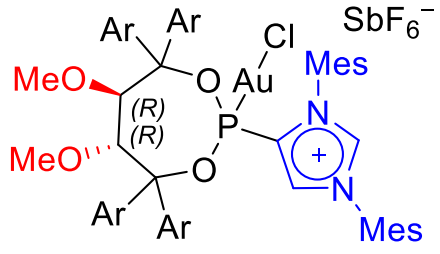

209a

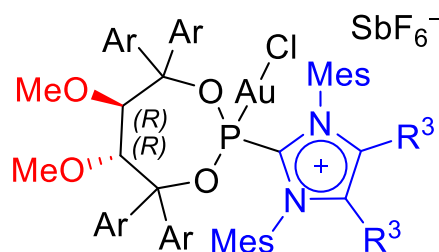

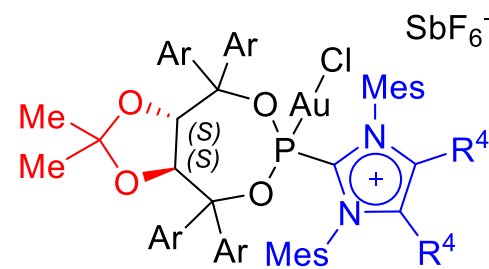

172s: $R^{1}=M e$

172t: $\mathrm{R}^{1}=\mathrm{Cl}$

$\mathrm{Ar}=4-\mathrm{CF}_{3}-\mathrm{C}_{6} \mathrm{H}_{4}-$

172a: $\mathrm{R}^{4}=\mathrm{H}$

172u: $R^{4}=\mathrm{Cl}$

$\mathrm{Ar}=4-\mathrm{BBu}^{-\mathrm{C}_{6} \mathrm{H}_{4}-}$

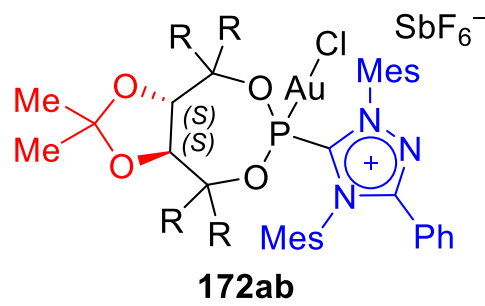

$172 \mathrm{ab}$

$\mathrm{Ar}=4-\mathrm{BBu}-\mathrm{C}_{6} \mathrm{H}_{4}-$

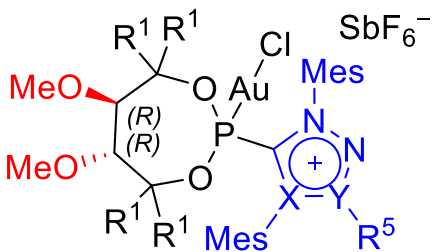

$\mathrm{Ar}=4-\mathrm{CF}_{3}-\mathrm{C}_{6} \mathrm{H}_{4}$ 172x: $X=N, Y=C, R^{5}=M e$ 172y: $X=N, Y=C, R^{5}=P h$ 172z: $X=C, Y=N, R^{5}=i P r$ 172aa: $X=C, Y=N, R^{5}=$ Mes

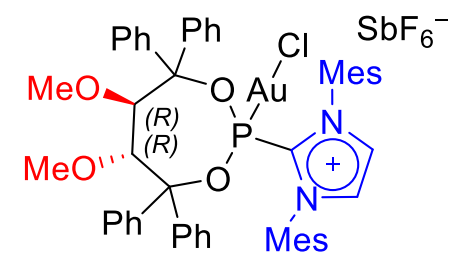

$172 q$

Figure 37. New cationic phosphonite gold(I) complexes evaluated in model studies.

Diyne $159 \mathrm{ab}$, being the most studied in this transformation, was a natural choice to evaluate the profile of the new generation of catalysts. However, as the substrates 159aa and 159ae had demonstrated respectively poorer ee's and conversion using the previous system, they 
were also chosen to find out how these aspects may also be improved. The results obtained with the new generation of catalysts in the cyclisation of $159 \mathrm{ab}$ are shown in Table 10.

Table 10. Results of screening for the enantioselective gold(I)-catalyzed cyclization of substrate 159ab.

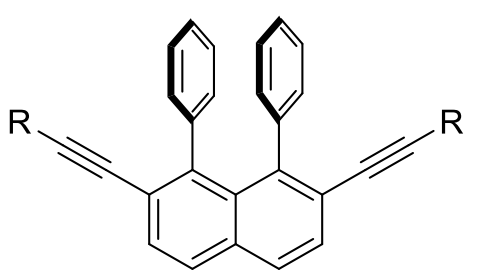

159ab: $R=p$-Tolyl (a)<smiles>[R]c1cc2ccc3cccc(-c4ccccc4)c3c2c2ccccc12</smiles>

$187 \mathrm{ab}$

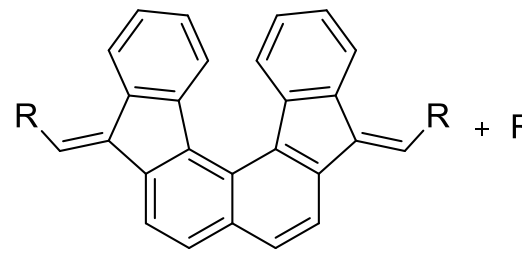

212ab<smiles></smiles>

$160 \mathrm{ab}$

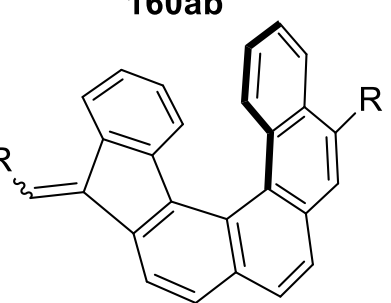

$188 \mathrm{ab}$

\begin{tabular}{cccccc}
\hline Entry & {$[\mathrm{Au}]$} & $\mathrm{T}\left({ }^{\circ} \mathrm{C}\right)$ & ${\text { Yield }(\%)^{\mathrm{a}}}^{\text {160ab: 188ab:187ab }(\%)^{\mathrm{b}}}$ & ee $(\%)^{\mathrm{c}}$ \\
\hline $1^{\mathrm{d}}$ & $\mathbf{1 7 2 i}$ & -20 & 88 & $97: 3: 0$ & +91 \\
$2^{\mathrm{d}}$ & $\mathbf{1 7 2}$ & 0 & 43 & Only 212ab & - \\
$3^{\mathrm{d}}$ & $\mathbf{1 7 2 0}$ & -20 & $98^{\mathrm{h}}$ & $45: 50: 5^{\mathrm{h}}$ & -60 \\
$4^{\mathrm{d}}$ & $\mathbf{2 0 9 a}$ & -20 & - & -9 & - \\
5 & $\mathbf{1 7 2 s}$ & -20 & 85 & $87: 13: 0$ & +82 \\
6 & $\mathbf{1 7 2 t}$ & -20 & 76 & $96: 4: 0$ & +84 \\
$7^{\mathrm{e}}$ & $\mathbf{1 7 2 y}$ & -20 & $>95$ & $98: 2: 0$ & +87 \\
$8^{\mathrm{e}}$ & $\mathbf{1 7 2 x}$ & -20 & $>95$ & $96: 4: 0$ & +84 \\
$9^{f}$ & $\mathbf{1 7 2 z}$ & -20 & $>95$ & $98: 2: 0$ & +82 \\
$10^{f}$ & $\mathbf{1 7 2 a a}$ & -20 & $>95$ & $98: 2: 0$ & +85 \\
\hline
\end{tabular}

Reaction conditions: (a) 159ab (1 equiv. 0.025 mmol), [Au] (10 mol\%), AgSbF 6 (10 mol\%), $\mathrm{C}_{6} \mathrm{H}_{5} \mathrm{~F}, \mathrm{~T}, 96 \mathrm{~h}$. ${ }^{\mathrm{a}} \mathrm{NMR}$ yield determined using dioxane (4 equiv.) as internal standard. ${ }^{b}$ Conversion and selectivity were determined using NMR/HPLC. 'Enantiomeric excess was determined using HPLC. dReaction conducted by Dr. E. GonzálezFernández. ${ }^{219,245]} \quad$ eReaction conducted by M. Marx MSc. ${ }^{244]}{ }^{f}$ Reaction conducted by T. Hartung MSc. 9 Combined yield determined as a mixture of isomers $160 \mathrm{ab}, 188 \mathrm{ab}$ and $187 \mathrm{ab}$. heaction conducted using $5 \mathrm{~mol} \%$ precatalyst and 5 mol\% $\mathrm{AgSbF}_{6}$ in $\mathrm{CH}_{2} \mathrm{Cl}_{2}$ for $72 \mathrm{~h}$.

For comparison, entry 1 shows the results obtained in this reaction using the precatalyst 172i. [219] In all cases, complete conversion of the starting material was observed. Interestingly, using the carbodiphosphonium derived precatalyst 159 I resulted in the exclusive formation of $212 \mathbf{a b} .{ }^{[219]}$ It is likely that the larger steric demand on the carbodiphosphonium substituent favours formation of the 5-exo-dig product. Additionally, 
209a, with an abnormally bound imidazolium unit failed to give any conversion in this transformation, while the complex 1720, with the smaller imidazolium substituent gave poor results. The complexes $\mathbf{1 7 2 s , t , y - a a}$ allowed a systematic comparison to $\mathbf{1 7 2 i}$, with the only change being in the cationic group. A decrease in selectivity was observed for 172s, with electron donating methyl groups at the backbone of the imidazolium moiety. Conversely, attachment of the more electron poor chloro substituents (172t, entry 6) maintained a regioselectivity comparable to $\mathbf{1 7 2} \mathbf{i}$. Similarly, for the complexes of 1,2,4-triazolium- and 1,2,3-triazolium-derived phosphonites, a trend in the increasing electron-withdrawing capacity of the cationic group and an increase in the regioselectivity towards the formation of the desired helicene could be observed, with the best results being obtained for phosphonite precatalysts 172y, 172z and 172a (entries 7, 9 and 10). However, the enantioselectivity displayed by the new generation of ligands slightly dropped in all cases, with the best ee of $87 \%$ for the new generation of catalyst obtained using $172 \mathrm{y}$ (entry 7 ).

While consistently giving higher regioselectivities, the propynyl-substituted naphthalene 167aa had consistently shown lower enantioselectivities compared to the substrates decorated with arylethynyl groups, the preparation of hexahelicenes with aliphatic chains using this methodology necessitates further optimization of the enantiomeric excess. It was decided to start with gold complexes that exhibited the best ee's in the initial screening studies conducted with this substrate, ${ }^{[219]}$ but to replace the solvent with fluorobenzene and carry out the reaction at $-20^{\circ} \mathrm{C}$. In addition, gold complexes of the cationic phosphonites of the new generation of precatalysts were also evaluated (Table 11).

The result obtained using the gold complex of cationic phosphonite $\mathbf{1 7 2} i$ under the optimized reaction conditions gave a moderate ee of $63 \%$ (entry 1 ). ${ }^{[219]}$ For all the complexes tested, the conversion and selectivity towards the desired hexahelicene 160aa remained excellent. The complexes 172s and 172t derived from 4,5-dichloro- and 4,5-dimethylsubstituted phosphonites gave respectively better and poorer selectivities, fitting with the trend found when using the model substrate 159ab discussed above. Unfortunately, these catalysts gave significantly poorer enantiomeric excesses, as did the precatalysts $172 \mathrm{n}$ and $\mathbf{1 7 2 q}$ (entries 2-5). Among the ligands tested, the tert-butylphenyl-substituted catalysts gave the highest ee's, with para-tertbutylphenyl substituted $172 \mathrm{a}$ and $146 \mathrm{ab}$ both giving ee's of $-70 \%$ (entries 6 and 8 ), improving marginally on the previous result. 
Table 11. Results of screening for the enantioselective gold(I)-catalyzed cyclisation of substrate 159aa.<smiles>CC#Cc1ccc2ccc(C#CC)c(-c3ccccc3)c2c1-c1ccccc1</smiles>

159aa (a)<smiles>CC#Cc1ccc2ccc3cc(C)c4cccc(c2-c2ccccc2)c4c3c1</smiles>

187 aa

\begin{tabular}{ccccc}
\hline Entry & $\mathbf{1 7 2}$ & ${\text { Yield }(\%)^{\mathrm{a}}}^{\text {160aa: 188aa:187aa }(\%)^{\mathrm{b}}}$ & ee $(\%)^{\mathrm{c}}$ \\
\hline $1^{\mathrm{d}}$ & $\mathbf{1 7 2 i}$ & 96 & $98: 2: 0$ & +63 \\
2 & $\mathbf{1 7 2 s}$ & 91 & $98: 1: 1$ & +21 \\
3 & $\mathbf{1 7 2 t}$ & 98 & $99: 1: 0$ & +14 \\
4 & $\mathbf{1 7 2 q}$ & 98 & $98: 2: 0$ & +22 \\
5 & $\mathbf{1 7 2 n}$ & 99 & $96: 2: 2$ & -18 \\
6 & $\mathbf{1 7 2 a}$ & 96 & $98: 1: 1$ & -70 \\
7 & $\mathbf{1 7 2 u}$ & 94 & $>99: 1: 0$ & -66 \\
$8^{\mathrm{e}}$ & $\mathbf{1 7 2 a b}$ & 95 & $>99: 1: 0$ & -70 \\
\hline
\end{tabular}

Reaction conditions: (a) 159aa (1 equiv. $0.025 \mathrm{mmol}), 172$ (10 mol\%), $\mathrm{AgSbF}_{6}(10 \mathrm{~mol} \%), \mathrm{C}_{6} \mathrm{H}_{5} \mathrm{~F},-20{ }^{\circ} \mathrm{C}, 96 \mathrm{~h}$. ${ }^{a}$ NMR yield determined using dioxane (4 equiv.) as internal standard. ${ }^{b}$ Conversion and selectivity determined using NMR/HPLC. 'Enantioselectivity determined using HPLC. dReaction conducted by Dr. E. GonzálezFernández. ${ }^{[219]}$ eReaction conducted by M. Marx MSc. ${ }^{244]}$

Finally, the reactivity of the new catalysts was further evaluated in the cyclisation of substrate 159ae bearing p-chlorophenyls (Table 12), which had given poor conversion using the catalyst 172i (entries 1 and 2). In dichloromethane, full conversion and an excellent selectivity of 98:2 could be reached when using 172t; however, the ee was only a moderate $77 \%$ (entry 3). In fluorobenzene, while the ee was increased to $87 \%$, the conversion was only $67 \%$ (entry 4). Interestingly, the same reaction with the triazolium derived catalyst 172y displayed a lower conversion, while the selectivity and enantioselectivity were improved (entry 5). 
Table 12. Results of screening for the enantioselective gold(I)-catalyzed cyclisation of substrate 159 ae.<smiles>[R]C#Cc1ccc2ccc(C#CF)c(-c3ccccc3)c2c1-c1ccccc1</smiles>

159ae: $\mathrm{R}=4-\mathrm{Cl}-\mathrm{C}_{6} \mathrm{H}_{4}^{-}$ (a)<smiles>[R]c1cc2ccc3ccc4cc([R])c5ccccc5c4c3c2c2ccccc12</smiles>

160ae<smiles>[R]C=C1c2ccccc2-c2c1ccc1ccc3cc([R])c4ccccc4c3c21</smiles>

188ae

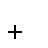

187 ae

\begin{tabular}{cccccc}
\hline Entry & $\mathbf{1 7 2}$ & Solvent & Yield (\%) $^{\mathrm{a}}$ & 160ae: 188ae:187ae $(\%)^{\mathrm{b}}$ & ee (\%) \\
\hline 1 & $\mathbf{1 7 2 i}$ & $\mathrm{CH}_{2} \mathrm{Cl}_{2}$ & $98^{\mathrm{e}}$ & $81: 3: 16^{\mathrm{f}}$ & +97 \\
2 & $\mathbf{1 7 2 i}$ & $\mathrm{C}_{6} \mathrm{H}_{5} \mathrm{~F}$ & $97^{\mathrm{e}}$ & $72: 6: 22^{\mathrm{g}}$ & +92 \\
3 & $\mathbf{1 7 2 t}$ & $\mathrm{CH}_{2} \mathrm{Cl}_{2}$ & 91 & $98: 2: 0$ & +77 \\
4 & $\mathbf{1 7 2 t}$ & $\mathrm{C}_{6} \mathrm{H}_{5} \mathrm{~F}$ & 37 & $85: 4: 11^{\mathrm{h}}$ & +87 \\
$5^{\mathrm{d}}$ & $\mathbf{1 7 2 y}$ & $\mathrm{C}_{6} \mathrm{H}_{5} \mathrm{~F}$ & 33 & $93: 2: 5^{\mathrm{i}}$ & +92 \\
\hline
\end{tabular}

Reaction conditions: (a) 159ae (1 equiv. $0.025 \mathrm{mmol}), 172$ (10 mol\%), AgSbF 6 (10 mol\%), solvent, $-20^{\circ} \mathrm{C}, 96 \mathrm{~h}$. aNMR yield determined using dioxane (4 equiv.) as internal standard. b Conversion and selectivity determined using NMR/HPLC. 'Enantioselectivity determined using HPLC. dReaction conducted by M. Marx MSc. ${ }^{[244]}$ eYield determined as a mixture in separable isomers. ${ }^{\dagger} 69 \%$ residual 159ae observed. $915 \%$ residual 159 ae observed. h37\% residual 159ae observed. ${ }^{5} 50 \%$ residual 159 ae observed.

The results of the model studies on substrates 159aa, 159ab and 159ae had shown that the new cationic phosphonite complexes were indeed more reactive, although equivocal results relating to the selectivities and enantioselectivities were obtained. For instance, a clear increase in conversion for both the precatalysts $172 \mathrm{t}$ and $172 \mathrm{y}$ as well as better selectivities in both cases were seen for the substrate 159ae in comparison to 172i. However, full conversion could still not be reached in fluorobenzene, which was necessary to give high ee values. Moreover, while marginally worse enantioselectivities were detected when using the substrate 159ab with 172y and 172aa in comparison to the complex 172i, slightly higher selectivities could be obtained. Overall, the second generation catalyst 172y, with a 1,4bis(mesityl)-3-phenyl-1,2,4-triazolium substituent, had displayed a promising profile regarding conversion, selectivity and enantioselectivity. It seemed that this triazolium substituent could have an especially positive effect on the selectivity and of the reaction. An increase in selectivity would circumvent problems with purification that occurred in purifying the helicenes when formed together with significant amounts of other isomers, which consistently 


\section{Enantioselective synthesis of [6]carbohelicenes}

required low-yielding preparative HPLC separations. Motivated by the possibility of optimizing this aspect, as well as aiming to further evaluate the effect on the comparable reactivity and enantioinduction of the new system, the cationic phosphonite gold(I) complex 172y was evaluated for a wider variety of substrates, including those newly accessed using the optimized substrate synthesis, as well as those that had been made with poorer selectivity when using the precatalyst $\mathbf{1 7 2 i}$.

\subsection{Substrate scope of new precursors and comparison studies using cationic phosphonite gold(I) complex 172y}

The results of the comparison study are shown in Table 13. Firstly, the substrate 159ob, which had shown almost no conversion with the precatalyst 172i, ${ }^{[219]}$ enabled another direct comparison with the reactivity of the new generation of triazolium derived gold catalysts. Impressively, the new precatalyst $\mathbf{1 7 2 y}$ afforded very good conversion towards helicene $160 \mathrm{ob}$, with only small amounts of other isomers $159 \mathrm{ob}, 188 \mathrm{ob}$ and $187 \mathrm{ob}$ observed (entry 12).

The new substrate synthesis of diynes 159 also enabled the easy modulation of the substituents at aryl $R^{2}$ and thus an evaluation on the change in selectivity and enantioselectivity in this reaction on variation of these groups. Previously, the effect of introducing methoxy groups at the positions 3 and 14 of the helicene had had a negative impact on the selectivity. However, when using the triazolium-derived catalyst 172y, a dramatic increase in selectivity towards the helicene was observed (entries 6 and 8). Similarly, a dramatic increase in selectivity was observed for both para-benzyloxy- and 3,5,dimethyl-phenylsubstituted 159jb and 159nb when using the catalyst 172y (entries 14 and 16). The same reactions catalyzed by $\mathbf{1 7 2} \mathbf{i}$ resulted in much poorer selectivity (entries 13 and 15).

Gratifyingly, in most cases of the substrates tested, the enantioselectivities remained high, lying between 85 and $88 \%$ for the dimethoxy-, dibenzyloxy- and tetramethylsubstituted helicenes 160bb, 160 jb and 160nb and even exceeding $90 \%$ ee for the chlorosubstituted helicenes 160ae and 160ob (entries 4 and 12). A moderate drop in enantioselectivity was apparent for the phenyl substituted helicene $160 \mathrm{cb}$ (entry 9 ). 
Table 13. Comparative study between gold(I) complexes $172 \mathbf{i}$ and $172 y$.

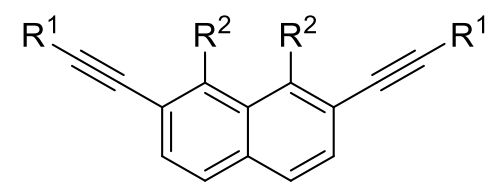

159 (a)

\begin{tabular}{|c|c|c|c|c|c|c|c|}
\hline Entry & 159 & $\mathrm{R}^{1}$ & $\mathrm{R}^{2}$ & 172 & Yield $(\%)^{a}$ & $\begin{array}{c}\text { 160: 188: } 187 \\
(\%)^{\mathrm{b}}\end{array}$ & $e e(\%)^{c}$ \\
\hline $1^{d}$ & 159ab & $p$-Tolyl & $\mathrm{Ph}$ & $172 i$ & 88 & $97: 3: 0$ & +91 \\
\hline $2^{\mathrm{e}}$ & $159 a b$ & $p$-Tolyl & $\mathrm{Ph}$ & $172 y$ & $>95$ & $98: 2: 0$ & +87 \\
\hline $5^{d}$ & $159 b b$ & $p$-Tolyl & $4-\mathrm{MeO}-\mathrm{C}_{6} \mathrm{H}_{4}-$ & $172 i$ & $>95^{\mathrm{h}}$ & $88: 12: 0$ & +78 \\
\hline 6 & $159 b b$ & $p$-Tolyl & 4-MeO- $\mathrm{C}_{6} \mathrm{H}_{4}-$ & $172 y$ & 83 & $96: 4: 0$ & +86 \\
\hline $7^{f}$ & 159be & 4- $\mathrm{Cl}-\mathrm{C}_{6} \mathrm{H}_{4-}$ & 4-MeO- $\mathrm{C}_{6} \mathrm{H}_{4-}$ & $172 \mathbf{i}$ & $92^{h}$ & $87: 13: 0$ & +95 \\
\hline 8 & 159be & $4-\mathrm{Cl}-\mathrm{C}_{6} \mathrm{H}_{4}-$ & 4-MeO- $\mathrm{C}_{6} \mathrm{H}_{4-}$ & $172 y$ & 93 & $95: 5: 0$ & +85 \\
\hline $9^{d}$ & $159 \mathrm{cb}$ & p-Tolyl & $4-\mathrm{Ph}-\mathrm{C}_{6} \mathrm{H}_{4-}$ & $172 i$ & $>95$ & $97: 3: 0$ & +82 \\
\hline 10 & $159 \mathrm{cb}$ & $p$-Tolyl & 4- $\mathrm{Ph}-\mathrm{C}_{6} \mathrm{H}_{4}-$ & $172 y$ & 99 & $98: 2: 0$ & +75 \\
\hline $11^{d}$ & $159 o b$ & $p$-Tolyl & 4- $\mathrm{Cl}-\mathrm{C}_{6} \mathrm{H}_{4-}$ & $172 i$ & - & - & - \\
\hline 12 & $159 o b$ & $p$-Tolyl & 4-Cl- $\mathrm{C}_{6} \mathrm{H}_{4-}$ & $172 y$ & $76^{i}$ & $86: 7: 7^{j}$ & +92 \\
\hline $13^{g}$ & $159 \mathrm{jb}$ & $p$-Tolyl & $4-\mathrm{BnO}-\mathrm{C}_{6} \mathrm{H}_{4-}$ & $172 i$ & $86^{i}$ & 87: 13: 0 & +75 \\
\hline 14 & $159 \mathrm{jb}$ & $p$-Tolyl & $4-\mathrm{BnO}-\mathrm{C}_{6} \mathrm{H}_{4}-$ & $172 y$ & 92 & 97: $3: 0$ & +88 \\
\hline $15^{\mathrm{g}}$ & $159 \mathrm{nb}$ & $p$-Tolyl & $3,5-(\mathrm{Me})_{2}-\mathrm{C}_{6} \mathrm{H}_{3}-$ & $172 \mathbf{i}$ & $84^{i}$ & 88: $12: 0$ & +75 \\
\hline 16 & $159 \mathrm{nb}$ & $p$-Tolyl & $3,5-(\mathrm{Me})_{2}-\mathrm{C}_{6} \mathrm{H}_{3^{-}}$ & $172 y$ & 90 & 97: $3: 0$ & +87 \\
\hline 17 & 159ib & $p$-Tolyl & 4-TMS- ${ }_{6} \mathrm{H}_{4-}$ & $172 y$ & $87^{i}$ & $89: 11: 0$ & +62 \\
\hline
\end{tabular}

Reagents and conditions: (a) 159 ( 1 equiv. $0.025 \mathrm{mmol}), 172$ (10 mol\%), $\mathrm{AgSbF}_{6}(10 \mathrm{~mol} \%), \mathrm{C}_{6} \mathrm{H}_{5} \mathrm{~F},-20{ }^{\circ} \mathrm{C}, 96 \mathrm{~h}$. alsolated yields, unless otherwise stated. 'belectivity determined by NMR and HPLC; ${ }^{c} e e$ determined by HPLC. ${ }^{\mathrm{d}}$ Reaction conducted by Dr. E. González-Fernández. ${ }^{[219]}{ }^{e}$ Reaction conducted by M. Marx MSc. ${ }^{[244]}{ }^{\mathrm{f}}$ Reaction conducted by L. Schaaf MSc. ${ }^{[220]}$ gReaction conducted by T. Hartung MSc. ${ }^{[245]}$ hYield reported as a mixture of isomers either/or 168, 182, 181 and/or 167. 'Yield determined using 1,4-dioxane as internal standard. 'j $6 \%$ residual 159 ob observed. ${ }^{\mathrm{k}} 73 \%$ residual $159 \mathrm{eb}$ observed. ${ }^{\mathrm{l}} \mathrm{92} \%$ residual $167 \mathrm{fb}$ observed. $\mathrm{m} 47 \%$ residual $159 \mathrm{gb}$ observed.

Having compared a wider range of substrates with both the triazolium derived $172 \mathrm{y}$ and imidazolium derived $\mathbf{1 7 2} \mathbf{i}$, it was clear that the triazolium derived catalyst exhibited an outstanding increase in selectivity compared to the previous system. This obviates the need for costly additional separations and opens the possibility of accessing a wider variety of enantiomerically enriched carbohelicenes. Additionally, in many cases, the enantioselectivity was also higher, indicating the high potential of this new generation of cationic phosphonites.

Attention then turned to the prospect of extending the synthesis to heterohelicenes. Accordingly, the thiophene and furan substituted diynes $159 \mathrm{eb}-\mathbf{h b}$ were submitted to the reaction conditions using $\mathbf{1 7 2 y}$ as a precatalyst. The results are summarised in Table 14. 
Interestingly, in no cases were any isomers relating to the 5-exo-dig/ 6-endo-dig product 188 observed. Unfortunately, in the reactions of 2-thienyl (159eb) and 2-furanyl (159fb) substituted conversion remained low, indicating that even the precatalyst $172 \mathrm{y}$ was not reactive enough to enact a cyclisation for these substrates. Furthermore, the enantioselectivities of the products was also much lower than for the [6]carbohelicenes 160 . For the 3-thienyl substituted diyne (159gb) conversion was high, although still incomplete, while for 3-furanyl substituted $159 \mathrm{hb}$ the only product detectable in the crude reaction mixture was the 3,12-dioxa helicene $160 \mathrm{hb}$, albeit with a low isolated yield of $33 \%$. In both cases, the regioselectivity with respect to the nucleophilic attack of the heteroatom to the alkyne was excellent, with the exclusive products arising from attack via the 2-position of the heteraromatics. On measurement of the enantiomeric excess of the resulting heterohelicenes, both $160 \mathrm{gb}$ and $160 \mathrm{hb}$ were racemic.

Table 14. Enantioselective gold(I) catalysed hydroarylation of precursors 159eb-hb bearing heteroaromatic groups.

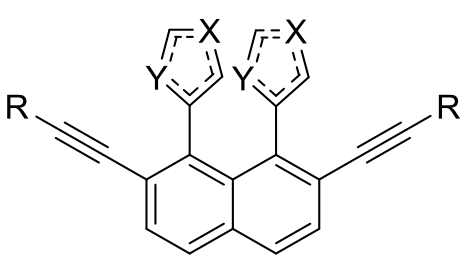

159

(a)

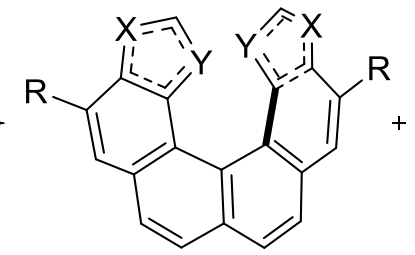

160

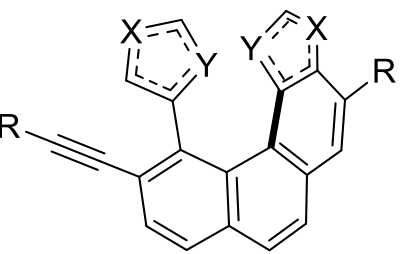

187

\begin{tabular}{|c|c|c|c|c|c|c|c|}
\hline Entry & 159 & $X=$ & $Y=$ & Yield (\%) & Conv. $(\%)^{b}$ & 160: $187(\%)^{\mathrm{c}}$ & $e e(\%)^{d}$ \\
\hline 1 & $159 \mathrm{eb}$ & $-\mathrm{C}-$ & -S- & 21 & 27 & $81: 19$ & +24 \\
\hline 2 & $159 \mathrm{fb}$ & $-\mathrm{C}-$ & $-\mathrm{O}-$ & 5 & 8 & $>99: 0$ & +24 \\
\hline 3 & $159 \mathrm{gb}$ & $-S-$ & $-C-$ & 16 & 53 & $38: 62$ & 0 \\
\hline 4 & $159 \mathrm{hb}$ & -0 & $-\mathrm{C}-$ & 33 & $>95$ & $>99: 0$ & 0 \\
\hline
\end{tabular}

Reagents and conditions: (a) 159 (1 equiv.), 172y (10 mol\%), AgSbF 6 (10 mol\%), $\mathrm{C}_{6} \mathrm{H}_{5} \mathrm{~F},-20{ }^{\circ} \mathrm{C}, 96 \mathrm{~h}$. a,bYields and conversion determined using 1,4-dioxane as internal standard. ${ }^{c}$ Selectivity determined by NMR and HPLC. dee determined by HPLC.

To the best of our knowledge, helicenes such as $160 \mathrm{eb}, 160 \mathrm{fb}$ and $160 \mathrm{hb}$ have until now not been described, although 3,12-dithia[6]helicene was originally reported by the group of Wynberg in 1973, ${ }^{[252]}$ and a quadruple helicene comprised of two 3,12-dithia[6]helicenes and two [5] helicenes was recently reported by the group of Itami. ${ }^{[230,253]}$ In this report, the two [5] helicene moieties were found to racemize on heating to $80^{\circ} \mathrm{C}$ for two days; however, the two dithia[6]helicene moieties remained configurationally stable. The structurally related 5,8dithia[6]helicene 111, which was determined not to be configurationally stable at room temperature with an experimentally determined barrier to racemization of $23.7 \mathrm{kcal} \cdot \mathrm{mol}^{-1}$ (cf. Figure 17), was also described by Wynberg in 1969. ${ }^{[134]}$ Nevertheless, the barrier to 
racemisation for any of the new heterohelicenes had not been previously described. As a low barrier to racemisation was a possible reason for the low ee values obtained, it was decided to perform a computational study to investigate this further.

Accordingly, the ground and transition state geometries for the helicenes $160 \mathrm{eb}-\mathrm{hb}$ were optimized using Gaussian ${ }^{[254]}$ at the B3LYP/6-31+g(d) level of theory, including [6]carbohelicene for comparative purposes. For the helicenes $160 \mathrm{eb}, 160 \mathrm{gb}$ and $160 \mathrm{hb}$, well defined transition state geometries could be optimized. All attempts to do the same for helicene $\mathbf{1 6 0 f b}$ were unsuccessful, and the reasons for this are not immediately clear. It is, however, possible that $\mathbf{1 6 0 f b}$ could racemize through an alternative pathway, distinctly different to the other helicenes. The optimized transition state geometries for [6]carbohelicene and heterohelicenes $160 \mathrm{eb}, 160 \mathrm{gb}$ and $160 \mathrm{hb}$ are shown in Figure 38.

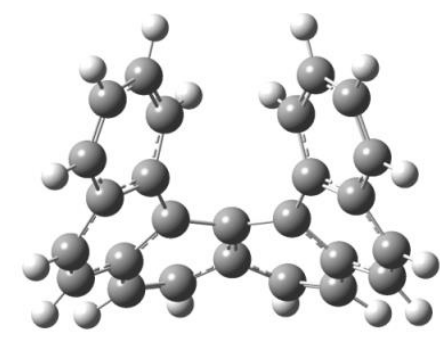

106: 36.31

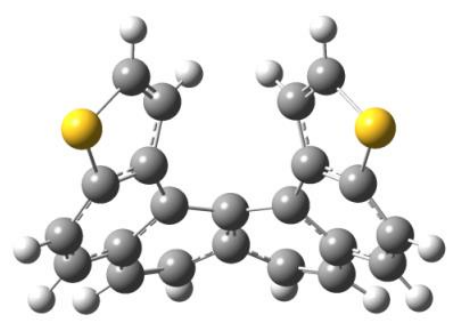

$160 \mathrm{gb}: 37.61$

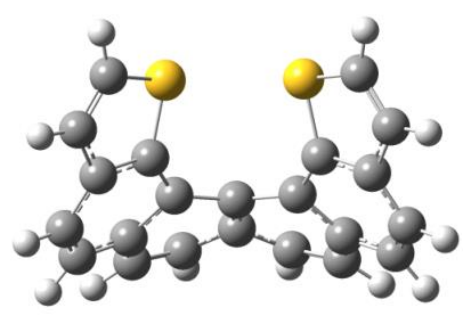

160eb: 41.09

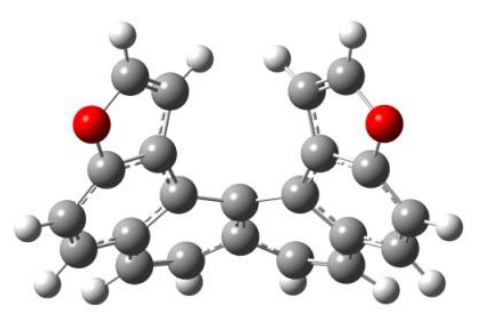

160hb: 35.02

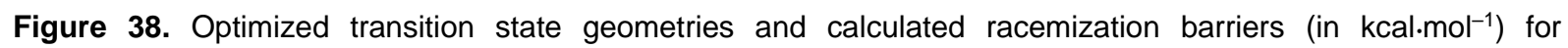
[6]carbohelicene 106 and heterohelicenes $160 \mathrm{eb}, 160 \mathrm{gb}$ and $160 \mathrm{hb}$.

The calculated racemization barrier of [6]carbohelicene 106 is in good agreement with a recently published literature value of $38.16 \mathrm{kcal} \cdot \mathrm{mol}^{-1} \cdot{ }^{[135]}$ The three calculated heterohelicenes showed barriers to racemization in the same range as that of [6]carbohelicene, indicating that they would be configurationally stable at room temperature and therefore suitable substrates for asymmetric catalysis. Helicene 160eb showed the highest barrier in the series, likely due to the large repulsion between the overlapping sulfur atoms at the periphery of the helix. Dithiahelicene $160 \mathrm{gb}$ shows a significantly higher barrier than its isomer, 5,8-dithia[6]helicene, 11 (cf. Figure 17, $23.7 \mathrm{kcal}^{-\mathrm{mol}^{-1}}$ ). It is likely that $\mathbf{1 1 1}$, 
with two internally embedded thiophene rings in the helical structure, is more flexible. As a result, it should approach the racemization transition state more easily than $160 \mathrm{gb}$, in which the thiophene rings are at the periphery of the helix. In light of the calculated racemisation barriers for the heterohelicenes, it is possible that another chiral catalyst would be more effective in catalysing an enantioselective hydroarylation reaction for the substrates $159 \mathrm{eb}-$ hb. However, considering the poor conversion of all the substrates tested in this study using chiral cationic phosphonites, this was not investigated further.

\subsection{Enantioselective hydroarylation of cyanated tetrahelicenes}

A highly selective cyanative chlorination of alkynes recently developed in the Alcarazo group by A. García-Barrado, ${ }^{[255]}$ mediated by boron trichloride and the electrophilic cyanating reagent $215,{ }^{[256]}$ has subsequently been expanded to give cyanated phenanthrenes. ${ }^{\text {[257] }}$ On submission of the helicene substrate 159ag to these conditions, even when using an excess of borane, the monocyanated intermediate 216a was selectively formed in excellent yield instead of the expected dicyanated helicene (Scheme 64).

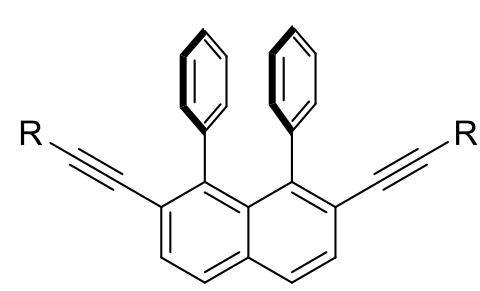

159ah: $R=\{$

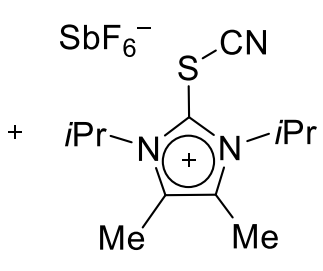

215 (a)

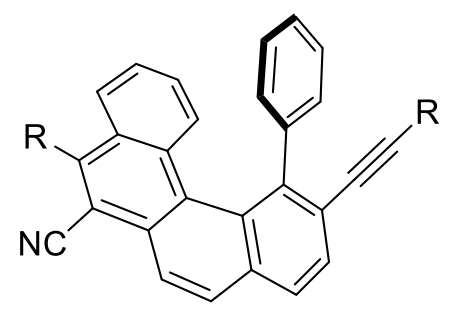

$216 a$

Scheme 64. Cyanative cyclisation of 159ag to give 216a using electrophilic cyanating reagent 215. Reagent and conditions (a) 159ag (1.0 equiv.), 215 (2.5 equiv.), $\mathrm{BCl}_{3}$ (2.5 equiv.), 2,6-di-tert-butylpyridine (2.0 equiv.), $\mathrm{CH}_{2} \mathrm{Cl}_{2}$, $0{ }^{\circ} \mathrm{C}, 1$ h. Reaction performed by A. García-Barrado. ${ }^{[257]}$

Tetrahelicenes such as $\mathbf{2 1 6} \mathbf{a}$ had been previously proven to easily convert between the two helically chiral enantiomers, even when cooled down to $-20^{\circ} \mathrm{C} \cdot{ }^{[219]} \mathrm{A}$ second cyclisation, this time mediated by chiral gold catalysts, would give access to cyanated, enantioenriched helicenes. Encouraged by this prospect, the tetrahelicene 216a was subjected to a variety of conditions using the cationic phosphonite gold complex 172y. The results are shown in Table 15. Firstly, the reactions were tried in both dichloromethane and fluorobenzene at room temperature and $0{ }^{\circ} \mathrm{C}$. In both solvents, the cyclizations were practically completed at room temperature, but enantioselectivities remained low (entries 1 and 2). Gratifyingly, no other isomers could be detected in the reaction mixtures. A stark increase in enantioselectivity was seen, especially in fluorobenzene (entries 3 and 4), when lowering the temperature to $0{ }^{\circ} \mathrm{C}$; however, incomplete conversions were observed in both cases. Increasing the temperature 
to $5^{\circ} \mathrm{C}$ in fluorobenzene led to a moderate conversion of $67 \%$, but still very good ee of $89 \%$. It is probable that the cyano group in the substrate inhibits reactivity of the catalyst by coordinating the cationic gold species, thus limiting conversion.

Table 15. Screening for the enantioselective intramolecular hydroarylation in [4]helicene 216a.<smiles>[R]C#Cc1ccc2ccc3c(C#N)c([R])c4ccccc4c3c2c1-c1ccccc1</smiles>

216a: $R=\xi O \mathrm{OMe}$ (a)<smiles>[R]c1ccc2c(ccc3ccc4c(C#N)c([R])c5ccccc5c4c32)c1</smiles>

217a

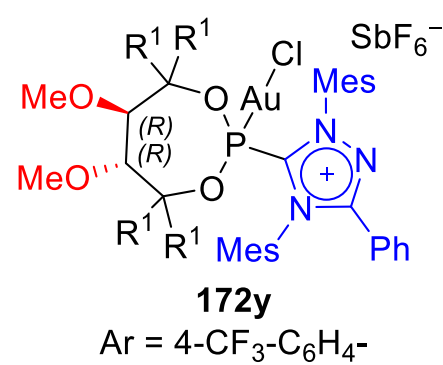

\begin{tabular}{ccccccc}
\hline Entry & Solvent & $\mathrm{T}\left({ }^{\circ} \mathrm{C}\right)$ & $\mathrm{t}(\mathrm{h})$ & ${\text { Conversion }(\%)^{\mathrm{a}}}$ & ${\text { Yield }(\%)^{\mathrm{b}}}$ & ee $(\%)^{\mathrm{c}}$ \\
\hline $1^{\mathrm{a}}$ & $\mathrm{CH}_{2} \mathrm{Cl}_{2}$ & 25 & 16 & $>95$ & 61 & +39 \\
2 & $\mathrm{C}_{6} \mathrm{H}_{5} \mathrm{~F}$ & 25 & 16 & 93 & 47 & +36 \\
3 & $\mathrm{CH}_{2} \mathrm{Cl}_{2}$ & 0 & 72 & 75 & 58 & +82 \\
4 & $\mathrm{C}_{6} \mathrm{H}_{5} \mathrm{~F}$ & 0 & 72 & 39 & 29 & +92 \\
5 & $\mathrm{C}_{6} \mathrm{H}_{5} \mathrm{~F}$ & 5 & 72 & 67 & 57 & +89 \\
\hline
\end{tabular}

Reagents and conditions: (a) 216a (1.0 equiv.), 172y (10 mol\%), AgSbF 6 (10 mol\%), solvent (0.05 M), T, t. a,b NMR yields and conversions determined using hexamethyltricyclotrisiloxane as internal standard. 'Enantioselectivities determined using HPLC.

In any case, the conditions of fluorobenzene at $5{ }^{\circ} \mathrm{C}$, which gave the best enantiomeric excess at a reasonable conversion, were chosen with an interest in further evaluating other substrates in a preliminary screening. From a variety of substrates $159 \mathrm{ag}-\mathbf{n g}$ tested in the

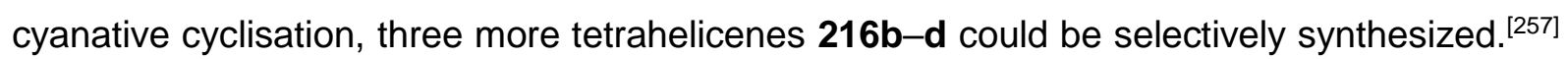
These were subsequently evaluated in the gold-catalyzed hydroarylation (Table 16).

The chlorinated tetrahelicenes 216c and 216d unfortunately displayed lower conversions in comparison to $\mathbf{2 1 6 a}$. Additionally, the enantiomeric excess measured for $217 \mathrm{c}$ was also very low. Cyclization of compound 216b with a 3,5-dimethylphenyl substituent proceeded to give excellent conversion to the hexahelicene product 217b. In this case, however, a lower enantiomeric excess was observed. Presumably the tetrahelicene 216b exhibits a higher inversion barrier than the other tetrahelicenes, because of the meta-substituted arene moieties pointing into the tetrahelicene core and the additional internal methyl group. In this case, the kinetic dynamic resolution to give the enantioenriched hexahelicene would likely proceed more slowly, and this is most likely a contributing factor to the low enantiomeric excess obtained. It is still interesting to note, however, that the structurally related substrate 
159nb was cyclized to give a good ee in the double hydroarylation reaction, as outlined in Table 13, entry 16. Perhaps in this case an enantiomerically enriched intermediate is formed after the first cyclisation.

Table 16. Enantioselective gold(I)-catalyzed cyclisation of tetrahelicenes 216a-d to give cyanated [6]carbohelicenes 217a-d.

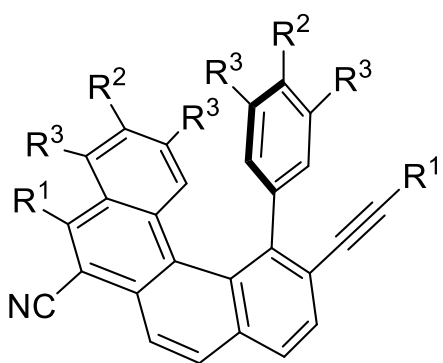

216a-d (a)<smiles></smiles>

$217 a-d$

\begin{tabular}{cccccccc}
\hline Entry & 216 $^{\mathrm{a}}$ & $\mathrm{R}^{1}$ & $\mathrm{R}^{2}$ & $\mathrm{R}^{3}$ & ${\text { Conversion }(\%)^{\mathrm{b}}}$ & ${\text { Yield }(\%)^{\mathrm{c}}}$ & ee $(\%)^{\mathrm{d}}$ \\
\hline 1 & $\mathbf{2 1 6 a}$ & $4-\mathrm{MeO}-\mathrm{C}_{6} \mathrm{H}_{4^{-}}$ & $\mathrm{H}$ & $\mathrm{H}$ & 67 & 57 & +89 \\
2 & 216b & $4-\mathrm{MeO}-\mathrm{C}_{6} \mathrm{H}_{4^{-}}$ & $\mathrm{H}$ & $\mathrm{Me}$ & $>95$ & $81^{\mathrm{e}}$ & +51 \\
3 & 216c & $4-\mathrm{MeO}-\mathrm{C}_{6} \mathrm{H}_{4^{-}}$ & $\mathrm{Cl}$ & $\mathrm{H}$ & 24 & 19 & +12 \\
4 & 216d & $p-T o l y l$ & $\mathrm{Cl}$ & $\mathrm{H}$ & $27^{\mathrm{f}}$ & traces & - \\
\hline
\end{tabular}

Reagents and conditions: (a) 216 (1.0 equiv.), 172y (10 mol\%), $\mathrm{AgSbF}_{6}(10 \mathrm{~mol} \%), \mathrm{C}_{6} \mathrm{H}_{5} \mathrm{~F}(0.05 \mathrm{M}), 5^{\circ} \mathrm{C}, 72 \mathrm{~h}$. aprepared by Alejandro García-Barrado.[257] b,cNMR yields and conversions determined using hexamethyltricyclotrisiloxane as internal standard. 'Enantioselectivity determined using HPLC. elsolated yield. fDetermined using 1,4-dioxane as internal standard.

Although the synthesis of cyanated helicenes is an interesting line of study, with the possibility of creating asymmetrically substituted push-pull helicenes and the high synthetic utility of the cyano substituent towards further derivatization, the likelihood is that the cyano group inhibits the reactivity of the gold catalyst too strongly to give any promising results in this type of transformation. Nevertheless, there appears to be an exploitable difference in reactivity between the substrate $159 \mathrm{ag}$ and its intermediate. It would be certainly worthwhile to asymmetrically functionalize the helicene using other electrophilic group transfer reagents. Interrupted cyclizations to give iodo, bromo, and sulfenyl substituted phenanthrenes have already been reported by the group of Larock ${ }^{[151]}$ and would make an ideal starting point for this line of research.

\subsection{Solid state structures}

The solid state structures for the helicene 160af, 160ae, 160ob and 160ag are shown in Table 17. The structural features of helicenes that are commonly compared are (i) the interplanar angle, defined by the angle between the planes set by each of the peripheral 
rings; (ii) the torsional angles of the inner helix and (iii) the distances between the innermost carbons of the inner helix (C1-C9, C2-C8). Many helicenes display unequal torsional angles in the solid state, as is the case for helicenes 160 af and $160 \mathrm{ob}$. This implies that each of the aromatic rings bears the strain of the helical axis differently.

Table 17. Solid state structures of 160af, 160ae, 160ag and 160ob

(A)

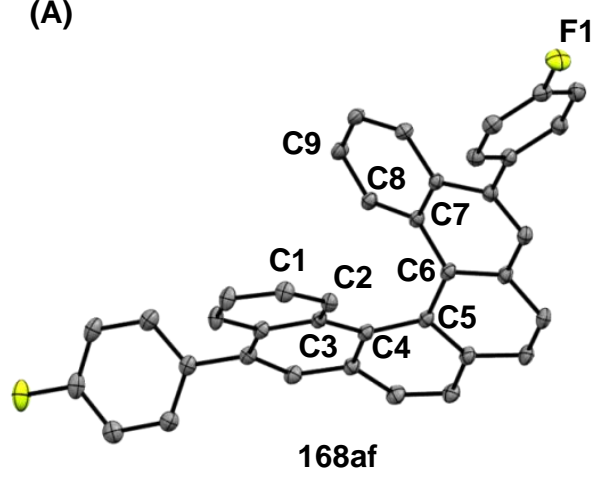

(C)

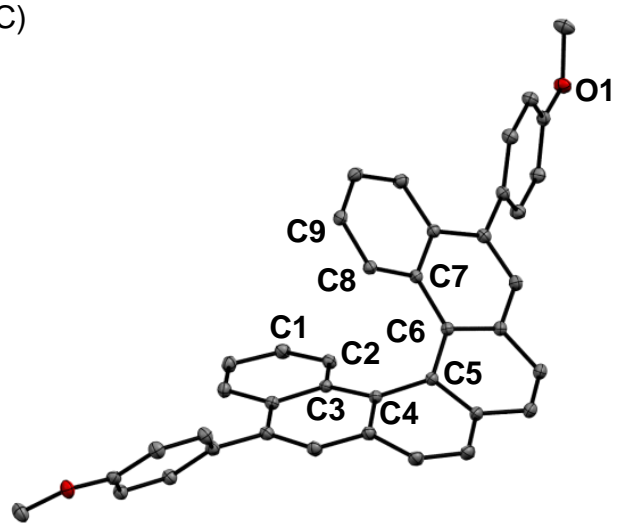

168ag

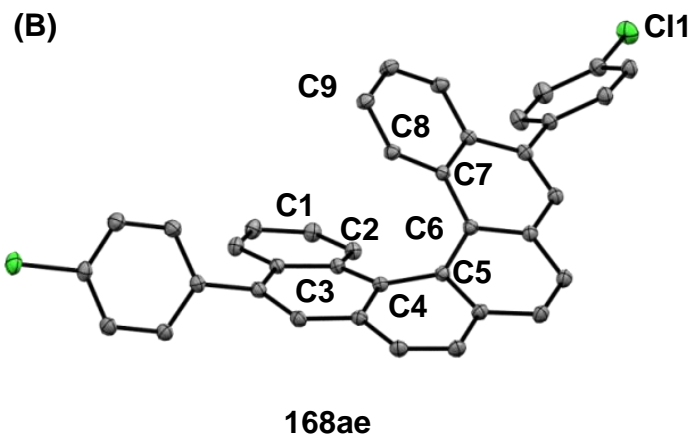

(D)

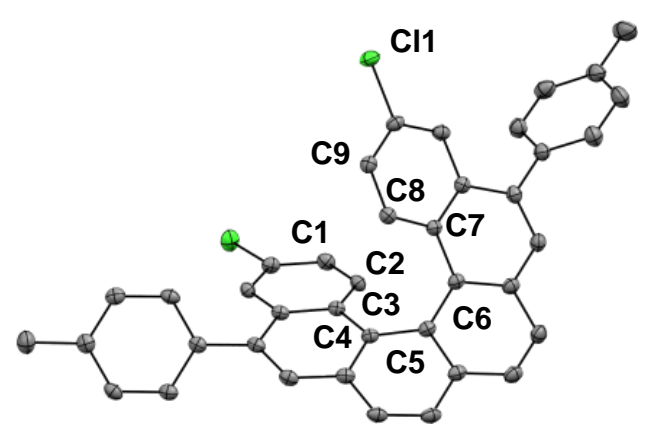

$160 \mathrm{ob}$

\begin{tabular}{ccccc}
\hline Selected features & 160af & 160ae & 160ag & 160ob \\
\hline Space group & $P 1$ & C2/c & C2/c & $P 2_{1} / \mathrm{c}$ \\
C2-C8 (Inner pitch) $(\AA)$ & $3.074(1)$ & $3.003(1)$ & $3.090(1)$ & $3.211(2)$ \\
C1-C9 (Å) & $4.225(1)$ & $3.978(1)$ & $4.367(1)$ & $4.566(1)$ \\
Interplanar angle $\left(^{\circ}\right)$ & 49.15 & 42.09 & 53.84 & 58.04 \\
$\Phi\left(\right.$ C2-C3-C4-C5) $\left(^{\circ}\right)$ & $12.6(2)$ & 19.1 & 7.9 & $16.3(2$ \\
$\Phi\left(\right.$ C3-C4-C5-C6) $\left(^{\circ}\right)$ & $28.5(2)$ & 23.9 & 30.5 & $28.0(2)$ \\
$\Phi\left(\right.$ C4-C5-C6-C7) $\left(^{\circ}\right)$ & $24.7(2)$ & 23.9 & 30.5 & $28.4(2)$ \\
$\Phi\left(\right.$ C5-C6-C7-C8) $\left(^{\circ}\right)$ & $18.9(2)$ & 19.1 & 7.9 & $18.2(2)$ \\
\hline
\end{tabular}

Solvent molecules are omitted for clarity. Thermal ellipsoids are set to $50 \%$ probability. The numbering does not correspond to the IUPAC rules.

Curiously, helicenes 160ae and 160ag crystallized in a $C_{2}$-symmetric space group, which is less commonly seen for helicenes. ${ }^{[120]}$ As can be seen in Table 17, despite the only 
difference in their respective structures being the substitution of fluorine for chlorine or methoxy groups, helicenes 160af, 160ae and 160ag display quite different interplanar angles, torsional angles and C2-C8 bond distances. However, this is likely due to differences in crystal packing effects, which can have a large influence on the flexible helix structure. ${ }^{[136]}$

The solid state structure of helicene 212an is shown in Figure 39. Here the poorer overlap of the peripheral aromatic rings can be seen, and is manifested by the dramatically reduced values for the outermost two torsional angles [C2-C3-C4-C5 = 5.0(6) ${ }^{\circ}$; C5-C6-C7-C8 = $10.0(6)^{\circ}$. The X-ray structure also confirms the geometry of the two exocyclic double bonds, with both shown to be $(E)$-configurated. This geometry is expected to be formed under the gold(I) catalysis conditions due to the anti mode of nucleophilic attack. It also indicates that no further isomerization occurs under the reaction conditions or upon workup.

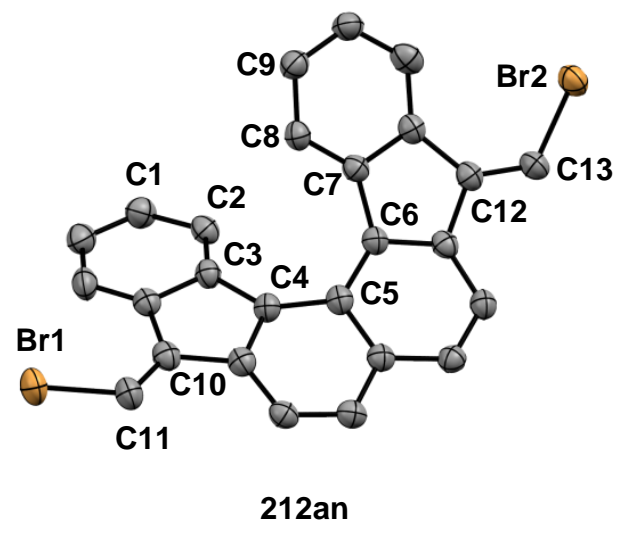

Figure 39. Solid state structure of 212an. Thermal ellipsoids set at $50 \%$ probability. The numbering does not correspond to the IUPAC rules. Space group $P 2_{1} / \mathrm{c}$. Selected bond lengths $(\AA) B r 1-C 11=1.878(4), B r 2-C 13=$ $1.877(3), \mathrm{C} 10-\mathrm{C} 11=1.339(5), \mathrm{C} 12-\mathrm{C} 13=1.342(5), \mathrm{C} 2-\mathrm{C} 8$ (inner pitch) = 3.034(5); interplanar angle = 54.20 ; torsional angles $\left(^{\circ}\right) \mathrm{C} 2-\mathrm{C} 3-\mathrm{C} 4-\mathrm{C} 5=5.0(6)$, C3-C4-C5-C6 = 22.3(6), C4-C5-C6-C7 = 25.2(6), C5-C6-C7-C8 $=10.0(6)$.

Dibenzohelicene $160 \mathrm{db}$ crystallizes in a $P 1$ space group, with two symmetry-independent molecules of each enantiomer occupying the asymmetric unit cell (Table 18). The annulated peripheral benzene rings have an interesting twisted structure, and actually bend in the opposite direction to the rings making up the central helicene. This can be seen by comparing the interplanar angle of these rings (shown as rings $A$ and $H$ in Table 18) with that of the helix (rings $B$ and $G$ ), where the planes formed by these rings intersect at opposite sides of the structure. This also has the effect of significantly reducing the $\mathrm{C} 4-\mathrm{C} 11$ distance in comparison to the other helicenes and may indicate some degree of electronic overlap between the $\pi$-systems of the two rings. 
Table 18. Structure of dibenzohelicene $160 \mathrm{db}$ in the solid state: selected features for both symmetry independent molecules.

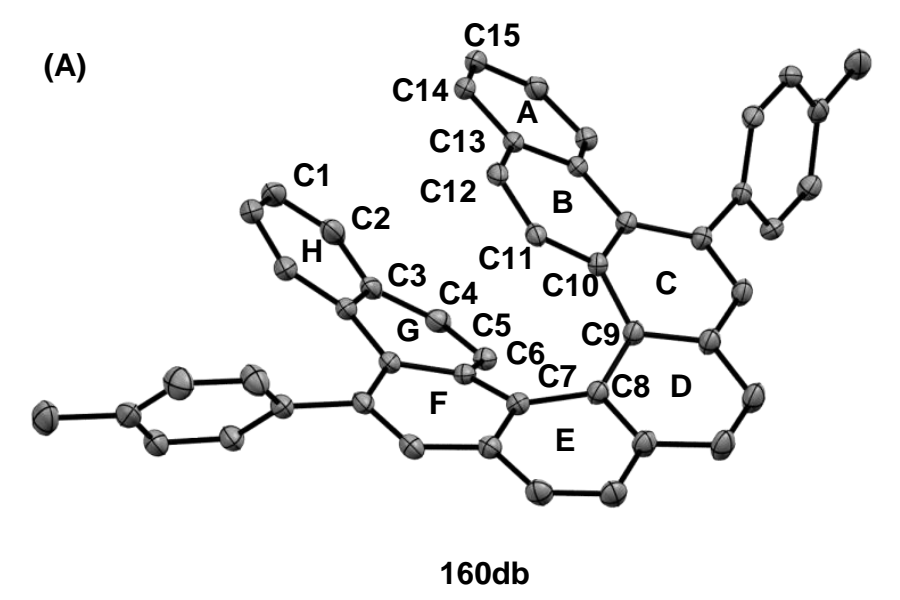

(B)

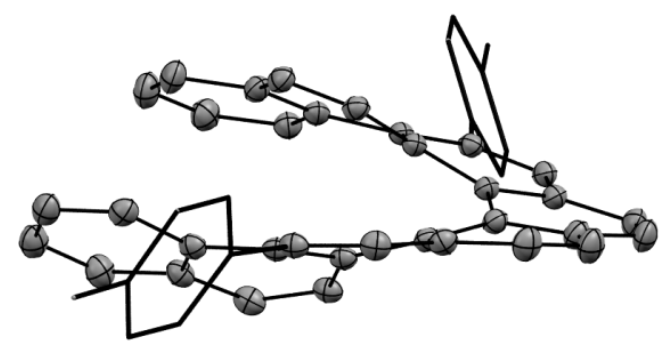

\begin{tabular}{|c|c|c|}
\hline Selected features & $168 \mathrm{db}(1)$ & $168 \mathrm{db}(2)$ \\
\hline C5-C10 $(\AA)$ & $2.954(2)$ & $2.968(2)$ \\
\hline $\mathrm{C} 4-\mathrm{C} 11(\AA)$ & $3.587(2)$ & $3.537(2)$ \\
\hline Interplanar angle (rings $B$ and $G$ ) $\left(^{\circ}\right.$ ) & 21.44 & 15.79 \\
\hline Interplanar angle (rings $\mathrm{A}$ and $\mathrm{H})\left(^{\circ}\right.$ ) & -9.17 & -6.52 \\
\hline$\Phi(\mathrm{C} 11-\mathrm{C} 10-\mathrm{C} 9-\mathrm{C} 8)\left(^{\circ}\right)$ & $19.4(2)$ & $26.7(2)$ \\
\hline$\Phi(\mathrm{C} 10-\mathrm{C} 9-\mathrm{C} 8-\mathrm{C} 7)\left(^{\circ}\right)$ & $20.8(2)$ & $20.3(2)$ \\
\hline$\Phi(\mathrm{C} 9-\mathrm{C} 8-\mathrm{C} 7-\mathrm{C} 6)\left(^{\circ}\right)$ & 24.1(2) & $20.2(2)$ \\
\hline$\Phi(\mathrm{C} 8-\mathrm{C} 7-\mathrm{C} 6-\mathrm{C} 5)\left(^{\circ}\right)$ & $24.0(2)$ & $26.0(2)$ \\
\hline
\end{tabular}

(A) One symmetry-independent molecule of $160 \mathrm{db}$. (B) Side on-view of $160 \mathrm{db}$, depicting twisting of the peripheral benzene rings of the helix; ancilliary $p$-tolyl rings set as capped sticks for clarity. Thermal ellipsoids set at $50 \%$ probability; the numbering does not correspond to the IUPAC rules.

The solid state structures of the new helicenes $168 \mathrm{eb}, 168 \mathrm{fb}, 168 \mathrm{gb}$ and $168 \mathrm{hb}$ are shown in Table 19. Helicene 168eb crystallized in a racemic space group $P 2_{1} / \mathrm{n}$, with three symmetry independent molecules making up the asymmetric unit cell, while helicene $168 \mathrm{fb}$ crystallized in a space group Pn consisting of two symmetry independent molecules in the asymmetric unit cell. A comparison of each of these symmetry-independent molecules of the same structure shows the helix to be differently twisted in all cases. Nevertheless, a clear steric repulsion between the two sulphur atoms in 168eb can be seen, shown by a large increase in the interplanar angle, torsional angles and the inner pitch of the helix compared to $168 \mathrm{gb}$, where the helix is much narrower. Differences between the helicenes $168 \mathrm{fb}$ and $\mathbf{1 6 8 \mathrm { hb }}$ are less manifested; presumably as the heteroatom is positioned outside the helix. 
Table 19. Solid state structures of the heterohelicenes $160 \mathrm{eb} 160 \mathrm{fb}, 160 \mathrm{gb}$ and $160 \mathrm{hb}$ and selected structural parameters.

(A)

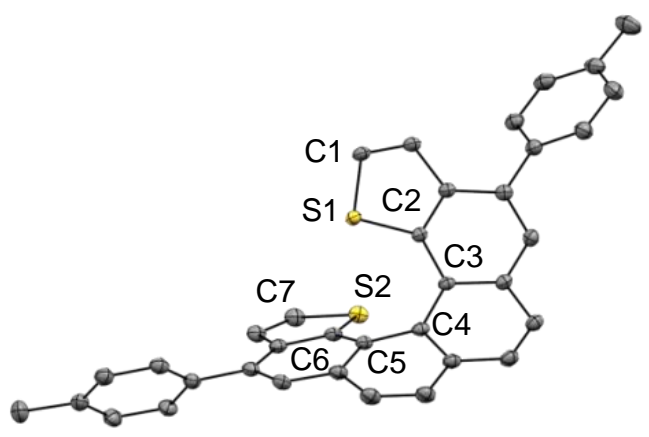

$160 \mathrm{eb}$

(C)

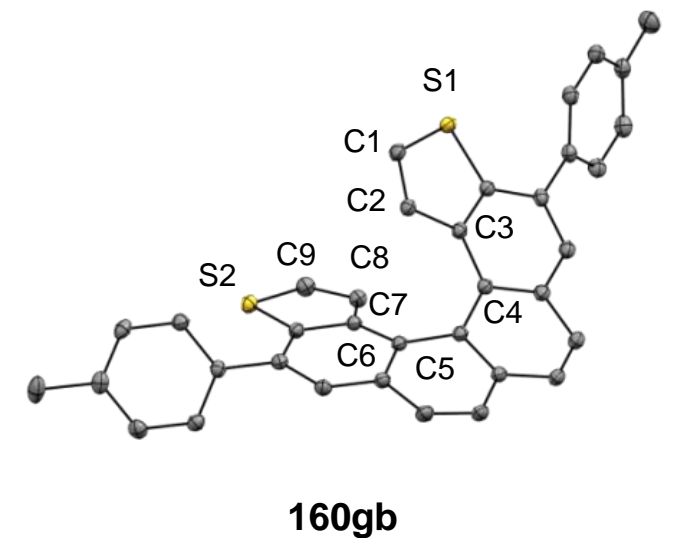

(B)

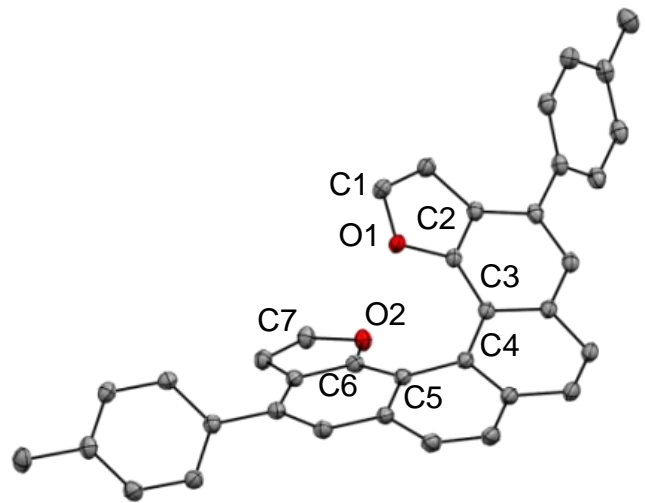

$160 \mathrm{fb}$
(D)

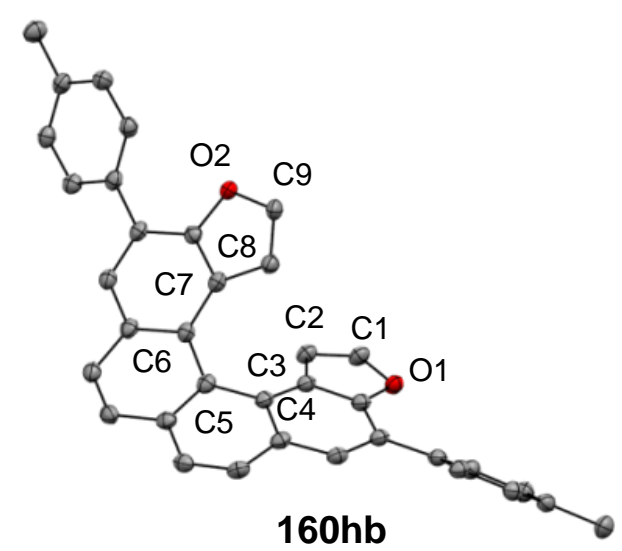

Table 19 (continued)

\begin{tabular}{llccccccc}
\hline \multicolumn{1}{c}{160} & $\begin{array}{c}\text { Space } \\
\text { group }\end{array}$ & $\begin{array}{c}\text { Inner } \\
\text { pitch }(\AA)\end{array}$ & $\begin{array}{c}\text { Inner pitch } \\
\text { (outer) }(\AA)\end{array}$ & $\begin{array}{c}\text { Interplanar } \\
\text { angle }\left({ }^{\circ}\right)\end{array}$ & $\Phi 1\left(^{\circ}\right)$ & $\Phi 2\left(^{\circ}\right)$ & $\Phi 3\left(^{\circ}\right)$ & $\Phi 4\left(^{\circ}\right)$ \\
\hline $\mathbf{1 6 0 e b}(1)$ & $P 2_{1} / \mathrm{n}$ & $3.2533(5)$ & $4.900(2)$ & 57.38 & $6.7(2)$ & $30.0(2)$ & $30.5(2)$ & $7.7(2)$ \\
$\mathbf{1 6 0 e b}(2)$ & $P 2_{1} / \mathrm{n}$ & $3.3149(5)$ & $4.979(2)$ & 60.89 & $8.9(2)$ & $33.8(2)$ & $27.5(2)$ & $7.4(2)$ \\
$\mathbf{1 6 0 e b}(3)$ & $P 2_{1} / \mathrm{n}$ & $3.2466(5)$ & $4.969(2)$ & 58.93 & $4.8(2)$ & $28.5(2)$ & $32.6(2)$ & $7.5(2)$ \\
$\mathbf{1 6 0 f b}$ & $P b c a$ & $2.607(1)$ & $3.906(2)$ & 47.90 & $5.4(2)$ & $25.5(2)$ & $24.0(2)$ & $8.6(2)$ \\
$\mathbf{1 6 0} \mathbf{g b}$ & $P b c a$ & $2.930(2)$ & $4.199(2)$ & 50.83 & $5.6(2)$ & $27.9(2)$ & $25.7(2)$ & $11.1(2)$ \\
$\mathbf{1 6 0 h b}(1)$ & $P n$ & $3.044(3)$ & $4.569(4)$ & 57.72 & $6.8(4)$ & $27.4(4)$ & $29.6(4)$ & $6.3(4)$ \\
$\mathbf{1 6 0 h b}(2)$ & $P n$ & $2.998(3)$ & $4.441(4)$ & 53.70 & $11.3(4)$ & $25.7(4)$ & $25.4(4)$ & $11.7(4)$ \\
\hline
\end{tabular}

Solvent molecules and additional symmetry independent molecules omitted for clarity. Thermal ellipsoids set at $50 \%$ probability. The numbering does not correspond to the IUPAC rules. Inner pitch defined as (160eb) S1-S2; (160fb) O1-O2; (160 gb, 160hb) C2-C8. Inner pitch (outer) defined as (160eb, 160fb) C1-C7;(160 gb, 160hb) C1-C9. Torsional angles of inner helix $(\Phi)$ measured in anticlockwise order. 


\subsection{Optical properties}

Because the synthesized helicenes systemically crystallized in racemic space groups, no information about which enantiomer was preferentially formed in the reaction could be inferred from their solid state structures. A characteristic chiroptical property of helicenes is their circular dichroism spectra, which have the same shape, but are oppositely directed, depending on the enantiomer being measured. Circular dichroism is the ability of enantioenriched chiral molecules to differently absorb left- or right-handed circularly polarized light. Many helicenes exhibit two characteristic transitions; for example, in [6]carbohelicene 106 one at 246 and another at 324nm can be seen (Figure 40). ${ }^{[258]}$ For the enantiomer $(P)$ 106, the circular dichroism is positive for the transition at $324 \mathrm{~nm}$ and negative for the transition at $246 \mathrm{~nm}$. This is equal and opposite for the $(M)$-enantiomer. By analyzing the known circular dichroism spectra (CDS) for both enantiomers of other similar compounds, the CDS of enantioenriched helicenes can be used as a tool to determine their absolute configuration. ${ }^{[200,258]}$ Accordingly, the CDS of helicenes 160af, 160ag and 160ah, as well as 160be, 160bi and 160b|[220] were measured and compared to that reported for hexahelicene 106 (Figure 41). The helicenes 160af, 160ag and 160ah showed a strong agreement to the $(P)$-enantiomer of hexahelicene $(P)$-106, with positive circular dichroisms for the transitions at between 324-366 and negative circular dichroisms at 246-250 nm being observed. The helicenes $160 \mathrm{be}, 160 \mathrm{bi}$ and $160 \mathrm{bl}$ also showed a strong similarity, with the transitions shifted roughly $20 \mathrm{~nm}$ to higher wavelengths, in the range of 264 and $344 \mathrm{~nm}$. Based on these findings, the absolute configuration of the helicenes formed using the $(R, R)$ precatalyst $(R, R)$-172i was assigned as the $(P)$-enantiomer.

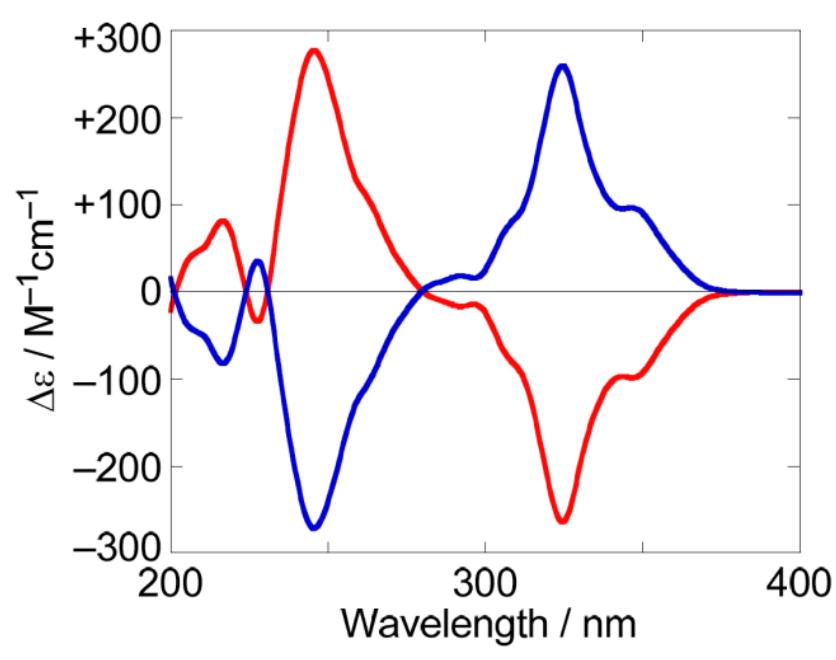

Figure 40. Experimentally determined circular dichroism spectra of optically resolved [6]carbohelicene: $(P)$ shown in blue; (M) shown in red. Figure taken from: Y. Nakai, T. Mori, Y. Inoue, J. Phys. Chem. A, 2012, 116, 7372. ${ }^{[202]}$ 


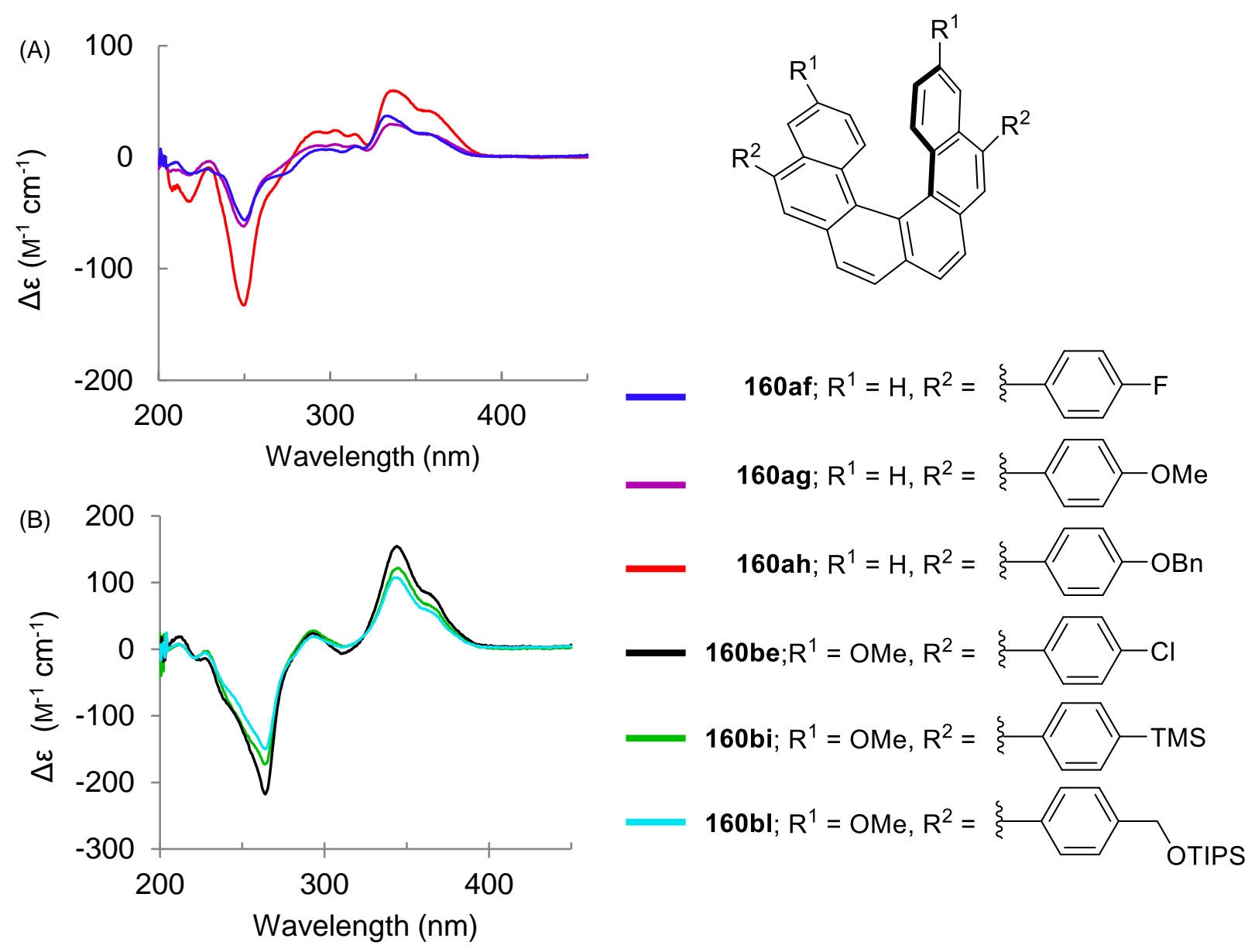

Figure 41. Experimentally determined CDS of helicenes synthesized through enantioselective gold(I) catalysis. Measured as solutions (100 $\mathrm{MM}$ in THF) of (A) compounds 160af, 160ag, and 160ah; (B) compounds 160be, $160 \mathrm{bi}$ and $160 \mathrm{bl}$. Helicenes $160 \mathrm{be}, 160 \mathrm{bi}$ and $160 \mathrm{bl}$ were prepared by Lukas Schaaf MSc. ${ }^{[220]}$

In addition, some photophysical properties for the helicenes formed using the precatalyst 172y were measured. In this instance, a comparison of how the substituents directly bound to the helicene termini affect photophysical properties such as circular dichroism, UV/Vis and fluorescence was possible. Although the helicenes were not formed with perfect enantiomeric excesses, a qualitative evaluation of the effect of different substituents can be seen from their respective spectra. The overlaid circular dichroism (CD) spectra for helicenes $160 \mathrm{ab}, 160 \mathrm{bb}, 160 \mathrm{jb}, 160 \mathrm{ob}$ and 160 $\mathrm{nb}$, which have comparable enantiomeric excesses are shown in Figure 42A. 
(A)

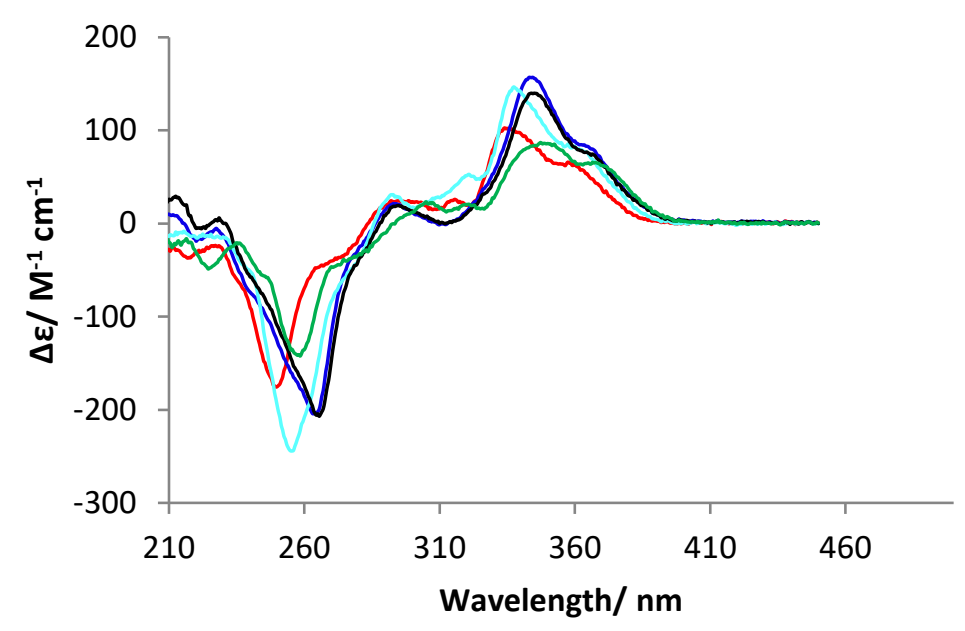

(B)

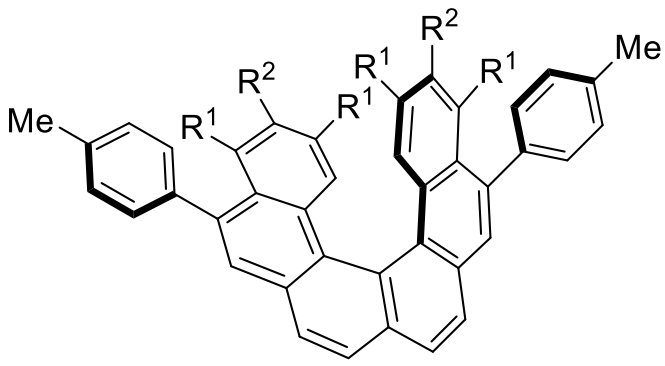

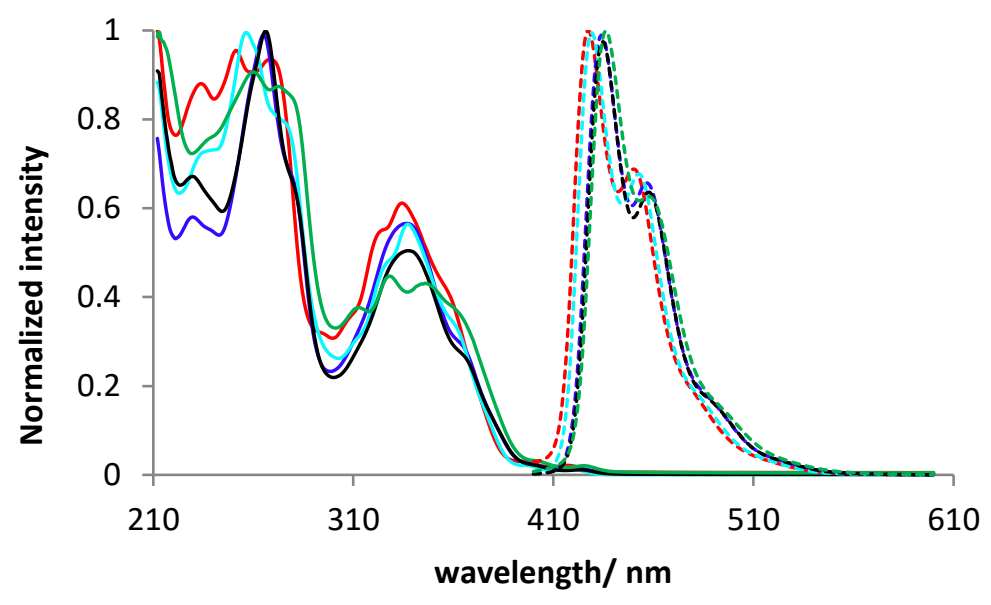

160ab: $R^{1}=H ; R^{2}=H$

160bb: $\mathrm{R}^{1}=\mathrm{H} ; \mathrm{R}^{2}=\mathrm{MeO}$

160jb: $R^{1}=H ; R^{2}=B n O$

160ob: $\mathrm{R}^{1}=\mathrm{H} ; \mathrm{R}^{2}=\mathrm{Cl}$

160nb: $R^{1}=M e ; R^{2}=H$

Figure 42. (A) Circular dichroism spectra of enantioenriched helicenes $160 \mathrm{ab}(87 \%$ ee) , 160bb $(86 \%$ ee), $160 \mathrm{jb}$ ( $88 \%$ ee $), 160 \mathrm{ob}(92 \%$ ee $)$ and 160 $\mathrm{nb}(87 \%$ ee). Samples measured in THF, $100 \mu \mathrm{M}$; (B) UV/Vis and fluorescence spectra of helicenes $160 \mathrm{ab} .160 \mathrm{bb}, 160 \mathrm{jb}, 160 \mathrm{ob}$ and $160 \mathrm{nb}$. Fluorescence shown as dashed lines. Spectra normalized to the relative maxima of each spectrum. Samples measured in THF.

In contrast to the previous series of helicenes (Figure 41), the positions of both maxima in the CD spectra are more significantly affected by the introduction of different functional groups directly on the helicene. Again, the spectra support the assignment of the $(P)$ enantiomer to the synthesized helicenes using $(R, R)-172 y$. Additionally, the UV/Vis absorbance spectra of the helicenes 168ab, 168bb, 168jb, 168ob and 168nb, as well as their fluorescence spectra were measured. An overlay of the normalized spectra is shown in Figure 42B. Wavelengths in the absorption band at approximately $340 \mathrm{~nm}$ were chosen in all cases as excitation wavelengths, and fluorescence was measured between 400 and $600 \mathrm{~nm}$. As can be seen, all samples fluoresced in the region of $430-460 \mathrm{~nm}$, with two maxima being observed. These resemble recently reported emission spectra for other [6]carbohelicenes. ${ }^{[232]}$ 


\subsection{Summary}

In summary, an expansion of the scope of the enantioselective gold(I) catalysed double hydroarylation of diynes 159 into 160 has been accomplished, and some additional variants on this reaction investigated. Firstly, a wider scope of the hydroarylation of diynes 159, with varying substituents at the alkyne substituents was evaluated using the chiral cationic phosphonite precatalyst 172i. Across a range of substrates, high yields, regio- and enantioselectivities could be obtained. Nevertheless, in instances where either the conversion of starting material 159 and intermediate 187, or regioselectivity over 6-endo-dig/ 5-exo-dig isomer 188 was not excellent, the desired helicene could only be separated by low yielding preparative HPLC separations.

Using a new selection of cationic phosphonite ligands, with a greater variety of cationic substituents, a series of model studies were carried out aiming to optimise the reactivity of the catalyst, in addition to achieve higher regio- and enantioselectivities in for a wider selection of substitution patterns. The 1,2,4-triazolium-based precatalyst 172y displayed a promising profile, and upon further evaluation across a range differently substituted precursors was shown to be highly reactive and consistently afforded helicenes $\mathbf{1 6 0}$ in excellent regioselectivities and enantioselectivites. Specifically, in challenging substrates where the precatalyst $\mathbf{1 7 2} \mathbf{i}$ afforded poor regioselectivities, $\mathbf{1 7 2 y}$ consistently resulted in dramatic improvements. Across a wide range of precursors 159, it is feasible to obtain [6]carbohelicenes $\mathbf{1 6 0}$ in high purity and enantiomeric excess (Chart 3). Although the [6]carbohelicenes consistently crystallised in racemic space groups, the absolute configuration could be assigned upon comparison of the CD spectra with Itierature reported values of [6]helicene. Therefore, when using the precatalyst $(R, R)-\mathbf{1 7 2 y}$, the $(P)$ [6]carbohelicenes $\mathbf{1 6 0}$ are formed. 


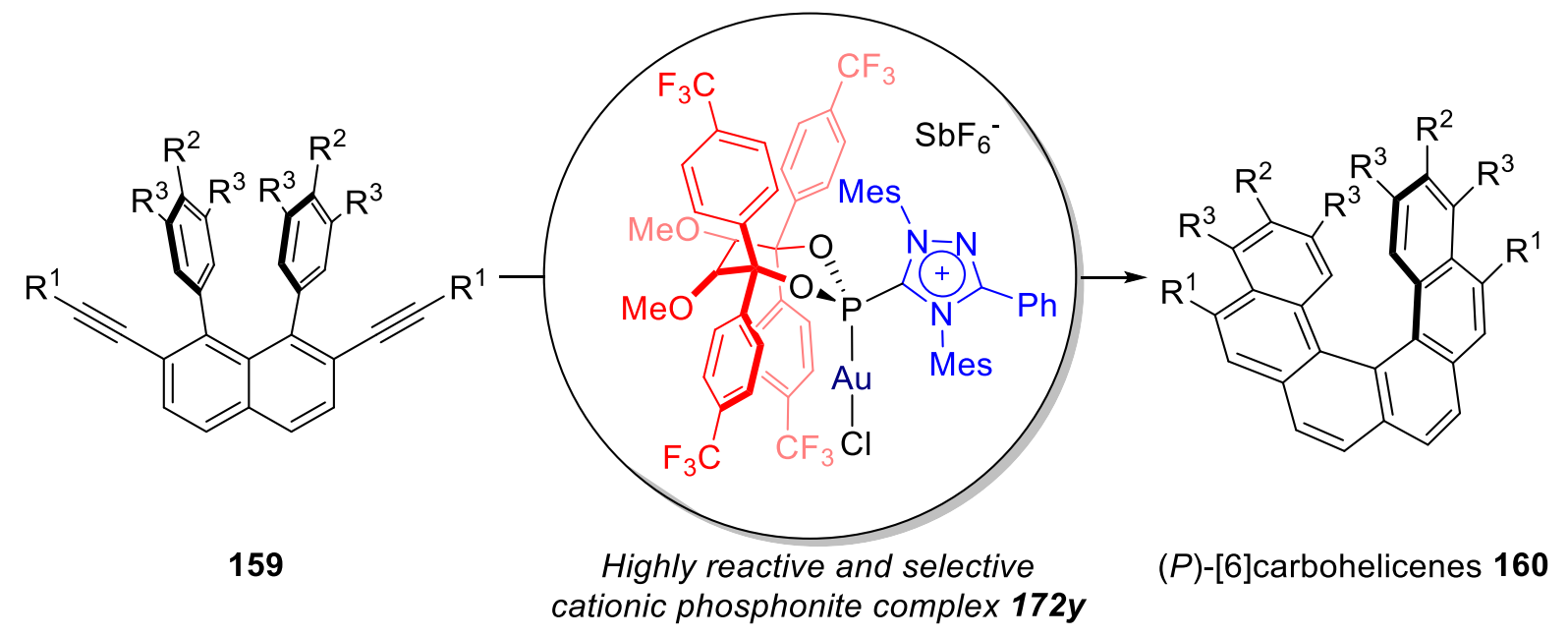

Chart 3. Gold complexes of first generation of chiral cationic phosphonites in twofold intramolecular hydroarylations towards [6]helicenes.

\section{Towards the enantioselective total synthesis of Monbarbatain A}

\subsection{Retrosynthetic analysis}

Because Monbarbatain A contains two symmetrically joined phenanthrene subunits, it was envisaged that a twofold gold-catalyzed hydroarylation reaction of diyne $\mathbf{2 1 9}$ could affect the key cyclization and introduce an axially chiral axis in the resulting product $\mathbf{2 1 8}$, as shown in the retrosynthetic analysis outlined in Scheme 65 . In addition, the natural product bears a series of free hydroxyl groups, which would most likely have to be protected during the gold catalysis step, so as not to allow a proton-catalyzed background reaction to take place due to coordination of the active gold species to any unprotected oxygen. A suitable protecting group, orthogonal to the two methoxy substituents, would therefore have to be chosen. 
7. Towards the enantioselective total synthesis of Monbarbatain A

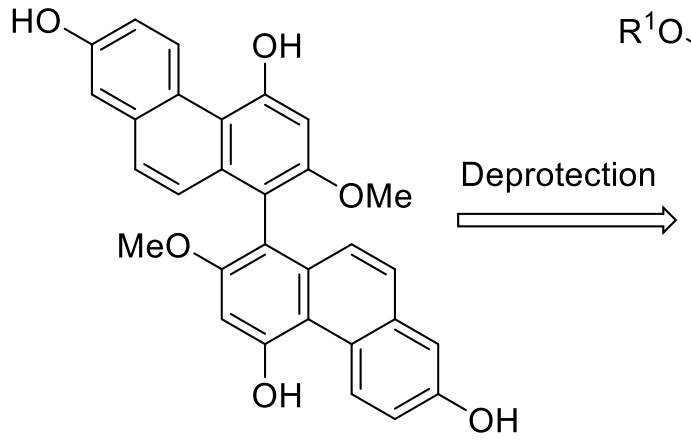

156a: Monbarbatain A<smiles>[R10]Oc1ccc2c(OCC)cc(OC)c(-c3c(OC)cc(OCC)c4c3ccc3cc(O)ccc34)c2c1</smiles>

218

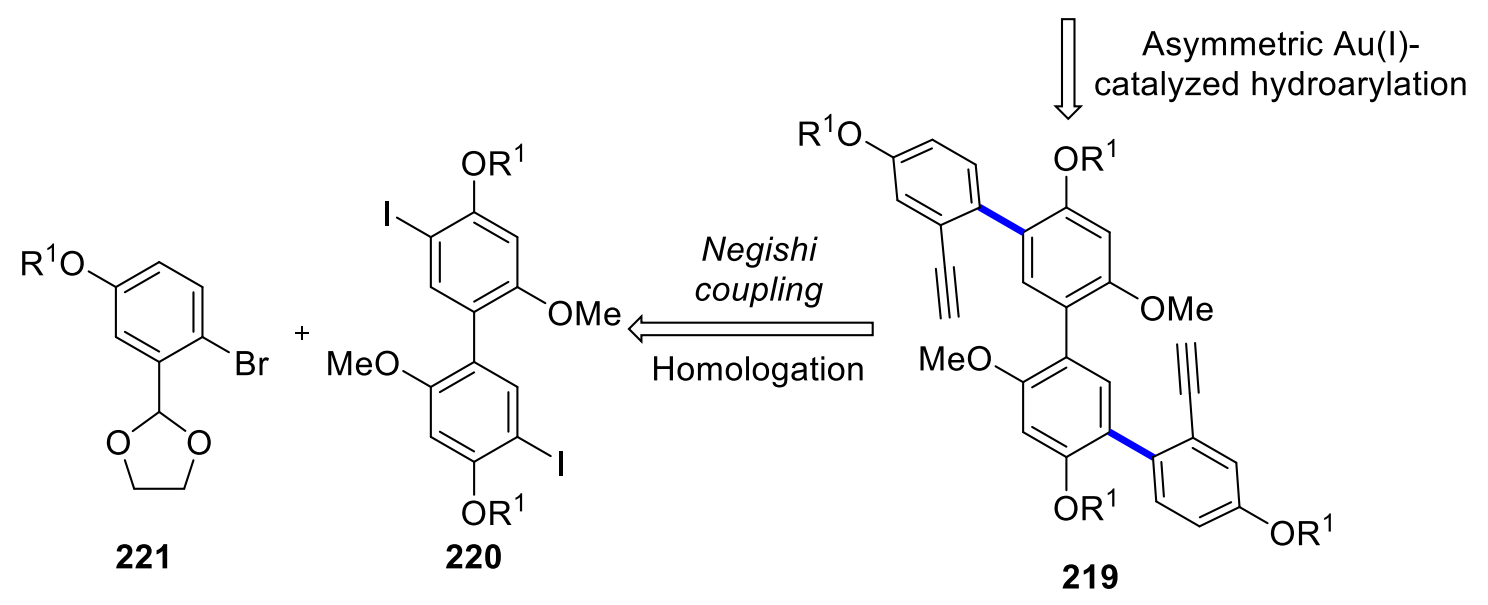

Scheme 65. Retrosynthetic analysis of Monbarbatain A.

It was envisaged that to access the terminal diyne substrate $\mathbf{2 1 9}$, a double cross-coupling of the biaryl diiodide 220 and arylbromide 221 would be suitable. Aryl bromide 221 would be easily accessible from the commercially available 2-bromo,5-hydroxybenzaldehyde; and biaryl iodide 220 through a closely related iodination ${ }^{[259]} /$ cross coupling $^{[260]}$ protocol (Scheme $66)$.<smiles>[R17]Oc1ccc(-c2ccc([R10])cc2OC)c(OC)c1</smiles>

Scheme 66. General strategy to access biaryl 220.

When choosing a suitable protecting group, previously obtained but unpublished results in the Alcarazo group indicated silyl groups to be particularly labile when using the cationic gold catalysts, and that significant decomposition of substrates bearing silyl ethers would occur 
unless the reactions were conducted at low temperatures. Otherwise, methoxymethylethers were thought to potentially cause issues relating to the catalytic activity, as the substrate 159am, bearing aliphatic ethers tested in this thesis gave low conversion, presumably through coordination of the oxygen to the highly electron deficient gold center in the catalyst (for the racemic reaction of $\mathbf{1 5 9} \mathbf{k b}$, also with aliphatic chains, see experimental section).

Two protecting group strategies that were hypothesized to tolerate the catalytic conditions, as well as to be selectively removed in the presence of the methyl ethers, were found. The robust benzyl protecting group had already shown high stability in other gold-catalyzed reactions conducted in the group, and it can be removed under mild hydrogenation conditions. Although the central double bond of phenanthrenes is known to be relatively reactive towards hydrogenation, ${ }^{[95]}$ a variety of phenanthrenes have successfully been synthesized using this strategy, including in the synthesis of the natural product confusarin with two benzyl ethers on one phenanthrene skeleton, ${ }^{[261]}$ and an analogue of Combretastatin $A-4^{[110]}$ (Scheme 67). On the other hand, partial reduction of the phenanthrene moieties in Monbarbatain A could lead to the other natural products Monbarbatain B (156b) and 157 (Figure 25).

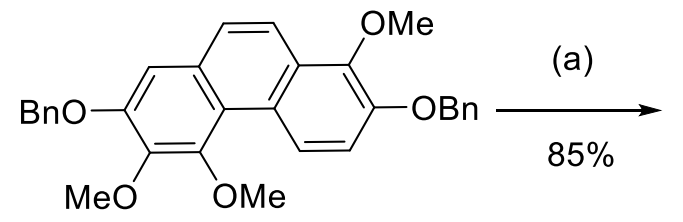

224

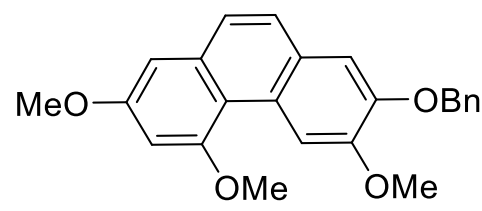

226<smiles>COc1c(O)cc2ccc3c(OC)c(O)ccc3c2c1OC</smiles>

225 (Confusarin)

(b)

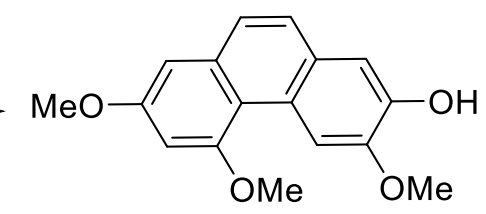

227

Scheme 67. Deprotection of benzylated phenanthrenes $225^{[261]}$ and 227. ${ }^{[110]}$ Reagents and conditions: (a) $\mathrm{H}_{2}$ (20 atm), 45 wt-\% Pd/C, EtOAc; (b) $\mathrm{H}_{2}$ (1 atm), 10 wt-\% Pd/C (0.1 equiv.), EtOAc.

Another possible orthogonal protecting group would be the isopropyl ether, which would likely sterically shield the oxygen from the large gold catalyst and not inhibit its reactivity or be deprotected. Amongst others, the natural product reports on Dictyodendrin $\mathrm{B}^{[262]}$, Dengibsin $^{[263]}$ as well as Fraxetin ${ }^{[264]}$ had also established that the isopropyl group could be selectively removed using boron trichloride at $0{ }^{\circ} \mathrm{C}$ in excellent yields, even in the presence of a two-to-one ratio of isopropyl to methoxy groups. 
7. Towards the enantioselective total synthesis of Monbarbatain A<smiles>COc1cc(OC(C)C)cc2c1-c1c(OC(C)C)cccc1C2=O</smiles>

228<smiles>COc1cc2ccc(=O)oc2c(OC(C)C)c1OC(C)C</smiles>

230 (a)<smiles>COc1cc(O)cc2c1-c1c(O)cccc1C2=O</smiles>

229: Dengibsin

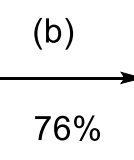<smiles>COc1cc2ccc(=O)oc2c(O)c1O</smiles>

231: Fraxetin

Scheme 68. Deprotection of isopropyl ethers in the synthesis of Dengibsin ${ }^{[263]}$ and Fraxetin. ${ }^{[264]}$ Reagents and conditions: (a) $\mathrm{BCl}_{3}\left(4.0\right.$ equiv.), $\mathrm{CH}_{2} \mathrm{Cl}_{2}, 0{ }^{\circ} \mathrm{C}$ to rt, $2 \mathrm{~h}$; (b) $\mathrm{BCl}_{3}$ (3.0 equiv.), $\mathrm{CH}_{2} \mathrm{Cl}_{2}, 18{ }^{\circ} \mathrm{C}, 3 \mathrm{~h}$.

While both protecting group strategies were deemed to viable, it was still unclear how each one would affect the outcome of the gold(I)-catalyzed hydroarylation. Because of this, both strategies were evaluated.

\subsection{Isopropyl group strategy}

The work described in this section was performed by A. Brennecke as a part of her bachelor thesis. ${ }^{[265]}$

Starting from commercially available 4-bromo-3-methoxyphenol, the free hydroxyl was protected as the isopropyl ether under basic conditions in excellent yield. This was followed by a Yamamoto homocoupling, ${ }^{[260]}$ giving biaryl 222a, which underwent iodination ${ }^{[259]}$ using in situ generated $\mathrm{HICl}_{2}$ giving 220a in excellent yield (Scheme 69). No iodination in the position between the isopropyl and methyl ethers was observed.<smiles>COc1cc(O)ccc1Br</smiles>

232<smiles>CCOc1ccc(Br)c(OC)c1</smiles>

$223 a$<smiles>COc1cc(OC(C)C)ccc1-c1ccc(OC(C)C)cc1OC</smiles>

(c)<smiles>COc1cc(OC)c(-c2cc(OC)c(OC(C)C)cc2I)cc1I</smiles>

Scheme 69. Synthesis of biaryl 222a. Reagents and conditions: (a) 232 (1.0 equiv.), $\mathrm{K}_{2} \mathrm{CO}_{3}(3.8$ equiv.), $\mathrm{PrBr}$ (1.9 equiv.), $100{ }^{\circ} \mathrm{C}, 16 \mathrm{~h}$; (b) 223a (1.0 equiv.), $\mathrm{PPh}_{3}$ (0.75 equiv.), $\mathrm{NiCl}_{2}(5 \mathrm{~mol} \%), \mathrm{BiPy}(5 \mathrm{~mol} \%), \mathrm{Zn}(1.5$ equiv.), DMAc, $90{ }^{\circ} \mathrm{C}, 16 \mathrm{~h}$; (c) 222a (1.0 equiv.), $\mathrm{I}_{2}$ (1.90 equiv.), $\mathrm{HCl}$ (0.9 equiv, 37\% aqueous), $\mathrm{H}_{2} \mathrm{O}_{2}(1.8$ equiv.), MeCN, rt, $16 \mathrm{~h}$. 
The aromatic building block 221a was prepared from the commercially available 2-bromo-5hydroxybenzaldehyde (233), firstly by forming the acetal with ethylene glycol under acidic catalysis to give phenol 234. It appeared that some of the starting material polymerized during this reaction, leading to a moderate yield of $50 \%$. After this, the hydroxyl group was protected as the isopropyl ether following standard conditions in excellent yield (Scheme 70).

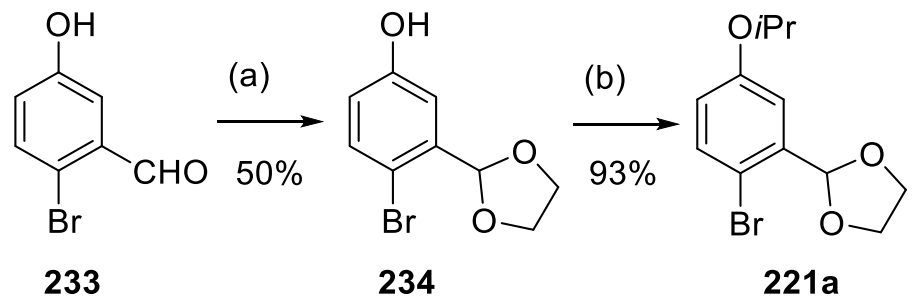

Scheme 70. Preparation of building block 221a starting from benzaldehyde 233 by acetal protection followed by isopropyl ether formation. Reagents and conditions: (a) 233 (1.0 equiv.), p-TSA. $\mathrm{H}_{2} \mathrm{O}$ (5 mol\%), ethylene glycol (1.3 equiv.), toluene, reflux 24h; (b) 234 (1.0 equiv.), $\mathrm{K}_{2} \mathrm{CO}_{3}$ (3.5 equiv.), $\mathrm{PrBr}\left(1.43\right.$ equiv.), $100{ }^{\circ} \mathrm{C}, 16 \mathrm{~h}$.

With both building blocks in hand, the aromatic bromide 221a was converted into the organozinc derivative 235a via lithiation followed by transmetalation with zinc chloride. This was followed by Negishi coupling with diiodide 220a using modified conditions by Buchwald, ${ }^{[266]}$ namely using $\mathrm{Pd}_{2}(\mathrm{dba})_{3}$ and SPhos. After work up, the reaction mixture was directly mixed with tetrahydrofuran and $1 \mathrm{M}$ hydrochloric acid to deprotect the formyl groups in situ yielding dialdehyde $\mathbf{2 3 6 a}$ in $51 \%$ yield after three-step synthesis (Scheme 71 ).<smiles>CC(C)Oc1ccc(Br)c(C2OCCO2)c1</smiles>

221a (a)<smiles>CCCOc1ccc([Ge]Cl)c(C2OCCO2)c1</smiles>

$235 a$

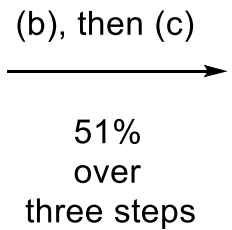

Scheme 71. Synthesis of dialdehyde 236a by Negishi coupling, followed by deprotection of the acetal under acidic conditions. Reagents and conditions: (a) 221a (1.0 equiv.), $n \mathrm{BuLi}\left(1.0\right.$ equiv.), THF, $-78^{\circ} \mathrm{C}, 2 \mathrm{~h}, \mathrm{hen} \mathrm{ZnCl}_{2}$ (2.5 equiv.), $-78^{\circ} \mathrm{C}$ to rt, $1.5 \mathrm{~h}$, directly used in next step; (b) 235a (3.0 equiv.), 220a (1.0 equiv.), $\mathrm{Pd}_{2}\left(\mathrm{dba}_{3}{ }_{3}(3\right.$ mol\%), SPhos (6 mol\%), THF, $70{ }^{\circ} \mathrm{C}, 18 \mathrm{~h}$; (c) THF, $\mathrm{HCl}(1 \mathrm{M}), 60^{\circ} \mathrm{C}, 2 \mathrm{~h}$.

The dialdehyde 236a was then subjected to the Seyferth-Gilbert homologation following the Bestmann-Ohira modification, which proceeded in a good yield (Scheme 72). It was found for this reaction that using a mixture of acetonitrile and methanol better solubilized the substrate and led to overall improved conversion. 
7. Towards the enantioselective total synthesis of Monbarbatain A
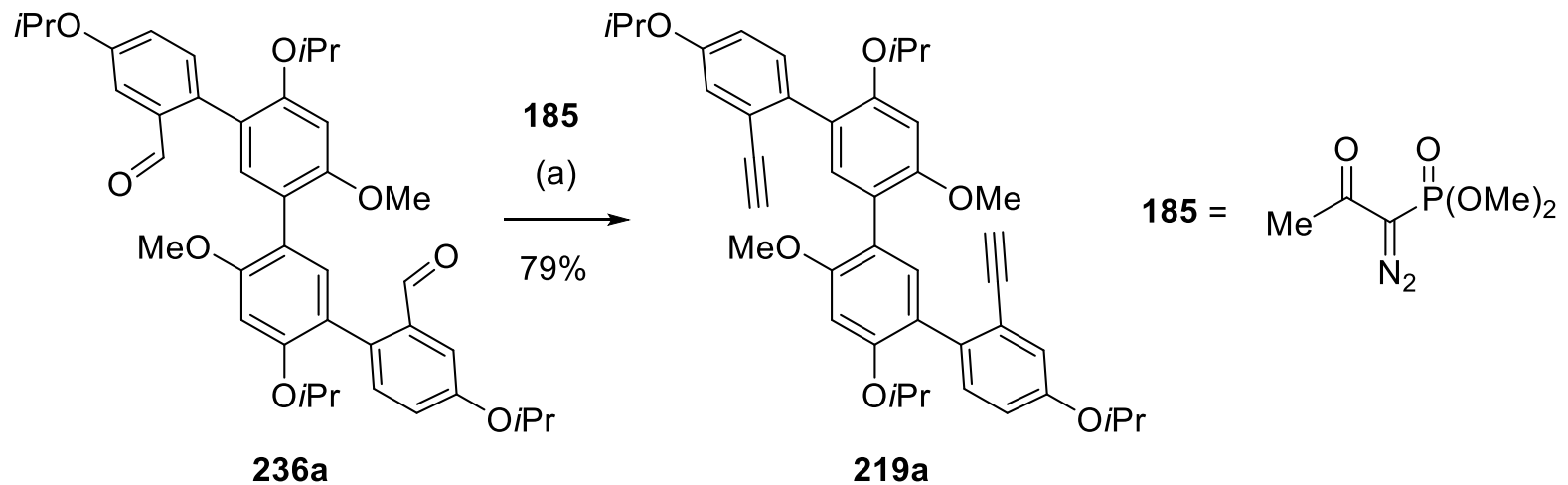

Scheme 72. Seyferth-Gilbert homologation using the Bestmann-Ohira modification to give 219a. Reagents and conditions: (a) 236a (1.0 equiv.), 185 (3.1 equiv.), $\mathrm{K}_{2} \mathrm{CO}_{3}$ ( 6.3 equiv.), $\mathrm{MeOH} / \mathrm{MeCN}$, rt, $16 \mathrm{~h}$.

This set the stage for the key gold(I)-catalyzed cyclization to give biphenanthrene 218a and form the chiral center in Monbarbatain A. As was the case for the [6]carbohelicene synthesis, it was likely that as well as the desired biphenanthrene 218a, additional products possibly identifiable in the reaction mixture would be the monocyclized intermediate $237 \mathbf{a}$, as well as the 6-endo-dig/5-exo-dig product 238a (Scheme 73).

The non-chiral gold $(\mathrm{I})$ catalyst $\mathbf{9 8 f}$, developed by Dr. H. Tinnermann, ${ }^{[87]}$ was tried as $\mathbf{9 8 f}$ had shown promising results in previous hydroarylation chemistry. On stirring diyne 219a, precatalyst $\mathbf{9 8 f}$ and silver hexafluoroantimonate in dichloromethane, a mixture of products was formed, which could be identified as 218a, 219a and 237a (12: 39: 49) according to the crude NMR spectrum. A mixture of compounds 218a and 237a was isolated after purification by column chromatography and submitted to the same reaction conditions again, using 10 mol\% catalyst loading for a second run. After work up and repeated purification by column chromatography, the desired product 218a was obtained, however, as inseparable mixture in a ratio of 78:22 with a new compound $\mathbf{2 3 8 a}$. The latter was tentatively assigned as the product of 6 -endo-dig/5-exo-dig cyclization. Both regioisomers could later be purified by semi-preparative HPLC, allowing the product $218 \mathrm{a}$ to be completely characterized. Unfortunately, the 6-endo/5-exo-dig product 238a proved to be unstable in solution and could only be recognized by its ${ }^{1} \mathrm{H}$ NMR spectrum. 
7. Towards the enantioselective total synthesis of Monbarbatain A<smiles>C#Cc1cc(OC(C)C)ccc1-c1cc(-c2cc(-c3ccc(OC(C)C)cc3C#C)c(OCC)cc2OC)c(OCC)cc1OC</smiles>

219a

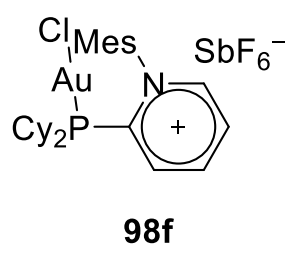

(a)<smiles>C#Cc1cc(OC(C)C)ccc1-c1cc(-c2c(OC)cc(OC(C)C)c3c2ccc2cc(OC(C)C)ccc23)c(OC)cc1OC(C)C</smiles>

237a<smiles>CCCOc1ccc2ccc3c(-c4c(OC)cc(OC(C)C)c5c4ccc4ccccc45)c(OC(C)C)cc(OC)c3c2c1</smiles>

218a: $(37 \%)$<smiles>COc1cc(OC(C)C)c2c(c1-c1c(OC)cc(OC(C)C)c3c1ccc1cc(OC(C)C)ccc13)C(=O)c1cc(OC(C)C)ccc1-2</smiles>

238a: $(10 \%)$

Scheme 73. Gold(I)-catalyzed hydroarylation of diyne $219 a$ to give the products $218 a, 237 a$ and $238 a$. Reagents and conditions: (a) 219a (1.0 equiv.), $98 \mathrm{f}$ (5 mol\%), $\mathrm{AgSbF}_{6}(5 \mathrm{~mol} \%), \mathrm{CH}_{2} \mathrm{Cl}_{2}$, rt, $48 \mathrm{~h}$; then $98 \mathrm{f}$ (10 mol\%), $\mathrm{AgSbF}_{6}(10 \mathrm{~mol} \%), \mathrm{CH}_{2} \mathrm{Cl}_{2}$, rt, $18 \mathrm{~h} ; 46 \%$ yield of $\mathbf{2 1 8 a} / \mathbf{2 3 8 a}$ mixure in ratio of 78:22.

The product 218a, as well as 238a displayed characteristic low-field doublets at 9.72, and 9.61 and $7.85 \mathrm{ppm}$, indicative of the phenanthrene ring having formed. 2D NMR assigned these low-field shifts to the inner $\mathrm{C}-\mathrm{H}$ of the phenanthrene bay area, which likely becomes deshielded due to an interaction with the proximal isopropyl ether oxygen.It is also easy to see a possible steric clash for the isopropyl group at the oxygen pointing into the phenanthrene bay area. In forming the 5-exo-dig isomer, this clash would be somewhat alleviated. 
7. Towards the enantioselective total synthesis of Monbarbatain A

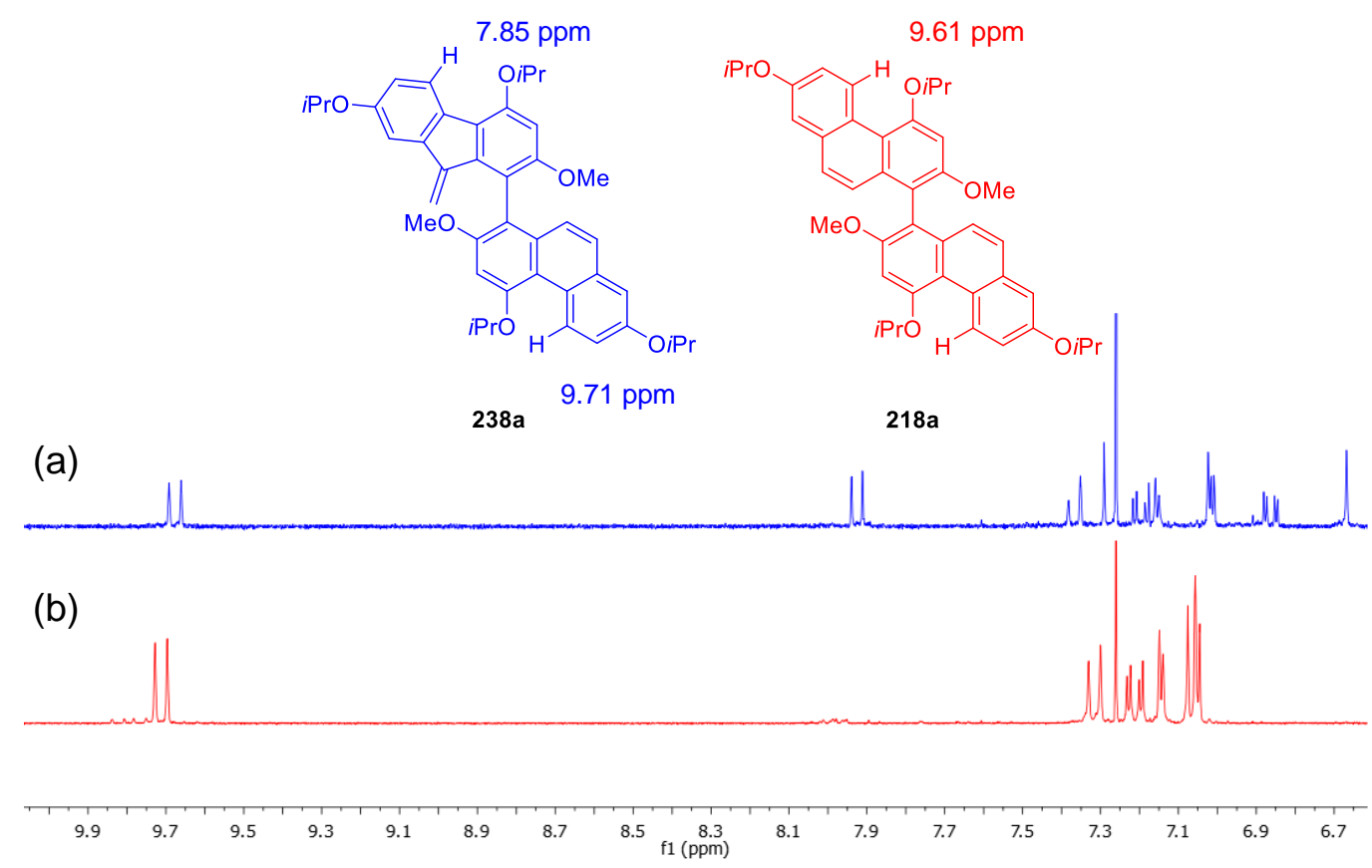

Figure 43. NMR spectra of compounds $238 \mathrm{a}(\mathrm{A})$ and $218 \mathrm{a}(\mathrm{B})\left(300 \mathrm{MHz}, \mathrm{CDCl}_{3}\right)$, indicating characteristic feature of phenanthrene core.

The preparative separation yielded enough substance to attempt the deprotection of $218 \mathbf{a}$ to give Monbarbatain A. Based upon previously reported conditions, ${ }^{[262]}$ it was decided to try first conservative conditions, treating 218a with $\mathrm{BCl}_{3}$ at $-20{ }^{\circ} \mathrm{C}$ in $\mathrm{CH}_{2} \mathrm{Cl}_{2}$ and quenching the reaction at this temperature after 30 minutes (Scheme 74 ).<smiles></smiles>

218a<smiles>COc1cc(O)c2c(ccc3cc(O)ccc32)c1-c1c(OC)cc(O)c2c1ccc1cc(O)ccc12</smiles>

$156 a$

Scheme 74. Attempted deprotection of $218 \mathrm{a}$ to give 157a. Reagents and conditions: (a) $218 \mathrm{a}\left(1.0\right.$ equiv.), $\mathrm{BCl}_{3}(8$ equiv.), $\mathrm{CH}_{2} \mathrm{Cl}_{2},-20^{\circ} \mathrm{C}, 30 \mathrm{~min}$.

On analysis by NMR, a large mixture of products could be seen. Comparison of the spectrum to that reported for Monbarbatain A by Yang et $a^{[215]}$ showed that only traces of the natural product 156a were present in the mixture. Attempts to purify by preparative TLC were unsuccessful, but analysis by HRMS confirmed the presence of not only the tri- and tetradeprotected 239a and 156a, but also the compounds 240a, 241a and 242a, where one or both methoxy groups were also removed (Figure 44). 
7. Towards the enantioselective total synthesis of Monbarbatain A

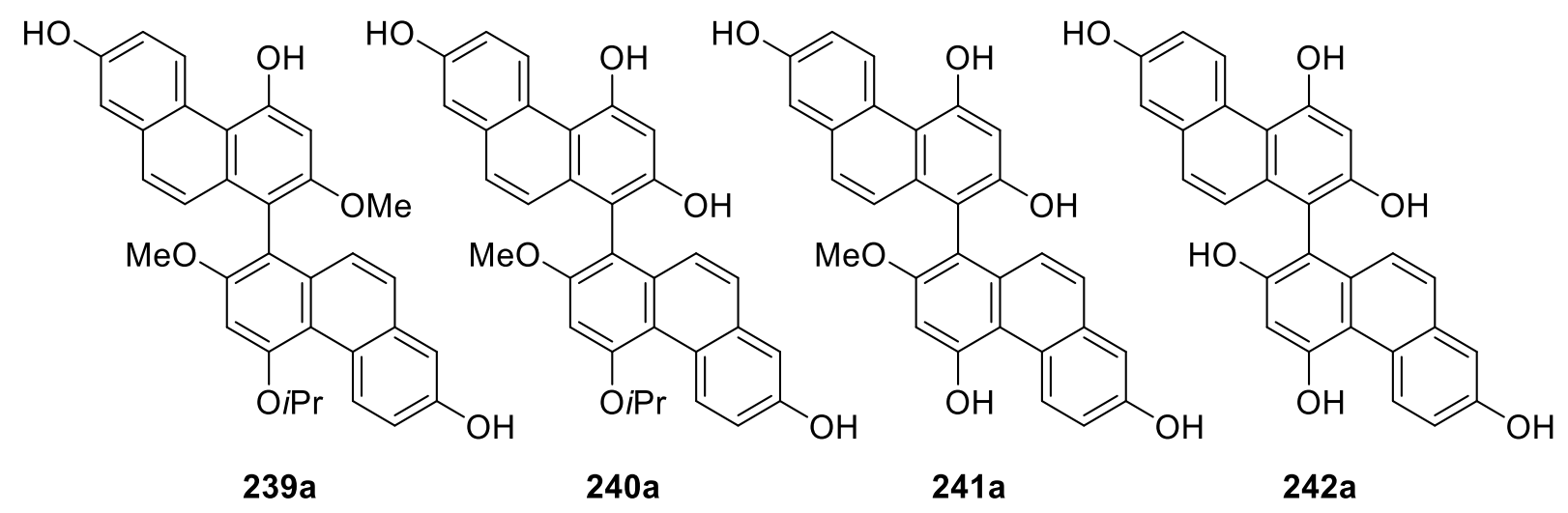

Figure 44. Possible products formed during the attempted deprotection reaction using $\mathrm{BCl}_{3}$.

The results of the deprotection, although not conclusive, indicated that partial removal of the methoxy protecting groups had occurred, even when using relatively conservative deprotection conditions. In addition, because of the poor conversion in the gold catalysis step and occurrence of the 5-exo-dig/6-endo-dig product 238a, it was decided to evaluate and compare the benzyl protecting group strategy.

\subsection{Benzyl protecting group strategy}

The synthesis of the benzyl-protected terminal diyne proceeded in a similar sequence to that described for isopropyl protected 218a. Starting from commercially available phenol $\mathbf{2 3 2}$, nucleophilic substitution with benzyl bromide proceeded in excellent yields to give benzylprotected compound 223b, which then smoothly underwent the reductive Yamamoto homocoupling to give biaryl 222b (Scheme 75). Attempts to iodinate 222b using the procedure outlined in Scheme 69 only led to decomposition. An alternative procedure, adding a solution of iodine in chloroform to a suspension of biaryl 222b and silver trifluoroacetate in chloroform, ${ }^{[267]}$ gave access to diiodide $220 \mathrm{~b}$ in excellent yield (Scheme 75).

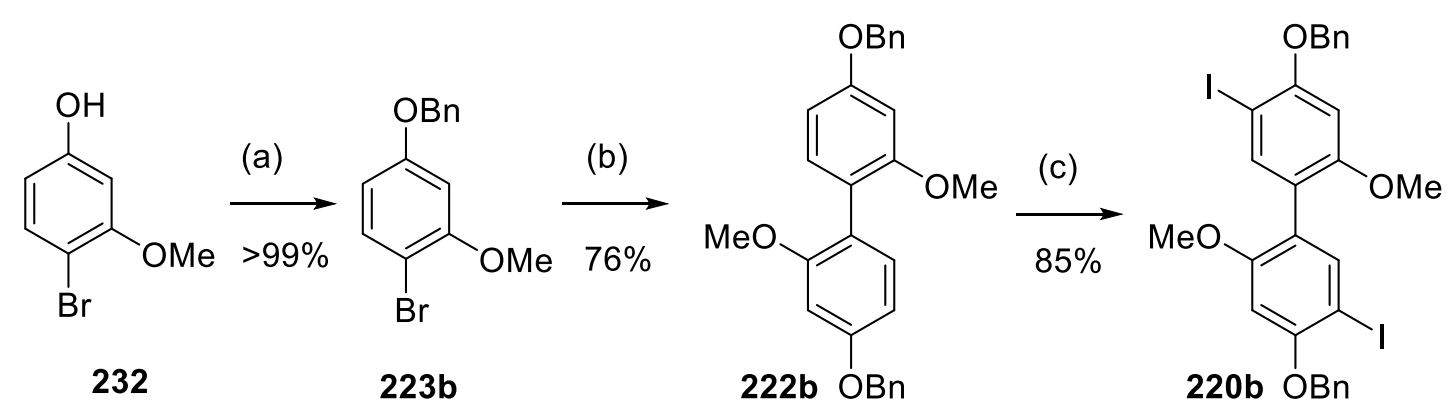

Scheme 75. Synthesis of diiodide 220b. Reagents and conditions: (a) 232 (1.0 equiv.), $\mathrm{BnBr}\left(1.0\right.$ equiv.), $\mathrm{K}_{2} \mathrm{CO}_{3}$ (3.0 equiv.), MeCN, rt, 16 h; (b) 223b (1.0 equiv.), $\mathrm{PPh}_{3}$ (0.75 equiv.), $\mathrm{NiCl}_{2}$ (5 mol\%), BiPy (5 mol\%), $\mathrm{Zn} \mathrm{(1.5}$ equiv.), DMAc, $90{ }^{\circ} \mathrm{C}, 16 \mathrm{~h}$; (c) $222 \mathrm{~b}$ (1.0 equiv.), $\mathrm{CF}_{3} \mathrm{CO}_{2} \mathrm{Ag}$ (2.4 equiv.), $\mathrm{I}_{2}$ (2.3 equiv.), $\mathrm{CHCl}_{3}, \mathrm{rt}, 3 \mathrm{~h}$. 
The synthesis of the protected aromatic aldehyde 221b was similarly straightforward. Because in the previous case the acetal formation did not proceed smoothly in the presence of the free phenol, this time the order of steps was reversed. Therefore, 2-bromo,5hydroxybenzaldehyde (233) was first protected as the benzyl ether, before making the acetal using ethylene glycol and catalytic para-toluenesulfonic acid applying a Dean-Stark apparatus (Scheme 76). Compound 221b was found to slowly decompose to the aldehyde $\mathbf{2 3 4 b}$ if not stored in the freezer at $-20^{\circ} \mathrm{C}$.

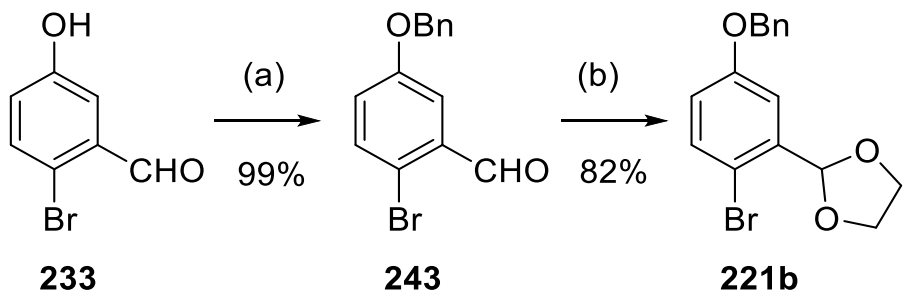

Scheme 76. Synthesis of 203b by benzyl protection followed by acetal formation. Reagents and conditions: (a) 233 (1.0 equiv.), $\mathrm{BnBr}$ (1.0 equiv.), $\mathrm{K}_{2} \mathrm{CO}_{3}$ (1.7 equiv.), $\mathrm{MeCN}, \mathrm{rt}, 16 \mathrm{~h}$; (b) 243 (1.0 equiv.), $p-\mathrm{TSA} \cdot \mathrm{H}_{2} \mathrm{O}$ (5 mol\%), ethylene glycol (1.3 equiv.), toluene, reflux.

Next, bromide 221b was converted into the organozinc derivative 235b via lithiation followed by transmetalation with zinc chloride. The Negishi coupling of 220b proceeded with good conversion. After work up, the crude product was directly mixed with tetrahydrofuran and $1 \mathrm{M}$ hydrochloric acid to cleave the acetal groups. In this case heating to $60{ }^{\circ} \mathrm{C}$ was necessary to give full conversion to the dialdehyde $\mathbf{2 3 6 b}$ (Scheme 77 ).<smiles>Brc1ccc(Br)c(C2OCCO2)c1</smiles>

221b (a)<smiles>Cl[Ge](Cl)c1ccc(OCc2ccccc2)cc1C1OCCO1</smiles>

235b (b), then (c)

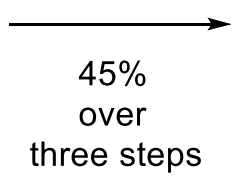

three steps<smiles>CCC(C)(C)Oc1cc(OC)c(-c2cc(-c3ccc(OCc4ccccc4)cc3C=O)c(OCc3ccccc3)cc2OC)cc1-c1ccc(OCc2ccccc2)cc1C=O</smiles>

Scheme 77. Synthesis of dialdehyde 236b by Negishi coupling followed by acetal deprotection. Reagents and conditions: (a) 221b (1.0 equiv.), $n \mathrm{BuLi}\left(1.0\right.$ equiv.), THF, $-78{ }^{\circ} \mathrm{C}, 2 \mathrm{~h}$, then $\mathrm{ZnCl}_{2}\left(2.5\right.$ equiv.), $-78{ }^{\circ} \mathrm{C}$ to rt, $1.5 \mathrm{~h}$, directly used in next step; (b) 235b (3.0 equiv.), 220b (1.0 equiv.), $\mathrm{Pd}_{2}(\mathrm{dba})_{3}$ (3 mol\%), SPhos (6 mol\%), THF, 70 ${ }^{\circ} \mathrm{C}, 18 \mathrm{~h}$; (c) THF, $\mathrm{HCl}(1 \mathrm{M}), 60^{\circ} \mathrm{C}, 2 \mathrm{~h}$.

Seyferth-Gilbert homologation following the Bestmann-Ohira modification then gave compound 219b (Scheme 78) in good yield, which set the stage for the key gold(I)-catalyzed cyclisation (Table 20). 
7. Towards the enantioselective total synthesis of Monbarbatain A<smiles>COc1cc(OC)c(-c2cc(-c3ccc(OCc4ccccc4)cc3C=O)c(OCc3ccccc3)cc2OC)cc1-c1ccc(OCc2ccccc2)cc1C=O</smiles>

236b<smiles>C#Cc1cc(OCc2ccccc2)ccc1-c1cc(-c2cc(OCc3ccccc3)c(OC)cc2OC)c(OC)cc1-c1cc(OCc2ccccc2)c(C#Cc2ccccc2)cc1OC</smiles>

Scheme 78. Seyferth-Gilbert homologation of $236 \mathrm{~b}$ to give diyne $219 \mathrm{~b}$. Reagents and conditions: (a) $236 \mathrm{~b}$ (1.0 equiv.), 185 (3.0 equiv.), $\mathrm{K}_{2} \mathrm{CO}_{3}$ (6.0 equiv.), $\mathrm{MeOH} / \mathrm{MeCN}$, rt, $16 \mathrm{~h}$.

Similarly, this reaction was first attempted with the non-chiral gold complex 98f. After stirring at room temperature for $16 \mathrm{~h}$ in dichloromethane, no signals which corresponded to product formation could be detected. The formation of a gold mirror on the inside of the reaction vessel also indicated decomposition of the catalyst. The chiral precatalysts $172 \mathrm{n}, 1720$ and 172t were then evaluated to gauge how the steric and electronic environment could influence conversion and selectivity. Unfortunately, in the best case $8 \%$ of product in comparison to starting material could be detected in the crude ${ }^{1} \mathrm{H}$ NMR. It seemed that the steric environment imposed by the benzyl protecting groups decreased the rate of reaction even more compared to the isopropyl group strategy. 
Table 20. Attempted gold(I)-catalyzed cyclisation of $\mathbf{2 1 9 b .}$<smiles>C#Cc1cc(OC)c(-c2cc(-c3ccc(OCc4ccccc4)cc3C#C)c(-c3cc(OCc4ccccc4)c(OC)cc3C#C)cc2OC)cc1OCc1ccccc1</smiles>

219b<smiles>C#Cc1cc(OCc2ccccc2)ccc1-c1cc(-c2c(OC)cc(OCc3ccccc3)c3c2ccc2cc(OCc4ccccc4)ccc23)c(OC)cc1OCc1ccccc1</smiles>

237b<smiles>COc1cc(COc2ccccc2)c2c(ccc3ccccc32)c1-c1c(OC)cc(OCc2ccccc2)c2c1ccc1cc(OCc3ccccc3)ccc12</smiles>

218b<smiles></smiles><smiles>Cn1cccc1C1(Cl)OP(Cl)(c2ccccc2)(c2ccccc2)OC(c2ccccc2)(c2ccccc2)C2OC(C)(C)OC21</smiles>

1720

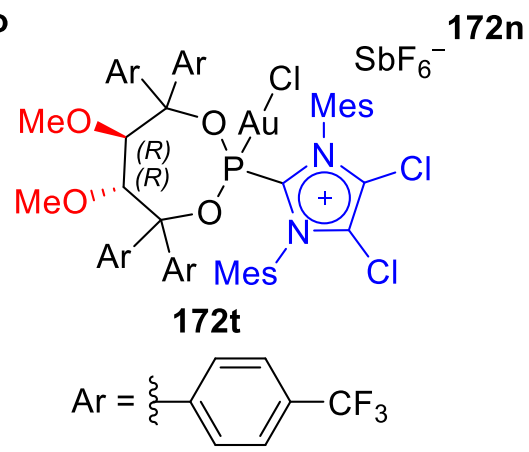<smiles>COc1cc(OCc2ccccc2)c2c(c1-c1c(OC)cc(OCc3ccccc3)c3c1ccc1cc(OCc4ccccc4)ccc13)C(=O)c1cc(CC(C)C)ccc1-2</smiles>

238b

\begin{tabular}{cccc}
\hline $\begin{array}{c}\text { Entr } \\
\mathrm{y}\end{array}$ & {$[\mathrm{Au}]$} & $\begin{array}{c}\mathrm{t} \\
(\mathrm{h})\end{array}$ & Comments \\
\hline $\mathbf{1}$ & $\mathbf{9 8 f}$ & 16 & No product detected. Partial decomposition of starting \\
material \\
$\mathbf{2}$ & $\mathbf{1 7 2 n}$ & 48 & $8 \%$ product detected \\
3 & $\mathbf{1 7 2 0}$ & 48 & $4 \%$ product detected, multiple side products \\
4 & $\mathbf{1 7 2 t}$ & 48 & No conversion \\
\hline
\end{tabular}

Reagents and conditions: 219b (1.0 equiv.), [Au] (5 mol\%), $\mathrm{AgSbF}_{6}(5 \mathrm{~mol} \%), \mathrm{CH}_{2} \mathrm{Cl}_{2}$, rt. After work up, reaction mixtures were analyzed by NMR.

It was clear at this stage that although the synthesis towards the starting diyne $219 \mathrm{~b}$ was straightforward, the key gold(I)-catalyzed hydroarylation required further optimization. According to the previous experience by the Alcarazo group, even highly sterically congested phenanthrenes could be synthesized using cationic phosphine complexes; therefore, the reasons for the low conversion in this reaction are not obvious. In efforts to further understand this transformation, it was decided to probe the effect of installing a methoxy 
group in place of the benzyl or isopropyl substituents at the phenanthrene core to see how reducing the steric bulk would influence conversion and the enantioselectivity of the reaction.

\subsection{Synthesis of dimethyl Monbarbatain A}

Starting from the cheap and commercially available 1-bromo-2,4-dimethoxybenzene (223c), the same synthetic sequence used above to access the diyne substrate 219 was applied (Scheme 79).<smiles>COc1ccc(Br)c(OC)c1</smiles>

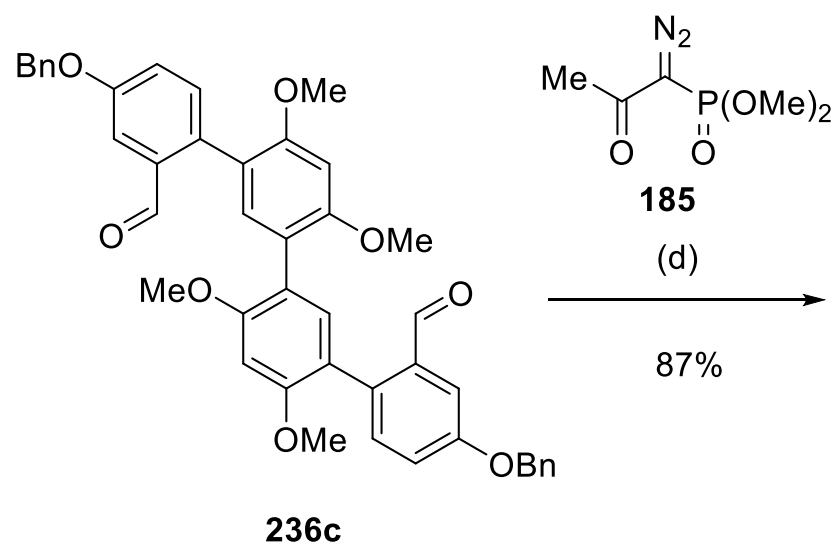<smiles>C#Cc1cc(OCc2ccccc2)ccc1-c1cc(-c2cc(-c3ccc(OCc4ccccc4)cc3C#C)c(OC)cc2OC)c(OC)cc1OC</smiles>

Scheme 79. Synthesis of diyne 219c starting from bromide 223c. Reagents and conditions: (a) 223c (1.0 equiv.), $\mathrm{PPh}_{3}\left(0.75\right.$ equiv.), $\mathrm{NiCl}_{2}$ (5 mol\%), BiPy (5 mol\%), $\mathrm{Zn}$ (1.5 equiv.), DMAc, $90{ }^{\circ} \mathrm{C}, 16 \mathrm{~h}$; (b) $222 \mathrm{c}\left(1.0\right.$ equiv.), $\mathrm{I}_{2}$ (1.20 equiv.), $\mathrm{HCl}$ (0.3 equiv, $37 \%$ aqueous), $\mathrm{H}_{2} \mathrm{O}_{2}$ (1.2 equiv.), $\mathrm{MeCN}, \mathrm{rt}, 16 \mathrm{~h}$; (c) $235 \mathrm{bc}$ (3.0 equiv.), 220c (1.0 equiv.), $\mathrm{Pd}_{2}(\mathrm{dba})_{3}(3 \mathrm{~mol} \%)$, SPhos $(6 \mathrm{~mol} \%), \mathrm{THF}, 70^{\circ} \mathrm{C}, 18 \mathrm{~h}$; then THF, $\mathrm{HCl}(1 \mathrm{M}), \mathrm{rt}, 2 \mathrm{~h}$; (d) $236 \mathrm{c}(1.0$ equiv.), 185 (3.0 equiv.), $\mathrm{K}_{2} \mathrm{CO}_{3}$ (6.0 equiv.), $\mathrm{MeOH} / \mathrm{MeCN}$, rt, $16 \mathrm{~h}$.

Homocoupling followed by iodination gave the diiodo $220 \mathrm{c}$ in $77 \%$ overall yield. The following Negishi coupling was then carried out using freshly prepared benzyl-protected organozinc compound $\mathbf{2 3 5 b}$, following the deprotection of formyl groups described above using a mixture of $1 \mathrm{M} \mathrm{HCl}$ and THF, providing the dialdehyde 236c in 76\% yield. Finally, SeyferthGilbert homologation provided the diyne $219 \mathrm{c}$ in very good yield. With the diyne 219c in hand, the gold(I) catalyzed cyclization was attempted using a variety of cationic ligands (Table 21). Gratifyingly, the substrate modification with sterically less demanding methoxy group led to much improved reactivity, and in most cases full conversion was seen after 
stirring for $144 \mathrm{~h}$ at $20^{\circ} \mathrm{C}$. Moreover, the 5-exo-dig/6-endo-dig isomer 238c could not be observed in the crude reaction mixtures. Additionally, the identity of the intermediate 237c, present in some reaction mixtures, could also be confirmed by full characterization after preparative HPLC separation. From these results, it is apparent that steric bulk in the parapositions of the biaryl core in diyne $\mathbf{2 1 9}$ had a large bearing on the outcome of the reaction.

Of the cationic ligands tested, the highest enantiomeric excesses were obtained for the gold complexes 172d (+47\%) and 172b (+34\%), bearing 2-naphthyl and 4-biphenyl substituents at the TADDOL backbone, respectively. In addition, the 2-naphthyl-substituted phosphoramidites $(R, R, S, S)$ - and $(S, S, S, S)-50 b$ were evaluated. Curiously, the reactions were finished much faster in these cases, although $8 \%$ of a by-product, which could possibly be the 5-exo-dig/6-endo-dig isomer, was detected in the reaction using (S,S,S,S)-50b, and the enantioselectivities in both cases were diminished. However, no catalyst gave particularly high levels of enantioselectivity, and this may indicate that a different ligand family may be beneficial in this type of transformation. Interestingly, steric effects in the catalyst appear to override any electronic impact, with the phosphoramidites giving complete conversion in appreciably shorter reaction times than the other cationic phosphonites. It may therefore be interesting to screen additional phosphoramidites in the future. Moreover, it is also clear that the choice of group in the para-position of the biaryl subunit in $\mathbf{2 1 9}$ is crucial to ensuring high conversion, with an apparent steric clash occurring between this group and the bay area of the formed phenanthrene ring in $\mathbf{2 1 8}$. 
7. Towards the enantioselective total synthesis of Monbarbatain A

Table 21. Gold(I)-catalyzed cyclisation of diyne 219c using cationic phosphonite-based gold complexes.<smiles>C#Cc1cc(OCc2ccccc2)ccc1-c1cc(OC)c(-c2cc(-c3ccc(OCc4ccccc4)cc3C#C)c(OC)cc2OC)cc1OC</smiles>

219c<smiles>C#Cc1cc(Cc2ccccc2)ccc1-c1cc(-c2c(OC)cc(OC)c3c2ccc2cc(OCc4ccccc4)ccc23)c(OC)cc1OC</smiles>

237c<smiles>COc1cc(OC)c2c(ccc3cc(OCc4ccccc4)ccc32)c1-c1c(OC)cc(OC)c2c1ccc1cc(OCc3ccccc3)ccc12</smiles>

$218 c$

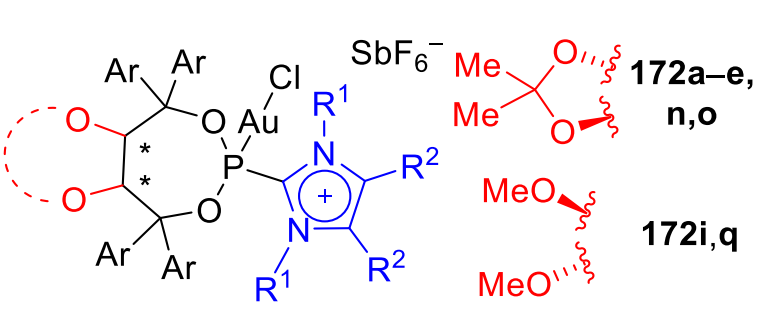

$\mathrm{R}$

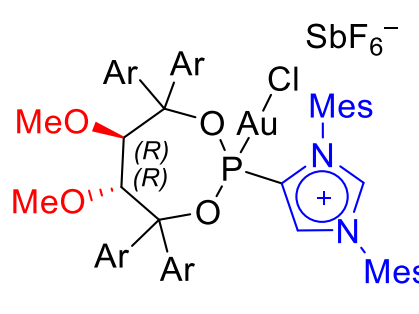

209b

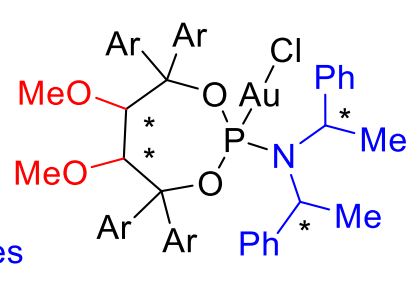

$\mathrm{Ar}=$ 2-naphthyl

50b: $(R, R, S, S)$

(S,S,S,S)

\begin{tabular}{|c|c|c|c|c|c|c|c|c|}
\hline Entry & {$[\mathrm{Au}]$} & $\mathrm{Ar}$ & $\mathrm{R}^{1}$ & $\mathrm{R}^{2}$ & $t(h)$ & $\begin{array}{c}\text { Conversion } \\
(\%)^{\mathrm{a}}\end{array}$ & 218c: $237 c^{b}$ & $e e(\%)^{c}$ \\
\hline 1 & - & - & - & - & 44 & 0 & - & - \\
\hline 2 & $172 n$ & $\mathrm{Ph}$ & Mes & $\mathrm{H}$ & 20 & $>95$ & $63: 8^{d}$ & +7 \\
\hline 3 & $172 d$ & 2-naphthyl & Mes & $\mathrm{H}$ & 75 & $>95$ & $96: 4$ & +47 \\
\hline 4 & $172 a$ & 4-tBu- $\mathrm{C}_{6} \mathrm{H}_{4}-$ & Mes & $\mathrm{H}$ & 24 & $>95$ & $99: 1$ & 0 \\
\hline 5 & $172 e$ & $3,5-\left(\mathrm{CF}_{3}\right)_{2}-\mathrm{C}_{6} \mathrm{H}_{3}-$ & Mes & $\mathrm{H}$ & 144 & 0 & - & - \\
\hline 6 & $172 b$ & 4-Ph- $\mathrm{C}_{6} \mathrm{H}_{4-}$ & Mes & $\mathrm{H}$ & 144 & $>95$ & $95: 5$ & +36 \\
\hline 7 & $172 c$ & $4-\mathrm{CF}_{3}-\mathrm{C}_{6} \mathrm{H}_{4-}$ & Mes & $\mathrm{H}$ & 144 & $>95$ & $54: 46$ & +3 \\
\hline 8 & 1720 & $\mathrm{Ph}$ & $\operatorname{Pr}$ & $\mathrm{Me}$ & 40 & $>95$ & 100:0 & +4 \\
\hline 9 & $172 q$ & $\mathrm{Ph}$ & Mes & $\mathrm{H}$ & 144 & $>95$ & $58: 42$ & +23 \\
\hline 10 & $172 i$ & $4-\mathrm{CF}_{3}-\mathrm{C}_{6} \mathrm{H}_{4-}^{-}$ & Mes & $\mathrm{H}$ & 144 & $>95$ & $56^{d}$ & 0 \\
\hline 11 & $209 b$ & $3,5-\left(\mathrm{CF}_{3}\right)_{2}-\mathrm{C}_{6} \mathrm{H}_{3}-$ & - & - & 144 & $>95$ & $93: 7$ & 12 \\
\hline 12 & & $(R, R, S, S)-50 \mathrm{~b}$ & & & 42 & $>95$ & $>99: 1$ & -16 \\
\hline 13 & & $(S, S, S, S)-\mathbf{5 0 b}$ & & & 23 & $>95$ & $92^{\mathrm{e}}$ & +23 \\
\hline
\end{tabular}

Reagents and conditions: 219c (1.0 equiv.), [Au] (5 mol\%), $\mathrm{AgSbF}_{6}(5 \mathrm{~mol} \%), \mathrm{CH}_{2} \mathrm{Cl}_{2}, 20{ }^{\circ} \mathrm{C}$, t. a,beactions were quenched then analyzed by NMR and HPLC to determine selectivity and conversion. ${ }^{c} e e$ determined by HPLC. ${ }^{d}$ Multiple other side products observed. ${ }^{\text {e }} \%$ of a single other isomer observed. 


\subsection{Summary}

To conclude, the total synthesis of the axially chiral, highly oxygenated biphenanthrene Monbarbatain A was attempted utilizing the newly developed chiral cationic phosphonites in a key gold (I)-catalyzed double hydroarylation reaction. Towards this goal, a versatile synthesis of a suitable diyne precursor was achieved. Two protecting group strategies, using either isopropyl or benzyl ethers, were then investigated. For both strategies, poor conversion of the gold(I) catalyzed key step limited the amount to which the final deprotection conditions could be evaluated.

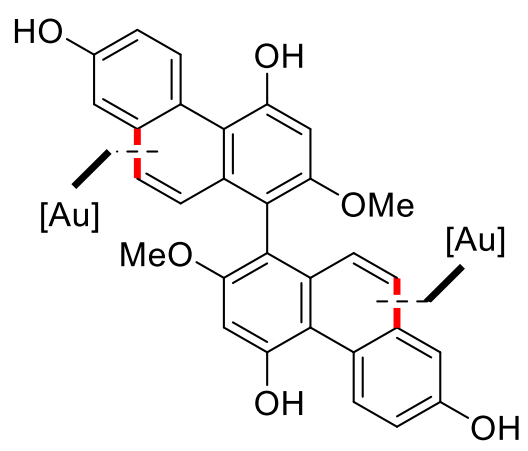

Monbarbatain A

In an effort to improve conversion, reduction of steric bulk by introducing a methoxy group in the para positions of the central biaryl unit led to much increased conversions, and the family of cationic phosphonites was evaluated in this reaction towards the dimethoxy derivative of Monbarbatain A, resulting in a best ee of $+47 \%$ for catalyst $172 \mathrm{~d}$. Interestingly, phoshoramidites tested in this transformation resulted in faster reaction times, indicating that the steric bulk of the catalyst overrides any electronic influence. Continued efforts in this area may focus on evaluating other phosphoramidites, or ligands with a reduced steric bulk. 


\section{Experimental}

\section{Experimental}

\subsection{General remarks}

\subsubsection{General working methods}

Unless otherwise stated, all reactions were carried in dried glassware under an atmosphere of argon using standard Schlenk techniques, or under an atmosphere of nitrogen in a MBraun UNIlab plus glovebox. Dry and degassed solvents were obtained by distillation over the appropriate drying agents and stored under argon. Tetrahydrofuran, diethyl ether (Mg/anthracene), toluene, pentane $(\mathrm{Na} / \mathrm{K})$, dichloromethane, fluorobenzene, pyridine, diisopropylamine, $\left(\mathrm{CaH}_{2}\right.$, then stored over $4 \AA$ molecular sieves), acetonitrile $\left(\mathrm{CaH}_{2}\right.$, then stored over $3 \AA$ molecular sieves), methanol (Mg, then stored over $3 \AA$ molecular sieves). ${ }^{[268]}$ Alternatively, dry solvents were obtained using an MBraun MB-SPS-800 solvent purification system (tetrahydrofuran, diethyl ether, toluene, pentane, dichloromethane, acetonitrile). Other solvents were dried by pouring the wet solvent into a dried Schlenk flask with activated molecular sieves, followed by degassing under flow of argon for 30 minutes $(N, N-$ dimethylformamide, $\mathrm{N}, \mathrm{N}$-dimethylacetamide, chlorobenzene, chloroform: $4 \AA$ molecular sieves). The water content of solvents was determined using a Karl Fischer titrator TitroLine R 7500 KF trace from SI Analytics. Flash chromatography was performed either on Merck 60 $(40-63 \mu \mathrm{m})$ or Macherey Nagel $60(40-63 \mu \mathrm{m})$ silica gel. Reactions were controlled by thin layer chromatography (TLC) analysis, performed using Merck silica gel 60 F254 TLC plates or polygram SIL G/UV254 from Macherey Nagel, and visualized by UV irradiation $(\lambda=254$ $\mathrm{nm}$ ) and/or phosphomolybdic acid or $\mathrm{KMnO}_{4}$ dip. When it was possible, the reactions were additionally followed by GC/MS measurements performed on either an Agilent Technology GC 6890 Series with MSD 5973 (carrier gas: helium), employing an MN Optima5 column $(30 \mathrm{~mm} \times 0.25 \mathrm{~mm}, 0.25 \mu \mathrm{m}$ particle size); or an Agilent Technology $7820 \mathrm{~A}$, with a $5977 \mathrm{E}$ MSD, employing a HP-5MS column $(30 \mathrm{~mm} \times 0.25 \mathrm{~mm}, 0.25 \mu \mathrm{m}$ particle size $)$.

\subsubsection{Starting materials}

Unless otherwise stated, all reagents were used as received from commercial suppliers (ABCR, Acros Organics, Alfa Aesar, Chempur GmbH, J and K Scientific, Sigma Aldrich, Thermo Fisher Scientific, Tokyo Chemical Industry). Molecular sieves were dried under high vacuum at $150{ }^{\circ} \mathrm{C}$ for two days before storing under argon and additionally dried at $650{ }^{\circ} \mathrm{C}$ under vacuum prior to use. $\mathrm{PCl}_{3}$ was distilled prior to use and stored under argon. Sodium hexafluoroantimonate was dried under high vacuum at $150{ }^{\circ} \mathrm{C}$ for $16 \mathrm{~h}$ and stored in a Schlenk flask under argon or a glovebox under nitrogen. Silver hexafluoroantimonate was 


\section{Experimental}

purchased from Sigma Aldrich, transferred into a glovebox and finely ground using a pestle and mortar. $\mathrm{N}$-bromosuccinimide was crystallized from boiling water, ground using a pestle and mortar and dried under high vacuum. Dimethyl (1-diazo-2-oxopropyl)phosphonate (185, the Bestmann-Ohira reagent), ${ }^{[269]}$ 1-(benzyloxy)-4-iodobenzene, ${ }^{[270]}$ 1-iodo-4(trimethylsilyl)benzene, ${ }^{[271]}$ were prepared according to literature procedures. Helicene precursors 186a, 186b, 186c, 181d, 159bb and 159be were prepared according to previously described procedures. ${ }^{[219,272]}$ Helicenes $160 \mathrm{be}$, bi and bl analysed by CD spectroscopy were prepared by L Schaaf MSc. ${ }^{[272]}$ The preparation of precatalysts $98 \mathrm{a}, \mathrm{e}-\mathrm{I}$ can be found in the literature. ${ }^{[86,87]}$ TADDOL derivatives $170 \mathbf{a},{ }^{[233]} \mathbf{1 7 0 b},{ }^{\left[{ }^{[273]}\right.} \mathbf{1 7 0} \mathbf{g},{ }^{[52]} \mathbf{1 7 0 i},{ }^{[272]}$ 170I, ${ }^{[233]} \mathbf{1 7 0 q},{ }^{[52]} \mathbf{1 7 0 t},{ }^{[234]}$ were prepared according to previously described procedures. Compound 201b was prepared according to a literature procedure. ${ }^{[52]}$ BINOL derivative 163 was prepared by Dr. Isaac Alonso according to a literature procedure. ${ }^{[242]}$ Carbenes 56a, $56 b,{ }^{[274]} 56 d,{ }^{[238]} 56 e^{[239]} 56 f^{[240]}$ and carbodiphosphorane ${ }^{202^{[236]}}$ were prepared according to literature procedures.

\subsubsection{General analytical methods}

NMR: spectra were recorded on a Bruker AV600, AV500, AV400, DPX300, Varian Mercury 300 or Varian Inova 500 spectrometers as stated for each case; ${ }^{1} \mathrm{H}$ and ${ }^{13} \mathrm{C}$ chemical shifts (ठ) are given in ppm relative to TMS, using the solvent signals as references and converting the chemical shifts to the TMS scale. ${ }^{31} \mathrm{P}$ and ${ }^{19} \mathrm{~F}$ chemical shifts $(\delta)$ are given in ppm relative to $\mathrm{H}_{3} \mathrm{PO}_{4}$ and $\mathrm{CFCl}_{3}$ respectively (external standard). Coupling constants $(\mathrm{J})$ are given in $\mathrm{Hz}$. Deuterated solvents $\left(\mathrm{CDCl}_{3}, \mathrm{C}_{6} \mathrm{D}_{6}, \mathrm{CD}_{3} \mathrm{CN}\right)$ were dried and distilled over $\mathrm{CaH}_{2}$ under argon and stored in Schlenk flasks over molecular sieves (4 $\AA$ : $\left.C^{2} C_{3}, C_{6} D_{6} ; 3 \AA: C D_{3} C N\right)$ or inside a glovebox.

HRMS: mass spectrometry analysis was performed either by the department for mass spectrometry at the Max-Planck-Institut für Kohlenforschung using the following equipment: Finnigan MAT 8200 (70 eV, EI), Finnigan MAT 95 (ESI) and Bruker APEX III FT-MS (7 T magnet, HRMS); or by the department for mass spectrometry at the Insitut für Organische und Biomolekulare Chemie, Georg-August-Universität Göttingen, Göttingen using the following equipment: Finnigan MAT 95 (70 eV, EI), Finnigan LCQ (ESI) and APEX IV 7T FTICR, Bruker Daltonic (HRMS).

IR: spectra were recorded on either Nicolet FT-7199, FT/IR-4100 (Jasco), or Bruker ALPHA FT-IR Platinum ATR spectrometers, wavenumbers $(\tilde{V})$ in $\mathrm{cm}^{-1}$.

HPLC: the relative ratios of products in catalysis reactions was determined by analytical HPLC using either a Shimadzu prominence LC-20A system with integrated downstream 
UV/Vis PDA detector; Shimadzu Nexera-i LC 2040 3D with integrated downstream UV/Vis PDA detector or a Shimadzu LC-2010-CHT with integrated downstream UV/Vis detector. Specific conditions, such as columns, eluent mixtures, flow rates and temperatures are stated for each case. System control and chromatogram analysis were carried out with LabSolutions software.

Chiral HPLC: the enantiomeric excesses of the products in catalysis reactions was determined using either a Shimadzu prominence LC-20A system with integrated downstream UV/Vis PDA detector; Shimadzu Nexera-i LC 2040 3D with integrated downstream UV/Vis PDA detector or Shimadzu LC-2010-CHT with integrated downstream UV/Vis detector. Specific conditions, such as columns, eluent mixtures, flow rates and temperatures are stated for each compound. System control and chromatogram analysis were carried out with LabSolutions software. When indicated, the enantiomeric excess was determined by the department for chromatography at the Max-Planck-Institut für Kohlenforschung by 2D HPLC using an Agilent 2D 1290 Infinity system. In the first dimension an achiral separation of the mixture of products was performed using the appropriate achiral column and elution conditions. The relevant peak was then transferred with a heart cut to a second dimension equipped with the appropriate chiral column. Specific elution conditions are stated for each case. Detection of both separations was performed via a diode array detector at the given wavelengths. System control and chromatogram analysis were carried out with the Agilent OpenLAB ChemStation software.

Semi-preparative HPLC: to obtain analytically pure samples, some compounds were separated by semi-preparative HPLC using a Jasco modular system (pump: PU-2087Plus, degasser: DG-2080-54, detector: UV-2077Plus, autosampler: AS-2055Plus, hardware interface: LC-NetII/ADC) connected to an Advantec fraction collector CHF12SC. System control and analysis was performed with Galaxie 1.10 chromotography software. Compound 237c was separated by the department for chromatography at the Max-Planck-institut für Kohlenforschung using a Shimadzu preparative LC-8A system connected to a Shimadzu FRC-10A fraction collector. Columns used for separation as well as specific conditions are stated for each case.

Circular dichroism: spectra were conducted on a Jasco $\mathrm{J}-1500$ spectrometer in tetrahydrofuran using a $1 \mathrm{~mm}$ quartz sample cell.

Specific rotations: were collected using Perkin Elmer 343 or Jasco P-2000 polarimeters at the stated temperature under a $\mathrm{Na} / \mathrm{Hg}$ lamp, $\lambda=589 \mathrm{~nm}(c$ in $\mathrm{g} / 100 \mathrm{ml})$. 


\section{Experimental}

Cyclic voltammetry: Cyclic voltammetry was conducted using a standard three electrode setup, consisting of a glassy carbon working electrode, $\mathrm{Ag} / \mathrm{AgNO}_{3}$ pseudo-reference electrode and a Pt counter electrode in combination with a VersaSTAT 4 potentiostat. $\mathrm{Bu}_{4} \mathrm{NPF}_{6}\left(0.2 \mathrm{M}\right.$ in $\mathrm{MeCN}$ or $0.1 \mathrm{M}$ in $\left.\mathrm{CH}_{2} \mathrm{Cl}_{2}\right)$ was used as the electrolyte. The voltammograms were measured in dry degassed $\mathrm{MeCN}$ or $\mathrm{CH}_{2} \mathrm{Cl}_{2}$ and referenced to the $\mathrm{Fc} / \mathrm{Fc}+$ couple via addition of ferrocene directly after the sample measurement. Square wave voltammetry was measured using the same experimental setup as for the cyclic voltammetry experiments. System control and analysis was performed using VersaStudio software from Princeton Applied Research.

UV/Vis: spectra were obtained using a Jasco V-630 spectrometer using a $1 \mathrm{~cm}$ quartz sample cell.

X-ray-diffraction: Single crystal structure determination for compounds was either performed by the department of chemical crystallography at the Max-Planck-Institut für Kohlenforschung or at The University of Göttingen. Conditions at the Max-Planck-Institut für Kohlenforschung: The X-ray intensity data were measured on a Bruker AXS Proteum X8, Bruker AXS KappaCCD and Bruker AXS Apex II diffractometers. The crystal structures were solved by direct methods using SHELXS-97 and refined with SHELXL-2014. Conditions at the University of Göttingen: structures were solved by Dr. Christopher Golz. Data collection was done on a Bruker D8 Venture four-circle-diffractometer from Bruker AXS GmbH; detector: Photon II from Bruker AXS $\mathrm{GmbH}$; X-ray sources: microfocus I $\mu S \mathrm{Cu} / \mathrm{Mo}$ from Incoatec $\mathrm{GmbH}$ with mirror optics HELIOS and single-hole collimator from Bruker AXS $\mathrm{GmbH}$. Used programs: APEX3 Suite (v2017.3-0) and therein integrated programs SAINT (Integration) und SADABS (Absorption correction) from Bruker AXS GmbH; structure solution was done with SHELXT, refinement with SHELXS; ${ }^{[275,275]}$ OLEX $^{2}$ was used for data finalization. ${ }^{[276]}$ Special Utilities: SMZ1270 stereomicroscope from Nikon Metrology GmbH was used for sample preparation; crystals were mounted on MicroMounts or MicroLoops from MiTeGen; for sensitive samples the X-TEMP 2 System was used for picking of crystals; ${ }^{[277]}$ crystals were cooled to given temperature with Cryostream 800 from Oxford

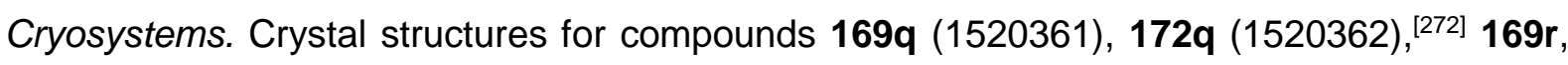
172s, 172t, 188b, 160ob ${ }^{[245]}$ can be found with their corresponding CCDC crystallographic database records.

Theoretical methods: Optimsied geometries were calculated by Dr. Christopher Golz using Gaussian 09 revision $E^{[221]}$ and GaussView (company: Semichem Inc. 2009). Starting geometries were based upon previously published coordinates for [6]carbohelicene. ${ }^{[103]}$ These were then optimized according to the B3LYP/6-31+G(d) level of theory. The optimized 
starting geometries were confirmed as local minima by calculating the molecular vibration frequencies, which gave no negative frequencies. Transition states were optimized using the qst2 methods, again starting from the previously published coordinates. From each transition state, one single imaginary frequency was obtained. The energies given are corrected by their zero point energy value. For the log files, see digital appendix.

\subsection{Synthesis of new compounds}

\subsubsection{Synthesis of helicene precursors}

\section{General procedure A (GPA)}

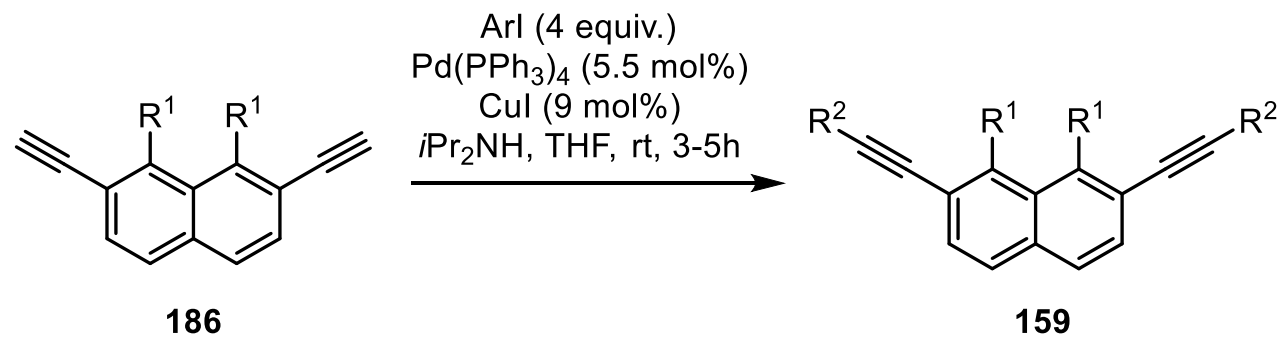

Based on the Sonogashira-Hagihara procedure reported by Starý for aryl-tethered triynes. ${ }^{[200]}$ A dry Schlenk flask equipped with a magnetic stirring bar and septum was charged with diyne $186^{[219]}$ (1.0 equiv.), the corresponding aromatic iodide (4.0 equiv.), $\mathrm{Pd}\left(\mathrm{PPh}_{3}\right)_{4}$ (5.5 mol\%), Cul (9 mol\%) and a mixture of $\operatorname{Pr}_{2} \mathrm{NH} / \mathrm{THF}(2: 1,0.047 \mathrm{M})$. The reaction mixture was degassed for 15 minutes under nitrogen flow and then stirred at room temperature for $3-5 \mathrm{~h}$. The solvents were removed in vacuo and the residue directly purified by column chromatography.

\section{2,7-Bis[(4-fluorophenyl)ethynyl]-1,8-diphenylnaphthalene (159af):}

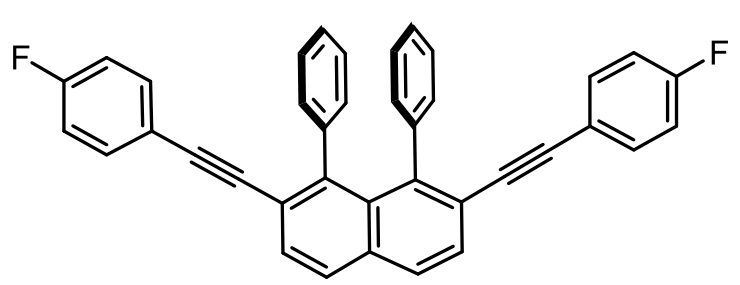

Prepared as a white solid $(60.0 \mathrm{mg}, 116 \mu \mathrm{mol}$, $76 \%$ yield) from alkyne $186 a$ ( 1.0 equiv, 50.0 $\mathrm{mg}, 152 \mu \mathrm{mol})$, 1-fluoro-4-iodobenzene (4.0 equiv, $135 \mathrm{mg}, 608 \mu \mathrm{mol}), \quad \mathrm{Pd}\left(\mathrm{PPh}_{3}\right)_{4} \quad(5.5$ mol\%, $9.7 \mathrm{mg}, 8.3 \mu \mathrm{mol})$, Cul $(8.9 \mathrm{~mol} \%, 2.6$

$\mathrm{mg}, 13.6 \mu \mathrm{mol}), i \mathrm{Pr}_{2} \mathrm{NH}(2.2 \mathrm{ml})$ and THF $(1.1 \mathrm{ml})$ according to GPA (stirring at room temperature for $3 \mathrm{~h}$, column chromatography: $20 \%$ toluene in petrol ether).

'H NMR: $\left(400 \mathrm{MHz}, \mathrm{CDCl}_{3}\right) \delta=7.87(\mathrm{~d}, J=8.4 \mathrm{~Hz}, 2 \mathrm{H}), 7.66(\mathrm{~d}, J=8.4 \mathrm{~Hz}, 2 \mathrm{H}), 7.06-6.81$ $(\mathrm{m}, 18 \mathrm{H}) ;{ }^{13} \mathrm{C}\left\{{ }^{1} \mathrm{H}\right\}$ NMR: $\left(101 \mathrm{MHz}, \mathrm{CDCl}_{3}\right) \delta=162.4\left(\mathrm{~d}, J_{C-F}=249.4 \mathrm{~Hz}\right), 144.0,141.3$, 133.9, $133.4\left(\mathrm{~d}, J_{C-F}=8.3 \mathrm{~Hz}\right), 131.3,130.8,128.8,128.4,127.0,126.2,124.0,119.5$ (d, $J_{C-F}$ $=3.5 \mathrm{~Hz}), 115.5\left(\mathrm{~d}, J_{C-F}=22.0 \mathrm{~Hz}\right), 93.5,89.8\left(\mathrm{~d}, J_{C-F}=1.5 \mathrm{~Hz}\right) ;{ }^{19} \mathrm{~F}$ NMR: $\left(282 \mathrm{MHz}, \mathrm{CDCl}_{3}\right)$ 


\section{Experimental}

$\delta=-109.9$ (tt, $J=8.6,5.5 \mathrm{~Hz}$ ); IR: (neat, $\mathrm{cm}^{-1}$ ) $\tilde{V}: 523,616,690,746,760,804,832,1154$, 1219, 1358, 1444, 1504, 1597, 3020, 3054; HRMS: calculated $m / z$ for $\mathrm{C}_{38} \mathrm{H}_{23} \mathrm{~F}_{2}{ }^{+}[\mathrm{M}+\mathrm{H}]^{+}$: 517.1762; found (ESI) 517.1767.

\section{2,7-Bis[(4-methoxyphenyl)ethynyl]-1,8-diphenylnaphthalene (159ag):}

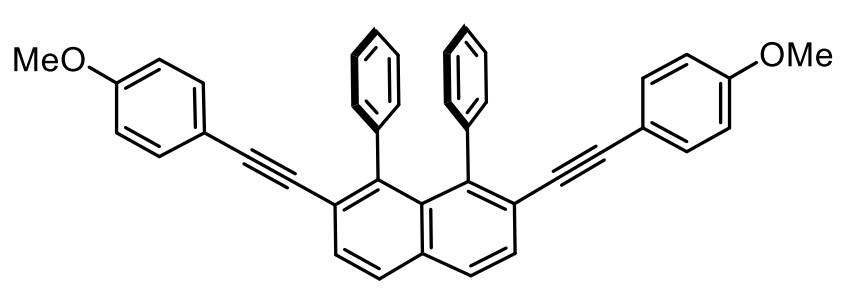

Prepared as a white solid $(84.0 \mathrm{mg}$, $155 \mu \mathrm{mol}, 85 \%$ yield) from alkyne 186a (1.0 equiv, $60.0 \mathrm{mg}, 182 \mu \mathrm{mol})$, 4iodoanisole (4.0 equiv, $171 \mathrm{mg}, 731$ $\mu \mathrm{mol}), \mathrm{Pd}\left(\mathrm{PPh}_{3}\right)_{4}(5.5 \mathrm{~mol} \%, 11.6 \mathrm{mg}$, $10.0 \mu \mathrm{mol})$, Cul (8.9 mol\%, $3.1 \mathrm{mg}, 16.2 \mu \mathrm{mol}), \operatorname{PPr}_{2} \mathrm{NH}(2.2 \mathrm{ml})$ and THF $(1.1 \mathrm{ml})$ according to GPA (stirring at room temperature for $3 \mathrm{~h}$, column chromatography: $50 \%$ toluene in petrol ether).

'H NMR: $\left(400 \mathrm{MHz}, \mathrm{CDCl}_{3}\right) \delta=7.84(\mathrm{~d}, J=8.5 \mathrm{~Hz}, 2 \mathrm{H}), 7.65(\mathrm{~d}, J=8.6 \mathrm{~Hz}, 2 \mathrm{H}), 7.06-6.87$ $(\mathrm{m}, 14 \mathrm{H}), 6.72(\mathrm{dd}, J=8.9,4.5 \mathrm{~Hz}, 4 \mathrm{H}), 3.75(\mathrm{~s}, 6 \mathrm{H}) \mathrm{ppm} ;{ }^{13} \mathrm{C}\left\{{ }^{1} \mathrm{H}\right\}$ NMR: $\left(101 \mathrm{MHz}, \mathrm{CDCl}_{3}\right) \delta$ $=159.5,143.4,141.5,133.6,133.0,131.4,130.9,128.7,128.2,127.0,126.1,124.4,115.6$, 113.9, 94.5, 89.0, 55.5 ppm; IR: (neat, $\left.\mathrm{cm}^{-1}\right) \tilde{v}=508,538,692,745,830,1027,1174,1245$, 1284, 1435, 1510, 1602, 1680, 2210, 2849, 2925, 3049; HRMS: calcd m/z for $\mathrm{C}_{40} \mathrm{H}_{29} \mathrm{O}_{2}{ }^{+}$ $[\mathrm{M}+\mathrm{H}]^{+}:$541.2162; found (ESI) 541.2149.

\section{2,7-Bis\{[4-(benzyloxy)phenyl]ethynyl\}-1,8-diphenylnaphthalene (159ah):}

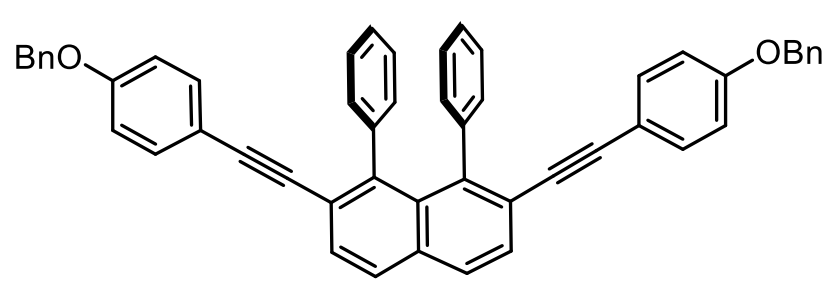

Prepared as a white solid $(77.0 \mathrm{mg}$, $111 \mu \mathrm{mol}, 73 \%$ yield) from alkyne $186 \mathrm{a}$ (1.0 equiv, $50.0 \mathrm{mg}, 152 \mu \mathrm{mol}$ ), 1 (benzyloxy)-4-iodobenzene (4.0 equiv, $189 \mathrm{mg}, 609 \mu \mathrm{mol}), \mathrm{Pd}\left(\mathrm{PPh}_{3}\right)_{4}(5.5 \mathrm{~mol} \%$,

$9.7 \mathrm{mg}, 8.4 \mu \mathrm{mol})$, Cul $(9.0 \mathrm{~mol} \%, 2.6 \mathrm{mg}, 13.7 \mu \mathrm{mol}), \operatorname{Pr}_{2} \mathrm{NH}(2.2 \mathrm{ml})$ and $\operatorname{THF}(1.1 \mathrm{ml})$ according to GPA (stirring at room temperature for $3 \mathrm{~h}$, column chromatography: $60 \%$ toluene in petrol ether).

'H NMR: $\left(400 \mathrm{MHz}, \mathrm{CDCl}_{3}\right) \delta=7.84(\mathrm{~d}, J=8.4 \mathrm{~Hz}, 2 \mathrm{H}), 7.65(\mathrm{~d}, J=8.4 \mathrm{~Hz}, 2 \mathrm{H}), 7.49-7.28$ $(\mathrm{m}, 10 \mathrm{H}), 7.07-6.88(\mathrm{~m}, 14 \mathrm{H}), 6.79(\mathrm{~d}, \mathrm{~J}=8.8 \mathrm{~Hz}, 4 \mathrm{H}), 5.01(\mathrm{~s}, 4 \mathrm{H}) \mathrm{ppm} ;{ }^{13} \mathrm{C}\left\{{ }^{1} \mathrm{H}\right\}$ NMR: $(101$ $\left.\mathrm{MHz} \mathrm{CDCl}_{3}\right) \delta=158.7,143.5,141.5,136.8,133.6,133.0,131.4,130.9,128.7,128.7,128.2$, 128.2, 127.5, 127.0, 126.1, 124.4, 115.9, 114.8, 94.5, 89.1, 70.1 ppm; IR: (neat, $\left.\mathrm{cm}^{-1}\right) \tilde{v}=$ 508, 538, 692, 745, 830, 1027, 1174, 1245, 1284, 1435, 1510, 1602, 1680, 2210, 2849, 2925, 3049; HRMS: calculated $\mathrm{m} / z$ for $\mathrm{C}_{52} \mathrm{H}_{36} \mathrm{NaO}_{2}{ }^{+}[\mathrm{M}+\mathrm{Na}]^{+}:$715.2608; found (ESI) 715.2594 . 


\section{Experimental}

\section{2,7-Bis\{[4-(trimethylsilyl)phenyl]ethynyl\}-1,8-diphenylnaphthalene (159ai):}

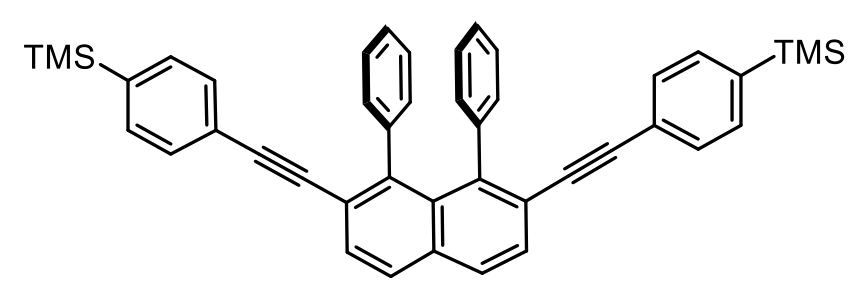

Prepared as a white solid $(50.0 \mathrm{mg}, 80$ $\mu \mathrm{mol}, 53 \%$ yield) from alkyne $186 \mathrm{a}(1.0$ equiv, $\quad 50.0 \mathrm{mg}, \quad 152 \mu \mathrm{mol}), \quad$ (4iodophenyl)trimethylsilane (4.0 equiv, $168 \mathrm{mg}, 609 \mu \mathrm{mol}), \mathrm{Pd}\left(\mathrm{PPh}_{3}\right)_{4}(5.5 \mathrm{~mol} \%$, $9.7 \mathrm{mg}, 8.4 \mu \mathrm{mol})$, Cul $(9.0 \mathrm{~mol} \%, 2.6 \mathrm{mg}, 13.7 \mu \mathrm{mol}), \operatorname{Pr}_{2} \mathrm{NH}(2.2 \mathrm{ml})$ and THF $(1.1 \mathrm{ml})$ according to GPA (stirring at $70{ }^{\circ} \mathrm{C}$ for $3 \mathrm{~h}$, then overnight at room temperature: column chromatography: $10-30 \%$ toluene in hexane).

'H NMR: $\left(400 \mathrm{MHz}, \mathrm{CDCl}_{3}\right) \delta=7.86(\mathrm{~d}, J=8.6 \mathrm{~Hz}, 2 \mathrm{H}), 7.74-7.65(\mathrm{~m}, 2 \mathrm{H}), 7.37-7.30(\mathrm{~m}$, $4 \mathrm{H}), 7.08-6.94(\mathrm{~m}, 10 \mathrm{H}), 6.94-6.87(\mathrm{~m}, 4 \mathrm{H}), 0.22(\mathrm{~s}, 18 \mathrm{H}) ;{ }^{13} \mathrm{C}\left\{{ }^{1} \mathrm{H}\right\} \mathbf{N M R}:(101 \mathrm{MHz}$, $\left.\mathrm{CDCl}_{3}\right) \delta=: 144.1,141.3,140.8,133.9,133.1,131.3,130.9,130.6,129.0,128.3,127.0$, 126.2, 124.2, 123.7, 94.7, 90.6, -1.1; IR: (neat, $\left.\mathrm{cm}^{-1}\right) \tilde{v}=536,571,295,609,630,691,716$, 759, 817, 836, 110, 1247, 1338, 1392, 1408, 1442, 1492, 1507, 1595, 2895, 2952, 3018, 3056; HRMS: calculated $m / z$ for $\mathrm{C}_{44} \mathrm{H}_{41} \mathrm{Si}_{2}{ }^{+}[\mathrm{M}+\mathrm{H}]^{+}: 625.2741$; found (ESI) 625.2752.

\section{2,7-Bis[(4-isopropylphenyl)ethynyl]-1,8-diphenylnaphthalene (159aj):}

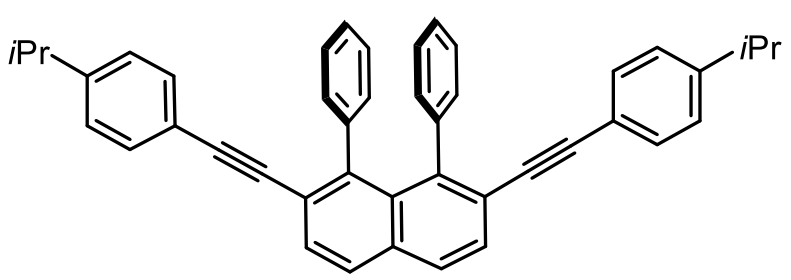

Prepared as a white solid (32 mg, 56.6 $\mu \mathrm{mol}, 37 \%$ yield) from alkyne 186a (1.0 equiv, $\quad 50.0 \mathrm{mg}, \quad 152.2 \mu \mathrm{mol}), \quad 1$-iodo-4isopropylbenzene (4.0 equiv, $150 \mathrm{mg}$, $610 \mu \mathrm{mol}), \operatorname{Pd}\left(\mathrm{PPh}_{3}\right)_{4}(5.5 \mathrm{~mol} \%, 9.7 \mathrm{mg}$, $8.4 \mu \mathrm{mol})$, Cul (9.0 mol\%, $2.6 \mathrm{mg}, 13.7 \mu \mathrm{mol}), \mathrm{Pr}_{2} \mathrm{NH}(2.2 \mathrm{ml})$ and THF $(1.1 \mathrm{ml})$ according to GPA, column chromatography (20\% toluene in petrol ether).

'H NMR: $\left(300 \mathrm{MHz}, \mathrm{CDCl}_{3}\right) \delta=7.85(\mathrm{~d}, J=8.5 \mathrm{~Hz}, 2 \mathrm{H}), 7.67(\mathrm{~d}, J=8.5 \mathrm{~Hz}, 2 \mathrm{H}), 7.11-6.84$ $(\mathrm{m}, 18 \mathrm{H}$ ), 2.83 (hept, $J=6.9 \mathrm{~Hz}, 2 \mathrm{H}), 1.19(\mathrm{~d}, J=6.9 \mathrm{~Hz}, 12 \mathrm{H})$ ppm; ${ }^{13} \mathbf{C}\left\{{ }^{1} \mathrm{H}\right\}$ NMR: $(126$ $\left.\mathrm{MHz}_{2} \mathrm{CDCl}_{3}\right) \delta=149.0,143.7,141.3,133.7,131.4,131.3,130.8,128.8,128.1,126.9,126.3$, 126.0, 124.2, 120.7, 94.7, 89.5, 34.2, 24.0 ppm; IR: (neat, $\mathrm{cm}^{-1}$ ) $\tilde{v}=502.4,556.4,621.0$, 691.4, 764.6, 807.1, 834.1, 1017.3, 1026.9, 1049.1, 1070.3, 1099.2, 1260.3, 1356.7, 1440.6, 1457.0, 1491.7, 1505.2, 1599.7, 2359.5, 2866.7, 2924.5, 2958.3, 3045.0; HRMS: calculated $\mathrm{m} / \mathrm{z}$ for $\mathrm{C}_{44} \mathrm{H}_{36}{ }^{+}[\mathrm{M}]^{+}: 564.2817$; found (EI) 564.2829. 


\section{Experimental}

\section{2,7-Bis[(3,4-dimethylphenyl)ethynyl]-1,8-diphenylnaphthalene (159ak):}

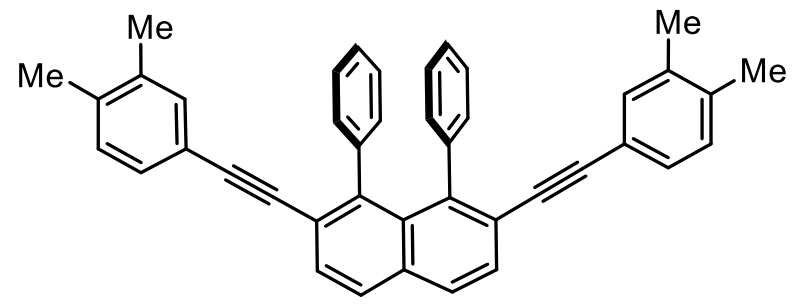

Prepared as a white solid (52 $\mathrm{mg}, 96.8$ umol, 64\% yield) from alkyne 186a (1.0 equiv, $\quad 50.0 \mathrm{mg}, \quad 152 \mu \mathrm{mol}), \quad 1$-iodo-3,4dimethylbenzene (4.0 equiv, $141 \mathrm{mg}$, $608 \mu \mathrm{mol}), \mathrm{Pd}\left(\mathrm{PPh}_{3}\right)_{4}(5.5 \mathrm{~mol} \%, 9.7 \mathrm{mg}$,

$8.4 \mu \mathrm{mol})$, Cul $(9.0 \mathrm{~mol} \%, 2.6 \mathrm{mg}, 13.7 \mu \mathrm{mol}), \mathrm{Pr}_{2} \mathrm{NH}(2.2 \mathrm{ml})$ and THF $(1.1 \mathrm{ml})$ according to GPA (column chromatography: $20-30 \%$ toluene in petrol ether).

'H NMR: $\left(300 \mathrm{MHz}, \mathrm{CDCl}_{3}\right) \delta=7.85(\mathrm{~d}, J=8.4 \mathrm{~Hz}, 2 \mathrm{H}), 7.65(\mathrm{~d}, J=8.4 \mathrm{~Hz}, 2 \mathrm{H}), 7.03-6.88$ $(\mathrm{m}, 12 \mathrm{H}), 6.81-6.71(\mathrm{~m}, 4 \mathrm{H}), 2.19(\mathrm{~s}, 6 \mathrm{H}), 2.14(\mathrm{~s}, 6 \mathrm{H}) \mathrm{ppm} ;{ }^{13} \mathrm{C}\left\{{ }^{1} \mathrm{H}\right\} \mathbf{N M R}:(126 \mathrm{MHz}$, $\left.\mathrm{CDCl}_{3}\right) \delta=143.6,141.4,136.9,136.4,133.6,132.6,131.3,130.9,129.4,128.9,128.7$, 128.1, 126.9, 126.0, 124.3, 120.6, 94.8, 89.4, 19.9, 19.7 ppm; IR: $\left(\right.$ neat, $\left.\mathrm{cm}^{-1}\right) \tilde{v}=528,577$, 634, 691, 744, 760, 806, 824, 845, 881, 1023, 1069, 1094, 1260, 1441, 1494, 1597, 2854, 2914, 2968, 3051; HRMS: calculated $\mathrm{m} / z$ for $\mathrm{C}_{42} \mathrm{H}_{32}{ }^{+}\left[\mathrm{M}^{+}:\right.$536.2504; found (EI) 536.2508.

\section{2,7-Bis\{[4-(triisopropylsilyloxymethyl)phenyl]ethynyl\}-1,8-diphenylnaphthalene (159al):}

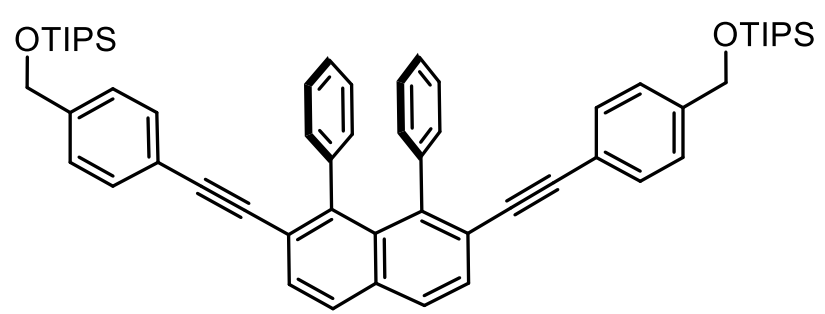

Prepared as a white solid (52 $\mathrm{mg}, 60.9$ $\mu \mathrm{mol}, 45 \%$ yield) from alkyne 186a (1.0 equiv, 50.0 mg, $152 \mu \mathrm{mol}), \quad[(4-$ iodobenzyl)oxy]triisopropylsilane

mol\%, $9.7 \mathrm{mg}, 8.4 \mu \mathrm{mol})$, Cul $(9.0 \mathrm{~mol} \%, 2.6 \mathrm{mg}, 13.7 \mu \mathrm{mol}), i \mathrm{Pr}_{2} \mathrm{NH}(2.2 \mathrm{ml})$ and THF (1.1 $\mathrm{ml}$ ) according to GPA (column chromatography: $30 \%$ toluene in hexane).

'H NMR: $\left(300 \mathrm{MHz}, \mathrm{CDCl}_{3}\right) \delta=7.86(\mathrm{~d}, J=8.5 \mathrm{~Hz}, 2 \mathrm{H}), 7.67(\mathrm{~d}, J=8.5 \mathrm{~Hz}, 2 \mathrm{H}), 7.21-7.13$ $(\mathrm{m}, 4 \mathrm{H}), 7.05-6.86(\mathrm{~m}, 14 \mathrm{H}), 4.76(\mathrm{~s}, 4 \mathrm{H}), 1.24-0.96(\mathrm{~m}, 42 \mathrm{H}) \mathrm{ppm} ;{ }^{13} \mathrm{C}\left\{{ }^{1} \mathrm{H}\right\}$ NMR: $(126$ $\left.\mathrm{MHz}, \mathrm{CDCl}_{3}\right) \delta=143.8,141.8,141.3,133.8,131.3,131.2,130.8,128.9,128.2,126.9,126.1$, 125.5, 124.2, 121.7, 94.6, 89.7, 64.9, 18.3, 12.3 ppm; IR: (neat, $\left.\mathrm{cm}^{-1}\right) \tilde{v}=528,565,659,763$, 797, 851, 882, 1012, 1066, 1093, 1204, 1254, 1370, 1642, 1516, 2865, 2926; HRMS: calculated $\mathrm{m} / \mathrm{z}$ for $\mathrm{C}_{58} \mathrm{H}_{68} \mathrm{O}_{2} \mathrm{NaSi}_{2}{ }^{+}:[\mathrm{M}+\mathrm{Na}]^{+:}$: 875.4650; found 875.4633.

\section{2,7-Bis\{[4-(methoxymethyl)phenyl]ethynyl\}-1,8-diphenylnaphthalene (159am):}

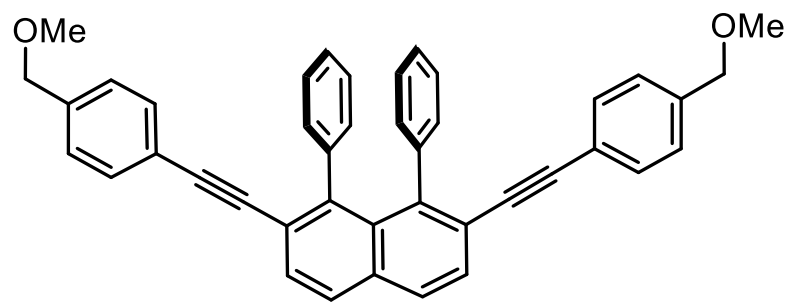

Prepared as a white solid $(65.0 \mathrm{mg}, 114$ $\mu \mathrm{mol}, 75 \%$ yield) from alkyne 186a (1.0 equiv, $50.0 \mathrm{mg}, 152 \mu \mathrm{mol}), \quad 1$-iodo-4(methoxymethyl)benzene (6.57 equiv, 248 
mg, $1.00 \mathrm{mmol}), \operatorname{Pd}\left(\mathrm{PPh}_{3}\right)_{4}(5.5 \mathrm{~mol} \%, 9.7 \mathrm{mg}, 8.4 \mu \mathrm{mol})$, Cul $(9.0 \mathrm{~mol} \%, 2.6 \mathrm{mg}, 13.7 \mu \mathrm{mol})$, $\mathrm{Pr}_{2} \mathrm{NH}(2.2 \mathrm{ml})$ and THF $(1.1 \mathrm{ml})$ according to GPA (stirring at $70{ }^{\circ} \mathrm{C}$ for $3 \mathrm{~h}$, then overnight at room temperature; column chromatography: 10-30\% toluene in hexane).

${ }^{1} \mathrm{H}$ NMR: $\left(300 \mathrm{MHz}, \mathrm{CDCl}_{3}\right) \delta=7.86(\mathrm{~d}, J=8.5 \mathrm{~Hz}, 2 \mathrm{H}), 7.67(\mathrm{~d}, J=8.6 \mathrm{~Hz}, 2 \mathrm{H}), 7.21-7.10$ $(\mathrm{m}, 4 \mathrm{H}), 7.07-6.87(\mathrm{~m}, 14 \mathrm{H}), 4.38(\mathrm{~s}, 4 \mathrm{H}), 3.34(\mathrm{~s}, 6 \mathrm{H}) \mathrm{ppm} ;{ }^{13} \mathbf{C}\left\{{ }^{1} \mathrm{H}\right\} \mathbf{N M R}:(126 \mathrm{MHz}$, $\left.\mathrm{CDCl}_{3}\right) \delta=143.9,141.2,138.2,133.8,131.5,131.2,130.8,128.8,128.2,127.4,126.9$, 126.1, 124.0, 122.6, 94.4, 90.2, 74.4, 58.3 ppm; IR: (neat, $\mathrm{cm}^{-1}$ ) $\tilde{\mathrm{v}}=525.5,696.3,760.8$, 806.1, 821.5, 844.7, 912.2, 965.2, 1026.9, 1071.3, 1088.6, 1183.1, 1201.4, 1356.7, 1440.6, 1490.7, 1510.0, 1597.7, 2820.4, 2854.1, 2922.6, 3049.9; HRMS: calcd $m / z$ for $\mathrm{C}_{42} \mathrm{H}_{33} \mathrm{O}_{2}{ }^{+}$ $[\mathrm{M}+\mathrm{H}]^{+}:$569.2475; found (ESI) 569.2474.

\section{2,7-Bis(bromoethynyl)-1,8-diphenylnaphthalene (159an):}

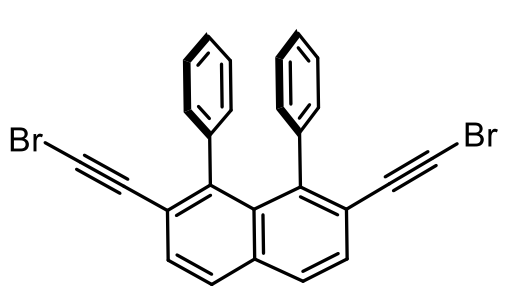

Prepared according to an adapted literature procedure. ${ }^{[278]}$ In a round bottom flask equipped with a magnetic stirrer, under air was added alkyne 186a (1.0 equiv, $50.0 \mathrm{mg}, 152 \mu \mathrm{mol}$ ), followed by acetone $(1.8 \mathrm{ml})$, Once the starting material had dissolved, silver nitrate ( 0.13 equiv, $3.4 \mathrm{mg}, 20.0 \mu \mathrm{mol})$ was added, followed by NBS (2.4 equiv, $65.8 \mathrm{mg}, 370 \mu \mathrm{mol}$ ) in portions. The reaction mixture was stirred for 30 minutes at room temperature, then concentrated in vacuo. The residue was diluted with dichloromethane $(30 \mathrm{ml})$ and successively washed extracted with $\mathrm{Na}_{2} \mathrm{~S}_{2} \mathrm{O}_{3}(3 \times$ $15 \mathrm{ml}$, sat. aqueous) and water (15 ml). The organic phase was dried over $\mathrm{Mg}_{2} \mathrm{SO}_{4}$, filtered and concentrated in in vacuo. Purification by column chromatography (10\% toluene in pentane) afforded compound $167 \mathrm{an}$ as a white solid $(65.0 \mathrm{mg}, 133 \mu \mathrm{mol}, 88 \%$ yield).

'H NMR: $\left(300 \mathrm{MHz}, \mathrm{CDCl}_{3}\right) \delta=7.80(\mathrm{~d}, J=8.5 \mathrm{~Hz}, 2 \mathrm{H}), 7.58(\mathrm{~d}, J=8.5 \mathrm{~Hz}, 2 \mathrm{H}), 7.00-6.88$ $(\mathrm{m}, 6 \mathrm{H}), 6.86-6.75(\mathrm{~m}, 4 \mathrm{H}) \mathrm{ppm} ;{ }^{13} \mathrm{C}\left\{{ }^{1} \mathrm{H}\right\}$ NMR: $\left(126 \mathrm{MHz}, \mathrm{CDCl}_{3}\right) \delta=144.6,140.2,134.0$, 130.6, 130.6, 129.4, 127.9, 126.8, 126.2, 123.3, 80.0, 53.9 ppm; IR: (neat, $\left.\mathrm{cm}^{-1}\right) \tilde{v}=577$, 608, 747, 804, 838, 1028, 1071, 1169, 1350, 1440, 1488, 1575, 1597, 2181, 2923, 3017, 3061; HRMS: calcd $m / z$ for $\mathrm{C}_{26} \mathrm{H}_{14} \mathrm{Br}_{2}{ }^{+}[\mathrm{M}]^{+}:$483.9462; found (EI) 483.9464 .

\section{2,7-Bis(trimethylsilylethynyl)-1,8-diphenylnaphthalene (159ao):}

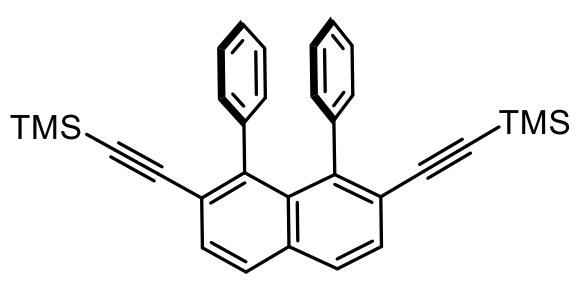

To the reaction mixture was then added chlorotrimethylsilane (2.20 equiv, $36.0 \mathrm{mg}, 331$

To a Schlenk flask equipped with magnetic stirring bar, was added the alkyne 186a (1.0 equiv, $50.0 \mathrm{mg}, 152$ $\mu \mathrm{mol})$ and THF $(0.5 \mathrm{ml})$, before cooling to $-78{ }^{\circ} \mathrm{C}$ and adding butyllithium ( 2.18 equiv, $0.21 \mathrm{ml}, 336 \mu \mathrm{mol}, 1.6 \mathrm{M}$ in hexanes) and allowing to stir at $-78{ }^{\circ} \mathrm{C}$ for one hour. 


\section{Experimental}

$\mu \mathrm{mol})$, allowing to stir for 30 minutes, before warming to room temperature and stirring for another $3 \mathrm{~h}$. The reaction was quenched by addition ammonium chloride (saturated, aqueous, $15 \mathrm{ml})$, then extracted with diethyl ether $(3 \times 20 \mathrm{ml})$. The combined organic layers were dried over $\mathrm{MgSO}_{4}$, filtered and concentrated in vacuo. After purification by column chromatography (10\% toluene in petrol ether), the product was isolated as a white solid (39.0 $\mathrm{mg}, 82.4 \mu \mathrm{mol}, 54 \%)$.

'H NMR: $\left(301 \mathrm{MHz}, \mathrm{CDCl}_{3}\right) \delta=7.78(\mathrm{~d}, J=8.5 \mathrm{~Hz}, 2 \mathrm{H}), 7.62-7.53(\mathrm{~m}, 2 \mathrm{H}), 6.99-6.84(\mathrm{~m}$, $6 \mathrm{H}), 6.84-6.74(\mathrm{~m}, 4 \mathrm{H}),-0.07(\mathrm{~d}, J=0.5 \mathrm{~Hz}, 18 \mathrm{H}) ;{ }^{13} \mathrm{C}\left\{{ }^{1} \mathrm{H}\right\}$ NMR: $\left(126 \mathrm{MHz}, \mathrm{CDCl}_{3}\right) \delta=$ $144.8,140.9,134.0,130.9,130.7,129.1,127.9,126.8,126.0,123.7,105.0,99.4,-0.1$ ppm; IR: $\left(\right.$ neat, $\left.\mathrm{cm}^{-1}\right) \tilde{v}=630,692,757,837,861,1022,1183,1246,1324,1352,1402,1438$, 1500, 1597, 2152, 2846, 2896, 2955, 3020, 3054; HRMS: calcd $m / z$ for $\mathrm{C}_{32} \mathrm{H}_{33} \mathrm{Si}_{2}{ }^{+}[\mathrm{M}+\mathrm{H}]^{+}$: 473.2115; found (ESI) 473.2122 .

\section{2,7-Bis[(triisopropylsilyl)ethynyl]-1,8-diphenylnaphthalene (159ap):}

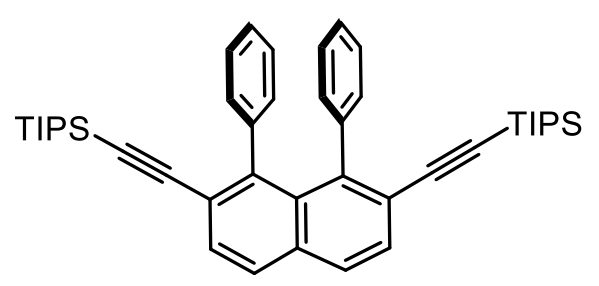

In a Schlenk flask equipped with magnetic stirring bar, was added the alkyne 186a (1.0 equiv, $50.0 \mathrm{mg}, 152$ $\mu \mathrm{mol})$, and THF $(0.5 \mathrm{ml})$, before cooling to $-78{ }^{\circ} \mathrm{C}$ before cooling to $-78{ }^{\circ} \mathrm{C}$ and adding butyllithium $(2.2$ equiv, $0.21 \mathrm{ml}, 336 \mu \mathrm{mol}, 1.6 \mathrm{M}$ in hexanes) and allowing to stir at $-78{ }^{\circ} \mathrm{C}$ for one hour. To the reaction was then added chlorotriisopropylsilane ( 2.2 equiv, $65.0 \mathrm{mg}, 337 \mu \mathrm{mol}$ ) allowing stirring for 30 minutes, before warming to room temperature and stirring for another $3 \mathrm{~h}$. The reaction was quenched by addition ammonium chloride (saturated, aqueous, $15 \mathrm{ml}$ ), then extracted with diethyl ether (3 $\times 20 \mathrm{ml}$ ). The combined organic layers were dried over $\mathrm{MgSO}_{4}$, filtered and concentrated in vacuo. After purification by column chromatography (10\% toluene in petrol ether) the product was isolated as a white solid (38.0 mg, $59.2 \mu \mathrm{mol}, 39 \%)$.

'H NMR: $\left(301 \mathrm{MHz}, \mathrm{CDCl}_{3}\right) \delta=7.77(\mathrm{~d}, J=8.4 \mathrm{~Hz}, 2 \mathrm{H}), 7.60(\mathrm{~d}, J=8.4 \mathrm{~Hz}, 2 \mathrm{H}), 6.90-6.72$ $(\mathrm{m}, 10 \mathrm{H}), 0.88(\mathrm{~d}, J=3.0 \mathrm{~Hz}, 41 \mathrm{H}) \mathrm{ppm} ;{ }^{13} \mathbf{C}\left\{{ }^{1} \mathrm{H}\right\}$ NMR: $\left(126 \mathrm{MHz}, \mathrm{CDCl}_{3}\right) \delta=144.2,141.0$, 133.9, 131.0, 130.8, 129.9, 127.8, 127.0, 126.0, 124.1, 106.8, 95.8, 18.8, 11.4 ppm; IR: $\left(\right.$ neat, $\mathrm{cm}^{-1}$ ) $\tilde{v}=565,604,624,658,676,748,842,878,919,994,1017,1071,1183,1253$, 1382, 1460, 1494, 1597, 2156, 2859, 2888, 2939, 3056; HRMS: calcd $\mathrm{m} / \mathrm{z}$ for $\mathrm{C}_{44} \mathrm{H}_{57} \mathrm{Si}_{2}{ }^{+}$ $[\mathrm{M}+\mathrm{H}]^{+}:$641.3993; found (ESI) 641.3981. 


\section{Experimental}

\section{2',7'-Diethynyl-2,1':8',2"-ternaphthalene (186d):}

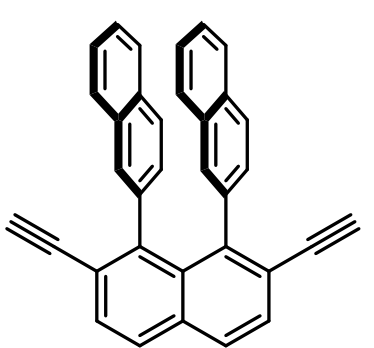

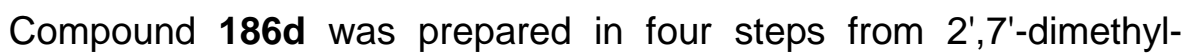
2,1':8',2"-ternaphthalene (181d). ${ }^{[219]}$ To a dry round bottom flask equipped with a stirring bar was added 181d (1.0 equiv, $321 \mathrm{mg}$, $786 \mu \mathrm{mol})$, followed by benzene $(16 \mathrm{ml}), \mathrm{N}$-bromosuccinimide $(2.2$ equiv, $308 \mathrm{mg}, 1.731 \mathrm{mmol})$, benzoyl peroxide $(6.7 \mathrm{~mol} \%, 12.7 \mathrm{mg}$, $52.4 \mu \mathrm{mol})$. The suspension was heated vigorously at reflux for $7 \mathrm{~h}$, before cooling to $0^{\circ} \mathrm{C}$ and filtering. The filtrate was concentrated in vacuo, taken up with chloroform and precipitated with hexanes. The resulting precipitate was filtered and the filtrate concentrated in vacuo, affording crude 2',7'-bis(bromomethyl)-2,1':8',2'ternaphthalene (182d) (395 mg, $695 \mu \mathrm{mol}, 89 \%$ crude yield), which was used in the next step without further purification. The compound 182d (1.0 equiv, $395 \mathrm{mg}, 694.7 \mu \mathrm{mol}$ ) was transferred to a round bottom flask; calcium carbonate $(5.5$ equiv, $384 \mathrm{mg}, 3.84 \mathrm{mmol}$ ), dioxane $(2 \mathrm{ml})$ and water $(0.5 \mathrm{ml})$ were added, and the reaction mixture was stirred under reflux for $48 \mathrm{~h}$, and then filtered hot, washing the filter cake with hot dioxane. The filtrate was concentrated in vacuo and acidified with aqueous hydrochloric acid (2M), whereupon a precipitate formed. The solid was filtered off, washed with water and dried under high vacuum, affording crude [2,1':8',2"-ternaphthalene]-2',7'-diyldimethanol (183d) (273 mg, 620 $\mu \mathrm{mol}, 89 \%$ crude yield), which was used without further purification. In a Schlenk flask equipped with stirring bar, compound 183d (1.0 equiv, $273 \mathrm{mg}, 620 \mu \mathrm{mol}$ ) was dissolved in anhydrous dichloromethane $(7.6 \mathrm{ml})$. The suspension was vigorously stirred at $40{ }^{\circ} \mathrm{C}$, before adding pyridinium chlorochromate (3.0 equiv, $402 \mathrm{mg}, 1.86 \mathrm{mmol}$ ) in one portion. The mixture was refluxed for 1 hour, before pouring into diethyl ether $(50 \mathrm{ml})$. Trituration afforded a solid, which was removed by filtration over a pad of silica, eluting with diethyl ether. The filtrate was concentrated in vacuo, affording crude [2,1':8',2"-ternaphthalene]-2',7'dicarbaldehyde (184d) as an orange solid (266 mg, $609.3 \mu \mathrm{mol}, 98 \%$ crude yield), which was used in the next step without further purification. To a Schlenk flask, equipped with stirring bar, was added dimethyl (1-diazo-2-oxopropyl)phosphonate (185) (3.0 equiv, 352 mg, $1.83 \mathrm{mmol})$, then methanol $(1.2 \mathrm{ml})$ and tetrahydrofuran $(1.2 \mathrm{ml})$. The solution was transferred to a pre-prepared second Schlenk flask containing 184d (1.0 equiv, $266 \mathrm{mg}$, $609.3 \mu \mathrm{mol})$ and potassium carbonate (4.0 equiv, $337 \mathrm{mg}, 2.44 \mathrm{mmol}$ ). The resulting mixture was stirred overnight at room temperature, before removing the solvent in vacuo and dissolving the residue in dichloromethane. The organic layer was washed with brine, extracting the aqueous layer twice with dichloromethane. The combined organic phases were combined, dried over $\mathrm{Na}_{2} \mathrm{SO}_{4}$ and concentrated in vacuo, before purifying by column 


\section{Experimental}

chromatography (20-30\% toluene in petrol ether), affording $\mathbf{1 8 6 \mathrm { d }}$ as a white solid (66 $\mathrm{mg}$, $154 \mu \mathrm{mol}, 25 \%$ yield, $20 \%$ yield over four steps), syn/anti $=44: 55$.

'H NMR: $\left(400 \mathrm{MHz}, \mathrm{CDCl}_{3}\right) \delta=7.88(\mathrm{~d}, J=8.5 \mathrm{~Hz}, 1 \mathrm{H}$, anti), $7.88(\mathrm{~d}, J=8.6 \mathrm{~Hz}, 0.81 \mathrm{H}$, syn), $7.68(\mathrm{~d}, J=8.4 \mathrm{~Hz}, 1 \mathrm{H}$, anti), $7.68(\mathrm{~d}, J=8.7 \mathrm{~Hz}, 0.81 \mathrm{H}$, syn), $7.53-7.47(\mathrm{~m}, 1 \mathrm{H}$, anti), $7.34-7.28(\mathrm{~m}, 2 \mathrm{H}$, anti), $7.24-7.12(\mathrm{~m}, 6 \mathrm{H}), 7.11(\mathrm{~d}, J=8.9 \mathrm{~Hz}, 1 \mathrm{H}$, anti), 7.06 (ddd, $J=$ 8.0, 6.5, $1.2 \mathrm{~Hz}, 0.81 \mathrm{H}, s y n), 6.90$ (dd, $J=8.4,1.6 \mathrm{~Hz}, 1 \mathrm{H}$, anti), 6.86 (dd, $J=8.3,1.7 \mathrm{~Hz}$, $0.81 \mathrm{H}$, syn), $6.81(\mathrm{~d}, J=8.2 \mathrm{~Hz}, 1 \mathrm{H}$, anti), $2.77(\mathrm{~s}, 1 \mathrm{H}$, anti), 2.76 (s, $0.81 \mathrm{H}$, syn) ppm; ${ }^{13} \mathrm{C}\left\{{ }^{1} \mathrm{H}\right\}$ NMR: $\left(101 \mathrm{MHz}, \mathrm{CDCl}_{3}\right) \delta=144.6,144.5,138.2,138.0,134.4,134.4,132.6,132.3$, $131.8,131.8,131.7,131.5,130.0,130.0,129.9,129.4,128.9,128.4,128.3,128.1,127.5$, $127.4,127.3,127.0,126.2,125.8,125.4,125.4,125.4,125.3,123.0,123.0,83.4,83.4,82.3$, 82.2 ppm; IR: $\left(\right.$ neat, $\left.\mathrm{cm}^{-1}\right) \tilde{v}=591,635,647,737,756,771,715,839,895,962,1012,1129$, 1254, 1321, 1500, 1602, 3018, 3052, 3276; HRMS: calculated $\mathrm{m} / \mathrm{z}$ for $\mathrm{C}_{34} \mathrm{H}_{20}{ }^{+}[\mathrm{M}]^{+}$: 428.1565; found (EI) 428.1569.

\section{2',7'-Bis(p-tolylethynyl)-2,1':8',2"'-ternaphthalene (159db):}

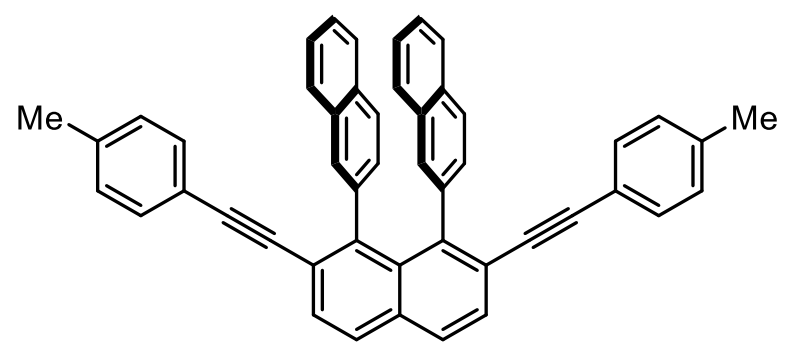

Prepared as a white solid [42.6 $\mathrm{mg}, 69.9$ $\mu \mathrm{mol},>99 \%$ yield; mixture of syn and anti $(45: 55)$ isomers] from alkyne $\mathbf{1 8 6 d}(1.0$ equiv, $30.0 \mathrm{mg}, 70.0 \mu \mathrm{mol})$, 4-iodotoluene (4.0 equiv, $61.1 \mathrm{mg}, 280 \mu \mathrm{mol}), \operatorname{Pd}\left(\mathrm{PPh}_{3}\right)_{4}$ (5.6 mol\%, $4.5 \mathrm{mg}, 3.9 \mu \mathrm{mmol})$, Cul $(9.0$ mol\%, $1.2 \mathrm{mg}, 6.3 \mu \mathrm{mol}), \operatorname{Pr}_{2} \mathrm{NH}(1: 0 \mathrm{ml})$ and THF $(0.5 \mathrm{ml})$ according to GPA, stirring at room temperature for $4 \mathrm{~h}$, column chromatography (5\% ethyl acetate in petrol ether),

'H NMR: $\left(301 \mathrm{MHz}, \mathrm{CDCl}_{3}\right) \delta=7.92(\mathrm{~d}, J=8.5 \mathrm{~Hz}, 1 \mathrm{H}$, anti), $7.91(\mathrm{~d}, J=8.6 \mathrm{~Hz}, 0.81 \mathrm{H}$, syn), 7.72 (d, $J=8.5 \mathrm{~Hz}, 1 \mathrm{H}$, anti), $7.72(\mathrm{~d}, J=8.4 \mathrm{~Hz}, 0.81 \mathrm{H}$, syn), 7.52 (dd, $J=8.2,1.1 \mathrm{~Hz}$, $1 \mathrm{H}$, anti), $7.39-7.27(\mathrm{~m}, 4 \mathrm{H}), 7.26-7.13(\mathrm{~m}, 4 \mathrm{H}), 7.09(\mathrm{dd}, J=6.8,1.4 \mathrm{~Hz}, 0.81 \mathrm{H}, s y n)$, 7.07 (dd, $J=8.4,1.6 \mathrm{~Hz}, 1 \mathrm{H}$, anti), 7.00 (dd, $J=8.3,1.7 \mathrm{~Hz}, 0.81 \mathrm{H}$, syn), 6.90 (d, $J=8.4 \mathrm{~Hz}$, $1 \mathrm{H}$, anti), $6.87-6.79(\mathrm{~m}, 4 \mathrm{H}), 6.70(\mathrm{dd}, J=8.2,1.7 \mathrm{~Hz}, 2 \mathrm{H}$, anti), $6.66(\mathrm{dd}, J=8.1,1.8 \mathrm{~Hz}$, 1.62H, syn), 2.19 (s, 3H, anti), $2.19\left(\mathrm{~s}, 2.43 \mathrm{H}\right.$, syn) ppm; ${ }^{13} \mathrm{C}\left\{{ }^{1} \mathrm{H}\right\}$ NMR: $\left(76 \mathrm{MHz} \mathrm{CDCl}_{3}\right) \delta=$ 143.3, 143.2, 138.7, 138.4, 138.0, 138.0, 133.8, 133.7, 132.5, 132.2, 131.8, 131.6, 131.5, $131.2,131.1,131.1,131.0,130.3,129.5,129.3,128.8,128.8,128.7,128.7,128.6,128.5$, $128.1,128.1,127.4,127.3,127.0,126.7,125.8,125.4,125.1,125.1,125.0,124.9,124.0$, 120.0, 120.0, 94.7, 94.7, 89.4, 89.4, 21.3, 21.3 ppm; IR: (neat, $\left.\mathrm{cm}^{-1}\right) \tilde{v}=523,668,704,740$, 810, 843, 891, 1017, 1338, 1437, 1512, 1597, 1907, 2210, 2854, 2916, 3014, 3051; HRMS: calculated $\mathrm{m} / \mathrm{z}$ for $\mathrm{C}_{48} \mathrm{H}_{32} \mathrm{Na}^{+}[\mathrm{M}+\mathrm{Na}]^{+}:$631.2396; found (ESI) 631.2375 . 


\section{Alternative four-step sequence to access helicene precursors 159}

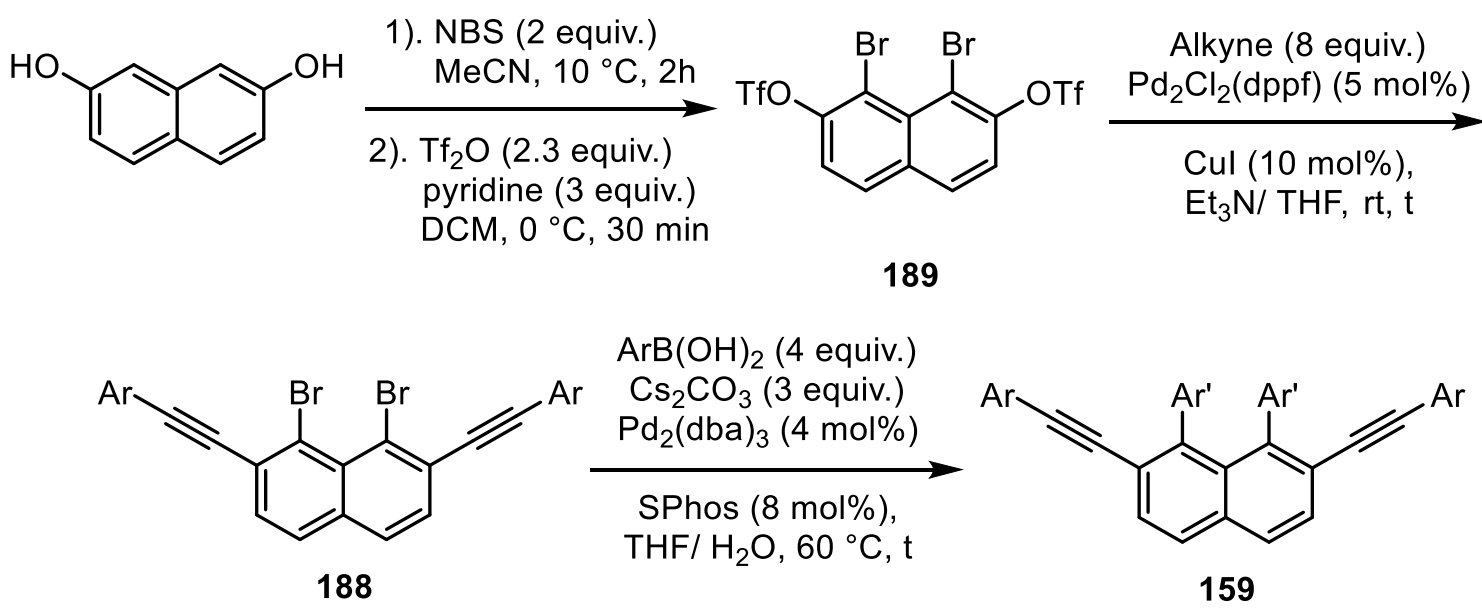

\section{1,8-Dibromonaphthalene-2,7-diyl Bis(trifluoromethanesulfonate) (189):}<smiles>Oc1ccc2ccc(Br)c(Br)c2c1Br</smiles>

Prepared over two steps according to adapted literature

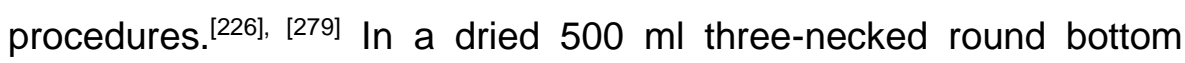
flask equipped with magnetic stirring bar was added 2,7dihydroxynaphthalene (1.0 equiv, $2.50 \mathrm{~g}, 15.6 \mathrm{mmol})$, followed by acetonitrile $(160 \mathrm{ml})$ under an argon atmosphere. The solution was cooled to $10^{\circ} \mathrm{C}$, before adding $\mathrm{N}$-bromosuccinimide (2.0 equiv, $5.56 \mathrm{~g}, 31.2 \mathrm{mmol}$ ) portionswise via argon trouser. On completion of the addition, the reaction was allowed to stir for $2 \mathrm{~h}$ at $10^{\circ} \mathrm{C}$. The yellow solution was quenched by addition of $\mathrm{H}_{2} \mathrm{SO}_{4}(2 \mathrm{M}, 200 \mathrm{ml})$. The mixture was transferred to a separatory funnel, rinsing the flask with dichloromethane and distilled water $(200 \mathrm{ml})$, then extracted with dichloromethane $(3 \times 650 \mathrm{ml})$. The combined organic layers were combined, dried over $\mathrm{MgSO}_{4}$ and directly filtered through a silica plug. The filtrate was evaporated and dried under high vacuum to give an orange/brown solid $(4.90 \mathrm{~g})$, which on analysis by ${ }^{1} \mathrm{H}$ NMR (DMSO$d_{6}$ ) contained approximately $70 \%$ of the desired 1,8-dibromonaphthalene-2,7-diol (190). The crude product was thoroughly dried under high vacuum then transferred to a pre-dried Schlenk flask, equipped with magnetic stirring bar, and the system was purged with argon before adding dry dichloromethane $(90 \mathrm{ml})$. The mixture was cooled to $0{ }^{\circ} \mathrm{C}$, then pyridine (3.0 equiv. $3.65 \mathrm{~g}, 46.1 \mathrm{mmol}$ ) followed by triflic anhydride (2.50 equiv. $10.9 \mathrm{~g}, 38.6 \mathrm{mmol}$ ) were added dropwise. The reaction was allowed to stir at $0{ }^{\circ} \mathrm{C}$ for 30 minutes, then quenched by careful addition of $\mathrm{NaHCO}_{3}$ solution (sat. aqueous) and extracted with dichloromethane. The organic layers were combined and washed with aqueous hydrochloric acid (10\%). The organic layer was dried over $\mathrm{MgSO}_{4}$ and evaporated to give the crude product, which could be purified by column chromatography ( 5 to $7 \%$ ethyl acetate in pentane) to give the product 189 as an off-white solid ( $2.38 \mathrm{~g}, 4.09 \mathrm{mmol}, 26 \%$ yield over two steps). 


\section{Experimental}

'H NMR: $\left(400 \mathrm{MHz}, \mathrm{CDCl}_{3}\right) \delta=7.97(\mathrm{~d}, J=9.2 \mathrm{~Hz}, 2 \mathrm{H}), 7.56(\mathrm{~d}, J=9.0 \mathrm{~Hz}, 2 \mathrm{H}) \mathrm{ppm}$; ${ }^{13} \mathrm{C}\left\{{ }^{1} \mathrm{H}\right\}$ NMR: $\left(126 \mathrm{MHz}, \mathrm{CDCl}_{3}\right) \delta=148.4,134.0,131.3,130.9,121.9,118.8$ (q, $J=320.3$ $\mathrm{Hz}), 115.9$ ppm; IR (neat, $\mathrm{cm}^{-1}$ ) $\tilde{v}=507,569,583,640,693,728,772,845,877,995,1131$, 1178, 1203, 1248, 1327, 1429; HRMS calcd. $\mathrm{m} / \mathrm{z}$ for $\mathrm{C}_{12} \mathrm{H}_{3} \mathrm{Br}_{2} \mathrm{~F}_{6} \mathrm{O}_{6} \mathrm{~S}_{2}{ }^{+}[\mathrm{M}-\mathrm{H}]^{+}:$578.7637; found (ESI) 578.7622 .

\section{General procedure B (GPB)}

In a dried Schlenk flask equipped with magnetic stirrer and septum was added triflate 189 (1.0 equiv.), followed by the alkyne (8.0-10.0 equiv.), $\mathrm{Et}_{3} \mathrm{~N}(0.37 \mathrm{M})$ and $\mathrm{DMF}(0.37 \mathrm{M})$. The mixture was degassed under argon flow for 15 minutes, before adding $\mathrm{PdCl}_{2}$ (dppf) (5 mol\%) and Cul (10 mol\%) and allowing to stir at room temperature for $3 \mathrm{~h}$. The volatiles were removed in vacuo and the crude product purified by column chromatography.

\section{1,8-Dibromo-2,7-bis[(trimethylsilyl)ethynyl]naphthalene (188a):}

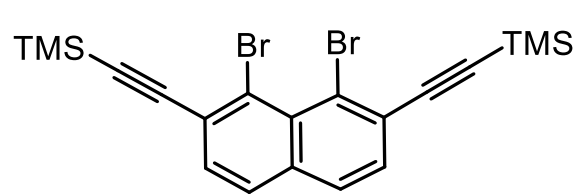

Prepared as a white solid (77 mg, $160.9 \mu \mathrm{mol}, 47 \%$ yield) using triflate 189 (1.0 equiv, $200 \mathrm{mg}, 344 \mu \mathrm{mol}$ ), (trimethylsilyl)acetylene (10 equiv, $338 \mathrm{mg}, 3.44 \mathrm{mmol}$ ), $\mathrm{PdCl}_{2}$ dppf) $(5.0 \mathrm{~mol} \%, 12.6 \mathrm{mg}, 17.2 \mu \mathrm{mol})$ and Cul $(10.1 \mathrm{~mol} \%, 6.6 \mathrm{mg}, 34.6 \mu \mathrm{mol})$ in $\mathrm{Et}_{3} \mathrm{~N}$ $(1.0 \mathrm{ml})$ and DMF $(1.0 \mathrm{ml})$ according to GPB after column chromatography $(5 \%$ dichloromethane in pentane).

'H NMR: $\left(600 \mathrm{MHz}, \mathrm{CDCl}_{3}\right) \delta=7.66(\mathrm{~d}, J=8.5 \mathrm{~Hz}, 2 \mathrm{H}), 7.51(\mathrm{~d}, J=8.5 \mathrm{~Hz}, 2 \mathrm{H}), 0.30$ (s, $18 \mathrm{H})$ ppm; ${ }^{13} \mathrm{C}\left\{{ }^{1} \mathrm{H}\right\}$ NMR: $\left(126 \mathrm{MHz}, \mathrm{CDCl}_{3}\right) \delta=135.3,130.4,130.2,128.2,128.1,123.7$, 104.8, 102.4, -0.1 ppm; IR: (neat, $\mathrm{cm}^{-1}$ ) $\tilde{v}=597,634,697,758,833,1137,1180,1218$, 1245, 1286, 1334, 1409, 1427, 1490, 1599, 2099, 2151, 2899, 2958; HRMS: calcd m/z for $\mathrm{C}_{20} \mathrm{H}_{22} \mathrm{Br}_{2} \mathrm{Si}_{2}{ }^{+}[\mathrm{M}]^{+}$: 475.9627 ; found (EI) 475.9627.

\section{1,8-Dibromo-7-[(trimethylsilyl)ethynyl]naphthalen-2-yl Trifluoromethanesulfonate} (191):<smiles>CCOc1ccc2ccc(C#CSC)c(Br)c2c1Br</smiles>

In a dried Schlenk flask equipped with magnetic stirrer and septum was added triflate 189 (1.0 equiv, $318 \mathrm{mg}, 546 \mu \mathrm{mol}$ ), followed by $\mathrm{Et}_{3} \mathrm{~N}(1.5 \mathrm{ml})$ and DMF $(1.5 \mathrm{ml})$, before degassing under argon flow for 20 minutes. (Trimethylsilyl)acetylene (2.0 equiv, $158 \mu \mathrm{l}, 109 \mathrm{mg}, 1.11$ $\mathrm{mmol}), \mathrm{PdCl}_{2}\left(\mathrm{PPh}_{3}\right)_{2}(5.0 \mathrm{~mol} \%, 19.2 \mathrm{mg}, 27.3 \mu \mathrm{mol})$ and Cul (10.0 mol\%, $10.4 \mathrm{mg}, 54.6$ $\mu \mathrm{mol})$ were then added, and the reaction mixture allowed to stir at room temperature for one hour. The mixture was diluted with aqueous hydrochloric acid $(1 \mathrm{M}, 50 \mathrm{ml})$ and extracted with dichloromethane $(3 \times 50 \mathrm{ml})$. The organic phases were combined, dried and concentrated in 
vacuo, before the residue was purified by column chromatography $(5 \%$ dichloromethane in pentane), to afford the product 191 as a white solid (95 mg, $179 \mu \mathrm{mol}, 33 \%$ yield).

'H NMR: $\left(300 \mathrm{MHz}, \mathrm{CDCl}_{3}\right) \delta=7.77(\mathrm{~d}, J=9.1 \mathrm{~Hz}, 1 \mathrm{H}), 7.69(\mathrm{~d}, J=8.6 \mathrm{~Hz}, 1 \mathrm{H}), 7.53(\mathrm{~d}, J=$ $8.4 \mathrm{~Hz}, 1 \mathrm{H}), 7.37(\mathrm{~d}, J=8.9 \mathrm{~Hz}, 1 \mathrm{H}), 0.25(\mathrm{~s}, 9 \mathrm{H}) \mathrm{ppm} ;{ }^{13} \mathrm{C}\left\{{ }^{1} \mathrm{H}\right\}$ NMR: $\left(126 \mathrm{MHz}, \mathrm{CDCl}_{3}\right) \delta=$ 147.6, 134.4, 131.0, 130.6, 130.4, 129.3, 128.3, 123.9, 122.4, 120.8, 119.9, 117.3, 115.2, 114.8, 104.3, 103.8, -0.2 ppm; IR: (neat, $\left.\mathrm{cm}^{-1}\right) \tilde{v}=585,624,666,702,759,840,869,984$, 1138, 1183, 1204, 1251, 1327, 1409, 1424, 1487, 1602, 2156, 2553, 2365, 2904, 2958; HRMS: calcd $m / z$ for $\mathrm{C}_{16} \mathrm{H}_{14} \mathrm{Br}_{2} \mathrm{~F}_{3} \mathrm{O}_{3} \mathrm{SSi}^{+}[\mathrm{M}+\mathrm{H}]^{+}: 530.8726$; found (ESI) 530.8726 .

\section{1,8-Dibromo-2,7-bis(p-tolylethynyl)naphthalene (188b):}

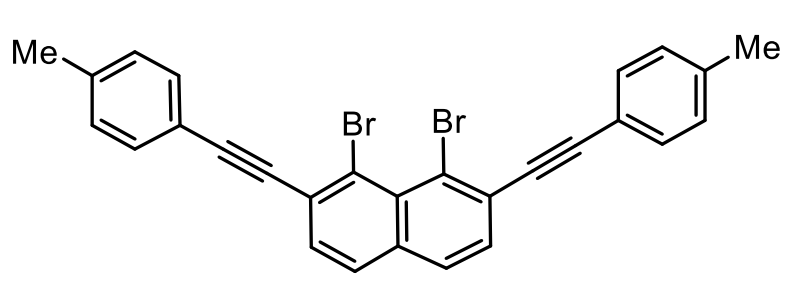

Prepared as a yellow/gold crystalline solid (108 mg, $210 \mu \mathrm{mol}, 82 \%$ yield) using triflate 189 (1.0 equiv, $150 \mathrm{mg}, 258 \mu \mathrm{mol})$, 4ethynyltoluene (7.70 equiv, $230 \mathrm{mg}, 1.98$ $\mathrm{mmol}$ ), $\mathrm{Pd}_{2} \mathrm{Cl}_{2}$ (dppf) (5.0 mol\%, $9.4 \mathrm{mg}, 12.8$ $\mu \mathrm{mol})$, Cul (10.0 mol\%, $4.9 \mathrm{mg}, 25.7 \mu \mathrm{mol}), \mathrm{Et}_{3} \mathrm{~N}(0.7 \mathrm{ml})$ and DMF $(0.7 \mathrm{ml})$ according to GPB after column chromatography (10-15\% dichloromethane in pentane). Crystals suitable for X-ray crystallographic analysis were grown by slowly cooling a hot saturated solution in ethyl acetate.

'H NMR: $\left(300 \mathrm{MHz}, \mathrm{CDCl}_{3}\right) \delta=7.72(\mathrm{~d}, J=8.6 \mathrm{~Hz}, 2 \mathrm{H}), 7.58(\mathrm{~d}, J=8.5 \mathrm{~Hz}, 2 \mathrm{H}), 7.53(\mathrm{dd}, J$ $=7.9,1.6 \mathrm{~Hz}, 4 \mathrm{H}), 7.23-7.17(\mathrm{~m}, 4 \mathrm{H}), 2.40(\mathrm{dd}, J=867.7,0.8 \mathrm{~Hz}, 6 \mathrm{H}) \mathrm{ppm} ;{ }^{13} \mathrm{C}\left\{{ }^{1} \mathrm{H}\right\} \mathrm{NMR}$ : $\left(126 \mathrm{MHz}, \mathrm{CDCl}_{3}\right) \delta=139.2,135.1,131.7,130.6,130.1,129.3,128.8,128.4,123.3,120.0$, 96.7, 89.9, 21.8 ppm; IR: (neat, $\mathrm{cm}^{-1}$ ) $\tilde{v}=523,573,692,765,809,819,844,1131,1245$, 1344, 1509, 1591, 2205, 2852, 2912, 2950, 3023, 3069; HRMS: calcd m/z for $\mathrm{C}_{28} \mathrm{H}_{19} \mathrm{Br}_{2}{ }^{+}$ $[\mathrm{M}+\mathrm{H}]^{+}: 512.9848$; found (ESI) 512.9831 .

\section{1,8-Dibromo-2,7-bis((4-methoxyphenyl)ethynyl)naphthalene (188c):}

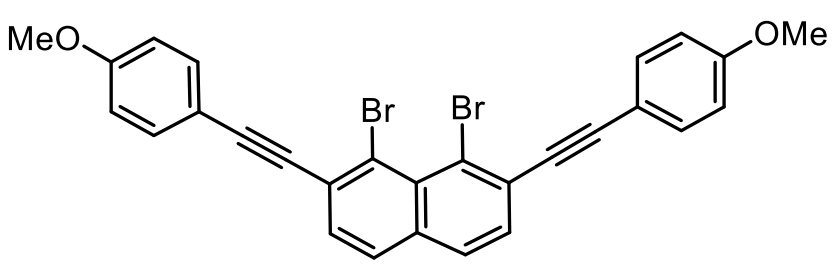

Prepared as a white solid (830 mg, 1.52 mmol, $86 \%$ yield) using triflate 189 (1.0 equiv, $1.03 \mathrm{~g}, \quad 1.77 \mathrm{mmol})$, 4ethynylanisole (7.5 equiv, $1.86 \mathrm{~g}, 14.1$ $\mathrm{mmol}$ ), $\mathrm{Pd}_{2} \mathrm{Cl}_{2}$ (dppf) (5.0 mol\%, $64.4 \mathrm{mg}$,

$88 \mu \mathrm{mol})$, Cul (9.9 mol\%, $33.5 \mathrm{mg}, 175.8 \mu \mathrm{mol}), \mathrm{Et}_{3} \mathrm{~N}(4.8 \mathrm{ml})$ and DMF $(4.8 \mathrm{ml})$ according to GPB after column chromatography (20-50\% dichloromethane in pentane). 


\section{Experimental}

'H NMR: $\left(300 \mathrm{MHz}, \mathrm{CDCl}_{3}\right) \delta=7.70(\mathrm{~d}, J=8.6 \mathrm{~Hz}, 2 \mathrm{H}), 7.61-7.53(\mathrm{~m}, 6 \mathrm{H}), 6.92(\mathrm{dd}, J=$ 8.7, $2.4 \mathrm{~Hz}, 4 \mathrm{H}), 3.85(\mathrm{~s}, 6 \mathrm{H}) \mathrm{ppm} ;{ }^{13} \mathrm{C}\left\{{ }^{1} \mathrm{H}\right\}$ NMR: $\left(75 \mathrm{MHz}, \mathrm{CDCl}_{3}\right) \delta=160.7,135.4,133.9$, $131.1,130.5,129.4,128.9,123.4,115.6,114.7,97.0,89.8,55.9$ ppm; IR: (neat, $\mathrm{cm}^{-1}$ ) $\tilde{v}=$ 530, 804, 821, 846, 1031, 1137, 1243, 1294, 1439, 1506, 1563, 1596, 2178, 2197, 2836, 2928, 2964, 3007, 3051; HRMS: calcd $\mathrm{m} / \mathrm{z}$ for $\mathrm{C}_{28} \mathrm{H}_{18} \mathrm{Br}_{2} \mathrm{NaO}_{2}{ }^{+}[\mathrm{M}+\mathrm{Na}]^{+}:$566.9566; found (ESI) 566.9537.

\section{General procedure C (GPC)}

Under argon, to a dried Schlenk flask equipped with a magnetic stirring bar was added the diyne 188 (1.0 equiv), followed by the boronic acid derivative (4.0 equiv.), cesium carbonate (3.0 equiv.), THF and $\mathrm{H}_{2} \mathrm{O}(10: 1,0.05 \mathrm{M})$. The mixture was degassed via three freeze-pumpthaw cycles, then $\mathrm{Pd}_{2}(\mathrm{dba})_{3}$ (4 mol\%), Sphos (8 mol\%) were added, a glass stopper fitted onto the Schlenk flask and the reaction mixture stirred at $60{ }^{\circ} \mathrm{C}$ for the stated amount of time. When the reaction had reached completion, it was allowed to cool to room temperature and filtered through a plug of silica, eluting with ethyl acetate. The solvents were removed in vacuo and the crude product purified by column chromatography.

\section{2,2'-[2,7-Bis( $p$-tolylethynyl)naphthalene-1,8-diyl]dithiophene (159eb):}

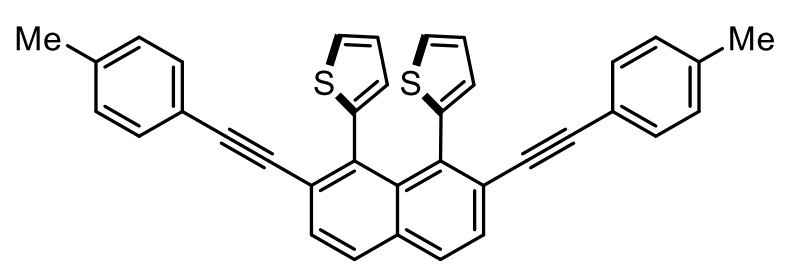

Prepared as an off-white solid (13 $\mathrm{mg}, 24.9$ $\mu \mathrm{mol}, 51 \%$ yield) using diyne $188 \mathrm{~b}$ (1.0 equiv, $25.0 \mathrm{mg}, 48.6 \mu \mathrm{mol})$, 2-thienylboronic acid (4.3 equiv, $26.8 \mathrm{mg}, 209 \mu \mathrm{mol})$, cesium carbonate (3.22 equiv, $51.1 \mathrm{mg}, 157 \mu \mathrm{mol}$ ),

THF (1 ml), $\mathrm{H}_{2} \mathrm{O}(0.1 \mathrm{ml}), \mathrm{Pd}_{2}(\mathrm{dba})_{3}(4.3 \mathrm{~mol} \%, 1.9 \mathrm{mg}, 2.1 \mu \mathrm{mol})$, Sphos $(8.4 \mathrm{~mol} \%, 1.7 \mathrm{mg}$, $4.1 \mu \mathrm{mol}$ ) according to GPE. The mixture was heated for $1 \mathrm{~h}$; column chromatography: $15 \%$ dichloromethane in pentane.

'H NMR: $\left(300 \mathrm{MHz}, \mathrm{CDCl}_{3}\right) \delta=7.86(\mathrm{~d}, J=8.5 \mathrm{~Hz}, 2 \mathrm{H}), 7.65(\mathrm{~d}, J=8.4 \mathrm{~Hz}, 2 \mathrm{H}), 7.18(\mathrm{dd}, J$ $=5.1,1.2 \mathrm{~Hz}, 2 \mathrm{H}), 7.04(\mathrm{~s}, 8 \mathrm{H}), 6.75(\mathrm{dd}, J=5.1,3.5 \mathrm{~Hz}, 2 \mathrm{H}), 6.59$ (dd, J=3.5, $1.2 \mathrm{~Hz}, 2 \mathrm{H}$ ), 2.30 (s, 6H) ppm; ${ }^{13} \mathrm{C}\left\{{ }^{1} \mathrm{H}\right\}$ NMR: $\left(126 \mathrm{MHz}, \mathrm{CDCl}_{3}\right) \delta=141.6,138.2,135.1,133.6,133.1$, 131.4, 129.3, 129.0, 128.8, 128.5, 126.7, 126.2, 125.0, 120.1, 95.5, 88.6, 21.6 ppm; IR: $\left(\right.$ neat, $\mathrm{cm}^{-1}$ ) $\tilde{v}=512,524,686,694,811,844,1018,1091,1260,1510,1591,2330,2360$, 2858, 2915, 2960; HRMS: calcd $m / z$ for $\mathrm{C}_{36} \mathrm{H}_{25} \mathrm{~S}_{2}{ }^{+}[\mathrm{M}+\mathrm{H}]^{+}: 521.1392$; found (El) 521.1385 . 


\section{Experimental}

\section{2,2'-[2,7-Bis(p-tolylethynyl)naphthalene-1,8-diyl]difuran (159fb):}

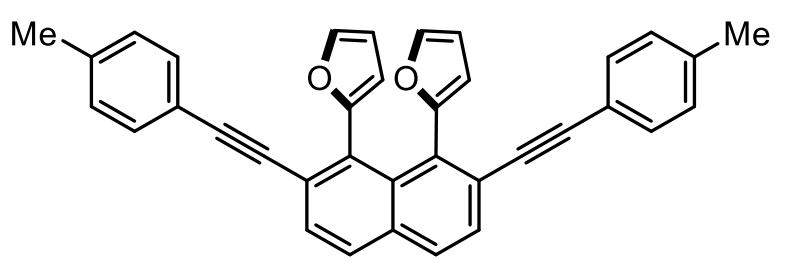

Prepared as a red/orange solid $(40.0 \mathrm{mg}$, $81.8 \mu \mathrm{mol}, 88 \%$ yield) using alkyne $188 \mathrm{~b}$ (1.0 equiv, $47.8 \mathrm{mg}, 92.9 \mu \mathrm{mol})$, 2-furanylboronic acid (4.0 equiv, $41.6 \mathrm{mg}, 372 \mu \mathrm{mol})$, cesium carbonate (3.0 equiv, $90.8 \mathrm{mg}, 279 \mu \mathrm{mol}$ ),

THF (2 ml), $\mathrm{H}_{2} \mathrm{O}(0.2 \mathrm{ml}), \mathrm{Pd}_{2}(\mathrm{dba})_{3}(4.0 \mathrm{~mol} \%, 3.4 \mathrm{mg}, 3.7 \mu \mathrm{mol})$, Sphos $(8.2 \mathrm{~mol} \%, 3.1 \mathrm{mg}$, $7.6 \mu \mathrm{mol}$ ) according to GPC. The mixture was heated for $1 \mathrm{~h}$; column chromatography: $30 \%$ dichloromethane in pentane.

'H NMR: $\left(300 \mathrm{MHz}, \mathrm{CDCl}_{3}\right) \delta=7.87(\mathrm{~d}, J=8.5 \mathrm{~Hz}, 2 \mathrm{H}), 7.66(\mathrm{~d}, J=8.5 \mathrm{~Hz}, 2 \mathrm{H}), 7.31$ (dd, $J$ $=1.8,0.8 \mathrm{~Hz}, 2 \mathrm{H}), 7.21(\mathrm{dd}, J=8.1,1.9 \mathrm{~Hz}, 4 \mathrm{H}), 7.13-7.03(\mathrm{~m}, 4 \mathrm{H}), 6.21(\mathrm{dd}, J=3.3,1.8$ $\mathrm{Hz}, 2 \mathrm{H}), 6.13(\mathrm{dd}, J=3.3,0.8 \mathrm{~Hz}, 2 \mathrm{H}), 2.33(\mathrm{~s}, 6 \mathrm{H}) ;{ }^{13} \mathrm{C}\left\{{ }^{1} \mathrm{H}\right\} \mathrm{NMR}:\left(126 \mathrm{MHz}, \mathrm{CDCl}_{3}\right) \delta=$ $150.8,140.8,138.6,133.3,133.2,131.7,131.6,129.6,129.1,129.0,126.5,120.2,111.2$, 109.8, 94.8, 88.3, 21.8; IR: (neat, $\mathrm{cm}^{-1}$ ) $\tilde{v}=526,613,730,814,846,991,1071,1152,1201$, 1342, 1494, 1510, 1597, 2208, 2855, 2917, 2958, 3056, 3115; HRMS: calcd $\mathrm{m} / \mathrm{z}$ for $\mathrm{C}_{36} \mathrm{H}_{25} \mathrm{O}_{2}{ }^{+}[\mathrm{M}+\mathrm{H}]^{+}: 489.1849$; found (ESI) 489.1852 .

\section{3,3'-[2,7-Bis( $p$-tolylethynyl)naphthalene-1,8-diyl]dithiophene (159gb):}

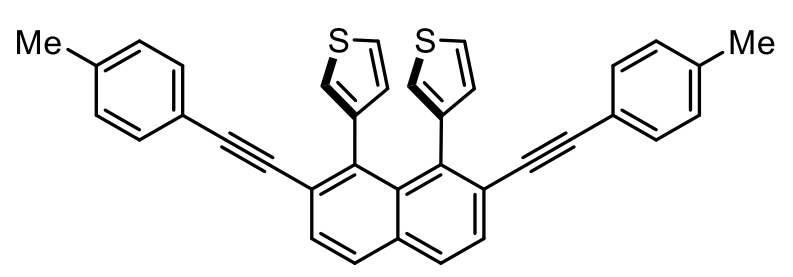

Prepared as a light yellow solid (23 mg, $44.1 \mu \mathrm{mol}, 57 \%$ yield) using alkyne $188 \mathrm{~b}$ (1.0 equiv, $40.0 \mathrm{mg}, \quad 77.7 \mu \mathrm{mol})$, 3thienylboronic acid (4.0 equiv, $39.8 \mathrm{mg}, 311$ $\mu \mathrm{mol})$, cesium carbonate (3.0 equiv, 75.9

$\mathrm{mg}, 233 \mu \mathrm{mol})$, THF $(1.7 \mathrm{ml}), \mathrm{H}_{2} \mathrm{O}(170 \mu \mathrm{l}), \mathrm{Pd}_{2}(\mathrm{dba})_{3}(4.0 \mathrm{~mol} \%, 2.8 \mathrm{mg}, 3.1 \mu \mathrm{mol})$, Sphos (8.1 mol\%, $2.6 \mathrm{mg}, 6.3 \mu \mathrm{mol}$ ) according to GPC. The mixture was heated for $1 \mathrm{~h}$; column chromatography: $20 \%$ toluene in pentane.

'H NMR: $\left(300 \mathrm{MHz}, \mathrm{C}_{6} \mathrm{D}_{6}\right) \delta=7.77(\mathrm{~d}, J=8.5 \mathrm{~Hz}, 2 \mathrm{H}), 7.53(\mathrm{~d}, J=8.5 \mathrm{~Hz}, 2 \mathrm{H}), 7.35$ (dd, $J=$ 8.1, $1.8 \mathrm{~Hz}, 4 \mathrm{H}), 6.91-6.83(\mathrm{~m}, 4 \mathrm{H}), 6.77(\mathrm{dd}, J=4.7,3.2 \mathrm{~Hz}, 2 \mathrm{H}), 6.70-6.60(\mathrm{~m}, 4 \mathrm{H})$, $2.01(\mathrm{~s}, 6 \mathrm{H})$ ppm; ${ }^{13} \mathrm{C}$ NMR: $\left(75 \mathrm{MHz}, \mathrm{C}_{6} \mathrm{D}_{6}\right) \delta=141.1,138.7,138.0,133.6,131.6,130.6$, 129.0, 128.7, 128.5, 126.8, 125.1, 124.5, 123.0, 120.7, 95.0, 89.6, 20.9 ppm; IR: (neat, $\mathrm{cm}^{-1}$ ) $\tilde{v}=524,659,766,788,812,842,1020,1034,1082,1258,1331,1413,1510,1585,2846$, 2915, 2958, 2990; HRMS: calcd $m / z$ for $\mathrm{C}_{36} \mathrm{H}_{24} \mathrm{~S}_{2}{ }^{+}[\mathrm{M}]^{+}: 520.1319$; found (EI) 52.1324. 


\section{Experimental}

\section{3,3'-[2,7-Bis(p-tolylethynyl)naphthalene-1,8-diyl]difuran (159hb):}

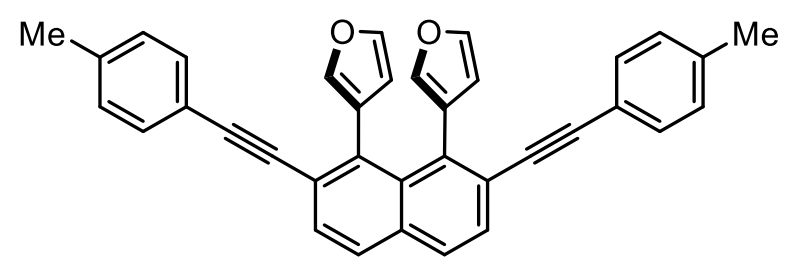

Prepared as a purple solid $(17.0 \mathrm{mg}, 34.7$ $\mu \mathrm{mol}, 45 \%$ yield) using alkyne 188b (1.0 equiv, $40.0 \mathrm{mg}, 77.7 \mu \mathrm{mol})$, 3-furanylboronic acid (4.0 equiv, $34.8 \mathrm{mg}, 311 \mu \mathrm{mol})$, cesium carbonate (3.0 equiv, $75.9 \mathrm{mg}, 233 \mu \mathrm{mol}$ ),

THF (1.7 ml), $\mathrm{H}_{2} \mathrm{O}(0.17 \mathrm{ml}), \mathrm{Pd}_{2}(\mathrm{dba})_{3}(4.0 \mathrm{~mol} \%, 2.8 \mathrm{mg}, 3.1 \mu \mathrm{mol})$, Sphos $(8.1 \mathrm{~mol} \%, 2.6$ $\mathrm{mg}, 6.3 \mu \mathrm{mol})$ according to GPC. The mixture was heated for $1 \mathrm{~h}$; column chromatography: $20 \%$ toluene in pentane.

'H NMR: $\left(300 \mathrm{MHz}, \mathrm{CDCl}_{3}\right) \delta=7.83(\mathrm{~d}, J=8.5 \mathrm{~Hz}, 2 \mathrm{H}), 7.67(\mathrm{~d}, J=8.5 \mathrm{~Hz}, 2 \mathrm{H}), 7.33(\mathrm{t}, J=$ $1.7 \mathrm{~Hz}, 2 \mathrm{H}$ ), 7.21 (dd, $J=8.1,1.8 \mathrm{~Hz}, 4 \mathrm{H}), 7.16-7.05(\mathrm{~m}, 6 \mathrm{H}), 6.27$ (dd, $J=1.8,0.8 \mathrm{~Hz}$, $2 \mathrm{H}), 2.36(\mathrm{~s}, 6 \mathrm{H}) \mathrm{ppm} ;{ }^{13} \mathrm{C}\left\{{ }^{1} \mathrm{H}\right\}$ NMR: $\left(126 \mathrm{MHz}, \mathrm{CDCl}_{3}\right) \delta=141.4,141.0,138.4,133.7$, 133.5, 133.2, 131.4, 129.1, 128.9, 128.4, 125.0, 125.0, 120.4, 113.6, 94.7, 89.3, 21.7 ppm; IR: $\left(\right.$ neat, $\left.\mathrm{cm}^{-1}\right) \tilde{v}=525,593,668,722,793,813,871,1021,1066,1161,1347,1442,1497$, 1510, 1567, 1594, 2208, 2858, 2920, 2958, 3031, 3113, 3140; HRMS: calcd $\mathrm{m} / \mathrm{z}$ for $\mathrm{C}_{36} \mathrm{H}_{24} \mathrm{NaO}_{2}{ }^{+}[\mathrm{M}+\mathrm{Na}]^{+}:$511.1669; found (ESI) 511.1670 .

\{[2,7-Bis(p-tolylethynyl)naphthalene-1,8-diyl]bis(4,1-phenylene)\}bis(trimethylsilane) (159ib):

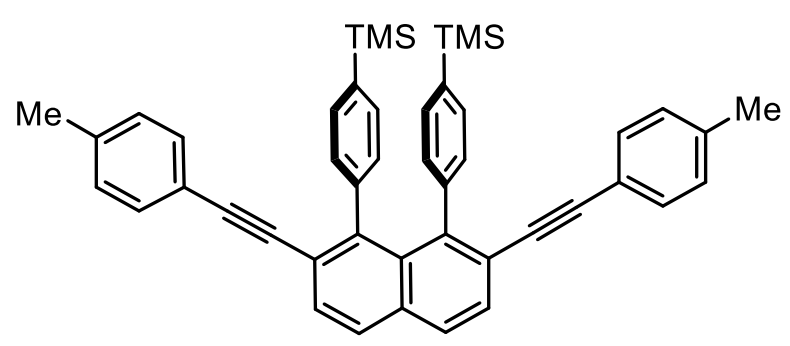

Prepared as a white solid (37.3 $\mathrm{mg}, 57.1$ $\mu \mathrm{mol}, 37 \%$ yield) using alkyne 188b (1.0 equiv, $80.0 \mathrm{mg}, \quad 156 \mu \mathrm{mol})$, 4(trimethylsilyl)phenylboronic acid (4.0 equiv, $120.0 \mathrm{mg}, 618 \mu \mathrm{mol})$, cesium carbonate $(3.0$ equiv, $152.0 \mathrm{mg}, 467 \mu \mathrm{mol})$, THF $(3.4 \mathrm{ml})$, $\mathrm{H}_{2} \mathrm{O}$ (0.34 ml), $\mathrm{Pd}_{2}(\mathrm{dba})_{3}(4.0 \mathrm{~mol} \%, 5.7 \mathrm{mg}, 6.2 \mu \mathrm{mol})$, Sphos (8.0 mol\%, $\left.5.1 \mathrm{mg}, 12.4 \mu \mathrm{mol}\right)$ according to GPC. The mixture was heated for $16 \mathrm{~h}$. Column chromatography $(10-15 \%$ toluene in pentane) was followed by crystallization from hexanes affording the product 159ib. Crystals suitable for X-ray crystallographic analysis were grown by slowly cooling a hot saturated hexane solution.

'H NMR: $\left(300 \mathrm{MHz}, \mathrm{CDCl}_{3}\right) \delta=7.84(\mathrm{~d}, J=8.6 \mathrm{~Hz}, 2 \mathrm{H}), 7.64(\mathrm{~d}, J=8.5 \mathrm{~Hz}, 2 \mathrm{H}), 7.13(\mathrm{dd}, J$ $=8.1,1.5 \mathrm{~Hz}, 4 \mathrm{H}), 6.98-6.93(\mathrm{~m}, 4 \mathrm{H}), 6.90(\mathrm{dd}, J=8.2,1.5 \mathrm{~Hz}, 4 \mathrm{H}), 6.83(\mathrm{dd}, J=8.2,1.7$ $\mathrm{Hz}, 4 \mathrm{H}), 2.27$ (s, 6H), $0.26(\mathrm{~s}, 18 \mathrm{H}) \mathrm{ppm} ;{ }^{13} \mathrm{C}\left\{{ }^{1} \mathrm{H}\right\}$ NMR: $\left(126 \mathrm{MHz}, \mathrm{CDCl}_{3}\right) \delta=143.9,142.0$, 138.1 , 137.2, 133.6, 131.7, 131.4, 130.9, 130.6, 128.8, 128.6, 128.0, 124.2, 120.3, 94.6, 89.6, 21.7, -0.6 ppm; IR: (neat, $\left.\mathrm{cm}^{-1}\right) \tilde{v}=524,631,721,753,814,837,1093,1121,1244$, 
1381, 1508, 2919, 2953, 3018, 3054; HRMS: calcd. $m / z$ for $\mathrm{C}_{46} \mathrm{H}_{44} \mathrm{NaSi}_{2}{ }^{+}[\mathrm{M}+\mathrm{Na}]^{+}:$675.2874; found (ESI) 675.2863 .

\section{1,8-Bis[4-(benzyloxy)phenyl]-2,7-bis(p-tolylethynyl)naphthalene (159jb)}

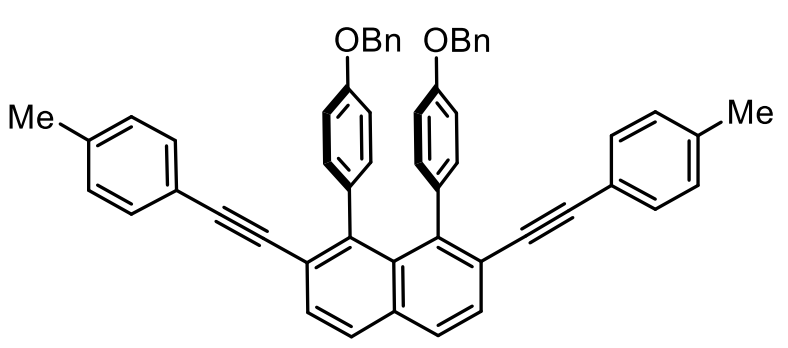

Prepared as a white solid (52.0 $\mathrm{mg}, 72.1$ $\mu \mathrm{mol}, 46 \%$ yield) using alkyne 188b (1.0 equiv, $80.0 \mathrm{mg}, \quad 156 \mu \mathrm{mol})$, 4(benzyloxy)phenylboronic acid (4.0 equiv, $141.0 \mathrm{mg}, 618 \mu \mathrm{mol})$, cesium carbonate $(3.0$ equiv, $152.0 \mathrm{mg}, 467 \mu \mathrm{mol})$, THF (3.4 ml), $\mathrm{H}_{2} \mathrm{O}$ (0.34 ml), $\mathrm{Pd}_{2}(\mathrm{dba})_{3}(4.0 \mathrm{~mol} \%, 5.7 \mathrm{mg}, 6.2 \mu \mathrm{mol})$, Sphos (8.0 mol\%, $\left.5.1 \mathrm{mg}, 12.4 \mu \mathrm{mol}\right)$ according to GPC. The mixture was heated for $16 \mathrm{~h}$; column chromatography: $35 \%$ toluene, then $40-60 \%$ toluene in pentane).

'H NMR: $\left(300 \mathrm{MHz}, \mathrm{C}_{6} \mathrm{D}_{6}\right) \delta=7.78(\mathrm{~d}, J=8.5 \mathrm{~Hz}, 2 \mathrm{H}), 7.53(\mathrm{~d}, J=8.5 \mathrm{~Hz}, 2 \mathrm{H}), 7.34-7.26$ $(\mathrm{m}, 4 \mathrm{H}), 7.25-7.17(\mathrm{~m}, 6 \mathrm{H}), 7.14-7.04(\mathrm{~m}, 4 \mathrm{H}), 6.86(\mathrm{dd}, J=8.8,2.3 \mathrm{~Hz}, 4 \mathrm{H}), 6.79-6.69$ $(\mathrm{m}, 4 \mathrm{H}), 6.60(\mathrm{dd}, J=8.8,2.3 \mathrm{~Hz}, 4 \mathrm{H}), 4.77(\mathrm{~s}, 4 \mathrm{H}), 1.91(\mathrm{~s}, 6 \mathrm{H}) \mathrm{ppm} ;{ }^{13} \mathrm{C}\left\{{ }^{1} \mathrm{H}\right\}$ NMR: $(75$ $\left.\mathrm{MHz}, \mathrm{C}_{6} \mathrm{D}_{6}\right) \delta=157.7,144.1,138.2,138.0,134.8,134.4,132.8,132.3,131.8,129.3,129.2$, 128.9, 128.7, 127.8, 127.6, 125.1, 121.2, 113.9, 95.4, 90.6, 70.3, 21.3 ppm; IR: (neat, $\left.\mathrm{cm}^{-1}\right) \tilde{v}$ $=531,697,737,804,817,851,1004,1030,1171,1241,1275,1373,1456,1502,1605$, 2857, 2911, 2953, 3031; HRMS: calcd $\mathrm{m} / \mathrm{z}$ for $\mathrm{C}_{54} \mathrm{H}_{40} \mathrm{NaO}_{2}{ }^{+}[\mathrm{M}+\mathrm{Na}]^{+}:$743.2921; found (ESI) 743.2932.

\section{1,8-Bis[4-(methoxymethyl)phenyl]-2,7-bis(p-tolylethynyl)naphthalene (159kb):}

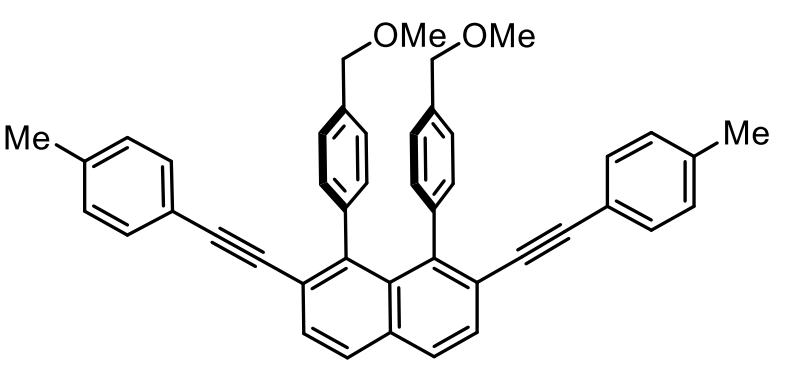

Prepared as a white solid (50.0 $\mathrm{mg}, 83.7$ $\mu \mathrm{mol}, 54 \%$ yield) using alkyne 188b (1.0 equiv, $80.0 \quad \mathrm{mg}, \quad 156 \mu \mathrm{mol})$, 4(methoxymethyl)phenylboronic acid (4.0 equiv, $103.0 \mathrm{mg}, 621 \mu \mathrm{mol})$, cesium carbonate (3.0 equiv, $152.0 \mathrm{mg}, 467 \mu \mathrm{mol}$ ),

THF (3.4 ml), $\mathrm{H}_{2} \mathrm{O}$ (0.34 ml), $\mathrm{Pd}_{2}(\mathrm{dba})_{3}(4.0 \mathrm{~mol} \%, 5.7 \mathrm{mg}, 6.2 \mu \mathrm{mol})$, Sphos $(8.0 \mathrm{~mol} \%, 5.1$ $\mathrm{mg}, 12.4 \mu \mathrm{mol}$ ) according to GPC. The mixture was heated for $16 \mathrm{~h}$; column chromatography: $40 \%$ ethyl acetate in pentane, then crystallization by layering pentane over a toluene solution.

'H NMR: $\left(400 \mathrm{MHz}, \mathrm{CDCl}_{3}\right) \delta=7.85(\mathrm{~d}, J=8.5 \mathrm{~Hz}, 2 \mathrm{H}), 7.66(\mathrm{~d}, J=8.5 \mathrm{~Hz}, 2 \mathrm{H}), 7.00-6.96$ $(\mathrm{m}, 4 \mathrm{H}), 6.96-6.86(\mathrm{~m}, 12 \mathrm{H}), 4.36(\mathrm{~s}, 4 \mathrm{H}), 3.34(\mathrm{~s}, 6 \mathrm{H}), 2.27(\mathrm{~s}, 6 \mathrm{H}) \mathrm{ppm} ;{ }^{13} \mathrm{C}\left\{{ }^{1} \mathrm{H}\right\}$ NMR: $(75$ $\left.\mathrm{MHz}, \mathrm{CDCl}_{3}\right) \delta=143.4,140.9,138.3,135.8,133.8,131.4,131.4,131.1,129.0,128.9,128.3$, 


\section{Experimental}

126.3, 124.3, 120.3, 94.7, 89.6, 74.6, 57.8, 21.6 ppm; IR: $\left(\right.$ neat, $\left.\mathrm{cm}^{-1}\right) \tilde{v}=521,611,729$, $817,849,918,1085,1178,1207,1329,1358,1404,1448,1514,2820$, 2846, 2899, 2995, 3046; HRMS: calcd. $\mathrm{m} / z$ for $\mathrm{C}_{44} \mathrm{H}_{36} \mathrm{NaO}_{2}{ }^{+}[\mathrm{M}+\mathrm{Na}]^{+}:$619.2608; found (ESI) 619.2603.

\section{1,8-Bis[4-(methoxymethyl)phenyl]-7,14-di(p-tolyl)-2,9-bis(p-tolylethynyl)pentaleno[2,1-} a:5,4-a']dinaphthalene (192):

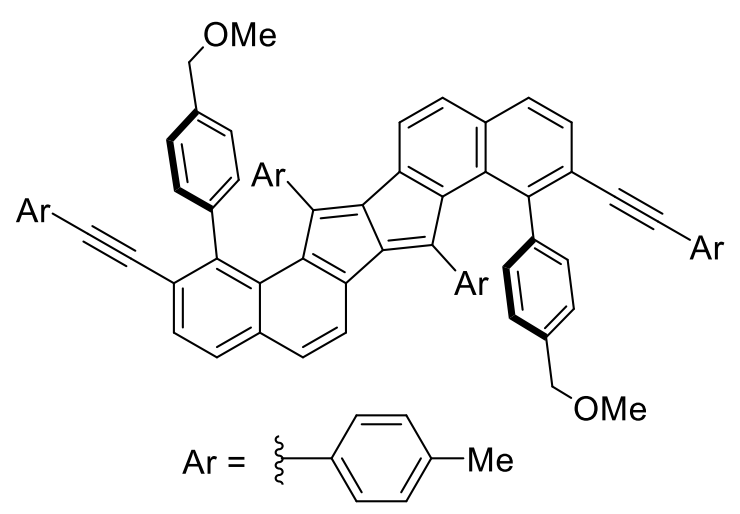

Isolated as a dark red solid $(1.7 \mathrm{mg}, 1.78 \mu \mathrm{mol}$, $2 \%$ yield) by recrystallizing the remainder of the mother liquor from toluene/pentane.

${ }^{1} \mathrm{H}$ NMR: $\left(400 \mathrm{MHz}, \mathrm{CDCl}_{3}\right) \delta=7.44(\mathrm{~d}, J=8.6$ $\mathrm{Hz}, 2 \mathrm{H}), 7.34(\mathrm{~d}, \mathrm{~J}=8.4 \mathrm{~Hz}, 2 \mathrm{H}), 7.18-7.11$ $(\mathrm{m}, 6 \mathrm{H}), 7.10-6.96(\mathrm{~m}, 14 \mathrm{H}), 6.91-6.85(\mathrm{~m}$, $4 \mathrm{H}), 6.57-6.50(\mathrm{~m}, 4 \mathrm{H}), 4.49(\mathrm{~s}, 4 \mathrm{H}), 3.46(\mathrm{~s}$, $6 \mathrm{H}), 2.37(\mathrm{~s}, 6 \mathrm{H}), 2.32(\mathrm{~s}, 6 \mathrm{H}) \mathrm{ppm} ;{ }^{13} \mathrm{C}\left\{{ }^{1} \mathrm{H}\right\}$ 137.2 , 135.9, 135.6, 133.1, 131.2, 130.3, 128.8, 128.7, 128.5, 128.4, 127.9, 126.2, 122.1 , 120.6, 119.2, 93.1, 90.5, 74.9, 58.2, 21.7, 21.7 ppm; IR: (neat, $\left.\mathrm{cm}^{-1}\right) \tilde{v}=522,727,814$, 1017, 1093, 1185, 1223, 1258, 1372, 1444, 1512, 1580, 2333, 2360, 2822, 2849, 2920, 3029; HRMS: calcd $m / z$ for $\mathrm{C}_{72} \mathrm{H}_{54} \mathrm{NaO}_{2}{ }^{+}[\mathrm{M}+\mathrm{Na}]^{+}:$973.4016; found (ESI) 973.4001 .

\section{1,8-Di([1,1'-biphenyl]-4-yl)-2,7-bis(p-tolylethynyl)naphthalene (159lb):}

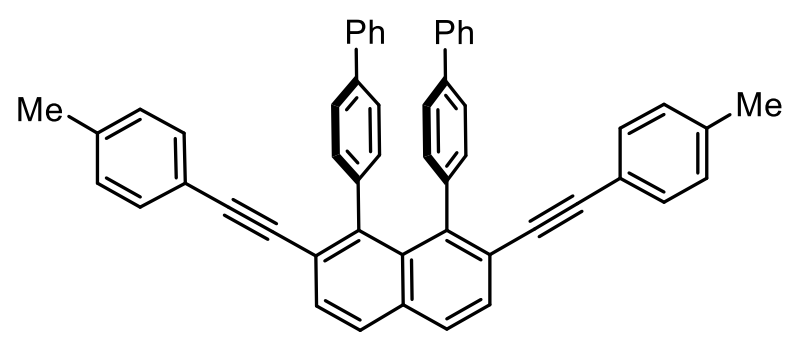

Prepared as a purple solid $(25.0 \mathrm{mg}, 37.8$ $\mu \mathrm{mol}, 24 \%$ yield) using alkyne 188b (1.0 equiv, $80.0 \mathrm{mg}, \quad 156 \mu \mathrm{mol})$, 4biphenylboronic acid (4.0 equiv, $124 \mathrm{mg}$, $626 \mu \mathrm{mol})$, cesium carbonate (3.0 equiv, $152.0 \mathrm{mg}, 467 \mu \mathrm{mol})$, THF $(3.4 \mathrm{ml}), \mathrm{H}_{2} \mathrm{O}$ (0.34 ml), $\mathrm{Pd}_{2}(\mathrm{dba})_{3}(4.0 \mathrm{~mol} \%, 5.7 \mathrm{mg}, 6.2 \mu \mathrm{mol})$, Sphos $(8.0 \mathrm{~mol} \%, 5.1 \mathrm{mg}, 12.4 \mu \mathrm{mol})$ according to GPC. The mixture was heated for $16 \mathrm{~h}$; column chromatography: $40 \%$ toluene in pentane.

Literature corresponded to those previously reported. ${ }^{[184]}$

'H NMR: $\left(400 \mathrm{MHz}, \mathrm{CDCl}_{3}\right) \delta=7.89(\mathrm{~d}, J=8.4 \mathrm{~Hz}, 2 \mathrm{H}), 7.70(\mathrm{~d}, J=8.4 \mathrm{~Hz}, 2 \mathrm{H}), 7.41-7.38$ (m, 4H), $7.36-7.31(\mathrm{~m}, 6 \mathrm{H}), 7.20(\mathrm{~d}, J=8.0 \mathrm{~Hz}, 4 \mathrm{H}), 7.00(\mathrm{~d}, J=8.0 \mathrm{~Hz}, 4 \mathrm{H}), 6.93(\mathrm{~d}, J=$ $7.9 \mathrm{~Hz}, 4 \mathrm{H}), 6.87$ (d, J = $7.9 \mathrm{~Hz}, 4 \mathrm{H}), 2.25$ (s, 6H) ppm; ${ }^{13} \mathrm{C} \mathrm{NMR:}\left(101 \mathrm{MHz}, \mathrm{CDCl}_{3}\right) \delta:=$ 143.4 , 141.6, 140.7, 139.0, 138.3, 133.7, 131.7, 131.4, 131.2, 129.0, 128.7, 128.7, 128.4, 


\section{Experimental}

127.4, 127.0, 125.8, 124.2, 120.2, 95.1, 89.7, 21.6 ppm; IR: $\left(\right.$ neat, $\left.\mathrm{cm}^{-1}\right) \tilde{v}=509,566,578$, $612,641,693,721,735,758,823,890,963,1021,1073,1107,1153,1181,1209,1261$, $1278,1305,1360,1378,1403,1429$, 1446, 1486, 1511, 1579, 1598, 2859, 2874, 2915, 2944, 2964, 3021; HRMS: calcd $m / z$ for $\mathrm{C}_{52} \mathrm{H}_{36}{ }^{+}[\mathrm{M}]^{+}: 660.2817$; found (EI) 660.2819.

\section{1,8-bis(3,5-Dimethoxyphenyl)-2,7-bis(p-tolylethynyl)naphthalene (159mb):}

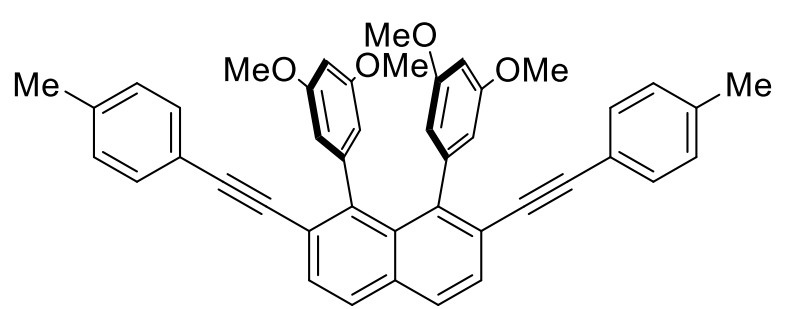

Prepared as an off-white solid $(62.0 \mathrm{mg}, 98.6$ $\mu \mathrm{mol}, 63 \%$ yield) using alkyne 188b (1.0 equiv, $80.0 \mathrm{mg}, 156 \mu \mathrm{mol})$, 3-5-dimethoxyphenylboronic acid (3.0 equiv, $84.9 \mathrm{mg}, 467$ $\mu \mathrm{mol})$, cesium carbonate (3.0 equiv, 152.0 $\mathrm{mg}, 467 \mu \mathrm{mol})$, THF (3.4 ml), $\mathrm{H}_{2} \mathrm{O}(0.34 \mathrm{ml}), \mathrm{Pd}_{2}(\mathrm{dba})_{3}(4.0 \mathrm{~mol} \%, 5.7 \mathrm{mg}, 6.2 \mu \mathrm{mol})$, Sphos $(8.0 \mathrm{~mol} \%, 5.1 \mathrm{mg}, 12.4 \mu \mathrm{mol})$ according to GPC. The mixture was heated for $16 \mathrm{~h}$; column chromatography: 0 to $1 \%$ ethyl acetate in toluene, then precipitation from dichloromethane/pentane.

'H NMR: $\left(300 \mathrm{MHz}, \mathrm{CDCl}_{3}\right) \delta=7.84(\mathrm{~d}, J=8.5 \mathrm{~Hz}, 2 \mathrm{H}), 7.66(\mathrm{~d}, J=8.5 \mathrm{~Hz}, 2 \mathrm{H}), 7.06-6.97$ $(\mathrm{m}, 8 \mathrm{H}), 6.19-6.13(\mathrm{~m}, 6 \mathrm{H}), 3.68(\mathrm{~s}, 12 \mathrm{H}), 2.30(\mathrm{~s}, 6 \mathrm{H}) \mathrm{ppm} ;{ }^{13} \mathbf{C}\left\{{ }^{1} \mathrm{H}\right\} \mathbf{N M R}:(75 \mathrm{MHz}$, $\left.\mathrm{CDCl}_{3}\right) \delta=159.1,143.2,142.7,138.3,133.8,131.5,130.9,129.1,128.9,128.3,123.8$, 120.4, 110.0, 99.3, 94.8, 89.5, 55.2, 21.6 ppm; IR: (neat, $\left.\mathrm{cm}^{-1}\right) \tilde{v}=521,697,924,1062$, 1152, 1201, 1365, 1417, 1454, 1516, 1591, 2836, 2911, 2935, 2984; HRMS: calcd m/z for $\mathrm{C}_{44} \mathrm{H}_{37} \mathrm{O}_{4}{ }^{+}[\mathrm{M}+\mathrm{H}]^{+}$: 629.2686; found (ESI) 629.2683.

\section{1,8-Bis(3,5-dimethylphenyl)-2,7-bis(p-tolylethynyl)naphthalene (159nb):}

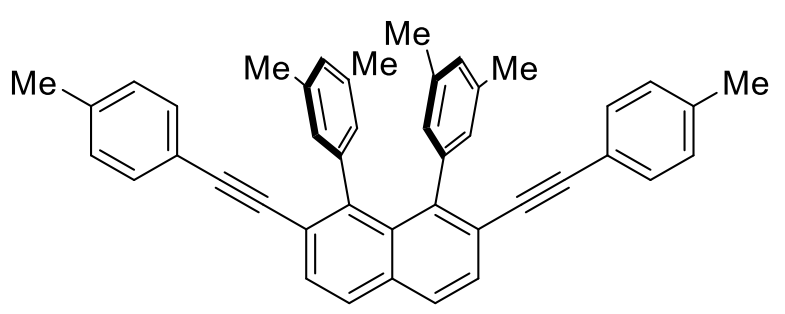

Prepared as an off-white solid (30.0 mg, 53.1 $\mu \mathrm{mol}, 34 \%$ yield) using alkyne 188b (1.0 equiv, $\quad 155.5 \quad \mu \mathrm{mol}), \quad 3-5-$ dimethylphenylboronic acid (3.0 equiv, 70.2 $\mathrm{mg}, 468 \mu \mathrm{mol})$, cesium carbonate (3.0 equiv,

$152.0 \mathrm{mg}, 466.5 \mu \mathrm{mol}), \mathrm{THF}(3.4 \mathrm{ml}), \mathrm{H}_{2} \mathrm{O}$ (0.34 ml), $\mathrm{Pd}_{2}(\mathrm{dba})_{3}(4.0 \mathrm{~mol} \%, 5.7 \mathrm{mg}, 6.2 \mu \mathrm{mol})$, Sphos $(8.0 \mathrm{~mol} \%, 5.1 \mathrm{mg}, 12.4 \mu \mathrm{mol})$ according to GPC. The mixture was heated for $16 \mathrm{~h}$; column chromatography: 20 to $40 \%$ toluene in pentane, then precipitation from dichloromethane/pentane.

'H NMR: $\left(300 \mathrm{MHz}, \mathrm{CDCl}_{3}\right) \delta=7.80(\mathrm{~d}, J=8.5 \mathrm{~Hz}, 2 \mathrm{H}), 7.64(\mathrm{~d}, J=8.4 \mathrm{~Hz}, 2 \mathrm{H}), 7.07-6.92$ $(\mathrm{m}, 8 \mathrm{H}), 6.62-6.58(\mathrm{~m}, 2 \mathrm{H}), 6.57-6.51(\mathrm{~m}, 4 \mathrm{H}), 2.29(\mathrm{~s}, 6 \mathrm{H}), 2.15(\mathrm{q}, J=0.6 \mathrm{~Hz}, 12 \mathrm{H})$ ppm; ${ }^{13} \mathrm{C}\left\{{ }^{1} \mathrm{H}\right\}$ NMR: $\left(101 \mathrm{MHz}, \mathrm{CDCl}_{3}\right) \delta=144.2,140.8,138.1,135.5,133.9,131.7,131.4$, 129.1, 129.0, 128.8, 127.8, 127.5, 123.6, 120.6, 94.3, 90.0, 21.6, 21.3 ppm; IR: (neat, $\left.\mathrm{cm}^{-1}\right) \tilde{v}$ 


\section{Experimental}

$=524,709,741,809,847,1036,1336,1374,1442,1512,1599,1907,2855,2915,2950$, 3023; HRMS: calcd. $m / z$ for $\mathrm{C}_{44} \mathrm{H}_{36}{ }^{+}[\mathrm{M}]^{+}:$564.2817; found (El) 564.2838.

\section{2,7-Bis((4-methoxyphenyl)ethynyl)-1,8-diphenylnaphthalene (159ag):}

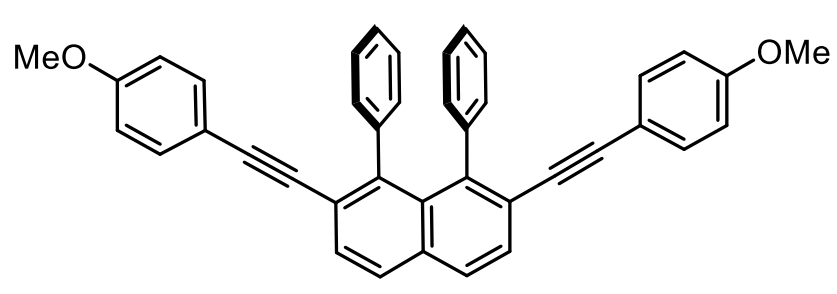

Prepared as a white solid $(52.0 \mathrm{mg}, 96.1$ $\mu \mathrm{mol}, 53 \%$ yield) using diyne $188 \mathrm{c}(1.0$ equiv, $100 \mathrm{mg}, 183 \mu \mathrm{mol})$, phenylboronic acid (4.0 equiv, $89.3 \mathrm{mg}, 732 \mu \mathrm{mol}$ ), cesium carbonate (3.0 equiv, $179 \mathrm{mg}$, $549 \mu \mathrm{mol})$, THF $(4 \mathrm{ml}), \mathrm{H}_{2} \mathrm{O}(0.4 \mathrm{ml}), \mathrm{Pd}_{2}(\mathrm{dba})_{3}(4.0 \mathrm{~mol} \%, 6.7 \mathrm{mg}, 7.3 \mu \mathrm{mol})$, Sphos $(8.0$ mol\%, $6.0 \mathrm{mg}, 14.6 \mu \mathrm{mol}$ ) according to GPC. The mixture was heated for $16 \mathrm{~h}$; column chromatography: $50 \%$ toluene in hexanes, then crystallization from dichloromethane/pentane.

Analytical data corresponded to those reported above in this thesis.

\section{2,7-Bis[(4-methoxyphenyl)ethynyl]-1,8-di-p-tolylnaphthalene (159pg):}

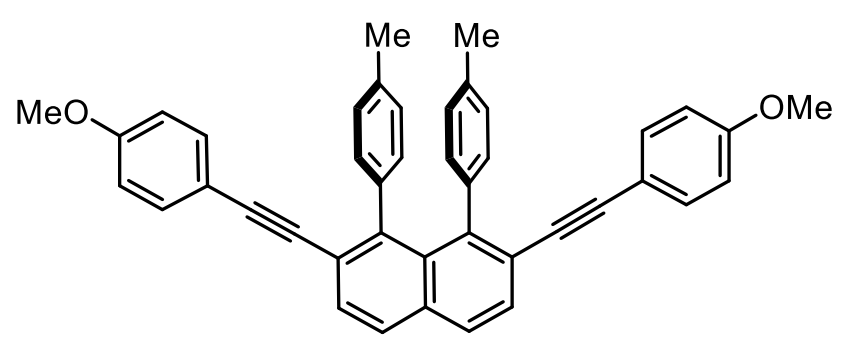

Prepared as a white solid (52.0 $\mathrm{mg}, 91.4$ $\mu \mathrm{mol}, 50 \%$ yield) using diyne $188 \mathrm{c}(1.0$ equiv, $100 \mathrm{mg}, 183 \mu \mathrm{mol})$, $p$-tolylboronic acid (4.0 equiv, $99.5 \mathrm{mg}, 731.8 \mu \mathrm{mol}$ ), cesium carbonate (3.0 equiv, $179 \mathrm{mg}$, $549 \mu \mathrm{mol}), \operatorname{THF}(4 \mathrm{ml}), \mathrm{H}_{2} \mathrm{O}(0.4 \mathrm{ml})$,

$\mathrm{Pd}_{2}(\mathrm{dba})_{3}(4.0 \mathrm{~mol} \%, 6.7 \mathrm{mg}, 7.3 \mu \mathrm{mol})$, Sphos $(8.0 \mathrm{~mol} \%, 6.0 \mathrm{mg}, 14.6 \mu \mathrm{mol})$ according to GPC. The mixture was heated for $16 \mathrm{~h}$; column chromatography: $50 \%$ toluene in hexanes, then crystallization from dichloromethane/pentane.

'H NMR: $\left(300 \mathrm{MHz}, \mathrm{CDCl}_{3}\right) \delta=7.82(\mathrm{~d}, J=8.5 \mathrm{~Hz}, 2 \mathrm{H}), 7.63(\mathrm{~d}, J=8.4 \mathrm{~Hz}, 2 \mathrm{H}), 6.95(\mathrm{dd}, J$ = 9.0, $2.3 \mathrm{~Hz}, 4 \mathrm{H}$ ), 6.76 (s, 8H), 6.71 (dd, J = 9.0, $2.5 \mathrm{~Hz}, 4 \mathrm{H}), 3.76(\mathrm{~s}, 6 \mathrm{H}), 2.28(\mathrm{~s}, 6 \mathrm{H}) \mathrm{ppm}$; ${ }^{13} \mathrm{C}\left\{{ }^{1} \mathrm{H}\right\}$ NMR: $\left(101 \mathrm{MHz}, \mathrm{CDCl}_{3}\right) \delta=159.5,143.7,138.6,135.2,133.0,131.1,128.6,128.1$, 127.4, 124.2, 115.8, 113.9, 94.3, 89.3, 55.4, 21.3 ppm; IR: $\left(\right.$ neat, $\left.\mathrm{cm}^{-1}\right) \tilde{v}=529,770,809$, $833,1028,1104,1174,1250,1287,1442,1458,1507,1602,2172,2203,2836,2920,2958$, 3001; HRMS: calcd $\mathrm{m} / \mathrm{z}$ for $\mathrm{C}_{42} \mathrm{H}_{32} \mathrm{NaO}_{2}{ }^{+}[\mathrm{M}+\mathrm{Na}]^{+}:$591.2295; found (ESI) 591.2284. 
[\{2,7-bis[(4-methoxyphenyl)ethynyl]naphthalene-1,8-diyl\}bis(4,1phenylene)]bis(trimethylsilane) (159ig):

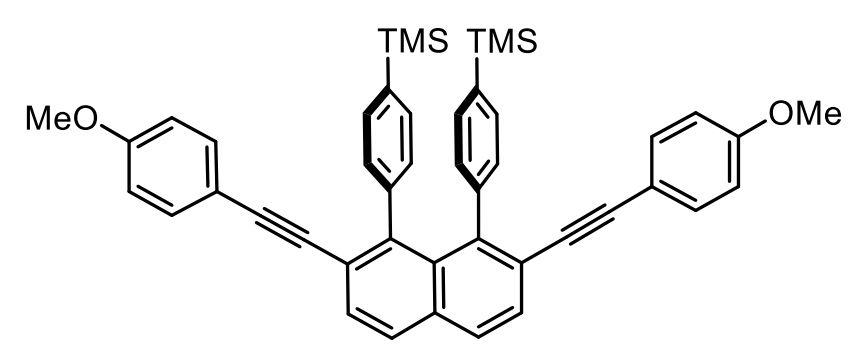

Prepared as a white solid (31.0 mg, 45.2 $\mu \mathrm{mol}, 25 \%$ yield) using alkyne $188 \mathrm{c}(1.0$ equiv, $100.0 \mathrm{mg}, 183 \mu \mathrm{mol})$, 4(trimethylsilyl)phenylboronic acid $(4.0$ equiv, $142 \mathrm{mg}, 732 \mu \mathrm{mol})$, cesium carbonate (3.0 equiv, $179 \mathrm{mg}, 549 \mu \mathrm{mol})$,

THF (4 ml), $\mathrm{H}_{2} \mathrm{O}(0.4 \mathrm{ml}), \mathrm{Pd}_{2}(\mathrm{dba})_{3}(4.0 \mathrm{~mol} \%, 6.7 \mathrm{mg}, 7.3 \mu \mathrm{mol})$, Sphos (8.0 mol\%, $6.0 \mathrm{mg}$, $14.6 \mu \mathrm{mol})$ according to GPC. The mixture was heated for $16 \mathrm{~h}$; column chromatography: $50 \%$ toluene in hexanes, then crystallization from dichloromethane/pentane.

'H NMR: $\left(300 \mathrm{MHz}, \mathrm{CDCl}_{3}\right) \delta=7.85(\mathrm{~d}, J=8.5 \mathrm{~Hz}, 2 \mathrm{H}), 7.65(\mathrm{~d}, J=8.4 \mathrm{~Hz}, 2 \mathrm{H}), 7.15$ (dd, $J$ $=8.0,1.3 \mathrm{~Hz}, 4 \mathrm{H}), 6.95-6.86(\mathrm{~m}, 8 \mathrm{H}), 6.70(\mathrm{dd}, J=8.9,2.5 \mathrm{~Hz}, 4 \mathrm{H}), 3.77(\mathrm{~s}, 6 \mathrm{H}), 0.28(\mathrm{~s}$, $18 \mathrm{H})$ ppm; ${ }^{13} \mathrm{C}\left\{{ }^{1} \mathrm{H}\right\}$ NMR: $\left(101 \mathrm{MHz}, \mathrm{CDCl}_{3}\right) \delta=159.5,143.7,142.2,137.2,133.0,131.7$, 130.7, 128.5, 128.1, 124.3, 115.6, 113.8, 94.4, 89.0, 55.4, -0.7 ppm; IR: (neat, $\mathrm{cm}^{-1}$ ) $\tilde{v}=$ 527, 631, 810, 826, 1031, 1248, 1283, 1509, 1566, 1601, 2202, 2213, 2831, 2893, 2948, 2994, 3061; HRMS: calcd $m / z$ for $\mathrm{C}_{46} \mathrm{H}_{44} \mathrm{NaO}_{2} \mathrm{Si}_{2}{ }^{+}[\mathrm{M}+\mathrm{Na}]^{+}:$707.2772; found (ESI) 707.2772.

\section{1,8-Bis(3,5-dimethylphenyl)-2,7-bis[(4-methoxyphenyl)ethynyl]naphthalene (159ng):}

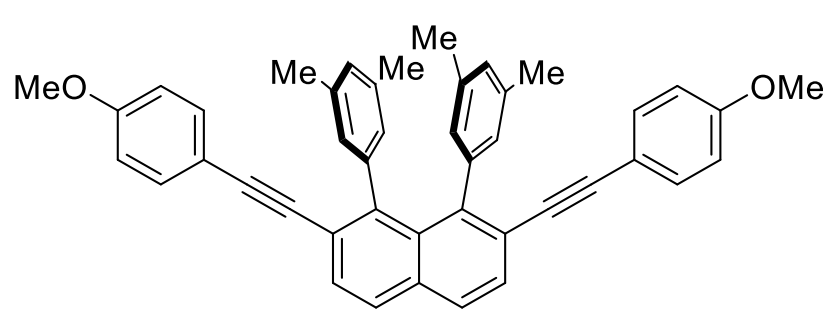

Prepared as a white solid $(43.0 \mathrm{mg}, 72.0$ $\mu \mathrm{mol}, 39 \%$ yield) using diyne 188c (1.0 equiv, $100 \mathrm{mg}, 183 \mu \mathrm{mol})$, 3,5dimethylphenylboronic acid (4.0 equiv, $110 \mathrm{mg}, 733 \mu \mathrm{mol})$, cesium carbonate

(3.0 equiv, $179 \mathrm{mg}, 549 \mu \mathrm{mol})$, THF (4 ml), $\mathrm{H}_{2} \mathrm{O}(0.4 \mathrm{ml}), \mathrm{Pd}_{2}(\mathrm{dba})_{3}(4.0 \mathrm{~mol} \%, 6.7 \mathrm{mg}, 7.3$ $\mu \mathrm{mol})$, Sphos (8.0 mol\%, $6.0 \mathrm{mg}, 14.6 \mu \mathrm{mol})$ according to GPC. The mixture was heated for $16 \mathrm{~h}$; column chromatography: $60 \%$ toluene in hexanes, then crystallization by slowly cooling a hot ethyl acetate solution to $-20^{\circ} \mathrm{C}$.

'H NMR: $\left(400 \mathrm{MHz}, \mathrm{CDCl}_{3}\right) \delta=7.79(\mathrm{~d}, J=8.4 \mathrm{~Hz}, 2 \mathrm{H}), 7.62(\mathrm{~d}, J=8.4 \mathrm{~Hz}, 2 \mathrm{H}), 7.00$ (dd, $J$ = 8.9, $2.5 \mathrm{~Hz}, 4 \mathrm{H}), 6.74(\mathrm{dd}, J=8.9,2.4 \mathrm{~Hz}, 4 \mathrm{H}), 6.61(\mathrm{~s}, 2 \mathrm{H}), 6.54(\mathrm{~s}, 4 \mathrm{H}), 3.77(\mathrm{~s}, 6 \mathrm{H}), 2.15$ $(\mathrm{s}, 12 \mathrm{H}) \mathrm{ppm} ;{ }^{13} \mathrm{C}\left\{{ }^{1} \mathrm{H}\right\}$ NMR: $\left(101 \mathrm{MHz}, \mathrm{CDCl}_{3}\right) \delta=159.5,143.8,140.9,135.5,132.9,129.1$, 128.6, 127.8, 127.5, 123.7, 115.9, 113.9, 94.2, 89.4, 55.4, 21.3 ppm; IR: (neat, $\mathrm{cm}^{-1}$ ) $\tilde{v}=$ 532, 701, 829, 849, 1025, 1107, 1169, 1243, 1288, 1512, 1602, 2199, 2831, 2918, 2964, 3007; HRMS: calcd $m / z$ for $\mathrm{C}_{44} \mathrm{H}_{36} \mathrm{NaO}_{2}{ }^{+}[\mathrm{M}+\mathrm{Na}]^{+}: 619.2608$; found (ESI) 619.2595. 


\section{Experimental}

\section{1,8-Bis(4-fluorophenyl)-2,7-bis[(4-methoxyphenyl)ethynyl]naphthalene (159qg):}

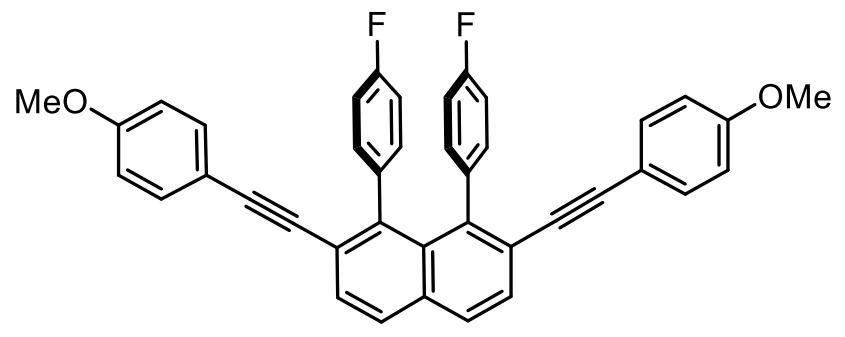

Prepared as a white solid $(23.0 \mathrm{mg}, 39.8$ $\mu \mathrm{mol}, 34 \%$ yield) using diyne 188c (1.0 equiv, $64.3 \mathrm{mg}, 118 \mu \mathrm{mol})$, fluorophenylboronic acid (4.0 equiv, 66.0 $\mathrm{mg}, 472 \mu \mathrm{mol})$, cesium carbonate (2.8 equiv, $107 \mathrm{mg}, 328 \mu \mathrm{mol})$, THF $(2.6 \mathrm{ml})$, $\mathrm{H}_{2} \mathrm{O}$ (0.26 ml), $\mathrm{Pd}_{2}(\mathrm{dba})_{3}(4.0 \mathrm{~mol} \%, 4.3 \mathrm{mg}, 4.69 \mu \mathrm{mol})$, Sphos $(8.1 \mathrm{~mol} \%, 3.9 \mathrm{mg}, 9.49$ $\mu \mathrm{mol})$ according to GPC. The mixture was heated for $16 \mathrm{~h}$; column chromatography: $60 \%$ toluene in hexanes, then crystallization from dichloromethane/pentane.

'H NMR: $\left(400 \mathrm{MHz}, \mathrm{CDCl}_{3}\right) \delta=7.85(\mathrm{~d}, J=8.5 \mathrm{~Hz}, 2 \mathrm{H}), 7.65(\mathrm{~d}, J=8.4 \mathrm{~Hz}, 2 \mathrm{H}), 6.99(\mathrm{dd}, J$ $=8.5,2.4 \mathrm{~Hz}, 4 \mathrm{H}$ ), 6.87 (ddd, $\left.J=8.9,2.4 \mathrm{~Hz}, J_{H-F}=5.6 \mathrm{~Hz}, 4 \mathrm{H}\right), 6.78-6.70(\mathrm{~m}, 8 \mathrm{H}), 3.77(\mathrm{~s}$, $6 \mathrm{H}) \mathrm{ppm} ;{ }^{13} \mathrm{C}\left\{{ }^{1} \mathrm{H}\right\}$ NMR: $\left(101 \mathrm{MHz}, \mathrm{CDCl}_{3}\right) \delta=161.3\left(\mathrm{~d}, J_{C-F}=245.2 \mathrm{~Hz}\right), 159.6,141.6,137.4$ $\left(\mathrm{d}, J_{C-F}=3.6 \mathrm{~Hz}\right), 133.4,132.8,132.7\left(\mathrm{~d}, J_{C-F}=8.6 \mathrm{~Hz}\right), 131.1,128.6,128.4,124.6,115.2$, 113.9, $113.8\left(\mathrm{~d}, J_{C-F}=21.9 \mathrm{~Hz}\right), 94.8,88.5,55.3 \mathrm{ppm} ;{ }^{19} \mathrm{~F}$ NMR: $\left(376 \mathrm{MHz}, \mathrm{CDCl}_{3}\right) \delta=-$ 116.6 (tt, $J=8.9,5.5 \mathrm{~Hz}$ ) ppm; IR: (neat, $\mathrm{cm}^{-1}$ ) $\tilde{v}=519,568,774,799,840,1016,1031$, 1152, 1177, 1218, 1245, 1288, 1500, 1512, 1593, 2162, 2211, 2842, 2904, 2939, 2967; HRMS: calcd $m / z$ for $\mathrm{C}_{40} \mathrm{H}_{26} \mathrm{~F}_{2} \mathrm{O}_{2}{ }^{+}[\mathrm{M}]^{+}:$576.1901; found (EI) 576.1906.

\section{2,7-Bis[(4-chlorophenyl)ethynyl]-1,8-bis(4-methoxyphenyl)naphthalene (159be):}

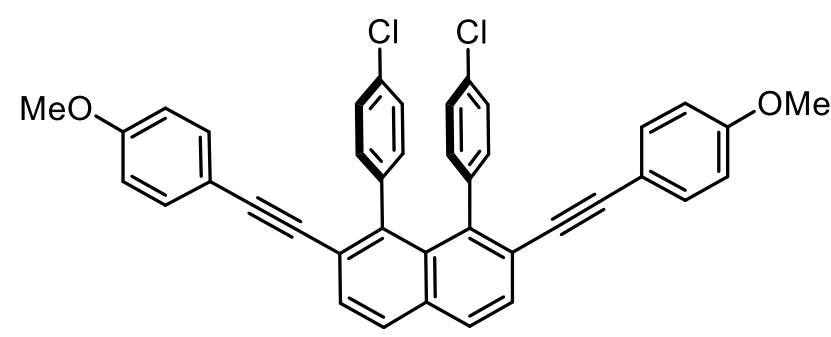

Prepared as a white solid (105 mg, 172 $\mu \mathrm{mol}, 63 \%$ yield) from diyne $186 \mathrm{c}^{[219]}(1.0$ equiv, $107 \mathrm{mg}, 275 \mu \mathrm{mol})$, 1-chloro-4iodobenzene (4.0 equiv, $252 \mathrm{mg}, 1.07$ $\mathrm{mmol}), \mathrm{Pd}\left(\mathrm{PPh}_{3}\right)_{4}(5.0 \mathrm{~mol} \%, 16.0 \mathrm{mg}$, $13.8 \mu \mathrm{mol})$, Cul $(9.5 \mathrm{~mol} \%, 5.0 \mathrm{mg}, 26.3$ $\mu \mathrm{mol}), \mathrm{Pr}_{2} \mathrm{NH}(4.9 \mathrm{ml})$ and THF $(2.4 \mathrm{ml})$ according to GPA; stirring at room temperature for 3 h, column chromatography: $60 \%$ toluene in pentane.

'H NMR: $\left(400 \mathrm{MHz}, \mathrm{CDCl}_{3}\right) \delta=7.85(\mathrm{~d}, J=8.5 \mathrm{~Hz}, 2 \mathrm{H}), 7.65(\mathrm{~d}, J=8.5 \mathrm{~Hz}, 2 \mathrm{H}), 7.03(\mathrm{dd}, J$ $=8.6,2.1 \mathrm{~Hz}, 4 \mathrm{H}), 6.97$ (dd, $J=8.9,2.5 \mathrm{~Hz}, 4 \mathrm{H}), 6.83(\mathrm{dd}, J=8.3,2.0 \mathrm{~Hz}, 4 \mathrm{H}), 6.75$ (dd, $J=$ 8.9, $2.3 \mathrm{~Hz}, 4 \mathrm{H}), 3.77(\mathrm{~s}, 6 \mathrm{H}) \mathrm{ppm} ;{ }^{13} \mathrm{C}\left\{{ }^{1} \mathrm{H}\right\}$ NMR: $\left(101 \mathrm{MHz}, \mathrm{CDCl}_{3}\right) \delta=159.8,141.4,140.0$, 133.5, 132.9, 132.7, 132.5, 130.9, 128.7, 128.7, 127.2, 124.5, 115.2, 114.1, 95.3, 88.6, 55.4; IR: $\left(\right.$ neat, $\left.\mathrm{cm}^{-1}\right) \tilde{v}=524,774,821,837,1014,1033,1041,1088,1167,1240,1256,1286$, 1485, 1509, 1604, 20165, 2197, 2836, 2928, 2958, 2994, 3067; HRMS: calcd. $\mathrm{m} / \mathrm{z}$ for $\mathrm{C}_{40} \mathrm{H}_{27} \mathrm{Cl}_{2} \mathrm{O}_{2}{ }^{+}[\mathrm{M}+\mathrm{H}]^{+}:$: 609.1383; found (ESI) 609.1380. 


\section{Experimental}

\subsubsection{Synthesis of chiral cationic phosphonites}

$\{[(3 a S, 8 a S)-2,2-D i m e t h y l-4,4,8,8-t e t r a p h e n y l t e t r a h y d r o-6$

$\lambda^{4}-[1,3]$ dioxolo[4,5e][1,3,2]dioxaphosphepin-6-ylidene](triphenyl- $\lambda^{4}$ phosphaneyl)methyl\}triphenylphosphonium Hexafluoroantimonate (169I):

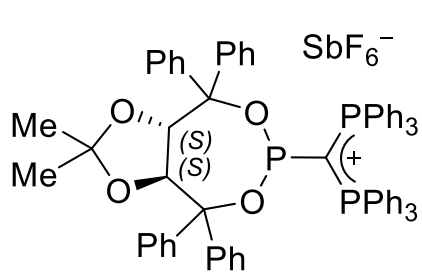

A dried Schlenk flask equipped with magnetic stirring bar was charged with the corresponding diol $(S, S)-\mathbf{1 7 0 |}^{[233]}$ (1.0 equiv, $300 \mathrm{mg}, 643 \mu \mathrm{mol})$, THF (5 ml) and triethylamine (2.19 equiv, $143 \mathrm{mg}, 1.41 \mathrm{mmol})$, before cooling to $-78^{\circ} \mathrm{C}$. In a second dried Schlenk flask, a solution of phosphorus trichloride (1.1 equiv, $97.3 \mathrm{mg}, 709 \mu \mathrm{mol})$ in THF $(5 \mathrm{ml})$ was prepared and added dropwise to the solution of the TADDOL 170I. The resulting white suspension was stirred at $-78{ }^{\circ} \mathrm{C}$ for $1 \mathrm{~h}$, then at room temperature for $1 \mathrm{~h}$, before filtering the liquid phase into a separate dried Schlenk flask. The solvent was removed in vacuo and the crude chlorophosphite dried under high vacuum, before dissolving in THF $(5 \mathrm{ml})$. A solution of hexaphenylcarbodiphosphorane $(202)^{[236]}(1.0$ equiv, $342 \mathrm{mg}, 637 \mu \mathrm{mol})$ in THF $(5 \mathrm{ml})$, prepared in a dried Schlenk flask, was added via canula at room temperature, whereby a white precipitate formed immediately. The reaction was allowed to stir overnight, before filtering and washing with diethyl ether $(3 \times 15 \mathrm{ml})$. The white solid was taken up in acetonitrile $(5 \mathrm{ml})$, and sodium hexafluoroantimonate (2.58 equiv, $430 \mathrm{mg}, 1.66 \mathrm{mmol}$ ) was added. The resulting suspension was stirred overnight, before removing solvent in vacuo and extracting the solid with dichloromethane $(3 \times 20 \mathrm{ml})$, filtering into a dried Schlenk flask. The combined washings were concentrated in vacuo, and the pure product was obtained by recrystallization from dichloromethane/pentane as a white powder (400 mg, $315 \mu \mathrm{mol}, 49 \%$ yield).

$[\alpha]_{20}^{D}:+73.1\left(c=1.00, \mathrm{CH}_{2} \mathrm{Cl}_{2}\right) ;{ }^{1} \mathrm{H}$ NMR: $\left(400 \mathrm{MHz}, \mathrm{CD}_{3} \mathrm{CN}\right) \delta=7.61-7.49(\mathrm{~m}, 18 \mathrm{H}), 7.35$ - $7.28(\mathrm{~m}, 12 \mathrm{H}), 7.27$ - $7.20(\mathrm{~m}, 3 \mathrm{H}), 7.21-7.12(\mathrm{~m}, 7 \mathrm{H}), 7.12-7.02(\mathrm{~m}, 6 \mathrm{H}), 6.98-6.92$ $(\mathrm{m}, 2 \mathrm{H}), 6.38-6.34(\mathrm{~m}, 2 \mathrm{H}), 4.80\left(\mathrm{dd}, J=8.3 \mathrm{~Hz}, J_{H-P}=5.9 \mathrm{~Hz}, 1 \mathrm{H}\right), 4.66(\mathrm{~d}, J=8.2 \mathrm{~Hz}$, $1 \mathrm{H}), 0.90(\mathrm{~s}, 3 \mathrm{H}), 0.21(\mathrm{~s}, 3 \mathrm{H}) \mathrm{ppm} ;{ }^{13} \mathrm{C}\left\{{ }^{1} \mathrm{H}\right\}$ NMR: $\left(101 \mathrm{MHz}, \mathrm{CD}_{3} \mathrm{CN}\right) \delta=146.6,146.0(\mathrm{~d}$, $\left.J_{C-P}=1.6 \mathrm{~Hz}\right), 142.1\left(\mathrm{~d}, J_{C-P}=2.5 \mathrm{~Hz}\right), 141.7,135.7-135.2(\mathrm{~m}), 133.9,130.3,130.0,130.0$ - 129.8 (m), 129.1, 128.9, 128.8, 128.7, 128.7, 128.4, 128.3, 128.2, 127.8, 127.7, 125.8 (dd, $\left.J_{C-P}=92.8,2.6 \mathrm{~Hz}\right), 112.9,86.0,84.5\left(\mathrm{~d}, J_{C-P}=13.4 \mathrm{~Hz}\right), 80.9\left(\mathrm{~d}, J_{C-P}=31.8 \mathrm{~Hz}\right), 80.1\left(\mathrm{~d}, J_{C-}\right.$ $P=3.5 \mathrm{~Hz}), 27.6,26.1,22.1\left(\mathrm{td}, J_{C-P}=84.6,70.4 \mathrm{~Hz}\right) \mathrm{ppm} ;{ }^{31} \mathrm{P}\left\{{ }^{1} \mathrm{H}\right\}$ NMR: $\left(162 \mathrm{MHz}, \mathrm{CD}_{3} \mathrm{CN}\right)$ $\delta=179.5(\mathrm{t}, J=91.8 \mathrm{~Hz}), 20.4(\mathrm{~d}, J=91.9 \mathrm{~Hz}) \mathrm{ppm} ;{ }^{19} \mathrm{~F} \mathrm{NMR:}\left(282 \mathrm{MHz}, \mathrm{CD}_{3} \mathrm{CN}\right) \delta=-$ 123.9 (sext, $J_{F-121 S b}=1938.0 \mathrm{~Hz}$ ), -123.9 (oct, $\left.J_{F-123 S b}=1062.1 \mathrm{~Hz}\right)$ ppm; IR: $\left(\right.$ neat, $\left.\mathrm{cm}^{-1}\right) \tilde{v}=$ 499, 654, 692, 742, 797, 877, 994, 1096, 1164, 1212, 1437, 1484, 1587, 3044, 3057; HRMS: calcd $\mathrm{m} / \mathrm{z}$ for $\mathrm{C}_{68} \mathrm{H}_{58} \mathrm{O}_{4} \mathrm{P}_{3}{ }^{+}\left[\mathrm{M}-\mathrm{SbF}_{6}\right]^{+:}$1031.3542; found (ESI) 1031.3546 . 


\section{Experimental}

\section{$\left\{\left[(5 R, 6 R)-5,6-\right.\right.$ Dimethoxy-4,4,7,7-tetra(4-tert-butylphenyl)-1,3,2 $\lambda^{4}$-dioxaphosphepan-2- ylidene)(triphenyl- $\lambda^{4}$-phosphaneyl]methyl\}triphenylphosphonium \\ Hexafluoroantimonate (169m):}

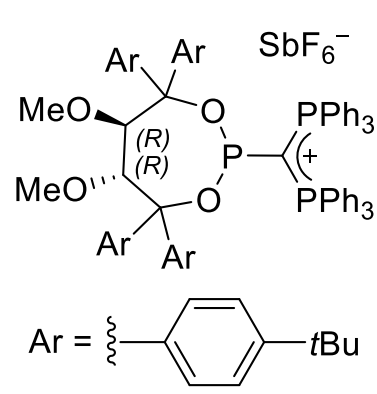

A dried Schlenk flask equipped with magnetic stirring bar was charged with the corresponding diol $\mathbf{1 7 0 g ^ { [ 2 3 4 ] }}$ (1.0 equiv, $400 \mathrm{mg}, 589$ $\mu \mathrm{mol})$, molecular sieves (4 $\mathrm{A}$, powdered, $100 \mathrm{mg}$ ), toluene $(30 \mathrm{ml})$ and triethylamine ( 3.31 equiv, $197 \mathrm{mg}, 1.95 \mathrm{mmol}$ ) before cooling to $0^{\circ}$ and adding phosphorus trichloride (1.32 equiv, $107 \mathrm{mg}, 779$ $\mu \mathrm{mol})$ dropwise via syringe. The mixture was stirred at $0{ }^{\circ} \mathrm{C}$ for a few minutes, then at $60{ }^{\circ} \mathrm{C}$ for one hour, where a white suspension formed. On cooling, the mixture was filtered and solvent removed in vacuo. The crude chlorophosphite was dried under high vacuum, then dissolved in THF $(10 \mathrm{ml})$ and added via cannula to a solution of hexaphenylcarbodiphosphorane $(202)^{[236]}(0.99$ equiv, $314 \mathrm{mg}, 585$ $\mu \mathrm{mol})$ in THF $(10 \mathrm{ml})$. The resulting mixture was allowed to stir overnight at room temperature and monitored by ${ }^{31} \mathrm{P}$ NMR. A second portion of hexaphenylcarbodiphosphorane (0.99 equiv, $314 \mathrm{mg}, 585 \mu \mathrm{mol})$ as a solution in THF (5 ml) was then added, and the reaction mixture allowed to stir at room temperature for $24 \mathrm{~h}$, then heated at $60{ }^{\circ} \mathrm{C}$ for an additional four more hours, whereby full conversion of the chlorophosphite was detected by ${ }^{31} \mathrm{P}$ NMR. The resulting white precipitate was filtered into a dried Schlenk flask and the residual solid was washed with diethyl ether $(2 \times 10 \mathrm{ml})$. The combined washings gave a second precipitate, which was again filtered, and the resulting slightly yellow solution was evaporated to dryness, affording a pale yellow solid. To the crude solid was added acetonitrile $(5 \mathrm{ml})$ and sodium hexafluoroantimonate $(2.78$ equiv, $425 \mathrm{mg}$, $1.64 \mathrm{mmol}$ ) and the resulting suspension stirred overnight, before removing solvent in vacuo and extracting with dichloromethane $(3 \times 15 \mathrm{ml})$. The combined washings were evaporated and the crude residue purified by column chromatography $(2 \%$ ethyl acetate in dichloromethane), affording a white solid (310 mg, $209 \mu \mathrm{mol}, 35 \%$ yield).

$[\alpha]_{20}^{D}:-42.1\left(c=1.04, \mathrm{CH}_{2} \mathrm{Cl}_{2}\right) ;{ }^{1} \mathrm{H}$ NMR: $\left(400 \mathrm{MHz}, \mathrm{CD}_{3} \mathrm{CN}\right) \delta=7.64-7.56(\mathrm{~m}, 6 \mathrm{H}), 7.51-$ $7.39(\mathrm{~m}, 12 \mathrm{H}), 7.39-7.29(\mathrm{~m}, 12 \mathrm{H}), 7.21-7.17(\mathrm{~m}, 2 \mathrm{H}), 7.15-7.07(\mathrm{~m}, 8 \mathrm{H}), 6.88-6.79$ (m, 2H), $6.76-6.66(\mathrm{~m}, 2 \mathrm{H}), 6.50-6.37(\mathrm{~m}, 2 \mathrm{H}), 4.29$ (dd, J = 6.8 Hz, JH-P $=8.5 \mathrm{~Hz}, 1 \mathrm{H})$, $3.84(\mathrm{~d}, J=6.9 \mathrm{~Hz}, 1 \mathrm{H}), 2.70(\mathrm{~s}, 3 \mathrm{H}), 2.40(\mathrm{~s}, 3 \mathrm{H}), 1.34(\mathrm{~s}, 9 \mathrm{H}), 1.30(\mathrm{~s}, 9 \mathrm{H}), 1.26(\mathrm{~s}, 9 \mathrm{H})$, $1.20(\mathrm{~s}, 9 \mathrm{H}) \mathrm{ppm} ;{ }^{13} \mathrm{C}\left\{{ }^{1} \mathrm{H}\right\}$ NMR: $\left(151 \mathrm{MHz}, \mathrm{CD}_{3} \mathrm{CN}\right) \delta=151.2,151.1,150.9,150.8,143.0$, $142.9\left(\mathrm{~d}, J_{C-P}=1.4 \mathrm{~Hz}\right), 134.0,140.0\left(\mathrm{~d}, J_{C-P}=1.7 \mathrm{~Hz}\right), 135.7-135.4(\mathrm{~m}), 133.7,130.0-$ $129.9(\mathrm{~m}), 129.8,129.1,129.1,129.0,129.0,126.0$ (dd, $\left.J_{C-P}=92.9,2.3 \mathrm{~Hz}\right), 125.6,125.0$, 124.8, 124.1, 87.5, $84.3\left(\mathrm{~d}, J_{C-P}=15.5 \mathrm{~Hz}\right), 81.7\left(\mathrm{~d}, J_{C-P}=31.6 \mathrm{~Hz}\right), 81.6,59.5,58.8,35.1$, 
35.0, 35.0, 35.0, 31.7, 31.6, 31.6, 31.5, $20.8\left(\mathrm{td}, J_{C-P}=84.1,71.4 \mathrm{~Hz}\right) \mathrm{ppm} ;{ }^{31} \mathbf{P}\left\{{ }^{1} \mathrm{H}\right\}$ NMR: $\left(162 \mathrm{MHz}, \mathrm{CD}_{3} \mathrm{CN}\right) \delta=175.1(\mathrm{t}, J=100.7 \mathrm{~Hz}), 20.6$ (d, $\left.J=100.7 \mathrm{~Hz}\right) \mathrm{ppm}$; ${ }^{19} \mathrm{~F}$ NMR: (282 $\mathrm{MHz}, \mathrm{CD}_{3} \mathrm{CN}$ ) $\delta=-123.9$ (sext, $J_{\mathrm{F}-121 \mathrm{Sb}}=1928.2 \mathrm{~Hz}$ ), -123.9 (oct, $J_{\mathrm{F}-123 \mathrm{Sb}}=1043.3 \mathrm{~Hz}$ ) ppm; IR: $\left(\right.$ neat, $\left.\mathrm{cm}^{-1}\right) \tilde{v}=500,522,654,691,745,842,897,995,1097,1269,1437,1482,2867$, 2903, 2957; HRMS: calcd $\mathrm{m} / z$ for $\mathrm{C}_{83} \mathrm{H}_{90} \mathrm{O}_{4} \mathrm{P}_{3}{ }^{+}\left[\mathrm{M}-\mathrm{SbF}_{6}\right]^{+}:$1243.6046; found (ESI) 1243.6043.

\section{General procedure D (GPD) for the preparation of cationic phosphonites}

A dried Schlenk flask, equipped with magnetic stirring bar and rubber septum, was charged with molecular sieves ( $4 \AA$, powdered) and the corresponding diol, before drying under vacuum for 30 minutes and purging with argon. Toluene was added through the septum and the mixture cooled to $0{ }^{\circ} \mathrm{C}$, before adding phosphorus trichloride, then pyridine. The mixture was stirred at $0{ }^{\circ} \mathrm{C}$ for a few minutes, then heated at $60{ }^{\circ} \mathrm{C}$ for one hour, then allowed to cool down to room temperature and filtered via cannula into a separate, dried Schlenk flask. The solvent was removed in vacuo, and the crude chlorophosphite dried under high vacuum, before dissolving in diethyl ether and cooling to $-78{ }^{\circ} \mathrm{C}$. In a separate Schlenk flask, a solution of the appropriate amount of the carbene in diethyl ether was prepared and added slowly, dropwise to the chlorophosphite via syringe, or cannula for a larger scale. The resulting suspension was allowed to stir at $-78^{\circ} \mathrm{C}$ for a few minutes, before adding sodium hexafluoroantimonate as a solid and allowing to warm to room temperature overnight. The liquid phase was then filtered off and the remaining solid washed twice with diethyl ether. The crude product was taken up in dichloromethane and filtered into a separate Schlenk flask, before removing the solvent in vacuo and purifying the crude product by column chromatography at $-10^{\circ} \mathrm{C}$.

\section{2-\{(3aS,8aS)-2,2-Dimethyl-4,4,8,8-tetraphenyltetrahydro-[1,3]dioxolo[4,5-}

e][1,3,2]dioxaphosphepin-6-yl\}-1,3-dimesityl-1 H-imidazol-3-ium Hexafluoroantimonate (169n):

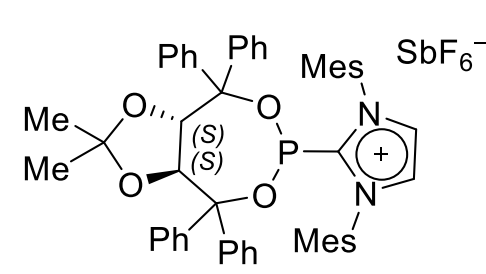

Prepared as a white solid (912 mg, $881 \mu \mathrm{mol}, 69 \%$ yield) from chiral diol $(S, S)-170{ }^{[233]}$ (1.0 equiv, $\left.600 \mathrm{mg}, 1.29 \mathrm{mmol}\right)$, molecular sieves (4 Å, powdered, $150 \mathrm{mg}$ ), pyridine (3.0 equiv, $313 \mathrm{mg}, 3.96 \mathrm{mmol}$ ), phosphorus trichloride (1.80 equiv, 315 $\mathrm{mg}, \quad 2.29 \mathrm{mmol})$ with 1,3-bis(2,4,6-trimethylphenyl)-1,3dihydro-2 $\mathrm{H}$-imidazol-2-ylidene $(\mathbf{5 6 b})^{[274]}(1.0$ equiv, $392 \mathrm{mg}, 1.29 \mathrm{mmol})$ and sodium hexafluoroantimonate (2.49 equiv, $832 \mathrm{mg}, 3.22 \mathrm{mmol}$ ) according to GPD after column chromatography ( 0 to $1.5 \%$ ethyl acetate in dichloromethane) at $-10^{\circ} \mathrm{C}$. 


\section{Experimental}

$[\alpha]_{20}^{D}:+87.1\left(c=1.03, \mathrm{CH}_{2} \mathrm{Cl}_{2}\right) ;{ }^{1} \mathrm{H}$ NMR: $\left(400 \mathrm{MHz}, \mathrm{CD}_{3} \mathrm{CN}\right) \delta=7.78(\mathrm{~s}, 2 \mathrm{H}), 7.44-7.34$ (m, 9H), $7.35-7.28(\mathrm{~m}, 5 \mathrm{H}), 7.27-7.21(\mathrm{~m}, 4 \mathrm{H}), 7.08(\mathrm{~s}, 2 \mathrm{H}), 6.93-6.85(\mathrm{~m}, 2 \mathrm{H}), 6.63(\mathrm{~d}$, $\mathrm{J}=8.0 \mathrm{~Hz}, 2 \mathrm{H}), 5.16\left(\mathrm{dd}, \mathrm{J}=8.0 \mathrm{~Hz}, J_{H-P}=5.6 \mathrm{~Hz} 1 \mathrm{H}\right), 4.66(\mathrm{~d}, \mathrm{~J}=8.5 \mathrm{~Hz}, 1 \mathrm{H}), 2.47(\mathrm{~s}, 6 \mathrm{H})$, $2.08(\mathrm{~s}, 6 \mathrm{H}), 1.71(\mathrm{~s}, 6 \mathrm{H}), 1.31(\mathrm{~s}, 3 \mathrm{H}), 0.00(\mathrm{~s}, 3 \mathrm{H}) \mathrm{ppm} ;{ }^{13} \mathrm{C}\left\{{ }^{1} \mathrm{H}\right\}$ NMR: $\left(101 \mathrm{MHz}, \mathrm{CD}_{3} \mathrm{CN}\right) \delta$ $=145.3\left(\mathrm{~d}, J_{C-P}=64.2 \mathrm{~Hz}\right), 144.7,143.9\left(\mathrm{~d}, J_{C-P}=4.5 \mathrm{~Hz}\right), 142.8,139.8\left(\mathrm{~d}, J_{C-P}=2.6 \mathrm{~Hz}\right)$, $139.2,135.9,135.9,132.0,130.9,130.7,130.2,129.9,129.7,129.7,129.6,129.3,129.3$, 129.2, 129.2, 129.0, 128.6, 128.4, 128.2, 113.7, 87.3 (d, $\left.J_{C-P}=11.6 \mathrm{~Hz}\right), 86.4\left(\mathrm{~d}, J_{C-P}=7.2\right.$ $\mathrm{Hz}), 83.1\left(\mathrm{~d}, J_{C-P}=3.2 \mathrm{~Hz}\right), 81.2\left(\mathrm{~d}, J_{C-P}=28.7 \mathrm{~Hz}\right), 27.6,24.8,21.3,18.1,17.8,17.8 \mathrm{ppm}$; ${ }^{31} \mathrm{P}\left\{{ }^{1} \mathrm{H}\right\}$ NMR: $\left(162 \mathrm{MHz}, \mathrm{CD}_{3} \mathrm{CN}\right) \delta=145.3 \mathrm{ppm} ;{ }^{19} \mathrm{~F}$ NMR: $\left(282 \mathrm{MHz}, \mathrm{CD}_{3} \mathrm{CN}\right) \delta=-124.0$ $\left(\mathrm{sext}, \mathrm{J}_{F-121 S b}=1930.0 \mathrm{~Hz}\right),-124.0\left(\right.$ oct, $\left.\mathrm{J}_{F-123 S b}=1050.0 \mathrm{~Hz}\right) \mathrm{ppm}$; IR (neat, $\left.\mathrm{cm}^{-1}\right) \tilde{v}=654$, 701, 733, 875, 983, 1086, 1113, 1221, 1372, 1447, 1481, 1605, 2918, 2995, 3058, 3169; HRMS calcd $\mathrm{m} / \mathrm{z}$ for $\mathrm{C}_{52} \mathrm{H}_{52} \mathrm{~N}_{2} \mathrm{O}_{4} \mathrm{P}^{+}: 799.3659$ [M-SbF$\left.]_{6}\right]^{+}$; found (ESI) 799.3656.

\section{2-\{(3aS,8aS)-2,2-Dimethyl-4,4,8,8-tetraphenyltetrahydro-[1,3]dioxolo[4,5-}

\section{e][1,3,2]dioxaphosphepin-6-yl\}-1,3-diisopropyl-4,5-dimethyl-1 H-imidazol-3-ium} Hexafluoroantimonate (169o):

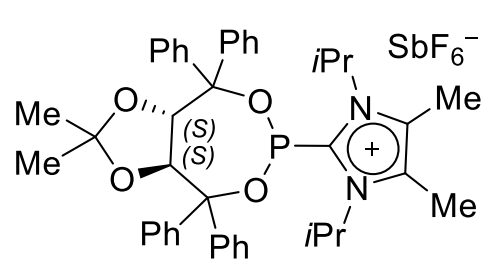

dihydro-2H-imidazol-2-ylidene $\quad \mathbf{5 6 d}^{[238]} \quad(1.0$ equiv, $155 \mathrm{mg}, 860 \mu \mathrm{mol})$, sodium hexafluoroantimonate (1.51 equiv, $333 \mathrm{mg}, 1.29 \mathrm{mmol}$ ) according to GPD after column chromatography ( 0 to $2 \%$ ethyl acetate in dichloromethane) at $-10^{\circ} \mathrm{C}$.

$[\alpha]_{20}^{D}:+87.6\left(c=1.03, \mathrm{CH}_{2} \mathrm{Cl}_{2}\right) ;{ }^{1} \mathrm{H}$ NMR: $\left(400 \mathrm{MHz}, \mathrm{CD}_{3} \mathrm{CN}, 70{ }^{\circ} \mathrm{C}\right) \delta=7.88-7.80(\mathrm{~m}, 2 \mathrm{H})$, $7.57-7.50(\mathrm{~m}, 2 \mathrm{H}), 7.50-7.43(\mathrm{~m}, 3 \mathrm{H}), 7.43-7.27(\mathrm{~m}, 13 \mathrm{H}), 5.57\left(\mathrm{dd}, J=8.5, J_{H-P}=4.3\right.$ $\mathrm{Hz}, 1 \mathrm{H}), 5.46$ (bs, 2H), $5.20(\mathrm{~d}, J=8.6 \mathrm{~Hz}, 1 \mathrm{H}), 2.40(\mathrm{~s}, 6 \mathrm{H}), 1.61-1.43(\mathrm{~m}, 15 \mathrm{H}), 0.34(\mathrm{~s}$, $3 \mathrm{H})$ ppm; ${ }^{13} \mathrm{C}\left\{{ }^{1} \mathrm{H}\right\}$ NMR: $\left(101 \mathrm{MHz}, \mathrm{CD}_{3} \mathrm{CN}, 70{ }^{\circ} \mathrm{C}\right) \delta=146.6,145.1\left(\mathrm{~d}, J_{C-P}=4.7 \mathrm{~Hz}\right), 141.8$ $\left(\mathrm{d}, J_{C-P}=57.6 \mathrm{~Hz}\right), 141.1\left(\mathrm{~d}, J_{C-P}=1.9 \mathrm{~Hz}\right), 140.9\left(\mathrm{~d}, J_{C-P}=3.3 \mathrm{~Hz}\right), 132.9,130.3,130.1$, 130.0, 129.9, 129.9, 129.9, 129.7, 129.7, 129.5, 129.4, 129.0, 128.9, 128.6, 128.6, 114.1, $87.6\left(\mathrm{~d}, J_{C-P}=12.9 \mathrm{~Hz}\right), 87.0\left(\mathrm{~d}, J_{C-P}=7.9 \mathrm{~Hz}\right), 84.5\left(\mathrm{~d}, J_{C-P}=3.9 \mathrm{~Hz}\right), 83.6\left(\mathrm{~d}, J_{C-P}=25.9\right.$ $\mathrm{Hz}), 54.4,54.2,28.2,25.4,22.6,22.6,22.4,22.3,11.2$ ppm; ${ }^{31} \mathrm{P}\left\{{ }^{1} \mathrm{H}\right\}$ NMR: $(162 \mathrm{MHz}$, $\left.\mathrm{CD}_{3} \mathrm{CN}\right) \delta=138.7 \mathrm{ppm} ;{ }^{19} \mathrm{~F}$ NMR: $\left(282 \mathrm{MHz}, \mathrm{CD}_{3} \mathrm{CN}\right) \delta=-124.1$ (sext, $\left.J_{F-121 S b}=1933.1 \mathrm{~Hz}\right)$, -124.1 (oct, $J_{F-123 S b}=1048.8 \mathrm{~Hz}$ ) ppm; IR: (neat, $\mathrm{cm}^{-1}$ ) $\tilde{v}=501,654,700,740,873,990$, 1087, 1164, 1215, 1374, 1447, 1614, 2937, 2988, 3060; HRMS: calcd $m / z$ for $\mathrm{C}_{42} \mathrm{H}_{48} \mathrm{~N}_{2} \mathrm{O}_{4} \mathrm{P}^{+}$ $\left[\mathrm{M}-\mathrm{SbF}_{6}\right]^{+}:$675.3346; found (ESI) 675.3351 . 


\section{Experimental}

\section{2-\{(3a $R, 8 \mathrm{a} R)-2,2-D i m e t h y l-4,4,8,8-$ tetra(biphen-4-yl)tetrahydro-[1,3]dioxolo[4,5-}

e][1,3,2]dioxaphosphepin-6-yl\}-1,3-di[(2,6-diisopropyl)phenyl]-1H-imidazol-3-ium Hexafluoroantimonate (169p):

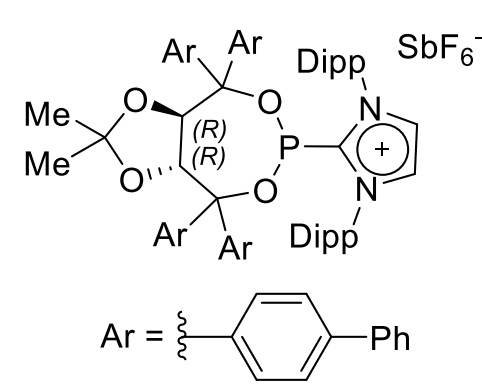

Prepared as a white solid (300 mg, $211 \mu \mathrm{mol}, 41 \%$ yield) from the corresponding chiral diol $\mathbf{1 7 0 b ^ { [ 2 3 4 ] }}$ (1.0 equiv, $400 \mathrm{mg}, 519$

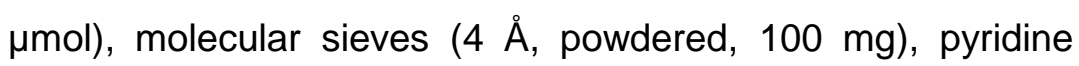
(2.9 equiv, $117 \mathrm{mg}, 1.48 \mathrm{mmol}$ ), phosphorus trichloride (1.3 equiv, $94.4 \mathrm{mg}, \quad 687 \mu \mathrm{mol})$ with 1,3-bis[(2,6diisopropyl)phenyl]-1,3-dihydro-2H-imidazol-2-ylidene $(56 \mathbf{a})^{[274]}$ (1.0 equiv, $204 \mathrm{mg}, 524 \mu \mathrm{mol}$ ) and sodium hexafluoroantimonate (3.24 equiv, $434 \mathrm{mg}$, $1.68 \mathrm{mmol}$ ) according to GPD, adding the carbene to the chlorophosphite at $-40^{\circ} \mathrm{C}$ and after allowing warming to room temperature overnight, further stirring for 24 hours. Column chromatography ( $40 \%$ ethyl acetate in hexanes) at $-10{ }^{\circ} \mathrm{C}$, Crystals suitable for X-ray crystallographic analysis were grown by layering a dichloromethane solution with pentane.

$[\alpha]_{20}^{D}:-141.3\left(c=1.01, \mathrm{CH}_{2} \mathrm{Cl}_{2}\right) ;{ }^{1} \mathrm{H}$ NMR: $\left(400 \mathrm{MHz}, \mathrm{CD}_{3} \mathrm{CN}\right) \delta=08.04(\mathrm{~s}, 2 \mathrm{H}), 7.89(\mathrm{t}, \mathrm{J}=$ $7.8 \mathrm{~Hz}, 2 \mathrm{H}), 7.69-7.60(\mathrm{~m}, 10 \mathrm{H}), 7.59-7.55(\mathrm{~m}, 2 \mathrm{H}), 7.55-7.51(\mathrm{~m}, 4 \mathrm{H}), 7.51-7.43(\mathrm{~m}$, $10 \mathrm{H}), 7.43-7.38(\mathrm{~m}, 4 \mathrm{H}), 7.36(\mathrm{~d}, J=8.4 \mathrm{~Hz}, 4 \mathrm{H}), 7.32(\mathrm{~d}, J=8.6 \mathrm{~Hz}, 2 \mathrm{H}), 7.09-7.04(\mathrm{~m}$, $2 \mathrm{H}), 6.46-6.40(\mathrm{~m}, 2 \mathrm{H}), 5.00(\mathrm{~d}, J=8.2 \mathrm{~Hz}, 1 \mathrm{H}), 4.89\left(\mathrm{dd}, J=8.2 \mathrm{~Hz}, J_{H-P}=4.3 \mathrm{~Hz}, 1 \mathrm{H}\right)$, 2.56 (p, $J=6.7 \mathrm{~Hz}, 2 \mathrm{H}), 2.48(\mathrm{p}, J=6.8 \mathrm{~Hz}, 2 \mathrm{H}), 1.28(\mathrm{~s}, 3 \mathrm{H}), 1.24(\mathrm{~d}, J=6.7 \mathrm{~Hz}, 6 \mathrm{H}), 1.12$ (d, $J=6.7 \mathrm{~Hz}, 6 \mathrm{H}), 0.99(\mathrm{~d}, J=6.8 \mathrm{~Hz}, 6 \mathrm{H}), 0.54(\mathrm{~d}, J=6.7 \mathrm{~Hz}, 6 \mathrm{H}), 0.36(\mathrm{~s}, 3 \mathrm{H}) \mathrm{ppm}$; ${ }^{13} \mathrm{C}\left\{{ }^{1} \mathrm{H}\right\}$ NMR: $\left(101 \mathrm{MHz}, \mathrm{CD}_{3} \mathrm{CN}\right) \delta=147.36\left(\mathrm{~d}, J_{C-P}=72.7 \mathrm{~Hz}\right), 146.3,145.8,143.4,142.6$ $\left(\mathrm{d}, J_{C-P}=3.0 \mathrm{~Hz}\right), 142.2,142.1,141.4,141.2,140.7,140.6,140.6,140.2,138.0$ (d, $J_{C-P}=3.1$ $\mathrm{Hz}), 137.9,133.6,132.0,130.5,130.0,129.9,129.9,129.8,129.8,129.6,129.2,129.1$, 128.8, 128.8, 128.7, 128.4, 128.2, 127.8, 127.8, 127.7, 127.7, 127.6, 127.0, 126.8, 126.8, 126.0, 114.2, $87.1\left(\mathrm{~d}, J_{C-P}=2.2 \mathrm{~Hz}\right), 86.8\left(\mathrm{~d}, J_{C-P}=13.1 \mathrm{~Hz}\right), 83.0\left(\mathrm{~d}, J_{C-P}=26.3 \mathrm{~Hz}\right), 81.2(\mathrm{~d}$, $J_{C-P}=3.5 \mathrm{~Hz}$ ), 30.3, 30.2, 29.9, 27.5, 26.2, 25.6, 25.3, 23.2, 21.0 ppm; ${ }^{31} \mathbf{P}\left\{{ }^{1} \mathrm{H}\right\}$ NMR: (162 $\left.\mathrm{MHz}, \mathrm{CD}_{3} \mathrm{CN}\right) \delta=153.0 \mathrm{ppm} ;{ }^{19} \mathrm{~F}$ NMR: $\left(282 \mathrm{MHz}, \mathrm{CD}_{3} \mathrm{CN}\right) \delta=-124.02$ (sext, $J_{F-121 S b}=$ 1927.4 Hz), -124.04 (oct, $J_{F-123 S b}=1050.1 \mathrm{~Hz}$ ) ppm; IR: (neat, $\mathrm{cm}^{-1}$ ) $\tilde{v}=654,696,744,759$, 802, 846, 877, 985, 1096, 1209, 1367, 1446, 1486, 1599, 1731, 2871, 2930, 2965, 3030; HRMS: calcd $m / z$ for $\mathrm{C}_{82} \mathrm{H}_{80} \mathrm{~N}_{2} \mathrm{O}_{4} \mathrm{P}^{+}\left[\mathrm{M}-\mathrm{SbF}_{6}\right]^{+}$: 1187.5850 ; found (ESI) 1187.5856 . 


\section{Experimental}

\section{2-[(5R,6R)-5,6-Dimethoxy-4,4,7,7-tetraphenyl-1,3,2-dioxaphosphepan-2-yl]-1,3-}

dimesityl-1H-imidazol-3-ium Hexafluoroantimonate (169q):

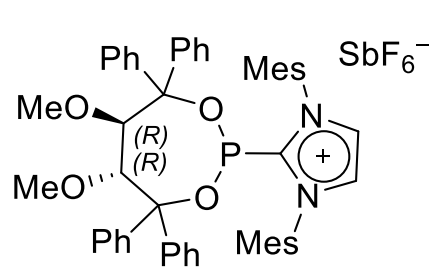

Prepared as a white solid (466 mg, $455 \mu \mathrm{mol}, 79 \%$ yield) from the corresponding diol $(R, R)-\mathbf{1 7 0} \mathrm{q}^{[234]}$ (1.25 equiv, $326 \mathrm{mg}, 717$ umol), molecular sieves ( $4 \AA$, powdered, $150 \mathrm{mg}$ ), pyridine (3.8 equiv, $169 \mathrm{mg}, 2,14 \mathrm{mmol}$ ), phosphorus trichloride (1.3 equiv, $105 \mathrm{mg}, \quad 765 \mu \mathrm{mol}$ ) with 1,3-bis(2,4,6-trimethylphenyl)-1,3dihydro-2H-imidazol-2-ylidene $(56 \mathbf{b})^{[274]}(1.0$ equiv, $175 \mathrm{mg}, 575 \mu \mathrm{mol})$ and sodium hexafluoroantimonate ( 3.8 equiv, $567 \mathrm{mg}, 2.19 \mathrm{mmol}$ ) according to GPD after column chromatography ( 0 to $0.5 \%$ ethyl acetate in dichloromethane) at $-10{ }^{\circ} \mathrm{C}$. Crystals suitable for $\mathrm{X}$-ray crystallographic analysis were grown by layering a dichloromethane solution with pentane.

$[\alpha]_{20}^{D}:-44.7\left(c=1.04, \mathrm{CH}_{2} \mathrm{Cl}_{2}\right) ;{ }^{1} \mathrm{H}$ NMR: $\left(400 \mathrm{MHz}, \mathrm{CD}_{3} \mathrm{CN}\right) \delta=7.73(\mathrm{~s}, 2 \mathrm{H}), 7.48-7.30$ $(\mathrm{m}, 12 \mathrm{H}), 7.29-7.21(\mathrm{~m}, 4 \mathrm{H}), 7.13(\mathrm{~s}, 2 \mathrm{H}), 7.06-7.01(\mathrm{~m}, 2 \mathrm{H}), 6.84-6.75(\mathrm{~m}, 4 \mathrm{H}), 4.58$ (dd, $\left.J=7.0 \mathrm{~Hz}, J_{H-P}=7.0 \mathrm{~Hz}, 1 \mathrm{H}\right), 3.98(\mathrm{~d}, J=7.3 \mathrm{~Hz}, 1 \mathrm{H}), 3.34(\mathrm{~s}, 3 \mathrm{H}), 2.50(\mathrm{~s}, 6 \mathrm{H}), 2.23$ (s, 3H), $2.00(\mathrm{~s}, 6 \mathrm{H}), 1.59(\mathrm{~s}, 6 \mathrm{H}) \mathrm{ppm} ;{ }^{13} \mathrm{C}\left\{{ }^{1} \mathrm{H}\right\}$ NMR: $\left(101 \mathrm{MHz}, \mathrm{CD}_{3} \mathrm{CN}\right) \delta=145.4\left(\mathrm{~d}, J_{C-P}=\right.$ $66.4 \mathrm{~Hz}), 144.2\left(\mathrm{~d}, J_{C-P}=4.5 \mathrm{~Hz}\right), 143.8,142.8,139.5$ (d, $\left.J_{C-P}=2.9 \mathrm{~Hz}\right), 139.3,135.9,132.1$, 130.8, 130.8, 130.2, 129.8, 129.7, 129.4, 129.3, 129.2, 129.1, 129.1, 129.0, 128.9, 128.8, 128.7, 127.5, $89.1\left(\mathrm{~d}, J_{C-P}=4.5 \mathrm{~Hz}\right), 87.2\left(\mathrm{~d}, J_{C-P}=14.5 \mathrm{~Hz}\right), 84.78\left(\mathrm{~d}, J_{C-P}=1.9 \mathrm{~Hz}\right), 81.0(\mathrm{~d}$, $\left.J_{C-P}=28.9 \mathrm{~Hz}\right), 61.1,60.0,21.4,17.9,17.4,17.3 \mathrm{ppm} ;{ }^{31} \mathbf{P}\left\{{ }^{1} \mathrm{H}\right\}$ NMR: $\left(162 \mathrm{MHz}, \mathrm{CD}_{3} \mathrm{CN}\right) \delta=$ 144.5 ppm; ${ }^{19} \mathrm{~F}$ NMR: $\left(282 \mathrm{MHz}, \mathrm{CD}_{3} \mathrm{CN}\right) \delta=-123.8$ (sext, $J_{F-121 S b}=1934.6 \mathrm{~Hz}$ ), -123.8 (oct, $\left.J_{F-123 S b}=1049.8 \mathrm{~Hz}\right)$ ppm; IR: $\left(\right.$ neat, $\left.\mathrm{cm}^{-1}\right) \tilde{v}=654,699,840,902,955,1016,1134,1187$, 1234, 1377, 1445, 1483, 1607, 2918, 2973, 3057, 3133, 3155; HRMS: calcd m/z for $\mathrm{C}_{51} \mathrm{H}_{52} \mathrm{~N}_{2} \mathrm{O}_{4} \mathrm{P}^{+}\left[\mathrm{M}-\mathrm{SbF}_{6}\right]^{+}:$787.3659; found (ESI) 787.3658.

\section{2-[(5R,6R)-5,6-dimethoxy-4,4,7,7-tetraphenyl-1,3,2-dioxaphosphepan-2-yl]-1,3- diisopropyl-4,5-dimethyl-1H-imidazol-3-ium Hexafluoroantimonate (169r)}

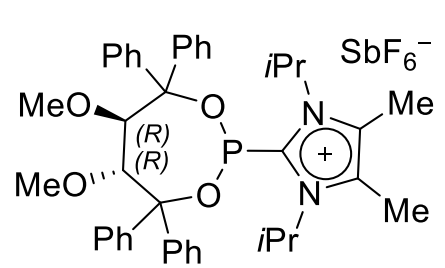

Prepared as a white solid (375 $\mathrm{mg}, 417 \mu \mathrm{mol}, 66 \%$ yield) from the corresponding diol $(R, R)-\mathbf{1 7 0} \mathbf{q}^{[234]}(1.11$ equiv, $320 \mathrm{mg}, 703.9$

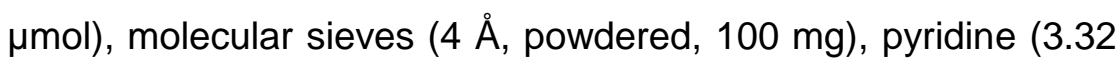
equiv, $166 \mathrm{mg}, 2.10 \mathrm{mmol}$ ), phosphorus trichloride (1.27 equiv, $110 \mathrm{mg}, 801 \mathrm{\mu mol}$ ) with 1,3-bis(isopropyl)-4,5-dimethyl-1,3-dihydro-2H-imidazol-2-ylidene $\mathbf{5 6 d}^{[238]}$ (1.0 equiv, $\left.114 \mathrm{mg}, 632 \mu \mathrm{mol}\right)$, sodium hexafluoroantimonate (1.68 equiv, $273 \mathrm{mg}$, $1.06 \mathrm{mmol}$ ) according to GPD after column chromatography (0 to $2 \%$ ethyl acetate in dichloromethane) at $-10^{\circ} \mathrm{C}$. Crystals suitable for $\mathrm{X}$-ray crystallographic analysis were grown by layering a dichloromethane solution with pentane. 
$[\alpha]_{20}^{D}:-54.1\left(c=1.05, \mathrm{CH}_{2} \mathrm{Cl}_{2}\right) ;{ }^{1} \mathrm{H}$ NMR: $\left(400 \mathrm{MHz}, \mathrm{CD}_{3} \mathrm{CN}, 70{ }^{\circ} \mathrm{C}\right) \delta=7.88-7.81(\mathrm{~m}, 2 \mathrm{H})$, $7.56-7.47(\mathrm{~m}, 5 \mathrm{H}), 7.47-7.28(\mathrm{~m}, 11 \mathrm{H}), 7.25-7.18(\mathrm{~m}, 2 \mathrm{H}), 5.41(\mathrm{~s}, 2 \mathrm{H}), 4.95(\mathrm{dd}, J=7.5$ $\left.\mathrm{Hz}, J_{H-P}=5.7 \mathrm{~Hz}, 1 \mathrm{H}\right), 4.56(\mathrm{~d}, J=7.6 \mathrm{~Hz}, 1 \mathrm{H}), 3.63(\mathrm{~s}, 3 \mathrm{H}), 2.65(\mathrm{~s}, 3 \mathrm{H}), 2.35(\mathrm{~s}, 6 \mathrm{H}), 1.48$ (bs, 6H), 1.41 (d, J = 7.0 Hz, 6H) ppm; ${ }^{13} \mathrm{C}\left\{{ }^{1} \mathrm{H}\right\}$ NMR: $\left(101 \mathrm{MHz}, \mathrm{CD}_{3} \mathrm{CN}, 70{ }^{\circ} \mathrm{C}\right) \delta=146.3$, $145.6\left(\mathrm{~d}, J_{C-P}=4.4 \mathrm{~Hz}\right), 142.0\left(\mathrm{~d}, J_{C-P}=62.0 \mathrm{~Hz}\right), 141.3\left(\mathrm{~d}, J_{C-P}=3.4 \mathrm{~Hz}\right), 140.9\left(\mathrm{~d}, J_{C-P}=1.4\right.$ $\mathrm{Hz}), 132.8,130.3,130.1,130.1,130.1,129.8,129.7,129.7,129.5,129.3,129.3,129.2$, 128.9, 128.8, 128.5, $90.0\left(\mathrm{~d}, J_{C-P}=5.5 \mathrm{~Hz}\right), 87.2\left(\mathrm{~d}, J_{C-P}=15.7 \mathrm{~Hz}\right), 86.7\left(\mathrm{~d}, J_{C-P}=2.4 \mathrm{~Hz}\right)$, $84.0\left(\mathrm{~d}, J_{C-P}=25.3 \mathrm{~Hz}\right), 61.6,61.1,54.2,54.1,22.5,22.5,22.3,22.3,11.2 \mathrm{ppm} ;{ }^{31} \mathrm{P}\left\{{ }^{1} \mathrm{H}\right\}$ NMR: $\left(162 \mathrm{MHz}, \mathrm{CD}_{3} \mathrm{CN}\right) \delta=138.1 \mathrm{ppm}$; IR: (neat, $\left.\mathrm{cm}^{-1}\right) \tilde{v}=484,654,698,829,938,996$, 1112, 1184, 1280, 1375, 1446, 1615, 2833, 2938, 2981; HRMS: calcd $m / z$ for $\mathrm{C}_{41} \mathrm{H}_{48} \mathrm{~N}_{2} \mathrm{O}_{4} \mathrm{P}^{+}$ $\left[\mathrm{M}-\mathrm{SbF}_{6}\right]^{+}:$663.3346; found (ESI) 663.3339.

\section{(2R,3R)-1,1,4,4-Tetrakis[3,5-bis(trifluoromethyl)phenyl]-2,3-dimethoxybutane-1,4-diol}

\section{$[(R, R)-170 s]:$}

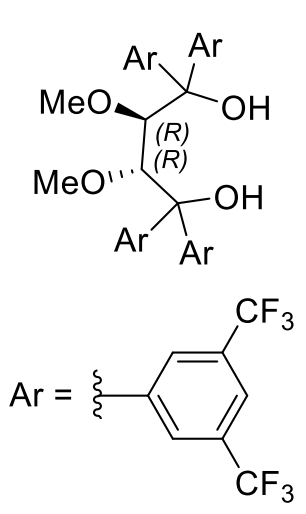

Prepared according to a modified literature procedure. ${ }^{[234]}$ A pre-dried three-necked flask, equipped with magnetic stirring bar and dropping funnel was charged with activated magnesium (5.0 equiv, $536 \mathrm{mg}, 22.05$ $\mathrm{mmol})$ and a spatula tip of elemental iodine. THF $(20 \mathrm{ml})$ was added, and the mixture allowed stirring for a few minutes. Under stirring, a solution of 1,3-bis(trifluoromethyl)-5-bromobenzene (5.0 equiv, $6.46 \mathrm{~g}, 22.0 \mathrm{mmol}$ ) in THF $(20 \mathrm{ml})$ was added slowly dropwise, using an ice bath to periodically funnel was replaced with a reflux condenser, and the suspension was stirred at reflux for an additional $2 \mathrm{~h}$. On cooling to $0^{\circ} \mathrm{C},(2 R, 3 R$ )-diethyl-2,3-dimethoxysuccinate (1.0 equiv, $1.03 \mathrm{~g}$, $4.40 \mathrm{mmol})$ was added via syringe as a solution in THF $(4.5 \mathrm{ml})$. On completion of the addition, the mixture was stirred at reflux for $2 \mathrm{~h}$. On cooling, a saturated aqueous solution of ammonium chloride $(50 \mathrm{ml})$ was slowly added, then aqueous hydrochloric acid $(1 \mathrm{M}, 5 \mathrm{ml})$. The mixture was transferred to a separatory funnel and extracted with diethyl ether $(3 \times 100$ $\mathrm{ml})$. The combined organic phases were dried $\left(\mathrm{MgSO}_{4}\right)$ and concentrated in vacuo. After purification of the crude product by column chromotography (15\% MTBE in hexanes), compound 170s was obtained as a white solid ( $3.15 \mathrm{~g}, 3.15 \mathrm{mmol}, 72 \%$ yield).

$[\alpha]_{20}^{D}:-17.8\left(c=1.02, \mathrm{CH}_{2} \mathrm{Cl}_{2}\right) ;{ }^{1} \mathrm{H}$ NMR: $\left(400 \mathrm{MHz}, \mathrm{CDCl}_{3}\right) \delta=8.08(\mathrm{~s}, 4 \mathrm{H}), 7.95(\mathrm{~s}, 4 \mathrm{H})$, $7.91(\mathrm{~s}, 2 \mathrm{H}), 7.85(\mathrm{~s}, 2 \mathrm{H}), 4.25(\mathrm{~s}, 2 \mathrm{H}), 4.25(\mathrm{~s}, 2 \mathrm{H}), 2.44(\mathrm{~s}, 6 \mathrm{H}) \mathrm{ppm} ;{ }^{13} \mathrm{C}\left\{{ }^{1} \mathrm{H}\right\}$ NMR: $(101$ $\left.\mathrm{MHz}, \mathrm{CDCl}_{3}\right) \delta=146.6,144.8,132.7$ (q, $J_{\mathrm{C}-\mathrm{F}}=34.0 \mathrm{~Hz}$ ), 132.0 (q, $J_{\mathrm{C}-\mathrm{F}}=33.9 \mathrm{~Hz}$ ), $127.3-$ $127.0(\mathrm{~m}), 126.7-126.4(\mathrm{~m}), 123.1$ (q, $\mathrm{J}_{\mathrm{C}-\mathrm{F}}=272.9 \mathrm{~Hz}$ ), 123.0 (q, J J-F $=272.7 \mathrm{~Hz}$ ), $122.7-$ $122.2(\mathrm{~m}), 84.1,78.7,60.7$ ppm; ${ }^{19} \mathrm{~F}$ NMR: $\left(282 \mathrm{MHz}, \mathrm{CDCl}_{3}\right) \delta=-63.10,-63.2$; IR: (neat, 


\section{Experimental}

$\left.\mathrm{cm}^{-1}\right) \tilde{v}=651,705,844,893,909,990,1075,1123,1164,1267,1369,1623,3467$; HRMS: calcd. $\mathrm{m} / \mathrm{z}$ for $\mathrm{C}_{38} \mathrm{H}_{22} \mathrm{~F}_{24} \mathrm{NaO}_{4}{ }^{+}[\mathrm{M}+\mathrm{Na}]^{+}:$1021.1027; found (ESI) 1021.1031.

\section{4-[(5R,6R)-5,6-dimethoxy-4,4,7,7-tetra[3,5-di(trifluoromethyl)phenyl-1,3,2-} dioxaphosphepan-2-yl]-1,3-dimesityl-1H-imidazol-3-ium Hexafluoroantimonate (203a):

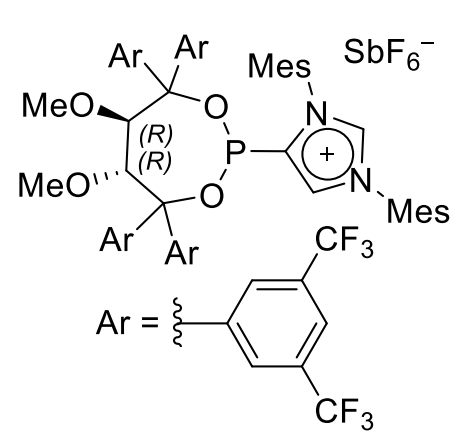

Prepared as a white solid (180 mg, $115 \mu \mathrm{mol}, 29 \%$ yield) from diol $(R, R)$-170s (1.0 equiv, $400 \mathrm{mg}, 401 \mu \mathrm{mol})$, molecular sieves (4 ̊̊, powdered, $100 \mathrm{mg}$ ), pyridine (3.1 equiv, $98 \mathrm{mg}, 1.24$ $\mathrm{mmol}$ ), phosphorus trichloride (1.43 equiv, $79 \mathrm{mg}, 575 \mu \mathrm{mol})$ with 1,3-bis(isopropyl)-4,5-dimethyl-1,3-dihydro-2H-imidazol-2ylidene $(\mathbf{5 6 b})^{[274]}$ (1.0 equiv, $\left.122 \mathrm{mg}, 401 \mu \mathrm{mol}\right)$, sodium hexafluoroantimonate (3.0 equiv, $311 \mathrm{mg}, 1.20 \mathrm{mmol}$ ) according to modified GPD. The product was partially soluble in diethyl ether and could be directly filtered from the inorganic salts, which were further washed with diethyl ether $(3 \times 10 \mathrm{ml})$. The ether washings were combined, concentrated under vacuum and purified by column chromatography $\left(0.5 \%\right.$ ethyl acetate in dichloromethane) at $-10{ }^{\circ} \mathrm{C}$. Crystals suitable for $\mathrm{X}$ ray crystallographic analysis were grown by layering a dichloromethane solution with pentane.

$[\alpha]_{20}^{D}:-19.0\left(c=1.03, \mathrm{CH}_{2} \mathrm{Cl}_{2}\right) ;{ }^{1} \mathrm{H}$ NMR: $\left(500 \mathrm{MHz}, \mathrm{CD}_{3} \mathrm{CN}\right) \delta=8.85(\mathrm{~s}, 1 \mathrm{H}), 8.38(\mathrm{~s}, 1 \mathrm{H})$, $8.21(\mathrm{~s}, 2 \mathrm{H}), 8.19(\mathrm{~s}, 1 \mathrm{H}), 8.10(\mathrm{~s}, 1 \mathrm{H}), 8.07(\mathrm{~s}, 2 \mathrm{H}), 8.06(\mathrm{~s}, 1 \mathrm{H}), 8.03(\mathrm{~s}, 1 \mathrm{H}), 7.72(\mathrm{~s}, 2 \mathrm{H})$, $7.66(\mathrm{~s}, 2 \mathrm{H}), 7.24(\mathrm{~s}, 1 \mathrm{H}), 7.23(\mathrm{~s}, 1 \mathrm{H}), 6.85(\mathrm{~s}, 1 \mathrm{H}), 6.69(\mathrm{~s}, 1 \mathrm{H}), 4.91\left(\mathrm{dd}, J=7.7 \mathrm{~Hz}, J_{H-P}=\right.$ $6.2 \mathrm{~Hz}, 1 \mathrm{H}), 3.67(\mathrm{~d}, J=7.6 \mathrm{~Hz}, 1 \mathrm{H}), 3.54(\mathrm{~s}, 3 \mathrm{H}), 2.40(\mathrm{~s}, 3 \mathrm{H}), 2.40(\mathrm{~s}, 3 \mathrm{H}), 2.21(\mathrm{~s}, 3 \mathrm{H})$, $2.19(\mathrm{~s}, 3 \mathrm{H}), 2.17(\mathrm{~s}, 3 \mathrm{H}), 1.87(\mathrm{~s}, 6 \mathrm{H}) \mathrm{ppm} ;{ }^{13} \mathrm{C}\left\{{ }^{1} \mathrm{H}\right\}$ NMR: $\left(126 \mathrm{MHz}, \mathrm{CD}_{3} \mathrm{CN}\right) \delta=145.9(\mathrm{~d}$, $\left.J_{C-P}=3.8 \mathrm{~Hz}\right), 145.1,143.3,143.1,141.8,141.7,141.5,135.8\left(\mathrm{~d}, J_{C-P}=23.1 \mathrm{~Hz}\right), 135.4$, 135.2, $133.0\left(q, J_{C-F}=33.6 \mathrm{~Hz}\right), 132.4\left(q, J_{C-F}=33.6 \mathrm{~Hz}\right), 132.2\left(q, J_{C-F}=33.6 \mathrm{~Hz}\right), 131.7$ (q, $\left.J_{C-F}=33.6 \mathrm{~Hz}\right), 131.3,130.8,130.5,130.5,129.9-129.7(\mathrm{~m}), 129.6,129.5-129.2(\mathrm{~m})$, $128.7-128.4(\mathrm{~m}), 125.1-124.7(\mathrm{~m}), 124.3\left(\mathrm{q}, J_{C-F}=272.0 \mathrm{~Hz}\right), 124.3\left(\mathrm{q}, J_{C-F}=272.0 \mathrm{~Hz}\right)$, $124.2\left(\mathrm{q}, J_{C-F}=272.0 \mathrm{~Hz}\right), 124.3-124.0(\mathrm{~m}), 124.0-123.6(\mathrm{~m}), 87.9,85.8\left(\mathrm{~d}, J_{C-P}=4.4 \mathrm{~Hz}\right)$, $84.8\left(\mathrm{~d}, J_{C-P}=10.0 \mathrm{~Hz}\right), 82.0\left(\mathrm{~d}, J_{C-P}=25.7 \mathrm{~Hz}\right), 61.7,61.6,21.2,21.0,17.5,17.5,17.4,17.4$, 17.3 ppm; ${ }^{31} \mathbf{P}\left\{{ }^{1} \mathrm{H}\right\}$ NMR: $\left(202 \mathrm{MHz}, \mathrm{CD}_{3} \mathrm{CN}\right) \delta=145.9 \mathrm{ppm} ;{ }^{19} \mathbf{F}$ NMR: $\left(470 \mathrm{MHz}, \mathrm{CD}_{3} \mathrm{CN}\right) \delta$ $=-63.1,-63.4,-63.6,-124.0\left(\mathrm{sext}, J_{F-121 S b}=1947.2 \mathrm{~Hz}\right),-124.0$ (oct, $\left.J_{F-123 S b}=1040.0 \mathrm{~Hz}\right)$ ppm; IR: $\left(\right.$ neat, $\left.\mathrm{cm}^{-1}\right) \tilde{v}=656,682,707,811,848,899,970,1032,1052,1130,1172,1277$, 1372, 1469, 1515, 1553, 1612, 2837, 2926, 2966, 3133; HRMS: calcd $\mathrm{m} / \mathrm{z}$ for $\mathrm{C}_{59} \mathrm{H}_{44} \mathrm{~F}_{24} \mathrm{~N}_{2} \mathrm{O}_{4} \mathrm{P}^{+}\left[\mathrm{M}-\mathrm{SbF}_{6}\right]^{+}:$1331.2650; found (ESI) 1331.2660 . 


\section{Experimental}

\section{1,3-Dimesityl-5-\{(3a' $\left.R, 8 \mathrm{a}^{\prime} R\right)-4^{\prime}, 4^{\prime}, 8^{\prime}, 8^{\prime}$-tetraphenyltetrahydrospiro[cyclohexane-1,2'- [1,3]dioxolo[4,5-e][1,3,2]dioxaphosphepin]-6'-yl\}-1H-imidazol-3-ium Hexafluoroantimonate (203b):}

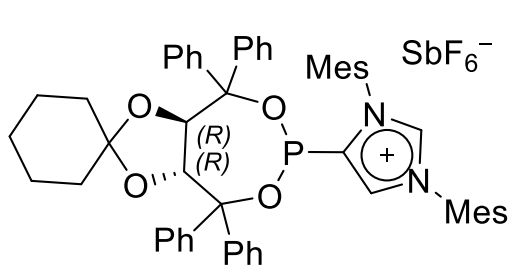

Prepared as a white solid (90 mg, $84 \mu \mathrm{mol}, 21 \%$ yield) from the corresponding diol $(R, R)-\mathbf{1 7 0 t}^{[234]}$ (1.0 equiv, $200 \mathrm{mg}$, $395 \mu \mathrm{mol})$, molecular sieves (4 $\mathrm{A}$, powdered, $100 \mathrm{mg}$ ),

pyridine (3.14 equiv, $97.8 \mathrm{mg}, 1.24 \mathrm{mmol}$ ), phosphorus trichloride (1.0 equiv, $55.1 \mathrm{mg}, 401 \mu \mathrm{mol})$ with 1,3-bis(2,4,6-trimethylphenyl)-1,3-dihydro- $2 \mathrm{H}$ imidazol-2-ylidene $(\mathbf{5 6 b})^{[274]}$ ( 1.0 equiv, $\left.120 \mathrm{mg}, 394 \mu \mathrm{mol}\right)$ and sodium hexafluoroantimonate (1.5 equiv, $153 \mathrm{mg}, 591 \mu \mathrm{mol}$ ) according to modified GPD. As the intermediate chlorophosphite 171t was not soluble in diethyl ether, it was dissolved in THF (4 ml), and the carbene solution in an equal volume of diethyl ether was added. The resulting white suspension was filtered, the filtrate evaporated and washed with diethyl ether $(2 \times 10 \mathrm{ml})$. The remaining solid was purified by column chromatography $(1 \%$ ethyl acetate in dichloromethane) at $-10^{\circ} \mathrm{C}$ affording $203 \mathrm{~b}$.

$[\alpha]_{20}^{D}:-50.1\left(c=1.04, \mathrm{CH}_{2} \mathrm{Cl}_{2}\right) ;{ }^{1} \mathrm{H}$ NMR: $\left(400 \mathrm{MHz}, \mathrm{CD}_{3} \mathrm{CN}\right) \delta=8.85\left(\mathrm{dd}, J=1.5 \mathrm{~Hz}, J_{H-P}=\right.$ $1.5 \mathrm{~Hz}, 1 \mathrm{H}), 8.32\left(\mathrm{dd}, J=1.5 \mathrm{~Hz}, J_{H-P}=1.2 \mathrm{~Hz}, 1 \mathrm{H}\right), 7.54-7.52(\mathrm{~m}, 1 \mathrm{H}), 7.52-7.50(\mathrm{~m}$, $1 \mathrm{H}), 7.45-7.43(\mathrm{~m}, 1 \mathrm{H}), 7.43-7.40(\mathrm{~m}, 1 \mathrm{H}), 7.38-7.28(\mathrm{~m}, 10 \mathrm{H}), 7.28-7.25(\mathrm{~m}, 2 \mathrm{H})$, $7.25-7.22(\mathrm{~m}, 4 \mathrm{H}), 7.22-7.18(\mathrm{~m}, 2 \mathrm{H}), 7.15-7.11(\mathrm{~m}, 1 \mathrm{H}), 7.09-7.06(\mathrm{~m}, 1 \mathrm{H}), 5.26$ (dd, $\left.J=8.6, \mathrm{~Hz}, J_{H-P}=4.1 \mathrm{~Hz}, 1 \mathrm{H}\right), 4.75(\mathrm{~d}, J=8.6 \mathrm{~Hz}, 1 \mathrm{H}), 2.40(\mathrm{~s}, 3 \mathrm{H}), 2.39(\mathrm{~s}, 3 \mathrm{H}), 2.28(\mathrm{~s}$, $3 \mathrm{H}), 2.17(\mathrm{~s}, 3 \mathrm{H}), 2.02(\mathrm{~s}, 3 \mathrm{H}), 1.94(\mathrm{~s}, 3 \mathrm{H}), 1.89-1.78(\mathrm{~m}, 1 \mathrm{H}), 1.78-1.68(\mathrm{~m}, 1 \mathrm{H}), 1.64-$ $1.45(\mathrm{~m}, 2 \mathrm{H}), 1.26-1.16(\mathrm{~m}, 1 \mathrm{H}), 1.16-0.94(\mathrm{~m}, 2 \mathrm{H}), 0.87-0.75(\mathrm{~m}, 1 \mathrm{H}), 0.37-0.23(\mathrm{~m}$, $1 \mathrm{H}), 0.20-0.07(\mathrm{~m}, 1 \mathrm{H}) \mathrm{ppm} ;{ }^{13} \mathrm{C}\left\{{ }^{1} \mathrm{H}\right\}$ NMR: $\left(101 \mathrm{MHz}, \mathrm{CD}_{3} \mathrm{CN}\right) \delta=146.3,144.8\left(\mathrm{~d}, J_{C-P}=\right.$ $4.5 \mathrm{~Hz}), 143.4,142.8,141.0,140.6\left(\mathrm{~d}, J_{C-P}=2.6 \mathrm{~Hz}\right), 140.3\left(\mathrm{~d}, J_{C-P}=1.8 \mathrm{~Hz}\right), 138.0\left(\mathrm{~d}, J_{C-P}=\right.$ $25.8 \mathrm{~Hz}), 136.5,135.8,135.6,135.5,131.5,130.9,130.8,130.6,130.6,130.0\left(\mathrm{~d}, J_{C-P}=8.3\right.$ $\mathrm{Hz}), 129.9,129.5,129.3,129.3,129.1,129.0,129.0,128.9,128.8,128.8,128.5,128.1$, 127.8, 113.9, $85.5\left(\mathrm{~d}, J_{C-P}=9.5 \mathrm{~Hz}\right), 84.8\left(\mathrm{~d}, J_{C-P}=5.3 \mathrm{~Hz}\right), 84.0\left(\mathrm{~d}, J_{C-P}=3.9 \mathrm{~Hz}\right), 83.4(\mathrm{~d}$, $\left.J_{C-P}=22.0 \mathrm{~Hz}\right), 37.8,34.9,25.3,24.7,24.7,21.3,21.2,17.8,17.8,17.8,17.8,17.7,17.6$ ppm; ${ }^{31} \mathrm{P}\left\{{ }^{1} \mathrm{H}\right\}$ NMR: $\left(162 \mathrm{MHz}, \mathrm{CD}_{3} \mathrm{CN}\right) \delta=143.1 \mathrm{ppm}$; IR: (neat, $\left.\mathrm{cm}^{-1}\right) \tilde{v}=654,699,738$, 823, 939, 1001, 1030, 1124, 1447, 1551, 1670, 2860, 2934, 3059, 3128; HRMS: calcd m/z for $\mathrm{C}_{55} \mathrm{H}_{56} \mathrm{~N}_{2} \mathrm{O}_{4} \mathrm{P}^{+}\left[\mathrm{M}-\mathrm{SbF}_{6}\right]^{+}$: 839.3972; found (ESI) 839.3979. 


\section{Experimental}

\section{2-\{(5R,6R)-5,6-Dimethoxy-4,4,7,7-tetrakis[4-(trifluoromethyl)phenyl]-1,3,2-}

dioxaphosphepan-2-yl\}-1,3-dimesityl-4,5-dimethyl-1H-imidazol-3-ium

Hexafluoroantimonate (169s):

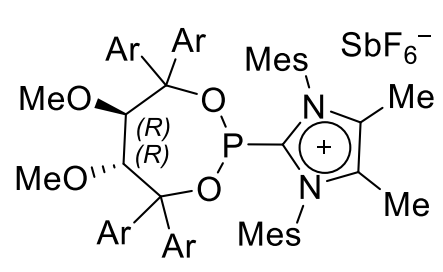

$\mathrm{Ar}=\xi-\mathrm{CF}_{3}$

Prepared as a white solid (215 mg, $162 \mu \mathrm{mol}, 67 \%$ yield) from the corresponding diol $(R, R)-170 \mathrm{i}^{[219]}(1.05$ equiv, $184 \mathrm{mg}, 253$

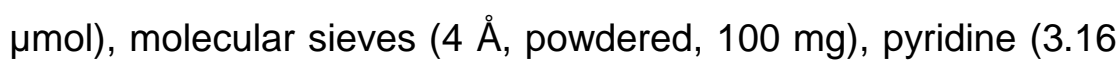
equiv, $60 \mathrm{mg}, 759 \mu \mathrm{mol})$, phosphorus trichloride (1.2 equiv, 38 $\mathrm{mg}, 0.28 \mathrm{mmol})$ with 1,3-bis(2,4,6-trimethylphenyl)-4,5-dimethyl1,3-dihydro-2 $H$-imidazol-2-ylidene $(56 \mathrm{e})^{[240]}(1.0$ equiv, $80 \mathrm{mg}$, $241 \mu \mathrm{mol})$, sodium hexafluoroantimonate $(2.1$ equiv, $131 \mathrm{mg}, 506 \mu \mathrm{mol}$ ) according to GPD after column chromatography ( $1 \%$ ethyl acetate in dichloromethane) at $-10{ }^{\circ} \mathrm{C}$. Crystals suitable for X-ray crystallographic analysis were grown by layering a dichloromethane solution with pentane.

$[\alpha]_{22}^{D}:-24.2\left(c=1.04, \mathrm{CH}_{2} \mathrm{Cl}_{2}\right) ;{ }^{1} \mathrm{H}$ NMR: $\left(300 \mathrm{MHz}, \mathrm{CD}_{3} \mathrm{CN}\right) \delta=7.74-7.64(\mathrm{~m}, 6 \mathrm{H}), 7.64-$ $7.57(\mathrm{~m}, 2 \mathrm{H}), 7.53$ (d, J = 8.3 Hz, 2H), 7.28 (s, 2H), 7.21 (d, J=8.4 Hz, 2H), $7.14(\mathrm{~s}, 2 \mathrm{H})$, $6.98(\mathrm{~d}, J=5.3 \mathrm{~Hz}, 2 \mathrm{H}), 6.95(\mathrm{~d}, J=5.4 \mathrm{~Hz}, 2 \mathrm{H}), 4.64\left(\mathrm{dd}, J=7.8 \mathrm{~Hz}, J_{H-P}=6.8 \mathrm{~Hz}, 1 \mathrm{H}\right)$, $3.84(\mathrm{~d}, J=7.6 \mathrm{~Hz}, 1 \mathrm{H}), 3.39$ (s, 3H), 2.49 (s, 6H), 2.28 (s, 3H), 1.95 (s, 6H), $1.93(\mathrm{~s}, 6 \mathrm{H})$, $1.56(\mathrm{~s}, 6 \mathrm{H}) \mathrm{ppm} ;{ }^{13} \mathrm{C}\left\{{ }^{1} \mathrm{H}\right\}$ NMR: $\left(126 \mathrm{MHz}, \mathrm{CD}_{3} \mathrm{CN}\right) \delta=147.5\left(\mathrm{~d}, J_{C-P}=4.6 \mathrm{~Hz}\right), 147.0$, $143.1,143.1\left(\mathrm{~d}, J_{C-P}=2.1 \mathrm{~Hz}\right), 143.1\left(\mathrm{~d}, J_{C-P}=1.5 \mathrm{~Hz}\right), 141.0\left(\mathrm{~d}, J_{C-P}=58.0 \mathrm{~Hz}\right), 136.2$, 136.2, 136.0, 136.0, 134.3, $131.5\left(\mathrm{q}, J_{C-F}=32.7 \mathrm{~Hz}\right), 131.3\left(\mathrm{q}, J_{C-F}=32.5 \mathrm{~Hz}\right), 131.3,131.2$, 131.0, $130.9\left(\mathrm{q}, J_{C-F}=32.2 \mathrm{~Hz}\right), 130.9\left(\mathrm{q}, J_{C-F}=32.3 \mathrm{~Hz}\right), 130.1\left(\mathrm{~d}, J_{C-P}=1.6 \mathrm{~Hz}\right), 130.0$, 130.0, 129.7, 129.7, 129.4, $126.6\left(\mathrm{q}, J_{C-F}=3.8 \mathrm{~Hz}\right), 126.4$ (q, $\left.J_{C-F}=3.8 \mathrm{~Hz}\right), 126.0$ (q, $J_{C-F}=$ $3.8 \mathrm{~Hz}), 125.2\left(\mathrm{q}, J_{C-F}=271.4 \mathrm{~Hz}\right), 125.1\left(\mathrm{q}, J_{C-F}=272.0 \mathrm{~Hz}\right), 125.1$ (q, $J_{C-F}=272.0 \mathrm{~Hz}$ ), $125.0\left(\mathrm{q}, J_{C-F}=271.6 \mathrm{~Hz}\right), 124.7\left(\mathrm{q}, J_{C-F}=3.8 \mathrm{~Hz}\right), 88.0\left(\mathrm{~d}, J_{C-P}=5.4 \mathrm{~Hz}\right), 86.3\left(\mathrm{~d}, J_{C-P}=15.2\right.$ $\mathrm{Hz}), 85.0\left(\mathrm{~d}, J_{C-P}=1.8 \mathrm{~Hz}\right), 80.5\left(\mathrm{~d}, J_{C-P}=29.1 \mathrm{~Hz}\right), 61.4,60.5,21.3,18.0,17.5,17.5,9.2$ ppm; ${ }^{31} \mathbf{P}\left\{{ }^{1} \mathrm{H}\right\}$ NMR: $\left(121 \mathrm{MHz}, \mathrm{CD}_{3} \mathrm{CN}\right) \delta=144.3$ ppm; ${ }^{19} \mathrm{~F}$ NMR: $\left(282 \mathrm{MHz}, \mathrm{CD}_{3} \mathrm{CN}\right) \delta=-$ 63.1, -63.1, -63.3, -63.3, -123.9 (sext, $J=1937.0 \mathrm{~Hz}$ ), -123.9 (oct, $J=1043.0 \mathrm{~Hz}$ ) ppm; IR: $\left(\right.$ neat, $\left.\mathrm{cm}^{-1}\right) \tilde{v}=656,854,934,999,1017,1069,1117,1167,1324,1406,1445,1483,1617$, 2834, 2930; HRMS: calcd $\mathrm{m} / \mathrm{z}$ for $\mathrm{C}_{57} \mathrm{H}_{52} \mathrm{~F}_{12} \mathrm{~N}_{2} \mathrm{O}_{4} \mathrm{P}^{+}\left[\mathrm{M}-\mathrm{SbF}_{6}\right]^{+}:$1087.3468; found (ESI) 1087.3472. 


\section{Experimental}

4,5-Dichloro-2-\{(5R,6R)-5,6-dimethoxy-4,4,7,7-tetrakis[4-(trifluoromethyl)phenyl]-1,3,2dioxaphosphepan-2-yl\}-1,3-dimesityl-1H-imidazol-3-ium Hexafluoroantimonate(169t):
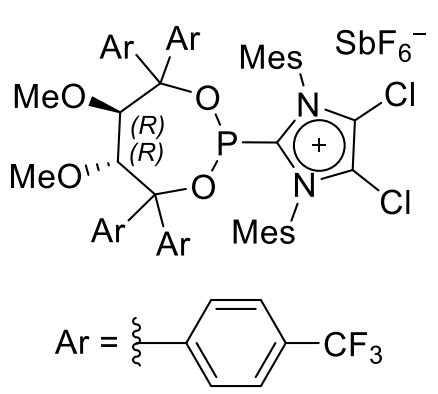

Prepared as a pale orange solid (55 $\mathrm{mg}, 30 \%$ yield) from the corresponding diol $(R, R)-170 \mathrm{i}^{[219]}$ (1.0 equiv, $\left.95 \mathrm{mg}, 130 \mu \mathrm{mol}\right)$, molecular sieves (4 $\AA$, powdered, $100 \mathrm{mg}$ ), pyridine (3.2equiv, $33 \mathrm{mg}, 417 \mu \mathrm{mol})$, phosphorus trichloride (1.20 equiv, $21 \mathrm{mg}$, $153 \mu \mathrm{mol}) \quad$ with 1,3-bis(2,4,6-trimethylphenyl),4,5-dichloro-1,3dihydro-2H-imidazol-2-ylidene (56f) ${ }^{[239]} \quad(1.0$ equiv, $48 \mathrm{mg}$, $129 \mu \mathrm{mol})$, sodium hexafluoroantimonate $(2.10$ equiv, $69 \mathrm{mg}$,

$267 \mu \mathrm{mol}$ ) according to modified GPD. After allowing warming to room temperature overnight, the mixture was stirred for a further 48 hours at room temperature. Column chromatography ( $1 \%$ ethyl acetate in dichloromethane) at $-10^{\circ} \mathrm{C}$ afforded compound $169 \mathrm{t}$.

$[\alpha]_{22}^{D}:-32.9\left(c=1.02, \mathrm{CH}_{2} \mathrm{Cl}_{2}\right) ;{ }^{1} \mathrm{H}$ NMR: $\left(400 \mathrm{MHz}, \mathrm{CD}_{3} \mathrm{CN}\right) \delta=7.75-7.65(\mathrm{~m}, 6 \mathrm{H}), 7.62$ $(\mathrm{d}, J=8.3 \mathrm{~Hz}, 2 \mathrm{H}), 7.44(\mathrm{~d}, J=8.2 \mathrm{~Hz}, 2 \mathrm{H}), 7.28(\mathrm{~s}, 2 \mathrm{H}), 7.24-7.14(\mathrm{~m}, 4 \mathrm{H}), 6.97(\mathrm{~d}, J=$ $8.1 \mathrm{~Hz}, 2 \mathrm{H}), 6.95(\mathrm{~d}, J=7.8 \mathrm{~Hz}, 2 \mathrm{H}), 4.62\left(\mathrm{dd}, J=7.0 \mathrm{~Hz}, J_{H-P}=7.8 \mathrm{~Hz}, 1 \mathrm{H}\right), 3.90(\mathrm{~d}, J=7.4$ $\mathrm{Hz}, 1 \mathrm{H}), 3.35(\mathrm{~s}, 3 \mathrm{H}), 2.49(\mathrm{~s}, 6 \mathrm{H}), 2.31(\mathrm{~s}, 3 \mathrm{H}), 1.95(\mathrm{~s}, 6 \mathrm{H}), 1.68(\mathrm{~s}, 6 \mathrm{H}) \mathrm{ppm} ;{ }^{13} \mathrm{C}\left\{{ }^{1} \mathrm{H}\right\}$ NMR: $\left(101 \mathrm{MHz}, \mathrm{CD}_{3} \mathrm{CN}\right) \delta=147.0\left(\mathrm{~d}, J_{C-P}=3.3 \mathrm{~Hz}\right), 146.4,144.6,144.3\left(\mathrm{~d}, J_{C-P}=75.1 \mathrm{~Hz}\right)$, 142.6, $142.5-142.4(\mathrm{~m}), 136.7,136.7,136.4,136.4,131.7$ (q, $\left.J_{C-F}=32.9 \mathrm{~Hz}\right), 131.5\left(\mathrm{q}, J_{C-F}\right.$ $=32.8 \mathrm{~Hz}$ ), 131.5, $131.2\left(\mathrm{q}, J_{C-F}=32.5 \mathrm{~Hz}\right), 131.0\left(\mathrm{q}, J_{C-F}=32.4 \mathrm{~Hz}\right), 130.8,129.9,129.8$, 129.8, 129.8, 129.5, $128.9\left(\mathrm{~d}, J_{C-P}=1.0 \mathrm{~Hz}\right), 126.7\left(\mathrm{q}, J_{C-F}=3.7 \mathrm{~Hz}\right), 126.5,126.5\left(\mathrm{q}, J_{C-F}=\right.$ $3.9 \mathrm{~Hz}$ ), $126.2\left(\mathrm{q}, J_{C-F}=4.0 \mathrm{~Hz}\right), 125.2\left(\mathrm{q}, J_{C-F}=271.3 \mathrm{~Hz}\right), 125.1\left(\mathrm{q}, J_{C-F}=271.6 \mathrm{~Hz}\right), 125.1$ $\left(\mathrm{q}, J_{C-F}=271.3 \mathrm{~Hz}\right), 125.0\left(\mathrm{q}, J_{C-F}=271.5 \mathrm{~Hz}\right), 124.9\left(\mathrm{q}, J_{C-F}=3.7 \mathrm{~Hz}\right), 88.9\left(\mathrm{~d}, J_{C-P}=4.7\right.$ $\mathrm{Hz}), 87.0\left(\mathrm{~d}, J_{C-P}=14.9 \mathrm{~Hz}\right), 84.5\left(\mathrm{~d}, J_{C-P}=1.4 \mathrm{~Hz}\right), 80.5\left(\mathrm{~d}, J_{C-P}=29.0 \mathrm{~Hz}\right), 61.4,60.5,21.4$, 18.2, 17.7, 17.7 ppm; ${ }^{31} \mathrm{P}\left\{{ }^{1} \mathrm{H}\right\}$ NMR: $\left(162 \mathrm{MHz}, \mathrm{CD}_{3} \mathrm{CN}\right) \delta=147.7 \mathrm{ppm} ;{ }^{19} \mathrm{~F}$ NMR: $(376 \mathrm{MHz}$, $\mathrm{CD}_{3} \mathrm{CN}$ ) $\delta=-63.1,-63.2,-63.3,-63.3,-123.9$ (oct, $J=1050.7 \mathrm{~Hz}$ ), -123.9 (sext, $J=1935.3$ Hz) ppm; IR: (neat, $\mathrm{cm}^{-1}$ ) $\tilde{v}=654,822,853,929,961,997,1017,1069,1117,1168,1323$, 1411, 1481, 1568, 1617, 2834, 2926, 2965; HRMS: calcd $m / z$ for $\mathrm{C}_{55} \mathrm{H}_{46} \mathrm{Cl}_{2} \mathrm{~F}_{12} \mathrm{~N}_{2} \mathrm{O}_{4} \mathrm{P}^{+}[\mathrm{M}-$ $\left.\mathrm{SbF}_{6}\right]^{+}:$1127.2375; found (ESI) 1127.2378. 


\section{Experimental}

\section{4,5-Dichloro-1,3-dimesityl-2-\{(3aS,8aS)-4,4,8,8-tetrakis[4-(tert-butyl)phenyl]-2,2-}

dimethyltetrahydro-[1,3]dioxolo[4,5-e][1,3,2]dioxaphosphepin-6-yl\}-1 H-imidazol-3-ium Hexafluoroantimonate (169u):

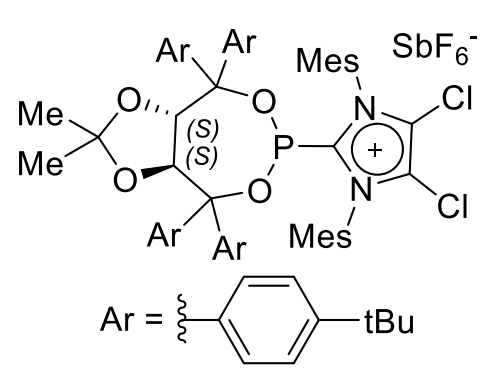

Prepared as a pale orange solid (110 mg, $83 \mu \mathrm{mol}, 31 \%$ yield) from the corresponding diol $(S, S)-170 a^{[233]}$ (1.0 equiv, $189 \mathrm{mg}$,

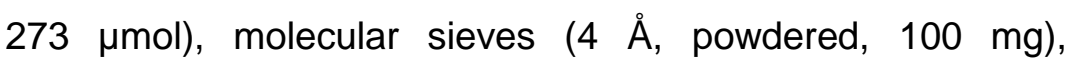
pyridine ( 3.15 equiv, $67 \mathrm{mg}, 847 \mu \mathrm{mol}$ ), phosphorus trichloride (1.16 equiv, $42.8 \mathrm{mg}, 312 \mu \mathrm{mol})$ with 1,3-bis(2,4,6trimethylphenyl),4,5-dichloro-1,3-dihydro-2H-imidazol-2-

ylidene $(\mathbf{5 6 f})^{[239]}$ (1.0 equiv, $\left.100 \mathrm{mg}, 269 \mu \mathrm{mol}\right)$, sodium hexafluoroantimonate (2.0 equiv, 141 $\mathrm{mg}, 545 \mu \mathrm{mol}$ ) according to modified GPD (after allowing warming to room temperature overnight, the mixture was stirred for a further 24 hours at room temperature). Column chromatography ( $1 \%$ ethyl acetate in dichloromethane) at $-10{ }^{\circ} \mathrm{C}$, followed by precipitating a saturated diethyl ether solution with pentane gave the product $169 \mathrm{u}$.

$[\alpha]_{20}^{D}:+65.0\left(c=0.955, \mathrm{CH}_{2} \mathrm{Cl}_{2}\right) ;{ }^{1} \mathrm{H}$ NMR: $\left(400 \mathrm{MHz}, \mathrm{CD}_{3} \mathrm{CN}\right) \delta=7.49-7.38(\mathrm{~m}, 4 \mathrm{H}), 7.38$ - $7.32(\mathrm{~m}, 4 \mathrm{H}), 7.31$ (s, 2H), $7.29-7.23(\mathrm{~m}, 4 \mathrm{H}), 7.16(\mathrm{~s}, 2 \mathrm{H}), 6.79(\mathrm{~d}, J=8.2 \mathrm{~Hz}, 2 \mathrm{H}), 6.50$ (d, $J=8.2 \mathrm{~Hz}, 2 \mathrm{H}$ ), 5.09 (dd, J = 8.2 Hz, JH-P $=4.8 \mathrm{~Hz}, 1 \mathrm{H}$ ), $4.63(\mathrm{~d}, J=8.5 \mathrm{~Hz}, 1 \mathrm{H}$ ), 2.51 (s, $6 \mathrm{H}), 2.11(\mathrm{~s}, 6 \mathrm{H}), 1.74(\mathrm{~s}, 6 \mathrm{H}), 1.34(\mathrm{~s}, 27 \mathrm{H}), 1.32(\mathrm{~s}, 3 \mathrm{H}), 1.31-1.27(\mathrm{~m}, 9 \mathrm{H}),-0.03(\mathrm{~s}, 3 \mathrm{H})$ ppm; ${ }^{13} \mathrm{C}\left\{{ }^{1} \mathrm{H}\right\}$ NMR: $\left(101 \mathrm{MHz}, \mathrm{CD}_{3} \mathrm{CN}\right) \delta=153.2,153.0,152.9,152.7,145.8\left(\mathrm{~d}, J_{C-P}=76.1\right.$ $\mathrm{Hz}$ ), 144.0, 141.6, 141.0 (d, $\left.J_{C-P}=4.5 \mathrm{~Hz}\right), 136.8,136.7,136.6$ (d, $J_{C-P}=2.5 \mathrm{~Hz}$ ), 136.1, $131.5,131.2$, 129.9, 129.1, 129.1, 129.0, 128.4, 128.1, 126.5, 126.2, 126.2, 125.9, 125.1 , 113.8, $87.7\left(\mathrm{~d}, J_{C-P}=11.2 \mathrm{~Hz}\right), 86.8\left(\mathrm{~d}, J_{C-P}=7.6 \mathrm{~Hz}\right), 83.2\left(\mathrm{~d}, J_{C-P}=2.6 \mathrm{~Hz}\right), 81.5\left(\mathrm{~d}, J_{C-P}=\right.$ $28.5 \mathrm{~Hz}), 35.3,35.3,35.2,31.4,27.6,24.6,21.6,18.4,18.2,18.2 \mathrm{ppm} ;{ }^{31} \mathbf{P}\left\{{ }^{1} \mathrm{H}\right\}$ NMR: (162 $\left.\mathrm{MHz}, \mathrm{CDCl}_{3}\right) \delta=148.9$ ppm; ${ }^{19} \mathrm{~F}$ NMR: $\left(376 \mathrm{MHz}, \mathrm{CD}_{3} \mathrm{CN}\right.$ ) $\delta=-123.97$ (sext, $J=1930.3$ $\mathrm{Hz}$ ), -123.98 (oct, $J=1051.0 \mathrm{~Hz}$ ) ppm; IR: (neat, $\mathrm{cm}^{-1}$ ) $\tilde{v}=562,598,655,841,877,955$, 979, 1088, 1112, 1201, 1379, 1411, 1473, 1512, 1566, 1606, 28623, 2907, 2958; HRMS: calcd. $\mathrm{m} / \mathrm{z}$ for $\mathrm{C}_{68} \mathrm{H}_{82} \mathrm{Cl}_{2} \mathrm{~N}_{2} \mathrm{O}_{4} \mathrm{P}^{+}\left[\mathrm{M}-\mathrm{SbF}_{6}\right]^{+:}$1091.5384; found (ESI) 1091.5385 .

Synthesis of (S)-2-(Dinaphtho[2,1-d:1',2'-f][1,3,2]dioxaphosphepin-4-yl)-1,3-dimesityl1H-imidazol-3-ium Hexafluoroantimonate (208a) and (S)-4-(Dinaphtho[2,1-d:1',2'$f][1,3,2]$ dioxaphosphepin-4-yl)-1,3-dimesityl-1 $H$-imidazol-3-ium Hexafluoroantimonate (208b):

From the corresponding diol $(S)-207^{[242]}$ (1.0 equiv, $200 \mathrm{mg}, 456 \mu \mathrm{mol}$ ), molecular sieves (4 Å, powdered, $50 \mathrm{mg}$ ), pyridine (3.0 equiv, $108 \mathrm{mg}, 1.37 \mathrm{mmol}$ ), phosphorus trichloride (1.5 equiv, $94 \mathrm{mg}, 684 \mu \mathrm{mol})$ with 1,3-bis(2,4,6-trimethylphenyl)-1,3-dihydro-2H-imidazol-2ylidene (56b) $)^{[274]}$ (1.0 equiv, $139 \mathrm{mg}, 457 \mu \mathrm{mol}$ ) and sodium hexafluoroantimonate (3.0 equiv, 


\section{Experimental}

$353 \mathrm{mg}, 1.36 \mathrm{mmol}$ ), a mixture of C-2 and C-4 adducts 208a and 208b was obtained according to GPD, along with several other unidentified compounds. Crystallization from dichloromethane/pentane separated the C-2 and C-4 adducts (fraction 1: 208a; fraction 2: 208b).

208a:

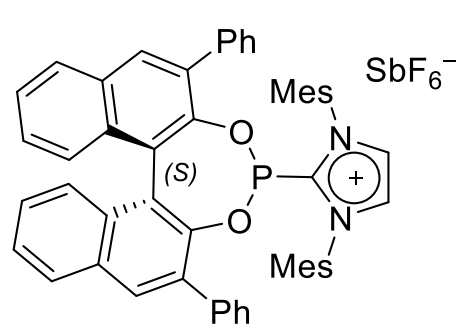

Further purification by column chromatography at $-10{ }^{\circ} \mathrm{C}(2 \%$ ethyl acetate in dichloromethane) afforded the product 208a as a white solid (40 mg, $40 \mu \mathrm{mol}, 9 \%$ yield). Crystals suitable for $X$ ray crystallographic analysis were grown by layering a dichloromethane solution with toluene.

$[\alpha]_{20}^{D}:+151.2\left(c=1.02, \mathrm{CH}_{2} \mathrm{Cl}_{2}\right) ;{ }^{1} \mathrm{H}$ NMR: $\left(400 \mathrm{MHz}, \mathrm{CD}_{3} \mathrm{CN}\right) \delta=8.12(\mathrm{~d}, J=8.4 \mathrm{~Hz}, 1 \mathrm{H})$, $8.12(\mathrm{~s}, 1 \mathrm{H}), 7.97(\mathrm{~d}, J=2.0 \mathrm{~Hz}, 1 \mathrm{H}), 7.91(\mathrm{dd}, J=8.4,2.7 \mathrm{~Hz}, 1 \mathrm{H}), 7.66-7.61(\mathrm{~m}, 2 \mathrm{H})$, $7.61-7.55(\mathrm{~m}, 2 \mathrm{H}), 7.55-7.44(\mathrm{~m}, 9 \mathrm{H}), 7.42(\mathrm{ddd}, J=8.5,6.9,2.3 \mathrm{~Hz}, 1 \mathrm{H}), 7.27(\mathrm{ddd}, J=$ 8.6, 7.0, 1.5 Hz, 1H), $7.13(\mathrm{~s}, 1 \mathrm{H}), 7.12-7.05(\mathrm{~m}, 1 \mathrm{H}), 6.96(\mathrm{~s}, 1 \mathrm{H}), 6.70(\mathrm{dd}, J=8.7,2.0 \mathrm{~Hz}$, $1 \mathrm{H}), 6.64(\mathrm{~d}, J=8.6 \mathrm{~Hz}, 1 \mathrm{H}), 6.55(\mathrm{~s}, 1 \mathrm{H}), 5.37(\mathrm{~s}, 1 \mathrm{H}), 2.36(\mathrm{~s}, 3 \mathrm{H}), 1.84(\mathrm{~s}, 3 \mathrm{H}), 1.71(\mathrm{~s}$, $3 \mathrm{H}), 1.39(\mathrm{~s}, 3 \mathrm{H}), 1.35(\mathrm{~s}, 3 \mathrm{H}), 1.01(\mathrm{~s}, 3 \mathrm{HH}) \mathrm{ppm} ;{ }^{13} \mathrm{C}\left\{{ }^{1} \mathrm{H}\right\} \mathrm{NMR}:\left(101 \mathrm{MHz}, \mathrm{CD}_{3} \mathrm{CN}\right) \delta=$ 146.0, 145.9, $145.7\left(\mathrm{~d}, J_{C-P}=118.0 \mathrm{~Hz}\right), 145.4,143.2,141.1,137.6,137.5,136.7\left(\mathrm{~d}, J_{C-P}=\right.$ $2.4 \mathrm{~Hz}$ ), 135.1 (d, $\left.J_{C-P}=3.6 \mathrm{~Hz}\right), 134.2\left(\mathrm{~d}, J_{C-P}=2.5 \mathrm{~Hz}\right), 133.4\left(\mathrm{~d}, J_{C-P}=1.0 \mathrm{~Hz}\right), 133.3$, 133.0, 132.9, 132.9, 132.4, 132.3, 132.1, 132.0, 130.5, 130.5, 130.5, 130.3, 130.2, 130.1 (d, $\left.J_{C-P}=2.2 \mathrm{~Hz}\right), 129.9,129.7,129.5,129.3,129.1,129.1,128.8,128.5,128.3,127.8,127.6$, 127.4, 127.2, 127.2, 127.1, 125.0 (d, $\left.J_{C-P}=5.7 \mathrm{~Hz}\right), 124.0$ (d, $\left.J_{C-P}=3.6 \mathrm{~Hz}\right), 21.1,21.1,18.6$, 17.9, 17.8, 17.2, 15.5 ppm; ${ }^{31} \mathbf{P}\left\{{ }^{1} \mathrm{H}\right\}$ NMR: $\left(162 \mathrm{MHz}, \mathrm{CD}_{3} \mathrm{CN}\right) \delta=142.0$ ppm; IR: (neat, $\left.\mathrm{cm}^{-1}\right)$ $\tilde{v}=652,701,76,821,854,962,1145,1192,1313,1407,1446,1481,1605,2920,3054$, 3152; HRMS: calcd $m / z$ for $\mathrm{C}_{53} \mathrm{H}_{44} \mathrm{~N}_{2} \mathrm{O}_{2} \mathrm{P}^{+}\left[\mathrm{M}-\mathrm{SbF}_{6}\right]^{+}:$771.3135; found (ESI) 771.3134.

208b:

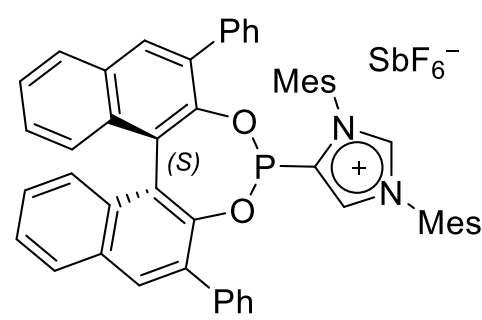

Evaporation of the second fraction, followed by column chromatography at $-10{ }^{\circ} \mathrm{C}(0$ to $1.5 \%$ ethyl acetate in dichloromethane), affording a white solid (15 mg, $15 \mu \mathrm{mol}$, $3 \%$ yield).

'H NMR: $\left(400 \mathrm{MHz}, \mathrm{CD}_{3} \mathrm{CN}\right) \delta=8.52\left(\mathrm{t}, J=1.7 \mathrm{~Hz}, J_{H-P}=1.7\right.$ $\mathrm{Hz}, 1 \mathrm{H}), 8.21(\mathrm{~s}, 1 \mathrm{H}), 8.17(\mathrm{~s}, 1 \mathrm{H}), 8.09(\mathrm{~d}, J=12.0 \mathrm{~Hz}, 1 \mathrm{H}), 8.07$ (d, J=12.5 Hz, 1H), 7.64 - $7.50(\mathrm{~m}, 6 \mathrm{H}), 7.49-7.44(\mathrm{~m}, 3 \mathrm{H}), 7.41-7.35(\mathrm{~m}, 4 \mathrm{H}), 7.35-7.29(\mathrm{~m}, 2 \mathrm{H}), 7.24(\mathrm{~d}, J=8.6$ $\mathrm{Hz}, 1 \mathrm{H}), 7.14(\mathrm{~s}, 1 \mathrm{H}), 7.05(\mathrm{~d}, J=1.7 \mathrm{~Hz}, 1 \mathrm{H}), 7.03(\mathrm{~s}, 1 \mathrm{H}), 6.95(\mathrm{~s}, 1 \mathrm{H}), 6.90(\mathrm{~s}, 1 \mathrm{H}), 2.32$ 


\section{Experimental}

(s, 3H), $2.27(\mathrm{~s}, 3 \mathrm{H}), 2.12(\mathrm{~s}, 3 \mathrm{H}), 1.86(\mathrm{~s}, 3 \mathrm{H}), 1.40(\mathrm{~s}, 3 \mathrm{H}), 1.20(\mathrm{~s}, 3 \mathrm{HH}) \mathrm{ppm} ;{ }^{13} \mathrm{C}\left\{{ }^{1} \mathrm{H}\right\}$ NMR: $\left(151 \mathrm{MHz}, \mathrm{CD}_{3} \mathrm{CN}\right) \delta=146.3\left(\mathrm{~d}, J_{C-P}=1.7 \mathrm{~Hz}\right), 145.1\left(\mathrm{~d}, J_{C-P}=6.6 \mathrm{~Hz}\right), 143.1,142.6$, 140.2, 137.6, 137.3, $136.5\left(\mathrm{~d}, J_{C-P}=63.7 \mathrm{~Hz}\right), 135.6,135.5\left(\mathrm{~d}, J_{C-P}=2.4 \mathrm{~Hz}\right), 135.2,134.5$, $134.2\left(\mathrm{~d}, J_{C-P}=2.1 \mathrm{~Hz}\right), 133.6,133.0,132.9,132.8,132.5,132.4,132.4,130.8,130.5,130.5$, $130.4,130.4$, 130.4, 130.3, 130.2, 130.0, 129.6, 129.6, 129.4, 129.4, 129.1, 128.9, 127.8, 127.7, 127.4, 127.2, 127.1, 127.0, $125.2\left(\mathrm{~d}, J_{C-P}=2.8 \mathrm{~Hz}\right), 124.6\left(\mathrm{~d}, J_{C-P}=19.6 \mathrm{~Hz}\right), 21.0$, 20.9, 18.1, 17.4, 17.2, 17.2, 16.5 ppm; ${ }^{31} \mathbf{P}\left\{{ }^{1} \mathrm{H}\right\}$ NMR: $\left(243 \mathrm{MHz}, \mathrm{CD}_{3} \mathrm{CN}\right) \delta=146.2$ ppm; IR: (neat, $\mathrm{cm}^{-1}$ ) $\tilde{v}=517,655,701,753,852,961,1077,1124,1193,1405,1497,1606,2853$, 2923, 3126; HRMS: calcd $m / z$ for $\mathrm{C}_{53} \mathrm{H}_{44} \mathrm{~N}_{2} \mathrm{O}_{2} \mathrm{P}^{+}\left[\mathrm{M}-\mathrm{SbF}_{6}\right]^{+}:$771.3135; found (ESI) 771.3146.

\section{(S)-2-(2,6-Diphenyldinaphtho[2,1-d:1',2'-f][1,3,2]dioxaphosphepin-4-yl)-1,3-dimesityl-}

\section{4,5-dimethyl-1H-imidazol-3-ium Hexafluoroantimonate (208c):}

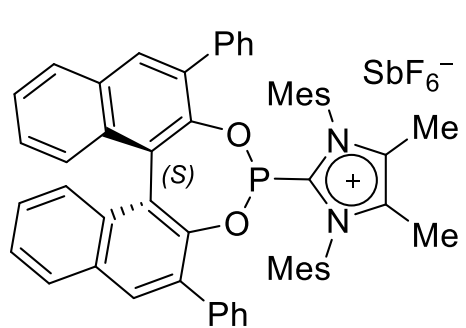

Compound 208c was prepared as a white solid $(300 \mathrm{mg}, 290$ $\mu \mathrm{mol}, 73 \%$ yield) from the corresponding diol $(S)-207^{[242]}(1.06$ equiv, $185 \mathrm{mg}, 422 \mu \mathrm{mol}$ ), molecular sieves (4 A, powdered, 50 $\mathrm{mg}$ ), pyridine (3.4 equiv, $108 \mathrm{mg}, 1.36 \mathrm{mmol}$ ), phosphorus trichloride (1.15 equiv, $63.0 \mathrm{mg}, 459 \mu \mathrm{mol})$ with 1,3-bis(2,4,6trimethylphenyl)4,5-dimethyl-1,3-dihydro-2H-imidazol-2-ylidene (56e) ${ }^{[240]}$ (1.0 equiv., $133 \mathrm{mg}$, $400 \mu \mathrm{mol}$ ) and sodium hexafluoroantimonate (1.5 equiv, $164 \mathrm{mg}, 634 \mu \mathrm{mol})$ according to GPD after column chromatography (1-1.5\% ethyl acetate in dichloromethane). Crystals suitable for X-ray crystallographic analysis were grown by layering a dichloromethane solution with toluene.

$[\alpha]_{20}^{D}:+188.4\left(c=1.00, \mathrm{CH}_{2} \mathrm{Cl}_{2}\right) ;{ }^{1} \mathrm{H}$ NMR: $\left(300 \mathrm{MHz}, \mathrm{CD}_{3} \mathrm{CN}\right) \delta=8.14(\mathrm{dt}, J=7.9,0.5 \mathrm{~Hz}$, $1 \mathrm{H}$ ), $8.13(\mathrm{~s}, 1 \mathrm{H}), 7.98(\mathrm{~s}, 1 \mathrm{H}), 7.95(\mathrm{dt}, J=8.2,0.5 \mathrm{~Hz}, 1 \mathrm{H}), 7.70-7.63(\mathrm{~m}, 2 \mathrm{H}), 7.59$ (ddd, $J=8.1,6.9,1.0 \mathrm{~Hz}, 1 \mathrm{H}$ ), $7.54-7.46(\mathrm{~m}, 8 \mathrm{H}$ ), 7.44 (ddd, $J=8.1,6.8,1.0 \mathrm{~Hz}, 1 \mathrm{H}$ ), 7.30 (ddd, $J=8.2,6.9,1.2 \mathrm{~Hz}, 1 \mathrm{H}), 7.16(\mathrm{~s}, 1 \mathrm{H}), 7.13(\mathrm{ddd}, J=7.1,5.4,1.3 \mathrm{~Hz}, 1 \mathrm{H}), 6.97(\mathrm{~s}, 1 \mathrm{H}), 6.72$ (dd, $J=8.0,0.6 \mathrm{~Hz}, 1 \mathrm{H}$ ), 6.67 (dd, $J=7.9,0.6 \mathrm{~Hz}, 1 \mathrm{H}), 6.58(\mathrm{~s}, 1 \mathrm{H}), 5.42(\mathrm{~s}, 1 \mathrm{H}), 2.38$ (s, $3 \mathrm{H}), 1.83(\mathrm{~s}, 6 \mathrm{H}), 1.72(\mathrm{~s}, 6 \mathrm{H}), 1.35(\mathrm{~s}, 3 \mathrm{H}), 1.33(\mathrm{~s}, 3 \mathrm{H}), 0.96(\mathrm{~s}, 3 \mathrm{H}) \mathrm{ppm} ;{ }^{13} \mathrm{C}\left\{{ }^{1} \mathrm{H}\right\}$ NMR: $\left(101 \mathrm{MHz}, \mathrm{CD}_{3} \mathrm{CN}\right) \delta=146.2\left(\mathrm{~d}, J_{C-P}=11.3 \mathrm{~Hz}\right), 145.8,143.2,142.9\left(\mathrm{~d}, J_{C-P}=112.7 \mathrm{~Hz}\right)$, 141.2, 137.9, $137.3\left(\mathrm{~d}, J_{C-P}=2.6 \mathrm{~Hz}\right), 135.4\left(\mathrm{~d}, J_{C-P}=3.6 \mathrm{~Hz}\right), 135.3,134.9\left(\mathrm{~d}, J_{C-P}=2.2 \mathrm{~Hz}\right)$, $134.4\left(\mathrm{~d}, J_{C-P}=2.6 \mathrm{~Hz}\right), 133.7,133.5,133.4,133.0,132.9,132.9,132.3,132.1,130.6,130.6$, $130.5,130.5,130.2,129.9,129.6,129.5,129.4,129.2,129.1,129.1,128.9,128.9,127.8$, 127.6, 127.5, 127.3, 127.2, 127.0, 125.1 (d, $\left.J_{C-P}=5.9 \mathrm{~Hz}\right), 124.0$ (d, $\left.J_{C-P}=2.9 \mathrm{~Hz}\right), 21.2$, 21.2, 18.9, 18.1, 18.0, 17.3, 15.5, 9.0 ppm; ${ }^{31} \mathrm{P}\left\{{ }^{1} \mathrm{H}\right\}$ NMR: $\left(122 \mathrm{MHz}, \mathrm{CD}_{3} \mathrm{CN}\right) \delta=142.4$ ppm; ${ }^{19}$ F NMR: $\left(282 \mathrm{MHz}, \mathrm{CD}_{3} \mathrm{CN}\right) \delta=-124.0$ (oct, $\left.J_{F-123 S b}=1048.4 \mathrm{~Hz}\right),-124.0$ (sext, $J_{F-121 S b}=$ $1926.5 \mathrm{~Hz}$ ) ppm; IR: (neat, $\left.\mathrm{cm}^{-1}\right) \tilde{v}=495,567,653,701,752,818,854,961,1172,1405$, 
1607, 2919, 3032, 3053; HRMS: calcd $\mathrm{m} / \mathrm{z}$ for $\mathrm{C}_{55} \mathrm{H}_{48} \mathrm{~N}_{2} \mathrm{O}_{2} \mathrm{P}^{+}\left[\mathrm{M}-\mathrm{SbF}_{6}\right]^{+:}$: 799.3448; found (ESI) 799.3454

\subsubsection{Synthesis of gold(I) complexes}

\section{General procedure E (GPE) for the preparation of gold(I) complexes}

A dried Schlenk flask equipped with a magnetic stirring bar and rubber septum was charged with the cationic phosphonite and dichloromethane, and then cooled to $-20{ }^{\circ} \mathrm{C}$. To the Schlenk flask was then added (dimethyl sulfide)gold(I) chloride as a solid. The resulting solution was allowed to stir at $-20^{\circ} \mathrm{C}$ for a few minutes, before warming to room temperature and stirring for between 30 minutes and one hour. The solvent was removed in vacuo and the product dried under high vacuum.

$\{(3 a S, 8 a S)-6-[B i s(t r i p h e n y l p h o s p h o n i o) m e t h y l e n e]-2,2-d i m e t h y l-4,4,8,8-$ tetraphenyltetrahydro-6 $\lambda^{5}$-[1,3]dioxolo[4,5-e][1,3,2]dioxaphosphepin-6-yl\}gold Chloride Hexafluoroantimonate (172I):

Prepared as a white solid (118 mg, $78.6 \mu \mathrm{mol},>99 \%$ yield)
from compound 169l (1.0 equiv, 92.0 mg, $72.5 \mu \mathrm{mol})$,
chloro(dimethyl sulfide)gold(I) (1.0 equiv, $21.4 \mathrm{mg}, 72.6 \mu \mathrm{mol})$ $[\alpha]_{20}^{D}:+78.7\left(c=1.03, \mathrm{CH}_{2} \mathrm{Cl}_{2}\right) ;{ }^{1} \mathrm{H}$ NMR: $\left(300 \mathrm{MHz}, \mathrm{CD}_{3} \mathrm{CN}\right) \delta=7.81(\mathrm{~d}, J=7.6 \mathrm{~Hz}, 2 \mathrm{H})$, $7.70-7.61(\mathrm{~m}, 8 \mathrm{H}), 7.61-7.50(\mathrm{~m}, 13 \mathrm{H}), 7.44-7.31(\mathrm{~m}, 15 \mathrm{H}), 7.31-7.21(\mathrm{~m}, 3 \mathrm{H}), 7.16(\mathrm{t}$, $J=7.4 \mathrm{~Hz}, 1 \mathrm{H}), 7.06(\mathrm{t}, J=7.6 \mathrm{~Hz}, 2 \mathrm{H}), 6.88(\mathrm{t}, J=7.7 \mathrm{~Hz}, 2 \mathrm{H}), 6.59(\mathrm{~d}, J=7.6 \mathrm{~Hz}, 2 \mathrm{H})$, $6.14(\mathrm{~d}, J=7.9 \mathrm{~Hz}, 2 \mathrm{H}), 5.52(\mathrm{~d}, J=7.8 \mathrm{~Hz}, 1 \mathrm{H}), 5.23(\mathrm{~d}, J=7.9 \mathrm{~Hz}, 1 \mathrm{H}), 0.96(\mathrm{~s}, 3 \mathrm{H}), 0.28$ $(\mathrm{s}, 3 \mathrm{HH})$ ppm; ${ }^{13} \mathrm{C}\left\{{ }^{1} \mathrm{H}\right\}$ NMR: $\left(101 \mathrm{MHz}, \mathrm{CD}_{3} \mathrm{CN}\right) \delta=145.7\left(\mathrm{~d}, J_{C-P}=4.8 \mathrm{~Hz}\right), 143.6,139.9(\mathrm{~d}$, $\left.J_{C-P}=6.6 \mathrm{~Hz}\right), 139.2,136.2-135.2(\mathrm{~m}), 134.7,130.7,130.6,130.6-130.3(\mathrm{~m}), 130.0$, 129.9, 129.8, 129.4, 129.4, 129.1, 129.0, 128.6, 128.2, 127.9, 125.2 (dd, $J_{C-P}=93.4,2.7 \mathrm{~Hz}$ ), 115.0, $94.6\left(\mathrm{~d}, J_{C-P}=13.8 \mathrm{~Hz}\right), 90.8,80.8\left(\mathrm{~d}, J_{C-P}=11.6 \mathrm{~Hz}\right), 79.8\left(\mathrm{~d}, J_{C-P}=2.5 \mathrm{~Hz}\right), 27.6$, 26.1, $25.6\left(\mathrm{td}, J_{C-P}=86.6,71.5 \mathrm{HzH}\right) \mathrm{ppm} ;{ }^{31} \mathrm{P}\left\{{ }^{1} \mathrm{H}\right\}$ NMR: $\left(162 \mathrm{MHz}, \mathrm{CD}_{3} \mathrm{CN}\right) \delta=132.5(\mathrm{t}, \mathrm{J}=$ $38.2 \mathrm{~Hz}$ ), 22.9 (d, $J=38.1 \mathrm{~Hz})$ ppm; ${ }^{19} \mathrm{~F}$ NMR: $\left(282 \mathrm{MHz}, \mathrm{CD}_{3} \mathrm{CN}\right) \delta=-124.0$ (sext, $J_{F-121 S b}=$ 1942.1 Hz), -124.0 (oct, $J_{F-123 s b}=1049.7 \mathrm{~Hz}$ ) ppm; IR: (neat, $\mathrm{cm}^{-1}$ ) $\tilde{v}=499,544,654,693$, 473, 885, 976, 1095, 1163, 1437, 1483, 2674, 2923, 3003, 3041; HRMS: calcd m/z for $\mathrm{C}_{68} \mathrm{H}_{58} \mathrm{AuClO}_{4} \mathrm{P}_{3}{ }^{+}\left[\mathrm{M}-\mathrm{SbF}_{6}\right]^{+:}$1263.2897; found (ESI) 1263.2900. 


\section{Experimental}

$\left\{(5 S, 6 S)-5,6-D i m e t h o x y-4,4,7,7-t e t r a(4-t e r t-b u t y l p h e n y l)-2-\left[\left(t r i p h e n y l-\lambda^{4}-\right.\right.\right.$ phosphaneyl)(triphenylphosphonio)methylene]-1,3,2 $\lambda^{5}$-dioxaphosphepan-2-yl\}gold Chloride Hexafluoroantimonate (172m):

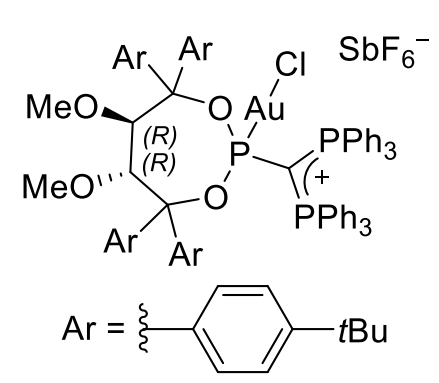

To a solution of compound $169 \mathrm{~m}(1.0$ equiv, $43.0 \mathrm{mg}, 29.0 \mu \mathrm{mol}$ ) in acetonitrile $(2 \mathrm{ml})$ at $-20^{\circ} \mathrm{C}$, was added solid chloro(dimethyl sulfide)gold(I) (1.0 equiv, $8.6 \mathrm{mg}, 29.1 \mu \mathrm{mol})$ according to modified GPE. The reaction mixture was allowed to stir at $-20^{\circ} \mathrm{C}$ for 30 minutes, then at room temperature for one hour. The solvent was removed in vacuo, affording the product as a white solid (49.0 mg, $28.6 \mu \mathrm{mol}, 99 \%$ yield).

$[\alpha]_{20}^{D}:-27.8\left(c=1.02, \mathrm{CH}_{2} \mathrm{Cl}_{2}\right) ;{ }^{1} \mathrm{H}$ NMR: $\left(400 \mathrm{MHz}, \mathrm{CD}_{3} \mathrm{CN}\right) \delta=7.66-7.61(\mathrm{~m}, 6 \mathrm{H}), 7.48-$ $7.41(\mathrm{~m}, 14 \mathrm{H}), 7.36-7.31(\mathrm{~m}, 12 \mathrm{H}), 7.21(\mathrm{~d}, J=8.5 \mathrm{~Hz}, 2 \mathrm{H}), 7.19-7.14(\mathrm{~m}, 6 \mathrm{H}), 7.02(\mathrm{~d}, J$ $=8.6 \mathrm{~Hz}, 2 \mathrm{H}), 6.57(\mathrm{~d}, J=8.5 \mathrm{~Hz}, 2 \mathrm{H}), 6.46(\mathrm{~d}, J=8.6 \mathrm{~Hz}, 2 \mathrm{H}), 4.91\left(\mathrm{dd}, J=6.6 \mathrm{~Hz}, J_{H-P}=\right.$ $2.7 \mathrm{~Hz}, 1 \mathrm{H}), 4.43(\mathrm{~d}, J=6.6 \mathrm{~Hz}, 1 \mathrm{H}), 2.73(\mathrm{~s}, 3 \mathrm{H}), 2.61(\mathrm{~s}, 3 \mathrm{H}), 1.37$ (s, 9H), $1.35(\mathrm{~s}, 9 \mathrm{H})$, $1.30(\mathrm{~s}, 9 \mathrm{H}), 1.26(\mathrm{~s}, 9 \mathrm{H}) \mathrm{ppm} ;{ }^{13} \mathrm{C}\left\{{ }^{1} \mathrm{H}\right\}$ NMR: $\left(101 \mathrm{MHz}, \mathrm{CD}_{3} \mathrm{CN}\right) \delta=152.5,152.5,152.4$, 152.0, $142.1\left(\mathrm{~d}, J_{C-P}=5.1 \mathrm{~Hz}\right), 139.9\left(\mathrm{~d}, J_{C-P}=1.5 \mathrm{~Hz}\right), 137.7\left(\mathrm{~d}, J_{C-P}=5.5 \mathrm{~Hz}\right), 137.1$ (d, $J_{C-P}$ $=2.0 \mathrm{~Hz}), 135.8-135.2(\mathrm{~m}), 134.6,130.6-130.2(\mathrm{~m}), 130.1,130.1,129.8,129.2,129.0$, 126.3, 126.1, 125.6 (ddd, $\left.J_{C-P}=97.7,4.7,2.1 \mathrm{~Hz}\right), 125.0,124.7,96.2\left(\mathrm{~d}, J_{C-P}=13.0 \mathrm{~Hz}\right.$ ), 91.5 $\left(\mathrm{d}, J_{C-P}=1.5 \mathrm{~Hz}\right), 81.2\left(\mathrm{~d}, J_{C-P}=11.1 \mathrm{~Hz}\right), 80.5,59.6,59.3,35.3,35.2,35.1,35.0,31.5,31.5$, 31.4, 23.7 (td, $\left.J_{C-P}=87.5,72.5 \mathrm{~Hz}\right) \mathrm{ppm} ;{ }^{31} \mathrm{P}\left\{{ }^{1} \mathrm{H}\right\}$ NMR: $\left(202 \mathrm{MHz}, \mathrm{CD}_{3} \mathrm{CN}\right) \delta=129.1(\mathrm{t}, J=$ $35.5 \mathrm{~Hz}$ ), 22.5 (d, $J=35.3 \mathrm{~Hz})$ ppm; ${ }^{19} \mathrm{~F}$ NMR: $\left(282 \mathrm{MHz}, \mathrm{CD}_{3} \mathrm{CN}\right) \delta=-123.3$ (sext, $J_{F-121 S b}=$ 1925.1 Hz), -125.9 (oct, $J_{F-123 S b}=1054.6 \mathrm{~Hz}$ ) ppm; IR: (neat, $\left.\mathrm{cm}^{-1}\right) \tilde{v}=501,526,655,746$, 918, 987, 1098, 1190, 1270, 1364, 1438, 1481, 2867, 2903, 2958;HRMS: calcd $\mathrm{m} / \mathrm{z}$ for $\mathrm{C}_{83} \mathrm{H}_{90} \mathrm{AuClO}_{4} \mathrm{P}_{3}+\left[\mathrm{M}-\mathrm{SbF}_{6}\right]^{+:}$1475.5401; found (ESI) 1475.5407.

\{2-[(3aS,8aS)-2,2-Dimethyl-4,4,8,8-tetraphenyltetrahydro-[1,3]dioxolo[4,5-e][1,3,2]dioxaphosphepin-6-yl]-1,3-dimesityl-1H-imidazol-3-ium\}gold Chloride Hexafluoroantimonate (172n)

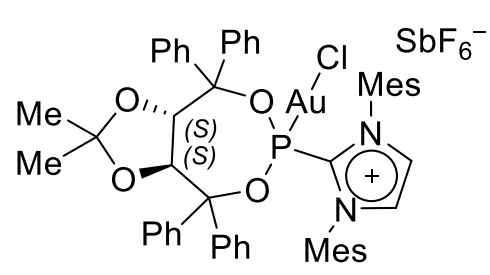

Prepared as a white solid (118 mg, $93.0 \mu \mathrm{mol}, 96 \%$ yield) from compound 169n (1.0 equiv, $100 \mathrm{mg}, 96.5 \mu \mathrm{mol})$, chloro(dimethyl sulfide)gold(I) (1.0 equiv, $28.4 \mathrm{mg}, 96.4 \mu \mathrm{mol})$ in dichloromethane $(3 \mathrm{ml})$ according to GPE.

$[\alpha]_{20}^{D}:+41.7\left(c=1.03, \mathrm{CH}_{2} \mathrm{Cl}_{2}\right){ }^{1}{ }^{1} \mathrm{H}$ NMR: $\left(300 \mathrm{MHz}, \mathrm{CD}_{3} \mathrm{CN}\right) \delta=7.93\left(\mathrm{~d}, J_{H-P}=2.4 \mathrm{~Hz}, 2 \mathrm{H}\right)$, $7.52-7.30(\mathrm{~m}, 14 \mathrm{H}), 7.23-7.19(\mathrm{~m}, 2 \mathrm{H}), 7.18(\mathrm{~s}, 2 \mathrm{H}), 7.14(\mathrm{~s}, 2 \mathrm{H}), 6.99-6.89(\mathrm{~m}, 2 \mathrm{H})$, $6.80-6.73(\mathrm{~m}, 2 \mathrm{H}), 6.28\left(\mathrm{dd}, J=8.6 \mathrm{~Hz}, J_{H-P}=2.0 \mathrm{~Hz}, 1 \mathrm{H}\right), 4.95(\mathrm{~d}, J=8.6 \mathrm{~Hz}, 1 \mathrm{H}), 2.40(\mathrm{~s}$, 
6H), $2.03(\mathrm{~s}, 6 \mathrm{H}), 2.00(\mathrm{~s}, 6 \mathrm{H}), 1.43(\mathrm{~s}, 3 \mathrm{H}), 0.00$ (s, 3H) ppm; ${ }^{13} \mathbf{C}\left\{{ }^{1} \mathrm{H}\right\}$ NMR: $(75 \mathrm{MHz}$, $\left.\mathrm{CD}_{3} \mathrm{CN}\right) \delta=143.9,142.0,141.9\left(\mathrm{~d}, J_{C-P}=8.5 \mathrm{~Hz}\right), 138.4\left(\mathrm{~d}, J_{C-P}=6.5 \mathrm{~Hz}\right), 138.3\left(\mathrm{~d}, J_{C-P}=\right.$ $102.4 \mathrm{~Hz}), 136.5$ (d, $\left.J_{C-P}=1.9 \mathrm{~Hz}\right), 135.9,135.8,132.3,132.2,131.7,131.6,131.6,131.3$, $131.0,130.8,130.7,130.1,130.0$ (bs), 130.0, 129.9, 129.8, 129.4, 129.3, 129.1, 128.7, 114.4, 93.7, $92.2\left(\mathrm{~d}, J_{C-P}=2.2 \mathrm{~Hz}\right), 83.2,80.7\left(\mathrm{~d}, J_{C-P}=13.4 \mathrm{~Hz}\right), 27.6,24.3,21.4,18.7,18.4$ ppm; ${ }^{31} \mathbf{P}\left\{{ }^{1} \mathrm{H}\right\}$ NMR: $\left(122 \mathrm{MHz}, \mathrm{CD}_{3} \mathrm{CN}\right) \delta=109.6 \mathrm{ppm} ;{ }^{19} \mathrm{~F}$ NMR: $\left(282 \mathrm{MHz}, \mathrm{CD}_{3} \mathrm{CN}\right) \delta=-$ 123.9 (sext, $J_{F-121 S b}=1933.7 \mathrm{~Hz}$ ), -123.9 (oct, $\left.J_{F-123 S b}=1053.2 \mathrm{~Hz}\right)$ ppm; IR: $\left(\right.$ neat, $\left.\mathrm{cm}^{-1}\right) \tilde{v}=$ 654, 699, 740, 910, 936, 1093, 1161, 1216, 1254, 1375, 1448, 1479, 1605, 2921, 2992, 3067, 3156; HRMS: calcd $m / z$ for $\mathrm{C}_{52} \mathrm{H}_{52} \mathrm{AuCIN}_{2} \mathrm{O}_{4} \mathrm{P}^{+}\left[\mathrm{M}-\mathrm{SbF}_{6}\right]^{+}$: 1031.3013; found (ESI 1031.3019 .

\{2-[(3aS,8aS)-2,2-Dimethyl-4,4,8,8-tetraphenyltetrahydro-[1,3]dioxolo[4,5-e][1,3,2]dioxaphosphepin-6-yl]-1,3-diisopropyl-4,5-dimethyl-1H-imidazol-3-ium\}gold Chloride Hexafluoroantimonate (1720):

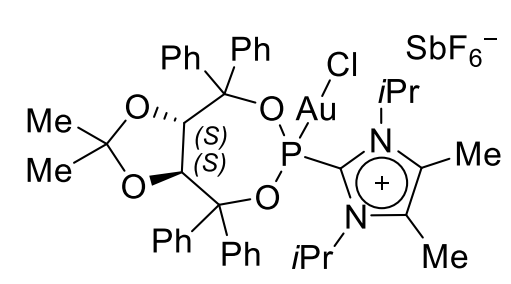

Prepared as a white solid (100 mg, $87.4 \mu \mathrm{mol}, 99 \%$ yield) from compound 1690 (1.0 equiv, $80.0 \mathrm{mg}, 87.7 \mu \mathrm{mol}$ ), chloro(dimethyl sulfide)gold(I) (1.0 equiv, $25.9 \mathrm{mg}, 87.9$ $\mu \mathrm{mol})$ in dichloromethane $(1.5 \mathrm{ml})$ according to GPE.

$[\alpha]_{20}^{D}:+26.8\left(c=1.01, \mathrm{CH}_{2} \mathrm{Cl}_{2}\right) ;{ }^{1} \mathrm{H}$ NMR: $\left(300 \mathrm{MHz}, \mathrm{CD}_{3} \mathrm{CN}\right) \delta=7.90-7.79(\mathrm{~m}, 2 \mathrm{H}), 7.62-$ $7.51(\mathrm{~m}, 5 \mathrm{H}), 7.51-7.38(\mathrm{~m}, 9 \mathrm{H}), 7.38-7.30(\mathrm{~m}, 4 \mathrm{H}), 6.57\left(\mathrm{dd}, J=8.6 \mathrm{~Hz}, J_{H-P}=1.8 \mathrm{~Hz}\right.$, $1 \mathrm{H}), 5.79\left(\mathrm{pd}, J=7.0 \mathrm{~Hz}, J_{H-P}=2.8 \mathrm{~Hz}, 2 \mathrm{H}\right), 5.19(\mathrm{~d}, J=8.5 \mathrm{~Hz}, 1 \mathrm{H}), 2.44(\mathrm{~s}, 6 \mathrm{H}), 1.59(\mathrm{~s}$, 7H), $1.57(\mathrm{~d}, J=7.0 \mathrm{~Hz}, 6 \mathrm{H}), 1.52(\mathrm{~d}, J=7.0 \mathrm{~Hz}, 6 \mathrm{H}), 0.22(\mathrm{~s}, 3 \mathrm{H}) \mathrm{ppm} ;{ }^{13} \mathrm{C}\left\{{ }^{1} \mathrm{H}\right\}$ NMR: $(101$ $\left.\mathrm{MHz}, \mathrm{CD}_{3} \mathrm{CN}\right) \delta=142.9(\mathrm{~d}, J=8.4 \mathrm{~Hz}), 142.8,138.8(\mathrm{~d}, J=6.3 \mathrm{~Hz}), 137.7(\mathrm{~d}, J=1.3 \mathrm{~Hz})$, $134.8(\mathrm{~d}, J=4.5 \mathrm{~Hz}), 133.6(\mathrm{~d}, J=122.5 \mathrm{~Hz}), 130.9,130.8,130.3,130.1,130.0,130.0$, 129.9, 129.8, 129.3, 129.1, 129.0, 128.6, 114.5, 92.5, 91.5 (d, $J=2.7 \mathrm{~Hz}), 83.3,81.1$ (d, $J=$ $12.3 \mathrm{~Hz}), 54.1,54.0,27.6,24.5,21.1,21.1,11.7 \mathrm{ppm} ;{ }^{31} \mathrm{P}\left\{{ }^{1} \mathrm{H}\right\} \mathrm{NMR}:\left(162 \mathrm{MHz}, \mathrm{CD}_{3} \mathrm{CN}\right) \delta=$ 110.6 ppm; ${ }^{19} \mathrm{~F}$ NMR: $\left(282 \mathrm{MHz}, \mathrm{CD}_{3} \mathrm{CN}\right) \delta=-124.0$ (sext, $J_{F-121 S b}=1935.1 \mathrm{~Hz}$ ), -124.0 (oct, $J_{F-123 S b}=1049.9 \mathrm{~Hz}$ ) ppm; IR: (neat, $\mathrm{cm}^{-1}$ ) $\tilde{v}=530,602,655,699,740,911,954,1089$, 1212, 1377, 1448, 1611, 2887, 2939, 2990, 3060; HRMS: calcd $m / z$ for $\mathrm{C}_{42} \mathrm{H}_{48} \mathrm{AuClN}_{2} \mathrm{O}_{4} \mathrm{P}^{+}$ $\left[\mathrm{M}-\mathrm{SbF}_{6}\right]^{+}:$907.2700; found (ESI) 907.2709.

\{2-[(5S,6S)-5,6-Dimethoxy-4,4,7,7-tetraphenyl-1,3,2-dioxaphosphepan-2-yl)-1,3dimesityl-1H-imidazol-3-ium\}gold Chloride Hexafluoroantimonate (172q):

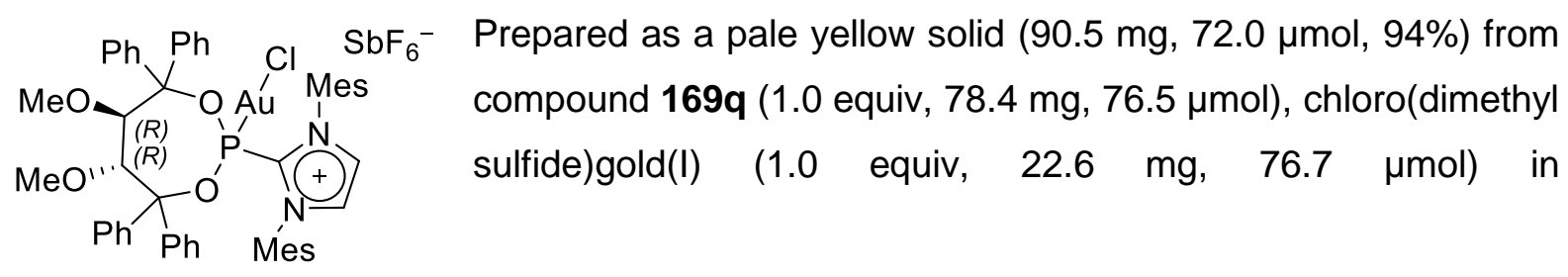




\section{Experimental}

dichloromethane $(1.5 \mathrm{ml})$ according to GPE. The solvent was removed in vacuo and the resulting residue re-precipitated from dichloromethane/ pentane. The solid was filtered and dried in vacuo to afford product 172q. Crystals suitable for X-ray crystallographic analysis were grown by layering a dichloromethane solution with pentane.

$[\alpha]_{D}^{20}:-47.3\left(c=1.14, \mathrm{CH}_{2} \mathrm{Cl}_{2}\right) ;{ }^{1} \mathrm{H}$ NMR: $\left(400 \mathrm{MHz}, \mathrm{CD}_{3} \mathrm{CN}\right) \delta=7.88\left(\mathrm{~d}, J_{H-P}=2.2 \mathrm{~Hz}, 2 \mathrm{H}\right)$, 7.50 (tt, $J=7.4,1.3 \mathrm{~Hz}, 1 \mathrm{H}), 7.47-7.36(\mathrm{~m}, 6 \mathrm{H}), 7.36-7.30(\mathrm{~m}, 6 \mathrm{H}), 7.30-7.16(\mathrm{~m}, 1 \mathrm{H})$, $7.21(\mathrm{~s}, 2 \mathrm{H}), 7.04(\mathrm{~s}, 2 \mathrm{H}), 7.00-6.96(\mathrm{~m}, 2 \mathrm{H}), 6.91-6.88(\mathrm{~m}, 2 \mathrm{H}), 6.85-6.82(\mathrm{~m}, 2 \mathrm{H}), 5.40$ (dd, $J=7.6 \mathrm{~Hz}, J_{H-P}=2.4 \mathrm{~Hz}, 1 \mathrm{H}$ ), $4.32(\mathrm{~d}, J=7.6 \mathrm{~Hz}, 1 \mathrm{H}), 3.58(\mathrm{~s}, 3 \mathrm{H}), 2.35(\mathrm{~s}, 6 \mathrm{H}), 2.32$ (s, 3H), 2.09 (s, 6H), $1.76(\mathrm{~s}, 6 \mathrm{H}) \mathrm{ppm} ;{ }^{13} \mathrm{C}\left\{{ }^{1} \mathrm{H}\right\}$ NMR: $\left(101 \mathrm{MHz}, \mathrm{CD}_{3} \mathrm{CN}\right) \delta=143.6,142.3(\mathrm{~d}$, $\left.J_{C-P}=8.5 \mathrm{~Hz}\right), 141.0,138.7\left(\mathrm{~d}, J_{C-P}=101.5 \mathrm{~Hz}\right), 138.1\left(\mathrm{~d}, J_{C-P}=6.9 \mathrm{~Hz}\right), 136.0,135.9,135.6$, $132.0,131.5,131.2,131.1,131.1,130.6,130.3,130.0,130.0$ (bs), 129.9, 129.8, 129.7, 129.6, 129.5, 129.2, 128.9, 127.7, 95.4 (d, $\left.J_{C-P}=4.2 \mathrm{~Hz}\right), 93.3,84.9,80.8$ (d, $\left.J_{C-P}=11.5 \mathrm{~Hz}\right)$, 61.7, 60.7, 21.3, 18.8, 18.0 ppm; ${ }^{31} \mathrm{P}\left\{{ }^{1} \mathrm{H}\right\}$ NMR: $\left(162 \mathrm{MHz}, \mathrm{CD}_{3} \mathrm{CN}\right) \delta=108.6 \mathrm{ppm} ;{ }^{19} \mathrm{~F}$ NMR: $\left(282 \mathrm{MHz}, \mathrm{CD}_{3} \mathrm{CN}\right) \delta=-124.0$ (oct, $J_{F-123 S b}=1044.9 \mathrm{~Hz}$ ), -124.1 (sext, $J_{F-121 S b}=1929.9 \mathrm{~Hz}$ ) ppm; IR: (neat, $\left.\mathrm{cm}^{-1}\right) \tilde{V}=617,654,734,789,865,939,1030,1129,1183,1264,1365,1445$, 1479, 1605, 2836, 2919, 3060, 3150; HRMS: calculated $\mathrm{m} / z$ for $\mathrm{C}_{51} \mathrm{H}_{52} \mathrm{AuClN}_{2} \mathrm{O}_{4} \mathrm{P}^{+}[\mathrm{M}-$ $\left.\mathrm{SbF}_{6}\right]^{+}:$1019.3013; found (ESI) 1019.3016.

\{4-[(5R,6R)-5,6-dimethoxy-4,4,7,7-tetra[3,5-di(trifluoromethyl)phenyl-1,3,2dioxaphosphepan-2-yl]-1,3-dimesityl-1H-imidazol-3-ium\}gold Chloride Hexafluoroantimonate (209a):

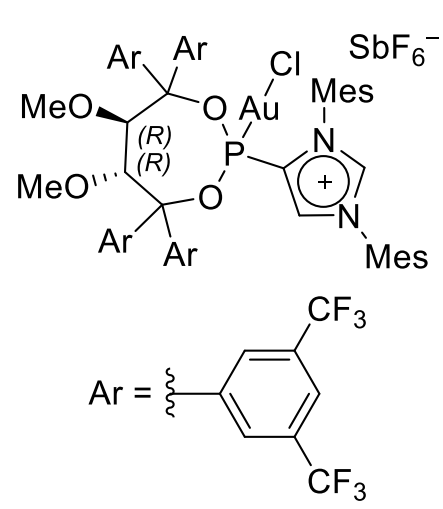

Prepared as a white solid (45.0 mg, $24.0 \mu \mathrm{mol}, 93 \%$ yield) from compound 203a (1.0 equiv, $40.7 \mathrm{mg}, 25.9 \mu \mathrm{mol}$ ), chloro(dimethyl sulfide)gold(I) (1.0, equiv, $7.6 \mathrm{mg}, 25.8 \mu \mathrm{mol})$ in dichloromethane $(1.5 \mathrm{ml})$ according to GPE.

$[\alpha]_{20}^{D}:-4.9\left(c=1.01, \mathrm{CH}_{2} \mathrm{Cl}_{2}\right) ;{ }^{1} \mathrm{H}$ NMR: $\left(400 \mathrm{MHz}, \mathrm{CD}_{3} \mathrm{CN}\right) \delta=$ $9.02\left(\mathrm{dd}, J=1.5 \mathrm{~Hz}, J_{H-P}=3.9 \mathrm{~Hz}, 1 \mathrm{H}\right), 8.76\left(\mathrm{dd}, J=1.5 \mathrm{~Hz}, J_{H-P}\right.$ $1.0 \mathrm{~Hz}, 1 \mathrm{H}), 8.18(\mathrm{~s}, 1 \mathrm{H}), 8.14(\mathrm{~s}, 1 \mathrm{H}), 8.13(\mathrm{~s}, 2 \mathrm{H}), 8.09-7.91$ (m, 4H), 7.88 (s, 2H), $7.78(\mathrm{~s}, 2 \mathrm{H}), 7.25$ (s, 2H), 7.04 (s, 1H), 7.03 (s, 1H), 5.11 (d, $J=7.3 \mathrm{~Hz}, 1 \mathrm{H}$ ), 4.31 (d, $J=7.2 \mathrm{~Hz}, 1 \mathrm{H}), 3.52$ (s, 3H), $2.66(\mathrm{~s}, 3 \mathrm{H}), 2.41$ (s, 3H), 2.30 (s, 3H), 2.20 (s, 3H), 2.08 (s, 3H), $2.03(\mathrm{~s}, 3 \mathrm{H}), 1.93(\mathrm{~s}, 3) \mathrm{ppm} ;{ }^{13} \mathrm{C}\left\{{ }^{1} \mathrm{H}\right\}$ NMR: $\left(101 \mathrm{MHz}, \mathrm{CD}_{3} \mathrm{CN}\right) \delta=144.6,143.5,143.5,143.5,143.4,143.2\left(\mathrm{~d}, J_{C-P}=5.0 \mathrm{~Hz}\right), 139.6(\mathrm{~d}$, $\left.J_{C-P}=51.8 \mathrm{~Hz}\right), 135.8,135.7,135.3,134.9,134.0,133.9,133.1\left(\mathrm{q}, J_{C-F}=33.8 \mathrm{~Hz}\right), 132.8(\mathrm{q}$, $\left.J_{C-F}=33.8 \mathrm{~Hz}\right), 132.3\left(\mathrm{q}, J_{C-F}=33.6 \mathrm{~Hz}\right), 131.5\left(\mathrm{q}, J_{C-F}=33.6 \mathrm{~Hz}\right), 131.4,131.0,130.8$, 130.7, 130.6, 130.3 - $130.1(\mathrm{~m}), 129.9$ - $129.8(\mathrm{~m}), 129.6$ - $129.5(\mathrm{~m}), 129.0$ - $128.9(\mathrm{~m})$, 
128.9, 125.6 - $125.5(\mathrm{~m}), 125.1$ - $124.9(\mathrm{~m}), 124.8$ - $124.6(\mathrm{~m}), 124.1$ (q, $\left.J_{C-F}=272.1 \mathrm{~Hz}\right)$, $123.9\left(\mathrm{q}, J_{C-F}=272.4 \mathrm{~Hz}\right.$ ), $123.9\left(\mathrm{q}, J_{C-F}=272.6 \mathrm{~Hz}\right), 91.9,87.9,83.9,81.0,61.6,61.3,21.2$, 21.1, 18.5, 17.9, 17.5, 17.4; ${ }^{31} \mathrm{P}\left\{{ }^{1} \mathrm{H}\right\}$ NMR: $\left(162 \mathrm{MHz}, \mathrm{CD}_{3} \mathrm{CN}\right) \delta=109.9$ ppm; ${ }^{19} \mathrm{~F}$ NMR: $(282$ $\mathrm{MHz}, \mathrm{CD}_{3} \mathrm{CN}$ ) $\delta=-63.2,-63.3,-63.3,-63.4,-124.1$ (sext, $J_{F-121 S b}=1925.0 \mathrm{~Hz}$ ), -124.1 (oct, $J_{F-123 S b}=1057.5 \mathrm{~Hz}$ ) ppm; IR: (neat, $\mathrm{cm}^{-1}$ ) $\tilde{v}=655,682,847,901,963,1025,1134$, 1169, 1310, 1373, 1469, 1549, 1611, 1626, 2838, 2929, 3128; HRMS: calcd. $\mathrm{m} / \mathrm{z}$ for $\mathrm{C}_{59} \mathrm{H}_{44} \mathrm{AuCIN}_{2} \mathrm{O}_{4} \mathrm{~F}_{24} \mathrm{P}^{+}\left[\mathrm{M}-\mathrm{SbF}_{6}\right]^{+:}: 1563.2004$; found (ESI) 1563.2017.

\{4-[(5R,6R)-5,6-dimethoxy-4,4,7,7-tetra[3,5-di(trifluoromethyl)phenyl-1,3,2dioxaphosphepan-2-yl]-1,3-dimesityl-1H-imidazol-3-ium\}gold

\section{Chloride} Hexafluoroantimonate (209b):

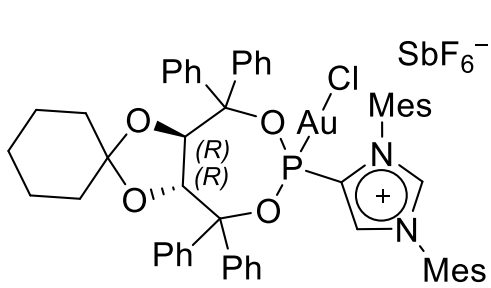

layering a dichloromethane solution with pentane.

$[\alpha]_{20}^{D}:-30.0\left(c=1.02, \mathrm{CH}_{2} \mathrm{Cl}_{2}\right) ;{ }^{1} \mathrm{H}$ NMR: $\left(300 \mathrm{MHz}, \mathrm{CD}_{3} \mathrm{CN}\right) \delta=8.99\left(\mathrm{dd}, J=1.5 \mathrm{~Hz}, J_{H-P}=\right.$ $3.4 \mathrm{~Hz}, 1 \mathrm{H}$ ), $8.58\left(\mathrm{dd}, J=1.2 \mathrm{~Hz}, J_{H-P}=1.2 \mathrm{~Hz}, 1 \mathrm{H}\right), 7.56-7.47(\mathrm{~m}, 2 \mathrm{H}), 7.44-7.35(\mathrm{~m}$, $13 \mathrm{H}), 7.35-7.33(\mathrm{~m}, 2 \mathrm{H}), 7.31(\mathrm{~s}, 1 \mathrm{H}), 7.29(\mathrm{~s}, 4 \mathrm{H}), 7.28-7.23(\mathrm{~m}, 3 \mathrm{H}), 7.22(\mathrm{~s}, 1 \mathrm{H}), 7.14$ $(\mathrm{s}, 1 \mathrm{H}), 7.06(\mathrm{~s}, 1 \mathrm{H}), 6.13\left(\mathrm{dd}, J=8.6 \mathrm{~Hz}, J_{H-P}=1.9 \mathrm{~Hz}, 1 \mathrm{H}\right), 4.92(\mathrm{~d}, J=8.5 \mathrm{~Hz}, 1 \mathrm{H}), 2.41$ (s, 6H), $2.30(\mathrm{~s}, 3 \mathrm{H}), 2.19(\mathrm{~s}, 3 \mathrm{H}), 2.10(\mathrm{~s}, 3 \mathrm{H}), 2.02(\mathrm{~s}, 3 \mathrm{H}), 1.91-1.80(\mathrm{~m}, 1 \mathrm{H}), 1.80-1.67$ $(\mathrm{m}, 1 \mathrm{H}), 1.67-1.41(\mathrm{~m}, 2 \mathrm{H}), 1.26-0.92(\mathrm{~m}, 3 \mathrm{H}), 0.85-0.68(\mathrm{~m}, 1 \mathrm{H}), 0.36-0.21(\mathrm{~m}, 1 \mathrm{H})$, $0.19-0.04(\mathrm{~m}, 1 \mathrm{H}) \mathrm{ppm} ;{ }^{13} \mathrm{C}\left\{{ }^{1} \mathrm{H}\right\}$ NMR: $\left(101 \mathrm{MHz}, \mathrm{CD}_{3} \mathrm{CN}\right) \delta=144.0,143.5,143.3\left(\mathrm{~d}, J_{C-P}=\right.$ $8.6 \mathrm{~Hz}), 143.1,143.0\left(\mathrm{~d}, J_{C-P}=4.8 \mathrm{~Hz}\right), 139.1\left(\mathrm{~d}, J_{C-P}=6.5 \mathrm{~Hz}\right), 137.8\left(\mathrm{~d}, J_{C-P}=2.0 \mathrm{~Hz}\right)$, 136.6, 136.4, 135.5, 135.4, 133.8 (d, $J_{C-P}=15.2 \mathrm{~Hz}$ ), 131.7, 131.3, 131.0, 130.9, 130.7, 130.3, $130.1\left(\mathrm{~d}, J_{C-P}=74.1 \mathrm{~Hz}\right), 130.1,129.8,129.8,129.7,129.6,129.6,129.5,129.0$, 128.8, 128.6, 128.3, 114.9, 90.4, $89.8\left(\mathrm{~d}, J_{C-P}=3.5 \mathrm{~Hz}\right), 83.4\left(\mathrm{~d}, J_{C-P}=1.8 \mathrm{~Hz}\right), 81.8\left(\mathrm{~d}, J_{C-P}=\right.$ $11.6 \mathrm{~Hz}), 37.6,34.7,25.1,24.6,24.6,21.3,21.1,19.1,18.2,17.9,17.9 ;{ }^{31} \mathrm{P}\left\{{ }^{1} \mathrm{H}\right\}$ NMR: (162 $\left.\mathrm{MHz}, \mathrm{CD}_{3} \mathrm{CN}\right) \delta=106.8 \mathrm{ppm} ;{ }^{19} \mathrm{~F}$ NMR: $\left(282 \mathrm{MHz}, \mathrm{CD}_{3} \mathrm{CN}\right) \delta=-123.9$ (oct, $J_{F-123 s b}=1054.1$ $\mathrm{Hz}$ ), -123.9 (sext, $J_{F-121 S b}=1927.7 \mathrm{~Hz}$ ) ppm; IR: (neat, $\mathrm{cm}^{-1}$ ) $\tilde{v}=533,611,654,698,909$, $945,981,1124,1447,1553,2859,2936, \quad 3058,3128$; HRMS: calcd $\mathrm{m} / \mathrm{z}$ for $\mathrm{C}_{55} \mathrm{H}_{56} \mathrm{AuCIN} \mathrm{O}_{4} \mathrm{P}^{+}\left[\mathrm{M}-\mathrm{SbF}_{6}\right]^{+}:$1071.3326; found (ESI) 1071.3341. 


\section{Experimental}

[2-\{(5R,6R)-5,6-Dimethoxy-4,4,7,7-tetrakis[4-(trifluoromethyl)phenyl]-1,3,2dioxaphosphepan-2-yl\}-1,3-dimesityl-4,5-dimethyl-1H-imidazol-3-ium]gold Hexafluoroantimonate (172s):
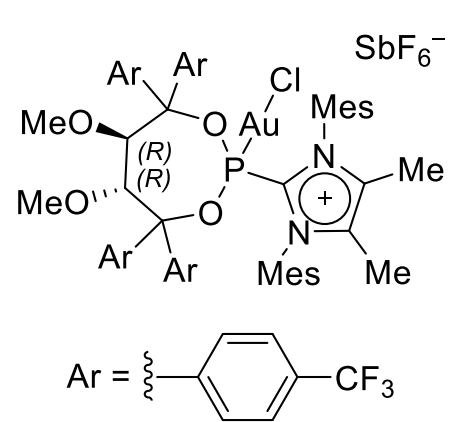

Prepared as a white solid (65.0 mg, $41.7 \mu \mathrm{mol}, 91 \%$ yield) from compound 169s (1.0 equiv, $60.7 \mathrm{mg}, 45.8 \mu \mathrm{mol}$ ), chloro(dimethyl sulfide)gold(I) (1.0, equiv, $13.5 \mathrm{mg}, 45.8 \mu \mathrm{mol})$ in dichloromethane $(2.0 \mathrm{ml})$ according to GPE. Crystals suitable for X-ray crystallographic analysis were grown by layering a dichloromethane solution with pentane.

$[\alpha]_{22}^{D}:-49.7\left(c=0.990, \mathrm{CH}_{2} \mathrm{Cl}_{2}\right) ;{ }^{1} \mathrm{H}$ NMR: $\left(400 \mathrm{MHz}, \mathrm{CD}_{3} \mathrm{CN}\right) \delta=7.78-7.73(\mathrm{~m}, 2 \mathrm{H}), 7.73$ - $7.68(\mathrm{~m}, 4 \mathrm{H}), 7.68-7.64(\mathrm{~m}, 2 \mathrm{H}), 7.51$ (bs, 2H), $7.26(\mathrm{~s}, 2 \mathrm{H}), 7.22$ (d, J=8.2 Hz, 2H), 7.16 $(\mathrm{s}, 2 \mathrm{H}), 7.06(\mathrm{~d}, J=7.7 \mathrm{~Hz}, 2 \mathrm{H}), 6.96(\mathrm{~d}, J=7.7 \mathrm{~Hz}, 2 \mathrm{H}), 5.54\left(\mathrm{dd}, J=8.0 \mathrm{~Hz}, J_{H-P}=2.1 \mathrm{~Hz}\right.$, $1 \mathrm{H}), 4.17(\mathrm{~d}, J=8.0 \mathrm{~Hz}, 1 \mathrm{H}), 3.69(\mathrm{~s}, 3 \mathrm{H}), 2.38(\mathrm{~s}, 9 \mathrm{H}), 2.00(\mathrm{~s}, 6 \mathrm{H}), 1.96(\mathrm{~s}, 6 \mathrm{H}), 1.81$ (s, $6 \mathrm{H})$ ppm; ${ }^{13} \mathrm{C}\left\{{ }^{1} \mathrm{H}\right\}$ NMR: $\left(126 \mathrm{MHz}, \mathrm{CD}_{3} \mathrm{CN}\right) \delta=145.6\left(\mathrm{~d}, J_{C-P}=8.7 \mathrm{~Hz}\right), 144.6\left(\mathrm{~d}, J_{C-P}=1.1\right.$ $\mathrm{Hz}), 144.1,141.7\left(\mathrm{~d}, J_{C-P}=7.6 \mathrm{~Hz}\right), 140.0\left(\mathrm{t}, J_{C-P}=1.4 \mathrm{~Hz}, J_{\mathrm{C}-\mathrm{F}}=1.4 \mathrm{~Hz}\right), 137.3,137.3$, 136.0, 135.9, 134.1 (d, $\left.J_{C-P}=112.5 \mathrm{~Hz}\right), 132.5$ (q, $\left.J_{C-F}=32.6 \mathrm{~Hz}\right), 132.2\left(\mathrm{q}, J_{\mathrm{C}-F}=32.8 \mathrm{~Hz}\right.$ ), 132.2, $131.8\left(q, J_{C-F}=32.6 \mathrm{~Hz}\right), 131.8,131.3\left(q, J_{C-F}=32.6,31.9 \mathrm{~Hz}\right), 130.8,130.7$ (bs), $130.5,130.0\left(\mathrm{~d}, J_{C-P}=1.1 \mathrm{~Hz}\right), 129.7,127.2\left(\mathrm{q}, J_{\mathrm{C}-\mathrm{F}}=3.8 \mathrm{~Hz}\right), 126.9\left(\mathrm{q}, J_{\mathrm{C}-\mathrm{F}}=3.8 \mathrm{~Hz}\right), 126.3$ $\left(q, J_{C-F}=3.8 \mathrm{~Hz}\right), 125.0\left(q, J_{C-F}=271.6 \mathrm{~Hz}\right), 124.9\left(q, J_{C-F}=3.8 \mathrm{~Hz}\right), 124.9\left(q, J_{C-F}=271.6\right.$ $\mathrm{Hz}), 124.9$ (q, $\left.J_{\mathrm{C}-\mathrm{F}}=271.7 \mathrm{~Hz}\right), 124.8\left(\mathrm{q}, J_{\mathrm{C}-\mathrm{F}}=271.7 \mathrm{~Hz}\right), 93.1\left(\mathrm{~d}, J_{C-P}=3.0 \mathrm{~Hz}\right), 91.5\left(\mathrm{~d}, J_{C-P}\right.$ $=1.6 \mathrm{~Hz}), 85.3,80.8\left(\mathrm{~d}, J_{C-P}=11.5 \mathrm{~Hz}\right), 62.1,61.4,21.3,18.8,18.2,9.9 ;{ }^{31} \mathbf{P}\left\{{ }^{1} \mathrm{H}\right\}$ NMR: $(162$ $\left.\mathrm{MHz}, \mathrm{CD}_{3} \mathrm{CN}\right) \delta=109.0$ ppm; ${ }^{19} \mathrm{~F}$ NMR: $\left(376 \mathrm{MHz}, \mathrm{CD}_{3} \mathrm{CN}\right) \delta=-63.2,-63.3,-63.3,-63.4,-$ 123.9 (oct, $\left.J_{F-123 S b}=1050.4 \mathrm{~Hz}\right),-123.9$ (sext, $\left.J_{F-121 S b}=1932.5 \mathrm{~Hz}\right)$ ppm; IR: $\left(\right.$ neat, $\left.\mathrm{cm}^{-1}\right) \tilde{v}=$ 656, 793, 932, 1015, 1069, 1092, 1259, 1325, 1407, 1141, 1479, 1617, 2846, 2918, 2963; HRMS: calcd $\mathrm{m} / \mathrm{z}$ for $\mathrm{C}_{57} \mathrm{H}_{52} \mathrm{AuCIF}_{12} \mathrm{~N}_{2} \mathrm{O}_{4} \mathrm{P}^{+}\left[\mathrm{M}-\mathrm{SbF}_{6}\right]^{+}:$1319.2822; found (ESI) 1319.2829.

[4,5-Dichloro-2-\{(5R,6R)-5,6-dimethoxy-4,4,7,7-tetrakis[4-(trifluoromethyl)phenyl]-1,3,2dioxaphosphepan-2-yl\}-1,3-dimesityl-1 $H$-imidazol-3-ium]gold

Chloride Hexafluoroantimonate(172t):

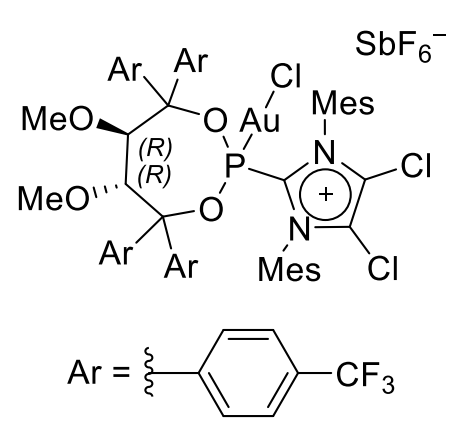

Prepared as a as a white solid (60.0 mg, $37.5 \mu \mathrm{mol}, 92 \%$ yield) from compound 169t (1.0 equiv, $47.2 \mathrm{mg}, 34.6 \mu \mathrm{mol}$ ), chloro(dimethyl sulfide)gold(I) (1.0, equiv, $10.2 \mathrm{mg}, 34.6 \mu \mathrm{mol})$ in dichloromethane $(2.0 \mathrm{ml})$ according to GPE. The solvent was removed in vacuo and the resulting solid re-precipitated from dichloromethane/pentane, filtered off and dried in vacuo to afford 


\section{Experimental}

the product 172t. Crystals suitable for X-ray crystallographic analysis were grown by layering a dichloromethane solution with pentane.

$[\alpha]_{23}^{D}:-60.8\left(c=0.950, \mathrm{CH}_{2} \mathrm{Cl}_{2}\right) ;{ }^{1} \mathrm{H}$ NMR: $\left(400 \mathrm{MHz}, \mathrm{CD}_{3} \mathrm{CN}\right) \delta=7.77(\mathrm{~d}, J=8.3 \mathrm{~Hz}, 2 \mathrm{H})$, $7.75-7.65$ (m, 6H), 7.44 (bs, 2H), 7.31 (s, 2H), 7.18 (d, J=8.3 Hz, 2H), 7.15 (s, 2H), 7.09 $(\mathrm{d}, J=8.3 \mathrm{~Hz}, 2 \mathrm{H}), 6.98(\mathrm{~d}, J=8.2 \mathrm{~Hz}, 2 \mathrm{H}), 5.42\left(\mathrm{dd}, J=7.8=\mathrm{Hz}, J_{C-P}=1.7 \mathrm{~Hz}, 1 \mathrm{H}\right), 4.25$ (d, J = $7.9 \mathrm{~Hz}, 1 \mathrm{H}), 3.66(\mathrm{~s}, 3 \mathrm{H}), 2.43(\mathrm{~s}, 3 \mathrm{H}), 2.37$ (s, 6H), 2.14 (s, 6H), 1.84 (s, 6H) ppm; ${ }^{13} \mathrm{C}\left\{{ }^{1} \mathrm{H}\right\}$ NMR: $\left(101 \mathrm{MHz}, \mathrm{CD}_{3} \mathrm{CN}\right) \delta=145.5,145.0\left(\mathrm{~d}, J_{C-P}=8.4 \mathrm{~Hz}\right), 144.1,141.2\left(\mathrm{~d}, J_{C-P}=\right.$ $7.3 \mathrm{~Hz}$ ), 139.4, 137.4 (d, $\left.J_{C-P}=98.2 \mathrm{~Hz}\right), 136.6,136.5,132.8$ (q, $\left.J_{C-F}=32.8 \mathrm{~Hz}\right), 132.5$ (q, $J_{C-}$ $F=32.6 \mathrm{~Hz}), 132.3,131.9,131.5\left(\mathrm{q}, J_{C-F}=32.4 \mathrm{~Hz}\right), 130.8,130.6,130.5,129.8,129.2\left(\mathrm{~d}, J_{C-}\right.$ $P=3.3 \mathrm{~Hz}), 129.0,127.3\left(\mathrm{q}, J_{C-F}=3.8 \mathrm{~Hz}\right), 127.1\left(\mathrm{q}, J_{C-F}=3.6 \mathrm{~Hz}\right), 126.5\left(\mathrm{q}, J_{C-F}=3.8 \mathrm{~Hz}\right)$, $125.1\left(\mathrm{q}, J_{C-F}=3.8 \mathrm{~Hz}\right), 125.0\left(\mathrm{q}, J_{C-F}=271.6 \mathrm{~Hz}\right), 124.9\left(\mathrm{q}, J_{C-F}=272.0 \mathrm{~Hz}\right), 124.9$ (q, $J_{C-F}=$ $271.9 \mathrm{~Hz}$ ), $124.8\left(\mathrm{q}, J_{C-F}=271.8 \mathrm{~Hz}\right), 94.5\left(\mathrm{~d}, J_{C-P}=3.2 \mathrm{~Hz}\right), 92.6,85.1,80.8\left(\mathrm{~d}, J_{C-P}=11.0\right.$ $\mathrm{Hz}), 62.1,61.5,21.3,19.2,18.5 \mathrm{ppm} ;{ }^{31} \mathrm{P}\left\{{ }^{1} \mathrm{H}\right\}$ NMR: $\left(162 \mathrm{MHz}, \mathrm{CD}_{3} \mathrm{CN}\right) \delta=111.2 \mathrm{ppm} ;{ }^{19} \mathrm{~F}$ NMR: $\left(376 \mathrm{MHz}, \mathrm{CD}_{3} \mathrm{CN}\right) \delta=-63.2,-63.3,-63.4,-63.4,-123.9$ (sext, $\left.J_{F-121 S b}=1929.4 \mathrm{~Hz}\right)$, -123.9 (oct, $J_{F-123 S b}=1045.7 \mathrm{~Hz}$ ) ppm; IR: $\left(\right.$ neat, $\mathrm{cm}^{-1}$ ) $\tilde{v}=594,824,932,1017,1071,1121$, 1168, 1324, 1381, 1410, 1471, 1565, 1615, 2844, 2929, 2997; HRMS: calcd $\mathrm{m} / \mathrm{z}$ for $\mathrm{C}_{55} \mathrm{H}_{46} \mathrm{AuCl}_{3} \mathrm{~F}_{12} \mathrm{~N}_{2} \mathrm{O}_{4} \mathrm{P}^{+}\left[\mathrm{M}-\mathrm{SbF}_{6}\right]^{+}:$1359.1729; found (ESI) 1359.1720 .

\section{[4,5-Dichloro-1,3-dimesityl-2-\{(3aS,8aS)-4,4,8,8-tetrakis[4-(tert-butyl)phenyl]-2,2-} dimethyltetrahydro-[1,3]dioxolo[4,5-e][1,3,2]dioxaphosphepin-6-yl\}-1 H-imidazol-3ium]gold Chloride Hexafluoroantimonate (172u):

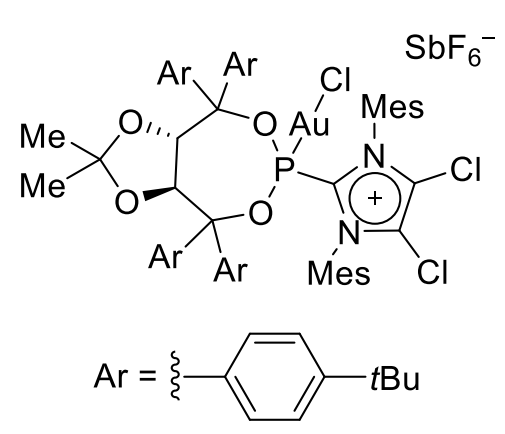

Prepared as a white solid (64.0 mg, $40.9 \mu \mathrm{mol}, 49 \%$ yield) from compound $169 \mathrm{u}$ (1.0 equiv, $110 \mathrm{mg}, 82.7 \mu \mathrm{mol}$ ), chloro(dimethyl sulfide)gold(I) (1.0, equiv, $24.4 \mathrm{mg}, 82.8$ $\mu \mathrm{mol})$ in dichloromethane $(2.0 \mathrm{ml})$ according to GPE. The solvent was removed in vacuo and the residue purified by column chromatography $(0.5 \%$ ethyl acetate in dichloromethane) at $-10^{\circ} \mathrm{C}$.

$[\alpha]_{20}^{D}:+36.6\left(c=0.94, \mathrm{CH}_{2} \mathrm{Cl}_{2}\right) ;{ }^{1} \mathbf{H}$ NMR: $\left(300 \mathrm{MHz}, \mathrm{CDCl}_{3}\right) \delta=7.44-7.35(\mathrm{~m}, 4 \mathrm{H}), 7.31(\mathrm{~s}$, $4 \mathrm{H}), 7.24(\mathrm{~d}, J=8.4 \mathrm{~Hz}, 2 \mathrm{H}), 7.19(\mathrm{~d}, J=8.3 \mathrm{~Hz}, 2 \mathrm{H}), 7.12-7.06(\mathrm{~m}, 4 \mathrm{H}), 6.84(\mathrm{dd}, J=8.7$, $4.0 \mathrm{~Hz}, 2 \mathrm{H}), 6.65-6.59(\mathrm{~m}, 2 \mathrm{H}), 6.31\left(\mathrm{dd}, J=8.6 \mathrm{~Hz}, J_{H-P}=2.0 \mathrm{~Hz}, 1 \mathrm{H}\right), 4.81(\mathrm{~d}, J=8.6 \mathrm{~Hz}$, $1 \mathrm{H}), 2.47(\mathrm{~s}, 6 \mathrm{H}), 2.04(\mathrm{~s}, 6 \mathrm{H}), 2.00(\mathrm{~s}, 6 \mathrm{H}), 1.39(\mathrm{~s}, 3 \mathrm{H}), 1.37(\mathrm{~s}, 9 \mathrm{H}), 1.35(\mathrm{~s}, 9 \mathrm{H}), 1.32(\mathrm{~s}$, $9 \mathrm{H}), 1.31(\mathrm{~s}, 9 \mathrm{H}),-0.03(\mathrm{~s}, 3 \mathrm{H}) ;{ }^{13} \mathrm{C}\left\{{ }^{1} \mathrm{H}\right\}$ NMR: $\left(126 \mathrm{MHz}, \mathrm{CDCl}_{3}\right) \delta=153.5,153.1,152.6$, 152.2, 143.6, 138.2 (d, $\left.J_{C-P}=81.3 \mathrm{~Hz}\right), 137.8,137.6,135.4,135.4,134.1$ (d, $\left.J_{C-P}=6.3 \mathrm{~Hz}\right)$, 132.3, 131.2, 130.8, 128.7, 128.6 (bs), 128.5, 128.1, 127.8, 127.7, 127.1 (d, $J_{C-P}=2.3 \mathrm{~Hz}$ ), 


\section{Experimental}

$125.8,125.8,125.1,124.1,113.1,93.4,92.3,82.0,79.8$ (d, $\left.J_{C-P}=13.9 \mathrm{~Hz}\right), 34.8,34.6,31.3$, 31.3, 27.5, 23.8, 21.6, 18.5, 18.1 ppm; ${ }^{31} \mathbf{P}\left\{{ }^{1} \mathrm{H}\right\}$ NMR $\left(162 \mathrm{MHz}, \mathrm{CDCl}_{3}\right) \delta=109.3$ ppm; IR: $\left(\right.$ neat, $\mathrm{cm}^{-1}$ ) $\tilde{v}=565,602,656,728,818,929,1019,1093,1212,1264,1373,1403,1471$, 1506, 1569, 1609, 2863, 2907, 2955; HRMS: calcd $m / z$ for $\mathrm{C}_{68} \mathrm{H}_{82} \mathrm{AuCl}_{3} \mathrm{~N}_{2} \mathrm{O}_{4} \mathrm{P}^{+}\left[\mathrm{M}-\mathrm{SbF}_{6}\right]^{+}$: 1323.4738; found (ESI) 1232.4736.

\section{$\left\{(S)-2-\left(2,6-D i p h e n y l d i n a p h t h o\left[2,1-d: 1^{\prime}, 2\right.\right.\right.$ '-f][1,3,2]dioxaphosphepin-4-yl)-1,3-dimesityl-} 4,5-dimethyl-1 $H$-imidazol-3-ium \}gold Chloride Hexafluoroantimonate (210a):

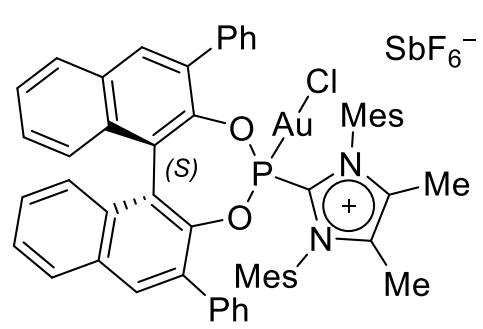

Prepared as a white solid (68.0 mg, $53.6 \mu \mathrm{mol}, 99 \%$ yield) from compound 208c (1.0 equiv, $56.0 \mathrm{mg}, 54.0 \mu \mathrm{mol}$ ), chloro(dimethyl sulfide)gold(I) (1.0 equiv, $15.9 \mathrm{mg}, 53.9 \mu \mathrm{mol})$ in dichloromethane $(1.5 \mathrm{ml})$ according to GPE.

$[\alpha]_{20}^{D}:+32.1\left(c=1.01, \mathrm{CH}_{2} \mathrm{Cl}_{2}\right) ;{ }^{1} \mathrm{H}$ NMR: $\left(400 \mathrm{MHz}, \mathrm{CD}_{3} \mathrm{CN}\right) \delta$ $=8.20(\mathrm{~s}, 2 \mathrm{H}), 8.20(\mathrm{~d}, J=7.8 \mathrm{~Hz}, 2 \mathrm{H}), 8.12(\mathrm{~s}, 1 \mathrm{H}), 7.99(\mathrm{~d}, J=8.2 \mathrm{~Hz}, 1 \mathrm{H}), 7.73-7.61(\mathrm{~m}$, $5 \mathrm{H}$ ), $7.56-7.44(\mathrm{~m}, 7 \mathrm{H}), 7.37$ (ddd, $J=8.3,6.8,1.2 \mathrm{~Hz}, 1 \mathrm{H}), 7.19(\mathrm{dt}, J=7.5,1.0,0.9 \mathrm{~Hz}$, $1 \mathrm{H}), 7.08(\mathrm{~s}, 2 \mathrm{H}), 7.05(\mathrm{~s}, 2 \mathrm{H}), 6.89(\mathrm{~d}, J=8.1 \mathrm{~Hz}, 1 \mathrm{H}), 6.77(\mathrm{~s}, 1 \mathrm{H}), 6.69(\mathrm{~d}, J=8.7 \mathrm{~Hz}, 1 \mathrm{H})$, $5.61(\mathrm{~s}, 1 \mathrm{H}), 2.36(\mathrm{~s}, 3 \mathrm{H}), 1.85(\mathrm{~s}, 6 \mathrm{H}), 1.76(\mathrm{~s}, 3 \mathrm{H}), 1.66(\mathrm{~s}, 3 \mathrm{H}), 1.55(\mathrm{~s}, 3 \mathrm{H}), 1.52(\mathrm{~s}, 3 \mathrm{H})$, $1.15(\mathrm{~s}, 3 \mathrm{H}) \mathrm{ppm} ;{ }^{13} \mathrm{C}\left\{{ }^{1} \mathrm{H}\right\}$ NMR: $\left(101 \mathrm{MHz}, \mathrm{CD}_{3} \mathrm{CN}\right) \delta=145.2\left(\mathrm{~d}, J_{C-P}=16.7 \mathrm{~Hz}\right), 144.7(\mathrm{~d}$, $\left.J_{C-P}=6.5 \mathrm{~Hz}\right), 144.4,142.6,138.0\left(\mathrm{~d}, J_{C-P}=2.4 \mathrm{~Hz}\right), 137.2\left(\mathrm{~d}, J_{C-P}=2.5 \mathrm{~Hz}\right), 136.8,136.2$, 136.1, 135.4, $135.0\left(\mathrm{~d}, J_{C-P}=0.9 \mathrm{~Hz}\right), 134.0\left(\mathrm{~d}, J_{C-P}=1.2 \mathrm{~Hz}\right), 133.9,133.9\left(\mathrm{~d}, J_{C-P}=53.5\right.$ $\mathrm{Hz}), 133.8\left(\mathrm{~d}, J_{C-P}=3.2 \mathrm{~Hz}\right), 133.6\left(\mathrm{~d}, J_{C-P}=1.8 \mathrm{~Hz}\right), 133.5,133.1\left(\mathrm{~d}, J_{C-P}=1.6 \mathrm{~Hz}\right), 133.0$ $\left(\mathrm{d}, J_{C-P}=2.2 \mathrm{~Hz}\right), 132.7\left(\mathrm{~d}, J_{C-P}=2.9 \mathrm{~Hz}\right), 132.6,132.5\left(\mathrm{~d}, J_{C-P}=0.6 \mathrm{~Hz}\right), 131.9,130.8$, 130.8, 129.9, 129.7, 129.6, 129.6, 129.6, 129.5, 129.4, 129.4, 128.9 (d, JC-P $=4.1 \mathrm{~Hz}), 128.5$, 128.3, 128.2, 127.9, 127.7, 127.6, $125.2\left(\mathrm{~d}, J_{C-P}=4.4 \mathrm{~Hz}\right), 122.3\left(\mathrm{~d}, J_{C-P}=4.0 \mathrm{~Hz}\right), 21.5$, $21.1,20.1,18.7,17.8,17.8,16.1,9.9,9.8,9.6$ ppm; ${ }^{31} \mathrm{P}\left\{{ }^{1} \mathrm{H}\right\}$ NMR: $\left(122 \mathrm{MHz}, \mathrm{CD}_{3} \mathrm{CN}\right) \delta=$ 112.8 ppm; ${ }^{19} \mathrm{~F}$ NMR: $\left(282 \mathrm{MHz}, \mathrm{CD}_{3} \mathrm{CN}\right.$ ) $\delta=-123.9$ (sext, $J_{F-121 S b}=1931.2 \mathrm{~Hz}$ ), -123.9 (oct, $\left.J_{F-123 S b}=1050.5 \mathrm{~Hz}\right)$ ppm; IR: $\left(\right.$ neat, $\left.\mathrm{cm}^{-1}\right) \tilde{v}=562,604,682,837,985,1001,1144,1185$, 1228, 1308, 1398, 1449, 1497, 2921, 2962, 3034, 3056; HRMS: calcd $\mathrm{m} / \mathrm{z}$ for $\mathrm{C}_{55} \mathrm{H}_{48} \mathrm{AuCIN} \mathrm{O}_{2} \mathrm{P}^{+}\left[\mathrm{M}-\mathrm{SbF}_{6}\right]^{+}:$1031.2802; found (ESI) 1031.2808.

Attempted preparation of the gold complex with ligand 169p. Isolation of compounds [2-(dihydroxyphosphaneyl)-1,3-bis(2,6-diisopropylphenyl)-1 H-imidazol-3-ium]gold chloride hexafluoroantimonate (175b) and 1,3,4-tri([1,1'-biphenyl]-4-yl)-7phenylnaphthalen-2-ol (174b):

To a dried Schlenk flask equipped with magnetic stirring bar was added 169p (1.00 equiv, $52.0 \mathrm{mg}, 36.5 \mu \mathrm{mol})$ followed by dichloromethane $(1 \mathrm{ml})$ under argon. The solution was 
cooled to $-20{ }^{\circ} \mathrm{C}$, and chloro(dimethyl sulfide)gold(I) (1.00 equiv, $\left.10.8 \mathrm{mg}, 36.6 \mu \mathrm{mol}\right)$ was added. The reaction mixture was allowed to stir at $-20^{\circ} \mathrm{C}$ for a few minutes, before warming to room temperature and stirring for an additional 30 minutes. The solvent was removed in vacuo, and the residue crystallized from dichloromethane/toluene.

\section{Compound 175b:}

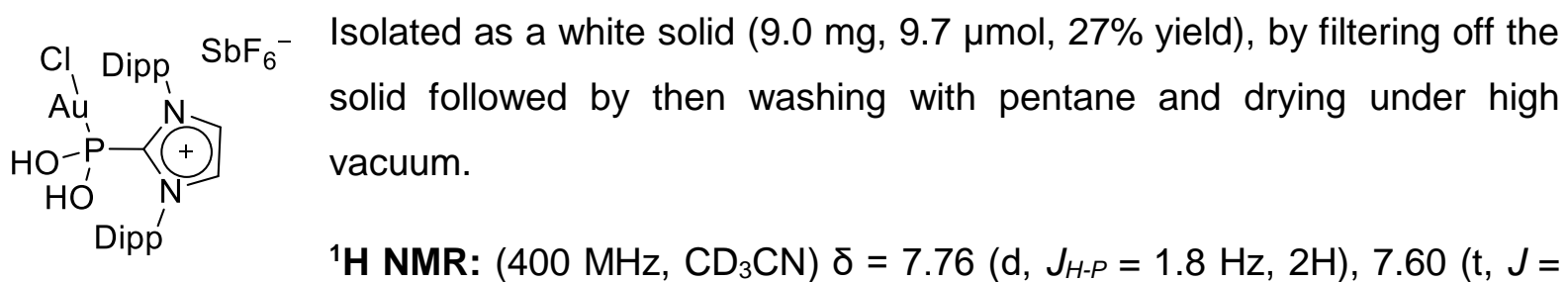
$7.8 \mathrm{~Hz}, 2 \mathrm{H}$ ), 7.36 (d, J=7.9 Hz, 4H), 2.34 (p, J=6.8 Hz, 4H), 1.25 (d, J=6.8 Hz, 12H), 1.12 $(\mathrm{d}, J=6.9 \mathrm{~Hz}, 12 \mathrm{H}) \mathrm{ppm} ;{ }^{13} \mathrm{C}\left\{{ }^{1} \mathrm{H}\right\}$ NMR: $\left(101 \mathrm{MHz}, \mathrm{CD}_{3} \mathrm{CN}\right) \delta=147.0\left(\mathrm{~d}, J_{C-P}=76.3 \mathrm{~Hz}\right)$, 146.1, 133.0, 131.8, 128.6, 125.5, 30.2, 25.5, 23.2 ppm; ${ }^{31} \mathrm{P}\left\{{ }^{1} \mathrm{H}\right\}$ NMR: $\left(162 \mathrm{MHz}, \mathrm{CD}_{3} \mathrm{CN}\right) \delta$ $=83.3$ (bs) ppm ${ }^{19} \mathrm{~F}$ NMR: $\left(376 \mathrm{MHz}, \mathrm{CD}_{3} \mathrm{CN}\right) \delta=-124.0$ (sext, $\left.J_{F-121 S b}=1940.7 \mathrm{~Hz}\right),-124.1$ (oct, $\left.J_{F-123 S b}=1038.8 \mathrm{~Hz}\right)$ ppm; IR: $\left(\right.$ neat, $\left.\mathrm{cm}^{-1}\right) \tilde{v}=557,652,747,799$, 880, 1025, 1061, 1098, 1258, 1455, 2871, 2928, 2964, 3138, 3168; HRMS: calcd $m / z$ for $\mathrm{C}_{54} \mathrm{H}_{75} \mathrm{Au}_{2} \mathrm{Cl}_{2} \mathrm{~N}_{4} \mathrm{O}_{4} \mathrm{P}_{2}{ }^{+}$ $\left[2 \mathrm{M}-2 \mathrm{SbF}_{6}-\mathrm{H}\right]^{+}:$1369.3966; found (ESI) 1369.3970.

\section{Compound 174b}

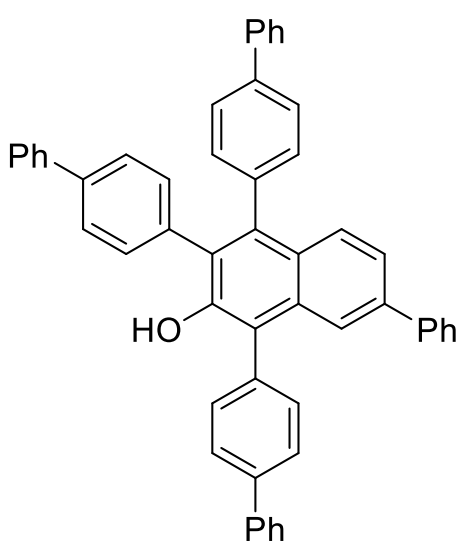
The combined washings were concentrated in vacuo and washed with acetonitrile affording $\mathbf{1 7 4 b}$ as a white powder $(9.0$ $\mathrm{mg}, 13.2 \mu \mathrm{mol}, 36 \%$ yield).

'H NMR: $\left(400 \mathrm{MHz}, \mathrm{CDCl}_{3}\right) \delta=7.92-7.88(\mathrm{~m}, 1 \mathrm{H}), 7.88-$ $7.82(\mathrm{~m}, 2 \mathrm{H}), 7.77-7.70(\mathrm{~m}, 3 \mathrm{H}), 7.70-7.65(\mathrm{~m}, 3 \mathrm{H}), 7.65-$ $7.60(\mathrm{~m}, 2 \mathrm{H}), 7.60-7.48(\mathrm{~m}, 10 \mathrm{H}), 7.48-7.27(\mathrm{~m}, 14 \mathrm{H}), 5.33$ (s, $1 \mathrm{H})$ ppm; ${ }^{13} \mathbf{C}\left\{{ }^{1} \mathrm{H}\right\}$ NMR: $\left(101 \mathrm{MHz}, \mathrm{CDCl}_{3}\right) \delta=147.9,141.3$, $141.0,140.8,140.7,140.6,140.1,139.9,139.4,137.7,136.5$, $135.0,134.1,132.5,131.7,131.7,131.5,129.9,129.0,128.9,128.9,128.8,128.5,128.0$, 127.7, 127.5, 127.4, 127.4, 127.3, 127.2, 127.1, 127.1, 127.0, 126.5, 126.3, 125.7, 125.3, 120.9 ppm; IR: $\left(\right.$ neat, $\left.\mathrm{cm}^{-1}\right) \tilde{v}=693,761,831,1007,1075,1094,1260,1386,1485,1598$, 2852, 2922, 2961, 3027, 3054, 3521; HRMS: calcd $m / z$ for $\mathrm{C}_{52} \mathrm{H}_{36} \mathrm{NaO}^{+}[\mathrm{M}+\mathrm{Na}]^{+}:$699.2658; found (ESI) 699.2658 . 


\section{Experimental}

\subsubsection{Achiral synthesis of helicenes}

\section{General procedure F (GPF) for racemic synthesis of helicenes:}

To a dried Schlenk flask equipped with magnetic stirrer was added the substrate (1.0 equiv.), followed by the gold precatalyst ( $5 \mathrm{~mol} \%$ ). A septum was fitted to the Schlenk flask and the contents dried under high vacuum for 30 minutes, before performing three argon purge cycles. Dichloromethane $(0.05 \mathrm{M})$ was added via syringe through the septum and the reaction mixture was allowed to stir at room temperature for 2 minutes at room temperature, before adding a solution of $\mathrm{AgSbF}_{6}(5 \mathrm{~mol} \%, 0.05 \mathrm{M}$ solution in dichloromethane) through the septum. The septum was exchanged for a greased glass stopper and the reaction mixture was allowed to stir at the specified temperature for the specified amount of time. The reaction mixture was filtered through a short pad of silica eluting with dichloromethane, and the solvent was removed in vacuo, before drying under high vacuum. The conversion and ratio of isomers was determined by NMR and/or HPLC.

\section{General procedure G (GPG) for racemic synthesis of helicenes when using a catalyst loading less than 5 mol\%:}

In a dried Schlenk equipped with magnetic stirrer was added the precursor 159 (1.0 equiv), a septum was fitted to the Schlenk and the contents dried under high vacuum for 30 minutes, before performing three argon purge cycles. Dichloromethane $(0.05 \mathrm{M})$ was added via syringe through the septum and the reaction was allowed to stir at room temperature for 2 minutes. Separately in a glovebox, a stock solution of the gold pre-catalyst $(0.006$ to $0.01 \mathrm{M}$ in dichloromethane) was prepared by weighing the gold catalyst into a volumetric flask (2 $\mathrm{ml})$, adding dichloromethane and transferring to a Schlenk. The required amount was added to the reaction via micro syringe, followed by a solution of silver hexafluoroantimonate $(0.01$ to $0.0125 \mathrm{M}$ in dichloromethane). The reaction was stirred for the specified amount of time at room temperature, before filtering through a short pad of silica, eluting with dichloromethane. The solvent was removed in vacuo before drying under high vacuum. The conversion and ratio of isomers was determined by NMR and HPLC. 
Table 22. Screening for racemic synthesis of [6]carbohelicenes.<smiles></smiles>

$98 f$<smiles></smiles>

98a<smiles>[R]C#Cc1ccc2ccc(C#C[R16])c(-c3ccccc3)c2c1-c1ccccc1</smiles>

(a)<smiles>[R17]C#Cc1ccc2ccc3cc([R1])c4ccccc4c3c2c1-c1ccccc1</smiles>

187<smiles>[R]C=C1c2ccccc2-c2c1ccc1ccc3c(c21)-c1ccccc1C3=C[R]</smiles><smiles>[R]C=C1c2ccccc2-c2c1ccc1ccc3cc([R])c4ccccc4c3c21</smiles>

188

\begin{tabular}{|c|c|c|c|c|}
\hline Entry & 159 & $\mathrm{R}^{1}$ & Yield (\%) ${ }^{a}$ & $160: 188: 187^{b}$ \\
\hline 1 & 159af & 4- $\mathrm{F}\left(\mathrm{C}_{6} \mathrm{H}_{4}\right)$ & $>99$ & 81: 19: 0 \\
\hline $2^{a}$ & 159ag & $4-\mathrm{MeO}\left(\mathrm{C}_{6} \mathrm{H}_{4}\right)$ & 98 & 92: 8: 0 \\
\hline 3 & 159ah & $4-\mathrm{BnO}\left(\mathrm{C}_{6} \mathrm{H}_{4}\right)$ & 98 & 93: 7: 0 \\
\hline 4 & 159ai & 4-TMS $\left(\mathrm{C}_{6} \mathrm{H}_{4}\right)$ & $>99$ & 83: 17: 0 \\
\hline 4 & 159aj & 4- $(\operatorname{Pr})\left(\mathrm{C}_{6} \mathrm{H}_{4}\right)$ & $>99$ & 87: 13: 0 \\
\hline 5 & 159ae & $4-\mathrm{Cl}\left(\mathrm{C}_{6} \mathrm{H}_{4}\right)$ & 99 & 82: 18: 0 \\
\hline 6 & 159ak & $3,4-(\mathrm{Me})_{2}\left(\mathrm{C}_{6} \mathrm{H}_{3}\right)$ & $>99$ & 92: 8: 0 \\
\hline 7 & 159al & 4- $\mathrm{CH}_{2} \mathrm{OTIPS}\left(\mathrm{C}_{6} \mathrm{H}_{4}\right)$ & - & - \\
\hline 8 & 159am & 4- $\mathrm{MeOCH}_{2}\left(\mathrm{C}_{6} \mathrm{H}_{4}\right)$ & $97^{\mathrm{c}}$ & 22: 9: 69 \\
\hline 9 & 159an & $\mathrm{Br}$ & $50^{d}$ & 212an \\
\hline 10 & 159ao & TMS & $85^{e}$ & n.d \\
\hline 11 & 159ap & TIPS & traces & - \\
\hline
\end{tabular}

Reagents and conditions (a) 159 (1 equiv.), 98 (5 mol\%), AgSbF6 ( $5 \mathrm{~mol} \%$ ), $\mathrm{CH}_{2} \mathrm{Cl}_{2}$, rt $1 \mathrm{~h}$. Yields determined of mixtures of inseparable isomers after work up. Conversion and selectivity determined by NMR and/ or HPLC. ${ }^{a}$ Reaction using 66a. ${ }^{b}$ Decomposition was observed. ${ }^{c} 36 \% 159 \mathrm{am}$ observable in reaction mixture. disolated yield after crystallisation. ${ }^{\mathrm{e}} \mathrm{80} \% \mathbf{1 5 9 a 0}$ observable in reaction mixture. 
Table 23. Screening of different $\mathrm{Au}(\mathrm{I})$ complexes in the achiral synthesis of [6]helicene 160ae.

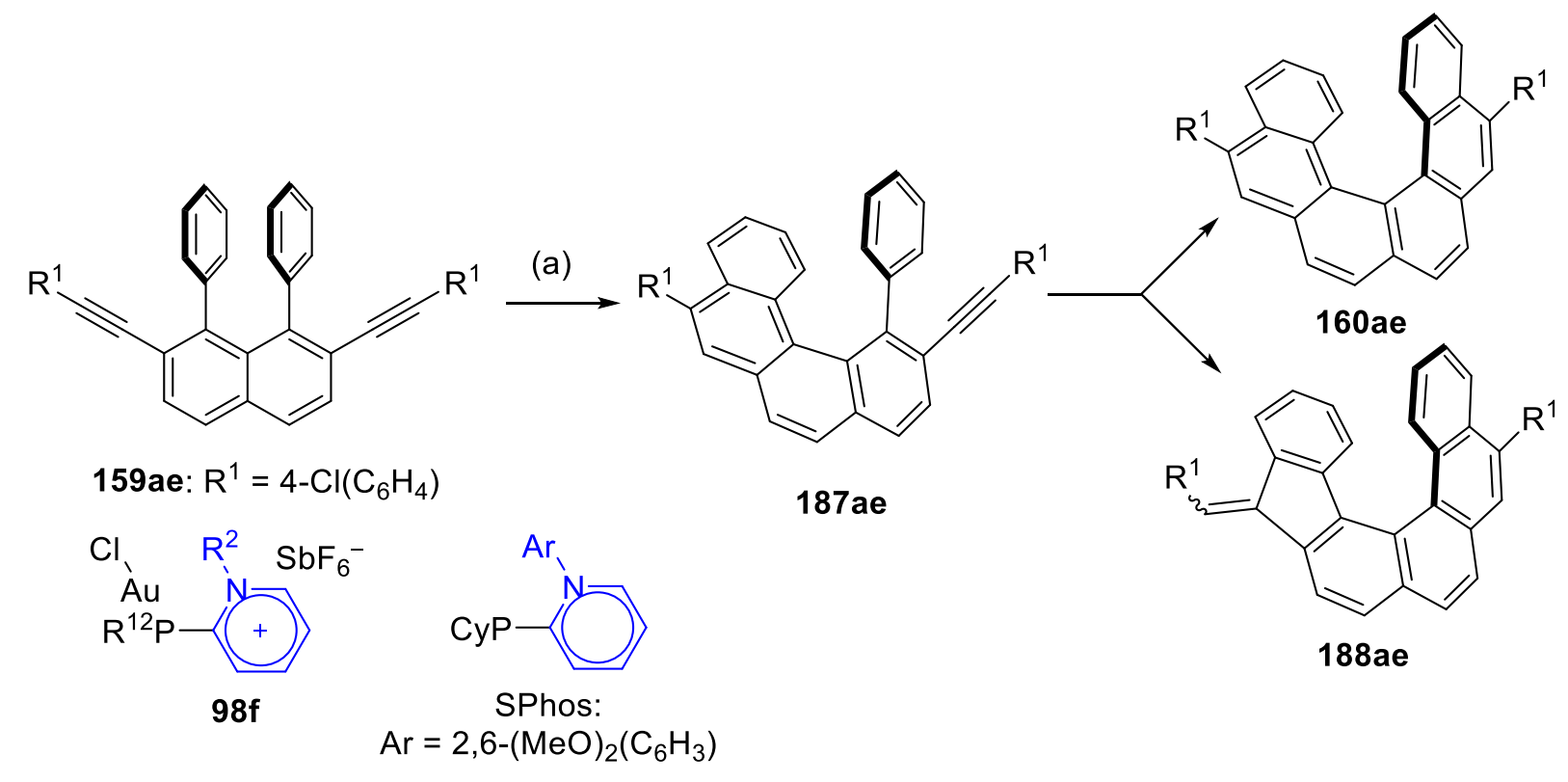

\begin{tabular}{|c|c|c|c|c|c|c|}
\hline Entry & {$[\mathrm{Au}]$} & $\mathrm{R}^{1}$ & $\mathrm{R}^{2}$ & $\begin{array}{c}\mathrm{mol} \\
\%\end{array}$ & $\begin{array}{c}\text { Conversion } \\
(\%)^{\mathrm{a}}\end{array}$ & $\begin{array}{c}\text { 160: } 188: \\
187^{b}\end{array}$ \\
\hline 1 & \multicolumn{3}{|c|}{$\mathrm{PPh}_{3} \cdot \mathrm{AuCl}$} & 5 & 49 & 22: 19: 59 \\
\hline 2 & \multicolumn{3}{|c|}{$\mathrm{P}(\mathrm{OPh})_{3} \cdot \mathrm{AuCl}$} & 5 & $>95$ & 65:34: 1 \\
\hline 3 & \multicolumn{3}{|c|}{ SPhos·AuCl } & 5 & 10 & 0: 0: 100 \\
\hline 4 & $98 f$ & Cy & Mes & 5 & $>95$ & 83: 17: 0 \\
\hline 5 & $98 \mathrm{e}$ & $\mathrm{Ph}$ & Mes & 5 & $>95$ & $44: 32: 22$ \\
\hline 6 & $98 \mathrm{i}$ & $\begin{array}{c}3,5- \\
\left(\mathrm{CF}_{3}\right)_{2}\left(\mathrm{C}_{6} \mathrm{H}_{3}\right)\end{array}$ & Mes & 5 & $>95$ & $31: 69: 0$ \\
\hline 7 & $98 \mathrm{~g}$ & Cy & $\stackrel{2,6-}{(\mathrm{MeO})_{2}\left(\mathrm{C}_{6} \mathrm{H}_{3}\right)}$ & 5 & $>95$ & 67: 33: 0 \\
\hline 8 & $98 \mathrm{~h}$ & $\begin{array}{c}3,5- \\
\left(\mathrm{CF}_{3}\right)_{2}\left(\mathrm{C}_{6} \mathrm{H}_{3}\right)\end{array}$ & $\begin{array}{c}2,6- \\
(\mathrm{MeO})_{2}\left(\mathrm{C}_{6} \mathrm{H}_{3}\right)\end{array}$ & 5 & $>95$ & 45: 55: 0 \\
\hline 9 & $98 a$ & $\mathrm{Ph}$ & $\mathrm{Me}$ & 5 & $>95$ & 82: 18: 0 \\
\hline $10^{c}$ & $98 b$ & Cy & $\mathrm{Me}$ & 5 & $>95$ & $79: 21: 0$ \\
\hline $11^{d}$ & $98 f$ & Cy & Mes & 5 & $>95$ & $84: 16: 0$ \\
\hline $12^{d}$ & $98 a$ & $\mathrm{Ph}$ & $\mathrm{Me}$ & 5 & $>95$ & $76: 17: 7$ \\
\hline 13 & $98 f$ & Cy & Mes & 2 & $>95$ & 83:17:0 \\
\hline 14 & $98 f$ & Cy & Mes & 1.5 & $>95$ & $78: 17: 5$ \\
\hline 15 & $98 a$ & $\mathrm{Ph}$ & $\mathrm{Me}$ & 1.5 & 22 & 6: $0: 94$ \\
\hline
\end{tabular}

Reagents and conditions (a) 159ae (1.0 equiv, $0.025 \mathrm{mmol}$ ), L.AuCl (x mol\%), $\mathrm{AgSbF}_{6}(\mathrm{x} \mathrm{mol} \%), \mathrm{CH}_{2} \mathrm{Cl}_{2}, \mathrm{rt}, 45$

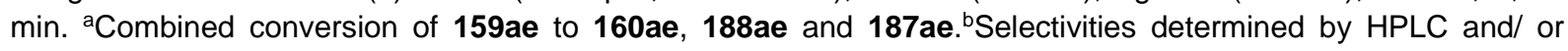
NMR. ${ }^{\mathrm{c}}$ Reaction performed by Tim Johannson. ${ }^{\mathrm{d}}$ Reactions conducted at $0{ }^{\circ} \mathrm{C}$, stirring for $2 \mathrm{~h}$. 
Table 24. Achiral synthesis of hexahelicenes using $98 \mathrm{f}$

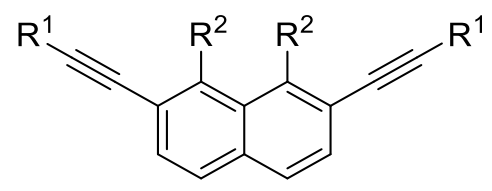

(a)

159

\begin{tabular}{|c|c|c|c|c|c|c|}
\hline \multicolumn{7}{|c|}{$\mathrm{R}^{1}=p$-Tolyl } \\
\hline Entry & 159 & $\mathrm{R}^{2}$ & $t(h)$ & Conversion (\%) & $\begin{array}{l}\text { Yield } \\
(\%)^{a}\end{array}$ & 160: 188: $187^{b}$ \\
\hline $1^{c}$ & $159 \mathrm{eb}$ & 2-thienyl & 1 & $>95$ & 59 & 100: $0: 0$ \\
\hline $2^{d}$ & $159 \mathrm{fb}$ & 2-furanyl & 1 & $>95$ & 46 & 100: 0: 0 \\
\hline 3 & $159 \mathrm{gb}$ & 3-thienyl & 2 & $>95$ & $34^{f}$ & 48: $3: 49$ \\
\hline $4^{e}$ & $159 \mathrm{gb}$ & 3-thienyl & 2 & $>95$ & 72 & 100: $0: 0$ \\
\hline 5 & $159 \mathrm{hb}$ & 3-furanyl & 2 & 88 & $42^{f}$ & $n . d^{g}$ \\
\hline $6^{e}$ & $159 \mathrm{hb}$ & 3-furanyl & 2 & $>95$ & 56 & 100: $0: 0$ \\
\hline 7 & 159ib & 4-TMS $\left(\mathrm{C}_{6} \mathrm{H}_{4}\right)$ & 2 & 68 & $9^{f}$ & n.d. ${ }^{\text {h }}$ \\
\hline 8 & 159jb & $4-\mathrm{BnO}\left(\mathrm{C}_{6} \mathrm{H}_{4}\right.$ & 2 & $>95$ & $52^{i}$ & $84: 16^{k}$ \\
\hline 9 & $159 n b$ & $3,5-\mathrm{Me}_{2}\left(\mathrm{C}_{6} \mathrm{H}_{3}\right)$ & 6 & $>95$ & 93 & 100: $0: 0$ \\
\hline 10 & $159 \mathrm{mb}$ & $3,5-(\mathrm{MeO})_{2}\left(\mathrm{C}_{6} \mathrm{H}_{3}\right)$ & 2 & $>95$ & 91 & 100: $0: 0$ \\
\hline
\end{tabular}

Reagents and conditions (a) 159 (1.5 mol\%), $\operatorname{AgSbF}_{6}$ (1.5 mol\%), $\mathrm{CH}_{2} \mathrm{Cl}_{2}(0.05 \mathrm{M})$, rt, t. alsolated yields. bSelectivity determined by NMR and/ or HPLC. ${ }^{c} 5$ mol \% catalyst loading, followed by re-run with 5 mol \% catalyst loading, then with $10 \mathrm{~mol} \%$ catalyst loading were necessary to give full conversion. ${ }^{\mathrm{d}}$ Two runs at 5 mol \% catalyst loading were necessary to give full conversion. ${ }^{\mathrm{S}} 5 \mathrm{~mol} \%$ catalyst loading used. ${ }^{\text {TYield }}$ calculated using 1,4dioxane as internal standard. 9Multiple unidentifiable side products apparent in reaction mixture. hMessy reaction mixture, $9 \%$ desilylated helicene 160ab identified. 'Yield calculated using hexamethylcyclotrisiloxane as internal standard. ${ }^{k}$ Multiple other impurities identifiable in reaction mixture.

(5E,10E)-5,10-Bis(bromomethylene)-5,10-dihydrofluoreno[3,4-c]fluorene (160an):<smiles>Br/C=C1\c2ccccc2-c2c1ccc1ccc3c(c21)-c1ccccc1/C3=C\Br</smiles>

Prepared using 159an (1.0 equiv, $12.2 \mathrm{mg}, 25.1 \mu \mathrm{mol}$ ), precatalyst $98 \mathrm{f} \quad(5.1 \mathrm{~mol} \%, \quad 1.1 \mathrm{mg}, \quad 1.27 \mu \mathrm{mol})$, dichloromethane $(0.5 \mathrm{ml})$ and $\mathrm{AgSbF}_{6}(4.8 \mathrm{~mol} \%, 1.2 \mu \mathrm{mol}, 25$ $\mu \mathrm{l}$ of a $0.05 \mathrm{M}$ solution in dichloromethane) according to GPF; stirring at room temperature for $2 \mathrm{~h}$. After work up, the product was obtained by crystallisation from hot toluene as an orange solid (6.4 mg, $13.2 \mu \mathrm{mol}, 53 \%$ yield).

'H NMR: $\left(400 \mathrm{MHz}, \mathrm{CDCl}_{3}\right) \delta=8.68-8.63(\mathrm{~m}, 2 \mathrm{H}), 7.80(\mathrm{~d}, J=8.4 \mathrm{~Hz}, 2 \mathrm{H}), 7.72(\mathrm{~d}, J=8.4$ $\mathrm{Hz}, 2 \mathrm{H}), 7.59$ (s, 2H), $7.35-7.29(\mathrm{~m}, 4 \mathrm{H}), 7.25-7.20$ (m, 2H) ppm; ${ }^{13} \mathrm{C}$ NMR: $(101 \mathrm{MHz}$, $\left.\mathrm{CDCl}_{3}\right) \delta=142.5,140.0,139.3,136.4,135.6,134.4,128.7,128.7,128.2,127.6,126.4$, 124.9, 122.8, 118.4, 107.4 ppm; IR: $\left(\right.$ neat, $\left.\mathrm{cm}^{-1}\right) \tilde{v}=584,600,646,700,741,770,838$, 


\section{Experimental}

1258, 1315, 1453, 1594, 1607, 3056; HRMS: calcd $m / z$ for $\mathrm{C}_{26} \mathrm{H}_{14} \mathrm{Br}_{2}{ }^{+}[\mathrm{M}]^{+}$: 483.9462; found (EI) 483.9462.

\section{rac-5,12-Di-p-tolylchryseno[3,4-c]chrysene (160db):}

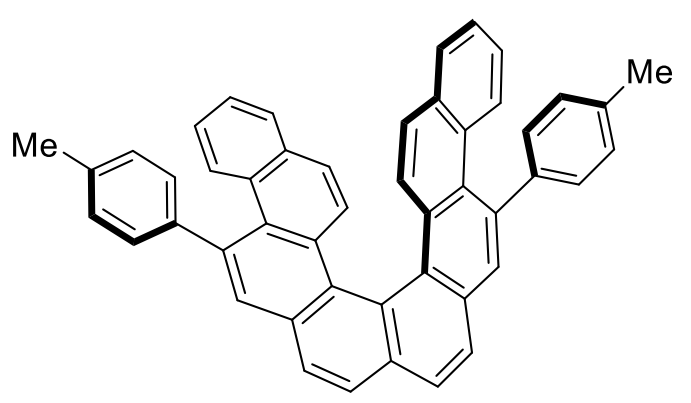

Prepared using 159db (1.0 equiv, $15.0 \mathrm{mg}, 14.7$ $\mu \mathrm{mol})$, precatalyst $98 \mathrm{f}(5.1 \mathrm{~mol} \%, 1.1 \mathrm{mg}, 1.27$ $\mu \mathrm{mol})$, dichloromethane $(0.5 \mathrm{ml})$ and $\mathrm{AgSbF}_{6}(4.8$ mol\%, $1.2 \mu \mathrm{mol}, 25 \mu \mathrm{l}$ of a $0.05 \mathrm{M}$ solution in dichloromethane) according to GPF; stirring at room temperature for $2 \mathrm{~h}$. After work up, the product was obtained by crystallisation from hot toluene as a brown solid (7.2 mg, $11.8 \mu \mathrm{mol}, 48 \%$ yield).

Analytical data corresponded to those reported for $(+)-160 \mathrm{db}$ in this thesis.

rac-11,12-Dithia-1,8-di-p-tolyl-11,12-dihydrocyclopenta[c]indeno[4,5- $g$ ]phenanthrene (160eb):

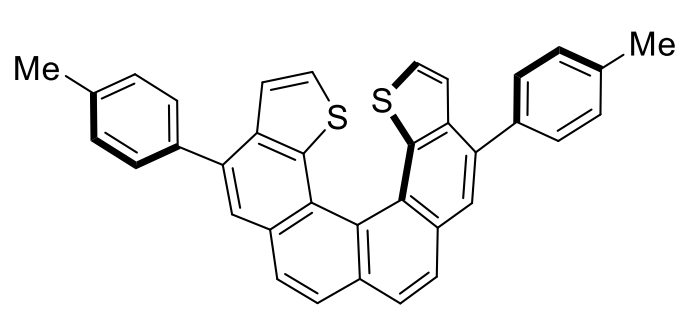

To a dried Schlenk flask equipped with magnetic stirrer was added the substrate $159 \mathrm{eb}$ (1.0 equiv, $10.0 \mathrm{mg}, \quad 19.2 \mu \mathrm{mol})$, followed by the gold precatalyst $98 \mathrm{f}$ (4.8 mol\%, $0.8 \mathrm{mg}, 0.93 \mu \mathrm{mol})$. A septum was fitted to the Schlenk flask and the

contents dried under high vacuum for 30 minutes, before performing three argon purge cycles. Dichloromethane $(0.4 \mathrm{ml})$ was added via syringe through the septum and the reaction mixture was allowed to stir at room temperature for 2 minutes. A solution of $\mathrm{AgSbF}_{6}$ (4.9 mol\%, $0.95 \mu \mathrm{mol}, 19 \mu \mathrm{l}$ of a $0.05 \mathrm{M}$ solution in dichloromethane) was added through the septum and the reaction was allowed to stir at room temperature for $1 \mathrm{~h}$. The reaction was filtered through a short pad of silica eluting with dichloromethane, and the solvent was removed in vacuo. ${ }^{1} \mathrm{H}$ NMR showed $50 \%$ conversion had occurred. The crude product was resubmitted to the reaction conditions, using gold precatalyst $98 \mathrm{f}(4.8 \mathrm{~mol} \%, 0.8 \mathrm{mg}, 0.93$ $\mu \mathrm{mol})$, dichloromethane $(0.4 \mathrm{ml})$ and $\mathrm{AgSbF}_{6}(4.9 \mathrm{~mol} \%, 0.95 \mu \mathrm{mol}, 19 \mu \mathrm{l}$ of a $0.05 \mathrm{M}$ solution in dichloromethane). The reaction mixture was stirred for $1 \mathrm{~h}$. at room temperature, before filtering through a silica plug eluting with dichloromethane. ${ }^{1} \mathrm{H}$ NMR showed that conversion was incomplete (proportion 160eb:187eb:159eb $=51: 24: 24$ ); therefore, the mixture was submitted to the same reaction conditions again, this time using gold precatalyst $98 \mathrm{f}$ (9.6 mol\%, $1.6 \mathrm{mg}, 1.85 \mu \mathrm{mol})$, dichloromethane $(0.4 \mathrm{ml})$ and $\mathrm{AgSbF}_{6}(9.9 \mathrm{~mol} \%, 1.90 \mu \mathrm{mol}, 38 \mu \mathrm{l}$ of a $0.05 \mathrm{M}$ solution in dichloromethane). After stirring for one $\mathrm{h}$ at room temperature and 


\section{Experimental}

workup, the product was purified by column chromatography $(20 \%$ dichloromethane in pentane), affording the compound $\mathbf{1 6 0 \mathrm { eb }}$ as a yellow solid (5.9 $\mathrm{mg}, 11.3 \mu \mathrm{mol}, 59 \%$ yield).

'H NMR: $\left(300 \mathrm{MHz}, \mathrm{CDCl}_{3}\right) \delta=8.07(\mathrm{~d}, J=8.4 \mathrm{~Hz}, 2 \mathrm{H}), 7.98(\mathrm{~s}, 2 \mathrm{H}), 7.88(\mathrm{~d}, J=8.4 \mathrm{~Hz}$, 2H), $7.78-7.68(\mathrm{~m}, 4 \mathrm{H}), 7.45(\mathrm{~d}, J=5.5 \mathrm{~Hz}, 2 \mathrm{H}), 7.42-7.35(\mathrm{~m}, 4 \mathrm{H}), 7.23(\mathrm{~d}, J=5.6 \mathrm{~Hz}$, 2H), 2.50 (s, 6H) ppm; ${ }^{13} \mathbf{C}\{\mathrm{H}\}$ NMR: $\left(126 \mathrm{MHz}, \mathrm{cdcl}_{3}\right) \delta=139.3,137.9,137.5,137.4,137.2$, 132.6, 131.3, 129.4, 129.4, 128.2, 125.6, 125.3, 124.5, 124.3, 124.0, 123.0, 21.5 ppm; IR: (neat, $\mathrm{cm}^{-1}$ ) $\tilde{\mathrm{v}}=588,700,755,801,817,880,1018,1261,1452,1509,2847,2918,2955$; HRMS: calcd $m / z$ for $\mathrm{C}_{36} \mathrm{H}_{24} \mathrm{~S}_{2}{ }^{+}[\mathrm{M}]^{+}: 520.1319$; found (EI) 520.1311.

rac-11,12-Dioxa-1,8-di-p-tolyl-11,12-dihydrocyclopenta[c]indeno[4,5-g]phenanthrene (160fb):

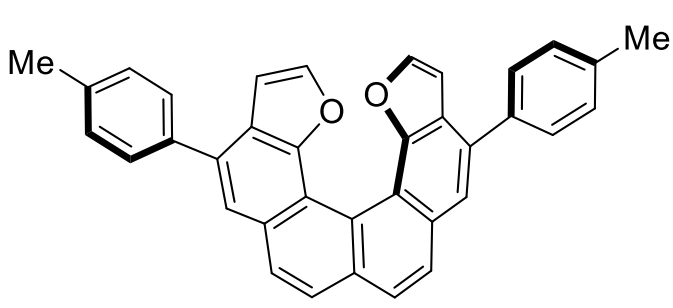

To a dried Schlenk flask equipped with magnetic stirrer was added the substrate $159 \mathrm{fb}$ (1.0 equiv, $12.2 \mathrm{mg}, 25.0 \mu \mathrm{mol})$, followed by the gold precatalyst $98 \mathrm{f}(5.1 \mathrm{~mol} \%, 1.1 \mathrm{mg}, 1.27 \mu \mathrm{mol})$. A septum was fitted to the Schlenk flask and the contents dried under high vacuum for 30 minutes, before performing three argon purge cycles. Dichloromethane $(0.5 \mathrm{ml})$ was added via syringe through the septum, and the reaction mixture was allowed to stir at room temperature for 2 minutes. A solution of $\mathrm{AgSbF}_{6}$ ( $5.0 \mathrm{~mol} \%, 1.25 \mu \mathrm{mol}, 25 \mu \mathrm{l}$ of a $0.05 \mathrm{M}$ solution in dichloromethane) was added through the septum, and the resulting mixture was allowed to stir at room temperature for $1 \mathrm{~h}$. The reaction mixture was filtered through a short pad of silica eluting with dichloromethane, and the solvent was removed in vacuo. ${ }^{1} \mathrm{H}$ NMR showed a mixture of products (proportion $160 \mathrm{fb}: 188 \mathrm{fb}: 159 \mathrm{fb}=28: 5: 67)$; therefore, the crude product was resubmitted to the reaction conditions, using gold precatalyst $98 \mathrm{f}(5.1 \mathrm{~mol} \%, 1.1 \mathrm{mg}, 1.27 \mu \mathrm{mol})$, dichloromethane $(0.5$ $\mathrm{ml})$ and $\mathrm{AgSbF}_{6}(5.0 \mathrm{~mol} \%, 1.25 \mu \mathrm{mol}, 25 \mu \mathrm{l}$ of a $0.05 \mathrm{M}$ solution in dichloromethane). The reaction mixture was stirred for 1 hour at room temperature, before the work-up as indicated above and purification by column chromatography ( $20 \%$ dichloromethane in pentane), affording the product $160 \mathrm{fb}$ as a yellow solid (5.6 $\mathrm{mg}, 11.5 \mu \mathrm{mol}, 46 \%$ yield).

'H NMR: $\left(300 \mathrm{MHz}, \mathrm{CDCl}_{3}\right) \delta=8.09(\mathrm{~d}, J=8.5 \mathrm{~Hz}, 2 \mathrm{H}), 7.96(\mathrm{~s}, 2 \mathrm{H}), 7.88(\mathrm{~d}, J=8.5 \mathrm{~Hz}$, $2 \mathrm{H}), 7.83-7.75(\mathrm{~m}, 4 \mathrm{H}), 7.43(\mathrm{~d}, J=2.1 \mathrm{~Hz}, 2 \mathrm{H}), 7.42-7.36(\mathrm{~m}, 4 \mathrm{H}), 7.06(\mathrm{~d}, J=2.1 \mathrm{~Hz}$, 2H), $2.49(\mathrm{~s}, 6 \mathrm{H}) ;{ }^{13} \mathrm{C}\{\mathrm{H}\}$ NMR: $\left(126 \mathrm{MHz}, \mathrm{CDCl}_{3}\right) \delta=152.6,143.3,137.3,137.0,134.1$, 131.5, 129.4, 128.6, 127.8, 125.3, 123.5, 121.5, 119.5, 117.5, 106.5, 21.4; IR: (neat, $\left.\mathrm{cm}^{-1}\right) \tilde{\mathrm{v}}$ $=578,717,729,759,798,824,874,906,1003,1017,1043,1091,1226,1260,1304,1363$, 1477, 1504, 2852, 2920, 2966, 3039, 3142; HRMS: calcd $m / z$ for $\mathrm{C}_{36} \mathrm{H}_{24} \mathrm{O}_{2}{ }^{+}[\mathrm{M}]^{+}: 488.1776$; found (El) 488.1764 . 


\section{Experimental}

rac-9,14-Dithia-1,8-di-p-tolyl-11,12-dihydrocyclopenta[c]indeno[4,5-g]phenanthrene (160gb):

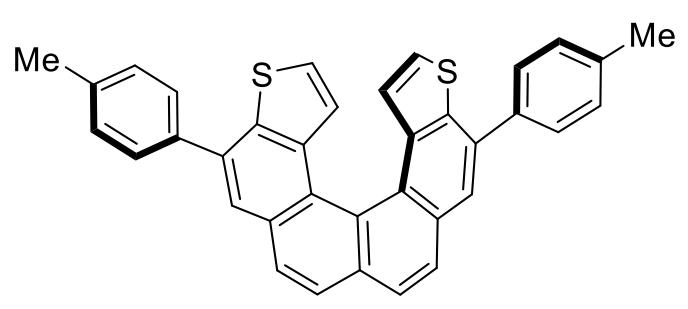

Prepared using 159gb (1.0 equiv, $9.0 \mathrm{mg}, 17.3$ $\mu \mathrm{mol})$, precatalyst $98 \mathrm{f}(4.7 \mathrm{~mol} \%, 0.7 \mathrm{mg}, 0.81$ $\mu \mathrm{mol})$, dichloromethane $(0.35 \mathrm{ml})$ and $\mathrm{AgSbF}_{6}(5.4$ mol\%, $0.93 \mu \mathrm{mol}, 49 \mu \mathrm{l}$ of a $0.05 \mathrm{M}$ solution in dichloromethane) according to GPF; stirring at room temperature for $2 \mathrm{~h}$. The product was obtained after purification by column chromatography ( $25 \%$ toluene in pentane) as a yellow solid $(6.5 \mathrm{mg}, 12.5 \mu \mathrm{mol}, 72 \%$ yield).

'H NMR: $\left(300 \mathrm{MHz}, \mathrm{CDCl}_{3}\right) \delta=8.07(\mathrm{~d}, J=8.3 \mathrm{~Hz}, 2 \mathrm{H}), 7.96(\mathrm{~s}, 2 \mathrm{H}), 7.91(\mathrm{~d}, J=8.4 \mathrm{~Hz}$, 2H), $7.89-7.81(\mathrm{~m}, 4 \mathrm{H}), 7.45-7.37(\mathrm{~m}, 4 \mathrm{H}), 7.19(\mathrm{~d}, J=5.6 \mathrm{~Hz}, 2 \mathrm{H}), 7.10(\mathrm{~d}, J=5.6 \mathrm{~Hz}$, $2 \mathrm{H}), 2.50(\mathrm{~s}, 6 \mathrm{H}) \mathrm{ppm} ;{ }^{13} \mathrm{C}\{\mathrm{H}\}$ NMR $\left(126 \mathrm{MHz}, \mathrm{CDCl}_{3}\right) \delta=138.1,138.0,137.6,137.3,135.9$, 132.3, 131.8, 129.6, 128.6, 127.8, 126.9, 126.1, 125.8, 125.0, 124.4, 123.6, 21.6 ppm; IR: $\left(\right.$ neat, $\mathrm{cm}^{-1}$ ) $\tilde{v}=586,681,749,793,816,898,1014,1093,1260,1334,1518,1740,2849$, 2914, 2953, 3044; HRMS: calcd $m / z$ for $\mathrm{C}_{36} \mathrm{H}_{24} \mathrm{~S}_{2}{ }^{+}[\mathrm{M}]^{+}:$520.1319; found (El) 520.1324.

rac-9,14-Dioxa-1,8-di-p-tolyl-11,12-dihydrocyclopenta[c]indeno[4,5- $g]$ phenanthrene (160hb):

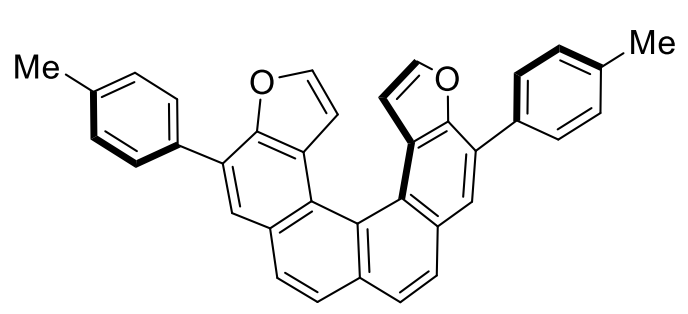

Prepared using 159hb (1.0 equiv, $5.9 \mathrm{mg}, 12.1$ umol), precatalyst $98 \mathrm{f}(4.8 \mathrm{~mol} \%, 0.5 \mathrm{mg}, 0.58$ $\mu \mathrm{mol})$, dichloromethane $(0.24 \mathrm{ml})$ and $\mathrm{AgSbF}_{6}(5.0$ mol\%, $0.60 \mu \mathrm{mol}, 12 \mu \mathrm{l}$ of a $0.05 \mathrm{M}$ solution in dichloromethane) according to GPF; stirring at room temperature for $2 \mathrm{~h}$. The product was obtained after purification by column chromatography ( $25 \%$ toluene in pentane) as a yellow solid ( $3.3 \mathrm{mg}, 6.75 \mu \mathrm{mol}, 56 \%$ yield).

'H NMR: $\left(300 \mathrm{MHz}, \mathrm{CDCl}_{3}\right) \delta=8.10(\mathrm{~d}, J=8.4 \mathrm{~Hz}, 2 \mathrm{H}), 8.10(\mathrm{~s}, 2 \mathrm{H}), 8.01$ (dd, $J=7.9,2.0$ $\mathrm{Hz}, 4 \mathrm{H}), 7.88(\mathrm{~d}, J=8.5 \mathrm{~Hz}, 2 \mathrm{H}), 7.55(\mathrm{dd}, J=2.2,0.4 \mathrm{~Hz}, 2 \mathrm{H}), 7.47-7.39(\mathrm{~m}, 4 \mathrm{H}), 6.63(\mathrm{~d}$, $J=2.2 \mathrm{~Hz}, 2 \mathrm{H}), 2.51(\mathrm{~s}, 6 \mathrm{H}) \mathrm{ppm} ;{ }^{13} \mathrm{C}\{\mathrm{H}\}$ NMR: $\left(126 \mathrm{MHz}, \mathrm{CDCl}_{3}\right) \delta=150.5,142.5,137.8$, 133.3, 131.7, 130.8, 129.4, 128.7, 127.9, 126.4, 125.2, 125.0, 124.9, 124.3, 123.4, 110.9, 21.4 ppm; IR: $\left(\right.$ neat, $\left.\mathrm{cm}^{-1}\right) \tilde{\mathrm{v}}=572,737,768,819,833,895,1047,1134,1191,1240,1373$, 1444, 1602, 1659, 1738, 2852, 2921; HRMS: calcd $m / z$ for $\mathrm{C}_{36} \mathrm{H}_{24} \mathrm{O}_{2}{ }^{+}[\mathrm{M}]^{+}:$488.1776; found (EI) 488.1768 . 


\section{Experimental}

rac-9,11,14,16-Tetramethoxy-1,8-di-p-tolylhexahelicene (168mb):

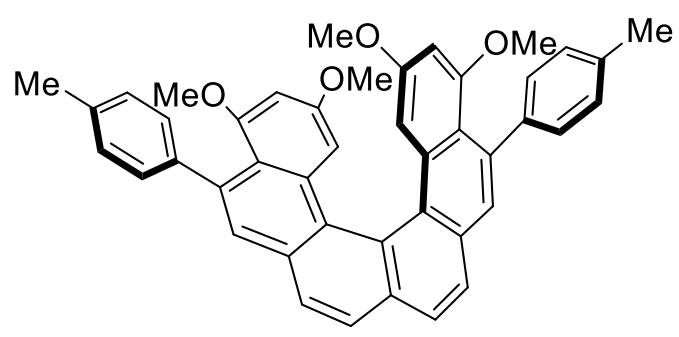

Prepared using 159mb (1.0 equiv, $15.7 \mathrm{mg}, 25.0$ $\mu \mathrm{mol})$, precatalyst $98 \mathrm{f}(1.50 \mathrm{~mol} \%, 0.37 \mu \mathrm{mol}, 107$ $\left.\mu \mathrm{l}, 3.5 \times 10^{-3} \mathrm{M}\right)$, dichloromethane $(0.5 \mathrm{ml})$ and $\mathrm{AgSbF}_{6}\left(1.3 \mathrm{~mol} \%, 0.31 \mu \mathrm{mol}, 25 \mu \mathrm{l}, 1.25 \times 10^{-2} \mathrm{M}\right)$ according to GPG; stirring at room temperature for $2 \mathrm{~h}$. The product was obtained as a yellow solid

(14.3 mg $22.7 \mu \mathrm{mol}, 91 \%$ yield).

'H NMR: $\left(400 \mathrm{MHz}, \mathrm{CDCl}_{3}\right) \delta=7.99(\mathrm{~d}, J=8.2 \mathrm{~Hz}, 2 \mathrm{H}), 7.90(\mathrm{~d}, J=8.2 \mathrm{~Hz}, 2 \mathrm{H}), 7.67$ (s, $2 \mathrm{H}), 7.51-7.27(\mathrm{~m}, 4 \mathrm{H}), 7.26-7.21(\mathrm{~m}, 4 \mathrm{H}), 6.99(\mathrm{~d}, J=2.4 \mathrm{~Hz}, 2 \mathrm{H}), 6.33(\mathrm{~d}, J=2.4 \mathrm{~Hz}$, $2 \mathrm{H}), 3.46(\mathrm{~s}, 6 \mathrm{H}), 3.15(\mathrm{~s}, 6 \mathrm{H}), 2.47(\mathrm{~s}, 6 \mathrm{H}) \mathrm{ppm} ;{ }^{13} \mathrm{C}\{\mathrm{H}\}$ NMR: $\left(101 \mathrm{MHz}, \mathrm{CDCl}_{3}\right) \delta=157.7$, 157.7, 142.5, 137.3, 135.4, 134.0, 133.4, 131.3, 128.1, 127.8, 127.8, 127.0, 126.9, 126.9, 124.5, 116.9, 100.8, 99.5, 55.5, 54.6, 21.4 ppm; IR: (neat, $\mathrm{cm}^{-1}$ ) $\tilde{\mathrm{v}}=$ 706, 820, 938, 1017, 1061, 1104, 1161, 1203, 1263, 1329, 1605, 2842, 2923, 2960; HRMS: calcd m/z for $\mathrm{C}_{44} \mathrm{H}_{37} \mathrm{O}_{4}{ }^{+}[\mathrm{M}+\mathrm{H}]^{+}$: 629.2686; found (ESI) 629.2673.

rac-9,11,14,16-Tetramethyl-1,8-di-p-tolylhexahelicene (168nb):

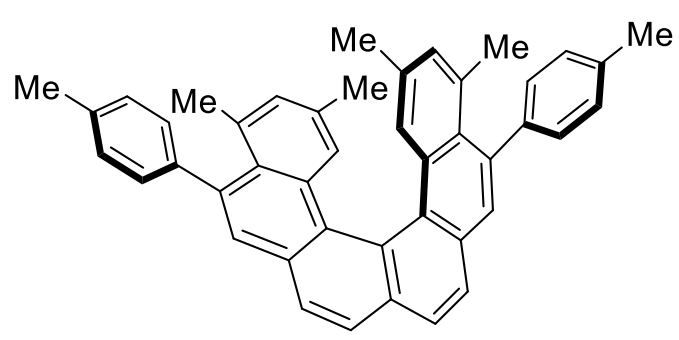

Prepared using 159nb (1.0 equiv, $5.7 \mathrm{mg}, 10.1$ $\mu \mathrm{mol})$, precatalyst $98 \mathrm{f}(1.50 \mathrm{~mol} \%, 0.15 \mu \mathrm{mol}, 35$ $\left.\mu \mathrm{l}, 4.4 \times 10^{-3} \mathrm{M}\right)$, dichloromethane $(0.2 \mathrm{ml})$ and $\mathrm{AgSbF}_{6}\left(1.5 \mathrm{~mol} \%, 0.15 \mu \mathrm{mol}, 12 \mu \mathrm{l}, 1.25 \times 10^{-2} \mathrm{M}\right)$ according to GPG; stirring at room temperature for $2 \mathrm{~h}$. The product was obtained as a yellow solid

(5.3 mg, $9.4 \mu \mathrm{mol}, 93 \%$ yield).

'H NMR: $\left(300 \mathrm{MHz}, \mathrm{CDCl}_{3}\right) \delta=7.98(\mathrm{~d}, J=8.2 \mathrm{~Hz}, 2 \mathrm{H}), 7.87(\mathrm{~d}, J=8.2 \mathrm{~Hz}, 2 \mathrm{H}), 7.72(\mathrm{~s}$, 2H), $7.55(\mathrm{~d}, J=7.7 \mathrm{~Hz}, 2 \mathrm{H}), 7.42(\mathrm{~s}, 2 \mathrm{H}), 7.32(\mathrm{~d}, J=8.2 \mathrm{~Hz}, 2 \mathrm{H}), 7.27(\mathrm{t}, J=1.3 \mathrm{~Hz}, 4 \mathrm{H})$, $6.81(\mathrm{~d}, J=1.8 \mathrm{~Hz}, 2 \mathrm{H}), 2.49(\mathrm{~s}, 6 \mathrm{H}), 2.03(\mathrm{~s}, 6 \mathrm{H}), 1.83(\mathrm{~s}, 6 \mathrm{H}) \mathrm{ppm} ;{ }^{13} \mathrm{C}\{\mathrm{H}\} \mathbf{N M R}:(75 \mathrm{MHz}$, $\left.\mathrm{CDCl}_{3}\right) \delta=142.3,138.9,136.3,134.0,133.6,132.9,131.6,131.2,129.9,129.8,129.0$, 128.3, 128.3, 128.2, 128.0, 127.7, 127.2, 126.7, 126.1, 124.2, 25.3, 21.3, 20.7 ppm; IR: $\left(\right.$ neat, $\left.\mathrm{cm}^{-1}\right) \tilde{v}=525,634,728,824,862,1028,1264, \mathrm{m1360}, 1433,1446,1509,1609,2852$, 2912, 2967, 3018; HRMS: calcd $\mathrm{m} / \mathrm{z}$ for $\mathrm{C}_{44} \mathrm{H}_{36} \mathrm{Na}^{+}[\mathrm{M}+\mathrm{Na}]^{+}$: 587.2709; found (ESI) 587.2704 . 


\subsubsection{Enantioselective synthesis of helicenes}

General procedure H (GPH) for the enantioselective synthesis of helicenes<smiles>[R]C#Cc1ccc2ccc(C#C)c(-c3ccc([R])c([R])c3)c2c1-c1ccc([R])c([R])c1</smiles>

159
[Au] $(10 \mathrm{~mol} \%)$ $\mathrm{AgSbF}_{6}$ (10 mol\%) solvent, $\mathrm{T}, \mathrm{t}$<smiles></smiles>

160<smiles>[R]C=C1c2ccc3ccc4cc([R])c5c([R])c([R])c([R])cc5c4c3c2-c2cc([R])c([R])c([R])c21</smiles>

188

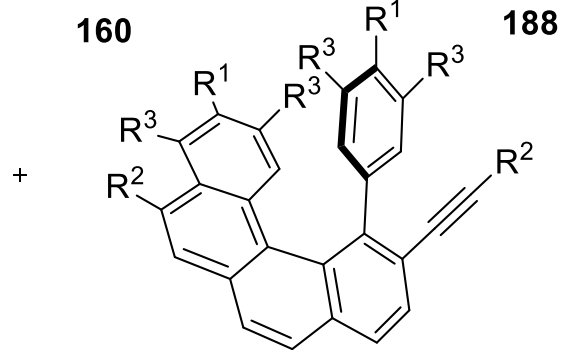

187

To a dried Schlenk flask equipped with magnetic stirrer was added the substrate (1.0 equiv.), followed by the gold precatalyst (10 mol\%). A septum was fitted to the Schlenk flask and the contents dried under high vacuum for 30 minutes, before performing three argon purge cycles. The reaction solvent $(0.05 \mathrm{M})$ was added via syringe through the septum and the reaction mixture was allowed to stir at room temperature for 2 minutes, before transferring to a pre-cooled isopropanol bath and stirred for 15 minutes to reach the required temperature. A solution of $\mathrm{AgSbF}_{6}$ (10 mol\%, 0.05M solution in dichloromethane) was added through the septum, the septum was exchanged for a greased glass stopper and the reaction mixture was allowed to stir at the stated temperature for the indicated time. The reaction mixture was filtered through a short pad of silica eluting with dichloromethane, and the solvent was removed in vacuo, before drying under high vacuum. The entire sample was then redissolved in a known amount of $\mathrm{CDCl}_{3}$, before an aliquot was taken for NMR and HPLC measurements. The conversion and ratio of isomers was determined by NMR and/or HPLC. The enantiomeric excess was determined by HPLC. In the case an internal standard was used to determine yield or conversion by ${ }^{1} \mathrm{H}$ NMR, the entire sample was dissolved in $\mathrm{CDCl}_{3}$ or $\mathrm{CD}_{2} \mathrm{Cl}_{2}$ and the internal standard (4.0 equiv.) was added, an aliquot was removed for NMR measurement. 


\section{Experimental}

\section{(P)-1,8-Bis(4-fluorophenyl)hexahelicene (160af):}

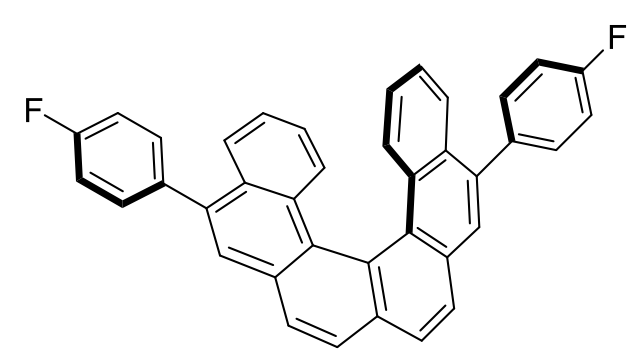

Prepared using 159af (1.0 equiv, $12.8 \mathrm{mg}, 24.7 \mu \mathrm{mol}$ ), precatalyst $172 \mathrm{i}(10 \mathrm{~mol} \%, 3.8 \mathrm{mg}, 2.44 \mu \mathrm{mol})$, fluorobenzene $(0.5 \mathrm{ml})$ and $\mathrm{AgSbF}_{6}(10 \mathrm{~mol} \%, 2.45$ $\mu \mathrm{mol}, 49 \mu \mathrm{l}$ of a $0.05 \mathrm{M}$ solution in dichloromethane) according to GPH; stirring at $-20{ }^{\circ} \mathrm{C}$ for $96 \mathrm{~h}$. The crude product was obtained as a yellow powder in $90 \%$ yield (mixture of 160af:188af:187af $=92: 7: 1,92 \%$ conversion).

In order to obtain a pure sample for characterization, the mixture was purified by semipreparative HPLC, affording $2.6 \mathrm{mg}(5.0 \mu \mathrm{mol}, 20 \%$ yield) of 160af. Separation conditions: $250 \times 4.6 \mathrm{~mm}$ YMC pack PVA-SIL $5 \mu \mathrm{m}$ column, hexanes/MTBE = 96/4 (v/v), $18.9 \mathrm{ml} \cdot \mathrm{min}^{-1}$, $2.4 \mathrm{MPa}, 333 \mathrm{~nm}$; HPLC: $250 \times 4.6 \mathrm{~mm}$ YMC-Pack PVA SIL-NP, $5 \mu \mathrm{m}$, hexanes/MTBE = 96/4 (v/v), $1.0 \mathrm{ml} \cdot \mathrm{min}^{-1}, 2.6 \mathrm{MPa}, 303 \mathrm{~K}, 246 \mathrm{~nm}$; helicene 160af: $t_{R}=14.51 \mathrm{~min}$, isomers 188af $(E / Z): t_{R}=15.15$ and $18.50 \mathrm{~min}$, intermediate 187af: $t_{R}=13.96 \mathrm{~min}$, starting material 159af: , $t_{R}=12.16 \mathrm{~min}$; Enantiomeric excess: $+90 \%$. Conditions for $2 \mathrm{D}$ separation: $50 \times 4.6$ mm Agilent Eclipse Plus C18 $1.8 \mu \mathrm{m}$ column, $\mathrm{CH}_{3} \mathrm{CN} / \mathrm{H}_{2} \mathrm{O}=85 / 15(\mathrm{v} / \mathrm{v}), 1.0 \mathrm{ml} \cdot \mathrm{min}^{-1}, 15.5$ $\mathrm{MPa}, 308 \mathrm{~K}, 333 \mathrm{~nm}$ helicene 160af $t_{R}=10.84 \mathrm{~min}$; then $150 \times 4.6 \mathrm{~mm}$ Chiralpak IC-3 column, $3 \mu \mathrm{m}, \mathrm{CH}_{3} \mathrm{CN} / \mathrm{H}_{2} \mathrm{O}=90 / 10(\mathrm{v} / \mathrm{v}), 1.0 \mathrm{ml} \cdot \mathrm{min}^{-1}, 10.3 \mathrm{MPa}, 298 \mathrm{~K}, 333 \mathrm{~nm}$; major enantiomer: $t_{R}=4.25 \mathrm{~min}$, minor enantiomer: $t_{R}=5.02 \mathrm{~min}$.

$[\alpha]_{D}^{19.5}:+1065.4\left(c=0.08, \mathrm{CH}_{2} \mathrm{Cl}_{2}\right) ;{ }^{1} \mathrm{H}$ NMR: $\left(400 \mathrm{MHz}, \mathrm{CDCl}_{3}\right) \delta=8.05(\mathrm{~d}, J=8.2 \mathrm{~Hz}, 2 \mathrm{H})$, $7.99(\mathrm{~d}, J=8.2 \mathrm{~Hz}, 2 \mathrm{H}), 7.89(\mathrm{~s}, 2 \mathrm{H}), 7.84-7.77(\mathrm{~m}, 4 \mathrm{H}), 7.68$ (ddd, $J=8.8,2.6 \mathrm{~Hz}, J_{H-F}=$ $5.3 \mathrm{~Hz}, 4 \mathrm{H}$ ), 7.28 (td, $J=8.5,2.4 \mathrm{~Hz}, J_{H-F}=8.5 \mathrm{~Hz}, 4 \mathrm{H}$ ), 7.18 (ddd, $J=8.2,6.9,1.3 \mathrm{~Hz}, 2 \mathrm{H}$ ), 6.74 (ddd, $J=8.3,6.8,1.4 \mathrm{~Hz}, 2 \mathrm{H}) \mathrm{ppm} ;{ }^{13} \mathrm{C}\left\{{ }^{1} \mathrm{H}\right\}$ NMR: $\left(126 \mathrm{MHz}, \mathrm{CDCl}_{3}\right) \delta=162.6\left(\mathrm{~d}, J_{C-F}=\right.$ $246.5 \mathrm{~Hz}), 138.7,136.6\left(\mathrm{~d}, J_{C-F}=3.3 \mathrm{~Hz}\right), 133.5,131.9\left(\mathrm{~d}, J_{C-F}=7.9 \mathrm{~Hz}\right), 130.9,130.6$, $130.5,128.2,127.8,127.6,127.3,126.9,125.8,125.7,124.7,123.9,115.5\left(\mathrm{~d}, J_{C-F}=21.3\right.$ $\mathrm{Hz})$ ppm; ${ }^{19}$ F NMR: $\left(376 \mathrm{MHz}, \mathrm{CDCl}_{3}\right) \delta=-115.1$ (tt, $\left.J_{F-H}=8.6,5.3 \mathrm{~Hz}\right)$ ppm; IR: (neat, $\mathrm{cm}^{-1}$ ) V: $115,506,517,572,608,734,766,766,836,887,1215,1401,1498,1508,1605,1766$, 1893, 2249, 2859, 2929, 3026; HRMS: calcd $m / z$ for $\mathrm{C}_{38} \mathrm{H}_{22} \mathrm{~F}_{2} \mathrm{Na}^{+}[\mathrm{M}+\mathrm{Na}]^{+}:$539.1582; found (ESI) 539.1560; UV/Vis: (THF): $\lambda_{\max }(\log \varepsilon)=230$ (4.79), 251 (4.76), 269 (4.69), 321 (4.44), 333 (4.48), 357 (4.29) nm. 


\section{Experimental}

\section{(P)-1,8-Bis(4-methoxyphenyl)hexahelicene (160ag):}

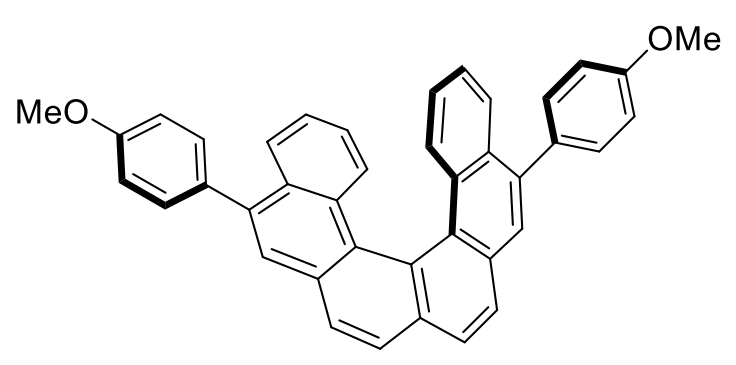

Prepared using 159ag (1.0 equiv, $13.4 \mathrm{mg}$, $24.7 \mu \mathrm{mol}$ ), precatalyst $172 \mathbf{i}(10 \mathrm{~mol} \%, 3.8 \mathrm{mg}$, $2.44 \mu \mathrm{mol})$, fluorobenzene $(0.5 \mathrm{ml})$ and $\mathrm{AgSbF}_{6}$ (10 mol\%, $2.45 \mu \mathrm{mol}, 49 \mu \mathrm{l}$ of a $0.05 \mathrm{M}$ solution in dichloromethane) according to GPH; stirring at $-20^{\circ} \mathrm{C}$ for $96 \mathrm{~h}$. The crude product was obtained as a yellow powder in 90\% yield (mixture of 160ag:188ag $=96: 4$ ).

HPLC: $50 \times 4.6 \mathrm{~mm}$ Agilent Eclipse Plus C18 $1.8 \mu \mathrm{m}$ column, $\mathrm{CH}_{3} \mathrm{CN} / \mathrm{H}_{2} \mathrm{O}=80 / 20(\mathrm{v} / \mathrm{v})$, $1.0 \mathrm{ml} \cdot \mathrm{min}^{-1}, 19.3 \mathrm{MPa}, 308 \mathrm{~K}, 193 \mathrm{~nm}$; helicene 160ag: $t_{R}=15.67 \mathrm{~min}$, isomers 188ag $(E / Z): t_{R}=16.61 \mathrm{~min}$; Enantiomeric excess: $+81 \%$. The ee was determined by chiral HPLC: $150 \times 4.6 \mathrm{~mm}$ Chiralpak IC-3 $3 \mu \mathrm{m}$ column, $\mathrm{CH}_{3} \mathrm{CN} / \mathrm{H}_{2} \mathrm{O}=80 / 20(\mathrm{v} / \mathrm{v}), 1.0 \mathrm{ml} \cdot \mathrm{min}^{-1}, 17.2$ $\mathrm{MPa}, 308 \mathrm{~K}, 193 \mathrm{~nm}$; major enantiomer: $t_{R}=9.90 \mathrm{~min}$, minor enantiomer: $t_{R}=14.00 \mathrm{~min}$.

$[\alpha]_{D}^{24}:+1099.1\left(c=0.518, \mathrm{CH}_{2} \mathrm{Cl}_{2}\right) ;{ }^{1} \mathrm{H}$ NMR: $\left(300 \mathrm{MHz}, \mathrm{CDCl}_{3}\right) \delta=8.03(\mathrm{~d}, J=8.2 \mathrm{~Hz}, 2 \mathrm{H})$, $7.98(\mathrm{~d}, J=8.3 \mathrm{~Hz}, 2 \mathrm{H}), 7.94-7.86(\mathrm{~m}, 4 \mathrm{H}), 7.81$ (ddd, $J=8.5,1.3,0.6 \mathrm{~Hz}, 2 \mathrm{H}), 7.66$ (dd, $J=$ 8.7, $2.5 \mathrm{~Hz}, 4 \mathrm{H}$ ), 7.22-7.09 (m, 6H), 6.74 (ddd, $J=8.3,6.8,1.4 \mathrm{~Hz}, 2 \mathrm{H}$ ), 3.96 (s, 6H) ppm; ${ }^{13} \mathrm{C}\left\{{ }^{1} \mathrm{H}\right\}$ NMR: $\left(126 \mathrm{MHz}, \mathrm{CDCl}_{3}\right) \delta=159.2,139.3,133.2,133.0,131.3,130.9,130.8,130.5$, 128.2, 127.5, 127.3, 127.1, 126.5, 125.9, 125.6, 124.5, 124.0, 114.0, 55.6 ppm; IR: (neat, $\left.\mathrm{cm}^{-1}\right) \tilde{v}=521,581,608,727,834,765,888,1031,1103,1174,1243,1286,1395,1460$, 1509, 1560, 2828, 2900, 2926, 2996, 3030; HRMS: calcd $m / z$ for $\mathrm{C}_{40} \mathrm{H}_{28} \mathrm{O}_{2}{ }^{+}[\mathrm{M}]^{+}:$540.2089; found (El) 540.2096; UV/Vis: (THF): $\lambda_{\max }(\log \varepsilon)=233$ (5.03), 249 (4.94), 269 (4.85), 323 (4.60), $336(4.65) \mathrm{nm}$.

\section{(P)-1,8-bis[4-(benzyloxy)phenyl]hexahelicene (160ah):}

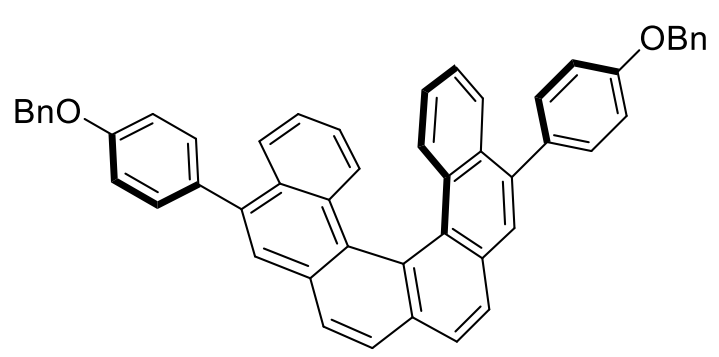

Prepared using 159ah (1.0 equiv, $17.1 \mathrm{mg}$, $24.7 \mu \mathrm{mol})$, precatalyst $172 \mathbf{i}(10 \mathrm{~mol} \%, 3.8 \mathrm{mg}$, $2.44 \mu \mathrm{mol})$, dichloromethane $(0.5 \mathrm{ml})$ and $\mathrm{AgSbF}_{6}(10 \mathrm{~mol} \%, 2.45 \mu \mathrm{mol}, 49 \mu \mathrm{l}$ of a $0.05 \mathrm{M}$ solution in dichloromethane) according to GPH; stirring at $-20^{\circ} \mathrm{C}$ for $96 \mathrm{~h}$. The crude product was obtained as a yellow powder, $98 \%$ yield (mixture of 160ah:188ah = 95:5).

HPLC: $50 \times 4.6 \mathrm{~mm}$ Agilent Eclipse Plus C18 $1.8 \mu \mathrm{m}$ column, $\mathrm{CH}_{3} \mathrm{CN} / \mathrm{H}_{2} \mathrm{O}=85 / 15(\mathrm{v} / \mathrm{v})$, $1.0 \mathrm{ml} \cdot \mathrm{min}^{-1}, 24.3 \mathrm{MPa}, 308 \mathrm{~K}, 250 \mathrm{~nm}$; helicene 160ah: $t_{R}=29.32 \mathrm{~min}$, isomers 188ah $(E / Z): t_{R}=30.81 \mathrm{~min}$; Enantiomeric excess: $+99 \%$. Conditions for $2 \mathrm{D}$ separation: $50 \times 4.6$ 
mm Agilent Eclipse Plus C18 $1.8 \mu \mathrm{m}$ column, $\mathrm{CH}_{3} \mathrm{CN} / \mathrm{H}_{2} \mathrm{O}=95 / 5(\mathrm{v} / \mathrm{v}), 1.0 \mathrm{ml} \cdot \mathrm{min}^{-1}, 12.6$ $\mathrm{MPa}, 308 \mathrm{~K}, 250 \mathrm{~nm}$, helicene 160ah: $t_{R}=5.02 \mathrm{~min}$; then $150 \times 4.6 \mathrm{~mm}$ Chiralpak IC-3 column, $3 \mu \mathrm{m}, \mathrm{CH}_{3} \mathrm{CN} / \mathrm{H}_{2} \mathrm{O}=90 / 10(\mathrm{v} / \mathrm{v}), 1.0 \mathrm{ml} \cdot \mathrm{min}^{-1}, 10.3 \mathrm{MPa}, 298 \mathrm{~K}, 250 \mathrm{~nm}$; major enantiomer: $t_{R}=6.92 \mathrm{~min}$, minor enantiomer: $t_{R}=8.08 \mathrm{~min}$.

$[\alpha]_{D}^{24.5}:+882.0\left(c=0.254, \mathrm{CH}_{2} \mathrm{Cl}_{2}\right) ;{ }^{1} \mathrm{H}$ NMR: $\left(300 \mathrm{MHz}, \mathrm{CDCl}_{3}\right) \delta=8.03(\mathrm{~d}, J=8.2 \mathrm{~Hz}, 2 \mathrm{H})$, $7.98(\mathrm{~d}, J=8.3 \mathrm{~Hz}, 2 \mathrm{H}), 7.92-7.87(\mathrm{~m}, 4 \mathrm{H}), 7.79(\mathrm{dd}, J=8.5,0.7 \mathrm{~Hz}, 2 \mathrm{H}), 7.65(\mathrm{dd}, J=8.8$, $2.5 \mathrm{~Hz}, 4 \mathrm{H}), 7.59-7.51(\mathrm{~m}, 4 \mathrm{H}), 7.50-7.36(\mathrm{~m}, 6 \mathrm{H}), 7.24-7.11(\mathrm{~m}, 6 \mathrm{H}), 6.73(\mathrm{ddd}, J=8.4$, 6.8, $1.3 \mathrm{~Hz}, 2 \mathrm{H}), 5.21(\mathrm{~s}, 4 \mathrm{H}) ;{ }^{13} \mathrm{C}\left\{{ }^{1} \mathrm{H}\right\}$ NMR: $\left(126 \mathrm{MHz}, \mathrm{CDCl}_{3}\right) \delta=158.4,139.3,137.1$, 133.3, 133.2, 131.3, 130.9, 130.8, 130.5, 128.7, 128.2, 128.1, 127.7, 127.5, 127.4, 127.2, 126.5, 125.9, 125.6, 124.5, 124.0, 114.9, 70.4; IR: (neat, $\mathrm{cm}^{-1}$ ) $\tilde{v}=750,833,879,958,1170$, 1246, 1287, 1384, 1454, 1509, 1607, 2859, 2914, 3027, 3057; HRMS: calcd $\mathrm{m} / \mathrm{z}$ for $\mathrm{C}_{52} \mathrm{H}_{36} \mathrm{NaO}_{2}{ }^{+}[\mathrm{M}+\mathrm{Na}]^{+}:$715.2608; found (ESI) 715.2590; UV/Vis: (THF): $\lambda_{\max }(\log \varepsilon)=233$ (4.94), 250 (4.86), 267 (4.79), 323 (4.52), 338 (4.58) nm.

\section{(P)-1,8-Bis[4-(trimethylsilyl)phenyl]hexahelicene (160ai):}

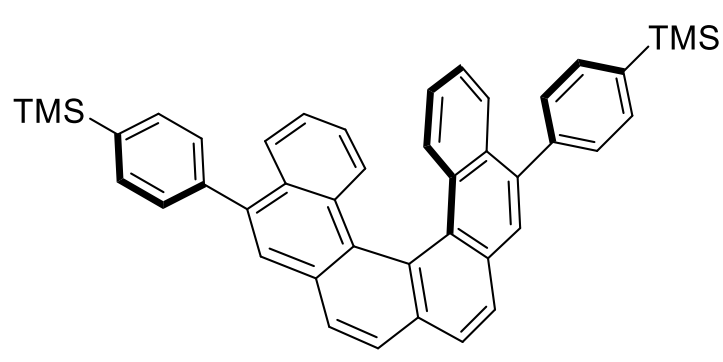

Prepared using 159ai (1.0 equiv, $15.4 \mathrm{mg}$, $24.6 \mu \mathrm{mol})$, precatalyst $172 \mathbf{i}(10 \mathrm{~mol} \%, 3.8 \mathrm{mg}$, $2.44 \mu \mathrm{mol})$, fluorobenzene $(0.5 \mathrm{ml})$ and $\mathrm{AgSbF}_{6}$ (10 mol\%, $2.45 \mu \mathrm{mol}, 49 \mu \mathrm{l}$ of a $0.05 \mathrm{M}$ solution in dichloromethane) according to GPH; stirring at $-20{ }^{\circ} \mathrm{C}$ for $96 \mathrm{~h}$. The crude product was obtained as a yellow powder in 76\% yield (mixture of 160ai:188ai:187ai = 84: 13:3).

In order to obtain a pure sample for characterization, the mixture was purified by semipreparative HPLC, obtaining $5.1 \mathrm{mg}(8.16 \mu \mathrm{mol}$, 33\% yield) of 160ai. Separation conditions: $250 \times 20 \mathrm{~mm}$ YMC-Pack PVA SIL, $5 \mu \mathrm{m}$, hexanes $/ \mathrm{MTBE}=97 / 3,18.9 \mathrm{ml} \cdot \mathrm{min}^{-1}, 4.2 \mathrm{MPa}$, 295 K, 246 nm; HPLC: $250 \times 4.6$ mm YMC-Pack PVA SIL, $5 \mu \mathrm{m}$, hexanes/MTBE = 94/6, 1.0 $\mathrm{ml} \cdot \mathrm{min}^{-1}$, $3.6 \mathrm{MPa}, 303 \mathrm{~K}, 246 \mathrm{~nm}$; helicene 160ai $t_{R}=6.28 \mathrm{~min}$, isomers 188ai $(E / Z): t_{R}=$ 8.17 and $4.48 \mathrm{~min}$, intermediate 187ai: $t_{R}=5.90$; Enantiomeric excess: $+87 \%$. The ee was determined by chiral HPLC: $150 \times 4.6 \mathrm{~mm}$ Chiralpak AS-3R column, $3 \mu \mathrm{m}, \mathrm{CH}_{3} \mathrm{CN} / \mathrm{H}_{2} \mathrm{O}=$ 90/10 ( $/ \mathrm{v}), 1.0 \mathrm{ml} \cdot \mathrm{min}^{-1}, 10.0 \mathrm{MPa}, 308 \mathrm{~K}, 246 \mathrm{~nm}$; major enantiomer: $t_{R}=4.37 \mathrm{~min}$, minor enantiomer: $t_{R}=5.19 \mathrm{~min}$.

$[\alpha]_{D}^{22}: 1125.0\left(c=0.23, \mathrm{CH}_{2} \mathrm{Cl}_{2}\right) ;{ }^{1} \mathrm{H}$ NMR: $\left(500 \mathrm{MHz}, \mathrm{CDCl}_{3}\right) \delta=8.04(\mathrm{~d}, J=8.2 \mathrm{~Hz}, 2 \mathrm{H})$, $7.99(\mathrm{~d}, J=8.2 \mathrm{~Hz}, 2 \mathrm{H}), 7.93-7.88(\mathrm{~m}, 4 \mathrm{H}), 7.81(\mathrm{dd}, J=8.5,0.6 \mathrm{~Hz}, 2 \mathrm{H}), 7.77-7.69(\mathrm{~m}$, $8 \mathrm{H}$ ), 7.17 (ddd, $J=8.2,6.8,1.3 \mathrm{~Hz}, 2 \mathrm{H}$ ), 6.74 (ddd, $J=8.4,6.8,1.3 \mathrm{~Hz}, 2 \mathrm{H}$ ), $0.39(\mathrm{~s}, 18 \mathrm{H}$ ) ppm; ${ }^{13} \mathbf{C}\left\{{ }^{1} \mathrm{H}\right\}$ NMR: $\left(126 \mathrm{MHz}, \mathrm{CDCl}_{3}\right) \delta=141.2,139.7,139.6,133.6,133.4,131.0,130.5$, 


\section{Experimental}

130.5, 129.6, 128.2, 127.7, 127.5, 127.4, 126.8, 126.0, 125.7, 124.7, 124.0, -0.8 ppm; IR: $\left(\right.$ neat, $\left.\mathrm{cm}^{-1}\right) \tilde{v}=610,696,722,746,769,825,1025,1095,1246,1310,1399,1594,2844$, 2917, 2951, 3010, 3046; HRMS: calcd $\mathrm{m} / \mathrm{z}$ for $\mathrm{C}_{44} \mathrm{H}_{40} \mathrm{NaSi}_{2}{ }^{+}[\mathrm{M}+\mathrm{Na}]^{+}: 647.2561$; found (ESI) 647.2538; UV/Vis: (THF): $\lambda_{\max }(\log \varepsilon)=227$ (5.14), 250 (5.00), 268 (4.92), 308 (4.47), 322 (4.63), 335 (4.68), 358 (4.52) nm.

\section{(P)-1,8-Bis(4-isopropylphenyl)hexahelicene (160aj):}

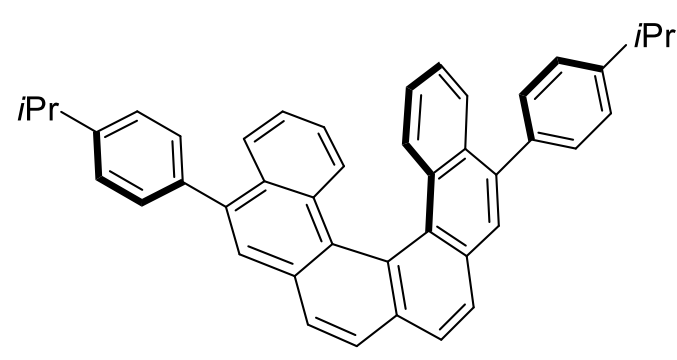

Prepared using 159aj (1.0 equiv, $13.9 \mathrm{mg}, 24.6$ $\mu \mathrm{mol})$, precatalyst $172 \mathrm{i}(10 \mathrm{~mol} \%, 3.8 \mathrm{mg}, 2.44$ $\mu \mathrm{mol})$, fluorobenzene $(0.5 \mathrm{ml})$ and $\mathrm{AgSbF}_{6}(10$ mol\%, $2.45 \mu \mathrm{mol}, 49 \mu \mathrm{l}$ of a $0.05 \mathrm{M}$ solution in dichloromethane) according to GPH; stirring at -20 ${ }^{\circ} \mathrm{C}$ for $96 \mathrm{~h}$. The crude product was obtained as a yellow powder in 99\% yield (mixture of 160aj:188aj:187aj = 87:8:5).

HPLC: $50 \times 4.6 \mathrm{~mm}$ Agilent Eclipse Plus C18 $1.8 \mu \mathrm{m}$ column, $\mathrm{CH}_{3} \mathrm{CN} / \mathrm{H}_{2} \mathrm{O}=95 / 5(v / v)$, $1.0 \mathrm{ml} \cdot \mathrm{min}^{-1}, 28.9 \mathrm{MPa}, 308 \mathrm{~K}, 190 \mathrm{~nm}$; helicene 160aj: $t_{R}=11.11 \mathrm{~min}$, isomers 188aj $(E / Z)$ : $t_{R}=11.41 \mathrm{~min}$ and $12.09 \mathrm{~min}$; intermediate 187aj: $t_{R}=8.88 \mathrm{~min}$; Enantiomeric excess: $+87 \%$. The ee was determined by chiral HPLC: $150 \times 4.6 \mathrm{~mm}$ Chiralpak IC-3 column, $3 \mu \mathrm{m}$, $\mathrm{CH}_{3} \mathrm{CN} / \mathrm{H}_{2} \mathrm{O}=80 / 20(\mathrm{v} / \mathrm{v}), 1.0 \mathrm{ml} \cdot \mathrm{min}^{-1}, 10.0 \mathrm{MPa}, 308 \mathrm{~K}, 246 \mathrm{~nm}$; major enantiomer: $t_{R}=$ $13.97 \mathrm{~min}$, minor enantiomer: $t_{R}=15.89 \mathrm{~min}$.

'H NMR: $\left(400 \mathrm{MHz}, \mathrm{CDCl}_{3}\right) \delta=8.03(\mathrm{~d}, J=8.2 \mathrm{~Hz}, 2 \mathrm{H}), 7.98(\mathrm{~d}, J=8.2 \mathrm{~Hz}, 2 \mathrm{H}), 7.95-7.89$ (m, 4H), 7.80 (dd, $J=8.6,1.1 \mathrm{~Hz}, 2 \mathrm{H}), 7.65$ (dd, $J=8.2,1.9 \mathrm{~Hz}, 4 \mathrm{H}), 7.45$ (dd, $J=8.1,1.8$ $\mathrm{Hz}, 4 \mathrm{H}$ ), 7.17 (ddd, $J=8.2,6.8,1.3 \mathrm{~Hz}, 2 \mathrm{H}$ ), 6.73 (ddd, $J=8.3,6.8,1.4 \mathrm{~Hz}, 2 \mathrm{H}$ ), 3.08 (p, $J=$ $6.9 \mathrm{~Hz}, 2 \mathrm{H}), 1.40(\mathrm{~d}, J=7.0 \mathrm{~Hz}, 12 \mathrm{H}) \mathrm{ppm} ;{ }^{13} \mathrm{C}\left\{{ }^{1} \mathrm{H}\right\}$ NMR: $\left(126 \mathrm{MHz}, \mathrm{CDCl}_{3}\right) \delta=148.2$, 139.7, 138.1, 133.3, 131.0, 130.7, 130.5, 130.2, 128.2, 127.6, 127.4, 127.3, 126.7, 126.6, 126.0, 125.6, 124.6, 124.0, 34.1, 24.3 ppm; IR: (neat, $\mathrm{cm}^{-1}$ ) $\tilde{\mathrm{v}}=536.1,591.1,612.3,748.2$, $771.4,810.9$, 835.0, 886.1, 1019.2, 1051.0, 110.2, 1364.4, 1410.7, 1457.0, 1509.0, 1604.5, 2359.5, 2861.8, 2923.6, 2955.4, 3020.0, 3037.3; HRMS: calcd $\mathrm{m} / \mathrm{z}$ for $\mathrm{C}_{44} \mathrm{H}_{36}{ }^{+}[\mathrm{M}]^{+}$: 564.2817 ; found (EI) 564.2838 .

(P)-1,8-Bis(4-chlorophenyl)hexahelicene (160ae):

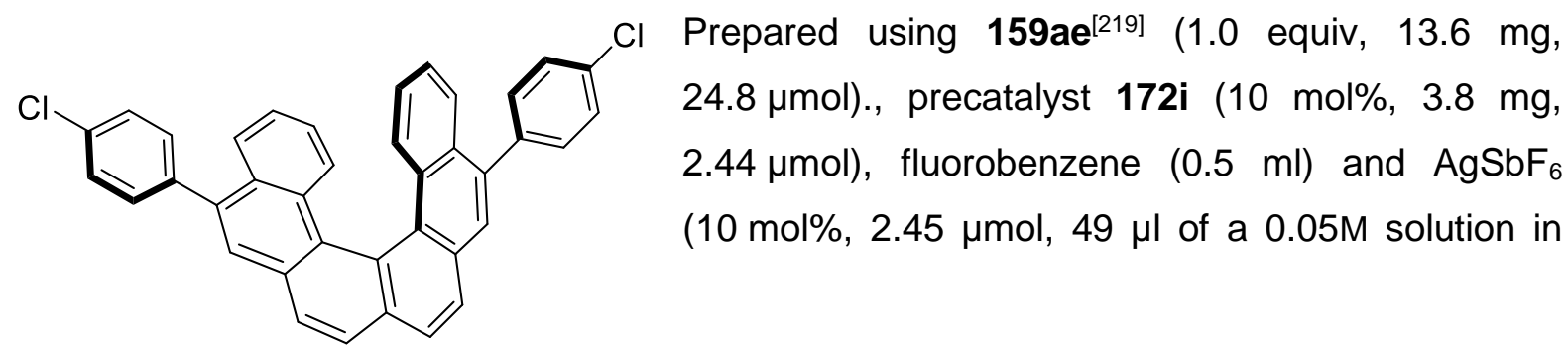




\section{Experimental}

dichloromethane) according to $\mathrm{GPH}$; stirring at $-20^{\circ} \mathrm{C}$ for $96 \mathrm{~h}$. The crude product was obtained as a yellow powder in 99\% yield (mixture of 160ae:188ae:187ae $=81: 3: 16 ; 30 \%$ conversion).

HPLC: $250 \times 4.6 \mathrm{~mm}$ YMC-Pack PVA SIL, $5 \mu \mathrm{m}$, hexanes/MTBE $=98 / 2,1.0 \mathrm{ml} \cdot \mathrm{min}^{-1}, 5.5$ $\mathrm{MPa}, 303 \mathrm{~K}, 195 \mathrm{~nm}$; helicene 160ae: $t_{R}=12.85 \mathrm{~min}$, isomers 188ae $t_{R}=17.27 \mathrm{~min}$, intermediate 187ae: $t_{R}=12.11 \mathrm{~min}$, starting material 159ae: $t_{R}=10.26 \mathrm{~min}$; Enantiomeric excess: $+97 \%$. $150 \times 4.6 \mathrm{~mm}$ Chiralpak IC-3 column, $3 \mu \mathrm{m}, \mathrm{CH}_{3} \mathrm{CN} / \mathrm{H}_{2} \mathrm{O}=90 / 10(v / v), 1.0$ $\mathrm{ml} \cdot \mathrm{min}^{-1}, 16.3 \mathrm{MPa}, 308 \mathrm{~K}, 193 \mathrm{~nm}$; major enantiomer: $t_{R}=5.68 \mathrm{~min}$, minor enantiomer: $t_{R}=$ $7.36 \mathrm{~min}$.

'H NMR: $\left(300 \mathrm{MHz}, \mathrm{CDCl}_{3}\right) \delta==8.06(\mathrm{~d}, J=8.2 \mathrm{~Hz}, 2 \mathrm{H}), 8.00(\mathrm{~d}, J=8.2 \mathrm{~Hz}, 2 \mathrm{H}), 7.89(\mathrm{~s}$, $2 \mathrm{H}), 7.85-7.74(\mathrm{~m}, 4 \mathrm{H}), 7.70-7.62(\mathrm{~m}, 4 \mathrm{H}), 7.61-7.53(\mathrm{~m}, 4 \mathrm{H}), 7.18$ (ddd, $J=8.3,6.9$, $1.3 \mathrm{~Hz}, 2 \mathrm{H}), 6.74$ (ddd, $J=8.4,6.9,1.4 \mathrm{~Hz}, 2 \mathrm{H}) \mathrm{ppm} ;{ }^{13} \mathrm{C}\left\{{ }^{1} \mathrm{H}\right\} \mathrm{NMR}:\left(126 \mathrm{MHz}, \mathrm{CDCl}_{3}\right) \delta==$ 138.97, 138.31, 133.50, 133.39, 131.44, 130.65, 130.33, 130.17, 128.62, 128.08, 127.66, 127.43, 127.23, 126.70, 125.72, 125.47, 124.65, 123.73, 77.24, 76.99, 76.74 ppm; IR: (neat, $\left.\mathrm{cm}^{-1}\right) \tilde{v}=580,594,609,717,747,772,831,887,1012,1085,1255,1361,1408,140,1600$, 3014, 3036, 3073; HRMS: calcd $m / z$ for $\mathrm{C}_{38} \mathrm{H}_{22} \mathrm{Cl}_{2}{ }^{+}[\mathrm{M}]^{+}:$548.1099; found (EI) 548.1099.

\section{(P)-1,8-Bis(3,4-dimethylphenyl)hexahelicene (160ak)}

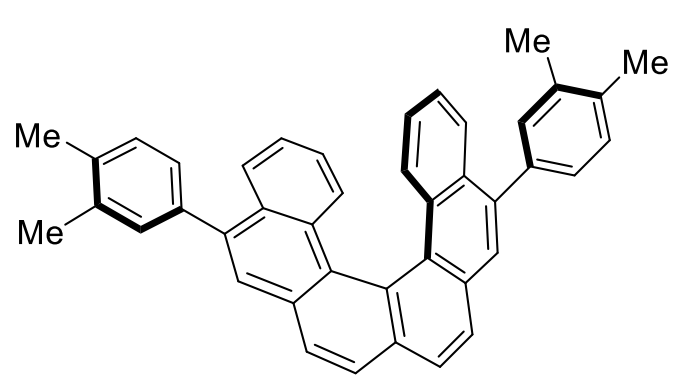

Prepared using 159ak (1.0 equiv, $13.3 \mathrm{mg}$, $24.8 \mu \mathrm{mol})$, precatalyst $172 \mathbf{i}(10 \mathrm{~mol} \%, 3.8 \mathrm{mg}$, $2.44 \mu \mathrm{mol})$, fluorobenzene $(0.5 \mathrm{ml})$ and $\mathrm{AgSbF}_{6}$ (10 mol\%, $2.45 \mu \mathrm{mol}, 49 \mu \mathrm{l}$ of a $0.05 \mathrm{M}$ solution in dichloromethane) according to GPH; stirring at -20 ${ }^{\circ} \mathrm{C}$ for $96 \mathrm{~h}$. The crude product was obtained as a yellow powder in 99\% yield (mixture of 160ak: 188ak, 95: 5).

HPLC: $50 \times 4.6 \mathrm{~mm}$ Agilent Eclipse Plus C18 $1.8 \mu \mathrm{m}$ column, $\mathrm{CH}_{3} \mathrm{CN} / \mathrm{H}_{2} \mathrm{O}=95 / 5(\mathrm{v} / \mathrm{v})$, $1.0 \mathrm{ml} \cdot \mathrm{min}^{-1}$, $19.8 \mathrm{MPa}, 308 \mathrm{~K}, 190 \mathrm{~nm}$; helicene 160ak: $t_{R}=7.89 \mathrm{~min}$, isomers 188ak $(E / Z)$ : $t_{R}=8.62 \mathrm{~min}$ and $9.08 \mathrm{~min}$; Enantiomeric excess: $+78 \%$. The ee was determined by chiral HPLC: $150 \times 4.6 \mathrm{~mm}$ Chiralpak IC-3 column, $3 \mu \mathrm{m}, \mathrm{CH}_{3} \mathrm{CN} / \mathrm{H}_{2} \mathrm{O}=80 / 20(\mathrm{v} / \mathrm{v}), 1.0 \mathrm{ml} \cdot \mathrm{min}^{-1}$, $15.9 \mathrm{MPa}, 308 \mathrm{~K}, 246 \mathrm{~nm}$; major enantiomer: $t_{R}=12.75 \mathrm{~min}$, minor enantiomer: $t_{R}=16.37$ $\min$.

'H NMR: $\left(500 \mathrm{MHz}, \mathrm{CDCl}_{3}\right) \delta=8.06-7.96(\mathrm{~m}, 4 \mathrm{H}), 7.93-7.88(\mathrm{~m}, 4 \mathrm{H}), 7.81(\mathrm{dd}, J=8.6$, $1.1 \mathrm{~Hz}, 2 \mathrm{H}), 7.51(\mathrm{~d}, J=1.8 \mathrm{~Hz}, 2 \mathrm{H}), 7.48-7.44(\mathrm{~m}, 2 \mathrm{H}), 7.36(\mathrm{~d}, J=7.6 \mathrm{~Hz}, 2 \mathrm{H}), 7.17$ (ddd, $J=8.2,6.8,1.3 \mathrm{~Hz}, 2 \mathrm{H}$ ), 6.75 (ddd, $J=8.4,6.8,1.4 \mathrm{~Hz}, 2 \mathrm{H}), 2.44(\mathrm{~s}, 6 \mathrm{H}), 2.43$ (s, 6H) ppm; 
${ }^{13} \mathrm{C}\left\{{ }^{1} \mathrm{H}\right\}$ NMR: $\left(126 \mathrm{MHz}, \mathrm{CDCl}_{3}\right) \delta=139.8,138.3,136.8,135.9,133.3,131.5,131.0,130.8$, 130.5, 129.8, 128.2, 127.8, 127.6, 127.4, 127.3, 126.6, 126.1, 125.6, 124.6, 124.0, 20.1, 19.8 ppm; IR: $\left(\right.$ neat, $\left.\mathrm{cm}^{-1}\right) \tilde{v}=587.2,609.4,622.9,730.9,747.3,765.6,812.8,881.3,906.4$, 1020.2, 1069.3, 1123.3, 1255.4, 1307.5, 1364.4, 1446.4, 1494.6, 1601.6, 2851.2, 2916.8, 3043.1; HRMS: calcd $\mathrm{m} / \mathrm{z}$ for $\mathrm{C}_{42} \mathrm{H}_{32}{ }^{+}\left[\mathrm{M}^{+}:\right.$536.2504; found (EI) 536.2507.

\section{(P)-1,8-Bis[4-\{[(triisopropylsilyl)oxy]methyl\}phenyl]hexahelicene (160al):}

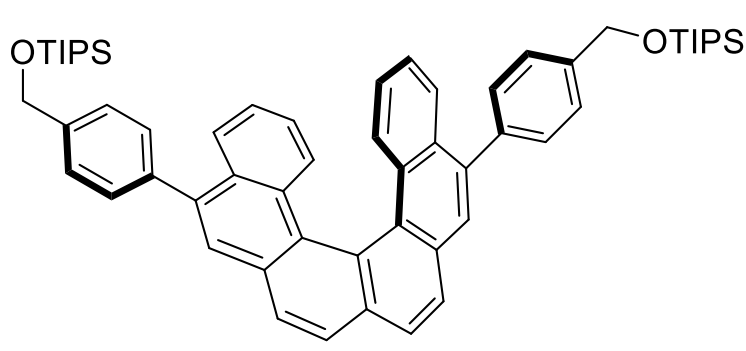

Prepared using 160al (1.0 equiv, $21.1 \mathrm{mg}$, $24.7 \mu \mathrm{mol})$, precatalyst $172 \mathbf{i}(10 \mathrm{~mol} \%, 3.8 \mathrm{mg}$, $2.44 \mu \mathrm{mol})$, fluorobenzene $(0.5 \mathrm{ml})$ and $\mathrm{AgSbF}_{6}(10.7 \mathrm{~mol} \%, 2.65 \mu \mathrm{mol}, 53 \mu \mathrm{l}$ of a $0.05 \mathrm{M}$ solution in dichloromethane) according to GPH. The crude product was obtained as a yellow solid in $98 \%$ yield (mixture of 160al:188al:187al $=87: 13: 0$ ).

HPLC: the selectivity was determined by HPLC: $250 \times 4.6 \mathrm{~mm}$ YMC-Pack PVA SIL, $5 \mu \mathrm{m}$, hexanes/MTBE $=94 / 6(\mathrm{v} / \mathrm{v}), 1.0 \mathrm{ml} \cdot \mathrm{min}^{-1}, 3.9 \mathrm{MPa}, 303 \mathrm{~K}, 250 \mathrm{~nm}$; helicene 160al: $t_{R}=6.85$ min, isomers 188al: $t_{R}=6.50,8.25 \mathrm{~min}$. Enantiomeric excess: $+86 \%$. Conditions for $2 \mathrm{D}$ chiral separation: Agilent $50 \times 4.6 \mathrm{~mm}$ Zorbax Eclipse Plus C8, $1.8 \mu \mathrm{m}$, pure $\mathrm{MeOH}, 1.0$ $\mathrm{ml} \cdot \mathrm{min}^{-1}, 19.5 \mathrm{MPa}, 308 \mathrm{~K}, 250 \mathrm{~nm}$; helicene 160al: $t_{R}=3.55 \mathrm{~min}$; then $150 \times 4.6 \mathrm{~mm}$ Chiralpak AS-3R column, $3 \mu \mathrm{m}, \mathrm{CH}_{3} \mathrm{CN} / \mathrm{H}_{2} \mathrm{O}=90 / 10(\mathrm{v} / \mathrm{v}), 1.0 \mathrm{ml} \cdot \mathrm{min}^{-1}, 10.6 \mathrm{MPa}, 298 \mathrm{~K}$, $254 \mathrm{~nm}$; major enantiomer: $t_{R}=10.18 \mathrm{~min}$, minor enantiomer: $t_{R}=11.90 \mathrm{~min}$

'H NMR: $\left(300 \mathrm{MHz}, \mathrm{CDCl}_{3}\right) \delta==8.04(\mathrm{~d}, J=8.2 \mathrm{~Hz}, 2 \mathrm{H}), 7.99(\mathrm{~d}, J=8.3 \mathrm{~Hz}, 2 \mathrm{H}), 7.94-$ $7.88(\mathrm{~m}, 4 \mathrm{H}), 7.83-7.78(\mathrm{~m}, 2 \mathrm{H}), 7.71(\mathrm{dd}, J=8.1,1.8 \mathrm{~Hz}, 4 \mathrm{H}), 7.63-7.56(\mathrm{~m}, 4 \mathrm{H}), 7.17$ (ddd, $J=8.2,6.9,1.3 \mathrm{~Hz}, 2 \mathrm{H}$ ), 6.75 (ddd, $J=8.4,6.8,1.4 \mathrm{~Hz}, 2 \mathrm{H}$ ), $5.02(\mathrm{~s}, 4 \mathrm{H}), 1.30-1.15$ $(\mathrm{m}, 42 \mathrm{H}) \mathrm{ppm} ;{ }^{13} \mathrm{C}\left\{{ }^{1} \mathrm{H}\right\}$ NMR: $\left(126 \mathrm{MHz}, \mathrm{CDCl}_{3}\right) \delta==141.0,139.6,139.2,133.3,130.9$, $130.7,130.5,130.1,128.1,127.6,127.3,127.2,126.6,125.9,125.9,125.6,124.6,124.0$, 65.2, 18.4, 12.4 ppm; IR: (neat, $\mathrm{cm}^{-1}$ ) $\tilde{\mathrm{v}}=610,657,681,747,768,798,881,999,1012$, 1066, 1092, 1119, 1258, 1368, 1464, 2857, 2891, 2937, 3046; HRMS: calcd $\mathrm{m} / \mathrm{z}$ for $\mathrm{C}_{58} \mathrm{H}_{72} \mathrm{NO}_{2} \mathrm{Si}_{2}{ }^{+}\left[\mathrm{M}+\mathrm{NH}_{4}\right]^{+}:$870.5096; found (ESI) 870.5073 .

(5E,10E)-5,10-Bis(bromomethylene)-5,10-dihydrofluoreno[3,4-c]fluorene (160an):

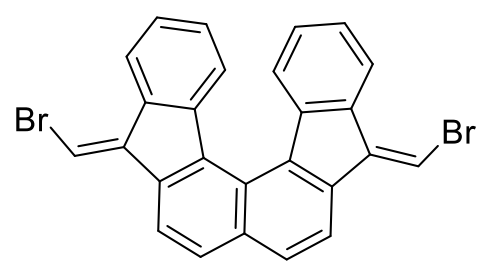

Prepared using 159an (1.0 equiv, $12.2 \mathrm{mg}, 25.1 \mu \mathrm{mol}$ ), precatalyst $172 \mathrm{i}(9.7 \mathrm{~mol} \%, 3.8 \mathrm{mg}, 2.44 \mu \mathrm{mol})$, fluorobenzene $(0.5 \mathrm{ml})$ and $\mathrm{AgSbF}_{6}(10 \mathrm{~mol} \%, 2.50 \mu \mathrm{mol}, 50 \mu \mathrm{l}$ of a $0.05 \mathrm{M}$ solution in dichloromethane) according to GPH. The crude 


\section{Experimental}

product was obtained as a yellow solid in $62 \%$ NMR yield.

Analytical data corresponded to those reported above for the synthesis of 160an using precatalyst $98 f$.

(+)-5,12-Di-p-tolylchryseno[3,4-c]chrysene (160db):

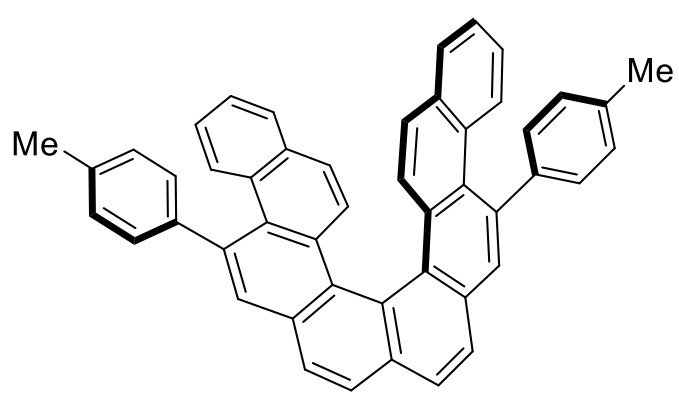

Prepared using 159db (1.0 equiv, $15.0 \mathrm{mg}$, $24.6 \mu \mathrm{mol})$, precatalyst $172 \mathbf{i}(10 \mathrm{~mol} \%, 3.8 \mathrm{mg}$, $2.44 \mu \mathrm{mol})$, fluorobenzene $(0.5 \mathrm{ml})$ and $\mathrm{AgSbF}_{6}$ (10 mol\%, $2.45 \mu \mathrm{mol}, 49 \mu \mathrm{l}$ of a $0.05 \mathrm{M}$ solution in dichloromethane) according to GPH; stirring at -20 ${ }^{\circ} \mathrm{C}$ for $96 \mathrm{~h}$. The crude mixture was obtained as a yellow powder in $99 \%$ combined yield and contained $68 \%$ of the desired product, as well as other unidentified side products.

In order to obtain a pure sample for characterization, the mixture was purified by semipreparative HPLC. Separation conditions: $250 \times 20 \mathrm{~mm}$ YMC-Pack PVA SIL, $5 \mu \mathrm{m}$, hexanes/MTBE = 99/1 ( $v / \mathrm{v}), 18.9 \mathrm{ml} \cdot \mathrm{min}^{-1}, 4.4 \mathrm{MPa}, 295 \mathrm{~K}, 280 \mathrm{~nm}$, affording $5.2 \mathrm{mg}$, (8.54 $\mu \mathrm{mol}, 35 \%$ yield) of $160 \mathrm{db}$; Enantiomeric excess: $+46 \%$. The ee was determined by chiral HPLC: $150 \times 4.6 \mathrm{~mm}$ Chiralpak IC-3 column, $3 \mu \mathrm{m}, \mathrm{CH}_{3} \mathrm{CN} / \mathrm{H}_{2} \mathrm{O}=80 / 20(\mathrm{v} / \mathrm{v}), 1.0 \mathrm{ml} \cdot \mathrm{min}^{-1}$, $15.5 \mathrm{MPa}, 308 \mathrm{~K}, 333 \mathrm{~nm}$; major enantiomer: $t_{R}=17.89 \mathrm{~min}$, minor enantiomer: $t_{R}=19.50$ $\min$.

$[\alpha]_{22}^{D}:+365.5\left(c=0.215, \mathrm{CH}_{2} \mathrm{Cl}_{2}\right) ;{ }^{1} \mathrm{H}$ NMR: $\left(300 \mathrm{MHz}, \mathrm{CDCl}_{3}\right) \delta=8.07(\mathrm{~d}, J=8.3 \mathrm{~Hz}, 2 \mathrm{H})$, $8.03(\mathrm{~d}, J=8.3 \mathrm{~Hz}, 2 \mathrm{H}), 7.98(\mathrm{~s}, 2 \mathrm{H}), 7.92(\mathrm{~d}, J=8.9 \mathrm{~Hz}, 2 \mathrm{H}), 7.68(\mathrm{~d}, J=5.9 \mathrm{~Hz}, 2 \mathrm{H}), 7.56$ (d, $J=9.1 \mathrm{~Hz}, 2 \mathrm{H}$ ), 7.43 (d, $J=7.4 \mathrm{~Hz}, 2 \mathrm{H}$ ), $7.36-7.22$ (m, 8H), 7.09 (ddd, $J=8.4,6.6,1.7$ $\mathrm{Hz}, 2 \mathrm{H}), 6.69(\mathrm{~d}, J=9.1 \mathrm{~Hz}, 2 \mathrm{H}), 2.53(\mathrm{~s}, 6 \mathrm{H}) \mathrm{ppm} ;{ }^{13} \mathrm{C}\{\mathrm{H}\}$ NMR: $\left(126 \mathrm{MHz}, \mathrm{CDCl}_{3}\right) \delta=$ 142.3, 139.0, 136.7, 133.4, 132.6, 130.6, 130.5, 130.1, 129.7, 129.7, 129.6, 129.2, 128.8, 128.5, 128.0, 127.6, 127.5, 127.1, 126.9, 125.9, 125.3, 125.0, 124.2, 123.9, 21.4; IR: (neat, $\left.\mathrm{cm}^{-1}\right) \tilde{V}=585.3,713,743,795,826,888,1017,1039,1258,1444,1512,1910,2854,2919$, 2950, 309, 3042; HRMS: calcd $m / z$ for $\mathrm{C}_{48} \mathrm{H}_{32}{ }^{+}$[M] $]^{+}: 608.2504$; found (El) 608.2497; UV/Vis: (THF): $\lambda_{\max }(\log \varepsilon)=257$ (4.46), 280 (4.65), 304 (4.57), 319 (4.54), 334 (4.50), 349 (4.45), $369(4.26) \mathrm{nm}$. 


\section{Experimental}

\section{(P)-10,15-Dimethoxy-1,8-di-p-tolylhexahelicene (160bb):}

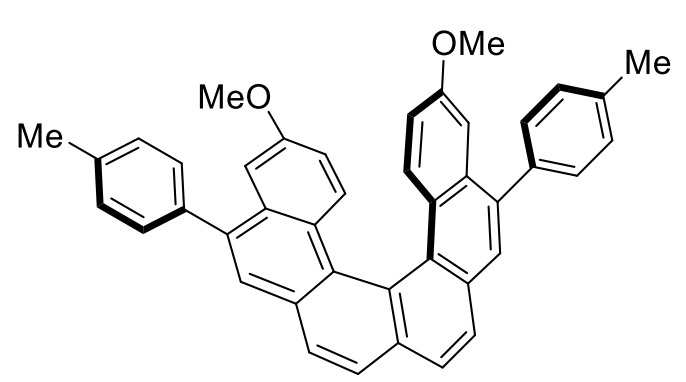

Prepared using 159bb (1.0 equiv, $12.9 \mathrm{mg}$, $22.7 \mu \mathrm{mol})$, precatalyst $172 \mathrm{y}(9.8 \mathrm{~mol} \%, 3.6 \mathrm{mg}$, $2.23 \mu \mathrm{mol})$, fluorobenzene $(0.46 \mathrm{ml})$ and $\mathrm{AgSbF}_{6}$ $(10.1 \mathrm{~mol} \%, 2.30 \mu \mathrm{mol}, 46 \mu \mathrm{l}$ of a $0.05 \mathrm{M}$ solution in dichloromethane) according to GPH. Purification by column chromatography $(50 \%$ toluene in petrol ether) afforded the product mixture $(10.8 \mathrm{mg}, 83 \%$ yield) in proportion $160 \mathrm{bb}: 188 \mathrm{bb}: 187 \mathrm{bb}=$ 96: 4: 0 as a yellow solid.

HPLC: The selectivity was determined by HPLC: Agilent $50 \times 4.6 \mathrm{~mm}$ Zorbax Eclipse Plus C18, $1.8 \mu \mathrm{m}, \mathrm{MeCN} / \mathrm{H}_{2} \mathrm{O}=90 / 10(\mathrm{v} / \mathrm{v}), 1.0 \mathrm{ml} \cdot \mathrm{min}^{-1}, 20.3 \mathrm{MPa}, 308 \mathrm{~K}, 254 \mathrm{~nm}$; helicene 160bb: $t_{R}=9.55 \mathrm{~min}$, isomers 188bb: $t_{R}=10.21$ and $18.54 \mathrm{~min}$. Enantiomeric excess: $+78 \%$. The ee was determined by chiral HPLC: $150 \times 4.6 \mathrm{~mm}$ Chiralpak IC-3 column, $3 \mu \mathrm{m}$, $\mathrm{MeCN} / \mathrm{H}_{2} \mathrm{O}=80 / 20(\mathrm{v} / \mathrm{v}), 1.0 \mathrm{ml} \cdot \mathrm{min}^{-1}, 15.4 \mathrm{MPa}, 308 \mathrm{~K}, 254 \mathrm{~nm}$; major enantiomer: $t_{R}=$ $11.48 \mathrm{~min}$, minor enantiomer: $t_{R}=14.46 \mathrm{~min}$.

$[\alpha]_{24}^{D}:(86 \%$ ee $)+1367\left(c=0.525, \mathrm{CH}_{2} \mathrm{Cl}_{2}\right) ; \mathrm{UV} /$ Vis: $(\mathrm{THF}): \lambda_{\max }(\log \varepsilon)=266(4.79), 279$ (4.64), 337 (4.59), 365 (4.30). Fluorescence: (THF $\left.\Lambda_{\mathrm{ex}} 337\right): \Lambda_{\max }\left(\mathrm{I}_{\text {rel }}\right) 434$ (0.99), 457 (0.66).

Analytical data corresponded to that previously reported. ${ }^{[219]}$

'H-NMR: $\left(400 \mathrm{MHz}, \mathrm{CDCl}_{3}\right.$ ) $\delta=7.94$ (AA'BB', 4H), 7.87 (s, 2H), 7.75 (d, J=9.3 Hz, 2H), 7.62 (d, J=8.0 Hz, 4H), 7.39 (d, J=7.8 Hz, 4H), 7.28 (d, J=2.7 Hz, 2H), 6.44 (dd, J= 9.3, $2.7 \mathrm{~Hz}$, 2H), 3.69 (s, 6H), 2.52 (s, 6H) ppm; ${ }^{13} \mathrm{C}\{\mathrm{H}\}-N M R:\left(101 \mathrm{MHz}, \mathrm{CDCl}_{3}\right) \delta=157.3,139.0,138.0$, 137.2 , 133.5, 132.2, 130.0, 129.9, 129.9, 129.3, 127.9, 127.2, 127.2, 126.4, 125.4, 123.5, 115.1, 106.23, 55.2, 21.5 ppm, IR: (neat, $\mathrm{cm}^{-1}$ ) $\widetilde{v}:$ 3035, 3021, 2996, 2967, 2943, 2923, 2873, 2859, 2838, 1558, 1498, 1469, 1449, 1428, 1404, 1374, 1327, 1288, 1262, 1230, 1199, 1180, 1150, 1090, 1078, 1032, 959, 898, 887, 870, 850, 824, 796, 756, 736, 721, 694, $671,612,584,550,521,503$, HRMS: calculated $\mathrm{m} / \mathrm{z}$ for $\mathrm{C}_{42} \mathrm{H}_{32} \mathrm{O}_{2} \mathrm{Na}^{+}$: 591.229449; found (ESI) 591.229610.

(P)-1,8-Bis(4-chlorophenyl)-10,15-dimethoxyhexahelicene (160be):

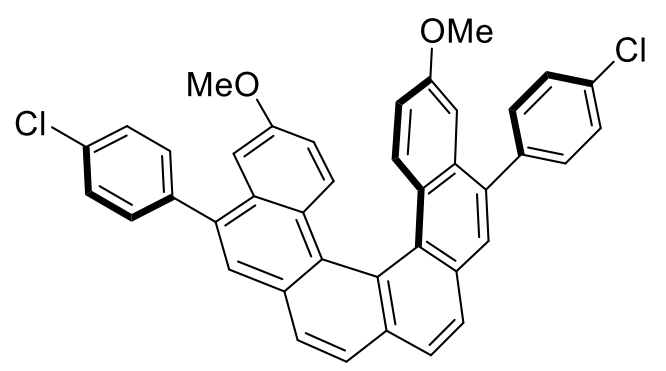

Prepared using 159be (1.0 equiv, $15.2 \mathrm{mg}, 24.9$ $\mu \mathrm{mol}$ ), precatalyst 172y (10 mol\%, $4.0 \mathrm{mg}, 2.48$ $\mu \mathrm{mol})$, fluorobenzene $(0.5 \mathrm{ml})$ and $\mathrm{AgSbF}_{6}(10$ mol\%, $2.50 \mu \mathrm{mol}, 50 \mu \mathrm{l}$ of a $0.05 \mathrm{M}$ solution in dichloromethane) according to GPH. Purification by 
column chromatography (50\% toluene in petrol ether) afforded the product mixture $(14.1 \mathrm{mg}$, $23.1 \mu \mathrm{mol}, 93 \%$ yield) in proportion $160 \mathrm{be}: 188 \mathrm{be}=95: 5$ as a yellow solid.

HPLC: 250 x $4.6 \mathrm{~mm}$ YMC-Pack PVA SIL, $5 \mu \mathrm{m}$, hexanes/MTBE = 92/8 $(\mathrm{v} / \mathrm{v}), 1.0 \mathrm{ml} \cdot \mathrm{min}^{-1}$, $5.4 \mathrm{MPa}, 303 \mathrm{~K}, 250 \mathrm{~nm}$; helicene 160be: $t_{R}=11.83 \mathrm{~min}$, isomers 188be: $t_{R}=16.23 \mathrm{~min}$; Enantiomeric excess: $+85 \%$. The ee was determined by chiral HPLC: $150 \times 4.6 \mathrm{~mm}$ Chiralpak IC-3 column, $3 \mu \mathrm{m}, \mathrm{MeCN} / \mathrm{H}_{2} \mathrm{O}=75 / 25(\mathrm{v} / \mathrm{v}), 1.0 \mathrm{ml} \cdot \mathrm{min}^{-1}, 18.4 \mathrm{MPa}, 308 \mathrm{~K}, 254$ $\mathrm{nm}$; major enantiomer: $t_{R}=28.94 \mathrm{~min}$, minor enantiomer $t_{R}=46.10 \mathrm{~min}$.

$[\alpha]_{24}^{D}:(+85 \%$ ee $)+1257\left(c=0.760, \mathrm{CH}_{2} \mathrm{Cl}_{2}\right)$

Analytical data corresponded to those previously reported. ${ }^{[272]}$

'H-NMR: $\left(500 \mathrm{MHz}, \mathrm{CDCl}_{3}\right): \delta=7.96(\mathrm{~d}, J=8.2 \mathrm{~Hz}, 2 \mathrm{H}), 7.94(\mathrm{~d}, J=8.2 \mathrm{~Hz}, 2 \mathrm{H}), 7.85(\mathrm{~s}$, 2H), $7.73(\mathrm{~d}, J=9.3 \mathrm{~Hz}, 2 \mathrm{H}), 7.66(\mathrm{dd}, J=8.4,2.2 \mathrm{~Hz}, 4 \mathrm{H}), 7.56$ (dd, $J=8.4,2.2 \mathrm{~Hz}, 4 \mathrm{H}$ ), $7.17(\mathrm{~d}, J=2.7 \mathrm{~Hz}, 2 \mathrm{H}), 6.44(\mathrm{dd}, J=9.3,2.7 \mathrm{~Hz}, 2 \mathrm{H}), 3.69(\mathrm{~s}, 6 \mathrm{H}) \mathrm{ppm} ;{ }^{13} \mathrm{C}\{\mathrm{H}\}-N M R:(126$ $\left.\mathrm{MHz}, \mathrm{CDCl}_{3}\right) \delta: 157.4,139.4,137.7,133.8,133.6,131.9,131.5,130.0,129.7,128.9,128.2$, 127.5, 127.4, 126.6, 125.3, 123.3, 115.4, 105.8, 55.3 ppm; IR: (neat, $\mathrm{cm}^{-1}$ ) v: 2923, 2855, 2358, 1613, 1504, 1488, 1458, 1429, 1398, 1373, 1329, 1261, 1230, 1193, 1132, 1090, 1036, 1013, 956, 891, 851, 826, 797, 749, 736, 720, 688, 632, 611, 595, 582, 528; HRMS: calculated $\mathrm{m} / \mathrm{z}$ for $\mathrm{C}_{40} \mathrm{H}_{27} \mathrm{O}_{2} \mathrm{Cl}_{2}{ }^{+}\left[\mathrm{M}_{+} \mathrm{H}^{+}\right.$]: 609.1382; found (ESI) 609.1346; UV/Vis: (THF): $\lambda_{\max }$ $(\log \varepsilon)=265$ (4.85), 282 (4.61), 336 (4.52), 366 (4.27) nm.

(P)-10,15-Diphenyl-1,8-di-p-tolylhexahelicene (160cb):

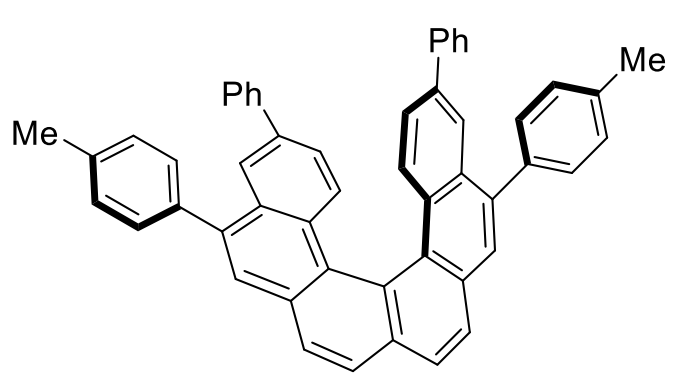

Prepared using $159 \mathrm{cb}$ (1.0 equiv, $11.6 \mathrm{mg}$, $17.6 \mu \mathrm{mol})$, precatalyst $172 \mathrm{y}(10 \mathrm{~mol} \%, 2.8 \mathrm{mg}$, $1.74 \mu \mathrm{mol})$, fluorobenzene $(0.35 \mathrm{ml})$ and $\mathrm{AgSbF}_{6}$ (9.9 mol\%, $1.76 \mu \mathrm{mol}, 35 \mu \mathrm{l}$ of a $0.05 \mathrm{M}$ solution in dichloromethane) according to GPH. Purification by column chromatography $(50 \%$ toluene in petrol ether) afforded the product mixture $(11.5 \mathrm{mg}, 17.4 \mu \mathrm{mol}$, 99\% yield) in proportion $160 \mathrm{cb}: 188 \mathrm{cb}=98: 3$ as a yellow solid.

HPLC: Agilent $50 \times 4.6 \mathrm{~mm}$ Zorbax Eclipse Plus C18, $1.8 \mu \mathrm{m}$, pure MeCN, $1.0 \cdot \mathrm{ml} \cdot \mathrm{min}^{-1}$, $14.9 \mathrm{MPa}, 308 \mathrm{~K}, 266 \mathrm{~nm}$; helicene $160 \mathrm{cb}: t_{R}=6.13 \mathrm{~min}$, isomer $188 \mathrm{cb}: t_{R}=6.86 \mathrm{~min}$; Enantiomeric excess: $+75 \%$. The ee was determined by chiral HPLC: $150 \times 4.6 \mathrm{~mm}$ Chiralpak IC-3 column, $3 \mu \mathrm{m}, \mathrm{MeCN} / \mathrm{H}_{2} \mathrm{O}=90 / 10(v / v), 1.0 \mathrm{ml} \cdot \mathrm{min}^{-1}, 12.3 \mathrm{MPa}, 308 \mathrm{~K}, 266$ $\mathrm{nm}$; major enantiomer: $t_{R}=8.32 \mathrm{~min}$, minor enantiomer: $t_{R}=12.03 \mathrm{~min}$. 


\section{Experimental}

$[\alpha]_{21}^{D}=+1304\left(c=0.495, \mathrm{CH}_{2} \mathrm{Cl}_{2}\right)$

Analytical data corresponded to those previously reported. ${ }^{[219]}$

${ }^{1} \mathrm{H}-\mathrm{NMR}:\left(400 \mathrm{MHz}, \mathrm{CDCl}_{3}\right) \delta=8.15(\mathrm{~d}, J=2.0 \mathrm{~Hz}, 2 \mathrm{H}), 8.06(\mathrm{~d}, J=8.2 \mathrm{~Hz}, 2 \mathrm{H}), 8.01(\mathrm{~d}, J$ $=8.2 \mathrm{~Hz}, 2 \mathrm{H}), 7.94(\mathrm{~s}, 2 \mathrm{H}), 7.90(\mathrm{~d}, J=8.8 \mathrm{~Hz}, 2 \mathrm{H}), 7.67(\mathrm{~d}, J=8.0 \mathrm{~Hz}, 4 \mathrm{H}), 7.42-7.39(\mathrm{~m}$, $8 \mathrm{H}), 7.33-7.28(\mathrm{~m}, 4 \mathrm{H}), 7.27-7.23(\mathrm{~m}, 2 \mathrm{H}), 6.99(\mathrm{dd}, J=8.8,2.0 \mathrm{~Hz}, 2 \mathrm{H}), 2.53(\mathrm{~s}, 6 \mathrm{H}) \mathrm{ppm}$; ${ }^{13} \mathrm{C}\{\mathrm{H}\}-N M R:\left(101 \mathrm{MHz}, \mathrm{CDCl}_{3}\right) \delta=143.4,141.1,139.9,138.1,137.7,137.4,133.4,131.1$, $131.0,130.2$, 129.8, 129.4, 128.8, 128.7, 127.5, 127.5, 127.4, 127.3, 127.2, 124.1, 124.0, 123.8, 21.5 ppm; IR: (neat, $\mathrm{cm}^{-1}$ ) $\widetilde{v}: 3021,2964,2944,2915,2874,2859,1598,1579,1511$, 1486, 1446, 1429, 1403, 1378, 1360, 1305, 1278, 1261, 1209, 1181, 1153, 1107, 1073, 1021, 963, 890, 823, 758, 735, 721, 693, 641, 612, 578, 566, 509; HRMS: calculated for $\mathrm{C}_{52} \mathrm{H}_{36}{ }^{+}: 660.281700$; found (EI) 660.281253.

\section{(P)-10,15-Dichloro-1,8-di-p-tolylhexahelicene (160ob):}

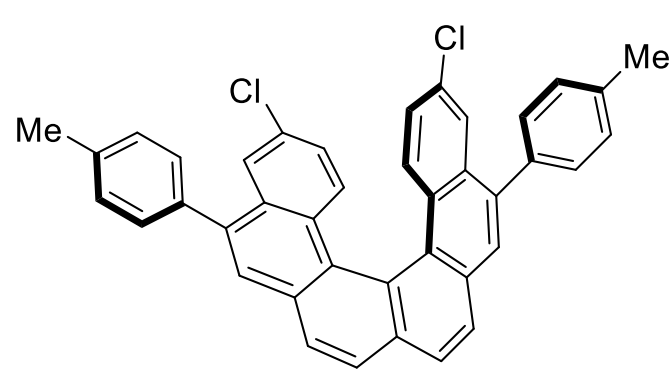

Prepared using 159ob (1.0 equiv, $14.4 \mathrm{mg}$, $24.9 \mu \mathrm{mol})$, precatalyst $172 \mathrm{y}(10 \mathrm{~mol} \%, 4.0 \mathrm{mg}$, $2.48 \mu \mathrm{mol})$, fluorobenzene $(0.5 \mathrm{ml})$ and $\mathrm{AgSbF}_{6}$ (10 mol\%, $2.50 \mu \mathrm{mol}, 50 \mu \mathrm{l}$ of a $0.05 \mathrm{M}$ solution in dichloromethane) according to GPH. The crude mixture was obtained as a yellow powder in $76 \%$ NMR yield (using 1,4-dioxane as an internal standard), conversion 94\%, proportion 160ob:188ob:187ob = 86:7: 7 .

In order to obtain a pure sample for characterization, the mixture was purified by semipreparative HPLC, obtaining $7.8 \mathrm{mg}$ of $160 \mathrm{ob}$ (13.5 $\mu \mathrm{mol}, 54 \%$ yield). Separation conditions: $250 \times 20 \mathrm{~mm}$ YMC-Pack PVA SIL, $5 \mu \mathrm{m}$, hexanes/MTBE $=99 / 1(v / v), 18.9 \mathrm{ml} \cdot \mathrm{min}^{-1}, 4.5$ MPa, $295 \mathrm{~K}, 210 \mathrm{~nm}$; HPLC: The ratio of isomers was determined by HPLC: Agilent $50 \times 4.6$ $\mathrm{mm}$ Zorbax Eclipse Plus C18, $1.8 \mu \mathrm{m}, \mathrm{MeCN}=100,1.0 \mathrm{ml} \cdot \mathrm{min}^{-1}, 17.5 \mathrm{MPa}, 308 \mathrm{~K}, 260 \mathrm{~nm}$; helicene $160 \mathrm{ob}: t_{R}=5.12 \mathrm{~min}$, isomers $188 \mathrm{ob}: t_{R}=5.59$ and $5.98 \mathrm{~min}$, intermediate $187 \mathrm{ob}$ : $t_{R}=3.26 \mathrm{~min}$, starting material 159ob: $t_{R}=2.54 \mathrm{~min}$. Enantiomeric excess: $+92 \%$. The ee was determined by chiral HPLC: $150 \times 4.6 \mathrm{~mm}$ Chiralpak IC-3 column, $3 \mu \mathrm{m}$, pure $\mathrm{CH}_{3} \mathrm{CN}$, $1.0 \mathrm{ml} \cdot \mathrm{min}^{-1}, 10.4 \mathrm{MPa}, 308 \mathrm{~K}, 210 \mathrm{~nm}$; major enantiomer: $t_{R}=3.67 \mathrm{~min}$, minor enantiomer: $t_{R}$ $=4.46 \mathrm{~min}$.

$[\alpha]_{23}^{D}:(92 \%$ ee $)+1684\left(c=0.390, \mathrm{CH}_{2} \mathrm{Cl}_{2}\right) .{ }^{1} \mathrm{H}$ NMR: $\left(400 \mathrm{MHz}, \mathrm{CDCl}_{3}\right) \delta=8.03(\mathrm{~d}, J=8.2$ $\mathrm{Hz}, 2 \mathrm{H}), 7.98(\mathrm{~d}, J=8.2 \mathrm{~Hz}, 2 \mathrm{H}), 7.92(\mathrm{~s}, 2 \mathrm{H}), 7.86(\mathrm{~d}, J=2.3 \mathrm{~Hz}, 2 \mathrm{H}), 7.70(\mathrm{~d}, J=9.1 \mathrm{~Hz}$, 2H), $7.58(\mathrm{dd}, J=8.1,1.9 \mathrm{~Hz}, 4 \mathrm{H}), 7.47-7.38(\mathrm{~m}, 4 \mathrm{H}), 6.74(\mathrm{dd}, J=9.1,2.3 \mathrm{~Hz}, 2 \mathrm{H}), 2.53$ 
(s, 6H) ppm; ${ }^{13} \mathrm{C}\left\{{ }^{1} \mathrm{H}\right\}$ NMR: $\left(101 \mathrm{MHz}, \mathrm{CDCl}_{3}\right) \delta==139.1,137.8,136.9,133.6,132.0,131.8$, 131.1, 130.1, 129.6, 129.5, 128.8, 127.8, 127.7, 127.5, 127.1, 125.4, 125.3, 123.6, 21.5 ppm; IR: $\left(\right.$ neat, $\left.\mathrm{cm}^{-1}\right) \tilde{v}=576,611,736,793,823,884,933,1025,1104,1182,1258,1296,1360$, 1426, 1488, 1512, 1599, 2858, 2920, 2960, 3020; HRMS: calcd $\mathrm{m} / \mathrm{z}$ for $\mathrm{C}_{40} \mathrm{H}_{26} \mathrm{Cl}_{2}{ }^{+}[\mathrm{M}]^{+}$: 576.1412; found (EI) 576.1423; UV/Vis: (THF): $\lambda_{\max }$ (log $\varepsilon$ ) $=235$ (4.64), 257 (4.78), 262 (4.76), 276 (4.68), 327 (4.46), 337 (4.53), 362 (4.30) nm; Fluorescence: (THF $\left.\Lambda_{\mathrm{ex}} 337\right): \Lambda_{\max }$ (I $I_{\text {rel }} 429$ (0.99), 454 (0.67).

(P)-10,15-Bis(benzyloxy)-1,8-di-p-tolylhexahelicene (160jb):

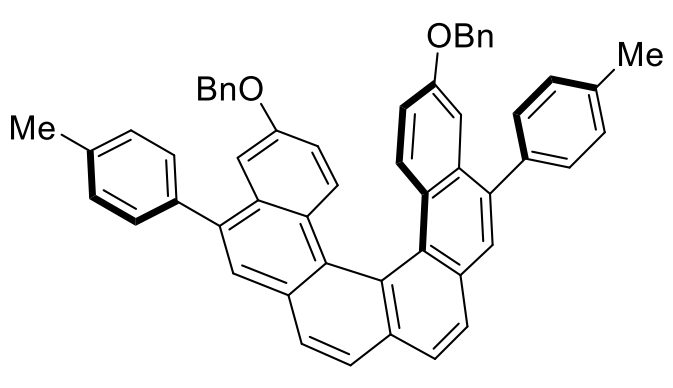

Prepared using 159jb (1.0 equiv, $18.0 \mathrm{mg}, 25.0$ $\mu \mathrm{mol})$, precatalyst 172y (9.9 mol\%, $4.0 \mathrm{mg}, 2.48$ $\mu \mathrm{mol})$, fluorobenzene $(0.5 \mathrm{ml})$ and $\mathrm{AgSbF}_{6}(10$ mol\%, $2.50 \mu \mathrm{mol}, 50 \mu \mathrm{l}$ of a $0.05 \mathrm{M}$ solution in dichloromethane) according to GPH. The product (16.6 mg, $23.0 \mu \mathrm{mol}, 92 \%$ yield) was obtained as a yellow powder, proportion 160jb:188jb = 97: 3 .

The ratio of isomers was determined by ${ }^{1} \mathrm{H}$ NMR. Enantiomeric excess: $+88 \%$. The ee was determined by chiral HPLC: $150 \times 4.6 \mathrm{~mm}$ Chiralpak IC-3 column, $3 \mu \mathrm{m}, \mathrm{CH}_{3} \mathrm{CN}=100,1.0$ $\mathrm{ml} \cdot \mathrm{min}^{-1}, 11.1 \mathrm{MPa}, 308 \mathrm{~K}, 255 \mathrm{~nm}$; major enantiomer: $t_{R}=2.97 \mathrm{~min}$, minor enantiomer: $t_{R}=$ $3.65 \mathrm{~min}$.

$[\alpha]_{22}^{D}:(88 \%$ ee $)+1261\left(c=0.510, \mathrm{CH}_{2} \mathrm{Cl}_{2}\right) .{ }^{1} \mathrm{H}$ NMR: $\left(400 \mathrm{MHz}, \mathrm{CDCl}_{3}\right) \delta==7.95-7.89(\mathrm{~m}$, 4H), $7.84(\mathrm{~s}, 2 \mathrm{H}), 7.72(\mathrm{~d}, J=9.2 \mathrm{~Hz}, 2 \mathrm{H}), 7.55-7.50(\mathrm{~m}, 4 \mathrm{H}), 7.36(\mathrm{~d}, J=7.8 \mathrm{~Hz}, 4 \mathrm{H}), 7.33$ - $7.25(\mathrm{~m}, 12 \mathrm{H}), 6.49$ (dd, $J=9.3,2.7 \mathrm{~Hz}, 2 \mathrm{H}), 4.97$ (d, $J=11.7 \mathrm{~Hz}, 2 \mathrm{H}), 4.91$ (d, J= 11.7 $\mathrm{Hz}, 2 \mathrm{H}), 2.51$ (s, 6H) ppm; ${ }^{13} \mathrm{C}\left\{{ }^{1} \mathrm{H}\right\}$ NMR: $\left(101 \mathrm{MHz}, \mathrm{CDCl}_{3}\right) \delta==156.3,139.0,137.9,137.2$, $137.0,133.5,132.1,130.0,130.0,129.9,129.4$, 128.6, 128.0, 127.9, 127.8, 127.2, 127.2, 126.5, 125.5, 123.5, 115.8, 107.6, 69.9, 21.5 ppm; IR: $\left(\right.$ neat, $\left.\mathrm{cm}^{-1}\right) \tilde{v}=611,692,724,824$, 887, 1019, 1179, 1226, 1263, 1377, 1442, 1501, 1610, 2861, 2915, 3029.HRMS: calcd m/z for $\mathrm{C}_{54} \mathrm{H}_{40} \mathrm{NaO}_{2}{ }^{+}[\mathrm{M}+\mathrm{Na}]^{+}:$743.2921; found (ESI) 743.2905; UV/Vis: (THF): $\lambda_{\max }(\log \varepsilon)=230$ (4.69), 266 (4.86), 280 (4.68), 338 (4.56), 366 (4.28); Fluorescence: (THF $\left.\Lambda_{\text {ex }} 338\right): \Lambda_{\max }\left(I_{\text {rel }}\right)$ 435 (0.97), 458 (0.63). 


\section{Experimental}

\section{(P)-9,11,14,16-Tetramethyl-1,8-di-p-tolylhexahelicene (160nb):}

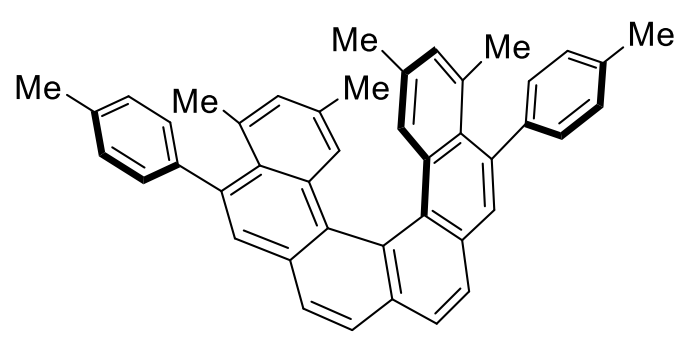

Prepared using 159nb (1.0 equiv, $14.1 \mathrm{mg}$, $25.0 \mu \mathrm{mol})$, precatalyst $172 \mathrm{y}(9.9 \mathrm{~mol} \%, 4.0 \mathrm{mg}$, $2.48 \mu \mathrm{mol})$, fluorobenzene $(0.5 \mathrm{ml})$ and $\mathrm{AgSbF}_{6}$ (10 mol\%, $2.50 \mu \mathrm{mol}, 50 \mu \mathrm{l}$ of a $0.05 \mathrm{M}$ solution in dichloromethane) according to GPH. The product (12.7 mg, $22.5 \mu \mathrm{mol}, 90 \%$ yield) was isolated as a yellow solid, proportion 160nb:188nb $=97: 3$.

HPLC: The selectivity of the reaction was determined by HPLC: Agilent $50 \times 4.6 \mathrm{~mm}$ Zorbax Eclipse Plus C8, $1.8 \mu \mathrm{m}, \mathrm{MeCN} / \mathrm{H}_{2} \mathrm{O}=90 / 10(\mathrm{v} / \mathrm{v}), 1.0 \mathrm{ml} \cdot \mathrm{min}^{-1}, 8.6 \mathrm{MPa}, 308 \mathrm{~K}, 2 \times 5 \mathrm{~nm}$; helicene 160nb: $t_{R}=6.80 \mathrm{~min}$, isomers 188nb: $t_{R}=8.91$ and $12.97 \mathrm{~min}$. Enantiomeric excess: $+87 \%$. The ee was determined by chiral HPLC: $150 \times 4.6 \mathrm{~mm}$ Chiralpak AS-3R column, $3 \mu \mathrm{m}, \mathrm{MeOH} / \mathrm{H}_{2} \mathrm{O}=95 / 5(v / v), 1.0 \mathrm{ml} \cdot \mathrm{min}^{-1}, 15.9 \mathrm{MPa}, 308 \mathrm{~K}, 255 \mathrm{~nm}$; major enantiomer: $t_{R}=10.43 \mathrm{~min}$, minor enantiomer: $t_{R}=14.78 \mathrm{~min}$.

Analytical data corresponded to those reported above for the synthesis of $160 \mathrm{nb}$ using precatalyst $\mathbf{9 8 f}$.

$[\alpha]_{24}^{D}:(87 \%$ ee $)+1174\left(c=0.225, \mathrm{CHCl}_{3}\right)$. UV/Vis: $(\mathrm{THF}): \lambda_{\max }(\log \varepsilon)=260(4.74), 273$ (4.72), 313 (4.36), 328 (4.43), 346 (4.42), 362 (4.35); Fluorescence: (THF $\left.\Lambda_{\text {ex }} 346\right): \Lambda_{\max }\left(I_{\text {rel }}\right)$ 436 (1.0), 458 (0.63).

(P)-10,15-Bis(trimethylsilyl)-1,8-di-p-tolylhexahelicene (160ib):

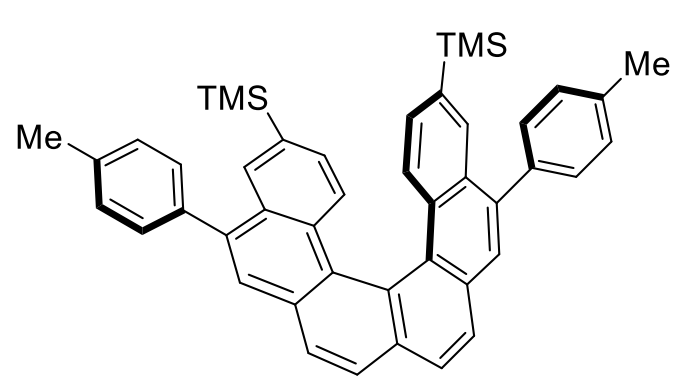

Prepared using 159ib (1.0 equiv, $16.3 \mathrm{mg}$, $25.0 \mu \mathrm{mol})$, precatalyst 172y $(9.9 \mathrm{~mol} \%, 4.0 \mathrm{mg}$, $2.48 \mu \mathrm{mol})$, fluorobenzene $(0.5 \mathrm{ml})$ and $\mathrm{AgSbF}_{6}$ (10 mol\%, $2.50 \mu \mathrm{mol}, 50 \mu \mathrm{l}$ of a $0.05 \mathrm{M}$ solution in dichloromethane) according to GPH; stirring at -20 ${ }^{\circ} \mathrm{C}$ for $96 \mathrm{~h}$. The crude product mixture was obtained as a yellow powder in 87\% NMR yield (using 1,4-dioxane as internal standard), proportion 160ib:188ib = 89:11.

In order to obtain a purer sample for characterization, the mixture was purified by semipreparative HPLC, obtaining $8.3 \mathrm{mg}$ (12.7 $\mu \mathrm{mol}, 51 \%$ yield) of 160ib. Separation conditions: $250 \times 20 \mathrm{~mm}$ YMC-Pack PVA SIL, $5 \mu \mathrm{m}$, hexanes/MTBE $=99 / 1(\mathrm{v} / \mathrm{v}), 18.9 \mathrm{ml} \cdot \mathrm{min}^{-1}, 4.5$ MPa, 295 K, $210 \mathrm{~nm}$. HPLC: $250 \times 4.6 \mathrm{~mm}$ YMC-Pack PVA SIL, $5 \mu \mathrm{m}$, hexanes/MTBE = 99/1 ( $/ \mathrm{v}), 1.0 \mathrm{ml} \cdot \mathrm{min}^{-1}, 3.9 \mathrm{MPa}, 303 \mathrm{~K}, 210 \mathrm{~nm}$; helicene 160ib: $t_{R}=9.91 \mathrm{~min}$, isomers 
188ib: $t_{R}=10.49$ and $10.91 \mathrm{~min}$; Enantiomeric excess: $+62 \%$. The ee was determined by chiral HPLC: $150 \times 4.6 \mathrm{~mm}$ Chiralpak IC-3 column, $3 \mu \mathrm{m}, \mathrm{CH}_{3} \mathrm{CN} / \mathrm{H}_{2} \mathrm{O}=80 / 20(\mathrm{v} / \mathrm{v}), 1.0$ $\mathrm{ml} \cdot \mathrm{min}^{-1}, 15.8 \mathrm{MPa}, 308 \mathrm{~K}, 255 \mathrm{~nm}$; major enantiomer: $t_{R}=16.24 \mathrm{~min}$, minor enantiomer: $t_{R}=$ $22.68 \mathrm{~min}$.

$[\alpha]_{25}^{D}:+923\left(c=0.415, \mathrm{CH}_{2} \mathrm{Cl}_{2}\right) ;{ }^{1} \mathrm{H}$ NMR: $\left(300 \mathrm{MHz}, \mathrm{CDCl}_{3}\right) \delta=8.13(\mathrm{~d}, J=24.1 \mathrm{~Hz}, 2 \mathrm{H})$, $8.03(\mathrm{~d}, J=8.2 \mathrm{~Hz}, 2 \mathrm{H}), 7.97(\mathrm{~d}, J=8.1 \mathrm{~Hz}, 2 \mathrm{H}), 7.88(\mathrm{~s}, 2 \mathrm{H}), 7.74$ (dd, $J=8.4,0.6 \mathrm{~Hz}, 2 \mathrm{H}$ ), $7.65(\mathrm{dd}, J=8.0,1.8 \mathrm{~Hz}, 4 \mathrm{H}), 7.47-7.38(\mathrm{~m}, 4 \mathrm{H}), 6.80(\mathrm{dd}, J=8.4,1.3 \mathrm{~Hz}, 2 \mathrm{H}$ ), 2.53 (s, $6 \mathrm{H}), 0.12(\mathrm{~s}, 18 \mathrm{H}) \mathrm{ppm} ;{ }^{13} \mathrm{C}\{\mathrm{H}\}$ NMR: $\left(75 \mathrm{MHz}, \mathrm{CDCl}_{3}\right) \delta=139.7,137.8,137.3,137.2,133.3$, $131.4,131.3,130.8,130.3,129.6,129.2,128.6,127.6,127.5,127.3,127.1,126.8,124.2$, 21.5, -1.0 ppm; IR: (neat, $\left.\mathrm{cm}^{-1}\right) \tilde{v}=612,752,802,823,854,1017,1077,1105,1123,1250$, 1258, 1512, 2953, 3018; HRMS: calcd $\mathrm{m} / \mathrm{z}$ for $\mathrm{C}_{36} \mathrm{H}_{44} \mathrm{Si}_{2}{ }^{+}[\mathrm{M}]^{+}:$652.2982; found (EI) 652.2993; UV/Vis: (THF): $\lambda_{\max }(\log \varepsilon)=237$ (4.72), 258 (4.86), 272 (4.76), 311 (4.35), 326 (4.51), 339 (4.55), 362 (4.38) $\mathrm{nm}$.

11,12-Dithia-1,8-di-p-tolyl-11,12-dihydrocyclopenta[c]indeno[4,5-g]phenanthrene (160eb) using 172y:

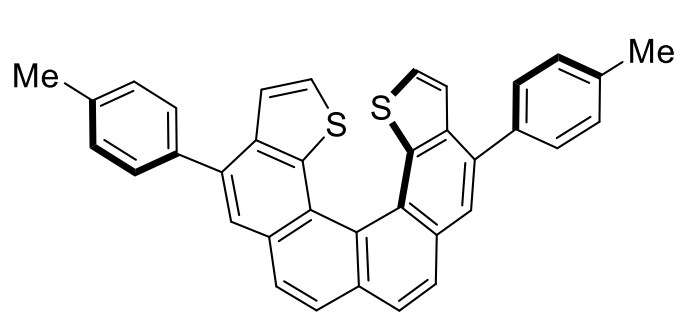

Prepared using 159eb (1.0 equiv, $13.0 \mathrm{mg}$, $25.0 \mu \mathrm{mol})$, precatalyst $172 \mathrm{y}(9.9 \mathrm{~mol} \%, 4.0 \mathrm{mg}$, $2.48 \mu \mathrm{mol})$, fluorobenzene $(0.5 \mathrm{ml})$ and $\mathrm{AgSbF}_{6}$ (10 mol\%, $2.50 \mu \mathrm{mol}, 50 \mu \mathrm{l}$ of a $0.05 \mathrm{M}$ solution in dichloromethane) according to GPH; stirring at $20{ }^{\circ} \mathrm{C}$ for $96 \mathrm{~h}$. The crude product mixture was obtained as a yellow powder in $21 \% \mathrm{NMR}$ yield (using 1,4-dioxane as internal standard), proportion 160eb:188eb:187eb = 81:0:19, $27 \%$ conversion.

The ratio of isomers was determined by ${ }^{1} \mathrm{H}$ NMR. Enantiomeric excess: $+24 \%$. The ee was determined by chiral HPLC: $150 \times 4.6 \mathrm{~mm}$ Chiralpak IA-3 column, $3 \mu \mathrm{m}$, hexane $/ \mathrm{CH}_{2} \mathrm{Cl}_{2}=$ $90 / 10(v / v), 1.0 \mathrm{ml} \cdot \mathrm{min}^{-1}, 6.6 \mathrm{MPa}, 303 \mathrm{~K}, 330 \mathrm{~nm}$; major enantiomer: $t_{R}=5.13 \mathrm{~min}$, minor enantiomer: $t_{R}=6.68 \mathrm{~min}$.

Analytical data corresponded to those reported above for $160 \mathrm{eb}$ made using $98 \mathrm{f}$. 


\section{Experimental}

\section{1,12-Dioxa-1,8-di-p-tolyl-11,12-dihydrocyclopenta[c]indeno[4,5- $g$ ]phenanthrene}

(160fb) using 172y:

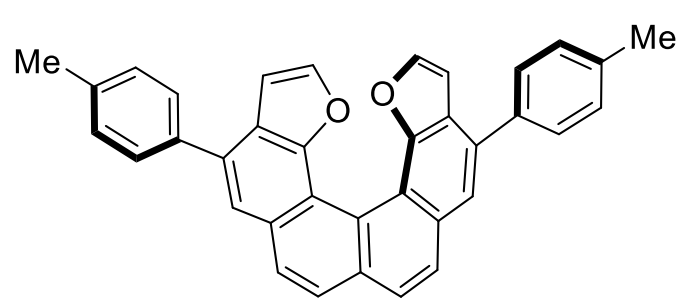

Prepared using 159fb (1.0 equiv, $12.2 \mathrm{mg}$, $25.0 \mu \mathrm{mol})$, precatalyst $172 \mathrm{y}(9.9 \mathrm{~mol} \%, 4.0 \mathrm{mg}$, $2.48 \mu \mathrm{mol})$, fluorobenzene $(0.5 \mathrm{ml})$ and $\mathrm{AgSbF}_{6}$ (10 mol\%, $2.50 \mu \mathrm{mol}, 50 \mu \mathrm{l}$ of a $0.05 \mathrm{M}$ solution in dichloromethane) according to GPH; stirring at -

$20{ }^{\circ} \mathrm{C}$ for $96 \mathrm{~h}$. The crude product mixture was obtained as a yellow powder in $5 \%$ NMR yield (using 1,4-dioxane as internal standard), proportion 160fb:188fb:187fb $=>99: 0: 0,8 \%$ conversion.

The ratio of isomers was determined by ${ }^{1} \mathrm{H}$ NMR. Enantiomeric excess: $+24 \%$. The ee was determined by chiral HPLC: $150 \times 4.6 \mathrm{~mm}$ Chiralpak IA-3 column, $3 \mu \mathrm{m}$, hexane/MTBE = $70 / 30(\mathrm{v} / \mathrm{v}), 1.0 \mathrm{ml} \cdot \mathrm{min}^{-1}, 6 \mathrm{MPa}, 303 \mathrm{~K}, 330 \mathrm{~nm}$; major enantiomer: $t_{R}=4.88 \mathrm{~min}$, minor enantiomer: $t_{R}=7.48 \mathrm{~min}$.

Analytical data corresponded to those reported above for $\mathbf{1 6 0 f b}$ made using $\mathbf{9 8 f}$.

\section{9,14-Dithia-1,8-di-p-tolyl-11,12-dihydrocyclopenta[c]indeno[4,5-g]phenanthrene} (160gb) using 172y:

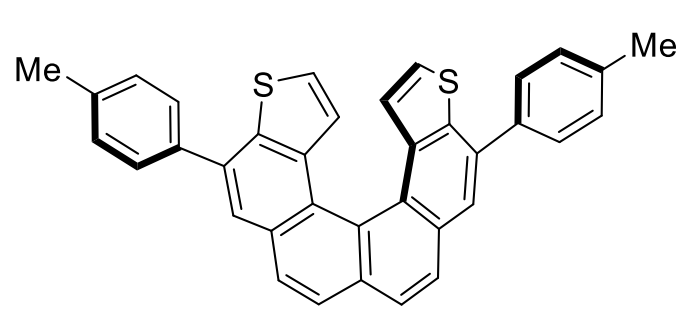

Prepared using 159gb (1.0 equiv, $11.1 \mathrm{mg}$, $21.3 \mu \mathrm{mol})$, precatalyst $172 \mathrm{y}(9.9 \mathrm{~mol} \%, 4.0 \mathrm{mg}$, $2.10 \mu \mathrm{mol})$, fluorobenzene $(0.5 \mathrm{ml})$ and $\mathrm{AgSbF}_{6}$ (10.1 mol\%, $2.15 \mu \mathrm{mol}, 43 \mu \mathrm{l}$ of a $0.05 \mathrm{M}$ solution in dichloromethane) according to GPH; stirring at -20 ${ }^{\circ} \mathrm{C}$ for $96 \mathrm{~h}$. The crude product mixture was obtained as a yellow powder in $16 \%$ NMR yield (using 1,4-dioxane as internal standard), proportion 160gb:188gb:187gb $=38: 0: 62,53 \%$ conversion.

The ratio of isomers was determined by ${ }^{1} \mathrm{H}$ NMR. Enantiomeric excess: $0 \%$. The ee was determined by chiral HPLC: $150 \times 4.6 \mathrm{~mm}$ Chiralpak IA-3 column, $3 \mu \mathrm{m}$, hexane $/ \mathrm{CH}_{2} \mathrm{Cl}_{2}=$ 95/5 $(\mathrm{v} / \mathrm{v}), 1.0 \mathrm{ml} \cdot \mathrm{min}^{-1}, 5.8 \mathrm{MPa}, 298 \mathrm{~K}, 330 \mathrm{~nm}$; major enantiomer: $t_{R}=8.70 \mathrm{~min}$, minor enantiomer: $t_{R}=13.59 \mathrm{~min}$.

Analytical data corresponded to those reported above for $160 \mathrm{gb}$ made using $98 \mathrm{f}$. 


\section{9,14-Dioxa-1,8-di-p-tolyl-11,12-dihydrocyclopenta[c]indeno[4,5-g]phenanthrene} (160hb) using 172y:

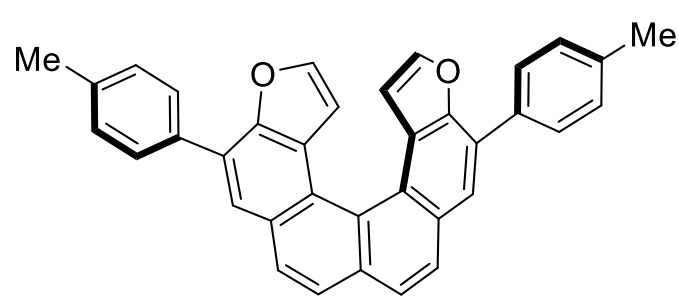

Prepared using $159 \mathrm{hb}(1.0$ equiv, $8.9 \mathrm{mg}$, $18.2 \mu \mathrm{mol})$, precatalyst $172 \mathrm{y}(9.9 \mathrm{~mol} \%, 2.9 \mathrm{mg}$, $1.79 \mu \mathrm{mol})$, fluorobenzene $(0.5 \mathrm{ml})$ and $\mathrm{AgSbF}_{6}$ (10 mol\%, $18.2 \mu \mathrm{mol}, 36 \mu \mathrm{l}$ of a $0.05 \mathrm{M}$ solution in dichloromethane) according to GPH; stirring at -20

${ }^{\circ} \mathrm{C}$ for $96 \mathrm{~h}$. The crude product mixture was obtained as a yellow powder in 33 isolated yield, proportion $160 \mathrm{gb}: 188 \mathrm{gb}: 187 \mathrm{gb}=>99: 0: 0$.

The ratio of isomers was determined by ${ }^{1} \mathrm{H}$ NMR. Enantiomeric excess: $0 \%$. The ee was determined by chiral HPLC: $150 \times 4.6 \mathrm{~mm}$ Chiralpak IA-3 column, $3 \mu \mathrm{m}$, hexane $/ \mathrm{CH}_{2} \mathrm{Cl}_{2}=$ $92 / 8(\mathrm{v} / \mathrm{v}), 1.0 \mathrm{ml} \cdot \mathrm{min}^{-1}, 6.1 \mathrm{MPa}, 330 \mathrm{~K}, 330 \mathrm{~nm}$; major enantiomer: $t_{R}=8.34 \mathrm{~min}$, minor enantiomer: $t_{R}=15.25 \mathrm{~min}$.

Analytical data corresponded to those reported above for $160 \mathrm{hb}$ made using $98 \mathrm{f}$.

(P)-1,8-Bis(4-methoxyphenyl)hexahelicene-2-carbonitrile (217a):

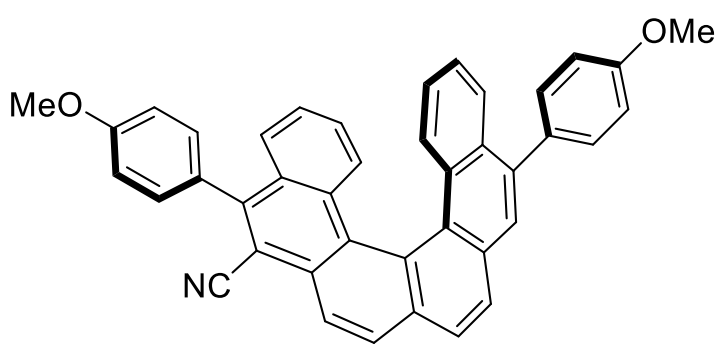

Prepared using 216a $\mathbf{a}^{[257]}$ (1.0 equiv, $8.0 \mathrm{mg}$, $14.1 \mu \mathrm{mol})$, precatalyst 172y (10.1 mol\%, 2.3 $\mathrm{mg}, 1.43 \mu \mathrm{mol})$, fluorobenzene $(0.28 \mathrm{ml})$ and $\mathrm{AgSbF}_{6}(9.9 \mathrm{~mol} \%, 1.40 \mu \mathrm{mol}, 28 \mu \mathrm{l}$ of a $0.05 \mathrm{M}$ solution in dichloromethane) according to GPH, (stirring at $5^{\circ} \mathrm{C}$ for $72 \mathrm{~h}$ ) as a yellow solid in

$57 \%$ NMR yield; $67 \%$ conversion.

In order to obtain a pure sample for characterization, the mixture was purified by semipreparative HPLC, obtaining $3.6 \mathrm{mg}(6.36 \mu \mathrm{mol}$, $45 \%$ yield $)$ of $\mathbf{2 1 7 a}$. Separation conditions: $250 \times 20 \mathrm{~mm}$ YMC-Pack PVA SIL, $5 \mu \mathrm{m}$, heptane/THF = 90/10 ( $/ \mathrm{v}), 18.9 \mathrm{ml} \cdot \mathrm{min}^{-1}, 5.6 \mathrm{MPa}$, $295 \mathrm{~K}, 246 \mathrm{~nm}$. Enantiomeric excess: $+89 \%$. The ee was determined by chiral HPLC: $150 \times$ 4.6 mm Chiralpak AS-3R column, $3 \mu \mathrm{m}, \mathrm{MeCN} / \mathrm{H}_{2} \mathrm{O}=75 / 25(v / v), 1.0 \mathrm{ml} \cdot \mathrm{min}^{-1}, 12.8 \mathrm{MPa}$, $308 \mathrm{~K}, 239 \mathrm{~nm}$; major enantiomer: $t_{R}=12.28 \mathrm{~min}$, minor enantiomer: $t_{R}=15.14 \mathrm{~min}$.

$[\alpha]_{23}^{D}:+1129\left(c=0.17, \mathrm{CH}_{2} \mathrm{Cl}_{2}\right) ;{ }^{1} \mathbf{H}$ NMR: $\left(400 \mathrm{MHz}, \mathrm{CDCl}_{3}\right) \delta=8.48(\mathrm{~d}, J=8.3 \mathrm{~Hz}, 1 \mathrm{H})$, $8.19(\mathrm{~d}, J=8.4 \mathrm{~Hz}, 1 \mathrm{H}), 8.08(\mathrm{~d}, J=8.2 \mathrm{~Hz}, 1 \mathrm{H}), 8.05$ (d, $J=8.3 \mathrm{~Hz}, 1 \mathrm{H}), 7.90$ (dd, $J=8.1$, $1.1 \mathrm{~Hz}, 1 \mathrm{H}), 7.90(\mathrm{~s}, 1 \mathrm{H}), 7.80-7.74(\mathrm{~m}, 1 \mathrm{H}), 7.73-7.67(\mathrm{~m}, 2 \mathrm{H}), 7.63(\mathrm{dd}, J=8.5,2.5 \mathrm{~Hz}$, 2H), 7.61 (d, J = 9.6 Hz, 2H), $7.22-7.15(\mathrm{~m}, 4 \mathrm{H}$ ), 7.12 (ddd, $J=13.9,8.6,2.5 \mathrm{~Hz}, 2 \mathrm{H}$ ), 6.82 (ddd, $J=8.3,6.8,1.3 \mathrm{~Hz}, 1 \mathrm{H}$ ), 6.73 (ddd, $J=8.3,6.8,1.3 \mathrm{~Hz}, 1 \mathrm{H}$ ), 3.97 (s, 3H), $3.95(\mathrm{~s}, 3 \mathrm{H}$ ) 


\section{Experimental}

ppm; ${ }^{13} \mathbf{C}\{\mathrm{H}\}$ NMR: $\left(126 \mathrm{MHz}, \mathrm{CD}_{2} \mathrm{Cl}_{2}\right) \delta=160.6,159.7,147.5,140.2,133.8,132.8,132.0$, $131.8,131.6,131.5,131.2,130.5,130.4,129.5,129.2,129.2,129.0,128.6,128.4,128.1$, $128.0,127.8,127.5,127.5,126.7,126.6,126.4,126.0,125.0,124.3,123.8,118.2,114.4$, 114.2, 109.3, 55.8, 55.7 ppm; IR: (neat, $\mathrm{cm}^{-1}$ ) $\tilde{v}=610,702,765,793,864,1013,1084$, 1175, 1257, 1444, 1511, 1606, 2853, 2923, 2961; HRMS: calcd $m / z$ for $\mathrm{C}_{41} \mathrm{H}_{27} \mathrm{NO}_{2}{ }^{+}[\mathrm{M}]^{+}$: 565.2042; found (EI) 565.2045.

\section{Compound 217b:}

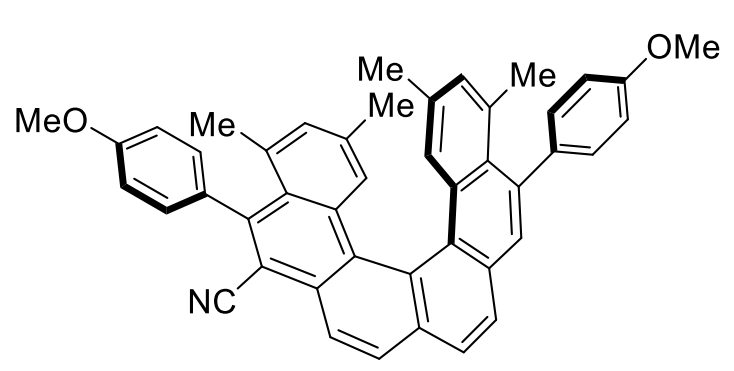

Prepared using $216 \mathbf{b}^{[257]}$ (1.0 equiv, $11.3 \mathrm{mg}$, $18.2 \mu \mathrm{mol})$, precatalyst $172 \mathrm{y}(9.9 \mathrm{~mol} \%, 2.9 \mathrm{mg}$, $1.80 \mu \mathrm{mol})$, fluorobenzene $(0.36 \mathrm{ml})$ and $\mathrm{AgSbF}_{6}$ $(9.9 \mathrm{~mol} \%, 1.80 \mu \mathrm{mol}, 36 \mu \mathrm{l}$ of a $0.05 \mathrm{M}$ solution in dichloromethane) according to GPH; stirring at $5{ }^{\circ} \mathrm{C}$ for $72 \mathrm{~h}$. Purification by column chromatography (toluene) afforded the product as a yellow solid $(9.1 \mathrm{mg}, 14.6 \mu \mathrm{mol}, 81 \%$ yield).

Enantiomeric excess: $+51 \%$. The ee was determined by chiral HPLC: $150 \times 4.6 \mathrm{~mm}$ Chiralpak IC-3 column, $3 \mu \mathrm{m}, \mathrm{MeCN} / \mathrm{H}_{2} \mathrm{O}=90 / 10(\mathrm{v} / \mathrm{v}), 1.0 \mathrm{ml} \cdot \mathrm{min}^{-1}, 12.9 \mathrm{MPa}, 308 \mathrm{~K}, 247$ $\mathrm{nm}$; major enantiomer: $t_{R}=7.05 \mathrm{~min}$, minor enantiomer: $t_{R}=8.05 \mathrm{~min}$.

$[\alpha]_{22}^{D}:+596\left(c=0.445, \mathrm{CH}_{2} \mathrm{Cl}_{2}\right)$; ${ }^{1} \mathrm{H}$ NMR: $\left(400 \mathrm{MHz}, \mathrm{CDCl}_{3}\right) \delta=8.37(\mathrm{~d}, J=8.4 \mathrm{~Hz}, 1 \mathrm{H})$, $8.13(\mathrm{~d}, J=8.4 \mathrm{~Hz}, 1 \mathrm{H}), 8.02(\mathrm{~d}, J=8.2 \mathrm{~Hz}, 1 \mathrm{H}), 7.93(\mathrm{~d}, J=8.2 \mathrm{~Hz}, 1 \mathrm{H}), 7.72(\mathrm{~s}, 1 \mathrm{H}), 7.57$ - $7.48(\mathrm{~m}, 2 \mathrm{H}), 7.40(\mathrm{~s}, 1 \mathrm{H}), 7.38-7.34(\mathrm{~m}, 1 \mathrm{H}), 7.33(\mathrm{~s}, 1 \mathrm{H}), 7.28(\mathrm{dd}, J=8.6,2.3 \mathrm{~Hz}, 1 \mathrm{H})$, $7.12-7.08(\mathrm{~m}, 2 \mathrm{H}), 7.07-6.98(\mathrm{~m}, 2 \mathrm{H}), 6.84(\mathrm{~s}, 1 \mathrm{H}), 6.80(\mathrm{~s}, 1 \mathrm{H}), 3.94(\mathrm{~s}, 3 \mathrm{H}), 3.93(\mathrm{~s}, 3 \mathrm{H})$, $2.03(\mathrm{~s}, 3 \mathrm{H}), 1.98(\mathrm{~s}, 3 \mathrm{H}), 1.82(\mathrm{~s}, 6 \mathrm{H}) \mathrm{ppm} ;{ }^{13} \mathrm{C}\{\mathrm{H}\}$ NMR: $\left(101 \mathrm{MHz}, \mathrm{CDCl}_{3}\right) \delta=160.0$, $158.9,146.6,139.3,137.4,137.1,135.9,134.5,134.0,133.5,133.4,133.2,132.5,131.6$, $131.5,131.0,131.0,130.8,130.3,129.6,129.0,128.5,128.3,128.2,128.0,127.9,127.4$, $127.3,127.3,127.2,126.4,124.1$, 123.5, 118.3, 114.1, 114.0, 113.8, 113.5, 110.5, 55.5, 55.5, 25.4, 25.0, 21.0, 20.8 ppm; IR: (neat, $\left.\mathrm{cm}^{-1}\right) \tilde{v}=532,733,830,1036,1174,1247,1287$, 1442, 1510, 1604, 2211, 2833, 2932, 2966; HRMS: calcd $\mathrm{m} / \mathrm{z}$ for $\mathrm{C}_{45} \mathrm{H}_{35} \mathrm{NO}_{2}{ }^{+}[\mathrm{M}]^{+}: 621.2668$; found (El) 621.2673; UV/Vis: (THF): $\lambda_{\max }(\log \varepsilon)=247$ (4.75), 289 (4.71), 343 (4.28), 355 (4.30), 373 (4.28) nm. 


\subsection{Towards the total synthesis of Monbarbatain A}

\section{1-Bromo-4-isopropoxy-2-methoxybenzene (223a):}<smiles>CCOc1cc(OC)ccc1Br</smiles>

Prepared according to a literature procedure. ${ }^{[280]}$ In a high pressure tube, equipped with a magnetic stirring bar, a suspension of 4-bromo-3methoxyphenol (232) (1.0 equiv, $1.88 \mathrm{~g}, 9.26 \mathrm{mmol}$ ), $\mathrm{K}_{2} \mathrm{CO}_{3}$ (3.80 equiv, 4.83 $\mathrm{g}, 34.9 \mathrm{mmol}$ ) and 2-bromopropane (1.27 equiv, $1.45 \mathrm{~g}, 11.8 \mathrm{mmol}$ ) in DMF $(20 \mathrm{ml})$ was stirred at $100{ }^{\circ} \mathrm{C}$. After three hours, 2-bromopropane (0.64 equiv, $730 \mathrm{mg}, 5.94$ $\mathrm{mmol}$ ) was added again and the reaction was stirred at $80^{\circ} \mathrm{C}$ over night. For the work up, water $(160 \mathrm{ml})$ was added, the aqueous layer was separated and extracted with MTBE $(3 \times$ $70 \mathrm{ml})$. The combined organic layers were washed with water $(5 \times 50 \mathrm{ml})$, aqueous $\mathrm{NaOH}$ solution $(1 \mathrm{M}, 50 \mathrm{ml})$ and dried over $\mathrm{Na}_{2} \mathrm{SO}_{4}$. The solvents were removed under reduced pressure to afford the product $223 \mathrm{a}$ as a yellow oil $(2.11 \mathrm{~g}, 8.61 \mathrm{mmol}, 93 \%$ yield).

Analytical data corresponded to that described in the literature. ${ }^{[280]}$

\section{4-(Benzyloxy)-1-bromo-2-methoxybenzene (223b):}<smiles>COc1cc(Br)ccc1Br</smiles>

In a round bottom flask, equipped with a magnetic stirring bar, 4-bromo-3methoxyphenol (232) (1.0 equiv, $2.30 \mathrm{~g}, 11.32 \mathrm{mmol}$ ), benzyl bromide (1.0 equiv, $1.94 \mathrm{~g}, 11.34 \mathrm{mmol}), \mathrm{K}_{2} \mathrm{CO}_{3}(3.0$ equiv, $4.7 \mathrm{~g}, 34.0 \mathrm{mmol}$ ) and acetonitrile $(38 \mathrm{ml})$ were added under air. The reaction mixture was stirred at room temperature for $16 \mathrm{~h}$, before diluting with ethyl acetate $(200 \mathrm{ml})$ and washing with water $(200 \mathrm{ml})$. The aqueous phase was extracted with ethyl acetate $(2 \times 200 \mathrm{ml})$; the combined organic phases were dried over $\mathrm{MgSO}_{4}$, filtered and concentrated in vacuo. The product (3.31 g, $11.29 \mathrm{mmol},>99 \%$ yield) was used without further purification.

Analytical data corresponded to those of the literature. ${ }^{[281]}$

\section{General procedure I (GPI)}

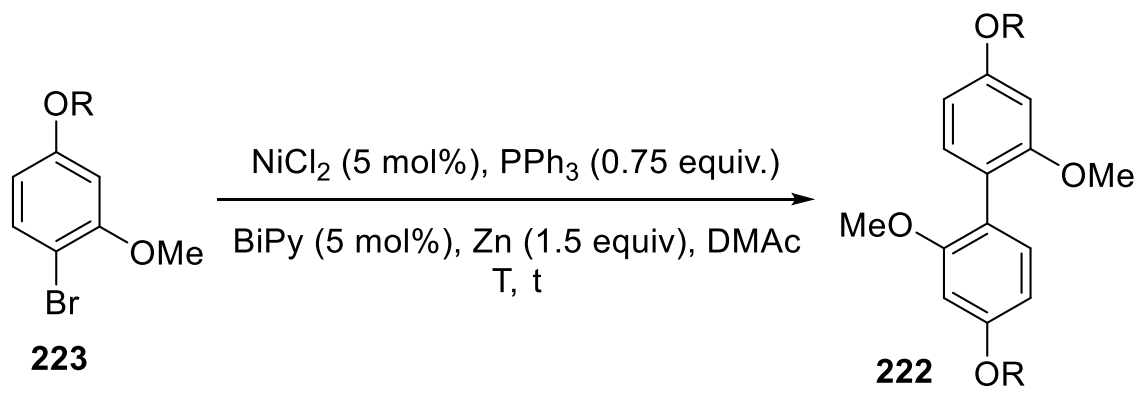

Following an adapted literature procedure, ${ }^{[260]}$ to a dried Schlenk flask, equipped with magnetic stirring bar were added $\mathrm{NiCl}_{2}$ (5 mol\%), $\mathrm{PPh}_{3}$ (0.75 equiv), 2-2'-bipyridine (5 mol\%), 


\section{Experimental}

Zn dust (1.5 equiv) and the aromatic bromide 223. The mixture was dried under high vacuum for 20 minutes, purging with argon, before adding dimethylacetamide $(0.088 \mathrm{M})$ and stirring at the specified temperature for the indicated time. The suspension was then diluted with chloroform and filtered through Celite ${ }^{\circledR}$. The filtrate was washed with aqueous hydrochloric acid $(1 \mathrm{M})$ and water, before drying over $\mathrm{Na}_{2} \mathrm{SO}_{4}$, filtering and removing volatiles in vacuo. The product was obtained by column chromatography.

\section{4,4'-Diisopropoxy-2,2'-dimethoxy-1,1'-biphenyl (222a):}

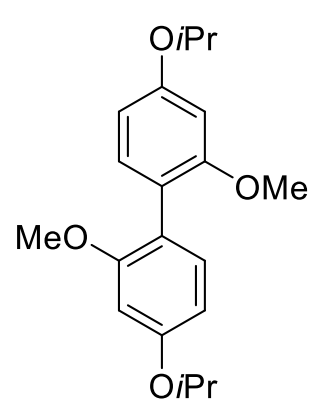

Prepared as a colorless solid (370 $\mathrm{mg}, 1.12 \mathrm{mmol}, 40 \%$ yield) from compound 223a (1.0 equiv, $1.36 \mathrm{~g}, 5.55 \mathrm{mmol}$ ) using $\mathrm{NiCl}_{2}(5.6 \mathrm{~mol} \%$, $40 \mathrm{mg}, 309 \mu \mathrm{mol}), \mathrm{PPh}_{3}$ (0.75 equiv, $1.09 \mathrm{~g}, 4.16 \mathrm{mmol}$ ), 2-2'-bipyridine (4.6 mol\%, $40.0 \mathrm{mg}, 256 \mu \mathrm{mol})$, Zn dust (1.5 equiv, $550 \mathrm{mg}, 8.41 \mathrm{mmol}$ ) in dimethylacetamide $(6.5 \mathrm{ml})$ according to GPI (stirring at $90{ }^{\circ} \mathrm{C}$ for 16 h; column chromatography: 8-10\% ethyl acetate in petrol ether).

'H NMR: $\left(300 \mathrm{MHz}, \mathrm{CDCl}_{3}\right) \delta=7.16-7.08(\mathrm{~m}, 2 \mathrm{H}), 6.56-6.48(\mathrm{~m}, 4 \mathrm{H}), 4.58$ (hept, $J=6.1$ $\mathrm{Hz}, 2 \mathrm{H}), 3.75(\mathrm{~s}, 6 \mathrm{H}), 1.38(\mathrm{~d}, J=6.1 \mathrm{~Hz}, 12 \mathrm{H})$ ppm; ${ }^{13} \mathrm{C}$ NMR: $\left(126 \mathrm{MHz}, \mathrm{CDCl}_{3}\right) \delta=158.1$, 157.9, 131.7, 119.8, 105.7, 100.5, 69.7, 55.6, 22.2 ppm; IR: (neat, $\left.\mathrm{cm}^{-1}\right) \tilde{v}=790,828,986$, 1036, 1113, 1127, 1158, 1196, 1269, 1305, 1415, 1455, 1490, 1576, 1609, 2977; HRMS: calcd $m / z$ for $\mathrm{C}_{20} \mathrm{H}_{27} \mathrm{O}_{4}[\mathrm{M}+\mathrm{H}]^{+}: 331.1904$, found: (ESI) 331.1903.

\section{4,4'-Bis(benzyloxy)-2,2'-dimethoxy-1,1'-biphenyl (222b):}

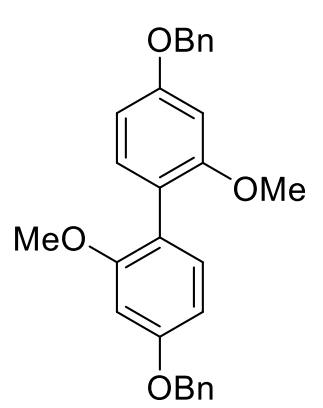

Prepared as a colorless solid $(1.84 \mathrm{~g}, 4.31 \mathrm{mmol}, 76 \%$ yield) from compound 223b (1.0 equiv, $3.31 \mathrm{~g}, 11.3 \mathrm{mmol})$ using $\mathrm{NiCl}_{2}(5.1 \mathrm{~mol} \%$, $75 \mathrm{mg}, 579 \mu \mathrm{mol}$ ), $\mathrm{PPh}_{3}$ (0.75 equiv, $2.22 \mathrm{~g}, 8.46 \mathrm{mmol}$ ), 2-2'-bipyridine (5.0 mol\%, $88 \mathrm{mg}, 563.4 \mu \mathrm{mol}), \mathrm{Zn}$ dust (1.53 equiv, $1.13 \mathrm{~g}, 17.28$ $\mathrm{mmol}$ ) in dimethylacetamide $\left(11.3 \mathrm{ml}\right.$ ) according to $\mathrm{GPI}$ (stirring at $90{ }^{\circ} \mathrm{C}$ for $16 \mathrm{~h}$; column chromatography: $10-60 \%$ ethyl acetate in petrol ether).

'H NMR: $\left(300 \mathrm{MHz}, \mathrm{CDCl}_{3}\right) \delta=7.55-7.31(\mathrm{~m}, 10 \mathrm{H}), 7.21-7.12(\mathrm{~m}, 2 \mathrm{H}), 6.72-6.58(\mathrm{~m}$, 4H), $5.11(\mathrm{~s}, 4 \mathrm{H}), 3.76(\mathrm{~s}, 6 \mathrm{H}) \mathrm{ppm} ;{ }^{13} \mathrm{C}$ NMR: $\left(75 \mathrm{MHz}, \mathrm{CDCl}_{3}\right) \delta=159.3,158.1,137.1$, 131.9, 128.6, 127.9, 127.6, 120.4, 105.0, 99.8, 70.2, 55.7 ppm; IR: $\left(\right.$ neat, $\left.\mathrm{cm}^{-1}\right) \tilde{v}=537,640$, 699, 827, 1021, 1133, 1189, 1286, 1382, 1457, 1492, 1573, 1606, 2876, 2929, 2954, 2997; HRMS: calcd $m / z$ for $\mathrm{C}_{28} \mathrm{H}_{26} \mathrm{NaO}_{4}[\mathrm{M}+\mathrm{Na}]^{+}:$449.1723, found (ESI) 449.1724. 


\section{2,2',4,4'-Tetramethoxy-1,1'-biphenyl (222c):}<smiles>COc1ccc(-c2ccc(OC)cc2OC)c(OC)c1</smiles>

Prepared as a colorless solid (5.0 g, $18.2 \mathrm{mmol}, 79 \%$ yield) from 1bromo-2,4-dimethoxybenzene (223c) (1.0 equiv, $10.0 \mathrm{~g}, 46.10 \mathrm{mmol}$ ) using $\mathrm{NiCl}_{2}$ (5.0 mol\%, $299 \mathrm{mg}, 2.31 \mathrm{mmol}$ ), $\mathrm{PPh}_{3}$ (0.749 equiv, $9.06 \mathrm{~g}$, $34.5 \mathrm{mmol}$ ), 2-2'-bipyridine (5.0 mol\%, $360 \mathrm{mg}, 2.31 \mathrm{mmol})$, Zn dust (1.50 equiv, $4.52 \mathrm{~g}, 69.13 \mathrm{mmol}$ ) in dimethylacetamide (45 ml) according to GPI (stirring at $90{ }^{\circ} \mathrm{C}$ for $16 \mathrm{~h}$; column chromatography: $20 \%$ ethyl acetate in petrol ether).

Analytical data corresponded to those reported. ${ }^{[282]}$

\section{General procedure J (GPJ)}<smiles>[R]Oc1ccc(-c2ccc([R20])cc2OC)c(OC)c1</smiles>

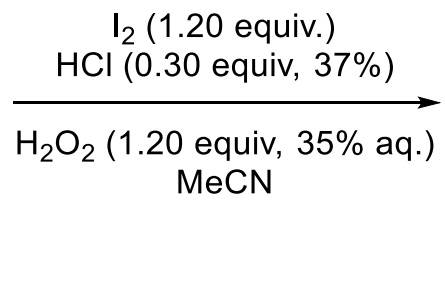<smiles>[R]Oc1cc(OC)c(-c2cc(OC)c(O[2H])cc2OC)cc1I</smiles>

Following an adapted literature procedure, ${ }^{[259]}$ to a round bottom flask, equipped with a magnetic stirrer, iodine (1.2 equiv.) in acetonitrile $(0.5 \mathrm{M})$, aqueous hydrochloric acid $(37 \%)$, hydrogen peroxide (1.2 equiv., 35\%) and the corresponding biaryl 222 (1.0 equiv) were added under air. The reaction mixture was stirred for $16 \mathrm{~h}$ at room temperature, monitoring the reaction by GCMS until completion and adding more equivalents of the reagents if necessary. The reaction mixture was then quenched by addition of water, sodium thiosulfate and sodium bicarbonate as saturated aqueous solutions. The phases were separated and the aqueous layer was extracted with dichloromethane. The combined organic layers were dried over $\mathrm{Na}_{2} \mathrm{SO}_{4}$, filtered and concentrated in vacuo. The product was used in the next step without further purification.

\section{5,5'-Diiodo-4,4'-diisopropoxy-2,2'-dimethoxy-1,1'-biphenyl (220a):}<smiles>COc1cc(I)c(-c2cc(I)c(OC(C)C)cc2OC)cc1OC</smiles>

Prepared as a light yellow solid (150 mg, $258 \mu \mathrm{mol}, 88 \%$ yield) from compound 222a (1.0 equiv, $97 \mathrm{mg}, 293 \mu \mathrm{mol}$ ) using iodine (1.21 equiv, $45 \mathrm{mg}, 177 \mu \mathrm{mol})$, aqueous hydrochloric acid (0.3 equiv, $88.0 \mu \mathrm{mol}$, $7.30 \mu \mathrm{l}$ of a $37 \%$ aq. solution) and hydrogen peroxide (1.2 equiv, 352 $\mu \mathrm{mol}, 30.3 \mu \mathrm{l}$ of a $35 \%$ aq. solution) in acetonitrile $(0.6 \mathrm{ml})$ according to 


\section{Experimental}

modified GPJ: after stirring at room temperature for $16 \mathrm{~h}$, iodine $(0.7$ equiv, $26 \mathrm{mg}, 102$ $\mu \mathrm{mol})$, aqueous hydrochloric acid ( 0.6 equiv, $176 \mu \mathrm{mol}, 14.6 \mu \mathrm{l}$ of a $37 \%$ aq. solution) and hydrogen peroxide ( 0.6 equiv, $176 \mu \mathrm{mol}, 15.2 \mu \mathrm{l}$ of a $35 \%$ aq. solution) were added, and the reaction mixture was stirred at room temperature for an additional $4 \mathrm{~h}$.

'H NMR: $\left(300 \mathrm{MHz}, \mathrm{CDCl}_{3}\right) \delta=7.54(\mathrm{~s}, 2 \mathrm{H}), 6.48(s, 2 \mathrm{H}), 4.58$ (hept, $\left.J=6.1 \mathrm{~Hz}, 2 \mathrm{H}\right), 3.75$ $(\mathrm{s}, 6 \mathrm{H}), 1.43(\mathrm{~d}, J=6.1 \mathrm{~Hz}, 12 \mathrm{H}) \mathrm{ppm} ;{ }^{13} \mathrm{C}$ NMR: $\left(101 \mathrm{MHz}, \mathrm{CDCl}_{3}\right) \delta=158.5,157.4,140.8$, 121.4, 99.5, 77.1, 72.8, 56.1, 22.4; IR: (neat, $\mathrm{cm}^{-1}$ ) $\tilde{v}=619,807,881,990,1023,1108$, 1132, 1170, 1196, 1255, 1347, 1370, 1482, 1589, 2935, 2971; HRMS: calcd m/z for $\mathrm{C}_{20} \mathrm{H}_{25} \mathrm{I}_{2} \mathrm{O}_{4}[\mathrm{M}+\mathrm{H}]^{+}:$582.9837, found (ESI) 582.9827.

\section{4,4'-Bis(benzyloxy)-5,5'-diiodo-2,2'-dimethoxy-1,1'-biphenyl (220b):}<smiles>COc1cc(-c2cc(I)c(OCc3ccccc3)cc2I)c(OC)cc1OCc1ccccc1</smiles>

According to a literature procedure, ${ }^{[232]}$ to a round bottom flask, equipped with magnetic stirring bar under air was added compound 222b (1.0 equiv, $1.62 \mathrm{~g}, 3.79 \mathrm{mmol}$ ), followed by chloroform (105 ml) and silver trifluoroacetate $(2.4$ equiv, $2.01 \mathrm{~g}, 9.10 \mathrm{mmol})$. The mixture was allowed to stir at room temperature for 5 minutes, before adding a solution of iodine (2.3 equiv, $2.21 \mathrm{~g}, 8.72 \mathrm{mmol}$ ) in chloroform $(484 \mathrm{ml})$ dropwise over $3 \mathrm{~h}$, giving a pale red solution with a white precipitate. The mixture was filtered, washing with chloroform and the organic phase was washed successively with sodium thiosulfate (saturated aqueous), sodium bicarbonate (saturated, aqueous) and water and brine. The organic phase was dried $\left(\mathrm{Mg}_{2} \mathrm{SO}_{4}\right)$ and concentrated in vacuo, then purified by column chromatography (50 to $100 \%$ dichloromethane in petrol ether). Any residual pink colour from the chromatographed compounds was extracted with sodium thiosulfate and water. Compound $\mathbf{2 0 2 b}$ was obtained as a white solid $(2.19 \mathrm{~g}, 3.23$ $\mathrm{mmol}, 85 \%$ yield).

'H NMR: $\left(300 \mathrm{MHz}, \mathrm{CDCl}_{3}\right) \delta=7.56(\mathrm{~s}, 2 \mathrm{H}), 7.56-7.50(\mathrm{~m}, 4 \mathrm{H}), 7.47-7.29(\mathrm{~m}, 6 \mathrm{H}), 6.51$ (s, 2H), $5.19(\mathrm{~s}, 4 \mathrm{H}), 3.72(\mathrm{~s}, 6 \mathrm{H}) \mathrm{ppm} ;{ }^{13} \mathbf{C}\{\mathrm{H}\}$ NMR: $\left(126 \mathrm{MHz}, \mathrm{CDCl}_{3}\right) \delta=158.5,157.8$, 140.9, 136.6, 128.8, 128.1, 127.2, 121.3, 98.0, 75.1, 71.4, 56.0 ppm; IR: (neat, $\mathrm{cm}^{-1}$ ) $\tilde{v}=591$, 619, 629, 696, 724, 813, 1009, 1170, 1194, 1217, 1267, 1309, 1366, 1385, 1440, 1459, 1552, 1584, 2845, 2928, 2966, 3004; HRMS: calcd $\mathrm{m} / \mathrm{z}$ for $\mathrm{C}_{28} \mathrm{H}_{24} \mathrm{I}_{2} \mathrm{O}_{4} \mathrm{Na}^{+}[\mathrm{M}+\mathrm{Na}]^{+}$: 700.9656, found (ESI) 700.9656 . 


\section{5,5'-Diiodo-2,2',4,4'-tetramethoxy-1,1'-biphenyl (220c):}<smiles>COc1cc(OC)c(-c2cc(I)c(OC)cc2OC)cc1I</smiles>

Prepared as a light yellow solid (9.34 g, $17.8 \mathrm{mmol}, 97 \%$ yield) from compound 222c (1.0 equiv, $5.00 \mathrm{~g}, 18.23 \mathrm{mmol}$ ) using iodine (1.2 equiv, $2.78 \mathrm{~g}, 10.9 \mathrm{mmol}$ ) aqueous hydrochloric acid (0.3 equiv, $5.47 \mathrm{mmol}$, $0.46 \mathrm{ml}$ of a $37 \%$ aq. solution) and hydrogen peroxide (1.2 equiv, $21.9 \mathrm{mmol}, 1.88 \mathrm{ml}$ of a $35 \%$ aq. solution) in acetonitrile $(44 \mathrm{ml})$ according to GPJ.

${ }^{1} \mathrm{H}$ NMR: $\left(400 \mathrm{MHz}, \mathrm{CDCl}_{3}\right) \delta=7.54(\mathrm{~s}, 2 \mathrm{H}), 6.48(\mathrm{~s}, 2 \mathrm{H}), 3.93(\mathrm{~s}, 6 \mathrm{H}), 3.79(\mathrm{~s}, 6 \mathrm{H}) \mathrm{ppm} ;{ }^{13} \mathrm{C}$ NMR: $\left(101 \mathrm{MHz}, \mathrm{CDCl}_{3}\right) \delta=158.6,158.6,140.8,120.8,95.9,77.5,77.4,56.6,56.1$ ppm; IR: $\left(\right.$ neat, $\left.\mathrm{cm}^{-1}\right) \tilde{v}=455,620,809,1021,1168,1204,1250,1348,1438,1457,1590,2838$, 2939, 3001; HRMS: calcd $\mathrm{m} / z$ for $\mathrm{C}_{16} \mathrm{H}_{16} \mathrm{I}_{2} \mathrm{NaO}_{4}{ }^{+}[\mathrm{M}+\mathrm{Na}]^{+}:$548.9030; found (ESI) 548.9033.

\section{General procedure K (GPK)}

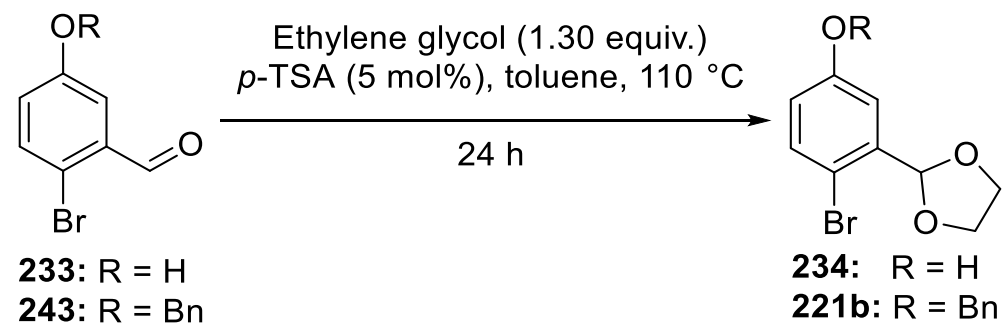

According to a literature procedure, ${ }^{[283]}$ to a round bottom flask equipped with magnetic stirrer, the respective aldehyde (1.0 equiv.), $p-\mathrm{TsOH} \cdot \mathrm{H}_{2} \mathrm{O}$ (5 mol\%), ethylene glycol (1.3 equiv.) and toluene $(0.45 \mathrm{M})$ were added under air. Using a Dean-Stark apparatus equipped with a reflux condenser, the reaction mixture was heated to reflux for $24 \mathrm{~h}$. After washing with a saturated aqueous solution of $\mathrm{NaHCO}_{3}$ and extracting the aqueous layer with dichloromethane, the combined organic layers were dried over $\mathrm{Na}_{2} \mathrm{SO}_{4}$, and the solvents were removed in vacuo. Purification by column chromatography afforded the product.

\section{4-Bromo-3-(1,3-dioxolan-2-yl)phenol (234)}<smiles>Oc1ccc(Br)c(C2OCCO2)c1</smiles>

ether.

Prepared as yellow crystals $(2.80 \mathrm{~g}, 11.43 \mathrm{mmol}, 50 \%$ yield) from compound 233 (1.0 equiv, $4.56 \mathrm{~g}, 22.7 \mathrm{mmol}), p-\mathrm{TsOH} \cdot \mathrm{H}_{2} \mathrm{O}(5 \mathrm{~mol} \%, 216 \mathrm{mg}, 1.13$ $\mathrm{mmol}$ ) and ethylene glycol (1.3 equiv, $1.83 \mathrm{~g}, 29.5 \mathrm{mmol})$ and toluene $(50 \mathrm{ml})$ according to GPK; column chromatography: $20-30 \%$ ethyl acetate in petrol

Analytical data corresponded to those described in the literature. ${ }^{[283]}$ 


\section{Experimental}

\section{2-(2-Bromo-5-isopropoxyphenyl)-1,3-dioxolane (221a)}<smiles>CC(C)Oc1ccc(Br)c(C2OCCO2)c1</smiles>

To a high pressure tube equipped with magnetic stirring bar was added compound 234 (1.00 equiv, $1.75 \mathrm{~g}, 7.14 \mathrm{mmol}$ ), $\mathrm{K}_{2} \mathrm{CO}_{3}$ (3.5 equiv, $3.41 \mathrm{~g}$, $24.7 \mathrm{mmol}$ ), iso-propyl bromide (1.3 equiv, $1.15 \mathrm{~g}, 9.35 \mathrm{mmol}$ ) and DMF (7 $\mathrm{ml})$, before stirring at $100^{\circ} \mathrm{C}$ overnight. On cooling, iso-propyl bromide $(0.13$ equiv, $0.24 \mathrm{~g}, 1.95 \mathrm{mmol}$ ) was added, and the suspension was stirred again for $2 \mathrm{~h}$ at 100 ${ }^{\circ} \mathrm{C}$. The reaction mixture was poured into water $(100 \mathrm{ml})$, and the aqueous phase was extracted with MTBE $(3 \times 40 \mathrm{ml})$. The combined organic layers were washed with water $(2 \times$ $50 \mathrm{ml}$ ), an aqueous solution of $\mathrm{NaOH}(1 \mathrm{M}, 50 \mathrm{ml})$ and dried over $\mathrm{Na}_{2} \mathrm{SO}_{4}$, before filtering and concentrating in vacuo. Purification by column chromatography $(10 \%$ ethyl acetate in petrol ether) afforded the product $221 \mathrm{a}(1.91 \mathrm{~g}, 6.65 \mathrm{mmol}, 93 \%$ yield) as a colorless oil.

'H NMR: $\left(300 \mathrm{MHz}, \mathrm{CDCl}_{3}\right) \delta=7.42(\mathrm{~d}, J=8.8 \mathrm{~Hz}, 1 \mathrm{H}), 7.14(\mathrm{~d}, J=3.1 \mathrm{~Hz}, 1 \mathrm{H}), 6.76(\mathrm{dd}, J$ $=8.8,3.1 \mathrm{~Hz}, 1 \mathrm{H}), 6.04(\mathrm{~s}, 1 \mathrm{H}), 4.54$ (hept, $J=6.1 \mathrm{~Hz}, 1 \mathrm{H}), 4.29-3.97(\mathrm{~m}, 4 \mathrm{H}), 1.32(\mathrm{~d}, J=$ 6.1 Hz, 6H) ppm; ${ }^{13} \mathrm{C}-\mathrm{NMR}:\left(126 \mathrm{MHz}, \mathrm{CDCl}_{3}\right) \delta=157.4,137.6,133.7,118.3,115.4,112.9$, 102.6, 70.5, 65.6, 22.2 ppm; IR: (neat, $\mathrm{cm}^{-1}$ ) $\tilde{v}=635,811,880,941,1027,1081,1111$, 1177, 1231, 1287, 1384, 1469, 1574, 1595, 2886, 2977, 3073; HRMS: calcd m/z for $\mathrm{C}_{12} \mathrm{H}_{15} \mathrm{BrO}_{3}{ }^{+}[\mathrm{M}]^{+}:$286.0205, found (EI) 286.0205.

\section{5-(Benzyloxy)-2-bromobenzaldehyde (243):}<smiles>O=Cc1cc(Br)ccc1Br</smiles>

To a round bottom flask equipped with a magnetic stirring bar, 2-bromo-5hydroxybenzaldehyde ( 1.0 equiv, $10.0 \mathrm{~g}, 49.8 \mathrm{mmol})$, acetonitrile $(60 \mathrm{ml})$ and $\mathrm{K}_{2} \mathrm{CO}_{3}$ (1.7 equiv, $11.7 \mathrm{~g}, 84.7 \mathrm{mmol}$ ) were added under air. The suspension was allowed to stir for a few minutes before adding benzyl bromide (1.05 equiv, $8.93 \mathrm{~g}$, $52.2 \mathrm{mmol}$ ) and allowed to stir at room temperature for 4 hours. The mixture was diluted with water and extracted with dichloromethane. The organic layers were combined, dried over $\mathrm{Na}_{2} \mathrm{SO}_{4}$, filtered and concentrated in vacuo. The product 243 was obtained as colorless oil (14.5 g, $49.8 \mathrm{mmol},>99 \%$ yield) and used without further purification.

Analytical data corresponded to those of the literature. ${ }^{[284]}$

\section{2-[5-(Benzyloxy)-2-bromophenyl]-1,3-dioxolane (221b):}

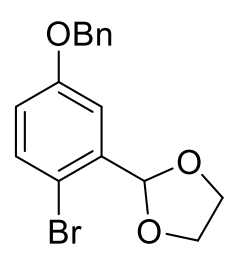

Prepared as a yellow solid (13.7 $\mathrm{g}, 40.9 \mathrm{mmol}, 82 \%$ yield) from compound 243 (1.0 equiv, $14.5 \mathrm{~g}, 49.8 \mathrm{mmol}$ ) using $p-\mathrm{TsOH} \cdot \mathrm{H}_{2} \mathrm{O}(5.2 \mathrm{~mol} \%, 490 \mathrm{mg}$, $2.58 \mathrm{mmol}$ ) and ethylene glycol (1.35 equiv, $4.16 \mathrm{~g}, 67.0 \mathrm{mmol}$ ) in toluene $(110 \mathrm{ml})$ according to GPK; column chromatography: 5-10\% ethyl acetate in hexanes. Compound $\mathbf{2 2 1 b}$ was stored in at $-20^{\circ} \mathrm{C}$ to avoid decomposition. 
Analytical data corresponded to those reported. ${ }^{[285]}$

\section{General procedure L (GPL)}<smiles>COc1cc(OC)c(-c2cc(OC)c(OC)cc2OC)cc1I</smiles><smiles>[R2]Oc1ccc([Ge]Cl)c(C2OCCO2)c1</smiles>

220a: $\mathrm{R}^{1}=\mathrm{iPr}$

220b: $\mathrm{R}^{1}=\mathrm{Bn}$

220c: $R^{1}=M e$ i). $\mathrm{Pd}_{2}(\mathrm{dba})_{3}(3 \mathrm{~mol} \%)$ SPhos (6 mol \%), THF $70{ }^{\circ} \mathrm{C}, 18 \mathrm{~h}$

ii). $\mathrm{HCl}(1 \mathrm{M}), \mathrm{THF}, \mathrm{T}, \mathrm{t}$<smiles>[R2]Oc1ccc(-c2cc(-c3cc(-c4cc(O[R2])ccc4OC)c(O[R1])cc3OC)c(C=O)cc2C=O)c(C=O)c1</smiles>

236a: $R^{1}=R^{2}=i P r$

236b: $R^{1}=R^{2}=B n$

236c: $R^{1}=M e, R^{2}=B n$

Following an adapted literature procedure, ${ }^{[266]}$ in a dried Schlenk flask equipped with a magnetic stirring bar, the aryl bromide 221 (3.0 equiv.) was dissolved in THF (0.5M) before cooling to $-78^{\circ} \mathrm{C}$ and adding nbutyllithium (3.4 equiv, $1.6 \mathrm{M}$ in hexanes) dropwise. The light yellow solution was allowed to stir at $-78{ }^{\circ} \mathrm{C}$ for $2 \mathrm{~h}$, before adding a freshly prepared solution of $\mathrm{ZnCl}_{2}$ in THF (7.4 equiv, $\left.1 \mathrm{M}\right)$ at $-78^{\circ} \mathrm{C}$. The reaction mixture was stirred for 30 min at $-78{ }^{\circ} \mathrm{C}$ and for $1.5 \mathrm{~h}$ at room temperature. Then $\mathrm{Pd}_{2}(\mathrm{dba})_{3}(3 \mathrm{~mol} \%)$, SPhos $(6 \mathrm{~mol} \%)$ and the aromatic iodide 220 (1.0 equiv) were added, before stirring at $70^{\circ} \mathrm{C}$ for $18 \mathrm{~h}$. On cooling, the reaction mixture was diluted with ethyl acetate and filtered through Celite ${ }^{\circledR}$, washing with ethyl acetate. The filtrate was washed with $\mathrm{NaHCO}_{3}$, extracting the aqueous layers with ethyl acetate. The combined organic layers were dried over $\mathrm{Na}_{2} \mathrm{SO}_{4}$, filtered and concentrated in vacuo.

The crude product was dissolved in THF $(0.015 \mathrm{M})$ and stirred with $1 \mathrm{M}$ aqueous hydrochloric acid at the specified temperature for the indicated time. Dichloromethane was then added, the layers were separated, and the organic layer was washed with water and brine. The aqueous layer was extracted with dichloromethane. The combined organic layers were dried over $\mathrm{MgSO}_{4}$, filtered and the volatiles removed in vacuo, before purification by column chromatography. 


\section{Experimental}

4,4",4"',6'-Tetraisopropoxy-4',6"-dimethoxy-[1,1':3',1":3",1"'-quaterphenyl]-2,2"'-dicarbaldehyde (236a):

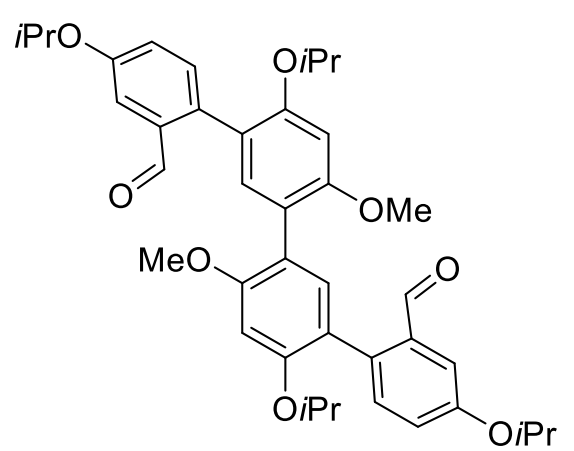

Prepared as a yellow solid ( $120 \mathrm{mg}, 183 \mu \mathrm{mol}, 51 \%$ yield over three steps) from compounds 220a (1.0 equiv, 208 $\mathrm{mg}, 357 \mu \mathrm{mol}$ ), 221a (3.07 equiv, $310 \mathrm{mg}, 1.08 \mathrm{mmol}$ ) in THF (2 ml), nBuLi (3.36 equiv, $1.20 \mathrm{mmol}, 0.75 \mathrm{ml}$ of a $1.6 \mathrm{M}$ solution in hexanes), $\mathrm{ZnCl}_{2}$ (7.28 equiv, $2.6 \mathrm{mmol}$, $2.6 \mathrm{ml}$ of a $1 \mathrm{M}$ solution in $\mathrm{THF}), \mathrm{Pd}_{2}(\mathrm{dba})_{3}(3.1 \mathrm{~mol} \%, 10$ $\mathrm{mg}, 10.9 \mu \mathrm{mol})$, SPhos (6.1 mol\%, $9 \mathrm{mg}, 21.9 \mu \mathrm{mol})$ according to GPL [stirring in THF (25 ml) and aqueous hydrochloric acid $(25 \mathrm{ml}, 1 \mathrm{M})$ at room temperature for $2 \mathrm{~h}$, column chromatography: 5-30\% ethyl acetate in petrol ether].

1'H NMR: $\left(300 \mathrm{MHz}, \mathrm{CDCl}_{3}\right) \delta=9.87(\mathrm{~s}, 2 \mathrm{H}), 7.46(\mathrm{~d}, J=2.8 \mathrm{~Hz}, 2 \mathrm{H}), 7.29(\mathrm{~d}, J=8.5 \mathrm{~Hz}$, 2H), 7.21 (s, 2H), 7.12 (dd, $J=8.5,2.8 \mathrm{~Hz}, 2 \mathrm{H}$ ), $6.61(\mathrm{~s}, 2 \mathrm{H}), 4.66$ (hept, $J=6.0 \mathrm{~Hz}, 2 \mathrm{H}$ ), 4.41 (hept, $J=6.0 \mathrm{~Hz}, 2 \mathrm{H}$ ), $3.85(\mathrm{~s}, 6 \mathrm{H}), 1.37(\mathrm{~d}, J=6.0 \mathrm{~Hz}, 12 \mathrm{H}), 1.25(\mathrm{~d}, J=6.0 \mathrm{~Hz}, 12 \mathrm{H}$ ); ${ }^{13} \mathrm{C}$ NMR: $\left(126 \mathrm{MHz}, \mathrm{CDCl}_{3}\right) \delta==157.1,155.6,155.03,134.2,133.3,129.8,127.8,125.4$, 125.2, 117.4, 114.7, 111.4, 99.1, 71.8, 69.9, 57.0, 22.7, 22.3; IR: $\left(\right.$ neat, $\left.\mathrm{cm}^{-1}\right) \tilde{v}=598,734$, $821,913,984,1042,1109,1162,1193,1240,1270,1385,1481,1601,1686,2972$; HRMS: calcd $m / z$ for $\mathrm{C}_{40} \mathrm{H}_{47} \mathrm{O}_{8}{ }^{+}[\mathrm{M}+\mathrm{H}]^{+}:$655.3265, found (ESI) 655.3256 .

\section{4,4",4"',6'-tetrakis(benzyloxy)-4',6"-dimethoxy-[1,1':3',1":3",1"'-quaterphenyl]-2,2'"- dicarbaldehyde (236b):}

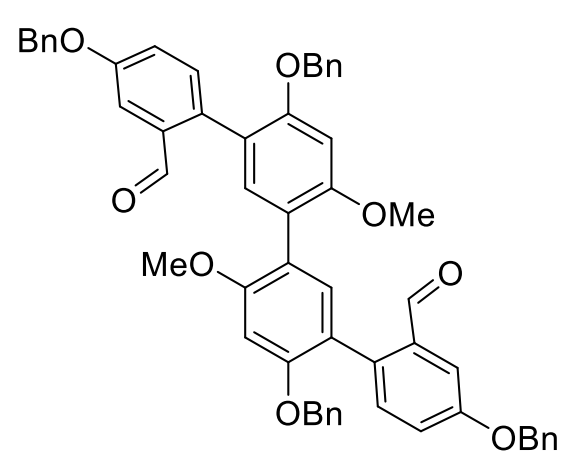

Prepared as a yellow solid (950 mg, $1.12 \mathrm{mmol}$, 45\% yield over three steps) using compounds $220 \mathrm{~b}$ ( 1.0 equiv, 1.69 $\mathrm{g}, 2.49 \mathrm{mmol}$ ), 221b (3.0 equiv, $2.50 \mathrm{~g}, 7.46 \mathrm{mmol}$ ) in THF $(15 \mathrm{ml}), n B u L i$ (3.08 equiv, $7.68 \mathrm{mmol}, 4.8 \mathrm{ml}$ of a $1.6 \mathrm{M}$ solution in hexanes), $\mathrm{ZnCl}_{2}$ (6.83 equiv, $17 \mathrm{mmol}, 17 \mathrm{ml}$ of a $1 \mathrm{M}$ solution in THF), $\mathrm{Pd}_{2}(\mathrm{dba})_{3}(3.0 \mathrm{~mol} \%, 68.4 \mathrm{mg}, 74.6$ $\mu \mathrm{mol})$, SPhos (6.0 mol\%, $61 \mathrm{mg}, 149 \mu \mathrm{mol})$ according to GPL [stirring in THF $(75 \mathrm{ml})$ and aqueous hydrochloric acid $(400 \mathrm{ml}, 1 \mathrm{M})$ at $60{ }^{\circ} \mathrm{C}$ for $2 \mathrm{~h}$, column chromatography: $20-30 \%$ ethyl acetate in petrol ether].

1'H NMR: $\left(500 \mathrm{MHz}, \mathrm{CDCl}_{3}\right) \delta=9.86(\mathrm{~s}, 2 \mathrm{H}), 7.50(\mathrm{~d}, J=2.8 \mathrm{~Hz}, 2 \mathrm{H}), 7.42-7.36(\mathrm{~m}, 4 \mathrm{H})$, $7.36-7.28(\mathrm{~m}, 6 \mathrm{H}), 7.21$ (ddt, $J=18.7,8.9,4.8 \mathrm{~Hz}, 14 \mathrm{H}), 7.12(\mathrm{~s}, 2 \mathrm{H}), 6.59(\mathrm{~s}, 2 \mathrm{H}), 5.07$ (s, $4 \mathrm{H}), 4.98$ (s, 4H), 3.72 (s, 6H).ppm; ${ }^{13} \mathrm{C}$ NMR: $\left(126 \mathrm{MHz}, \mathrm{CDCl}_{3}\right) \delta=192.9,158.0,157.9$, 156.0, 136.6, 136.5, 135.0, 134.9, 134.9, 133.0, 128.6, 128.5, 128.1, 127.8, 127.6, 127.0, 121.7, 119.4, 118.7, 110.3, 97.6, 70.9, 70.2, 55.9. ppm; IR: $\left(\right.$ neat, $\left.\mathrm{cm}^{-1}\right) \tilde{v}=694,731,817$, 


\section{Experimental}

$999,1014,1163,1190,1236,1269,1382,1452,1483,1603,1681,2747,2839,2934,3030$, 3063; HRMS: calcd $m / z$ for $\mathrm{C}_{56} \mathrm{H}_{46} \mathrm{NaO}_{8}{ }^{+}[\mathrm{M}+\mathrm{Na}]^{+}$: 869.3085; found (ESI) 869.3076.

\section{4,4"'-Bis(benzyloxy)-4',4",6',6"-tetramethoxy-[1,1':3',1":3",1"'-quaterphenyl]-2,2"'-- dicarbaldehyde (236c):}

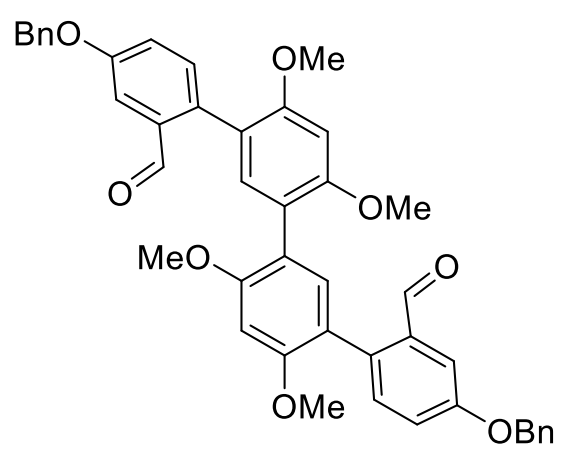

Prepared as a yellow foam $(1.31 \mathrm{~g}, 1.89 \mathrm{mmol}, 76 \%$ yield over three steps) from compounds $220 \mathrm{c}$ (1.0 equiv, 1.31 $\mathrm{g}, 2.49 \mathrm{mmol}$ ), 221b (3.0 equiv, $2.50 \mathrm{~g}, 7.46 \mathrm{mmol}$ ) in THF (15 ml), nBuLi (3.40 equiv, $8.38 \mathrm{mmol}, 5.24 \mathrm{ml}$ of a $1.6 \mathrm{M}$ solution in hexanes), $\mathrm{ZnCl}_{2}(7.30$ equiv, $18.2 \mathrm{mmol}$, $18.2 \mathrm{ml}$ of a $1 \mathrm{M}$ solution in THF), $\mathrm{Pd}_{2}(\mathrm{dba})_{3}(2.50 \mathrm{~mol} \%$, $56.9 \mathrm{mg}, 62.1 \mu \mathrm{mol})$, SPhos $(6.0 \mathrm{~mol} \%, 61.2 \mathrm{mg}, 149$ $\mu \mathrm{mol})$ according to GPL [stirring in THF $(25 \mathrm{ml})$ and aqueous hydrochloric acid $(100 \mathrm{ml}, 1 \mathrm{M})$ at room temperature for $2 \mathrm{~h}$, column chromatography: 0-40\% ethyl acetate in hexanes].

'H NMR: $\left(400 \mathrm{MHz}, \mathrm{CDCl}_{3}\right) \delta=9.86(\mathrm{~s}, 2 \mathrm{H}), 7.58(\mathrm{~d}, J=2.8 \mathrm{~Hz}, 2 \mathrm{H}), 7.50-7.44(\mathrm{~m}, 4 \mathrm{H})$, $7.44-7.37(\mathrm{~m}, 4 \mathrm{H}), 7.37-7.32(\mathrm{~m}, 4 \mathrm{H}), 7.27-7.23(\mathrm{~m}, 2 \mathrm{H}), 7.21(\mathrm{~s}, 2 \mathrm{H}), 6.62(\mathrm{~s}, 2 \mathrm{H}), 5.14$ (s, 4H), 3.89 (s, 6H), 3.81 (s, 6H) ppm; ${ }^{13} \mathrm{C}$ NMR: $\left(101 \mathrm{MHz}, \mathrm{CDCl}_{3}\right) \delta=193.0,158.2,158.1$, 156.9, 136.6, 134.9, 134.8, 134.7, 133.0, 128.8, 128.3, 127.8, 122.0, 119.1, 118.1, 110.3, 95.3, 70.3, 56.1, 55.7 ppm; IR: (neat, $\mathrm{cm}^{-1}$ ) $\tilde{v}=455,597,696,1027,1172,1203,1283$, 1466, 1481, 1603, 1682, 2827, 2883, 2934; HRMS: calcd $\mathrm{m} / \mathrm{z}$ for $\mathrm{C}_{44} \mathrm{H}_{38} \mathrm{NaO}_{8}{ }^{+}[\mathrm{M}+\mathrm{Na}]^{+}$: 717.2459; found (ESI) 717.2460.

\section{General procedure M (GPM)}

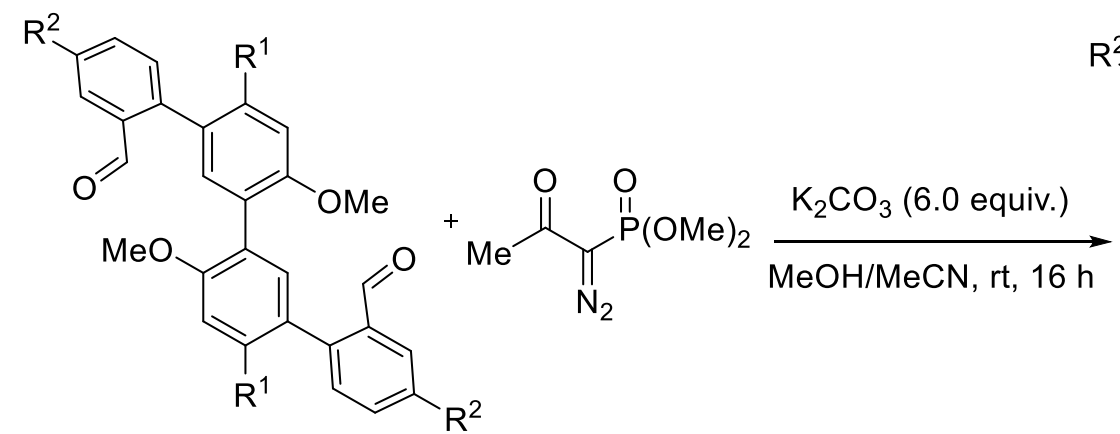

236a: $R^{1}=R^{2}=i P r$

236b: $R^{1}=R^{2}=B n$

236c: $R^{1}=M e, R^{2}=B n$

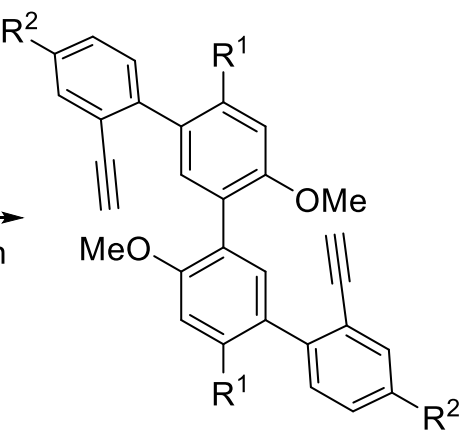

219a-c

To a dried Schlenk flask equipped with a magnetic stirring bar was added $\mathrm{K}_{2} \mathrm{CO}_{3}$ (6.0 equiv.), before drying under vacuum at $500{ }^{\circ} \mathrm{C}$ for $5 \mathrm{~min}$. After cooling to room temperature, the aldehyde 236 (1.0 equiv.), dry methanol ( $0.2 \mathrm{M})$ and the Ohira-Bestmann reagent (3.0 equiv.) 


\section{Experimental}

were added. A bubbler was attached to the flask, and the reaction was stirred overnight at room temperature. To quench the reaction, water was slowly added at $0{ }^{\circ} \mathrm{C}$ followed by dichloromethane. The aqueous layer was extracted with dichloromethane, the combined organic layers were dried over $\mathrm{Na}_{2} \mathrm{SO}_{4}$ and the solvents were removed under reduced pressure, before purifying by column chromatography.

\section{2,2"'-Diethynyl-4,4",4"',6'-tetraisopropoxy-4',6"-dimethoxy-1,1':3',1":3",1"'- quaterphenyl (219a):}

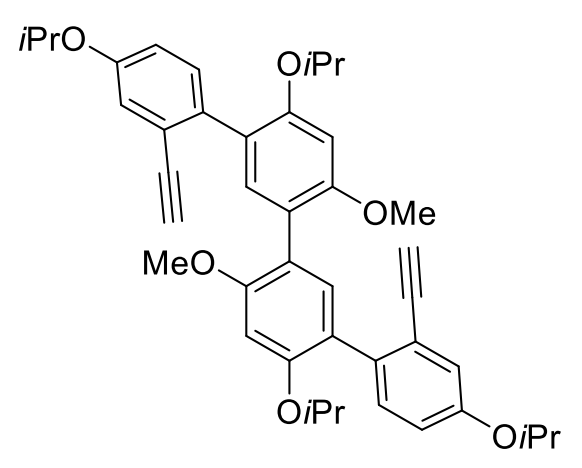

Prepared as a yellow solid (94.0 mg, $145.3 \mu \mathrm{mol}, 79 \%$ yield) from aldehyde 236a (1.0 equiv, $120 \mathrm{mg}, 183 \mu \mathrm{mol}$ ) using $\mathrm{K}_{2} \mathrm{CO}_{3}$ (6.3 equiv, $160 \mathrm{mg}, 1.16 \mathrm{mmol}$ ) and the Ohira-Bestmann reagent (3.1 equiv, $109 \mathrm{mg}, 567 \mu \mathrm{mol}$ ) in anhydrous dry methanol ( $1 \mathrm{ml}$ ) according to GPM (column chromatography: $20 \%$ ethyl acetate in petrol ether).

'H NMR: $\left(300 \mathrm{MHz}, \mathrm{CDCl}_{3}\right) \delta=7.28(\mathrm{~d}, J=8.6,0.5 \mathrm{~Hz}$, 2H), 7.26 (s, 2H), 7.07 (d, J = 2.7 Hz, 2H), 6.87 (dd, J = 8.6, 2.7 Hz, 2H), $6.61(s, 2 \mathrm{H}), 4.55$ (hept, $J=6.1 \mathrm{~Hz}, 2 \mathrm{H}$ ), 4.31 (hept, $J=6.1 \mathrm{~Hz}, 2 \mathrm{H}$ ), $3.81(\mathrm{~s}, 6 \mathrm{H}), 2.94(\mathrm{~d}, J=0.5 \mathrm{~Hz}, 2 \mathrm{H}$ ), 1.35 $(\mathrm{d}, J=6.1 \mathrm{~Hz}, 12 \mathrm{H}), 1.22(\mathrm{~d}, J=6.1 \mathrm{~Hz}, 12 \mathrm{H})$ ppm; ${ }^{13} \mathrm{C}-\mathrm{NMR}:\left(75 \mathrm{MHz}, \mathrm{CDCl}_{3}\right) \delta=157.4$, $156.2,155.5,135.0,134.4,132.3,123.0,122.7,120.1,119.5,116.9,100.4,83.9,79.3,72.0$, 70.2, 56.1, 22.4, 22.2 ppm; IR: neat) $\tilde{v}=631,118,908,981,1040,1110,1193,1239,1278$, 1383, 1478, 1599, 1681, 2975, 3277; HRMS: calcd $\mathrm{m} / \mathrm{z}$ for $\mathrm{C}_{42} \mathrm{H}_{47} \mathrm{O}_{6}[\mathrm{M}+\mathrm{H}]^{+}: 647.3367$, found(ESI) 647.3361 .

\section{4,4",4"',6'-Tetrakis(benzyloxy)-2,2"'-diethynyl-4',6"-dimethoxy-1,1':3',1":3",1"'-quarter- phenyl (219b):}

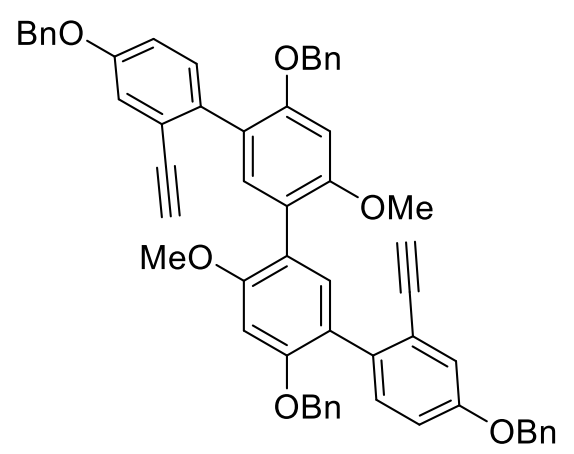

Prepared as a white solid (383 $\mathrm{mg}, 456 \mu \mathrm{mol}, 77 \%$ yield) from aldehyde 236b (1.0 equiv, $500 \mathrm{mg}, 590 \mu \mathrm{mol}$ ) using the Ohira-Bestmann reagent (3.0 equiv, $340 \mathrm{mg}, 1.77$ $\mathrm{mmol}$ ) and $\mathrm{K}_{2} \mathrm{CO}_{3}$ (6.0 equiv, $489 \mathrm{mg}, 3.54 \mathrm{mmol}$ ) in methanol $(2 \mathrm{ml})$ according to GPM [acetonitrile $(2 \mathrm{ml})$ was added to solubilize the substrate; column chromatography: $25 \%$ ethyl acetate in petrol ether].

'H NMR: $\left(300 \mathrm{MHz}, \mathrm{CDCl}_{3}\right) \delta=7.50-7.26(\mathrm{~m}, 24 \mathrm{H}), 7.20(\mathrm{~d}, \mathrm{~J}=2.7 \mathrm{~Hz}, 2 \mathrm{H}), 6.99(\mathrm{dd}, J=$ 8.6, $2.7 \mathrm{~Hz}, 2 \mathrm{H}), 6.65$ (s, 2H), 5.08 (s, 8H), 3.78 (s, 6H), 2.97 (s, 2H) ppm; ${ }^{13} \mathrm{C}$ NMR: (126 $\left.\mathrm{MHz}, \mathrm{CDCl}_{3}\right) \delta=157.3,157.0,156.0,137.4,136.7,134.5,134.3,132.0,128.5,128.3,127.9$, 127.4, 126.9, 122.8, 121.7, 119.7, 118.4, 115.8, 98.2, 83.6, 79.3, 70.9, 70.2, 55.9; IR: (neat, 
$\left.\mathrm{cm}^{-1}\right) \tilde{v}=624,694,733,816,1002,1022,1162,1191,1234,1283,1379,1452,1485,1599$, 2333, 2360, 2909, 2931, 3027, 3282 ppm; HRMS: calcd $\mathrm{m} / \mathrm{z}$ for $\mathrm{C}_{58} \mathrm{H}_{46} \mathrm{NaO}_{6}{ }^{+}[\mathrm{M}+\mathrm{Na}]^{+}$: 861.3187; found (ESI) 861.3189.

4,4"'-Bis(benzyloxy)-2,2"'-diethynyl-4',4",6',6"-tetramethoxy-1,1':3',1":3",1'"quaterphenyl (219c):

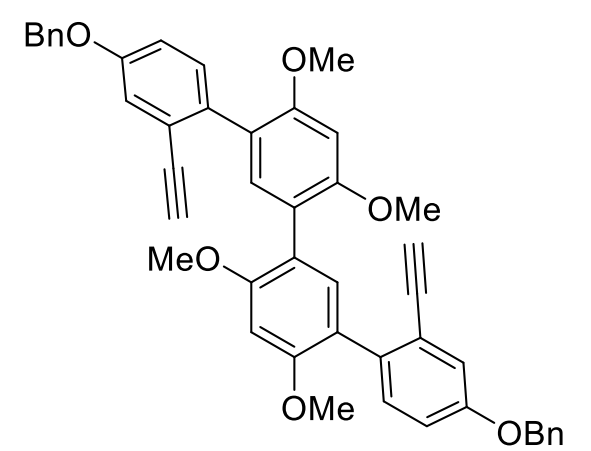

Prepared as a white solid (1.05 g, $1.53 \mathrm{mmol}, 87 \%$ yield) from aldehyde $236 \mathrm{c}$ ( 1.0 equiv, $1.22 \mathrm{~g}, 1.76 \mathrm{mmol}$ ) using the Ohira-Bestmann reagent (3.0 equiv, $1.02 \mathrm{~g}, 5.31$ $\mathrm{mmol})$ and $\mathrm{K}_{2} \mathrm{CO}_{3}(6.0$ equiv, $1.46 \mathrm{~g}, 10.6 \mathrm{mmol})$ in anhydrous dry methanol $(5 \mathrm{ml})$ and according to GPM [acetonitrile $(5 \mathrm{ml})$ was added to increase solubility of the in hexanes, then precipitation from hot ethyl acetate].

'H NMR: $\left(400 \mathrm{MHz}, \mathrm{CD}_{3} \mathrm{CN}\right) \delta=7.48-7.43(\mathrm{~m}, 4 \mathrm{H}), 7.43-7.31(\mathrm{~m}, 6 \mathrm{H}), 7.23(\mathrm{~d}, J=8.6$ $\mathrm{Hz}, 2 \mathrm{H}), 7.15$ (d, J = 2.7 Hz, 2H), $7.04(\mathrm{~s}, 2 \mathrm{H}), 7.01$ (dd, $J=8.6,2.8 \mathrm{~Hz}, 2 \mathrm{H}), 6.72(\mathrm{~s}, 2 \mathrm{H})$, $5.11(\mathrm{~s}, 4 \mathrm{H}), 3.82(\mathrm{~s}, 6 \mathrm{H}), 3.80(\mathrm{~s}, 6 \mathrm{H}), 3.24(\mathrm{~s}, 2 \mathrm{H}) \mathrm{ppm} ;{ }^{13} \mathrm{C}$ NMR: $\left(101 \mathrm{MHz}, \mathrm{CD}_{3} \mathrm{CN}\right) \delta=$ 158.7, 158.0, 157.9, 138.0, 134.9, 134.6, 132.9, 129.4, 128.9, 128.5, 123.7, 121.2, 119.7, 119.2, 116.7, 96.8, 84.0, 80.6, 70.5, 56.3, 56.1 ppm; IR: (neat, $\left.\mathrm{cm}^{-1}\right) \tilde{v}=532,662,695,814$, 901, 1028, 1097, 1155, 1201, 1298, 1453, 1599, 2839, 2927, 3257, 3352; HRMS: calcd m/z for $\mathrm{C}_{46} \mathrm{H}_{38} \mathrm{NaO}_{6}{ }^{+}[\mathrm{M}+\mathrm{Na}]^{+}:$709.2561; found (ESI) 709.2566. 


\section{Experimental}

\section{General procedure N (GPN)}

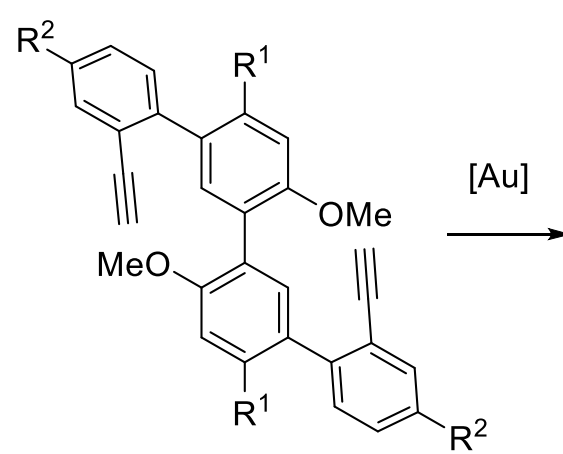

219a: $R^{1}=R^{2}=i \operatorname{Pr}$

219b: $R^{1}=R^{2}=B n$

219c: $R^{1}=M e, R^{2}=B n$

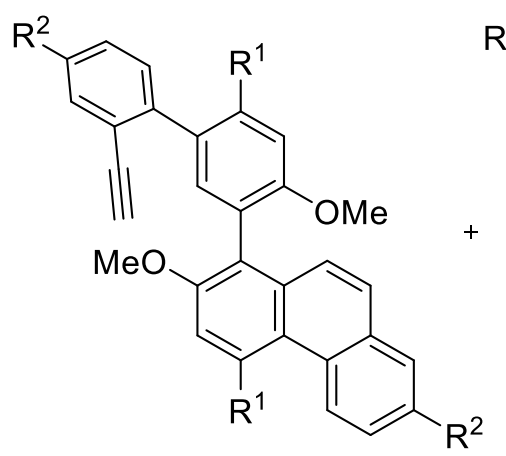

$237 a-c$<smiles></smiles>

218a-c

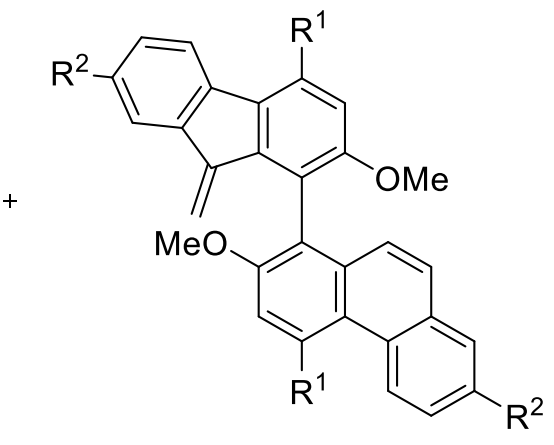

$238 a-c$

In a dried Schlenk flask equipped with a magnetic stirrer was added the substrate (1.0 equiv.), followed by the gold precatalyst ( $5 \mathrm{~mol} \%$ ). A septum was fitted to the Schlenk flask and the contents dried under high vacuum for 30 minutes, before performing three argon purge cycles. The reaction solvent $(0.05 \mathrm{M})$ was added via syringe through the septum and the reaction mixture was allowed to stir at room temperature for 2 minutes, before transferring to a pre-cooled bath and stirred for 15 minutes to reach the required temperature. A solution of $\mathrm{AgSbF}_{6}$ (5 mol\%, 0.05M in dichloromethane) was added through the septum, the septum was replaced with a greased glass stopper and the reaction was allowed to stir at the stated temperature for the indicated time. The reaction mixture was filtered through a short pad of silica eluting with dichloromethane, and the solvent was removed in vacuo, before drying under high vacuum. The conversion and ratio of isomers was determined by NMR and/or HPLC. The enantiomeric excess was determined by HPLC.

Reaction of 219a under gold catalysis:

Prepared from compound $219 \mathrm{a}(1.0$ equiv, $61.0 \mathrm{mg}, 94.3 \mu \mathrm{mol})$ using precatalyst $98 \mathrm{ff}$ (5.0 mol\%, $4.1 \mathrm{mg}, 4.75 \mu \mathrm{mol})$ and $\mathrm{AgSbF}_{6}(5.0 \mathrm{~mol} \%, 4.7 \mu \mathrm{mol}, 94 \mu \mathrm{l}$ of a $0.05 \mathrm{M}$ solution in dichloromethane) in dichloromethane $(1.9 \mathrm{ml}$ ) according to GPN (stirring for $45 \mathrm{~h}$ at room temperature). After purification by column chromatography $(40 \%$ ethyl acetate in petrol ether), the crude residue was resubmitted to the reaction conditions, using precatalyst (10.0 


\section{Experimental}

mol\%, $8.2 \mathrm{mg}, 9.50 \mu \mathrm{mol})$ and $\mathrm{AgSbF}_{6}(10.0 \mathrm{~mol} \%, 9.50 \mu \mathrm{mol}, 0.19 \mathrm{ml}$ of a $0.05 \mathrm{M}$ solution in dichloromethane) in dichloromethane $(1.9 \mathrm{ml})$, stirring at room temperature for $18 \mathrm{~h}$. The reaction was quenched by filtering through a silica plug, eluting with dichloromethane and concentrating the filtrate in vacuo. The crude mixture was purified by column chromatography (40\% ethyl acetate in petrol ether), affording a mixture of the product 218a and the 6-endo-dig/5-exo-dig isomer 238a (78:21), yellow foam (28.0 mg, $43.3 \mu \mathrm{mol}, 46 \%$ yield).

\section{4,4',7,7'-Tetraisopropoxy-2,2'-dimethoxy-1,1'-biphenanthrene (218a):}

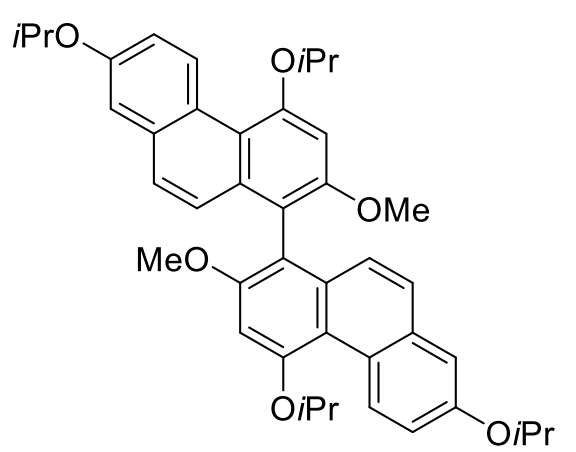

To obtain samples suitable for characterization, the mixture of isomers was purified by preparative HPLC. Separation conditions: $250 \times 20.0 \mathrm{~mm}$ YMC-Pack PVASIL column, particle size $5 \mu \mathrm{m}$, hexane/MTBE $=85: 15$ (v/v), $18.9 \mathrm{ml} \cdot \mathrm{min}^{-1}, 5.0 \mathrm{MPa}, 303 \mathrm{~K}, 254 \mathrm{~nm}$; HPLC: 250 $\mathrm{mm} \times 4.6 \mathrm{~mm}, 5 \mu \mathrm{m}$ YMC-pak PVA-sil column, hexane/MTBE $=85: 15,1 \mathrm{ml} \cdot \mathrm{min}^{-1}, 5.0 \mathrm{MPa}, 303 \mathrm{~K}, 254$ $\mathrm{nm}$; biphenanthrene 218a: $t_{R}=20.42 \mathrm{~min}$, isomer 238a: $t_{R}=21.30 \mathrm{~min}$.

'H NMR: $\left(300 \mathrm{MHz}, \mathrm{CDCl}_{3}\right) \delta=9.71(\mathrm{~d}, J=9.4 \mathrm{~Hz}, 2 \mathrm{H}), 7.32(\mathrm{~d}, \mathrm{~J}=9.1 \mathrm{~Hz}, 2 \mathrm{H}), 7.21$ (dd, $J$ = 9.4, $2.8 \mathrm{~Hz}, 2 \mathrm{H}), 7.14(\mathrm{~d}, J=2.8 \mathrm{~Hz}, 2 \mathrm{H}), 7.06(\mathrm{~d}, J=9.1 \mathrm{~Hz}, 2 \mathrm{H}), 7.06(\mathrm{~s}, 2 \mathrm{H}), 5.04-4.90$ $(\mathrm{m}, 2 \mathrm{H}), 4.71$ (hept, $J=6.0 \mathrm{~Hz}, 2 \mathrm{H}), 3.75(\mathrm{~s}, 6 \mathrm{H}), 1.65(\mathrm{~d}, J=6.0,0.8 \mathrm{~Hz}, 12 \mathrm{H}), 1.39(\mathrm{~d}, J=$ 6.0, 12H) ppm; ${ }^{13} \mathrm{C}$ NMR: $\left(126 \mathrm{MHz}, \mathrm{CDCl}_{3}\right) \delta=157.0,155.5,155.0,134.2,133.3,129.7$, 127.8, 125.4, 125.2, 117.4, 117.0, 114.8, 111.4, 99.2, 71.8, 70.0, 57.0, 22.8, 22.4 ppm; IR: neat) $\tilde{v}=565,726,827,908,966,1107,1197,1267,1325,1383,1460,1575,1606,2926$, 2975; HRMS: calcd $m / z$ for $\mathrm{C}_{42} \mathrm{H}_{47} \mathrm{O}_{6}{ }^{+}[\mathrm{M}+\mathrm{H}]^{+}:$647.3367, found (ESI) 647.3351.

\section{1-(4,7-Diisopropoxy-2-methoxy-9-methylene-9H-fluoren-1-yl)-4,7-diisopropoxy-2-} methoxyphenanthrene (238a):

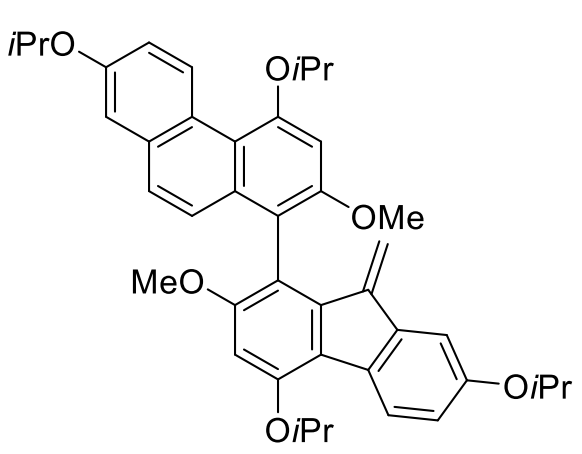

The 5-exo-dig/6-endo-dig product 238a could also be isolated from the semi-preparative HPLC separation; however, unfortunately it decomposed before could be fully characterized.

'H NMR: $\left(300 \mathrm{MHz}, \mathrm{CDCl}_{3}\right) \delta=9.68(\mathrm{~d}, J=9.8 \mathrm{~Hz}, 1 \mathrm{H})$, $7.93(\mathrm{~d}, J=8.6 \mathrm{~Hz}, 1 \mathrm{H}), 7.37(\mathrm{~d}, J=8.9 \mathrm{~Hz}, 1 \mathrm{H}), 7.29$ (d, $J=8.9 \mathrm{~Hz}, 1 \mathrm{H}), 7.20(\mathrm{dd}, J=9.8,2.9 \mathrm{~Hz}, 1 \mathrm{H}), 7.16(\mathrm{~d}, J$ $=2.9 \mathrm{~Hz}, 1 \mathrm{H}), 7.02(\mathrm{~s}, 1 \mathrm{H}), 7.01(\mathrm{~d}, J=2.4 \mathrm{~Hz}, 1 \mathrm{H}), 6.86(\mathrm{dd}, J=8.6,2.4 \mathrm{~Hz}, 1 \mathrm{H}), 6.67$ (s, $1 \mathrm{H}$ ), $5.50(\mathrm{~s}, 1 \mathrm{H}$ ), 4.94 (hept, $J=6.0 \mathrm{~Hz}, 1 \mathrm{H}$ ), 4.80 (hept, $J=6.0 \mathrm{~Hz}, 1 \mathrm{H}$ ), 4.71 (hept, $J=6.1$ 


\section{Experimental}

Hz, 1H), $4.53(\mathrm{~s}, 1 \mathrm{H}), 4.52$ (hept, $J=6.0 \mathrm{~Hz}, 1 \mathrm{H}), 3.78(\mathrm{~s}, 3 \mathrm{H}), 3.66(\mathrm{~s}, 3 \mathrm{H}), 1.62(\mathrm{~d}, \mathrm{~J}=6.0$ $\mathrm{Hz}, 6 \mathrm{H}), 1.56(\mathrm{~d}, J=6.0,6 \mathrm{H}), 1.39(\mathrm{dd}, J=6.1,1.0 \mathrm{~Hz}, 6 \mathrm{H}), 1.31(\mathrm{~d}, J=6.0 \mathrm{~Hz}, 6 \mathrm{H}) \mathrm{ppm}$.

\section{7,7'-Bis(benzyloxy)-2,2',4,4'-tetramethoxy-1,1'-biphenanthrene (218c):}

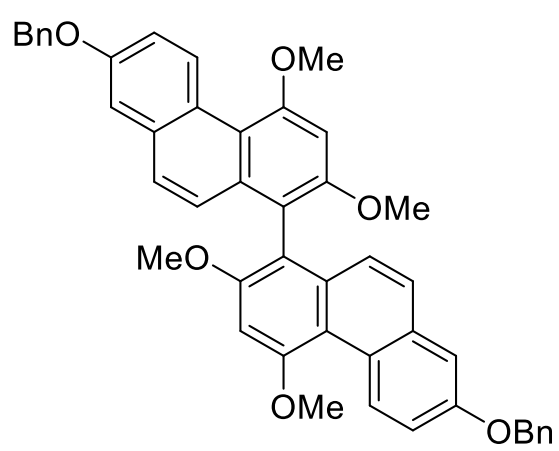

Prepared using 219c (1.0 equiv, $20.0 \mathrm{mg}, 29.1 \mu \mathrm{mol})$, precatalyst 172d (4.9 mol\%, $2.1 \mathrm{mg}, 1.43 \mu \mathrm{mol})$, dichloromethane $(0.58 \mathrm{ml})$ and $\mathrm{AgSbF}_{6}(5.0 \mathrm{~mol} \%, 1.45$ $\mu \mathrm{mol}, 29 \mu \mathrm{l}$ of a $0.05 \mathrm{M}$ solution in dichloromethane) according to GPN, affording the product mixture $(19.5 \mathrm{mg}$, $28.4 \mu \mathrm{mol}, 98 \%$ yield) in proportion $218 \mathrm{c}: 237 \mathrm{c}=96: 4$ as an off-white solid. Enantiomeric excess $=+47 \%$.

HPLC: Conditions for 2D separation: $50 \times 4.6 \mathrm{~mm}$ Agilent Eclipse Plus C8 $1.8 \mu \mathrm{m}$ column, $\mathrm{CH}_{3} \mathrm{CN} / \mathrm{H}_{2} \mathrm{O}=90 / 10(\mathrm{v} / \mathrm{v}), 1.0 \mathrm{ml} \cdot \mathrm{min}^{-1}$, $18.5 \mathrm{MPa}, 298 \mathrm{~K}, 244 \mathrm{~nm}, 218 \mathrm{c}: t_{R}=1.15 \mathrm{~min}, 237 \mathrm{c}$ $t_{R}=0.98 \mathrm{~min}$; then $150 \times 4.6 \mathrm{~mm}$ Chiralpak AS-3R column, $3 \mu \mathrm{m}, \mathrm{CH}_{3} \mathrm{CN} / \mathrm{H}_{2} \mathrm{O}=75 / 25-10^{\prime}$ - 90/10 $\mathrm{CH}_{3} \mathrm{CN} / \mathrm{H}_{2} \mathrm{O}(\mathrm{v} / \mathrm{v}), 1.0 \mathrm{ml} \cdot \mathrm{min}^{-1}, 14.5 \mathrm{MPa}, 298 \mathrm{~K}, 244 \mathrm{~nm}$; major enantiomer 218c: $t_{R}$ $=8.16 \mathrm{~min}$, minor enantiomer: $t_{R}=8.92 \mathrm{~min}$.

'H NMR: $\left(400 \mathrm{MHz}, \mathrm{CDCl}_{3}\right) \delta=9.58(\mathrm{~d}, J=9.5 \mathrm{~Hz}, 2 \mathrm{H}), 7.54-7.46(\mathrm{~m}, 4 \mathrm{H}), 7.45-7.30(\mathrm{~m}$, $10 \mathrm{H}$ ), 7.24 (d, J = $2.9 \mathrm{~Hz}, 2 \mathrm{H}$ ), 7.07 (d, J = $9.2 \mathrm{~Hz}, 2 \mathrm{H}$ ), 7.05 (s, 2H), 5.19 (s, 4H), 4.24 (s, $6 \mathrm{H}), 3.80$ (s, 6H) ppm; ${ }^{13} \mathrm{C}$ NMR: $\left(101 \mathrm{MHz} \mathrm{CDCl}_{3}\right) \delta=159.1,156.0,155.6,137.2,134.1$, 133.2, 129.7, 128.7, 128.1, 128.0, 127.7, 125.4, 125.3, 116.9, 116.0, 114.3, 109.8, 96.3, 70.0, 56.9, 55.9 ppm; IR: (neat, $\left.\mathrm{cm}^{-1}\right) \tilde{v}=500,638,697,742,781,824,859,963,1024$, 1087, 1169, 1204, 1276, 1335, 1455, 1470, 1576, 1608, 2840, 2929, 2996, 3032; HRMS: calcd $m / z$ for $\mathrm{C}_{46} \mathrm{H}_{39} \mathrm{O}_{6}{ }^{+}[\mathrm{M}+\mathrm{H}]^{+}: 687.2741$; found (ESI) 687.2739 .

\section{7-(Benzyloxy)-1-(4'-(benzyloxy)-2'-ethynyl-4,6-dimethoxy-[1,1'-biphenyl]-3-yl)-2,4-} dimethoxyphenanthrene (237c):

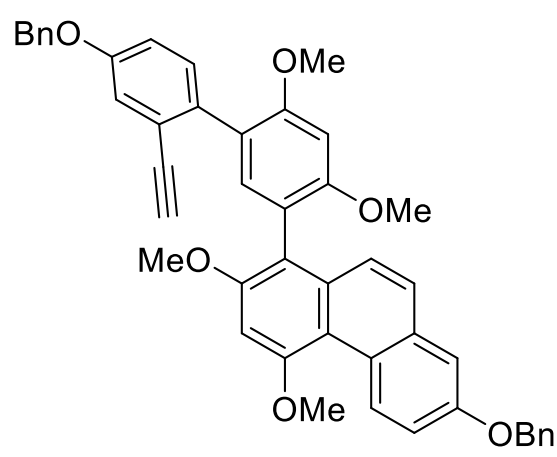

Prepared using 219c (1.0 equiv, $200 \mathrm{mg}, 291 \mu \mathrm{mol}$ ), precatalyst 172n (5.0 mol\%, $18.5 \mathrm{mg}, 14: 6 \mu \mathrm{mol})$, dichloromethane (5.8 ml) and $\mathrm{AgSbF}_{6}(5.0 \mathrm{~mol} \%, 5.0 \mathrm{mg}$, $14.6 \mu \mathrm{mol})$ according to GPN. After $4 \mathrm{~h}(\mathrm{~F} 1)$ and $20 \mathrm{~h}$ (F2), a $1 \mathrm{ml}$ aliquot was taken and eluted through a pad of silica, affording product mixtures in proportion 218c:237c:238c $=(F 1)$ 2: 34: 64 and (F2) 42: 55: 3, respectively. After $44 \mathrm{~h}$, the remainder of the reaction was quenched by eluting through a pad of silica and evaporating to dryness, affording $218 \mathrm{c}$ as an off white solid. 
A sample of 237c suitable for analysis was obtained by semi-preparative HPLC of F2. Separation conditions: $150 \times 20.0 \mathrm{~mm}$ YMC-ODS-A column (particle size $5 \mu \mathrm{m}$ ), 100\% acetonitrile, $10 \mathrm{ml} \cdot \mathrm{min}^{-1}, 1.7 \mathrm{MPa}, 308 \mathrm{~K}, 244 \mathrm{~nm}, \mathrm{t}_{\mathrm{R}}=4.60 \mathrm{~min}$.

'H NMR: $\left(400 \mathrm{MHz}, \mathrm{CDCl}_{3}\right) \delta=9.53(\mathrm{~d}, J=9.3 \mathrm{~Hz}, 1 \mathrm{H}), 7.54-7.48(\mathrm{~m}, 2 \mathrm{H}), 7.47(\mathrm{~s}, 2 \mathrm{H})$, $7.46-7.40(\mathrm{~m}, 4 \mathrm{H}), 7.39(\mathrm{dt}, J=2.7,1.2 \mathrm{~Hz}, 2 \mathrm{H}), 7.38-7.31(\mathrm{~m}, 4 \mathrm{H}), 7.28(\mathrm{t}, J=3.2 \mathrm{~Hz}$, $1 \mathrm{H}), 7.19(\mathrm{~s}, 1 \mathrm{H}), 7.19(\mathrm{~s}, 1 \mathrm{H}), 7.00(\mathrm{dd}, J=8.6,2.8 \mathrm{~Hz}, 1 \mathrm{H}), 6.96(\mathrm{~s}, 1 \mathrm{H}), 6.72(\mathrm{~s}, 1 \mathrm{H}), 5.21$ $(\mathrm{s}, 2 \mathrm{H}), 5.06(\mathrm{~s}, 2 \mathrm{H}), 4.17(\mathrm{~s}, 3 \mathrm{H}), 3.90(\mathrm{~s}, 3 \mathrm{H}), 3.88(\mathrm{~s}, 3 \mathrm{H}), 3.75(\mathrm{~s}, 3 \mathrm{H}), 2.99(\mathrm{~s}, 1 \mathrm{H}) \mathrm{ppm}$; ${ }^{13} \mathrm{C}$ NMR: $\left(101 \mathrm{MHz}, \mathrm{CDCl}_{3}\right) \delta=158.8,158.4,157.3,157.3,156.1,155.1,137.3,136.9$, $135.9,134.3,133.7,133.1,132.2,129.7,128.7,128.7,128.7,128.2,128.1,127.7,127.7$, 127.7, 127.6, 125.8, 125.2, 123.0, 121.1, 118.6, 117.4, 116.8, 116.3, 116.0, 115.9, 109.8, 96.4, 96.1, 83.6, 79.3, 70.2, 70.1, 57.0, 56.1, 55.9, 55.9; IR: (neat, $\left.\mathrm{cm}^{-1}\right) \tilde{v}=648,696,729$, 907, 1027, 1088, 1163, 1201, 1277, 1350, 1454, 1578, 1605, 2240, 2841, 2929, 2999, 3033, 3289; HRMS: calcd $m / z$ for $\mathrm{C}_{46} \mathrm{H}_{38} \mathrm{NaO}_{6}{ }^{+}[\mathrm{M}+\mathrm{Na}]^{+}:$709.2561; found (ESI) 709.2561. 


\section{References}

[1] B. Lindström, L. J. Pettersson, CATTECH 2003, 7, 130.

[2] A. de Meijere, S. Bräse, M. Oestreich (Eds.) Metal-Catalyzed Cross-Coupling Reactions and More., Wiley-VCH Verlag GmbH, Weinheim, Germany, 2014.

[3] R. Noyori, H. Takaya, Acc. Chem. Res. 2002, 23, 345.

[4] W. S. Knowles, Angew. Chem. Int. Ed. 2002, 41, 1998.

[5] K. B. Sharpless, Angew. Chem. Int. Ed. 2002, 41, 2024.

[6] B. Armer, H. Schmidbaur, Angew. Chem. Int. Ed. Engl., 9, 101.

[7] A. S. K. Hashmi, G. J. Hutchings, Angew. Chem. Int. Ed. 2006, 45, 7896.

[8] H. Schmidbaur, Interdisc. Sci. Rev. 2013, 17, 213.

[9] G. C. Bond, P. A. Sermon, G. Webb, D. A. Buchanan, P. B. Wells, J. Chem. Soc., Chem. Commun. 1973, 444b-445.

[10] M. Haruta, T. Kobayashi, H. Sano, N. Yamada, Chem. Lett. 1987, 16, 405.

[11] G. J. Hutchings, J. Catal. 1985, 96, 292.

[12] Y. Ito, M. Sawamura, T. Hayashi, J. Am. Chem. Soc. 1986, 108, 6405.

[13] a) C. Bour, V. Gandon, Synlett 2015, 26, 1427; b) A. Fürstner, Chem. Soc. Rev. 2009, 38, 3208; c) C. Nieto-Oberhuber, S. López, E. Jiménez-Núñez, A. M. Echavarren, Chem. Eur. J. 2006, 12, 5916; d) E. Jiménez-Núñez, A. M. Echavarren, Chem. Rev. 2008, 108, 3326.

[14] A. Fürstner, P. W. Davies, Angew. Chem. Int. Ed. 2007, 46, 3410.

[15] Y. Fukuda, K. Utimoto, H. Nozaki, HETEROCYCLES 1987, 25, 297.

[16]Y. Fukuda, K. Utimoto, J. Org. Chem. 1991, 56, 3729.

[17] J. H. Teles, S. Brode, M. Chabanas, Angew. Chem. Int. Ed. 1998, 37, 1415.

[18] a) A. S. K. Hashmi, L. Schwarz, J.-H. Choi, T. M. Frost, Angew. Chem. Int. Ed. 2000, 39, 2285; for recent reviews see also: b) D. P. Day, P. W. Hong Chan, Adv. Synth. Catal. 2016, 358, 1368; c) A. M. Asiri, A. S. K. Hashmi, Chem. Soc. Rev. 2016, 45, 4471.

[19] A. S. K. Hashmi, T. M. Frost, J. W. Bats, J. Am. Chem. Soc. 2000, 122, 11553.

[20] G. Dyker, Angew. Chem. Int. Ed. 2000, 39, 4237.

[21] T. F. Dean, S. K. A. Hashmi, A. M. Echavarren, Adv. Synth. Catal. 2016, 358, 1347.

[22] H. Schmidbaur, Angew. Chem. Int. Ed. Engl. 1976, 15, 728.

[23] D. J. Gorin, F. D. Toste, Nature 2007, 446, 395.

[24] P. Pyykkö, Angew. Chem. Int. Ed. 2004, 43, 4412.

[25] B. Ranieri, I. Escofet, A. M. Echavarren, Org. Biomol. Chem. 2015, 13, 7103.

[26] a) R. Dorel, A. M. Echavarren, Chem. Rev. 2015, 115, 9028; b) C. Obradors, A. M.

Echavarren, Chem. Commun. 2014, 50, 16; c) C. Obradors, A. M. Echavarren, Acc. Chem. Res. 2014, 47, 902. 


\section{References}

[27] a) Z. Lu, J. Han, G. B. Hammond, B. Xu, Org. Lett. 2015, 17, 4534; b) D. Wang, R. Cai,

S. Sharma, J. Jirak, S. K. Thummanapelli, N. G. Akhmedov, H. Zhang, X. Liu, J. L.

Petersen, X. Shi, J. Am. Chem. Soc. 2012, 134, 9012.

[28]a) J. Chatt, L. A. Duncanson, J. Chem. Soc. 1953, 2939; b) M. Dewar, Bull. Soc. Chim. Fr. 1951, 18, 79.

[29] a) M. S. Nechaev, V. M. Rayón, G. Frenking, J. Phys. Chem. A 2004, 108, 3134; b) R. H. Hertwig, W. Koch, D. Schröder, H. Schwarz, J. Hrušák, P. Schwerdtfeger, J. Phys. Chem. 1996, 100, 12253.

[30]S. Flügge, A. Anoop, R. Goddard, W. Thiel, A. Fürstner, Chem. Eur. J. 2009, 15, 8558. [31]Z. Li, C. Brouwer, C. He, Chem. Rev. 2008, 108, 3239.

[32] a) D. B. Huple, S. Ghorpade, R.-S. Liu, Adv. Synth. Catal. 2016, 358, 1348; b) W.

Debrouwer, T. S. A. Heugebaert, B. I. Roman, C. V. Stevens, Adv. Synth. Catal. 2015, 357, 2975; c) L. Fensterbank, M. Malacria, Acc. Chem. Res. 2014, 47, 953.

[33]R. J. Harris, R. A. Widenhoefer, Chem. Soc. Rev. 2016, 45, 4533.

[34]Y. Wang, M. E. Muratore, A. M. Echavarren, Chem. Eur. J. 2015, 21, 7332.

[35]D. Qian, J. Zhang, Chem. Soc. Rev. 2015, 44, 677.

[36] a) D. J. Gorin, B. D. Sherry, F. D. Toste, Chem. Rev. 2008, 108, 3351; b) W. Wang, G.

B. Hammond, B. Xu, J. Am. Chem. Soc. 2012, 134, 5697.

[37] P. Mauleón, R. M. Zeldin, A. Z. González, F. D. Toste, J. Am. Chem. Soc. 2009, 131, 6348.

[38]I. Alonso, B. Trillo, F. López, S. Montserrat, G. Ujaque, L. Castedo, A. Lledós, J. L. Mascareñas, J. Am. Chem. Soc. 2009, 131, 13020.

[39]D. Benitez, E. Tkatchouk, A. Z. Gonzalez, W. A. Goddard, F. D. Toste, Org. Lett. 2009, $11,4798$.

[40]W. Zi, F. D. Toste, Chem. Soc. Rev. 2016, 45, 4567.

[41]Y. Li, W. Li, J. Zhang, Chem. Eur. J. 2016, 23, 467.

[42] R. A. Widenhoefer, Chem. Eur. J. 2008, 14, 5382.

[43] M. P. Muñoz, J. Adrio, J. C. Carretero, A. M. Echavarren, Organometallics 2005, 24, 1293.

[44]Y.-M. Wang, A. D. Lackner, F. D. Toste, Acc. Chem. Res. 2014, 47, 889.

[45] A. Pradal, P. Toullec, V. Michelet, Synthesis 2011, 2011, 1501.

[46]S. Sengupta, X. Shi, ChemCatChem 2010, 2, 609.

[47] a) T. P. Yoon, E. N. Jacobsen, Science 2003, 299, 1691; b) H. Shimizu, I. Nagasaki, K. Matsumura, N. Sayo, T. Saito, Acc. Chem. Res. 2007, 40, 1385; c) W. Zhang, Y. Chi, X. Zhang, Acc. Chem. Res. 2007, 40, 1278; d) A. Börner (Ed.) Phosphorus Ligands in Asymmetric Catalysis, Wiley-VCH Verlag GmbH, Weinheim, Germany, 2008; e) F. L. Lam, F. Y. Kwong, A. S. C. Chan, Top. Organomet. Chem. 2011, 36, 29. 
[48] R. L. LaLonde, B. D. Sherry, E. J. Kang, F. D. Toste, J. Am. Chem. Soc. 2007, 129, 2452.

[49] D. H. Miles, M. Veguillas, F. D. Toste, Chem. Sci. 2013, 4, 3427.

[50] S. G. Sethofer, T. Mayer, F. D. Toste, J. Am. Chem. Soc. 2010, 132, 8276.

[51] For alternative enantioselective gold catalysts in this reaction, see also: C. Bartolomé, D. García-Cuadrado, Z. Ramiro, P. Espinet, Inorg. Chem. 2010, 49, 9758.

[52] H. Teller, S. Flügge, R. Goddard, A. Fürstner, Angew. Chem. Int. Ed. 2010, 49, 1949.

[53] G. L. Hamilton, E. J. Kang, M. Mba, F. D. Toste, Science 2007, 317, 496.

[54] R. L. Lalonde, Z. J. Wang, M. Mba, A. D. Lackner, F. D. Toste, Angew. Chem. Int. Ed. 2010, 49, 598.

[55] Y.-M. Wang, C. N. Kuzniewski, V. Rauniyar, C. Hoong, F. D. Toste, J. Am. Chem. Soc. 2011, 133, 12972.

[56] J. Francos, F. Grande-Carmona, H. Faustino, J. Iglesias-Sigüenza, E. Díez, I. Alonso, R. Fernández, J. M. Lassaletta, F. López, J. L. Mascareñas, J. Am. Chem. Soc. 2012, 134, 14322.

[57] a) H. Faustino, F. López, L. Castedo, J. L. Mascareñas, Chem. Sci. 2011, 2, 633. On the other hand, the [2+2] cycloaddition could observed, but using SEGPHOS: b) M. Jia, M. Monari, Q.-Q. Yang, M. Bandini, Chem. Commun. 2015, 51, 2320.

[58] J. F. Teichert, B. L. Feringa, Angew. Chem. Int. Ed. 2010, 49, 2486.

[59] A. Z. González, F. D. Toste, Org. Lett. 2010, 12, 200.

[60] I. Alonso, H. Faustino, F. López, J. L. Mascareñas, Angew. Chem. Int. Ed. 2011, 50, 11496.

[61] H. Teller, M. Corbet, L. Mantilli, G. Gopakumar, R. Goddard, W. Thiel, A. Fürstner, J. Am. Chem. Soc. 2012, 134, 15331.

[62] P. Chen, Angew. Chem. Int. Ed. 2003, 42, 2832.

[63] K. H. Shaughnessy, Chem. Rev. 2009, 109, 643.

[64] a) K. Ohmatsu, M. Ito, T. Kunieda, T. Ooi, J. Am. Chem. Soc. 2013, 135, 590; b) K.

Ohmatsu, M. Ito, T. Kunieda, T. Ooi, Nat. Chem. 2012, 4, 473.

[65]U. Zoller, Tetrahedron 1988, 44, 7413.

[66] I. V. Komarov, M. Y. Kornilov, A. A. Tolmachev, A. A. Yurchenko, E. B. Rusanov, A. N.

Chernega, Tetrahedron 1995, 51, 11271.

[67] N. Kuhn, J. Fahl, D. Bläser, R. Boese, Z. anorg. allg. Chem. 1999, 625, 729.

[68] D. Mendoza-Espinosa, B. Donnadieu, G. Bertrand, J. Am. Chem. Soc. 2010, 132, 7264.

[69] L. Gu, Y. Zheng, E. Haldón, R. Goddard, E. Bill, W. Thiel, M. Alcarazo, Angew. Chem. Int. Ed. 2017, 56, 8790.

[70] M. Mehta, T. C. Johnstone, J. Lam, B. Bagh, A. Hermannsdorfer, M. Driess, D. W. Stephan, Dalton Trans. 2017, 46, 14149. 


\section{References}

[71]M. H. Holthausen, M. Mehta, D. W. Stephan, Angew. Chem. Int. Ed. 2014, 53, 6538.

[72] C. Maaliki, C. Lepetit, Y. Canac, C. Bijani, C. Duhayon, R. Chauvin, Chem. Eur. J. 2012, 18, 7705.

[73]J. Sirieix, M. Oßberger, B. Betzemeier, P. Knochel, Synlett 2000, 2000, 1613.

[74]J. Ruiz, A. F. Mesa, D. Sol, Organometallics 2015, 34, 5129.

[75]R. Javier, M. A. F, Chem. Eur. J. 2012, 18, 4485.

[76]K. Schwedtmann, R. Schoemaker, F. Hennersdorf, A. Bauza, A. Frontera, R. Weiss, J. J. Weigand, Dalton Trans. 2016, 45, 11384.

[77] K. Schwedtmann, M. H. Holthausen, K.-O. Feldmann, J. J. Weigand, Angew. Chem. Int. Ed. 2013, 52, 14204.

[78]M. Azouri, J. Andrieu, M. Picquet, P. Richard, B. Hanquet, I. Tkatchenko, Eur. J. Inorg. Chem. 2007, 2007, 4877.

[79]J. J. Weigand, K.-O. Feldmann, F. D. Henne, J. Am. Chem. Soc. 2010, 132, 16321.

[80]U. Hintermair, U. Englert, W. Leitner, Organometallics 2011, 30, 3726.

[81] a) A. A. Tolmachev, A. A. Yurchenko, A. S. Merculov, M. G. Semenova, E. V.

Zarudnitskii, V. V. Ivanov, A. M. Pinchuk, Heteroatom Chem. 1999, 10, 585; b) Y. Canac,

C. Maaliki, I. Abdellah, R. Chauvin, New J. Chem. 2012, 36, 17; c) V. Karthik, V. Gupta,

G. Anantharaman, Organometallics 2015, 34, 3713.

[82]Á. Kozma, T. Deden, J. Carreras, C. Wille, J. Petuškova, J. Rust, M. Alcarazo, Chem. Eur. J. 2014, 20, 2208.

[83]L. Gu, L. M. Wolf, A. Zieliński, W. Thiel, M. Alcarazo, J. Am. Chem. Soc. 2017, 139, 4948.

[84]E. Haldón, Á. Kozma, H. Tinnermann, L. Gu, R. Goddard, M. Alcarazo, Dalton Trans. 2016, 45, 1872.

[85] J. W. Dube, Y. Zheng, W. Thiel, M. Alcarazo, J. Am. Chem. Soc. 2016, 138, 6869.

[86] H. Tinnermann, C. Wille, M. Alcarazo, Angew. Chem. Int. Ed. 2014, 53, 8732.

[87]H. Tinnermann, Dissertation, Georg-August-Universität Göttingen, Göttingen, 2017.

[88]M. Alcarazo, Acc. Chem. Res. 2016, 49, 1797.

[89]M. Azouri, J. Andrieu, M. Picquet, H. Cattey, Inorg. Chem. 2009, 48, 1236.

[90]F. D. Henne, A. T. Dickschat, F. Hennersdorf, K.-O. Feldmann, J. J. Weigand, Inorg. Chem. 2015, 54, 6849.

[91] C. Maaliki, Y. Canac, C. Lepetit, C. Duhayon, R. Chauvin, RSC Adv. 2013, 3, 20391.

[92] G. Mehler, P. Linowski, J. Carreras, A. Zanardi, J. W. Dube, M. Alcarazo, Chem. Eur. J. 2016, 22, 15320.

[93] C. D. Mboyi, C. Maaliki, A. Mankou Makaya, Y. Canac, C. Duhayon, R. Chauvin, Inorg. Chem. 2016, 55, 11018.

[94]B. D. Ellis, C. A. Dyker, A. Decken, C. L. B. Macdonald, Chem. Commun. 2005, 1965. 
[95] J. Carreras, G. Gopakumar, L. Gu, L. Gu, A. Gimeno, P. Linowski, J. Petuškova, W.

Thiel, M. Alcarazo, J. Am. Chem. Soc. 2013, 135, 18815.

[96] L. Gu, L. M. Wolf, W. Thiel, C. W. Lehmann, M. Alcarazo, Organometallics 2017, 37, 665.

[97] J. Petuškova, M. Patil, S. Holle, C. W. Lehmann, W. Thiel, M. Alcarazo, J. Am. Chem. Soc. 2011, 133, 20758.

[98] C. A. Tolman, J. Am. Chem. Soc. 1970, 92, 2953.

[99] D. G. Gusev, Organometallics 2009, 28, 763.

[100] V. V. Pavlishchuk, A. W. Addison, Inorg. Chim. Acta 2000, 298, 97.

[101] X. Marset, A. Khoshnood, L. Sotorríos, E. Gómez-Bengoa, D. A. Alonso, D. J.

Ramón, ChemCatChem 2017, 9, 1269.

[102] S. Saleh, E. Fayad, M. Azouri, J.-C. Hierso, J. Andrieu, M. Picquet, Adv. Synth. Catal. 2009, 351, 1621.

[103] D. J. Brauer, K. W. Kottsieper, C. Liek, O. Stelzer, H. Waffenschmidt, P.

Wasserscheid, J. Organomet. Chem. 2001, 630, 177.

[104] J. Li, J. Peng, Y. Bai, G. Zhang, G. Lai, X. Li, J. Organomet. Chem. 2010, 695, 431.

[105] J. Petuškova, H. Bruns, M. Alcarazo, Angew. Chem. Int. Ed. 2011, 50, 3799.

[106] M. Alcarazo, Chem. Eur. J. 2014, 20, 7868.

[107] F. Inagaki, C. Matsumoto, Y. Okada, N. Maruyama, C. Mukai, Angew. Chem. Int. Ed. 2015, 54, 818.

[108] A. Fürstner, H. Szillat, F. Stelzer, J. Am. Chem. Soc. 2000, 122, 6785.

[109] S. M. Kim, S. I. Lee, Y. K. Chung, Org. Lett. 2006, 8, 5425.

[110] V. Mamane, P. Hannen, A. Fürstner, Chem. Eur. J. 2004, 10, 4556.

[111] J. Carreras, M. Patil, W. Thiel, M. Alcarazo, J. Am. Chem. Soc. 2012, 134, 16753.

[112] P. L. Majumder, A. Kar, J. N. Shoolery, Phytochemistry 1985, 24, 2083.

[113] P. Majumder, D. Bandyopadhyay, S. Joardar, J. Chem. Soc., Perkin Trans. 11982 , 1131.

[114] W.-K. Li, J.-Q. Pan, M.-J. Lü, R.-Y. Zhang, P.-G. Xiao, Phytochemistry 1995, 39, 231.

[115] C.-L. Lee, F.-R. Chang, M.-H. Yen, D. Yu, Y.-N. Liu, K. F. Bastow, S. L. Morris-

Natschke, Y.-C. Wu, K.-H. Lee, J. Nat. Prod. 2009, 72, 210.

[116] M. Gingras, Chem. Soc. Rev. 2013, 42, 1051.

[117] M. Gingras, G. Félix, R. Peresutti, Chem. Soc. Rev. 2013, 42, 1007.

[118] M. Gingras, Chem. Soc. Rev. 2013, 42, 968.

[119] C.-F. Chen, Y. Shen, Helicene Chemistry. From Synthesis to Applications, Springer

Berlin Heidelberg, Berlin, Heidelberg, s.l., 2017.

[120] Y. Shen, C.-F. Chen, Chem. Rev. 2012, 112, 1463.

[121] K. Watanabe, K. Suda, K. Akagi, J. Mater. Chem. C 2013, 1, 2797. 


\section{References}

[122] M. Rickhaus, M. Mayor, M. Juríček, Chem. Soc. Rev. 2016, 45, 1542.

[123] A. Link, C. Sparr, Chem. Soc. Rev. 2018, 47, 3804.

[124] Siegel, Tobe, Shinkai (Eds.) Science of Synthesis. Product Class 21: Phenanthrenes, Helicenes, and Other Angular Acenes, Georg Thieme Verlag, Stuttgart, 2009.

[125] R. S. Cahn, C. Ingold, V. Prelog, Angew. Chem. Int. Ed. Engl. 1966, 5, 385.

[126] C. Goedicke, H. Stegemeyer, Tetrahedron Lett. 1970, 11, 937.

[127] J. P. Gao, V. Grand, S. MacKinnon, T. P. Bender, X. S. Meng, Z. Y. Wang, (None), Chem. Commun. 1999, 1281.

[128] R. H. Martin, M.-J. Marchant, Tetrahedron Lett. 1972, 13, 3707.

[129] R. H. Martin, M. J. Marchant, Tetrahedron 1974, 30, 343.

[130] H. Scherübl, U. Fritzsche, A. Mannschreck, Chem. Ber. 1984, 117, 336.

[131] P. Ravat, R. Hinkelmann, D. Steinebrunner, A. Prescimone, I. Bodoky, M. Juríček, Org. Lett. 2017, 19, 3707.

[132] M. S. Newman, D. Lednicer, J. Am. Chem. Soc. 1956, 78, 4765.

[133] R. H. Martin, V. Libert, J. Chem. Res. Miniprint 1980, 1940.

[134] H. Wynberg, M. B. Groen, J. Chem. Soc. D 1969, 964.

[135] J. Barroso, J. L. Cabellos, S. Pan, F. Murillo, X. Zarate, M. A. Fernandez-Herrera, G. Merino, Chem. Commun. 2018, 54, 188.

[136] M. Dračínský, J. Storch, V. Církva, I. Císařová, J. Sýkora, Phys. Chem. Chem. Phys. 2017, 19, 2900.

[137] J. M. Schulman, R. L. Disch, J. Phys. Chem. A 1999, 103, 6669.

[138] Y.-H. Tian, G. Park, M. Kertesz, Chem. Mater. 2008, 20, 3266.

[139] A. Bossi, L. Falciola, C. Graiff, S. Maiorana, C. Rigamonti, A. Tiripicchio, E. Licandro, P. R. Mussini, Electrochim. Acta 2009, 54, 5083.

[140] Y. Hu, X.-Y. Wang, P.-X. Peng, X.-C. Wang, X.-Y. Cao, X. Feng, K. Müllen, A. Narita, Angew. Chem. Int. Ed. 2017, 56, 3374.

[141] C.-Z. Wang, R. Kihara, X. Feng, P. Thuéry, C. Redshaw, T. Yamato, ChemistrySelect 2017, 2, 1436.

[142] H. Sakai, T. Kubota, J. Yuasa, Y. Araki, T. Sakanoue, T. Takenobu, T. Wada, T. Kawai, T. Hasobe, Org. Biomol. Chem. 2016, 14, 6738.

[143] J. Liu, J. Ma, K. Zhang, P. Ravat, P. Machata, S. Avdoshenko, F. Hennersdorf, H. Komber, W. Pisula, J. J. Weigand, A. A. Popov, R. Berger, K. Müllen, X. Feng, J. Am. Chem. Soc. 2017, 139, 7513.

[144] J. B. Birks, D.J.S. Birch, E. Cordemans, E. Vander Donckt, Chem. Phys. Lett. 1976, 43, 33.

[145] Y. Ooyama, Y. Shimada, Y. Kagawa, Y. Yamada, I. Imae, K. Komaguchi, Y. Harima, Tetrahedron Lett. 2007, 48, 9167. 
[146] H. Sakai, S. Shinto, Y. Araki, T. Wada, T. Sakanoue, T. Takenobu, T. Hasobe, Chem. Eur. J., 20, 10099.

[147] M. Li, Y. Niu, X. Zhu, Q. Peng, H.-Y. Lu, A. Xia, C.-F. Chen, Chem. Commun. 2014, 50, 2993.

[148] N. Saleh, C. Shen, J. Crassous, Chem. Sci. 2014, 5, 3680.

[149] P. Aillard, A. Voituriez, A. Marinetti, Dalton Trans. 2014, 43, 15263.

[150] M. Gicquel, Y. Zhang, P. Aillard, P. Retailleau, A. Voituriez, A. Marinetti, Angew. Chem. Int. Ed. 2015, 54, 5470.

[151] T. Yao, M. A. Campo, R. C. Larock, J. Org. Chem. 2005, 70, 3511.

[152] a) P. Aillard, D. Dova, V. Magné, P. Retailleau, S. Cauteruccio, E. Licandro, A. Voituriez, A. Marinetti, Chem. Commun. 2016, 52, 10984; b) P. Aillard, A. Voituriez, D.

Dova, S. Cauteruccio, E. Licandro, A. Marinetti, Chem. Eur. J. 2014, 20, 12373.

[153] K. Yavari, P. Aillard, Y. Zhang, F. Nuter, P. Retailleau, A. Voituriez, A. Marinetti, Angew. Chem. Int. Ed. 2014, 53, 861.

[154] A. U. Malik, F. Gan, C. Shen, N. Yu, R. Wang, J. Crassous, M. Shu, H. Qiu, J. Am. Chem. Soc. 2018, 140, 2769.

[155] a) L. Chen, P. S. Reiss, S. Y. Chong, D. Holden, K. E. Jelfs, T. Hasell, M. A. Little, A. Kewley, M. E. Briggs, A. Stephenson, K. M. Thomas, J. A. Armstrong, J. Bell, J. Busto,

R. Noel, J. Liu, D. M. Strachan, P. K. Thallapally, A. I. Cooper, Nat. Mater. 2014, 13, 954;

b) W. Xuan, M. Zhang, Y. Liu, Z. Chen, Y. Cui, J. Am. Chem. Soc. 2012, 134, 6904.

[156] N. D. Willmore, L. Liu, T. J. Katz, Angew. Chem. Int. Ed. Engl. 1992, 31, 1093.

[157] C. Nuckolls, T. J. Katz, L. Castellanos, J. Am. Chem. Soc. 1996, 118, 3767.

[158] C. Nuckolls, T. J. Katz, G. Katz, P. J. Collings, L. Castellanos, J. Am. Chem. Soc. 1999, 121, 79.

[159] A. J. Lovinger, C. Nuckolls, T. J. Katz, J. Am. Chem. Soc. 1998, 120, 264.

[160] C. Nuckolls, T. J. Katz, T. Verbiest, S. Van Elshocht, H.-G. Kuball, S. Kiesewalter, A. J. Lovinger, A. Persoons, J. Am. Chem. Soc. 1998, 120, 8656.

[161] T. Verbiest, S. Van Elshocht, M. Kauranen, L. Hellemens, J. Snauwaert, C. Nuckolls,

T. J. Katz, A. Persoons, Science 1998, 282, 913.

[162] R. Fasel, M. Parschau, K.-H. Ernst, Nature 2006, 439, 449.

[163] Y. Yang, R. C. da Costa, D.-M. Smilgies, A. J. Campbell, M. J. Fuchter, Adv. Mater. 2013, 25, 2624.

[164] a) Y. Geng, A. Trajkovska, S. W. Culligan, J. J. Ou, H. M. P. Chen, D. Katsis, S. H. Chen, J. Am. Chem. Soc. 2003, 125, 14032; b) Y. Geng, A. Trajkovska, D. Katsis, J. J. Ou, S. W. Culligan, S. H. Chen, J. Am. Chem. Soc. 2002, 124, 8337.

[165] a) Y. Yang, R. C. da Costa, M. J. Fuchter, A. J. Campbell, Nat. Photonics 2013, 7, 634 EP -; b) Y. Yang, B. Rice, X. Shi, J. R. Brandt, R. Correa da Costa, G. J. Hedley, D.- 


\section{References}

M. Smilgies, J. M. Frost, I. D. W. Samuel, A. Otero-de-la-Roza et al., ACS nano 2017, 11, 8329; c) Y. Zhou, T. Lei, L. Wang, J. Pei, Y. Cao, J. Wang, Adv. Mater. 2010, 22, 1484.

[166] a) J. Storch, J. Zadny, T. Strasak, M. Kubala, J. Sykora, M. Dusek, V. Cirkva, P. Matejka, M. Krbal, J. Vacek, Chem. Eur. J. 2015, 21, 2343; b) J. Vacek, J. V. Chocholoušová, I. G. Stará, I. Starý, Y. Dubi, Nanoscale 2015, 7, 8793.

[167] a) Y. Ooyama, Y. Harima, Eur. J. Org. Chem. 2009, 2009, 2903; b) Y. Ooyama, S. Inoue, R. Asada, G. Ito, K. Kushimoto, K. Komaguchi, I. Imae, Y. Harima, Eur. J. Org. Chem. 2010, 2010, 92; c) Y. Ooyama, G. Ito, K. Kushimoto, K. Komaguchi, I. Imae, Y. Harima, Org. Biomol. Chem. 2010, 8, 2756; d) Y. Ooyama, Y. Shimada, Y. Kagawa, I. Imae, Y. Harima, Org. Biomol. Chem. 2007, 5, 2046; e) P. Josse, L. Favereau, C. Shen, S. Dabos-Seignon, P. Blanchard, C. Cabanetos, J. Crassous, Chem. Eur. J. 2017, 23, 6277.

[168] V. Kiran, S. P. Mathew, S. R. Cohen, I. Hernández Delgado, J. Lacour, R. Naaman, Adv. Mater. 2016, 28, 1957.

[169] a) Z. Y. Wang, E. K. Todd, X. S. Meng, J. P. Gao, J. Am. Chem. Soc. 2005, 127, 11552; b) T. B. Norsten, A. Peters, R. McDonald, M. Wang, N. R. Branda, J. Am. Chem. Soc. 2001, 123, 7447; c) T. J. Wigglesworth, D. Sud, T. B. Norsten, V. S. Lekhi, N. R. Branda, J. Am. Chem. Soc. 2005, 127, 7272; d) T. Okuyama, Y. Tani, K. Miyake, Y. Yokoyama, J. Org. Chem. 2007, 72, 1634; e) Y. Tani, T. Ubukata, Y. Yokoyama, Y. Yokoyama, J. Org. Chem. 2007, 72, 1639; f) J.-i. Nishida, T. Suzuki, M. Ohkita, T. Tsuji, Angew. Chem. Int. Ed. 2001, 40, 3251; g) H. Higuchi, E. Ohta, H. Kawai, K. Fujiwara, T. Tsuji, T. Suzuki, J. Org. Chem. 2003, 68, 6605; h) J. N. Moorthy, P. Venkatakrishnan, S. Sengupta, M. Baidya, Org. Lett. 2006, 8, 4891; i) D. Schweinfurth, M. Zalibera, M. Kathan, C. Shen, M. Mazzolini, N. Trapp, J. Crassous, G. Gescheidt, F. Diederich, J. Am. Chem. Soc. 2014, 136, 13045; j) M. Srebro, E. Anger, B. Moore, N. Vanthuyne, C. Roussel, R. Réau, J. Autschbach, J. Crassous, Chem. Eur. J. 2015, 21, 17100.

[170] a) A. Babič, S. Pascal, R. Duwald, D. Moreau, J. Lacour, E. Allémann, Adv. Funct. Mater. 2017, 27, 1701839; b) M. Li, L.-H. Feng, H.-Y. Lu, S. Wang, C.-F. Chen, Adv. Funct. Mater. 2014, 24, 4405.

[171] M. S. Newman, W. B. Lutz, D. Lednicer, J. Am. Chem. Soc. 1955, 77, 3420.

[172] M. Scholz, M. Mühlstädt, F. Dietz, Tetrahedron Lett. 1967, 8, 665.

[173] M. Flammang-Barbieux, J. Nasielski, R. H. Martin, Tetrahedron Lett. 1967, 8, 743.

[174] C. Stammel, R. Fröhlich, C. Wolff, H. Wenck, A. d. Meijere, J. Mattay, Eur. J. Org. Chem., 1999, 1709.

[175] R. H. Martin, M. Flammang-Barbieux, J. P. Cosyn, M. Gelbcke, Tetrahedron Lett. 1968, 9, 3507. 
[176] R. H. Martin, G. Morren, J. J. Schurter, Tetrahedron Lett. 1969, 10, 3683.

[177] R. H. Martin, M. Baes, Tetrahedron 1975, 31, 2135.

[178] K. Mori, T. Murase, M. Fujita, Angew. Chem. Int. Ed. 2015, 54, 6847.

[179] R. H. Martin, C. Eyndels, N. Defay, Tetrahedron Lett. 1972, 13, 2731.

[180] a) L. Liu, T. J. Katz, Tetrahedron Lett. 1991, 32, 6831; b) L. Liu, B. Yang, T. J. Katz, M. K. Poindexter, J. Org. Chem. 1991, 56, 3769.

[181] A. Sudhakar, T. J. Katz, Tetrahedron Lett. 1986, 27, 2231.

[182] A. Sudhakar, T. J. Katz, J. Am. Chem. Soc. 1986, 108, 179.

[183] N. Hoffmann, Journal of Photochemistry and Photobiology C: Photochemistry

Reviews 2014, 19, 1.

[184] a) T. J. Katz, L. Liu, N. D. Willmore, J. M. Fox, A. L. Rheingold, S. Shi, C. Nuckolls, B. H. Rickman, J. Am. Chem. Soc. 1997, 119, 10054; b) L. Liu, T. J. Katz, Tetrahedron Lett. 1990, 31, 3983.

[185] M. C. Carreño, R. Hernández-Sánchez, J. Mahugo, A. Urbano, J. Org. Chem. 1999, $64,1387$.

[186] a) M. C. Carreno, S. Garcia-Cerrada, A. Urbano, Chem. Commun. 2002, 1412; b) M.

C. Carreño, S. García-Cerrada, A. Urbano, J. Am. Chem. Soc. 2001, 123, 7929; c) M. C.

Carreño, S. García-Cerrada, A. Urbano, Chem. Eur. J., 9, 4118.

[187] a) M. C. Carreno, S. Garcia-Cerrada, M. J. Sanz-Cuesta, A. Urbano, Chem.

Commun. 2001, 1452; b) M. C. Carreño, Á. Enríquez, S. García-Cerrada, M. J. Sanz-

Cuesta, A. Urbano, F. Maseras, A. Nonell-Canals, Chem. Eur. J. 2008, 14, 603.

[188] M. C. Carreno, M. Gonzalez-Lopez, A. Urbano, Chem. Commun. 2005, 611.

[189] a) A. A. Ruch, S. Handa, F. Kong, V. N. Nesterov, D. R. Pahls, T. R. Cundari, L. M.

Slaughter, Org. Biomol. Chem. 2016, 14, 8123; b) K. Kamikawa, I. Takemoto, S.

Takemoto, H. Matsuzaka, J. Org. Chem. 2007, 72, 7406.

[190] I. G. Stará, I. Starý, A. Kollárovič, F. Teplý, D. Šaman, M. Tichý, J. Org. Chem. 1998, 63, 4046.

[191] I. G. Stará, I. Starý, A. Kollárovič, F. Teplý, Š. Vyskočil, D. Šaman, Tetrahedron Lett. 1999, 40, 1993.

[192] F. Teplý, I. G. Stará, I. Starý, A. Kollárovič, D. Šaman, Š. Vyskočil, P. Fiedler, J. Org. Chem. 2003, 68, 5193.

[193] F. Teplý, I. G. Stará, I. Starý, A. Kollárovič, D. Šaman, L. Rulíšek, P. Fiedler, J. Am. Chem. Soc. 2002, 124, 9175.

[194] P. Sehnal, I. G. Stará, D. Saman, M. Tichy, J. Mísek, J. Cvacka, L. Rulísek, J. Chocholousová, J. Vacek, G. Goryl et al., Proc. Natl. Acad. Sci. USA. 2009, 106, 13169. 


\section{References}

[195] J. Nejedlý, M. Šámal, J. Rybáček, M. Tobrmanová, F. Szydlo, C. Coudret, M.

Neumeier, J. Vacek, J. Vacek Chocholoušová, M. Buděšínský et al., Angew. Chem. Int. Ed. 2017, 129, 5933.

[196] J. Klívar, A. Jančařík, D. Šaman, R. Pohl, P. Fiedler, L. Bednárová, I. Starý, I. G.

Stará, Chem. Eur. J. 2016, 22, 14401.

[197] S. Chercheja, J. Klívar, A. Jančařík, J. Rybáček, S. Salzl, J. Tarábek, L. Pospíšil, J.

Vacek Chocholoušová, J. Vacek, R. Pohl et al., Chem. Eur. J. 2014, 20, 8477.

[198] M. Šámal, S. Chercheja, J. Rybáček, J. Vacek Chocholoušová, J. Vacek, L.

Bednárová, D. Šaman, I. G. Stará, I. Starý, J. Am. Chem. Soc. 2015, 137, 8469.

[199] a) M. Karras, J. Holec, L. Bednarova, R. Pohl, B. Schmidt, I. G. Stará, I. Stary, J. Org.

Chem. 2018; b) I. Starý, I. G. Stará, Z. Alexandrová, P. Sehnal, F. Teplý, D. Šaman, L.

Rulíšek, Pure Appl. Chem. 2006, 78, 495.

[200] A. Jančařík, J. Rybáček, K. Cocq, J. Vacek Chocholoušová, J. Vacek, R. Pohl, L.

Bednárová, P. Fiedler, I. Císařová, I. G. Stará et al., Angew. Chem. Int. Ed. 2013, 52, 9970.

[201] I. G. Sanchez, M. Samal, J. Nejedly, M. Karras, J. Klivar, J. Rybacek, M. Budesinsky,

L. Bednarova, B. Seidlerova, I. G. Stara et al., Chem. Commun. 2017, 53, 4370.

[202] R. Yamano, Y. Shibata, K. Tanaka, Chem. Eur. J. 2018.

[203] J. Caeiro, D. Peña, A. Cobas, D. Pérez, E. Guitián, Adv. Synth. Catal. 2006, 348, 2466.

[204] a) S. K. Collins, A. Grandbois, M. P. Vachon, J. Côté, Angew. Chem. Int. Ed. 2006, 45, 2923; b) J. Côté, S. K. Collins, Synthesis 2009, 2009, 1499.

[205] A. Grandbois, S. K. Collins, Chem. Eur. J. 2008, 14, 9323.

[206] C. C. McAtee, P. S. Riehl, C. S. Schindler, J. Am. Chem. Soc. 2017, 139, 2960.

[207] a) J. Storch, J. Čermák, J. Karban, Tetrahedron Lett. 2007, 48, 6814; b) J. Storch, J. Sýkora, J. Cermák, J. Karban, I. Císarová, A. Růzicka, J. Org. Chem. 2009, 74, 3090; c) H. Oyama, M. Akiyama, K. Nakano, M. Naito, K. Nobusawa, K. Nozaki, Org. Lett. 2016, 18, 3654; d) K. Hirano, Y. Inaba, K. Takasu, S. Oishi, Y. Takemoto, N. Fujii, H. Ohno, J. Org. Chem. 2011, 76, 9068; e) J. Matsuoka, Y. Matsuda, Y. Kawada, S. Oishi, H. Ohno, Angew. Chem. Int. Ed. 2017, 56, 7444; f) M. B. Goldfinger, K. B. Crawford, T. M.

Swager, J. Am. Chem. Soc. 1997, 119, 4578.

[208] K. Nakamura, S. Furumi, M. Takeuchi, T. Shibuya, K. Tanaka, J. Am. Chem. Soc. 2014, 136, 5555.

[209] M. Tanaka, Y. Shibata, K. Nakamura, K. Teraoka, H. Uekusa, K. Nakazono, T.

Takata, K. Tanaka, Chem. Eur. J. 2016, 22, 9537.

[210] A. Kovács, A. Vasas, J. Hohmann, Phytochemistry 2008, 69, 1084.

[211] B. Tóth, J. Hohmann, A. Vasas, J. Nat. Prod. 2018, 81, 661. 
[212] M. Yamaki, L. Bai, K. Inoue, S. Takagi, Phytochemistry 1989, 28, 3503.

[213] T. Hattori, Y. Shimazumi, H. Goto, O. Yamabe, N. Morohashi, W. Kawai, S. Miyano, J. Org. Chem. 2003, 68, 2099.

[214] K. Natori, T. Iwayama, O. Yamabe, Y. Kitamoto, H. Ikeda, K. Sakamoto, T. Hattori, S. Miyano, Chirality 2015, 27, 479.

[215] M. Yang, Le Cai, Z. Tai, X. Zeng, Z. Ding, Fitoterapia 2010, 81, 992.

[216] M. Yamaki, C. Honda, Phytochemistry 1996, 43, 207.

[217] R. M. Perez Gutierrez, A. M. N. Gonzalez, E. Garcia Baez, S. Lugardo Diaz, Chem. Nat. Comp. 2010, 46, 554.

[218] a) H. W. Lam, Synthesis 2011, 13, 2011; b) M. T. Reetz, Angew. Chem. Int. Ed. 2008, 47, 2556; c) A. J. Minnaard, B. L. Feringa, L. Lefort, J. G. de Vries, Acc. Chem. Res. 2007, 40, 1267.

[219] E. González Fernández, Dissertation, Georg-August-Universität Göttingen, Göttingen, 2017.

[220] Lukas Schaaf, Master's dissertation, Georg-August-Universität Göttingen, Göttingen, 2016.

[221] D. Seebach, P. B. Rheiner, A. K. Beck, F. M. N. Kühnle, B. Jaun, Pol. J. Chem. 1994, 68, 2397.

[222] W. H. Laarhoven, W.H.M. Peters, A.H.A. Tinnemans, Tetrahedron 1978, 34, 769.

[223] N. Chernyak, V. Gevorgyan, J. Am. Chem. Soc. 2008, 130, 5636.

[224] J. Panteleev, K. Geyer, A. Aguilar-Aguilar, L. Wang, M. Lautens, Org. Lett. 2010, 12, 5092.

[225] C. Thirsk, G. E. Hawkes, R. T. Kroemer, K. R. Liedl, T. Loerting, R. Nasser, R. G. Pritchard, M. Steele, J. E. Warren, A. Whiting ${ }^{\star}$, J. Chem. Soc., Perkin Trans. 22002 , 1510.

[226] I. Pozo, A. Cobas, D. Peña, E. Guitián, D. Pérez, Chem. Commun. 2016, 52, 5534.

[227] T. E. Barder, S. D. Walker, J. R. Martinelli, S. L. Buchwald, J. Am. Chem. Soc. 2005, $127,4685$.

[228] A. G. Fix, P. E. Deal, C. L. Vonnegut, B. D. Rose, L. N. Zakharov, M. M. Haley, Org. Lett. 2013, 15, 1362.

[229] Z. U. Levi, T. D. Tilley, J. Am. Chem. Soc. 2009, 131, 2796.

[230] T. Fujikawa, Y. Segawa, K. Itami, J. Am. Chem. Soc. 2015, 137, 7763.

[231] For selected recent communications see: a) X. Gu, X. Xu, H. Li, Z. Liu, Q. Miao, J. Am. Chem. Soc. 2015, 137, 16203; b) T. Katayama, S. Nakatsuka, H. Hirai, N. Yasuda, J. Kumar, T. Kawai, T. Hatakeyama, J. Am. Chem. Soc. 2016, 138, 5210; c) D. Meng, H. Fu, C. Xiao, X. Meng, T. Winands, W. Ma, W. Wei, B. Fan, L. Huo, N. L. Doltsinis et al., J. Am. Chem. Soc. 2016, 138, 10184; d) X.-Y. Wang, X.-C. Wang, A. Narita, M. Wagner, 


\section{References}

X.-Y. Cao, X. Feng, K. Müllen, J. Am. Chem. Soc. 2016, 138, 12783; e) T. Hosokawa, Y.

Takahashi, T. Matsushima, S. Watanabe, S. Kikkawa, I. Azumaya, A. Tsurusaki, K.

Kamikawa, J. Am. Chem. Soc. 2017, 139, 18512; f) V. Berezhnaia, M. Roy, N.

Vanthuyne, M. Villa, J.-V. Naubron, J. Rodriguez, Y. Coquerel, M. Gingras, J. Am. Chem.

Soc. 2017, 139, 18508; g) Y. Zhu, Z. Xia, Z. Cai, Z. Yuan, N. Jiang, T. Li, Y. Wang, X.

Guo, Z. Li, S. Ma et al., J. Am. Chem. Soc. 2018, 140, 4222; h) M. Ferreira, G. Naulet, H. Gallardo, P. Dechambenoit, H. Bock, F. Durola, Angew. Chem. Int. Ed. 2017, 56, 3379; i)

K. Kato, Y. Segawa, L. T. Scott, K. Itami, Angew. Chem. Int. Ed. 2018, 57, 1337.

[232] M. Satoh, Y. Shibata, K. Tanaka, Chem. Eur. J. 2018.

[233] X. Gao, J. Han, L. Wang, Org. Lett. 2015, 17, 4596.

[234] H. Teller, Dissertation, Max-Planck-Institut für Kohlenforschung, Mülheim an der

Ruhr, 2013.

[235] F. Ramirez, N. B. Desai, B. Hansen, N. McKelvie, J. Am. Chem. Soc. 1961, 83, 3539.

[236] C. Zybill, G. Mueller, Organometallics 1987, 6, 2489.

[237] A. J. Arduengo, H. V. R. Dias, R. L. Harlow, M. Kline, J. Am. Chem. Soc. 1992, 114, 5530.

[238] N. Kuhn, T. Kratz, Synthesis 1993, 1993, 561.

[239] A. J. Arduengo, F. Davidson, H. V. R. Dias, J. R. Goerlich, D. Khasnis, W. J.

Marshall, T. K. Prakasha, J. Am. Chem. Soc. 1997, 119, 12742.

[240] K. Hirano, S. Urban, C. Wang, F. Glorius, Org. Lett. 2009, 11, 1019.

[241] E. Aldeco-Perez, A. J. Rosenthal, B. Donnadieu, P. Parameswaran, G. Frenking, G.

Bertrand, Science (New York, N.Y.) 2009, 326, 556.

[242] K. B. Simonsen, K. V. Gothelf, K. A. Jørgensen, J. Org. Chem. 1998, 63, 7536.

[243] J. Petuškova, Dissertation, Max-Planck-Institut für Kohlenforschung, Mülheim an der Ruhr, 2012.

[244] Maximilian Marx, Master's dissertation, Georg-August-Universität Göttingen, Göttingen, 2017.

[245] L. D. M. Nicholls, M. Marx, T. Hartung, E. González-Fernández, C. Golz, M. Alcarazo, ACS Catal. 2018, 8, 6079.

[246] J. Bouffard, B. K. Keitz, R. Tonner, V. Lavallo, G. Guisado-Barrios, G. Frenking, R. H. Grubbs, G. Bertrand, Organometallics 2011, 30, 2617.

[247] V. R. Yatham, W. Harnying, D. Kootz, J.-M. Neudörfl, N. E. Schlörer, A. Berkessel, J. Am. Chem. Soc. 2016, 138, 2670.

[248] D. Enders, K. Breuer, G. Raabe, J. Simonet, A. Ghanimi, H. B. Stegmann, J.H. Teles, Tetrahedron Lett. 1997, 38, 2833.

[249] C. Hansch, A. Leo, R. W. Taft, Chem. Rev. 1991, 91, 165.

[250] E. Soriano, J. Marco-Contelles, Organometallics 2006, 25, 4542. 
[251] a) I. V. Seregin, V. Gevorgyan, J. Am. Chem. Soc. 2006, 128, 12050; b) I. V. Seregin, A. W. Schammel, V. Gevorgyan, Tetrahedron 2008, 64, 6876; c) V. Lavallo, G. D. Frey, S. Kousar, B. Donnadieu, G. Bertrand, Proceedings of the National Academy of Sciences 2007, 104, 13569; d) W. Debrouwer, A. Fürstner, Chem. Eur. J. 2017, 23, 4271.

[252] J. H. Dopper, D. Oudman, H. Wynberg, J. Am. Chem. Soc. 1973, 95, 3692.

[253] T. Fujikawa, Y. Segawa, K. Itami, J. Am. Chem. Soc. 2016, 138, 3587.

[254] M. J. Frisch, G. W. Trucks, H. B. Schlegel, G. E. Scuseria, M. A. Robb, J. R. Cheeseman, G. Scalmani, V. Barone, G. A. Petersson, H. Nakatsuji, X. Li, M. Caricato, A. V. Marenich, J. Bloino, B. G. Janesko, R. Gomperts, B. Mennucci, H. P. Hratchian, J. V. Ortiz, A. F. Izmaylov, J. L. Sonnenberg, D. Williams-Young, F. Ding, F. Lipparini, F. Egidi, J. Goings, B. Peng, A. Petrone, T. Henderson, D. Ranasinghe, V. G. Zakrzewski, J. Gao, N. Rega, G. Zheng, W. Liang, M. Hada, M. Ehara, K. Toyota, R. Fukuda, J. Hasegawa, M. Ishida, T. Nakajima, Y. Honda, O. Kitao, H. Nakai, T. Vreven, K. Throssell, J. A. Montgomery, Jr., J. E. Peralta, F. Ogliaro, M. J. Bearpark, J. J. Heyd, E. N. Brothers, K. N. Kudin, V. N. Staroverov, T. A. Keith, R. Kobayashi, J. Normand, K. Raghavachari, A. P. Rendell, J. C. Burant, S. S. lyengar, J. Tomasi, M. Cossi, J. M. Millam, M. Klene, C. Adamo, R. Cammi, J. W. Ochterski, R. L. Martin, K. Morokuma, O. Farkas, J. B. Foresman, D. J. Fox, Gaussian, Inc., Rev. B.01, Wallingford, CT, 2016. [255] A. G. Barrado, A. Zieliński, R. Goddard, M. Alcarazo, Angew. Chem. Int. Ed. 2017, 56, 13401.

[256] G. Talavera, J. Peña, M. Alcarazo, J. Am. Chem. Soc. 2015, 137, 8704.

[257] A. García Barrado, Dissertation, Georg-August-Universität Göttingen, Göttingen, 2018.

[258] Y. Nakai, T. Mori, Y. Inoue, J. Phys. Chem. A 2012, 116, 7372.

[259] L. Bedrač, J. Iskra, Adv. Synth. Catal. 2013, 355, 1243.

[260] T. Okada, N. Fujiwara, T. Ogata, O. Haba, M. Ueda, J. Polym. Sci. A Polym. Chem. 1997, 35, 2259.

[261] S. Radix, R. Barret, Tetrahedron 2007, 63, 12379.

[262] A. Fürstner, M. M. Domostoj, B. Scheiper, J. Am. Chem. Soc. 2005, 127, 11620.

[263] W. D. Jones, F. L. Ciske, J. Org. Chem. 1996, 61, 3920.

[264] A. Cervi, P. Aillard, N. Hazeri, L. Petit, C. L. L. Chai, A. C. Willis, M. G. Banwell, J. Org. Chem. 2013, 78, 9876.

[265] A. Brennecke, Bachelor Thesis, Georg-August-Universität Göttingen, Göttingen, 2017.

[266] J. E. Milne, S. L. Buchwald, J. Am. Chem. Soc. 2004, 126, 13028.

[267] M. A. Selepe, S. E. Drewes, F. R. van Heerden, J. Nat. Prod. 2010, 73, 1680. 


\section{References}

[268] W. L. M. Amarego, C. L. L. Chai (Eds.) Purification of Laboratory Chemicals (Fifth Edition), Butterworth-Heinemann, Burlington, 2003.

[269] J. Pietruszka, A. Witt, Synthesis 2006, 2006, 4266.

[270] T. Furuyama, M. Yonehara, S. Arimoto, M. Kobayashi, Y. Matsumoto, M. Uchiyama, Chem. Eur. J. 2008, 14, 10348.

[271] L. Doszczak, P. Kraft, H.-P. Weber, R. Bertermann, A. Triller, H. Hatt, R. Tacke, Angew. Chem. Int. Ed. 2007, 46, 3367.

[272] E. González-Fernández, L. D. M. Nicholls, L. D. Schaaf, C. Farès, C. W. Lehmann, M. Alcarazo, J. Am. Chem. Soc. 2017, 139, 1428.

[273] S. Allmendinger, H. Kinuta, B. Breit, Adv. Synth. Catal. 2014, 357, 41.

[274] X. Bantreil, S. P. Nolan, Nat. Protoc. 2011, 6, 69.

[275] G. M. Sheldrick, Acta Crystallographica Section A 2008, 64, 112.

[276] O. V. Dolomanov, L. J. Bourhis, R. J. Gildea, J. A. K. Howard, H. Puschmann, Journal of Applied Crystallography 2009, 42, 339.

[277] T. Kottke, D. Stalke, Journal of Applied Crystallography 1993, 26, 615.

[278] G. R. Schaller, F. Topić, K. Rissanen, Y. Okamoto, J. Shen, R. Herges, Nat. Chem. 2014, 6, 608.

[279] H. Toledo, M. Amar, S. Bar, M. A. Iron, N. Fridman, B. Tumanskii, L. J. W. Shimon, M. Botoshansky, A. M. Szpilman, Org. Biomol. Chem. 2015, 13, 10726.

[280] C. A. James, V. Snieckus, J. Org. Chem. 2009, 74, 4080.

[281] Y. Zhao, Z. Li, C. Yang, R. Lin, W. Xia, Beilstein J. Org. Chem. 2014, 10, 622.

[282] J. Buter, D. Heijnen, C. Vila, V. Hornillos, E. Otten, M. Giannerini, A. J. Minnaard, B.

L. Feringa, Angew. Chem. Int. Ed. 2016, 55, 3620.

[283] T. Akama, S. J. Baker, Y.-K. Zhang, V. Hernandez, H. Zhou, V. Sanders, Y. Freund, R. Kimura, K. R. Maples, J. J. Plattner, Bioorg. Med. Chem. Lett. 2009, 19, 2129.

[284] A. F. Ku, G. D. Cuny, J. Org. Chem. 2016, 81, 10062.

[285] M.-H. Kim, Y.-L. Choi, J.-N. Heo, Y.-K. Min, S.-H. Kim, B. Korean Chem. Soc. 2010, $31,2047$. 
9. References 


\section{Curriculum Vitae. Leo D. M. Nicholls}

Nationality: British

Address: Stegemühlenweg 28, Göttingen, 37083, DE

Telephone: (+49) 15759710265

E-mail: Inichol1@gwdg.de

Languages: English (native), German (advanced), French (elementary)

\section{Research Experience}

PhD in chemistry

Georg-August-Universität Göttingen, Göttingen, DE: Prof. Manuel Alcarazo

2016-2018

Max-Planck-Institut für Kohlenforschung, Mülheim an der Ruhr, DE: Prof. Alois

2014-2015

Fürstner, Prof. Manuel Alcarazo

Medicinal Chemistry Internship

$2013-2014$

Hoffmann-La Roche AG, Basel, CH: Dr. Mark Rogers-Evans

Master's project

$2012-2013$

The University of Edinburgh, Edinburgh, UK: Dr. Uwe Schneider

Research internship

2011-2012

Nanyang Technological University, Singapore: Prof. Francois Mathey

\section{Education}

PhD in Chemistry (Dr. rer. nat.), Max-Planck-Institut für Kohlenforschung and

2014-present Georg-August-Universität Göttingen, Göttingen, DE.

MChem with year abroad (Singapore), $1^{\text {st }}$ class honours, The University of

$2009-2013$ Edinburgh, Edinburgh, UK.

\section{Publications and patents}

- L. D. M. Nicholls, T. Hartung, M. Marx, E. González-Fernández, C. Golz, M. Alcarazo, TADDOLderived Cationic Phosphonites: Towards an Effective Enantioselective Synthesis of [6]Helicenes via Au-catalyzed Alkyne Hydroarylation, ACS Catal. 2018, 8, 6079-6085.

- E. González-Fernández, ${ }^{\S}$ L. D. M. Nicholls, ${ }^{\S}$ L. D. Schaaf, C. Farés, C. C. Lehmann, M. Alcarazo, Enantioselective Synthesis of [6]carbohelicenes, J. Am. Chem. Soc. 2017, 139, 1428. ${ }^{\S}$ Equal author contribution. (Highlighted in: Synfacts , 2017, 13, 505).

- S. M. Ametamy, J. Fingerle, L. Gobbi, U. Grether, A: Haider, T. Hartung, L. Mu, L. Nicholls, M. Rogers-Evans, C. Ullmer, Radiolabeled Cannabinoid Receptor 2 Ligand, WO2016066534, 2016.

- F. Mathey, M. P. Duffy, L. Yu Ting, L. Nicholls, Y. Li, R. Ganguly, Reactions of Terminal Phosphinidenes with Dihydrogen, Organometallics 2012, 31, 2936.

\section{Conferences and Public Presentations}

- Poster presenter, Jungchemikerforum Frühjahrssymposium, Constance, Mar. 2018.

- Short talk and poster presenter: Blue Danube Symposium on Heterocyclic Chemistry, Linz, Austria, Aug. 2017: awarded GDCh travel grant.

- Oral presentation: Göttingen Weihnachtskolloquium, Dec. 2016: represented the organic chemistry faculty.

- Three additional poster presentations at local conferences (Göttingen, Mülheim an der Ruhr). 